\title{
Robust UV-Curable Antimicrobial Polymeric Coatings for Medical Plastics Prepared by Controlled Radical Polymerization
}

\author{
by \\ Rachel L. Shum \\ Honours Bachelor of Science \\ McMaster University, Hamilton, Ontario, Canada 2017 \\ A thesis presented to Ryerson University \\ in partial fulfillment of the \\ requirements for the degree of \\ Master of Science \\ in the program of Molecular Science
}

Toronto, Ontario, Canada 2019

(C) Rachel L. Shum, 2019 


\section{AUTHOR'S DECLARATION}

I hereby declare that I am the sole author of this thesis. This is a true copy of the thesis including any required final revisions, as accepted by my examiners.

I authorize Ryerson University to lend this thesis to other institutions or individuals for the purpose of scholarly research.

I further authorize Ryerson University to reproduce this thesis by photocopying or by other means, in total or in part, at the request of other institutions or individuals for the purpose of scholarly research.

I understand that my thesis may be made electronically available to the public. 


\title{
Robust UV-Curable Antimicrobial Polymeric Coatings for Medical Plastics Prepared by Controlled Radical Polymerization
}

\author{
Rachel L. Shum \\ Master of Science - Molecular Science, Ryerson University 2019
}

\begin{abstract}
In response to hospital acquired infections stemming from biofilms and the impending antibiotic resistance crisis, the development of non-traditional, non-leachable antimicrobials have gained significant traction. Contact-active antimicrobial coatings physically attached to surfaces with cationic active sites, such as ammonium and phosphonium, are of particular interest in the prevention of pathogenic bacterial transfer. Previously reported antimicrobial coatings are found to be susceptible to abrasion, significantly limiting their potential applications. In this work, a range of robust, antimicrobial polymeric coatings synthesized by control radical polymerization are presented. Polymeric thin film coatings possessing cationic groups with $n$-alkyl substituents of $n \leq 4$ demonstrated antimicrobial properties against gram-positive bacteria, while species containing bulkier substituents were biologically inactive, contradictory of previously reported monomeric coatings. Cationic polymeric brush coatings were found to have a higher antibacterial activity against the gram-positive model compared to its non-brush equivalent, but failed against the gram-negative model. These polymeric thin films demonstrate the complexity of antimicrobial coating designs and facilitates the investigation into the architecture of these coatings.
\end{abstract}




\title{
ACKNOWLEDGEMENTS
}

First and foremost, I would like to express my greatest appreciation to my advisor, Professor Daniel Foucher, for providing a wonderful research opportunity. His continuous guidance, support, and patience helped me achieve success throughout my M.Sc. thesis. I cannot imagine having a better supervisor and mentor.

I would like to convey acknowledgments to Dr. Gideon Wolfaardt for usage of biological research space and Dr. Alan Lough for X-ray crystallography data. I would also like to thank my thesis committee members, Dr. Andrew McWilliams and Dr. Gideon Wolfaardt, for their research suggestions and direction.

My experience and understanding of chemistry have benefited immensely from interactions with my labmates and coworkers. With me, they shared their ambition, knowledge, and support. Over these short years, this lab has become my family.

\author{
Joseph Bedard \\ Desiree Bender \\ Alex Caschera \\ Gloria D'Amaral \\ Siobhan Liu \\ Julie Loungxay \\ Kathy May \\ Jeff Pau \\ Lukasz Porosa \\ Thershan Satkunarajah
}




\section{DEDICATION}

For my parents, Benny and Mandy Shum,

my brother Roscoe,

and my best friend, Domenic. 


\section{TABLE OF CONTENTS}

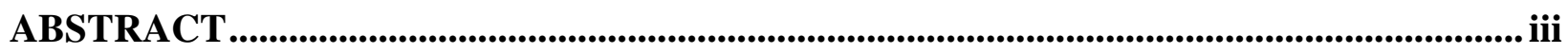

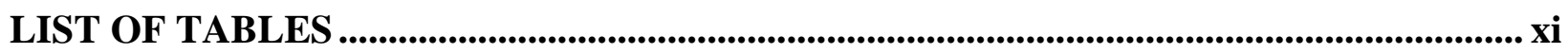

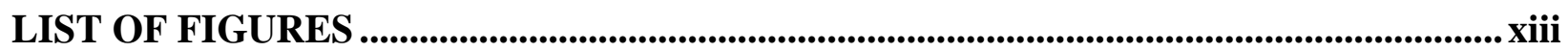

LIST OF SCHEMES .........................................................................................................

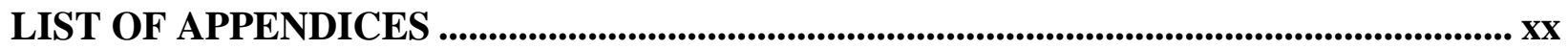

LIST OF ABBREVIATIONS ..................................................................................................... $\mathrm{xxV}$

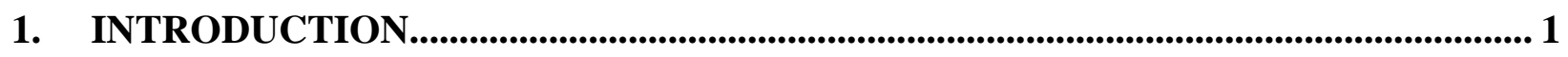

1.1. Quaternary Ammonium Containing Polymers............................................................... 1

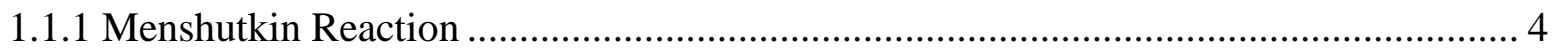

1.1.2 Mechanism of Action of Unbound QACs and PolyQACs ........................................... 5

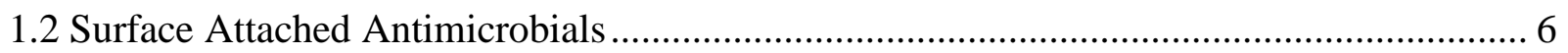

1.3 Methods to Grafting on Surfaces: "Grafting from" and "Grafting to" ................................ 9

1.4 Controlled/Living Radical Polymerization ................................................................ 10

1.4.1 Nitroxide Mediated Polymerization............................................................................. 11

1.4.2 Atom-Transfer Radical Polymerization ....................................................................... 12

1.4.3 Reversible Addition-Fragmentation Chain-Transfer Polymerization........................... 13

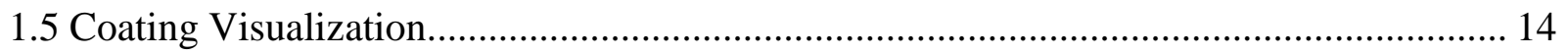

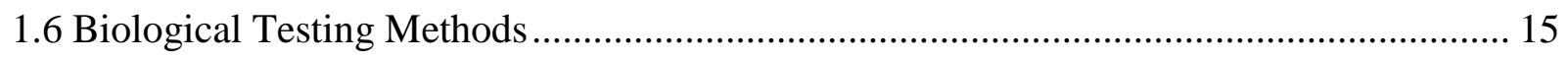

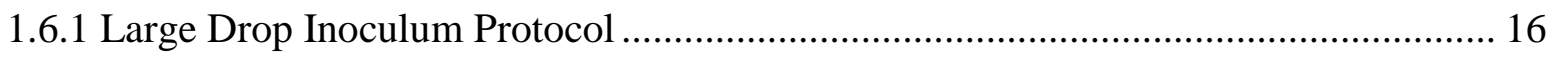

1.6.2 Dynamic Shake Flask Method ............................................................................... 16

1.7 Mechanism of Action of Immobilized Antimicrobials ...................................................... 16

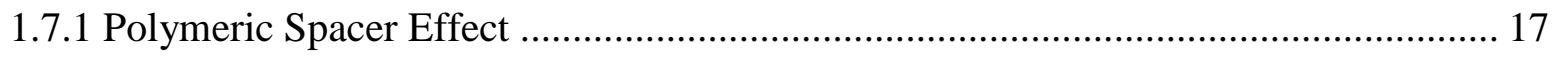

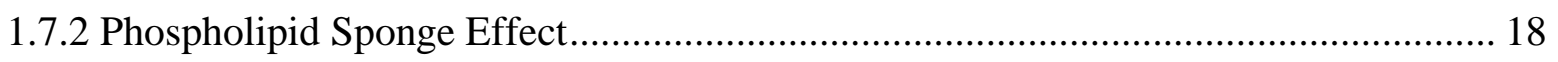

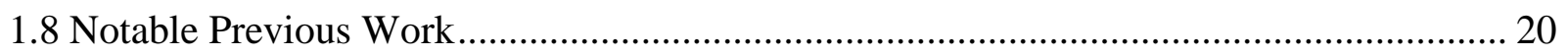


1.9 Research Objective

2. RESULTS AND DISCUSSION ................................................................................. 26

2.1 Quaternary Ammonium and Phosphonium Random Block Copolymer Coatings ............ 26

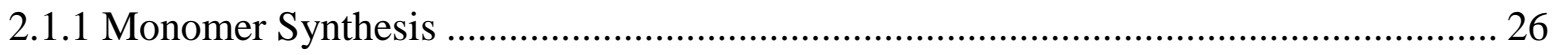

2.1.2 Controlled Radical Polymerization of VBBP and VBC Monomers......................... 28

2.1.4 Post-polymerization Quaternization of Poly(VBC-BP) Random Copolymer ............ 36

2.15 Preparation of Quaternary Polymer Coatings ................................................... 43

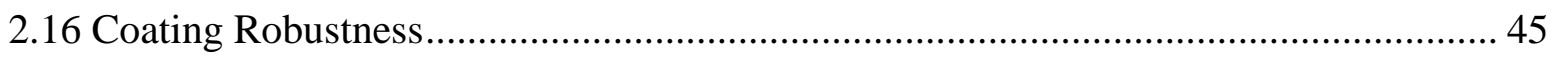

2.17 Characterization of Polymeric Coatings ........................................................... 47

2.18 Biological Testing of Coated Surfaces ................................................................. 50

2.2 Low Quaternary Ammonium Loading Random Block Copolymer ................................ 60

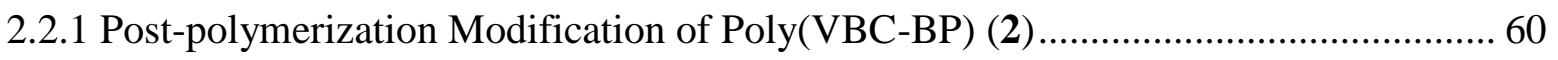

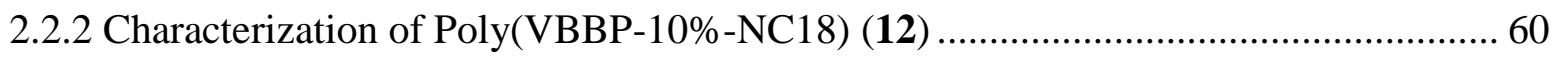

2.2.3 Coating Formulation of Poly(VBBP-10\%-NC18) (12) ...................................... 61

2.2.4 Physical and Biological Properties Poly(VBBP-10\%-NC18) (12).......................... 62

2.3 Quaternary Phosphonium Diblock Copolymer Brush Coatings .................................... 64

2.3.1 RAFT Polymerization for Synthesis of Poly(VBBP-b-VBC) (14)........................... 65

2.3.2 Post-polymerization Quaternization of Poly(VBBP-b-VBC) (14) and Poly(VBC-BP)

2.3.3 Characterization of Poly(VBBP-b-P $\left.\mathrm{P}^{\mathrm{n}} \mathrm{Bu}_{3}\right)(\mathbf{1 5})$ and Poly $\left(\mathrm{VBBP}-\mathrm{r}-\mathrm{P}^{\mathrm{n}} \mathrm{Bu}_{3}\right)(\mathbf{1 6}) \ldots \ldots . . . .69$

2.3.4 Coating Preparation of Poly(VBBP-b-P $\left.\mathrm{Pu}_{3}\right)(\mathbf{1 5})$ and Poly(VBBP-r-P $\left.\mathrm{Pu}_{3}\right)(\mathbf{1 6}) \ldots .70$

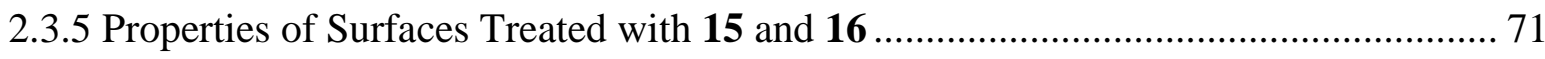

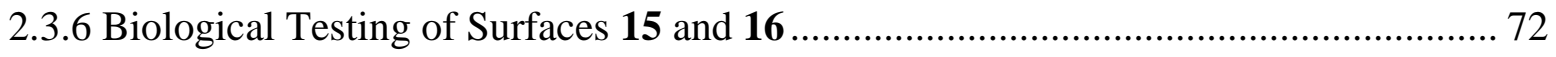

2.4 Quaternary Ammonium/Phosphonium Monomers and Polymerizations ........................ 74 
2.4.1 Synthesis of Quaternary Ammonium and Phosphonium Monomers

2.4.2 NMP of Quaternary Ammonium/Phosphonium Monomers .................................... 83

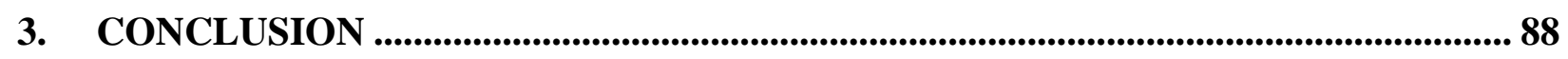

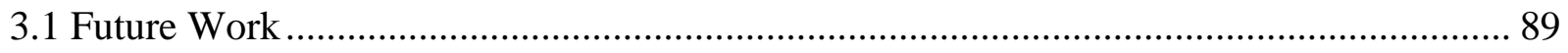

4. EXPERIMENTAL METHODS ..................................................................................... 93

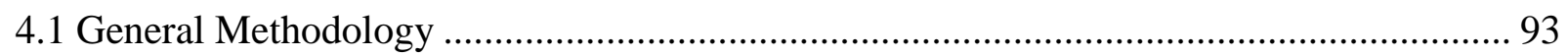

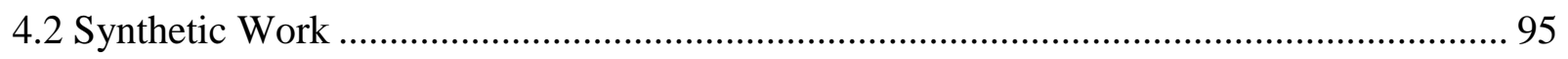

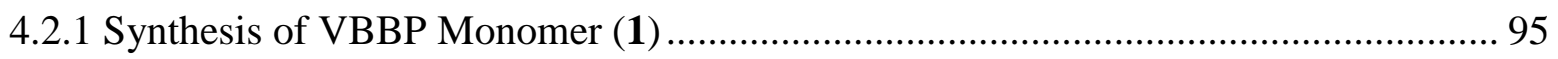

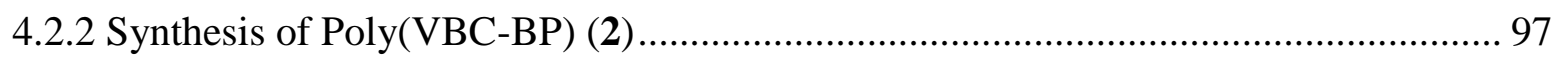

4.2.3 General Synthesis of Poly(VBC) by RAFT Polymerization (3-5) .......................... 98

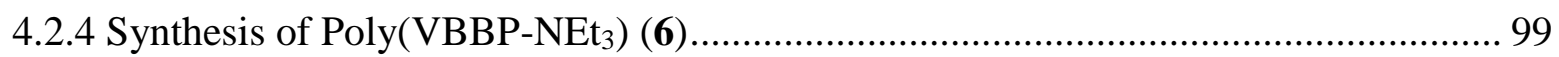

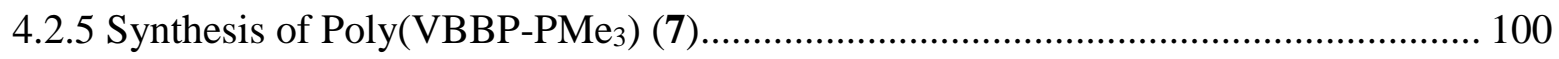

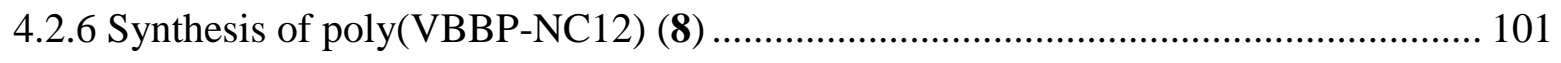

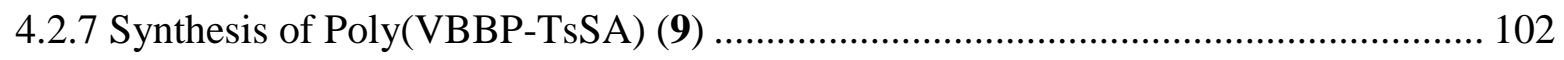

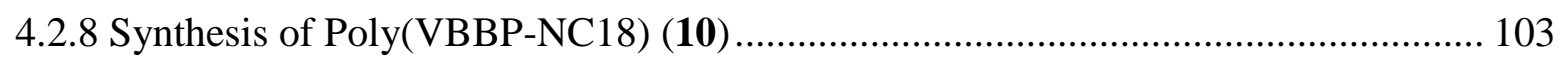

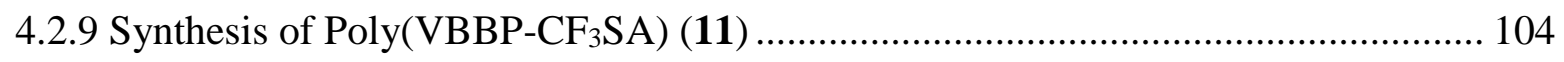

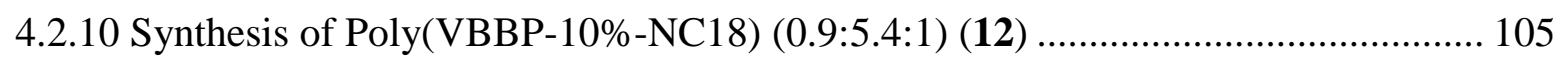

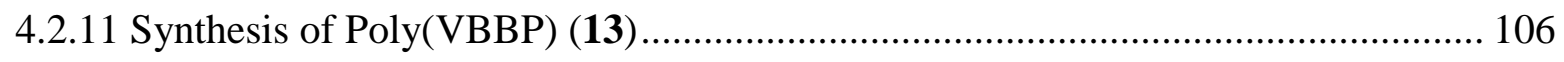

4.2.12 Synthesis of Diblock Copolymer Poly(VBBP-b-VBC) $(2: 31)(\mathbf{1 4})$....................... 107

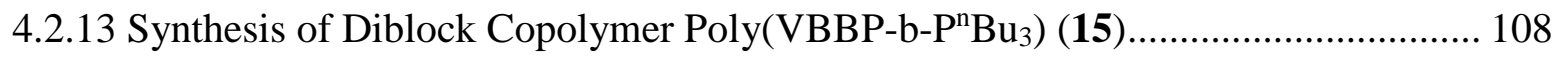

4.2.14 Synthesis of Random Block Copolymer Poly(VBBP-r-P $\left.\mathrm{Bu}_{3}\right)(\mathbf{1 6}) \ldots \ldots \ldots \ldots \ldots \ldots . . . . . . . . .109$

4.2.15 Synthesis of 4-Vinylbenzyl Triethylammonium Chloride (17) ........................... 110

4.2.16 Synthesis of 4-Vinylbenzyl Tributylphosphonium Chloride (18) ......................... 111

4.2.17 Synthesis of of 4-Vinylbenzyl Trimethylphosphonium Chloride (19) .................. 112

4.2.18 Synthesis of 4-Vinylbenzyl Diethylsulfonium Chloride (20) .............................. 113 
4.2.19 Synthesis of 4-Vinylbenzyl Dimethyloctadecylammonium Chloride (21) 114

4.2.20 Synthesis of 4-Vinylbenzyl Diethylamine (22) ........................................... 115

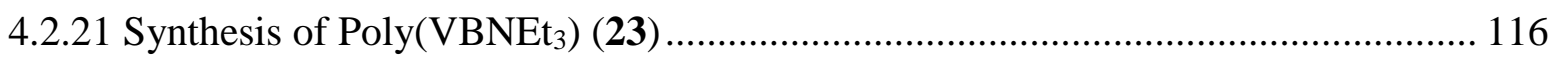

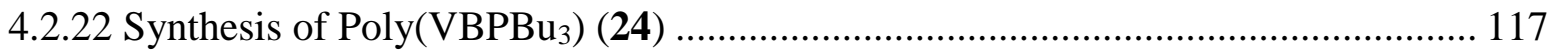

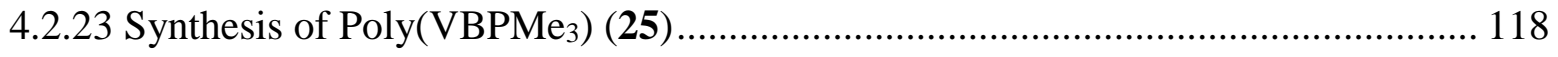

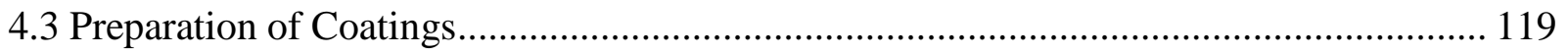

4.4 Antibacterial Testing of Coated Surfaces ....................................................... 120

4.41 Large Drop Inoculum Antimicrobial Testing of Coated Surfaces.......................... 120

4.42 Dynamic shake flask testing method of coated surfaces ................................... 121

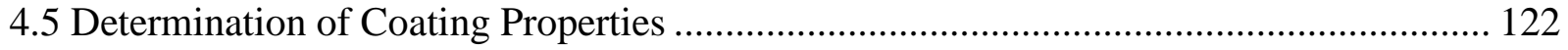

4.51 Cationic Charge Density Determination ...................................................... 122

4.52 Advancing Water Contact Angle Measurements.............................................. 123

4.53 Coating Preparation for AFM imaging ........................................................ 123

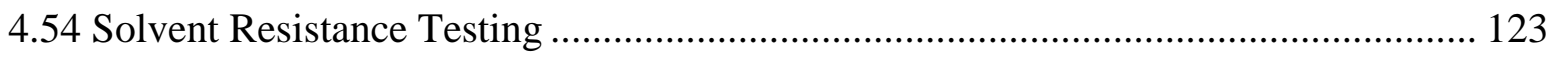

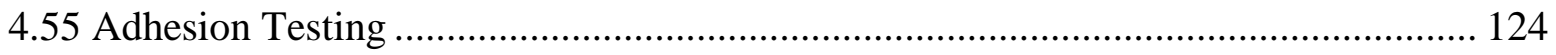

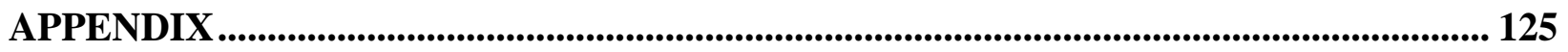

Appendix A: NMR Spectra and Mass Spectra .......................................................... 125

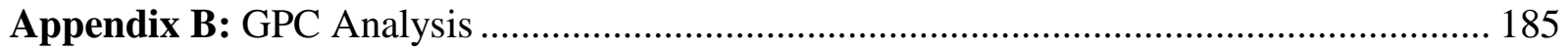

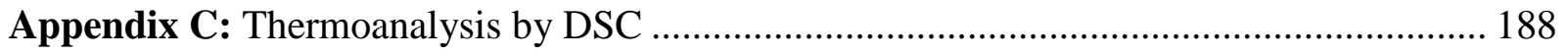

Appendix D: Composition Estimation by 1H NMR and Elemental Analysis.................... 192

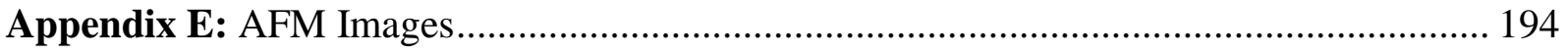

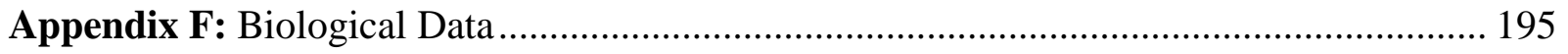

Appendix G: X-Ray Crystallography Data _............................................................. 199 


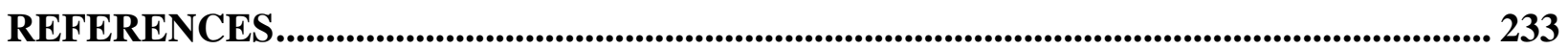




\section{LIST OF TABLES}

Table 2.1 Summary of NMP conditions in the synthesis of poly(VBC-BP) (2) ........................ 28

Table 2.2 Summary of estimated monomer loading in the poly(VBC-BP) random copolymer by

${ }^{1} \mathrm{H}$ NMR 32

Table 2.3 Elemental analysis results of poly(VBC-BP) random copolymer in estimation of benzophenone loading 32

Table 2.4 Summary of RAFT polymerization conditions initiated by BPO and resultant conversion, dispersity, and molecular weight of $\mathbf{3}$ 34

Table 2.5 RAFT polymerization conditions of VBC initiated by BPO with RAFT agent CTA2 and subsequent molecular weight and dispersity of $\mathbf{4}$

Table 2.6 RAFT polymerization conditions of VBC initiated by BPO with RAFT agent CTA1 and CTA2 for $5 \mathrm{~h}$ and resulting polymer weight, dispersity, and conversion of $\mathbf{5}$. 36

Table 2.7 Summary of estimated functionality composition of the quaternized random copolymers 6-11. 38

Table 2.8 Thermal events observed from DSC analysis ..... 42

Table 2.9 Charge density and advancing contact angle of PS substrate coated with 6-11 48

Table 2.10 RMS Roughness and coating thicknesses of uncoated control, single coated 6-9, and small molecule references on PS 49

Table 2.11 Advancing contact angle of $\mathbf{2}$ and $\mathbf{1 2}$ double coated on PP 62

Table 2.12 Polymer properties before and after continued bulk copolymerization 67

Table 2.13 Advancing water contact angle and cationic charge density of PS substrates coated with $\mathrm{P}^{n} \mathrm{Bu}_{3}$ species 15 and $\mathbf{1 6}$. 72

Table 2.14 Reaction and work-up conditions and subsequent percent yield to synthesize (17).. 77 
Table 2.15 Reaction conditions for the synthesis of monomer 19...................................... 80

Table 3.1 Advancing water contact angle of PS substrates coated with $\mathbf{1 5}$ by spin-coating and

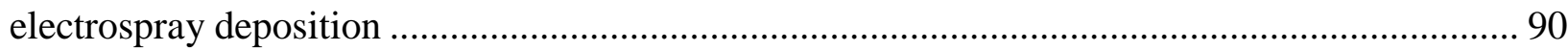




\section{LIST OF FIGURES}

Figure 1.1 QACs (a) small molecule disinfectant benzalkonium chloride (b) poly(QAC)

PAMBM blended with PLGA for suture coating

Figure 1.2 Synthetic AMP mimetics (a) PMMA based mimics with cationic and hydrophobic substituents $^{21}$ (b) Equivalent side-chain and main-chain QAC polymers ${ }^{22}$ (c) polynorbornene based AMP mimics with cationic pendants ${ }^{25}$

Figure 1.3 Gram-positive and -negative bacterial cell walls and key negative components (Adapted from ref. ${ }^{1}$ ).

Figure 1.4 The two categories of antimicrobial coatings: repelling and killing coatings (adapted from ref. $\left.^{27}\right)$

Figure 1.5 Surface-attached QACs grafted by (a) organosilane functionality on porous surfaces ${ }^{35}$

(b) phosphinate functionality on metal surfaces ${ }^{36}$ 8

Figure 1.6 Photo-initiated radical crosslinking via $\mathrm{C}-\mathrm{H}$ insertion mechanism from aryl ketone of the benzophenone to form carbon-carbon bond with the substrate surface. 9

Figure 1.7 Surface grafting methods (a) "grafting from" and (b) "grafting to", 10

Figure 1.8 Stable nitroxide radicals for versatile NMP 12

Figure 1.9 Classes of RAFT agents 13

Figure 1.10 (a) RAFT agent 4-Cyano-4-(phenylcarbonothioylthio)pentanoic acid seen as a bright pink powder (b) resulting 4-vinylbenzyl chloride homopolymer prepared by RAFT polymerization that appears pink 14

Figure 1.11 Polymeric spacer effect (Adapted from ref. ${ }^{27}$ ) ……………………………......... 18

Figure 1.12 Phospholipid sponge effect (Adapted from ref. ${ }^{27}$ )................................................. 18 
Figure 1.13 Illustration the phospholipid sponge effect on different cross-linking densities.

(Adapted from ref. ${ }^{69}$ )

Figure 1.14 Previously reported QAC coatings surface attached and cross-linked with benzophenone (a) QAC polymer brushes synthesized by surface initiated ATRP using a "grafting from" approach ${ }^{74}$ (b) QAC polymer brushes synthesized by UV initiation of the surface, PEEK, which closely resembles benzophenone using a "grafting from" approach" ${ }^{75}$ (c) C12 and C18 QAC small molecule coating synthesized by “grafting to" technique ${ }^{53,76}$ (d) Quaternized PEI coating "grafted to" surface ${ }^{77}$ 22

Figure 1.15 Mesityl-, tosyl-, $\mathrm{CF}_{3}$-benzylsulfonamide small molecule surface attached coatings prepared by UV cure recently reported by the Foucher group ${ }^{79}$........ 23

Figure 1.16 Notable quaternary phosphonium surfaces that has previously been reported to demonstrate antibacterial properties (a) Styrenic based quaternary phosphonium polymer brushes synthesized by surface initiated uncontrolled free radical polymerization in a "grafting from" approach (b) A film consisting of phosphonium polymer networks prepared by UV-initiated crosslinking of the polyphosphonium and diacrylate. 24

Figure 2.1 Stacked ${ }^{1} \mathrm{H}$ NMR spectra of $\mathbf{1}$ and corresponding VBC starting material $\left(\mathrm{CDCl}_{3}\right) \ldots 27$ Figure 2.2 Stacked ${ }^{1} \mathrm{H}$ NMR spectra of poly(VBC-BP) 2 against VBC and VBBP (1) monomers

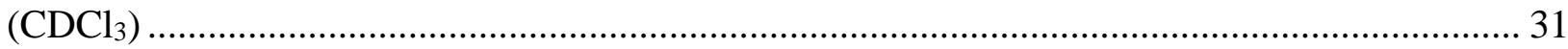

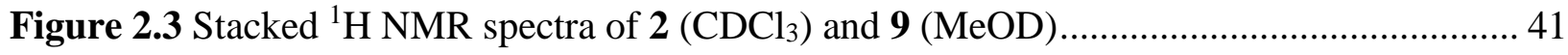

Figure 2.4 Small molecule 4-vinylbenzyl triethylammonium chloride ................................. 42

Figure 2.5 Thermal events observed from DSC trace (a) $T_{g}$ events of polymer 6 and 9 (b) $T_{m}$ events of polymer $\mathbf{6}$ and $\mathbf{8}$ 
Figure 2.6 PS substrates coated with polymers 6-11 before and after BPB staining compared to the uncoated control 44

Figure 2.7 C18 BP-QAC small molecule coating on PS after subject to solvent resistance testing followed by BPB staining. Treated surfaces were double rubbed once with a cotton fabric soaked with $\mathrm{dH}_{2} \mathrm{O}$, IPA, $\mathrm{MeOH}$, and Windex ${ }^{\circledR}$ with a pressure of $\sim 10 \mathrm{~N}$ 45

Figure 2.8 Polymeric coatings of 6 on PS after undergoing solvent resistance testing with $\mathrm{dH}_{2} \mathrm{O}$, IPA, $\mathrm{MeOH}$, and Windex ${ }^{\circledR}$ followed by BPB staining. 46

Figure 2.9 Results of the tape test for adhesion evaluation of double coating 6 as a representative sample 47

Figure 2.10 AFM images of uncoated PS control (left), PS coated with poly(VBBP-NEt 3 ) (6) (middle) and poly(VBBP-TsSA) (9) (right). The treated surfaces are seen to be smoother than the uncoated PS surface.

Figure 2.11 Graphs depicting average bacterial cell survival on coatings 6-11 against; (a)

Arthrobacter sp. and (b) E. coli wt36. The surfaces were inoculated with a $100 \mu \mathrm{L}$ droplet of $10^{7}$ CFU bacterial suspension for $3 \mathrm{~h}$ until desiccation. Surfaces of $\mathbf{6}$ and $\mathbf{7}$ demonstrated antibacterial activity only against the gram-positive model as seen by the lack of cell survival in comparison to the control surface. The E. coli on coatings 6-11 experienced survived over the testing period, indicating an inactivity against gram-negative bacteria. (t-test, ${ }^{*} p<0.05$, ns $p>$ $0.95)$

Figure 2.12 Graph depicting coating 9 tested against Arthrobacter sp. at the $3 \mathrm{~h}$ and $24 \mathrm{~h}$ time point. Coatings were found to significantly reduce the bacterial load. (t-test, $\left.*_{p}<0.05\right)$...... 52 Figure 2.13 Illustration depicting (a) cationic charge burial, hindering cell wall interaction with cationic active sites and (b) cationic active sites self-enriched at the surface. 54 
Figure 2.14 Illustration of a coating of high cationic loading depicting hydrophobic chain aggregation preventing charge availability at the surface 57

Figure 2.15 Graph depicting average bacterial cell survival of coating 8 against Arthrobacter sp. Film coatings were grounded to a powder and inoculated with $100 \mu \mathrm{L}$ of $10^{5} \mathrm{CFU}$ bacterial suspension, shaken for 1 h. Coating $\mathbf{8}$ demonstrated antibacterial activity only against the grampositive model as seen by the lack of cell survival of the treated sample in comparison to the control. (t-test, $* p<0.05)$ 59

Figure 2.16 PP substrates coated with 2 and 12 before and after BPB staining..... 62

Figure 2.17 Graph depicting average bacterial cell survival over $3 \mathrm{~h}$ period of control and treated surfaces of coatings 2 and 12 on PP against $10^{7}$ CFU loading of Arthrobacter sp. There was no significant cell reduction observed for both coatings. (t-test, $* p<0.05$, ns $p>0.95$ ) 63

Figure 2.18 Illustration of cationic copolymer brush coatings 64

Figure 2.19 Stacked ${ }^{1} \mathrm{H}$ NMR spectra of VBBP monomer 1 , homopolymer $\mathbf{1 3}$, and diblock copolymer $14\left(\mathrm{CDCl}_{3}\right)$

Figure 2.20 $\mathrm{P}^{n} \mathrm{Bu}_{3}$ species 15 and 16 coated and BPB stained PS substrates . 71

Figure 2.21 Graphs depicting bacterial cell survival when coatings of $\mathrm{P}^{n} \mathrm{Bu}_{3}$ species were tested against (a) Arthrobacter sp. and (b) E. coli wt36. Surfaces were inoculated with $100 \mu \mathrm{L}$ droplet of $10^{7} \mathrm{CFU}$ bacterial suspension for $3 \mathrm{~h}$ until desiccation. Brushes of $\mathbf{1 5}$ reduced all grampositive bacteria on the surface while 16 significantly reduced bacteria. Both $\mathrm{P}^{n} \mathrm{Bu}_{3}$ species failed

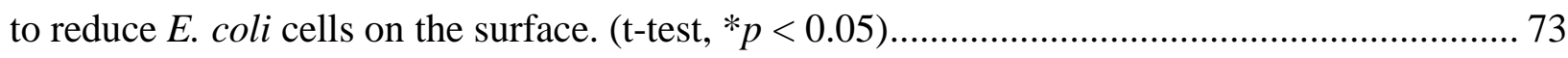
Figure 2.22 Micelle formation from A-B diblock copolymers in a polar solution where the Ablock is the hydrophobic benzophenone repeat units (grey) and the B-block is the hydrophilic cationic species (red). 75 
Figure 2.23 Left: $\mathrm{P}^{n} \mathrm{Bu}_{3}$ diblock copolymer 15 in $10 \mathrm{w} / \mathrm{v} \%$ EtOH solution presented as a light blue tinge, indicting micelle formation. Right: $\mathrm{P}^{n} \mathrm{Bu}_{3}$ random block copolymer 16 in $10 \mathrm{w} / \mathrm{v} \%$

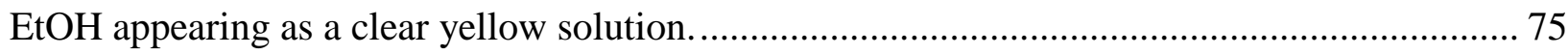

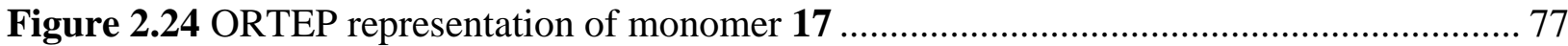

Figure 2.25 ORTEP representation of tributylphosphonium monomer (18) ......................... 79

Figure 2.26 Stacked ${ }^{31} \mathrm{P}$ NMR spectra of monomer 18 and polymer $\mathbf{2 4}\left(\mathrm{D}_{2} \mathrm{O}\right) \ldots \ldots \ldots \ldots \ldots \ldots \ldots . . . . . . . . . . . . .55$

Figure 3.1 PS substrate coated by electrospray deposition of diblock copolymer $\mathbf{1 5}$ and stained

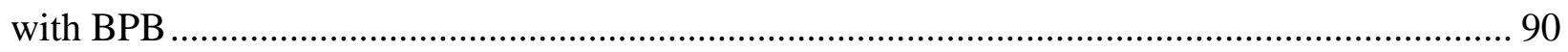

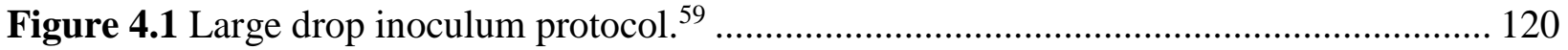

Figure 4.2 Dynamic shake flask method............................................................. 121 


\section{LIST OF SCHEMES}

Scheme 1.1 Menshutkin reaction ................................................................................. 5

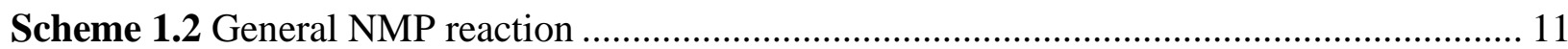

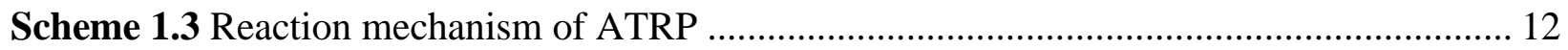

Scheme 1.4 Reaction mechanism of RAFT polymerization .................................................... 13

Scheme 1.5 Coating visualization by BPB staining. ................................................................ 15

Scheme 2.1 The Williamson Ether reaction between VBC and 4-hydroxybenzophenone to

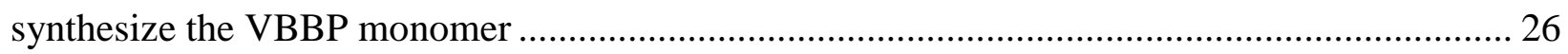

Scheme 2.2 Nitroxide mediated copolymerization of VBBC and VBC initiated by BPO and

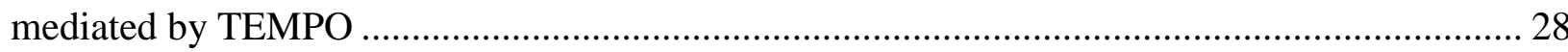

Scheme 2.3 Attempted partial substitution of acrylic acid on VBC homopolymer by Williamson

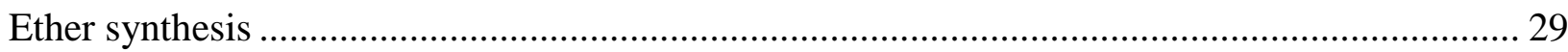

Scheme 2.4 RAFT polymerization initiated by BPO and mediated by CTA1 ............................. 34

Scheme 2.5 RAFT polymerization of VBC, initiated by BPO with RAFT agent CTA2 ………. 35

Scheme 2.6 RAFT polymerization of VBC initiated by BPO at $5 \mathrm{~h}$ time point .......................... 35

Scheme 2.7 Amination and phosphination reactions of $\mathbf{2}$ to synthesize quaternary ammonium

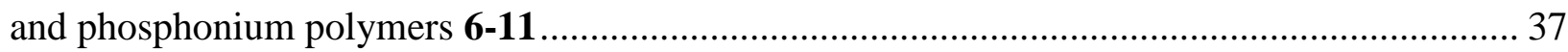

Scheme 2.8 Post-polymerization modification for synthesis of poly(VBBP-10\%-NC18) (12) .. 60 Scheme 2.9 RAFT polymerization for the synthesis of low molecular weight poly(VBBP)

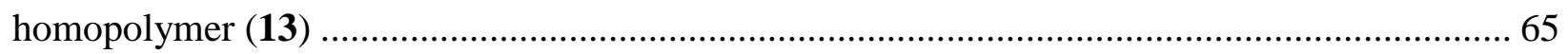

Scheme 2.10 Continued bulk RAFT copolymerization of poly(VBBP) (13) with VBC in the

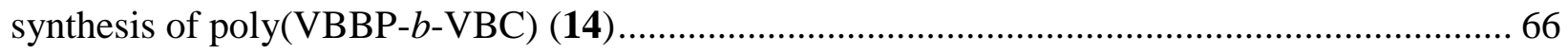


Scheme 2.11 Partial quaternization of (a) 14 to synthesize poly(VBBP- $b$ - $\left.\mathrm{P}^{n} \mathrm{Bu}_{3}\right)$ (15) and (b) 2 to

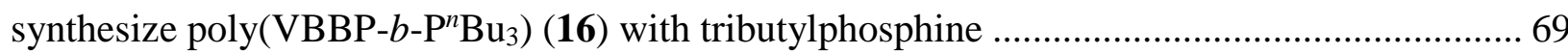

Scheme 2.12 Quaternization reaction of triethylamine with VBC for synthesis of $17 \ldots \ldots . . . . . . .76$

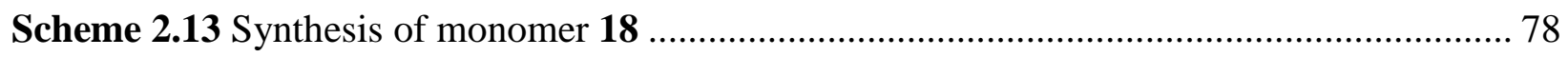

Scheme 2.14 Reaction scheme for synthesis of monomer 19 ......................................... 80

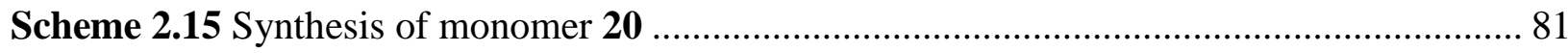

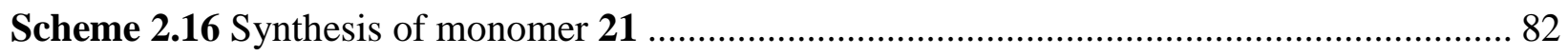

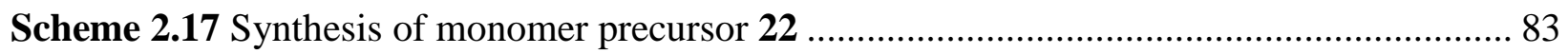

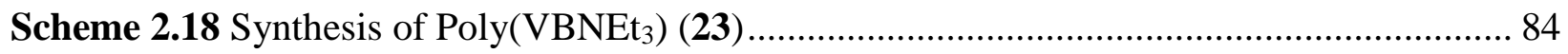

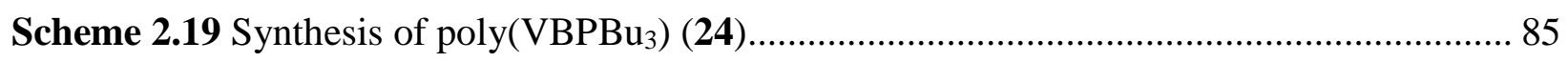

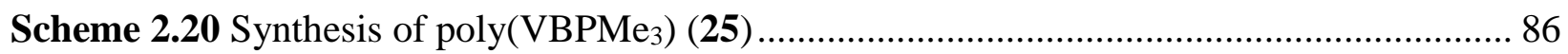

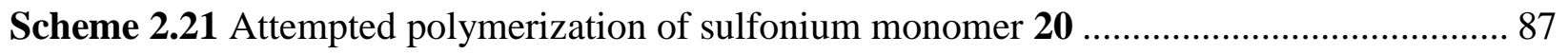

Scheme 3.1 Quaternization reaction for synthesis of benzophenone quaternary ammonium

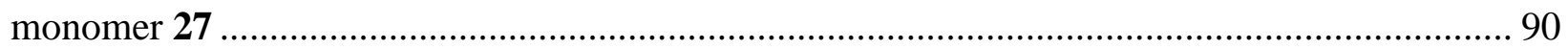

Scheme 3.2 RAFT polymerization of 21 for synthesis of homopolymer 26......................... 91

Scheme 3.3 Continued RAFT copolymerization to incorporate benzophenone monomer into

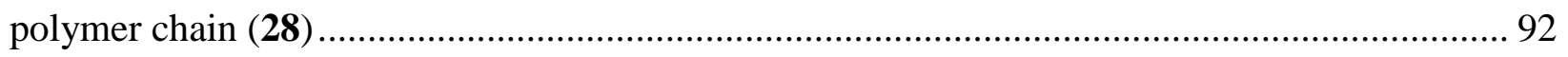




\section{LIST OF APPENDICES}

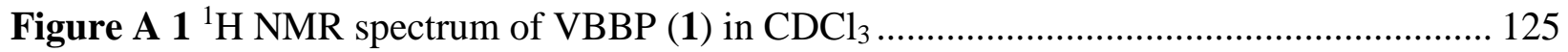

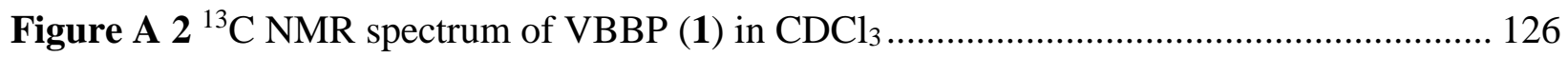

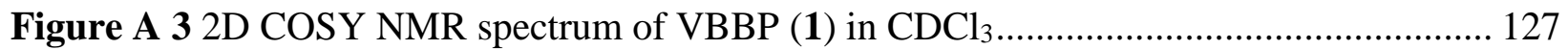

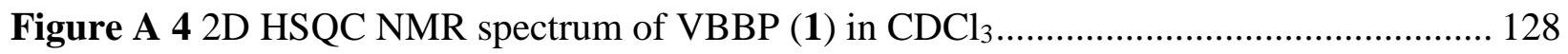

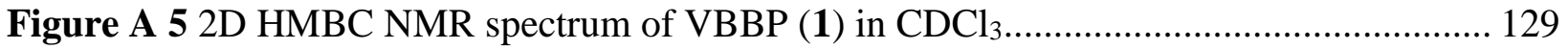

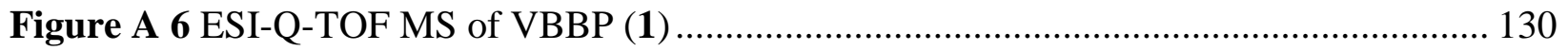

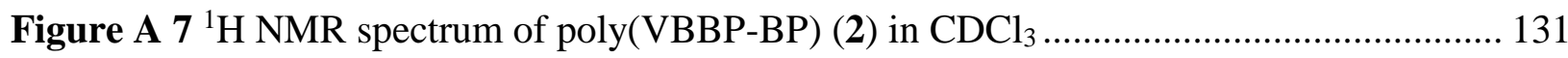

Figure A 8 2D COSY NMR spectrum of poly(VBC-BP) (2) in $\mathrm{CDCl}_{3} \ldots \ldots \ldots \ldots \ldots \ldots \ldots \ldots \ldots \ldots \ldots . . . . . . . . . . . . . . . . .132$

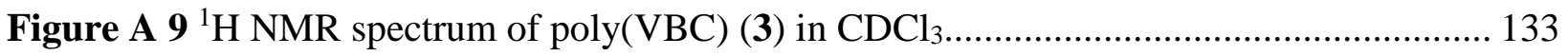

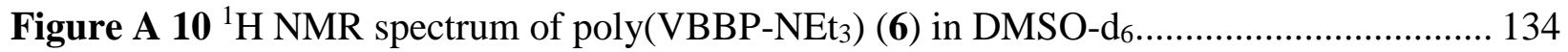

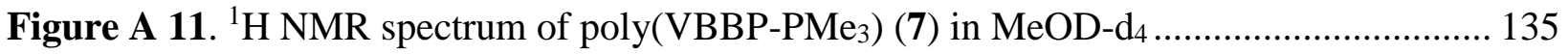

Figure A 12. ${ }^{31} \mathrm{P}$ NMR spectrum of poly(VBBP-PMe 3 ) (7) in MeOD-d $\mathrm{d}_{4} \ldots \ldots \ldots \ldots \ldots \ldots \ldots \ldots \ldots \ldots . . . . . . . . . . . . . . . .136$

Figure A 13. ${ }^{1} \mathrm{H}$ NMR spectrum of poly(VBBP-NC12) (8) in MeOD-d $\mathrm{d}_{4} \ldots \ldots \ldots \ldots \ldots \ldots \ldots \ldots \ldots \ldots \ldots . . . . . . . . . . . . . . .137$

Figure A 14. 2D COSY NMR spectrum of poly(VBBP-NC12) (8) in MeOD-d …................. 138

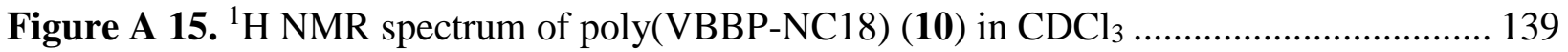

Figure A 16. ${ }^{19} \mathrm{~F}$ NMR spectrum of poly $\left(\mathrm{VBBP}-\mathrm{CF}_{3} \mathrm{SA}\right)(\mathbf{1 1})$ in MeOD-d $\mathrm{d}_{4} \ldots \ldots \ldots \ldots \ldots \ldots \ldots \ldots \ldots . . . . . . . . . . . . .140$

Figure A 17. ${ }^{1} \mathrm{H}$ NMR spectrum of poly(VBBP-10\%-NC18) (12) in $\mathrm{CDCl}_{3} \ldots \ldots \ldots \ldots \ldots \ldots \ldots \ldots \ldots . . . . . . . . . . . .141$

Figure A 18. 2D COSY NMR spectrum of poly(VBBP-10\%-NC18) (12) in $\mathrm{CDCl}_{3} \ldots \ldots \ldots \ldots . . . . .142$

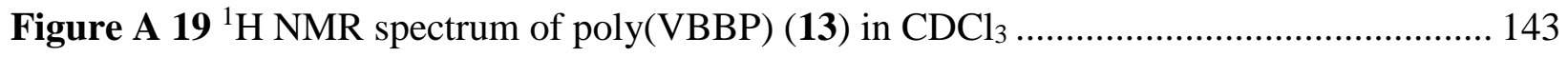

Figure A $20{ }^{1} \mathrm{H}$ NMR spectrum of diblock copolymer poly(VBBP- $b$-VBC) (14) in $\mathrm{CDCl}_{3} \ldots . .144$

Figure A $21{ }^{1} \mathrm{H}$ NMR spectrum of diblock copolymer poly(VBBP- $\left.b-\mathrm{P}^{n} \mathrm{Bu}_{3}\right)(\mathbf{1 5})$ in $\mathrm{CDCl}_{3} \ldots 145$

Figure A $22{ }^{31} \mathrm{P}$ NMR spectrum of poly(VBBP- $\left.b-\mathrm{P}^{n} \mathrm{Bu}_{3}\right)(\mathbf{1 5})$ in $\mathrm{CDCl}_{3} \ldots \ldots \ldots \ldots \ldots \ldots \ldots \ldots \ldots \ldots . . . . . . . . . . . . . . .146$ 


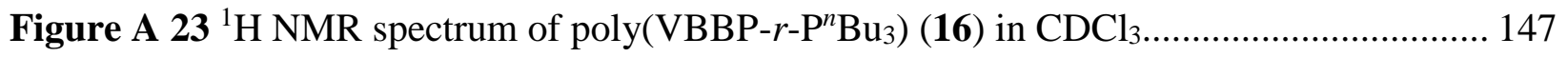

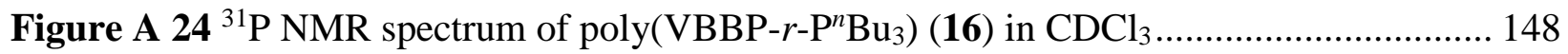

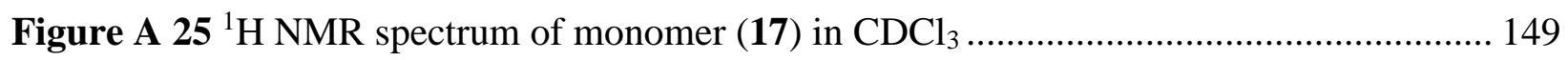

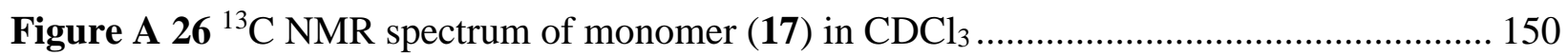

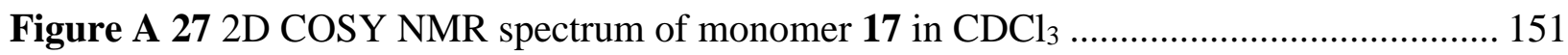

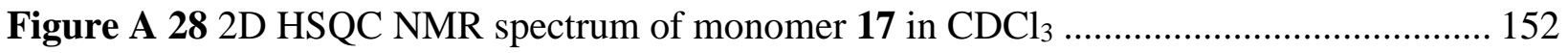

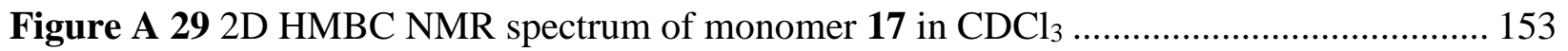

Figure A 30 HRMS ESI-Q-TOF spectrum of monomer 17................................................. 154

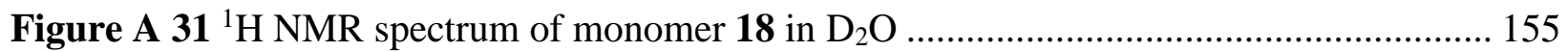

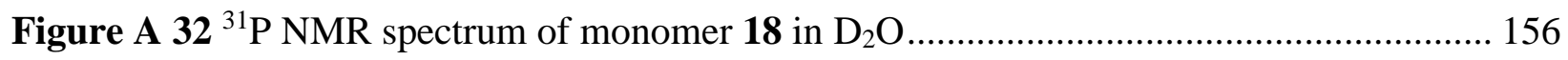

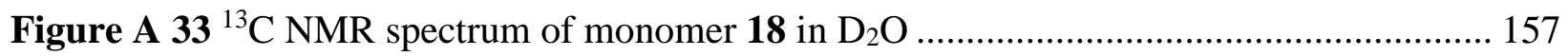

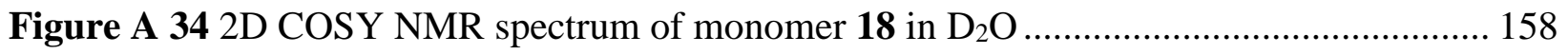

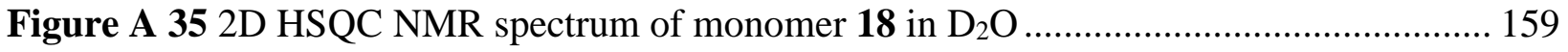

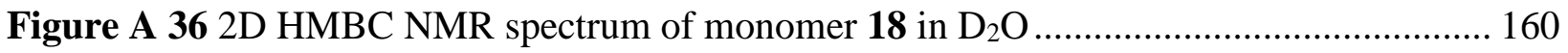

Figure A 37 HRMS ESI-Q-TOF spectrum of monomer 18.................................................. 161

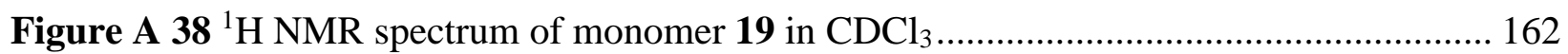

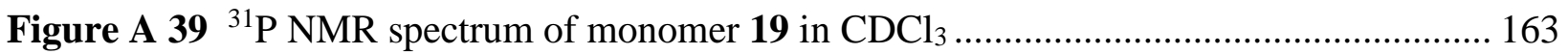

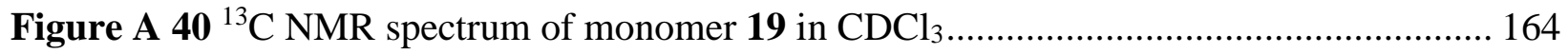

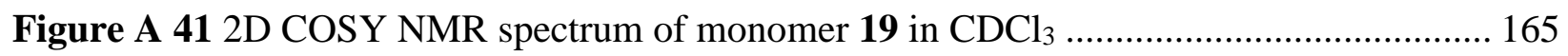

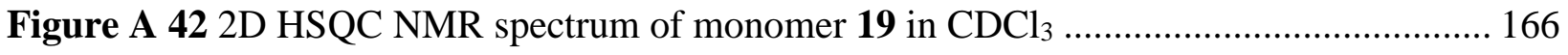

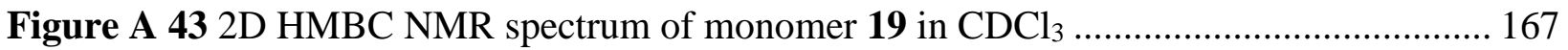

Figure A 44 HRMS ESI-Q-TOF spectrum of monomer 19................................................. 168

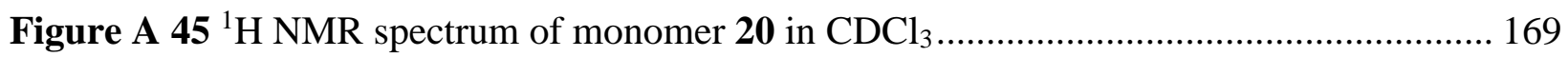




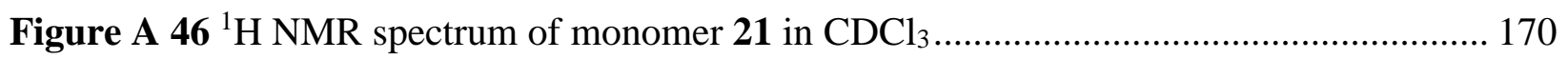

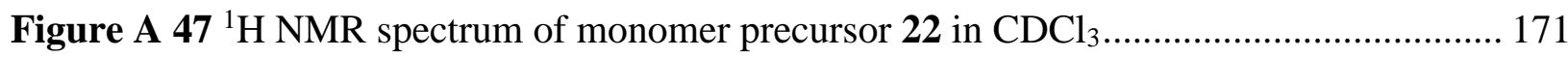

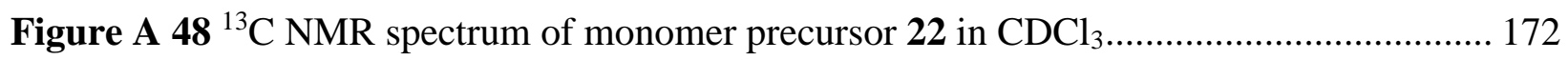

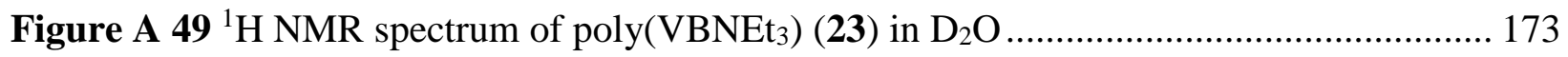

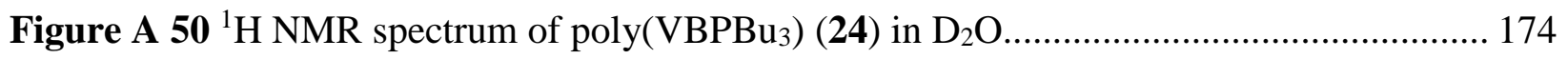

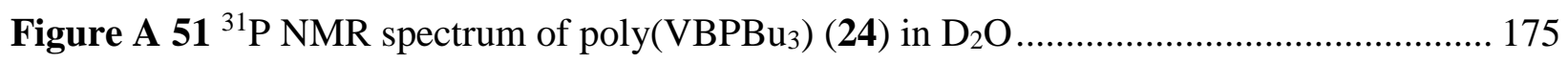

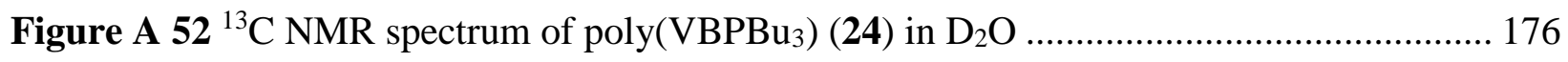

Figure A 53 2D HSQC NMR spectrum of poly(VBPBu$)(\mathbf{2 4})$ in $\mathrm{D}_{2} \mathrm{O} \ldots \ldots \ldots \ldots \ldots \ldots \ldots \ldots \ldots \ldots \ldots \ldots . . . . . . . . . . . . . . .177$

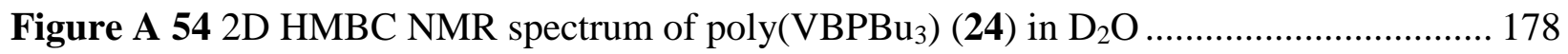

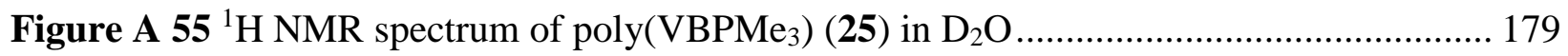

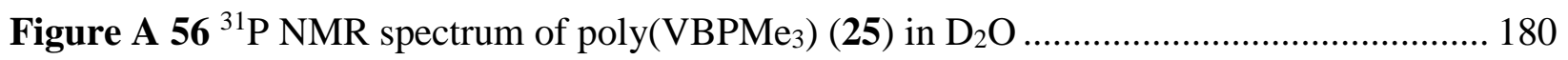

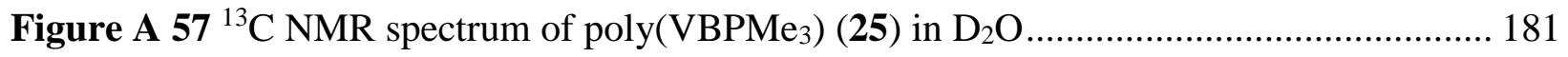

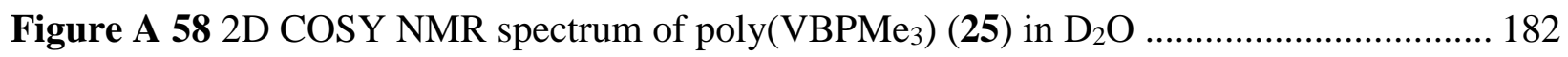

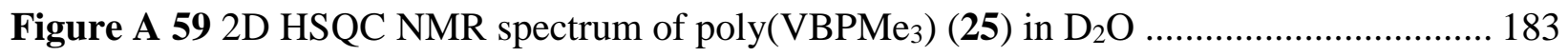

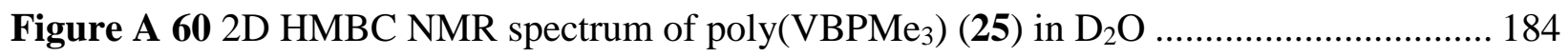

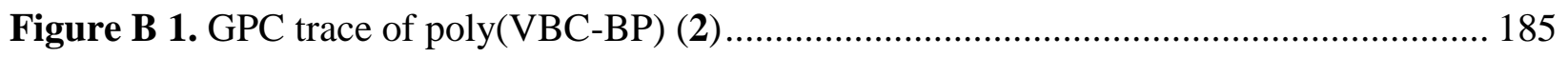

Figure B 2. GPC trace of polymers 3 synthesized by RAFT polymerization with varying reaction conditions using RAFT agent CTA1.................................................................... 185

Figure B 3 GPC trace of polymers 4 synthesized by RAFT polymerization with varying reaction

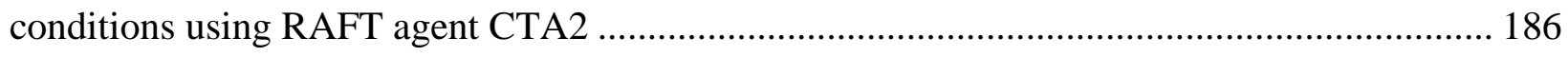

Figure B 4 GPC trace of polymers 5 synthesized by RAFT polymerization mediated by CTA1

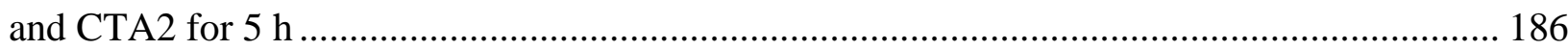


Figure B 5 GPC trace of starting homopolymer $\mathbf{1 3}$ and diblock copolymer after copolymerization $(\mathbf{1 4})$ 187

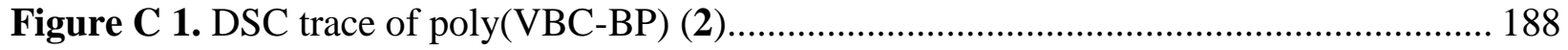

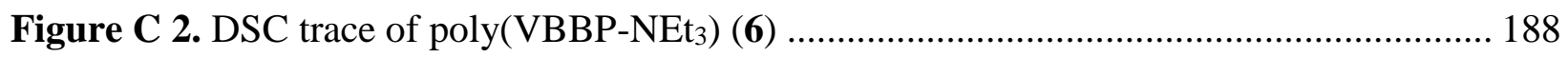

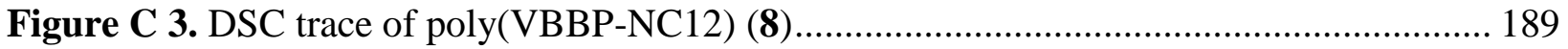

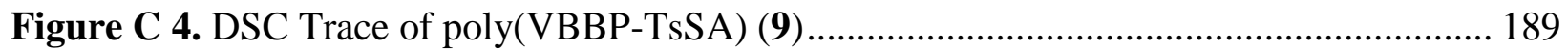

Figure C 5 DSC trace of poly(VBBP) (13) homopolymer .............................................. 190

Figure C 6 DSC trace of diblock copolymer poly(VBBC-b-VBC) (14) ............................... 190

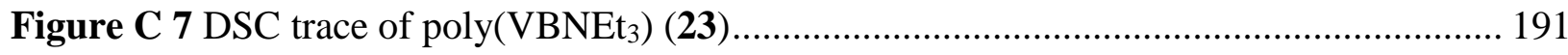

Table D 1 Data obtained from proton integration analysis for composition estimates of polymers

6-11 192

Table D 2 Elemental analysis data of C, H, and N for estimation of polymer composition (6-11)

Table D 3 Elemental analysis data of $\mathrm{C}$ and $\mathrm{H}$ for estimation for composition estimations of polymers 15 and $\mathbf{1 6}$ 193

Table D 4 Proton integration data for composition estimates of polymer $\mathbf{1 6}$ 193

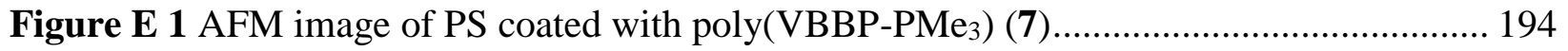

Figure E 2 AFM image of PS coated with poly(VBBP-NC12) (8) ..................................... 194 
Table F 1 Colony-forming unit (CFU) from antibacterial testing by LDI method against Arthrobacter sp. Control and treated surfaces (coatings $\mathbf{2 , 6 - 1 2 , 1 5 , 1 6 )}$ was inoculated with 10 $\mu \mathrm{L}$ droplet of $10^{7} \mathrm{CFU}$ in sterile tap water for $3 \mathrm{~h}$. 195

Table F 2 Colony-forming unit (CFU) from antibacterial testing by LDI method against E. coli

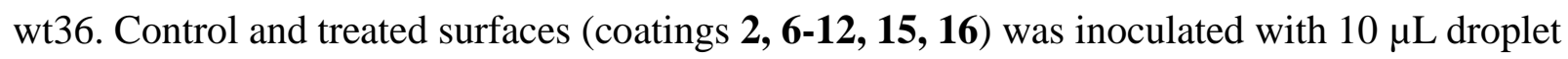
of $10^{7} \mathrm{CFU}$ in sterile tap water for $3 \mathrm{~h}$. 197

Table F 3 Colony-forming unit (CFU) from antibacterial testing by DSF method against Arthrobacter sp. Control and treated film (coating 8) was inoculated with $10 \mu \mathrm{L}$ solution of $10^{5}$

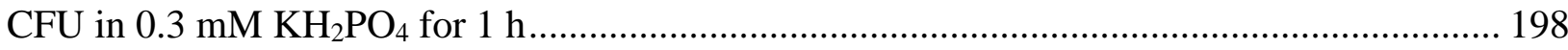

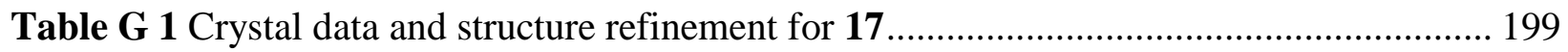

Table G 2 Atomic coordinated and equivalent isotropic displacement parameters for 17 ....... 200

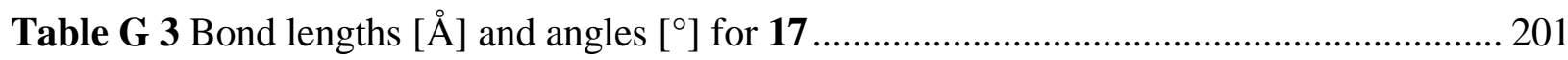

Table G 4 Anisotropic displacement parameters for 17 ................................................. 206

Table G 5 Hydrogen coordinates and isotropic displacement parameters for $\mathbf{1 7}$................. 207

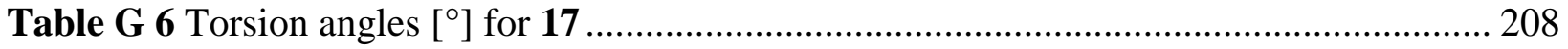

Table G 7 Crystal data and structure refinement for 19............................................ 210

Table G 8 Atomic coordinates and equivalent isotropic displacement parameters for 19 ....... 211

Table G 9 Bond lengths $[\AA ̊$

Table G 10 Anisotropic displacement parameters for 19 ................................................ 225

Table G 11 Hydrogen coordinates and isotropic displacement parameters for 19 ................ 227

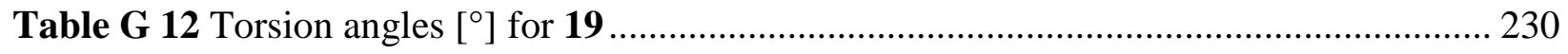




\section{LIST OF ABBREVIATIONS}

AFM

AIBN

AMP

ATRP

BAPO

BPB

BPO

$\mathrm{BuOH}$

$\mathrm{CDCl}_{3}$

CFU

$\mathrm{CHCl}_{3}$

COSY

CRP

CTA1

CTA2

CTA3

$Ð$

$\mathrm{D}_{2} \mathrm{O}$

DCM

$\mathrm{dH}_{2} \mathrm{O}$

DMBQPEI
Atomic force microscopy

Azobisisobutyronitrile

Antimicrobial peptide

Atom transfer radical polymerization

Phenylbis(2,4,6-trimethylbenzoyl)phosphine oxide

Bromophenol blue

Benzoyl peroxide

2-Butanol

Deuterated chloroform

Colony-forming unit

Chloroform

Correlation spectroscopy

Controlled radical polymerization

4-Cyano-4-(phenylcarbonothioylthio)pentanoic acid

4-Cyano-4-[(dodecylsulfanylthiocarbonyl)sulfanyl]pentanoic acid

2-Methyl-2-[(dodecylsulfanylthiocarbonyl)sulfanyl]propanoic acid

Dispersity

Deuterium oxide

Dichloromethane

Distilled water

$N, N$-dodecyl methyl-co- $N, N$-methylbenzophenone methyl quaternary

polyethylenimine 


$\begin{array}{ll}\text { DMF } & \text { Dimethylformamide } \\ \text { DMSO } & \text { Dimethylsulfoxide } \\ \text { DSC } & \text { Differential scanning calorimetry } \\ \text { DSF } & \text { Dynamic shake flask } \\ \text { EPS } & \text { Exopolysaccharide } \\ \text { ESI-Q-TOF } & \text { Electrospray-ionization quadrupole time-of-flight } \\ \text { Et } 2 \mathrm{O} & \text { Diethyl ether } \\ \text { EtOAc } & \text { Ethyl acetate } \\ \text { EtOH } & \text { Ethanol } \\ \text { FRP } & \text { Free radical polymerization } \\ \text { GPC } & \text { Gel permeation chromatography } \\ \text { h } & \text { hour } \\ \text { HAIs } & \text { Healthcare-associated infections } \\ \text { HDPE } & \text { High density polyethylene } \\ \text { HMBC } & \text { Heteronuclear multiple bond correlation } \\ \text { HRMS } & \text { High resolution mass spectrometry } \\ \text { HSQC } & \text { Heteronuclear single quantum coherence } \\ \text { IPA } & \text { Isopropyl alcohol } \\ \text { LDI } & \text { Large drop inoculum } \\ \text { LDPE } & \text { Low density polyethylene } \\ \text { MeCN } & \text { Acetonitrile } \\ \text { MeOH } & \text { Methanol } \\ \text { Magnesium sulfate } \\ \text { Hor }\end{array}$




\begin{tabular}{ll} 
M $_{\mathrm{w}}$ & Molecular weight \\
NMP & Nitroxide mediated polymerization \\
NMR & Nuclear magnetic resonance \\
ORTEP & Oak Ridge thermal ellipsoid plot \\
PAMBM & Poly[(aminoethyl methacrylate)-co-(butyl methacrylate) \\
PBS & Phosphate buffer solution \\
PEEK & Poly(ether-ether-ketone) \\
PLGA & Poly(lactic-co-glycolic acid) \\
PMMA & Poly(methylmethacrylate) \\
PolyQAC & Quaternary ammonium containing polymer \\
PP & Polypropylene \\
PQA & Polyquaternary ammonium \\
PS & Polystyrene \\
PVC & Polyvinyl chloride \\
PVP & Poly(vinylpyridine) \\
PVP+ & Poly(vinylpyridinium) \\
Q & Quaternary ammonium/phosphonium group \\
QAC & Quaternary ammonium compounds \\
RAFT & Reversible addition-fragmentation chain-transfer \\
ref. & Reference \\
RMS & Root mean square \\
TEMPO & (2,2,6,6-Tetramethylpiperidin-1-yl)oxyl \\
TFT & Too few to count \\
\hline
\end{tabular}




$\begin{array}{ll}T_{g} & \text { Glass transition temperature } \\ \text { THF } & \text { Tetrahydrofuran } \\ \text { TLC } & \text { Thin layer chromatography } \\ T_{m} & \text { Melt temperature } \\ \text { Tol } & \text { Toluene } \\ \text { VBC } & \text { 4-Vinylbenzyl chloride } \\ \text { wt } & \text { Wild type }\end{array}$




\section{INTRODUCTION}

Microbial aggregates in which bacterial cells are embedded within a self-produced exopolysaccharide (EPS) matrix promote cell cohesion and adhesion to surfaces. ${ }^{1,2}$ These biofilms form distinct microenvironments and complex structures that facilitate microbial proliferation as well as protect bacteria from environmental stresses, antibiotics, and disinfection. Biofilms and the pathogenic bacteria they house have been found to be implicated in a high percentage of healthcare-associated infections (HAIs), annually affecting hundreds of millions of patients worldwide. ${ }^{3,4}$ Each year, over 200,000 Canadians acquire an HAI resulting in 8,000 deaths. ${ }^{5}$ Of these infections, $50 \%$ are resistant to at least one type of antibiotic. ${ }^{6}$ The overprescription of antibiotics in livestock and by healthcare professionals alike have accelerated the development of antibiotic resistant bacterial strains, making antibiotic resistance one of most prevalent public health concerns in modern medicine. ${ }^{7,8}$ At the current rate of antibiotic resistance progression, it is expected to cost up to $\$ 100$ trillion dollars and result in 50 million deaths per year by $2050 .{ }^{9}$ In hopes of countering HAIs and the looming antibiotic resistance crisis, the development of nontraditional, non-leachable antimicrobials have gained traction.,

\subsection{Quaternary Ammonium Containing Polymers}

Synthetic antimicrobial polymers have been studied extensively since the mid-1960s with the reported synthesis and antibacterial activity of 2-methacryloxytropone polymers by Cornell and Donaruma. ${ }^{10}$ With the popularity of quaternary ammonium compounds (QACs) in a range of commercial disinfectants such as benzyalkonium chloride (Figure 1.1(a)), quaternary ammonium containing polymers (polyQACs) have been of wide interest due to their promising properties of higher antimicrobial efficacy, lower cell toxicity, and increased stability in comparison to their 
monomeric counterparts. ${ }^{11-15}$ Recent reviews on the synthesis and biocidal properties of polyQACs have demonstrated their promise in a number of antimicrobial material applications. ${ }^{16-}$ 18 The commercialized antimicrobial polymer, poly[(aminoethyl methacrylate)-co-(butyl methacrylate)] (PAMBM) (Figure 1.1(b)), was blended with poly(lactic-co-glycolic acid) (PLGA) and coated on sutures. These PAMBM coated sutures demonstrated high antibacterial activity against $S$. aureus at low concentrations in comparison to the conventional triclosan coated VICRYL® Plus Antibacterial sutures, which instead possessed bacteriostatic properties. ${ }^{19}$

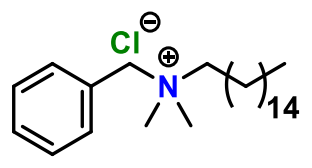

Popular disinfectant benzalkonium chloride

(a)

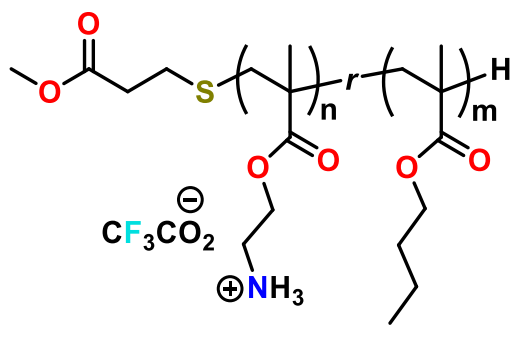

PAMBM suture coating Li et al. Langmuir 2012

(b)

Figure 1.1 QACs (a) small molecule disinfectant benzalkonium chloride (b) poly(QAC) PAMBM blended with PLGA for suture coating

Much work in quaternary ammonium polymers has been inspired by naturally occurring antimicrobial peptides (AMPs), leading to the field of AMP mimetics. Traditionally, common building blocks for AMP mimetics include peptoids, $\beta$-peptides, arylamides, or phenylene ethynylenes. ${ }^{20}$ Recent work by Takahashi and colleagues described AMP mimics expanding to the use of synthetic random copolymers with cationic charges, illustrated in Figure 1.2(a). Methylmethacrylate polymerization (PMMA) by reversible addition-fragmentation chain-transfer (RAFT) polymerization produced a 2-3 $\mathrm{kDa}$ polymer to mimic the size of $\alpha$-helical AMPs. ${ }^{21}$ Additionally, studies of the structure-activity relationship between the polyQAC backbone and antimicrobial activity are well reported. Significant investigations into AMP mimetics have 
demonstrated that the balance between hydrophobic and cationic groups are necessary to achieve high antibacterial and non-hemolytic properties. ${ }^{12,21}$ Recent work by Guo et al. comparing the antimicrobial and non-hemolytic properties of a series of small molecule QACs to their side- and main-chain polymer counterparts (Figure 1.2(b)) indicated that polymeric structures where QACs are situated in the backbone (ie. Polyquaternary ammoniums, PQAs) demonstrate higher antimicrobial activity and lower hemolytic properties than polymeric QACs with quaternary ammonium pendants. ${ }^{22}$ The reason for reduced hemolytic properties are unclear and investigations are ongoing. Conversely, extensive work done by Tew and colleagues on polynorbornene based AMP mimetics have demonstrated that hydrophobicity of polymeric QACs are more tuneable with a hydrophobic polymer backbone and QAC side chains (Figure 1.2(c)). ${ }^{12,23-25}$ 


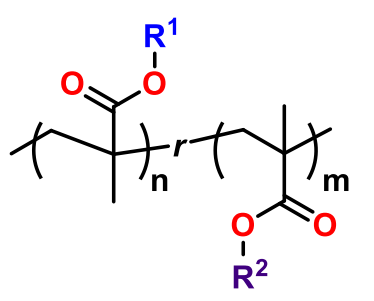

PMMA synthetic AMPs Takahashi et al. Bioconjug. 2017

(a)

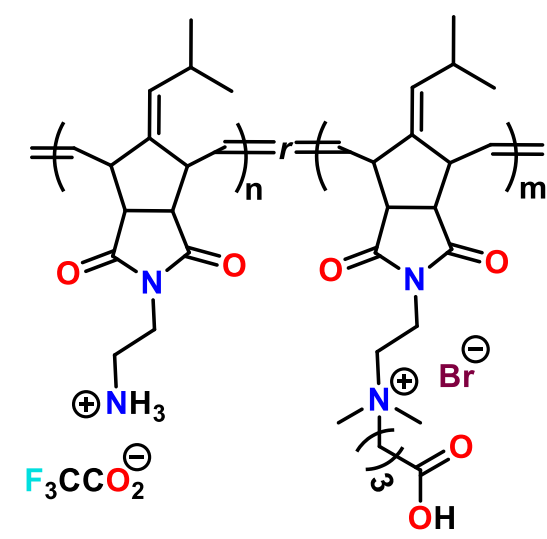

Polynorbornene synthetic AMP mimetics Tew et al. Biomacromolecules 2009

(c)

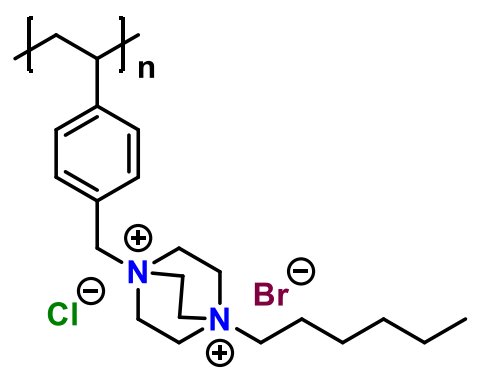

Side-chain polyQAC

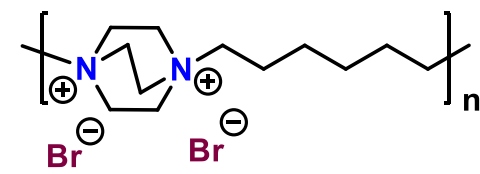

Main-chain polyQAC (PQA)

Guo et al. Polym. Chem. 2018

(b)

Figure 1.2 Synthetic AMP mimetics (a) PMMA based mimics with cationic and hydrophobic substituents $^{21}$ (b) Equivalent side-chain and main-chain QAC polymers ${ }^{22}$ (c) polynorbornene based AMP mimics with cationic pendants ${ }^{25}$

\subsubsection{Menshutkin Reaction}

The synthesis of quaternary ammonium groups are primarily carried out by the Menshutkin reaction. Here, the tertiary amine undergoes alkylation with an alkyl halide to synthesize the equivalent quaternary ammonium salt and counteranion, illustrated in Scheme 1.1. The reaction is carried out under $\mathrm{S}_{\mathrm{N}} 2$-like conditions, but with the use of more polar solvents such as alcohols, the reaction was found to have a decreased energy barrier and increased reaction rate, uncharacteristic of typical $\mathrm{S}_{\mathrm{N}} 2$ reactions which require polar aprotic solvents. ${ }^{26}$ 


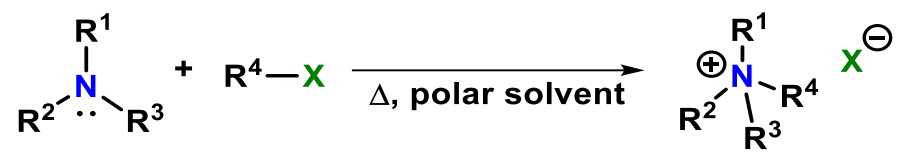

Scheme 1.1 Menshutkin reaction

\subsubsection{Mechanism of Action of Unbound QACs and PolyQACs}

Biological bilayers are composed of amphiphilic phospholipids with a hydrophilic phosphate head and hydrophobic tails of two fatty acid chains. Bacterial cells carry a net negative charge from the negatively charged head group, stabilized by cationic magnesium and calcium. ${ }^{27}$ Bacterial cell walls possess an overall negative charge. Gram-positive bacteria have teichoic acid embedded in the peptidoglycan layer, illustrated in Figure 1.3(a). Lipopolysaccharide (LPS) present in the outer membrane of gram-negative bacteria bestow negative charge to the cell surface, seen in Figure 1.3(b). ${ }^{27}$ QACs characteristically have at least one quaternary ammonium moiety and a hydrophobic alkyl chain, allowing for surfactant like interactions with the negatively charged bacterial cell wall. The cationic nitrogen interacts with the negative components of the cell wall and hydrophilic phosphate head. The hydrophobic tail of the QAC interact with the cell membrane by interlocking with the fatty acid chains to form pores in the cell membrane. At high concentrations, the compound effectively solubilizes the membrane leading to cell leakage and death. ${ }^{28}$ 


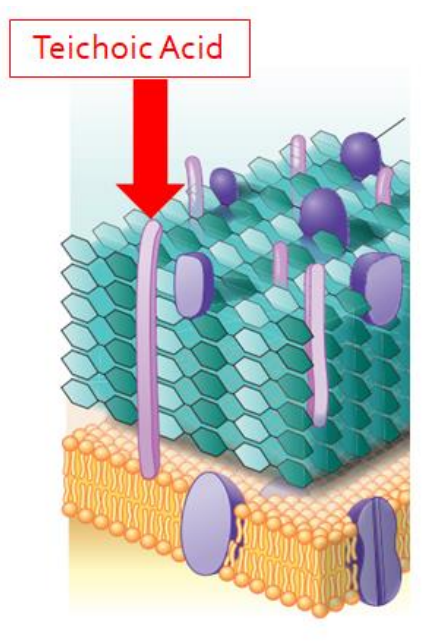

Gram-positive cell wall

(a)

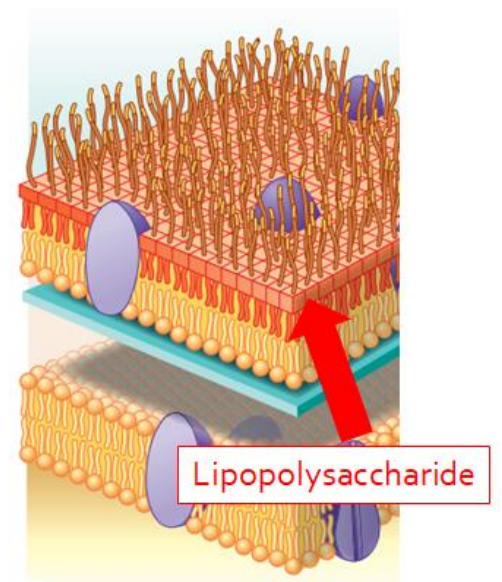

Gram-negative cell wall

(b)

Figure 1.3 Gram-positive and -negative bacterial cell walls and key negative components (Adapted from ref. ${ }^{1}$ )

\subsection{Surface Attached Antimicrobials}

QACs and polyQACs are extremely well-studied for their use in solution as disinfectants and antiseptics. Their use in antimicrobial surfaces are also a very popular investigative field. There are two categories of antimicrobial surfaces; repelling and killing surfaces, illustrated in Figure 1.4. Repelling surfaces rely on deterring bacterial attachment to the surface by means of steric or electrostatic repulsion or surfaces of low surface energy. ${ }^{27}$ While successful as an antimicrobial surface, these surfaces do not reduce bacterial cells, allowing for bacterial transfer in high traffic areas to unprotected surfaces. For this reason, bacteria killing surfaces have been the main focus in our work. 


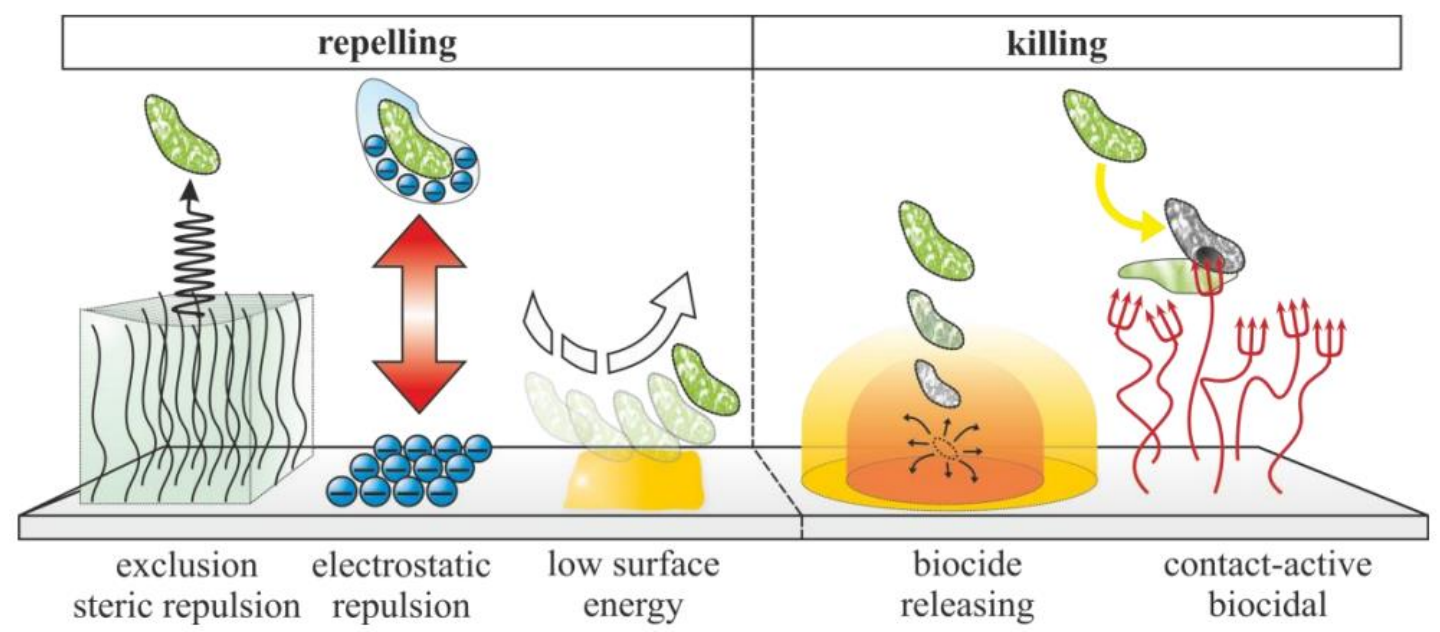

Figure 1.4 The two categories of antimicrobial coatings: repelling and killing coatings (adapted from ref. ${ }^{27}$ )

Conventional antimicrobial surfaces discharge biocides or antibiotics for bacterial cell uptake. ${ }^{29,30}$ These biocide releasing coatings include triclosan, silver sulfadiazine nanosilver, tributyltin (TBT), cetyl trimethyl ammonium chloride (CTAC), and antibiotics such as ciprofloxacine and chlorhexidine. ${ }^{30}$ Although effective, these antimicrobial surfaces lose efficacy over time. Frequent usage of these compounds, especially triclosan and tributyltin, have been proven to pose significant harm to waterways and aquatic life. ${ }^{31}$ Repeated usage and discharge into the environment also contribute to the formation of resistant antimicrobial strains. ${ }^{32-34}$

Surface-attached contact active antimicrobials provide non-leaching alternatives for the prevention of biofilm formation and discourage bacterial transfer. The first popular QAC physically attached to the surface was an anti-biofilm small molecule coating with an organosilane functionality reported by Isquith et al.(Figure 1.5(a)). ${ }^{35}$ The QAC was readily grafted onto porous surfaces such as textiles by chemically altering the surface to become physically attached. The Foucher group functionalized the QAC small molecule with a phosphinate group that covalently bonded to metal surfaces for successful formulation of an antimicrobial coating. (Figure 1.5(b)). ${ }^{36}$ 


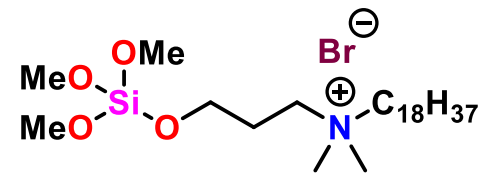

QAC grafted on porous surfaces Isquith et. al, Appl. Microbiol. 1972

(a)

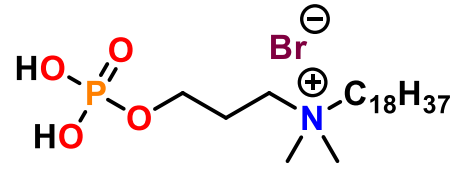

QAC grafted on metal surfaces Porosa et. al, J. Mater. Chem. B 2014

(b)

Figure 1.5 Surface-attached QACs grafted by (a) organosilane functionality on porous surfaces ${ }^{35}$ (b) phosphinate functionality on metal surfaces ${ }^{36}$

Plastic materials are thoroughly integrated into various industries and everyday products, especially in medical and food industries where plastics are incorporated into medical devices and food packaging, and thus susceptible to biofilm formation. ${ }^{37}$ The first plastic surface grafted with anti-biofilm technology was reported by Gottenbos et al. ${ }^{38}$ QACs were grafted onto an oxidized hydroxyl terminated silicone rubber surface. Although successful and antimicrobial, the inert C-H terminated surface of the silicone rubber required argon plasma activation prior to grafting. Plasma activation requires extremely high temperatures risking damage and etching of plastic surfaces. Expensive equipment is also required to carry out plasma activation, making this technique unrealistic for commercial use on most plastics.

Investigations into benzophenone recognized its characteristic behaviour when exposed to UV light. This type II photoinitiator forms a di-radical under UV light and behaves as a crosslinker for grafting onto plastic substrates via a C-H insertion mechanism, illustrated in Figure 1.6. ${ }^{39}$ The diradical benzophenone species abstracts a hydrogen atom from the $\mathrm{C}-\mathrm{H}$ group at the surface, producing a surface radical and ketyl radical. ${ }^{40}$ The radicals combine to form a carbon-carbon bond between the surface and the benzophenone molecule leading to successful surface attachment. 


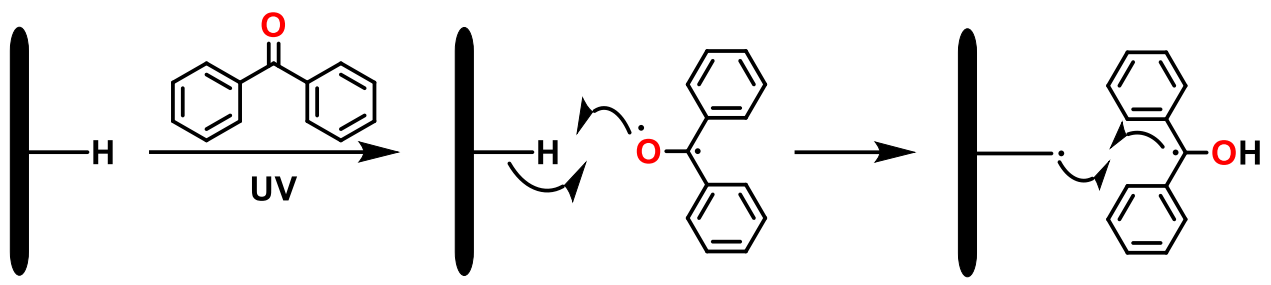

Figure 1.6 Photo-initiated radical crosslinking via $\mathrm{C}-\mathrm{H}$ insertion mechanism from aryl ketone of the benzophenone to form carbon-carbon bond with the substrate surface.

1.3 Methods to Grafting on Surfaces: "Grafting from" and "Grafting to"

Surface attachment of thin films on solid surfaces are successfully carried out by graft polymerization techniques, "grafting from" and "grafting to". Commonly used for attachment of polymer brushes, these techniques have also been used for small molecule compounds and nonbrush polymers.

The "grafting from" method of surface modification describes compound attachment at locations of fixed or tethered surface initiators. The initiating functionality at the surface initiates polymerization of free monomers and propagation of the polymeric chain leading to brush structures, illustrated in Figure 1.7(a). The formation of well-defined polymeric brushes on solid surfaces have been achieved by a range of polymerization techniques, but the most thoroughly investigated method is by atom-transfer radical polymerization (ATRP). ${ }^{41}$

In the "grafting to" approach, polymers are pre-prepared with reactive groups as a polymer end group or side chain functionality to be covalently bonded to surfaces, depicted in Figure 1.7(b). Reactive end group sites of polymers tether to the surface to form brushes, while side chain functionalization with reactive groups throughout the chain form non-brush polymeric coatings. 
(a) "Grafting from"
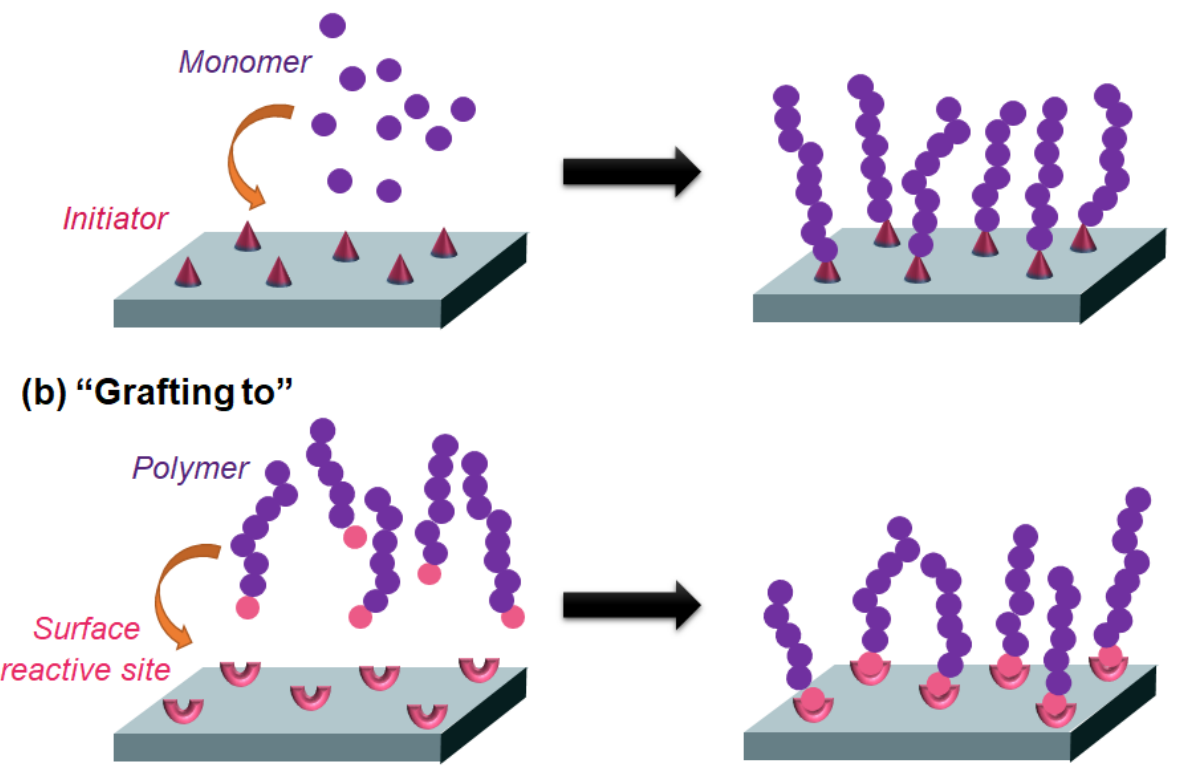

Figure 1.7 Surface grafting methods (a) "grafting from" and (b) "grafting to"

\subsection{Controlled/Living Radical Polymerization}

Conventional free radical polymerization (FRP) is extremely popular in commercial preparation of polymeric materials, representing $45-50 \%$ of all industrially prepared polymers. ${ }^{42}$ FRP offers rather tolerant reaction conditions for polymerizations of vinyl monomers, and considering that the most important plastics today are polypropylene (PP), high density polyethylene (HDPE), linear low density polyethylene (LLDPE), LDPE, and polyvinyl chloride (PVC), it is unsurprising that FRP is well-known. ${ }^{42,43}$ The major disadvantage of FRP is the lack of control over the reaction hindering preparation of well-defined polymers specified by controlled molecular weight, narrow dispersity $(\bigoplus)$, and chain architecture such as block, comb, or star branched polymers. This shortcoming was quickly remedied with the proposal of reversible deactivation of growing chains by Otsu et al. in 1982 and investigations that have since followed. ${ }^{42,44}$ Today, a range of controlled or living radical polymerization (CRP) techniques have been reported and explored. 


\subsubsection{Nitroxide Mediated Polymerization}

One of the early investigations of CRP began with the study of stable free radical polymerizations (SFRP) using nitroxide-mediated radical polymerization (NMP). Here, the reversible deactivation of the growing chain relies on the reversible coupling with stable nitroxide radicals, depicted in Scheme 1.2. Georges et al. notably demonstrated the potential for NMP by his work in styrene polymerization. ${ }^{45}$ The NMP reaction in Scheme 1.2 uses $(2,2,6,6-$ tetramethylpiperidin-1-yl)oxyl (TEMPO) as the nitroxide mediator, a rather inexpensive controlling agent that performs well with styrenic monomers.

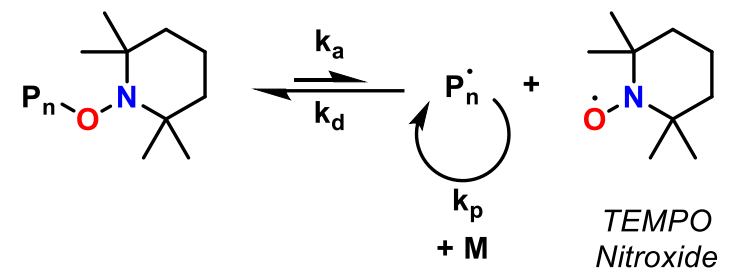

Scheme 1.2 General NMP reaction

Unfortunately, traditional TEMPO-like nitroxides have proven to be rather selective, useful with only a narrow range of monomers and require high polymerization temperatures (125-145 $\left.{ }^{\circ} \mathrm{C}\right)$. New exploration into other nitroxide species capable of wider monomer range and lower temperatures have been a key study in the NMP field. ${ }^{46}$ Nitroxides such as SG-1 and BlocBuilder ${ }^{\circledR}$ (Figure 1.8) have recently become commercially available for the polymerization of styrenic acrylic and acrylamido monomers. ${ }^{42}$ 


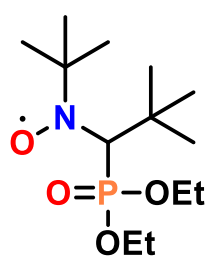

SG-1

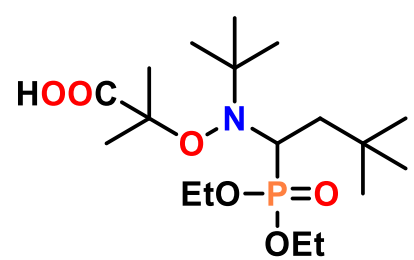

Blocbuilder $^{\circledR}$

(b)

Figure 1.8 Stable nitroxide radicals for versatile NMP

\subsubsection{Atom-Transfer Radical Polymerization}

Atom-transfer radical polymerization (ATRP) is a considerably more versatile CRP method, with applications for a range of monomers in a range of reaction conditions for complex polymer architectures. ${ }^{42}$ In ATRP, polymer chain deactivation depends on reversible atom transfer catalyzed by a transition-metal complex, illustrated in Scheme 1.3. Kato and colleagues described the use of a ruthenium based catalyst $\left(\left(\mathrm{RuCl}_{2} / \mathrm{PPh}_{3}\right)_{3}\right)$ to polymerize MMA, initiated by carbon tetrachloride. The system requires activation by aluminum alkoxides with an unclear reaction mechanism. ${ }^{47}$ Soon after, Matyjaszewski reported ATRP using a copper based controlling agent $(\mathrm{CuX} / 2 \mathrm{bpy}) .{ }^{48}$ The system was found to be highly successful with the polymerization of styrenes, acrylates, acrylonitriles, and other monomers.

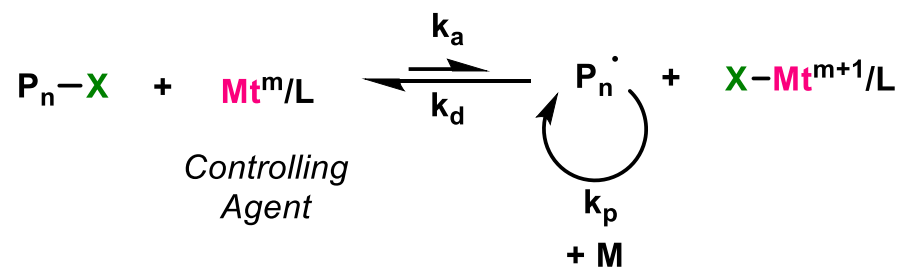

Scheme 1.3 Reaction mechanism of ATRP

While extremely versatile, the majority of ATRP systems require a metal-based catalyst which is environmentally problematic due to residual metals present in the polymer product. 
Lingering metals trapped within the polymer matrix limit applications for ATRP, making it unsuitable for medical and biomedical plastics.

\subsubsection{Reversible Addition-Fragmentation Chain-Transfer Polymerization}

The most versatile of the discussed CRP methods is the reversible addition-fragmentation chain-transfer (RAFT) polymerization, compatible with a range of monomers with mild reaction conditions. ${ }^{42}$ The chain deactivation and activation is reliant on the degenerative transfer of a group, depicted in Scheme 1.4.

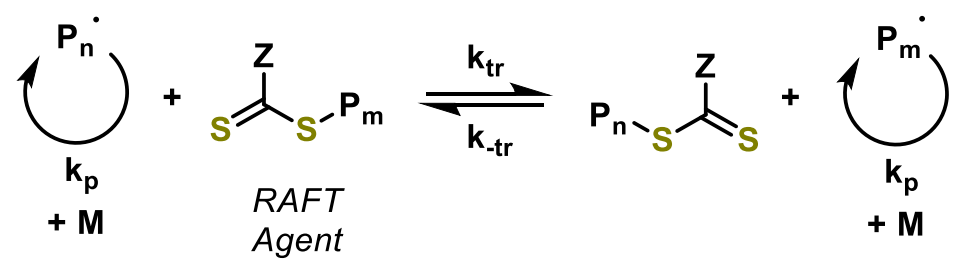

Scheme 1.4 Reaction mechanism of RAFT polymerization

Chiefari et al. ${ }^{49}$, Le et $a l .{ }^{50}$, and Corpart et al. ${ }^{51}$ reported the earliest RAFT polymerization with a thio-based RAFT agent in the late 1990s, and since then, there has been extensive reports and investigations into RAFT agent design. The most common classes of RAFT agents are dithiobenzoates, trithiocarbonates, and dithiocarbamates, shown in Figure 1.9. These RAFT agents have tunable $\mathrm{R}$ and $\mathrm{Z}$ groups for compatibility with a wide range of monomers.<smiles>[R]SC(=S)c1ccccc1</smiles>

Dithiobenzoates<smiles>[Z]SC(=S)S[Z]</smiles>

Trithiocarbonates<smiles>[R]SC(=S)N([Z1])[Z]</smiles>

Dithiocarbamates

Figure 1.9 Classes of RAFT agents 
Polymers synthesized by RAFT polymerization require end group modification steps. As many RAFT agents are highly pigmented red or yellow compounds (demonstrated in Figure 1.10(a)), the resultants polymer products are often also coloured (Figure 1.10(b)), necessitating end group modification. RAFT polymer end groups are reactive thiocarbonylthio structures that can host side reactions, warranting extra steps for removal. End group modification is well studied, with extensive summaries and reviews available for reference. ${ }^{52}$

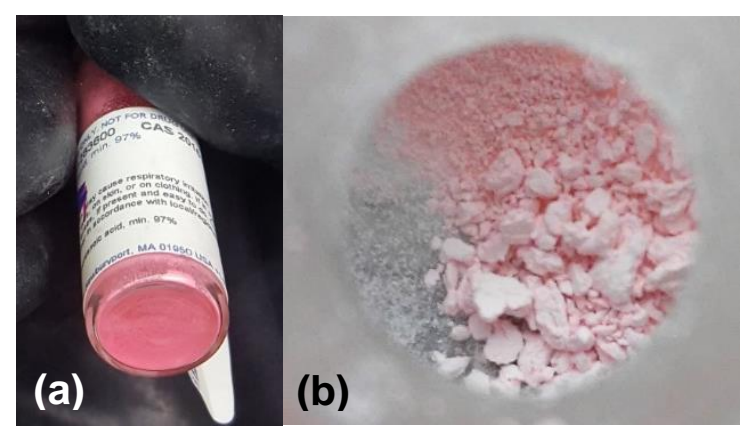

Figure 1.10 (a) RAFT agent 4-Cyano-4-(phenylcarbonothioylthio)pentanoic acid seen as a bright pink powder (b) resulting 4-vinylbenzyl chloride homopolymer prepared by RAFT polymerization that appears pink

While all CRP methods present their own advantages and unique challenges, NMP and RAFT polymerization are worthy for further investigations for the work in this thesis. The residual metal in ATRP make this CRP method incompatible for coatings formulations intended for medical environments.

\subsection{Coating Visualization}

Surfaces treated with antimicrobial compound can be visualized by bromophenol blue (BPB) staining. This qualitative technique will indicate the quality of the coating and whether the cationic compound is present on the surface. BPB is an anionic indicator dye that will ion-exchange with the counteranion of the compound to form an ionic pair, resulting in a blue colour (Scheme 
1.5). Treated surfaces are exposed to a solution of BPB and the surface will visually stain blue to indicate the presence of the compound. ${ }^{53,54}$ This technique can confirm the presence of the antimicrobial compound prior to biological testing.

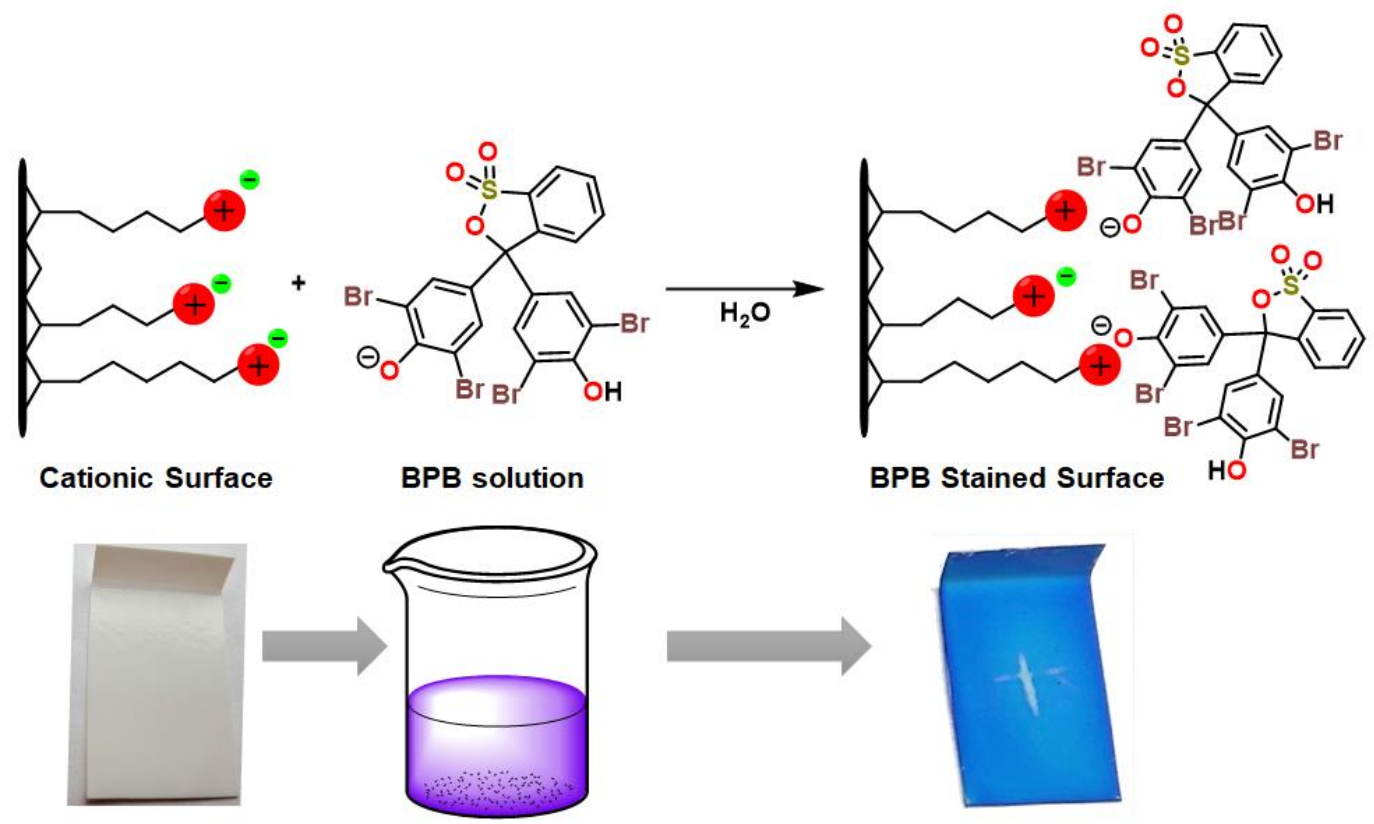

Scheme 1.5 Coating visualization by BPB staining.

\subsection{Biological Testing Methods}

Current and common established methods in determining antibacterial properties of surfaces include the dynamic shake flask (DSF) method (ASTM E2149-13a) ${ }^{55}$ and ISO $22196 .^{56}$ In the DSF method, the treated surface is submerged in the inoculum and shaken over a period of time. While helpful for antibacterial determination of materials for solution applications, this method does not reflect the bioactivity at the solid/air interface. Similarly, the ISO 22169 method fails to consider the drying of the droplet by using a coverslip to cover inoculated surfaces, maintaining a wet surface. These testing methods do not consider or mimic real-world conditions for bacterial transmission by droplet transmission. Infectious materials are largely transferred by 
suspension in aerosol and droplet from sneezing, coughing, and vomiting, with additional propagation by ventilation, nebulizers and air-conditioning systems. ${ }^{57,58}$ In this work, surfaces will be evaluated for the antibacterial activity at the solid/air interface by performing the large drop inoculum (LDI) protocol.

\subsubsection{Large Drop Inoculum Protocol}

Developed by Ronan et al..$^{59}$, the LDI method provides a more accurate representation of real life bacterial transfer by relying on inoculation by large droplet. Unlike the similar aerosol inoculation method ${ }^{60}$, the usage of large droplets allows for more control over the total volume of bacterial load deposited on the surface.

\subsubsection{Dynamic Shake Flask Method}

Campos et al. reported high variability of results between protocols ISO 22196 and DSF when testing for the antibacterial efficacy of surfaces against select gram-negative and -positive species, suggesting that antibacterial efficacy cannot be reproduced or accurately compared. ${ }^{58}$ As there are a variety of antibacterial test methods available, researchers rarely use the same method, making accurate comparisons and development of antibacterial surfaces difficult to achieve. In this work, the DSF method will also be used for select samples to allow for comparisons to literature.

\subsection{Mechanism of Action of Immobilized Antimicrobials}

The mechanism of action for immobilized antimicrobials has been a major area of interest for the discovery of antimicrobial surfaces. Mechanism elucidation can aid in structure-activity 
investigations and consequently lead to the development of more potent antimicrobial surfaces. These immobilized, cationic polymers with major hydrophobic substituents have been found to interrupt bacterial cell membranes with little to no effect on mammalian cells. ${ }^{61,62}$ Although the true mechanism has not been confirmed with confidence, the density of cationic charge ${ }^{63}$, alkyl chain length ${ }^{64}$, and surface topography ${ }^{65}$ have been factors contributing to the antibacterial efficacy of treated surfaces. While there are many mechanisms proposed in literature, the most popular contact-killing postulates are the polymeric spacer effect and the phospholipid sponge effect.

\subsubsection{Polymeric Spacer Effect}

Proposed by Tiller et al. in 2001, the polymeric spacer effect suggests that the bacteriocidal polymer surface has the ability to penetrate the bacterial cell wall of adhered microbes. At a significant polymer length, the cytoplasmic membrane can be disrupted leading to cell death, illustrated in Figure 1.11. Tiller et al. demonstrated this effect by grafting varying lengths of poly(vinylpyridine) (PVP) chains onto highly quaternized poly(vinyl- $N$-hexylpyridinium) slides. ${ }^{64}$ Polymer surfaces of shorter PVP chains at $60 \mathrm{~kg} / \mathrm{mol}$ had $<64 \%$ reduction of deposited S. aureus cells after $24 \mathrm{~h}$, while longer PVP chains of $160 \mathrm{~kg} / \mathrm{mol} \mathrm{had} \mathrm{a} 94 \%$ reduction of cells after the same period of time. ${ }^{64}$ However, the polymeric spacer effect is inapplicable for antibacterial surfaces that feature short alkyl chains. ${ }^{66}$ 


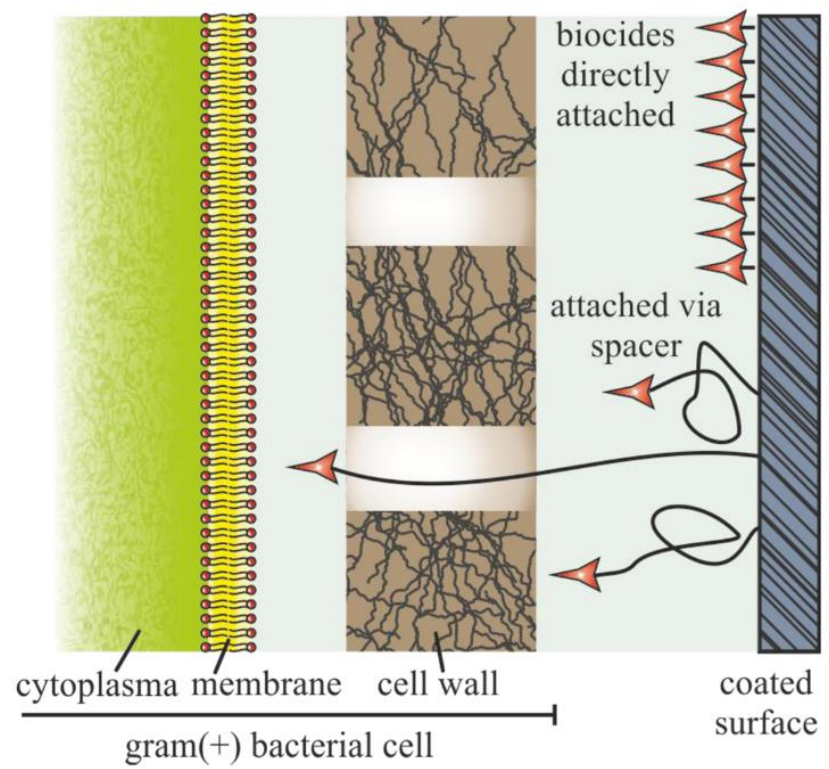

Figure 1.11 Polymeric spacer effect (Adapted from ref. ${ }^{27}$ )

\subsubsection{Phospholipid Sponge Effect}

In the phospholipid sponge effect, it is hypothesized that the cationic surfaces draw and pull apart fragments of the water insoluble, negatively charged phospholipid and LPS of the bacterial cell membrane, effectively destroying the microbe (Figure 1.12). ${ }^{27,67}$

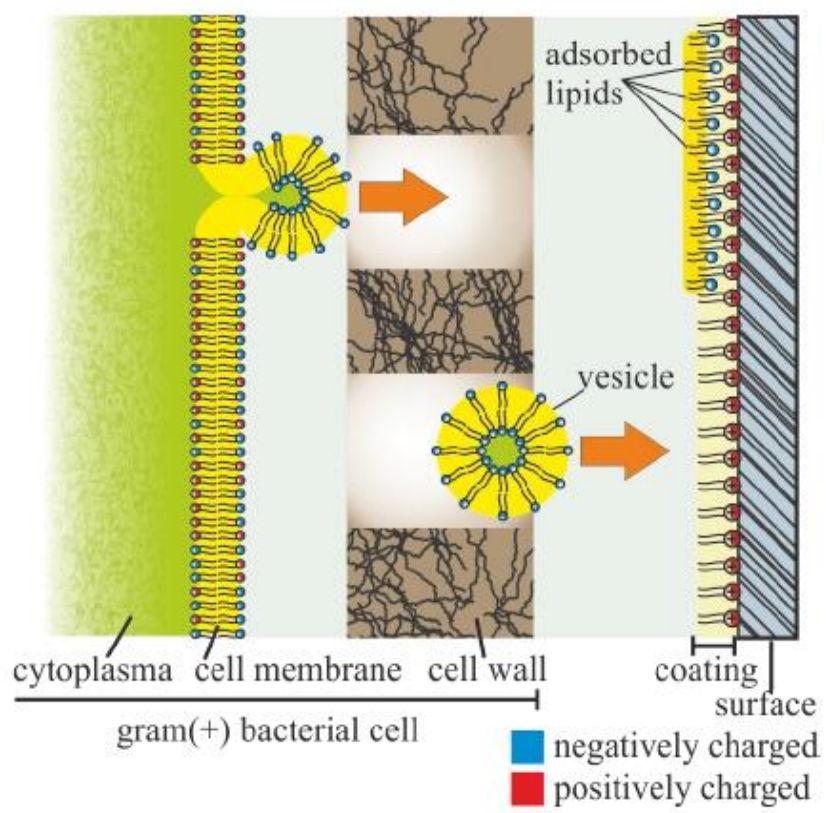

Figure 1.12 Phospholipid sponge effect (Adapted from ref. ${ }^{27}$ ) 
In 2010, Li et al. synthesized an antimicrobial hydrogel surface from quaternized ammonium chitosan-grafted-poly(ethylene glycol) methacrylate (qC-g-EM) ${ }^{68}$ The hydrogel possessed nanopores and lacked long alkyl chains. Using computer simulations, Li demonstrated that larger pore sizes and a higher charge density had higher biocidal efficacy. ${ }^{68}$ More recently, Gao et al. also demonstrated this effect using surface-bound $N, N$-dodecyl methyl-co- $N, N$ methylbenzophenone methyl quaternary polyethylenimine (DMBQPEI). ${ }^{69}$ The cationic polymer was UV-curable and was cross-linked to form polymer networks. At varying UV-cure dosages, different levels of crosslinking density were achieved. At lower UV-cure dosages, the DMBQPEI network formed lower crosslinking densities, leaving large pores in the network. Lower crosslinking densities were reported to have higher biocidal abilities. The group hypothesized that the large pores allowed for higher, more intense phospholipid sponge effect, where more of the bacterial membrane could be pulled into the network. Higher crosslink densities formed smaller spaces in the network, leaving less space for fragments of the cell membrane, illustrated in Figure 1.13. ${ }^{69}$

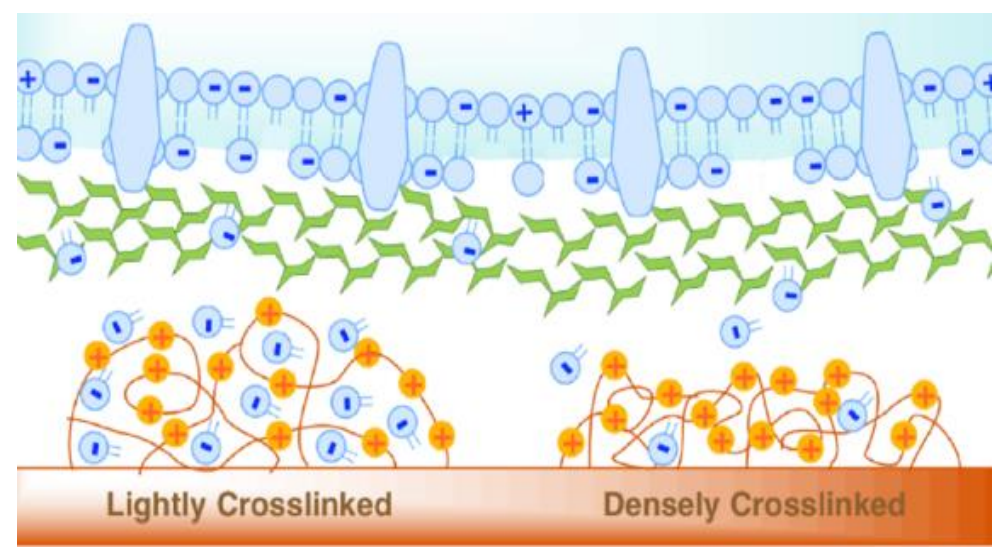

Figure 1.13 Illustration the phospholipid sponge effect on different cross-linking densities. (Adapted from ref. ${ }^{69}$ ) 
Although promising, the pathway of the water-insoluble phospholipids across the cell wall to the cationic surface is unclear. A possible hypothesis for this pathway is the migration of phospholipids as liposomes through holes in the bacterial cell wall. ${ }^{27}$ These effects of the contactactive kill mechanism are debated in literature, and alternate, less popular mechanisms should not be dismissed. ${ }^{27}$

\subsection{Notable Previous Work}

Contact active antimicrobial surfaces with cationic active sites such as quaternary ammonium and phosphonium are of particular interest in the prevention of biofilm formation and bacterial transfer. Since the formulation of the first notable antimicrobial coating based on quaternary ammonium silanes by Dow in the 1970's that readily graft to porous surfaces such as fabrics, QACs have been extensively studied for use as biocidal coatings while related phosphonium coatings are relatively unknown. ${ }^{14,35}$

While there are a number of covalently attached QAC coatings reported in literature ${ }^{36,70-}$ 73 , only a few incorporate a benzophenone functionality as the surface attachment moiety as part of the compound. Benzophenone, when exposed to UV light, grafts onto plastic substrates and itself via a $\mathrm{C}-\mathrm{H}$ insertion mechanism forming a carbon-carbon bond leading to successful surface attachment and cross-linking, negating the need for a photoactive initiator additive. ${ }^{39,40}$ Seminal work by Matyjaszewski et al. reported the first benzophenone system illustrated in Figure 1.14(a), where quaternary ammonium polymeric brushes of poly(2-dimethylaminoethyl methacrylate) (PDMAEMA) were synthesized by surface initiated atom ATRP. ${ }^{74}$ While PDMAEMA brushes of $10 \mathrm{kDa}$ and higher demonstrated complete kill against Escherichia. coli via the dynamic shake flask method, the three-step "grafting from" approach is impractical on an industrial scale. In a 
two-step self-initiated surface "grafting from" method, Ishihara and colleagues formulated a QAC coating based on 2-methacryloyloxyethyl phosphorylcholine (QAC-MAOPC) by UV-initiation of the diphenylketone backbone of the poly(ether-ether-ketone) (PEEK) substrate which closely resembles benzophenone (Figure 1.14(b)). ${ }^{75}$ Biocompatibility of the surface was anticipated, but antimicrobial activity was not determined.

A one-step "grafting to" system reported by Locklin et al. described a QAC functionalized with benzophenone and $\mathrm{C}_{12}$-alkyl chain was surface attached onto PP, PVC, glass and cotton substrates. Through qualitative antibacterial analysis via modified spray inoculation methods, the coated surfaces demonstrated activity against Staphylococcus aureus and E. coli. ${ }^{76}$ At the same time, the Foucher Group described the preparation of $\mathrm{C}_{12}$ and $\mathrm{C}_{18}$-alkyl chain quaternary ammonium self-assembled coatings with a benzophenone functionality that was readily grafted onto plastics, shown in Figure 1.14(c) ${ }^{53}$ These compounds in water/ethanol formulation were aerosol spray coated and UV-cured onto a variety of plastic substrates; including PP, polyethylene (PE), polystyrene (PS), PVC, and PEEK. Quantitative bioactivity analysis by large drop inoculum (LDI) protocol $^{59}$ of these coated surfaces demonstrated efficacy against gram-positive (Arthrobacter sp., Listeria monocytogenes) and gram-negative (Pseudomonas aeruginosa) bacteria at a solid/air interface after a three hour period. ${ }^{53}$

The one-step "grafting to" benzophenone functionalized QAC system has mainly been limited to small molecule compounds as seen in the work by the Foucher and Locklin Groups with only one known example of a polymeric based QAC system. In previous work by Locklin et al. in 2011, the bioactivity of surface attached polyquaternary ammonium (PQA) synthesized from commercially available polyethylenimine (PEI) and quaternized with $\mathrm{C}_{12}$-alkyl chains and benzophenone was reported (Figure 1.14(d)). ${ }^{77,78}$ The PQA was spray-coated and UV-cured onto 
PP, PVC, PE, and cotton. Through qualitative antibacterial analysis via spray inoculation methods, the coated surfaces demonstrated activity against $S$. aureus and E. coli. ${ }^{77}$ The polymeric counterparts of these QAC benzophenone systems can allow for lower benzophenone loading and higher charge to attachment moiety ratios.

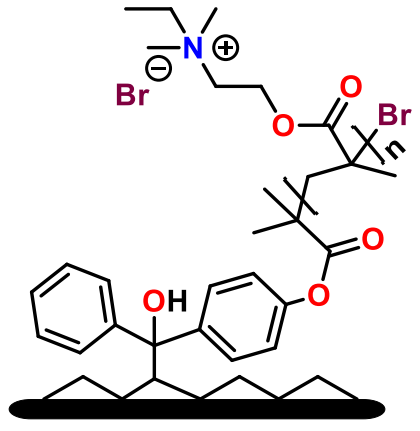

PDMAEMA brushes on PP Matyjaszewski et al. Biomacromolecules 2007

(a)

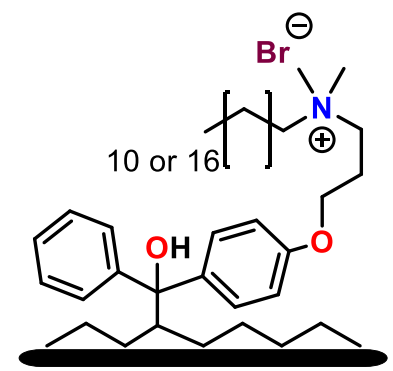

C12 and C18 BP-QAC on PP, PE, PS, PVC, PEEK, glass, cotton

Locklin et al. ACS Biomater. Sci. Eng. 2016 Foucher et al. ACS Appl. Mater. Interfaces 2017

(c)

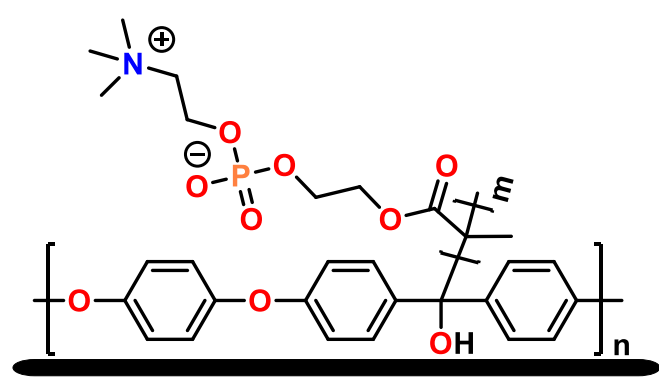

QAC-MAOPC on PEEK

Ishihara et al. Biomaterials 2010

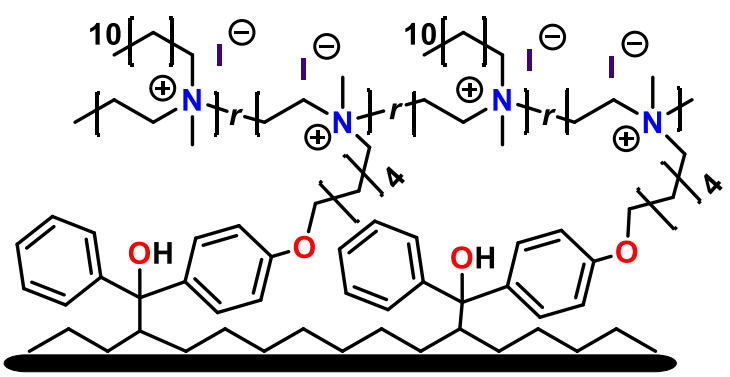

PQA-PEI on PP, PVC, PE, cotton Locklin et al. ACS Appl. Mater. Interfaces $\mathbf{2 0 1 1}$

(d)

Figure 1.14 Previously reported QAC coatings surface attached and cross-linked with benzophenone (a) QAC polymer brushes synthesized by surface initiated ATRP using a "grafting from" approach ${ }^{74}$ (b) QAC polymer brushes synthesized by UV initiation of the surface, PEEK, which closely resembles benzophenone using a "grafting from" approach" (c) C12 and C18 QAC small molecule coating synthesized by "grafting to" technique ${ }^{53,76}$ (d) Quaternized PEI coating "grafted to" surface ${ }^{77}$ 
More recent work by the Foucher group reported the formulation of surface attached sulfonamide substituted quaternary ammonium coatings (Figure 1.15). Benzophenone functionalized sulfonamide analogs were spray coated on PS and immobilized to the surface by UV curing. Mesityl-, tosyl-, and $\mathrm{CF}_{3}$ benzylsulfonamide all demonstrated complete reduction of S. aureus, Arthrobacter sp., and E. coli at the solid/air interface over a $3 \mathrm{~h}$ period. ${ }^{79}$ The sulfonamide coatings were found to be effective at a cationic charge density lower than the suggested minimum charge density for kill of $5 \times 10^{15}\left[\mathrm{Q}^{+}\right] \mathrm{cm}^{-2} .{ }^{63}$ The high antibacterial activity and low cationic charge density make the sulfonamide group on the quaternary ammonium moiety an interesting substituent for investigations.

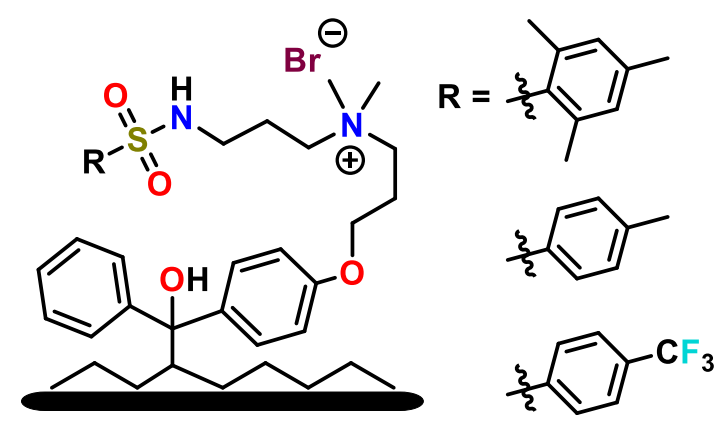

Sulfonamide analogs on PS

Caschera et al. RCS Adv. 2019

Figure 1.15 Mesityl-, tosyl-, $\mathrm{CF}_{3}$-benzylsulfonamide small molecule surface attached coatings prepared by UV cure recently reported by the Foucher group ${ }^{79}$

The first notable studies of antimicrobial polymeric phosphonium salts were conducted by Kanazawa in the mid-1990s. They compared polymeric ammonium salts to their phosphonium counterparts and reported higher antibacterial efficacy and thermal stability from polymeric phosphonium. ${ }^{13}$ Kanazawa et al. continued work on polymeric phosphonium and investigated the role of alkyl chain lengths and compared these to their monomeric counterparts. ${ }^{13,80}$ Interestingly, Kanazawa et al. also reported the earliest surface attached phosphonium polymer film, seen in Figure 1.16(a) ${ }^{81}$ Vinylbenzyl chloride (VBC) underwent graft polymerization onto a PP surface 
via photoinitation of benzophenone. Phosphonium salt was then immobilized to the surface by quaternization of tributyl or trioctylphosphine. The surface demonstrated antibacterial activity against $E$. coli and $S$. aureus through a modified dynamic contact antimicrobial testing method. ${ }^{81}$ Recently, the Ragogna and Gillies Group reported the synthesis and antibacterial activity of a polyphosphonium semi-interpenetrating polymer network (SIPN), illustrated in Figure 1.16(b). ${ }^{82}$ The polyphosphonium was synthesized by a controlled radical polymerization method, reversible addition-fragmentation chain-transfer (RAFT) polymerization. Through the addition of a crosslinker and UV-active photoinitiator, the SIPN was created as non-adhering film on a glass substrate. In their work, it was demonstrated that the alkyl chain length on the quaternary phosphonium had implications on biocidal activity against gram-negative and gram-positive bacteria, surface biofouling, and distribution of the cationic polymer in the network. ${ }^{82}$

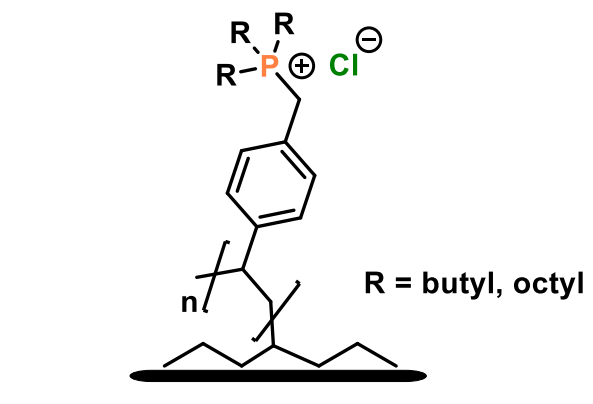

PS-Phosphonium brushes Kanazawa et al. Polym. Sci. Part A Polym. Chem. 1993<smiles>[R][PH]([R])(CCl)Cc1ccc(C(C)CC)cc1</smiles>

Polyphosphonium SIPN Surfaces Cuthbert et al. J. Mater. Chem. B 2016

(a)

Figure 1.16 Notable quaternary phosphonium surfaces that has previously been reported to demonstrate antibacterial properties (a) Styrenic based quaternary phosphonium polymer brushes synthesized by surface initiated uncontrolled free radical polymerization in a "grafting from" approach (b) A film consisting of phosphonium polymer networks prepared by UV-initiated crosslinking of the polyphosphonium and diacrylate.

While these coatings are reported to be successful against gram-positive and negative bacteria, these coatings did not undergo robustness testing, limiting potential applications in high 
traffic areas and long-term usage. Exploration into forming polymeric coatings that are solvent resistant and robust will allow for applications in the real world.

\subsection{Research Objective}

In this work, the synthesis of polymeric antimicrobial coatings by CRP methods for narrow polydispersity and high molecular weight is reported. NMP polymerization of VBC and its derivatives is revisited since the seminal work by Georges et al. ${ }^{83}$ Parallel RAFT polymerization was also explored for these monomers. Through post-polymerization modifications, the polymers are quaternized with amines and phosphines for the creation of quaternary ammonium and phosphonium coatings suitable for plastic substrates. These antimicrobial surfaces were tested against gram-negative and gram-positive bacteria by the LDI protocol ${ }^{59}$ to examine bioactivity at the solid/air interface. The one step "grafting to" approach of polymeric compounds aim to formulate uniform coatings or additives that possess higher durability properties to withstand high traffic microbial environments. 


\section{RESULTS AND DISCUSSION}

2.1 Quaternary Ammonium and Phosphonium Random Block Copolymer Coatings

\subsubsection{Monomer Synthesis}

Vinylbenzylbenzophenone monomer (VBBP, 1) was synthesized following a modified procedure previously described by Lin et al. ${ }^{84}$ The Williamson Ether reaction (Scheme 2.1) was carried out using 4-hydroxybenzophenone and VBC with potassium iodide (KI) as a catalyst and recrystallized twice from petroleum ether with a yield of $82 \%$.

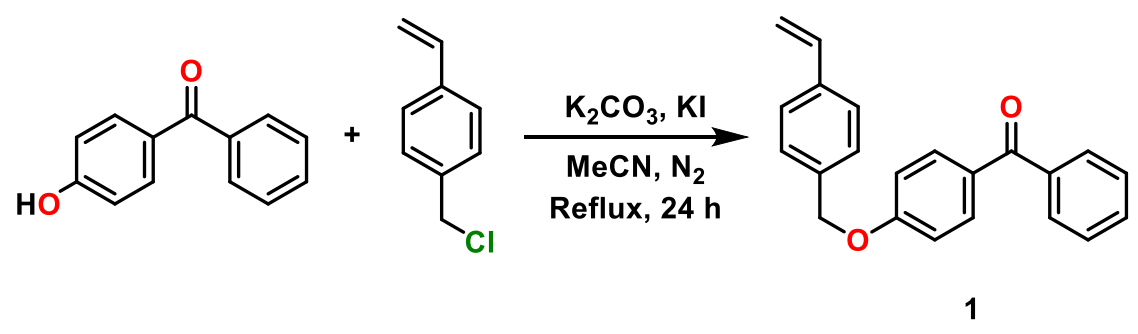

Scheme 2.1 The Williamson Ether reaction between VBC and 4-hydroxybenzophenone to synthesize the VBBP monomer

NMR analysis confirmed the synthesis of monomer 1. On the ${ }^{1} \mathrm{H}$ NMR spectrum (Figure A 1), the most indicative signal corresponds to the $\mathrm{CH}_{2}$ adjacent to the benzophenone substituent, highlighted in Figure 2.1. The $\mathrm{CH}_{2}$ signal of monomer $\mathbf{1}$ appears at $5.13 \mathrm{ppm}$, more downfield than the $\mathrm{CH}_{2}$ of the $\mathrm{VBC}$ starting material, $4.59 \mathrm{ppm}$, suggesting that the benzophenone substituent is more electron withdrawing than the chlorine group. The remaining proton signals correspond to the remainder of the monomer, with additional aryl proton signals in the 7.3-8.0 ppm range, corresponding to the benzophenone group. 


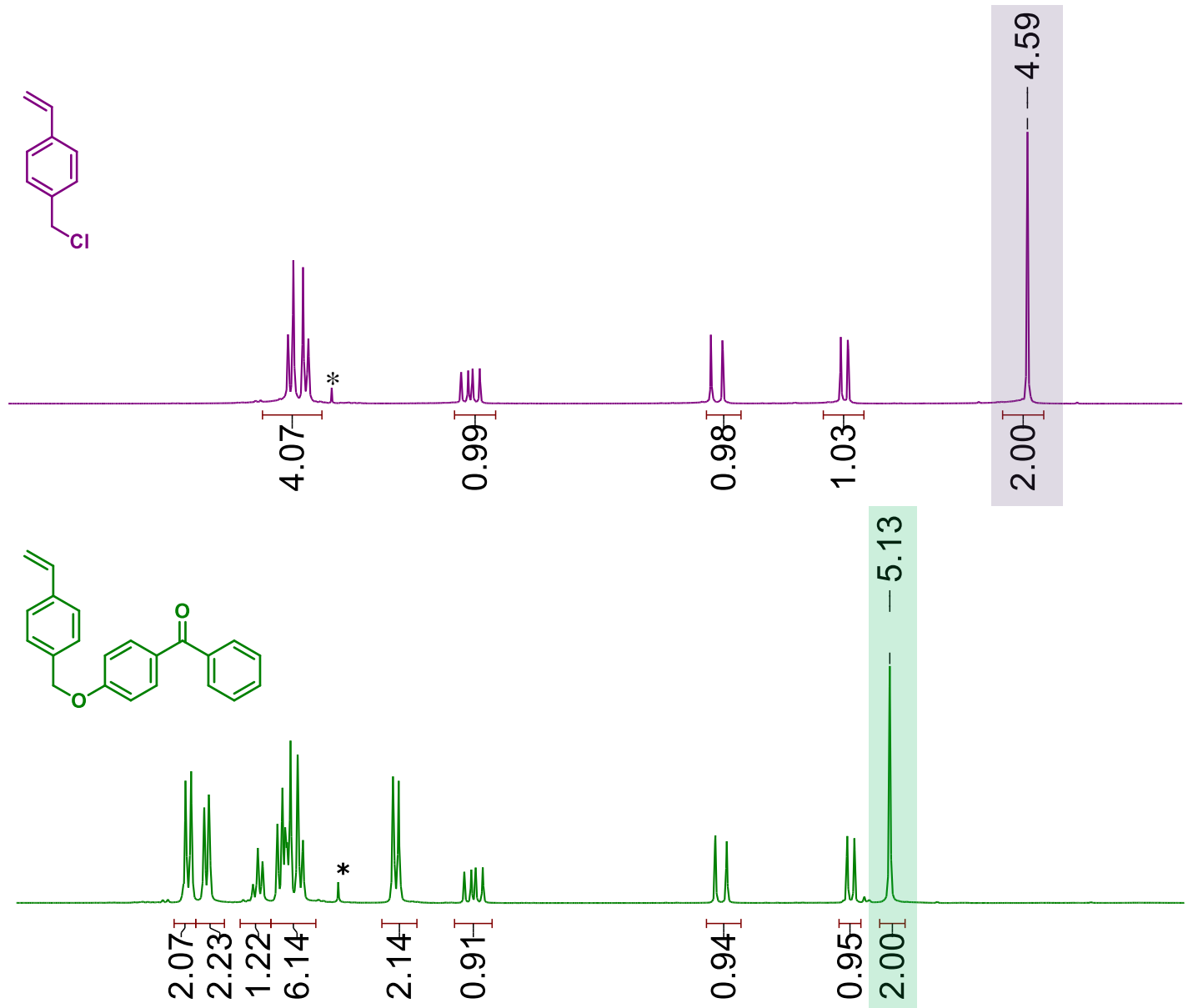

Figure 2.1 Stacked ${ }^{1} \mathrm{H}$ NMR spectra of $\mathbf{1}$ and corresponding VBC starting material $\left(\mathrm{CDCl}_{3}\right)$

Carbon NMR analysis of $\mathbf{1}$ also confirmed the monomer structure (Figure A 2). The signal at $195.49 \mathrm{ppm}$ corresponding to the carbonyl group of the benzophenone moiety is most revealing for structure confirmation. The remaining carbon signals were assigned with aid from 2D NMR analysis, seen in Figure A 3-Figure A 5. Mass spectrometry analysis by ESI-Q-TOF further established the synthesis of 1 (Figure A 6). 


\subsubsection{Controlled Radical Polymerization of VBBP and VBC Monomers}

\subsubsection{Nitroxide Mediated Polymerization}

Poly(VBC-BP) random copolymer (2) was synthesized by nitroxide mediated polymerization (NMP) of monomers $\mathrm{VBC}$ and $\mathbf{1}$ initiated by benzoyl peroxide (BPO) and mediated by (2,2,6,6-tetramethylpiperidin-1-yl)oxyl (TEMPO), illustrated in Scheme 2.2. Experiments using varying molar equivalents of monomer and reagent and different solvents resulted in polymers of different molecular weights $\left(\mathrm{M}_{\mathrm{w}}\right)$, dispersities $(\nexists)$ and yield, summarized in Table 2.1.
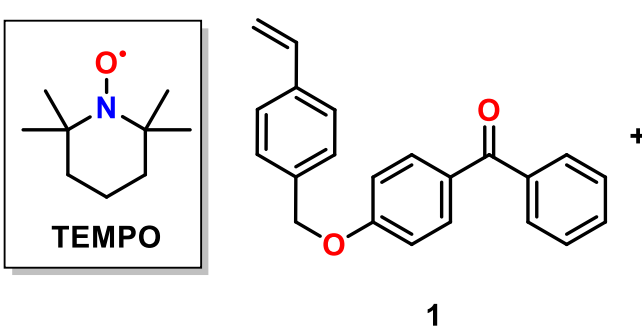
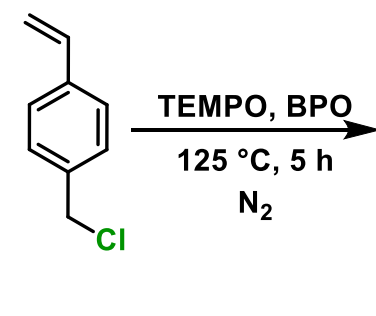

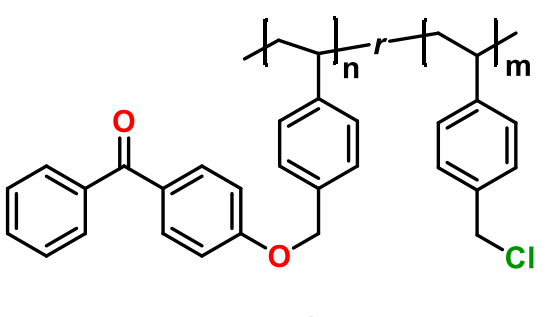

2

Scheme 2.2 Nitroxide mediated copolymerization of VBBC and VBC initiated by BPO and mediated by TEMPO

Table 2.1 Summary of NMP conditions in the synthesis of poly(VBC-BP) (2)

\begin{tabular}{|c|c|c|c|c|c|c|c|c|}
\hline Entry & $\begin{array}{c}\text { VBBP } \\
(\mathbf{m o l} \text { \%) }\end{array}$ & $\begin{array}{c}\text { VBC } \\
(\mathbf{m o l} \text { \%) }\end{array}$ & $\begin{array}{c}\text { TEMPO } \\
(\mathbf{m o l} \%)\end{array}$ & $\begin{array}{c}\text { BPO } \\
(\mathbf{m o l} \%)\end{array}$ & Solvent & $\begin{array}{c}\text { Yield by } \\
\mathbf{1}^{\text {H NMR }}\end{array}$ & $\begin{array}{c}\mathbf{M}_{\mathbf{w}} \\
(\mathbf{k D a})\end{array}$ & $\boldsymbol{D}$ \\
\hline 1 & 0 & 100 & 0.73 & 0.58 & Neat & $40 \%$ & 34.3 & 1.12 \\
\hline 2 & 0 & 100 & 0.58 & 0.73 & Neat & $87 \%$ & 109.0 & 1.16 \\
\hline 3 & 20 & 80 & 0.58 & 0.73 & Neat $^{a}$ & $33 \%$ & 25.2 & 1.03 \\
\hline 4 & 20 & 80 & 0.58 & 0.73 & Tol & $25 \%$ & 21.7 & $1.11^{b}$ \\
\hline 5 & 5 & 95 & 0.58 & 0.73 & Neat & $77 \%$ & 38.8 & 1.07 \\
\hline 6 & 5 & 95 & 0.73 & 0.58 & Neat & $67 \%$ & 15.1 & 1.02 \\
\hline
\end{tabular}

${ }^{a}$ Low solubility, ${ }^{b}$ Bimodal

The polymerization of monomers began with revisiting the homopolymerization of VBC, previously reported by Georges et al. ${ }^{83}$ The bulk polymerization was carried out at $125{ }^{\circ} \mathrm{C}$ with a 
TEMPO/BPO ratio of 1.3 (Table 2.1, Entry 1). After $5 \mathrm{~h}$, there was a $40 \%$ monomer conversion determined by ${ }^{1} \mathrm{H}$ NMR analysis. The polymer was found to have an absolute molecular weight $\mathrm{M}_{\mathrm{w}}=34.3 \mathrm{kDa}$ with well controlled molecular weight distribution of $Ð=1.12$ by GPC analysis compared to broad and narrow PS standards. At a TEMPO/BPO ratio of 0.75 , the reaction mixture after $5 \mathrm{~h}$ was a resin, while the previous TEMPO/BPO ratio gave a viscous gel. The molecular weight was significantly increased to $109.0 \mathrm{kDa}$ with a broader but modest molecular weight distribution of 1.16 at $87 \%$ monomer conversion. The impressive molecular weight surpassed previously reported polymerizations of VBC mediated by TEMPO of $20.2 \mathrm{kDa}$ at $Ð=1.34^{83}, 33.1$ $\mathrm{kDa}$ at $Ð=1.24$, and $71.7 \mathrm{kDa}$ at $Ð=1.57 .^{45}$ With this successful synthesis of high molecular weight, controlled homopolymerization of VBC mediated by TEMPO, incorporation of the benzophenone moiety onto the polymer could be carried out by Williamson Ether reaction in the post-polymerization modification step. However, this step was found to be problematic as the homopolymer backbone was found to be base sensitive, resulting in polymer chain cleavage and low molecular weight chains. The base, $\mathrm{K}_{2} \mathrm{CO}_{3}$, was used in the attempted partial substitution of acrylic acid (Scheme 2.3) by Williamson Ether synthesis. The VBC homopolymer starting material had a molecular weight of $61.7 \mathrm{kDa}$, but the resulting polymer was found to have a reduced molecular weight of $1.5 \mathrm{kDa}$, indication of polymer degradation. An alternative route to include the benzophenone moiety is to copolymerize VBC and benzophenone monomers.

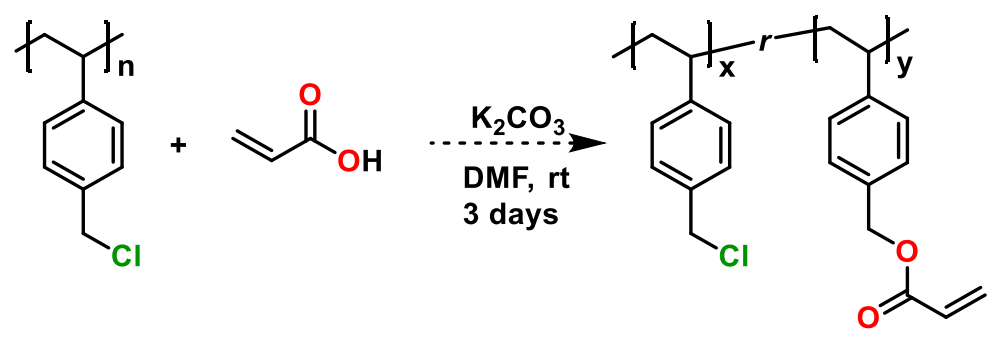

Scheme 2.3 Attempted partial substitution of acrylic acid on VBC homopolymer by Williamson Ether synthesis 
The first copolymerization attempt was a bulk polymerization with a $20 \mathrm{~mol} \%$ VBBP loading with a 0.75 TEMPO/BPO ratio (Table 2.1, Entry 3 ). While the resulting polymer molecular weight distribution was well-controlled at $Ð=1.03$ with a modest molecular weight of $25.2 \mathrm{kDa}$, a 20 mol \% VBBP loading was too high for good solubilization in VBC, resulting in low monomer conversion of $33 \%$. In hopes of increasing solubilization, solution polymerization of VBBP at 20 mol $\%$ and VBC was attempted at a 0.75 TEMPO/BPO ratio in toluene (Entry 4, Table 2.1). After $5 \mathrm{~h}$, the polymer was found to have a promising molecular weight of $21.7 \mathrm{kDa}$ with a $25 \%$ monomer conversion. With longer reaction times, the low monomer conversion could most likely be increased as the polymerization was diluted in solvent. While molecular weight and yields were promising, further GPC analysis indicated that the polymer was bimodal with $Ð=1.11$, suggesting continued solubility issues of the monomers in toluene. The solubility problem was ultimately addressed with a lower VBBP loading (5 mol \%) and bulk polymerization with VBC at a 0.75 TEMPO/BPO ratio, yielding a monomodal molecular weight distribution of $\oslash=1.07$ with modest molecular weight of $38.8 \mathrm{kDa}$ at $77 \%$ monomer conversion. When the copolymerization was carried out with Georges et al. 1.3 TEMPO/BPO ratio, a low molecular weight of $15.1 \mathrm{kDa}$ was found instead, making the copolymerization following the conditions listed in Entry 5, Table 2.1 the most ideal. The GPC trace for polymerization can be seen in Figure B 1.

NMR analysis confirmed the formation of polymer due to broadened signals and the disappearance of the vinyl signals in the 5.3-6.0 ppm range, emphasized in the proton NMR spectra in Figure 2.2(a). Presence of both monomers in copolymer $\mathbf{2}$ was indicated by the signals assigned to the $\mathrm{CH}_{2}$ group adjacent to the chlorine and benzophenone group. The more upfield signal in the 5.0-5.1 ppm range corresponds to the methylene protons adjacent to the benzophenone functionality (Figure 2.2(b)), in agreement with its respective electron withdrawing properties in 
comparison to the chlorine. As a result, the downfield signal present at the 4.5-4.7 ppm range corresponds to the methylene protons adjacent to the chlorine, accentuated in Figure 2.2(c). Proton integrations obtained from ${ }^{1} \mathrm{H}$ NMR spectrum and elemental analysis were utilized to estimate the resultant VBBP loading in copolymer 2.

(a) (b) (c)

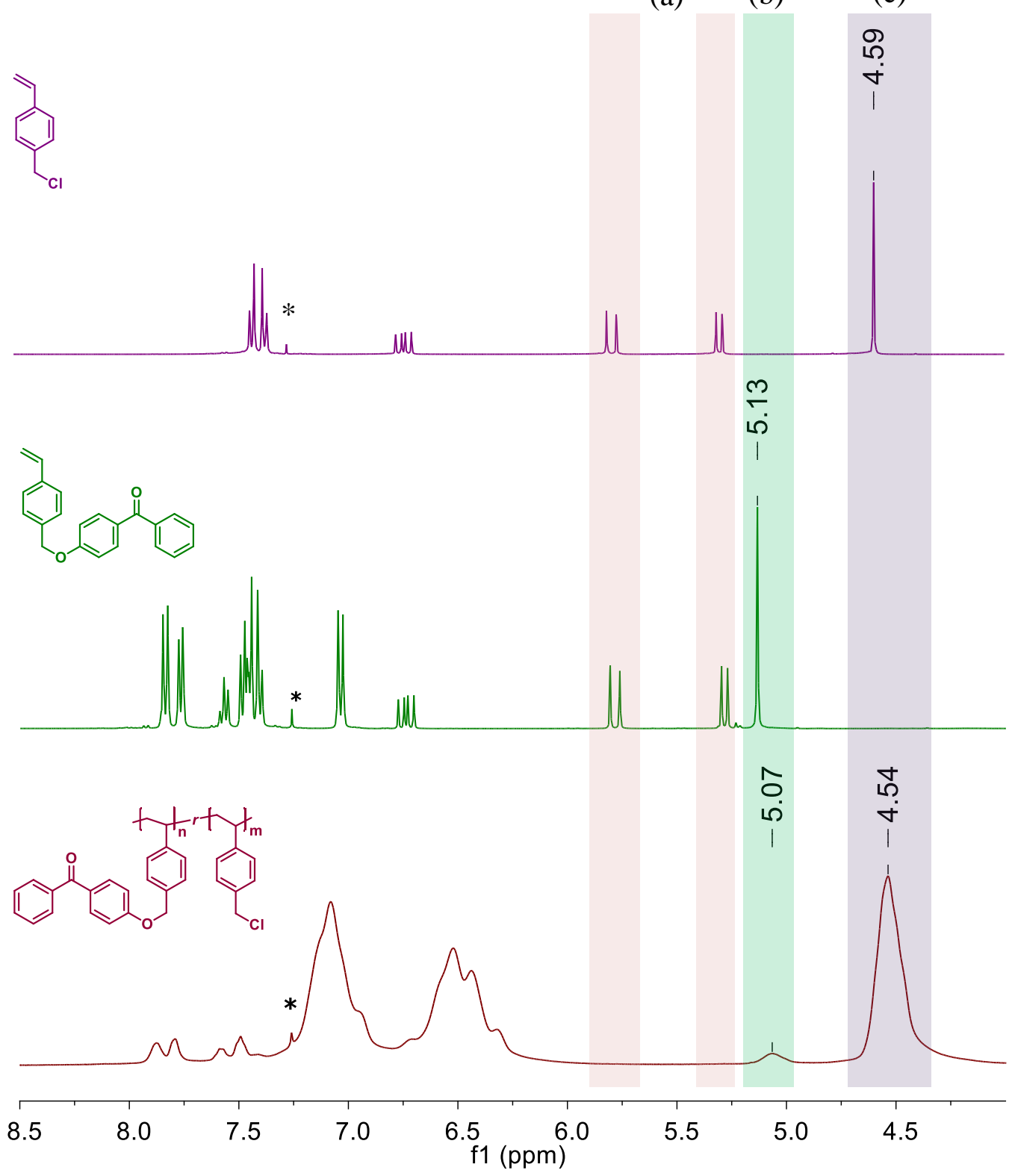

Figure 2.2 Stacked ${ }^{1} \mathrm{H}$ NMR spectra of poly(VBC-BP) 2 against VBC and VBBP (1) monomers $\left(\mathrm{CDCl}_{3}\right)$ 
Proton integration of the signals corresponding to the $\mathrm{CH}_{2}$ protons adjacent to the chlorine or benzophenone substituent were used, and these signals in $\mathrm{CDCl}_{3}$ and $\mathrm{DMSO}-\mathrm{d}_{6}$ can be found summarized in Table 2.2. From the proton integrations of the ${ }^{1} \mathrm{H} \mathrm{NMR}\left(\mathrm{CDCl}_{3}\right)$ spectrum of 2 , the random copolymer had an estimated resultant $\mathrm{VBBP}$ percentage of $\sim 4 \%$ making the $\mathrm{VBBP}$ to VBC ratio 1:27. When the ${ }^{1} \mathrm{H}$ NMR analysis of 2 was performed in DMSO- $\mathrm{d}_{6}$, the VBBP loading was estimated to be $\sim 8 \%$ with a VBBP:VBC ratio of 1:12. The varying proton integrations between the two solvents is the result of differing solubilities of the copolymer in chloroform and DMSO, making ${ }^{1} \mathrm{H}$ NMR analysis an approximate estimation.

Table 2.2 Summary of estimated monomer loading in the poly(VBC-BP) random copolymer by ${ }^{1} \mathrm{H}$ NMR

\begin{tabular}{|l|c|c|c|c|}
\hline & \multicolumn{2}{|c|}{$\mathbf{1}^{\mathbf{H}}\left(\mathbf{C H}_{2}\right) \boldsymbol{\delta}$} & \multirow{2}{*}{$\begin{array}{c}\text { Estimated VBBP \% } \\
\text { Loading }\end{array}$} & \multirow{2}{*}{ VBBP:VBC } \\
\hline Deuterated Solvent & Benzophenone & Chlorine & $4 \%$ & $1: 27$ \\
\hline CDCl3 & 5.07 & 4.54 & $8 \%$ & $1: 12$ \\
\hline DMSO-d6 & 5.11 & 4.65 & \\
\hline
\end{tabular}

In hopes of obtaining a more accurate representation of VBBP loading, elemental analysis of carbon and hydrogen was attained for further estimation of benzophenone composition (Table 2.3). Elemental analysis of $\mathbf{2}$ indicated that the random copolymer had a final VBBP loading of $6 \%$, resulting in a $\mathrm{VBBP}: \mathrm{VBC}$ ratio of $1: 16$, the average of the percent compositions obtained by proton NMR. Thermoanalysis of $\mathbf{2}$ by differential scanning calorimetry (DSC) (Figure C 1) found a glass transition temperature $\left(T_{g}\right)$ of $110.8^{\circ} \mathrm{C}$, indicating an amorphous polymer.

Table 2.3 Elemental analysis results of poly(VBC-BP) random copolymer in estimation of benzophenone loading

\begin{tabular}{|l|c|c|c|c|}
\hline & C & H & Estimated VBBP \% Loading & VBBP:VBC \\
\hline Found & 72.34 & 5.85 & $6 \%$ & $1: 16$ \\
\hline Calculated & 72.34 & 5.92 & 6 & \\
\hline
\end{tabular}




\subsubsection{Reversible Addition-Fragmentation Chain-Transfer Polymerization}

RAFT polymerization conditions were explored by homopolymerization of VBC to synthesize poly(VBC) (3), illustrated in Scheme 2.4. Varying mole concentrations of initiators, BPO or azobisisobutyronitrile (AIBN), and dithiobenzoate RAFT agent 4-cyano-4(phenylcarbonothioylthio)pentanoic acid (CTA1) and subsequent molecular weight and dispersity are summarized in Table 2.4. In the first trial of this bulk polymerization, VBC was initiated with $0.4 \mathrm{~mol} \%$ of AIBN and mediated with $0.6 \mathrm{~mol} \%$ of CTA1 at $80{ }^{\circ} \mathrm{C}$ for $24 \mathrm{~h}$ (Entry 1, Table 2.4). The reaction had a fairly low monomer conversion and resulted in a low molecular weight homopolymer of $11.1 \mathrm{kDa}$ with a narrow $Đ$ of 1.04. These conditions were attempted again (Entry 2, Table 2.4) but initiated by $\mathrm{BPO}$ at $100{ }^{\circ} \mathrm{C}$. In comparison to AIBN, the polymerization had a higher monomer conversion at $62 \%$ and higher molecular weight of $23.5 \mathrm{kDa}$ while maintaining a narrow dispersity of 1.04. Two more attempts with varying initiator and RAFT agent mole ratios were carried out in the same reaction conditions to yield homopolymers with molecular weights, dispersities, and monomer conversions similar to the second attempt (Entry $3 \& 4$, Table 2.4). GPC analysis of these samples exhibited bimodality, indicating the presence of oligomers and nonuniform polymer chains (Figure B 2). 

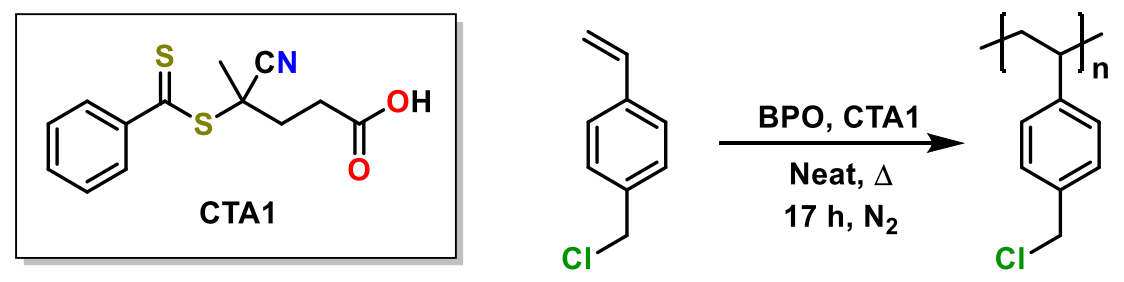

3

Scheme 2.4 RAFT polymerization initiated by BPO and mediated by CTA1

Table 2.4 Summary of RAFT polymerization conditions initiated by BPO and resultant conversion, dispersity, and molecular weight of $\mathbf{3}$

\begin{tabular}{|c|c|c|c|c|c|c|c|}
\hline Entry & $\begin{array}{c}\text { Initiator } \\
\text { mol \% }\end{array}$ & $\begin{array}{c}\text { CTA1 mol \% } \\
\text { RAFT Agent }\end{array}$ & Temp & $\begin{array}{c}\text { \% } \\
\text { Conversion }\end{array}$ & $\begin{array}{c}\text { Repeat } \\
\text { Units }\end{array}$ & $\mathbf{M}_{\mathbf{w}}$ & $\boldsymbol{D}$ \\
\hline $\mathbf{1}$ & $0.4 \mathrm{~mol} \%$ & $0.6 \mathrm{~mol} \%$ & $80^{\circ} \mathrm{C}$ & $51 \%$ & 73 & $11.1 \mathrm{kDa}$ & 1.04 \\
\hline $\mathbf{2}$ & $0.4 \mathrm{~mol} \%$ & $0.6 \mathrm{~mol} \%$ & $100{ }^{\circ} \mathrm{C}$ & $62 \%$ & 154 & $23.5 \mathrm{kDa}$ & 1.04 \\
\hline $\mathbf{3}$ & $0.4 \mathrm{~mol} \%$ & $0.4 \mathrm{~mol} \%$ & $100{ }^{\circ} \mathrm{C}$ & $57 \%$ & 145 & $22.1 \mathrm{kDa}^{b}$ & $1.04^{c}$ \\
\hline $\mathbf{4}$ & $0.6 \mathrm{~mol} \%$ & $0.4 \mathrm{~mol} \%$ & $100{ }^{\circ} \mathrm{C}$ & $57 \%$ & 195 & $29.7 \mathrm{kDa}^{b}$ & $1.03^{c}$ \\
\hline
\end{tabular}

${ }^{a}$ Initiated by AIBN $\quad{ }^{b} \mathrm{M}_{\mathrm{w}}$ combined $\quad{ }^{c}$ Bimodal - only main peak analyzed for $\theta$

Similar RAFT polymerization conditions of VBC with RAFT agent 4-cyano-4[(dodecylsulfanylthiocarbonyl)sulfanyl]pentanoic acid (CTA2) initiated by BPO to synthesize poly(VBC) (4) (Scheme 2.5) were also explored. Initiator and RAFT agent equivalencies and resulting polymer weight and dispersity are tabulated in Table 2.5. At equal equivalents of initiator and RAFT agent (Entry 1, Table 2.5), there was a modest monomer conversion of $77 \%$ with a $\mathrm{M}_{\mathrm{w}}$ $=19.3 \mathrm{kDa}$ and narrow dispersity of 1.02 , comparable to the CTA1 mediated reactions discussed previously. Other AIBN and CTA2 ratios (Entry $2 \& 3$, Table 2.5) resulted in polymers of bimodal distribution, indicated by the GPC trace, Figure B 3. 

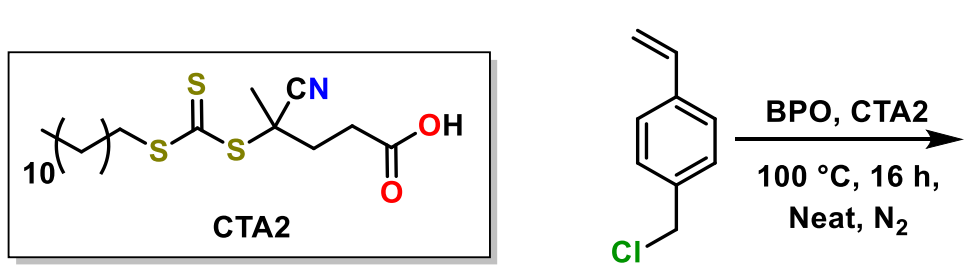<smiles>CC(C)(C)C(C)(C)C(C)(C)C</smiles>

4

Scheme 2.5 RAFT polymerization of VBC, initiated by BPO with RAFT agent CTA2

Table 2.5 RAFT polymerization conditions of VBC initiated by BPO with RAFT agent CTA2 and subsequent molecular weight and dispersity of $\mathbf{4}$

\begin{tabular}{|c|c|c|c|c|c|c|}
\hline Entry & Initiator mol \% & $\begin{array}{c}\text { CTA2 mol \% } \\
\text { RAFT Agent }\end{array}$ & \% Conversion & $\begin{array}{c}\text { Repeat } \\
\text { Units }\end{array}$ & $\mathbf{M}_{\mathbf{w}}$ & $\boldsymbol{\#}$ \\
\hline 1 & $0.4 \mathrm{~mol} \%$ & $0.4 \mathrm{~mol} \%$ & $77 \%$ & 126 & $19.3 \mathrm{kDa}$ & 1.02 \\
\hline 2 & $0.4 \mathrm{~mol} \%$ & $0.6 \mathrm{~mol} \%$ & $63 \%$ & 98 & $14.9 \mathrm{kDa}^{a}$ & $1.03^{b}$ \\
\hline 3 & $0.6 \mathrm{~mol} \%$ & $0.4 \mathrm{~mol} \%$ & $82 \%$ & 229 & $35.0 \mathrm{kDa}^{a}$ & $1.02^{b}$ \\
\hline
\end{tabular}

${ }^{a} \mathrm{M}_{\mathrm{w}}$ combined $\quad{ }^{b}$ Bimodal - only main peak analyzed for $\oslash$

For comparisons to the NMP for the synthesis of $\mathbf{3}$, RAFT polymerization of VBC initiated by $\mathrm{BPO}$ and mediated with CTA1 or CTA2 were attempted under a $5 \mathrm{~h}$ reaction time (Scheme 2.6). Resulting molecular weights and dispersities of homopolymer poly(VBC) (5) are tabulated in Table 2.6. At $5 \mathrm{~h}$, the polymerization was incomplete with a low monomer conversion and low molecular weights for both RAFT agents. While the dispersities for both conditions were narrow, the GPC trace exhibit bimodality (Figure B 4).

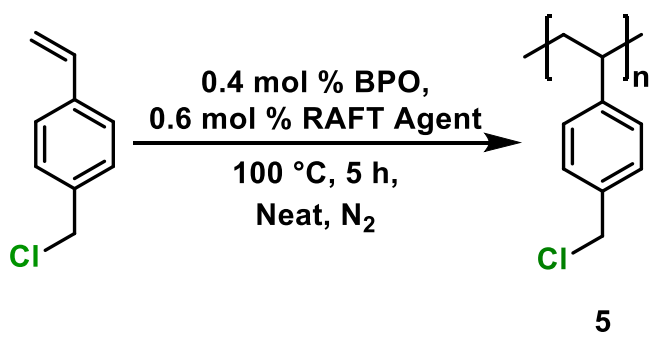

Scheme 2.6 RAFT polymerization of VBC initiated by BPO at $5 \mathrm{~h}$ time point 
Table 2.6 RAFT polymerization conditions of VBC initiated by BPO with RAFT agent CTA1 and CTA 2 for $5 \mathrm{~h}$ and resulting polymer weight, dispersity, and conversion of $\mathbf{5}$.

\begin{tabular}{|c|c|c|c|c|}
\hline RAFT Agent & \% Conversion & Repeat Units & $\mathbf{M}_{\mathbf{w}}$ & $\boldsymbol{D}$ \\
\hline CTA1 & $28 \%$ & 59 & $9.0 \mathrm{kDa}^{a}$ & $1.07^{b}$ \\
\hline CTA2 & $43 \%$ & 27 & $4.2 \mathrm{kDa}^{a}$ & $1.02^{b}$ \\
\hline
\end{tabular}

${ }^{a} \mathrm{M}_{\mathrm{w}}$ combined ${ }^{b}$ Bimodal

While RAFT polymerizations of VBC in the work gave superior, narrow dispersities, NMP experiments presented the highest molecular weight polymer of $109.0 \mathrm{kDa}$ at a shorter reaction time. Consequently, copolymer 3 synthesized by NMP was used as the foundation for the formulation of cationic coatings.

\subsubsection{Post-polymerization Quaternization of Poly(VBC-BP) Random Copolymer}

\subsubsection{Synthesis}

The poly(VBC-BP) (2) copolymer underwent quaternization by post-polymerization modification, illustrated in Scheme 2.7. The chlorine on the VBC repeat units of the copolymer 2 was aminated/phosphosinated with triethylamine (poly(VBBP-NEt 3 ), 6), trimethylphosphine (poly(VBBP-PMe $), \quad 7), \quad N, N$-dimethyldodecylamine $\quad($ poly(VBBP-NC12), $\quad 8), \quad N-(3-$ (dimethylamino)propyl)-4-methylbenzenesulfonamide $\quad(\operatorname{poly}(\mathrm{VBBP}-\mathrm{Ts} \mathrm{SA}), \quad$ 9), $\quad \mathrm{N}, \mathrm{N}-$ dimethyloctadecylamine (poly(VBBP-NC18), 10), or $\quad N$-(3-(dimethylamino)propyl)-4(trifluoromethyl)benzenesulfonamide (poly $\left(\mathrm{VBBP}-\mathrm{CF}_{3} \mathrm{SA}, \quad 11\right)$ refluxed in a methanol $(\mathrm{MeOH}) /$ acetone mixture or acetonitrile $(\mathrm{MeCN})$ for 1-24 h to yield compounds 6-11 as white or off-white fine powders. These polymers were purified by precipitation into cold diethyl ether $\left(\mathrm{Et}_{2} \mathrm{O}\right)$ or hexanes from $\mathrm{MeOH}$ or ethanol $(\mathrm{EtOH})$. The molecular weights of polymers 6-11 were expected to be the same as the initial presubstituted polymer. The ionic nature of the quaternary ammonium/phosphonium active sites attract water, and thus the polymers were found to be 
extremely hygroscopic, readily absorbing moisture from the air to eventually convert to a gel when improperly stored.

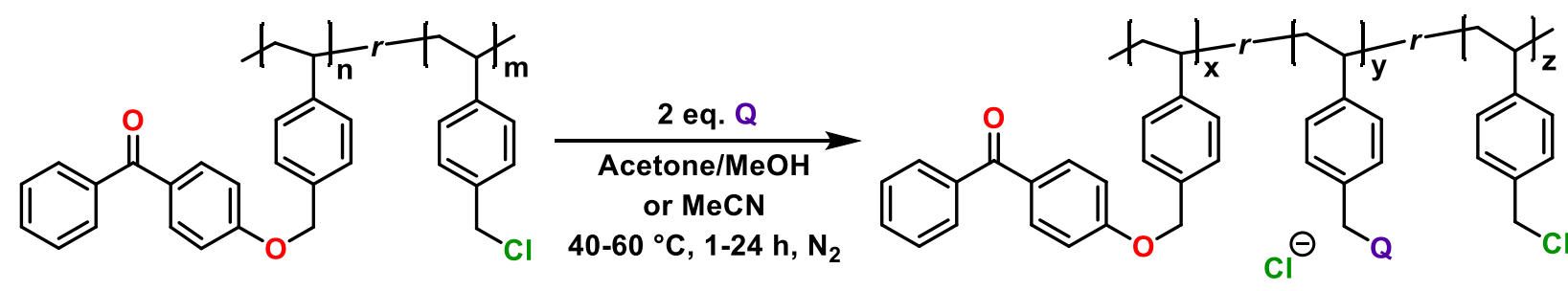

2

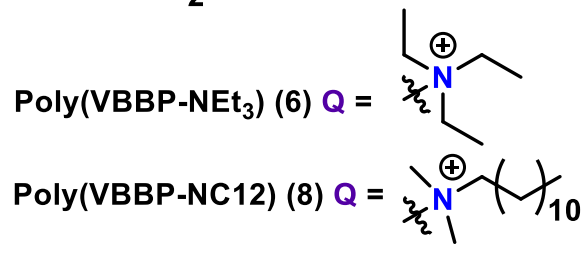

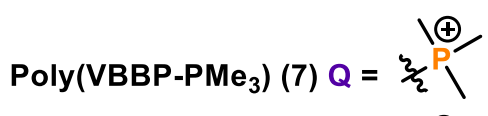<smiles>Cc1ccc(S(=O)(=O)NCCC[N+](C)(C)C=[Ge][Ba][Ba])cc1</smiles>

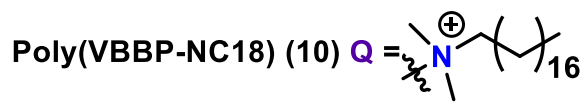<smiles>C[N+](C)(C)CCCNS(=O)(=O)c1ccc(C(F)(F)F)cc1</smiles>

Scheme 2.7 Amination and phosphination reactions of $\mathbf{2}$ to synthesize quaternary ammonium and phosphonium polymers 6-11

\subsubsection{Characterization}

${ }^{1} \mathrm{H}$ NMR spectroscopy of all polymers were used for structural determination. Due to steric hindrance, it is expected that not all alkylchloride substituents of $\mathbf{2}$ will undergo amination/phosphination. Partial substitution of quaternary groups on the polymer ensures that the polymer will possess three functionalities; chlorine of $\mathrm{VBC}$, benzophenone of $\mathrm{VBBP}$, and a cationic quaternary compound (Q). The three different functionalities of the polymer have varying degrees of polarity, resulting in incompatible solubilities. The cationic group is considerably more polar than the benzophenone and chlorine groups, subsequently leading to partial solubilization of the polymer in polar solvents, while other portions were insoluble. While solubility issues did not 
hinder the synthesis or work-up of the reaction, it was especially problematic when determining the compositions of the polymer, thus only an approximate estimation of percent composition was determined via ${ }^{1} \mathrm{H}$ NMR. Elemental analysis was also conducted to obtain percent composition of the polymer functionalities. From NMR analysis, residual solvent was seen to be trapped by the polymer, even after days under high vacuum drying at $60{ }^{\circ} \mathrm{C}$. Residual solvent in the polymer sample consequently contributed to complications in accurately determining percent composition by elemental analysis, again only providing an approximate estimation of functionality composition with consideration of residual solvent indicated by NMR analysis. The final functionality composition of the quaternized polymers were estimated by ${ }^{1} \mathrm{H}$ NMR and elemental analysis and summarized on Table 2.7 .

Table 2.7 Summary of estimated functionality composition of the quaternized random copolymers 6-11

\begin{tabular}{|c|c|c|c|c|c|c|}
\hline \multirow{3}{*}{ Polymer } & \multicolumn{3}{|c|}{${ }^{1}$ H NMR Analysis } & \multicolumn{3}{|c|}{ Elemental Analysis } \\
\hline & \multicolumn{2}{|c|}{$\begin{array}{c}\text { Estimated \% } \\
\text { Loading }\end{array}$} & \multirow{2}{*}{ VBBP:VBC:Q } & \multicolumn{2}{|c|}{$\begin{array}{l}\text { Estimated \% } \\
\text { Loading }\end{array}$} & \multirow[t]{2}{*}{ VBBP:VBC:Q } \\
\hline & $V B B P$ & $Q$ & & $V B B P$ & $Q$ & \\
\hline \multirow{2}{*}{6} & $12 \%$ & $60 \%$ & $2: 5: 10$ & \multirow{2}{*}{$9.5 \%$} & \multirow{2}{*}{$81 \%$} & \multirow{2}{*}{$2: 2: 17$} \\
\hline & $7.7 \%$ & $69 \%$ & $1: 3: 9$ & & & \\
\hline 7 & $4.7 \%$ & $87 \%$ & $1: 2: 19$ & $4.7 \%$ & $88 \%$ & $2: 3: 38$ \\
\hline 8 & $5.7 \%$ & $49 \%$ & $1: 8: 8.6$ & $8.0 \%$ & $75 \%$ & $1: 2: 9.5$ \\
\hline 9 & - & - & - & $5.0 \%$ & $85 \%$ & $1: 2: 17$ \\
\hline 10 & - & $55 \%$ & $4: 5$ & $7.7 \%$ & $62 \%$ & $1: 4: 8$ \\
\hline 11 & - & - & - & $5.1 \%$ & $85 \%$ & $1: 2: 16.5$ \\
\hline
\end{tabular}

- Undetermined due to solubility issues

${ }^{1} \mathrm{H}$ NMR analysis of 6 (Figure A 10) was conducted in MeOD and DMSO- $\mathrm{d}_{6}$, and from proton integrations, the functionality composition was estimated. Similar to the ${ }^{1} \mathrm{H}$ NMR signals 
of 2 , the most upfield signal in the $4.5-5.3 \mathrm{ppm}$ range corresponds to the methylene protons adjacent to the electron withdrawing benzophenone substituent. The signal of the methylene protons adjacent to the chlorine and ammonium groups overlap downfield at 4.5-4.7 ppm. The composition of the quaternized copolymer estimated by ${ }^{1} \mathrm{H}$ NMR in DMSO- $\mathrm{d}_{6}$ indicated a benzophenone and triethylammonium percentage of $12 \%$ and $60 \%$, respectively. When ${ }^{1} \mathrm{H} \mathrm{NMR}$ studies were conducted in MeOD, benzophenone and triethylammonium percent loadings were found to be $7.7 \%$ and $69 \%$, respectively. The differing percent loading in MeOD versus DMSO$\mathrm{d}_{6}$ evidently demonstrates the differing solubilities of the functionalities in the different solvent. Elemental analysis data provided an estimated benzophenone percentage of $9.5 \%$ and quaternary ammonium percentage of $81 \%$.

The proton spectrum of 7 (Figure A 11) was similar to 6. Methylene protons adjacent to the phosphonium corresponded to the most upfield resonance at $3.86 \mathrm{ppm}$, and the protons adjacent to the benzophenone functionality were found downfield at $5.22 \mathrm{ppm}$, consistent with their respective electronegativity. The more upfield $\mathrm{CH}_{2}$ signal indicates that trimethylphosphonium is less electronegative in comparison to the triethylammonium group of 6. Proton integration data from ${ }^{1} \mathrm{H}$ NMR analysis estimated the benzophenone and trimethylphosphonium composition to be $4.7 \%$ and $87 \%$, respectively. Phosphorous NMR (Figure A 12) confirmed one phosphorous environment at $26.91 \mathrm{ppm}$, in agreement with a quaternary phosphonium shift. Elemental analysis of 7 found the VBBP and phosphonium percent loading to be $4.7 \%$ and $88 \%$, respectively and aligns with NMR approximations.

NMR analysis of polymer 8 was performed in MeOD (Figure A 13). The quaternary ammonium group was found to be least electronegative compared to the chlorine and benzophenone group, specified by the upfield methylene shift at $3.48 \mathrm{ppm}$. Based off the proton 
integration of the $\mathrm{CH}_{2}$ group as well as the protons on the terminal carbon of the alkyl chain most upfield at $0.91 \mathrm{ppm}, \mathbf{8}$ was estimated to have a VBBP and quaternary ammonium percent loading of $5.7 \%$ and $49 \%$. The low degree of quaternary ammonium composition of $\mathbf{8}$ may be the result of steric hindrance brought about by the bulky $\mathrm{C} 12$ alkyl chains of $N, N$-dimethyldodecylamine substituent, preventing amination of the alkylchloride compared to the short alkyl species of $\mathbf{6}$ and 7. Elemental analysis of $\mathbf{8}$ determined VBBP composition of $5 \%$ and quaternary ammonium of $85 \%$. The quaternary ammonium composition was found to be significantly higher than the estimate provided by the ${ }^{1} \mathrm{H}$ NMR integrations. The large discrepancy between the two results are inexplicable and warrant further investigations.

Poly(VBBP-TsSA) (9) was found to be only soluble in MeOH, but NMR analysis in MeOD found that 9 was insufficiently soluble for accurate interpretation. The proton spectrum indicated MeOD only solubilized the TsSA group of 9 as the signals corresponding to the styrenic backbone are not present. Emphasized in Figure 2.3, the polymeric backbone seen in the proton NMR of 2 is not seen in the proton spectrum of 9. The unreliable proton spectrum prevented polymer composition determination by NMR, thus relying heavily on elemental analysis. Elemental analysis of $\mathrm{C}, \mathrm{H}$, and $\mathrm{N}$ indicated a VBBP loading of $5 \%$ and sulfonamide percentage of $85 \%$.

Composition of poly(VBBP-NC18) (10) was analyzed by proton NMR (Figure A 15) in the same method carried out on $\mathbf{8}$. The quaternary ammonium sites were found to have a percent loading of $55 \%$, however due to partial solubility, the proton signals were too broad and unresolved for determination of VBBP loading. Elemental analysis estimated the VBBP and quaternary ammonium loading to be $7.7 \%$ and $62 \%$. 


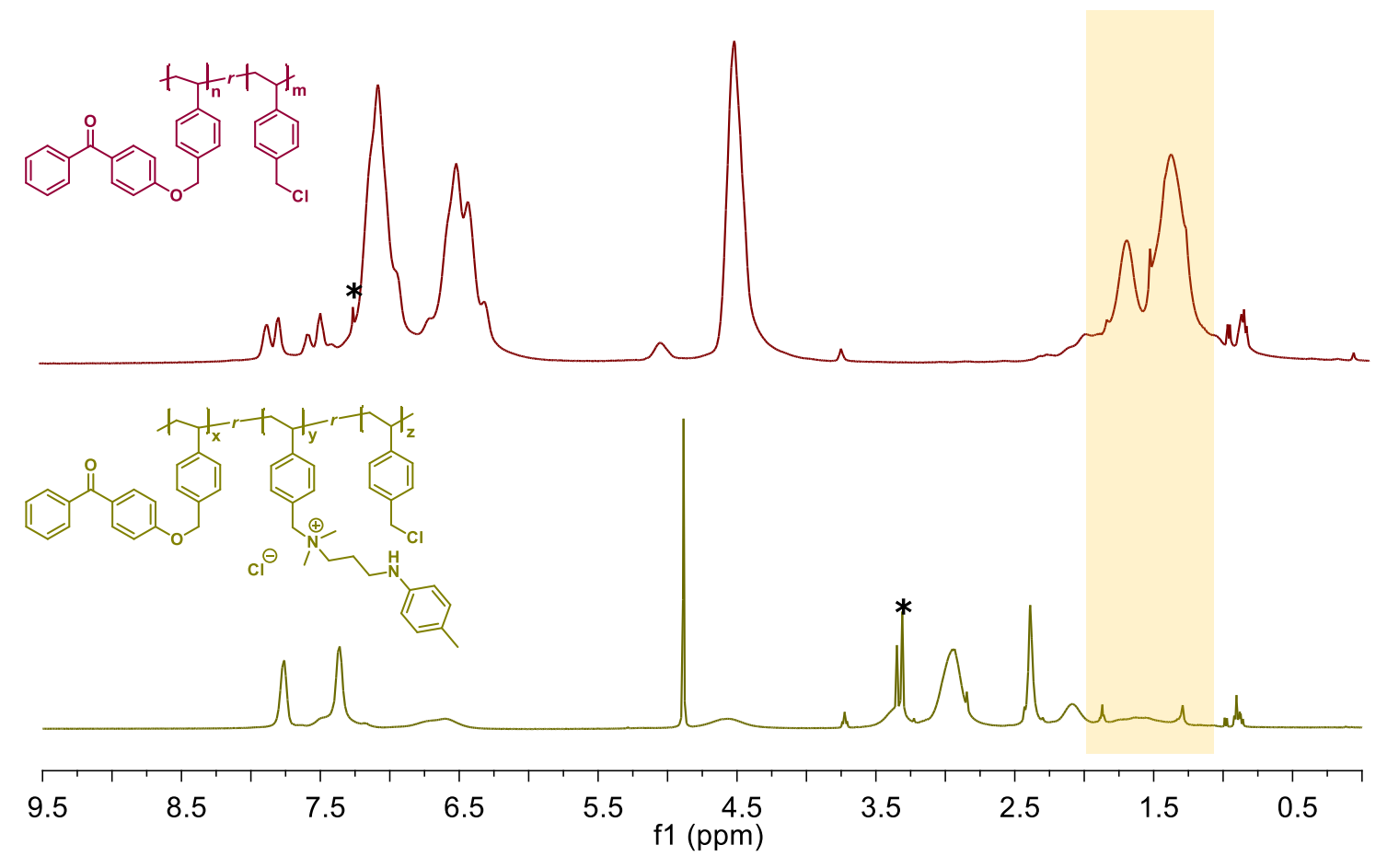

Figure 2.3 Stacked ${ }^{1} \mathrm{H}$ NMR spectra of $\mathbf{2}\left(\mathrm{CDCl}_{3}\right)$ and $\mathbf{9}(\mathrm{MeOD})$

Copolymer 11, similar to 9 , was not adequately soluble for NMR analysis. ${ }^{19} \mathrm{~F}$ NMR was obtained and formation of fluorine containing polymer was observed by appearance of a broad peak at $64.32 \mathrm{ppm}$ (Figure A 16). Residual starting material trapped in the polymer chain was also detected. Elemental analysis of $\mathrm{C}, \mathrm{H}$, and $\mathrm{N}$ determined a VBBP and sulfonamide loading of 5.1 $\%$ and $85 \%$, respectively. From these analyses, polymers 6-11 were determined to have a quaternary ammonium/phosphonium loading between 49-88 \%.

Thermoanalysis of cationic polymers were also investigated by DSC. Thermoanalysis of $\mathbf{6}$ indicated the presence of a glass transition temperature $\left(T_{g}\right)$ and melt temperature $\left(T_{m}\right)$ of $92.5^{\circ} \mathrm{C}$ and $218.5{ }^{\circ} \mathrm{C}$, respectively (Figure $\mathrm{C} 2$ ), suggesting a semi-crystalline polymer. The thermostability of $\mathbf{6}$ is surprising as the quaternary ammonium monomer, 4-vinylbenzyl triethylammonium chloride (Figure 2.4), was found to decompose at $\sim 200{ }^{\circ} \mathrm{C}$. While the monomeric form of the quaternary ammonium compound would not be suitable for participation 
in plastic extrusion or injection molding, polymer $\mathbf{6}$ is a promising candidate as an additive for thermoforming manufacture processes.

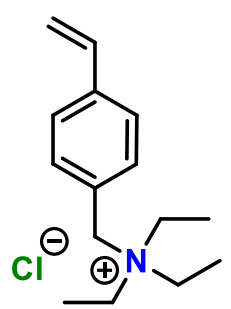

Figure 2.4 Small molecule 4-vinylbenzyl triethylammonium chloride

Polymer 7 did not present a $T_{g}$ or $T_{m}$ from DSC analysis. Poly(VBBP-NC12) (8) presented a $T_{m}$ at $210.9^{\circ} \mathrm{C}$ and is considered as another suitable candidate for injection molding (Figure $\mathrm{C}$ 3). Thermoanalysis by DSC of 9 indicated a $T_{g}$ at $119.9^{\circ} \mathrm{C}$ and a second $T_{g}$ at $158.5^{\circ} \mathrm{C}$ (Figure $\mathrm{C}$ 4). The presence of two $T_{g}$ events suggest phase separation. The phase separation may be between the sulfonamide substituent from the polymer backbone, indicated by the partial solubility in proton NMR analysis. Thermal events of these polymers are tabulated in Table 2.8 and stacked in Figure 2.5.

Table 2.8 Thermal events observed from DSC analysis

\begin{tabular}{|c|c|c|}
\hline Polymer & $\boldsymbol{T}_{\boldsymbol{g}}\left({ }^{\circ} \mathbf{C}\right)$ & $\boldsymbol{T}_{\boldsymbol{m}}\left({ }^{\circ} \mathbf{C}\right)$ \\
\hline $\mathbf{6}$ & 92.5 & 218.5 \\
\hline $\mathbf{8}$ & - & 210.9 \\
\hline $\mathbf{9}$ & 119.9 & - \\
\hline
\end{tabular}


(a) $T_{g}$ Oberserved from DSC Trace of $\mathbf{6}$ and 9
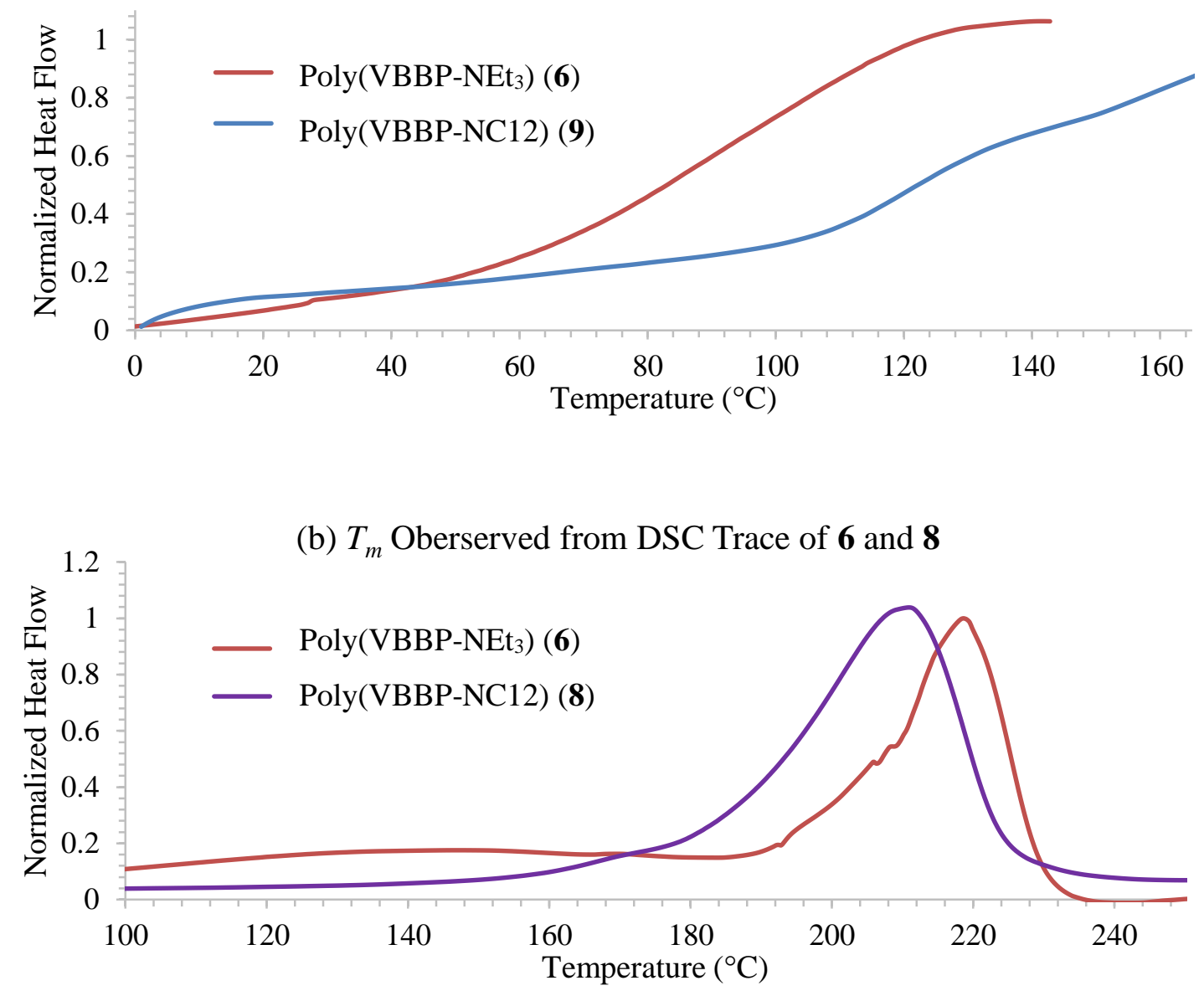

Figure 2.5 Thermal events observed from DSC trace (a) $T_{g}$ events of polymer 6 and 9 (b) $T_{m}$ events of polymer $\mathbf{6}$ and $\mathbf{8}$

\subsection{Preparation of Quaternary Polymer Coatings}

Polymers were dissolved at $10 \mathrm{w} / \mathrm{v} \%$ in EtOH for 6, a 1:1 MeOH/EtOH mixture for 7-9, 11, and $s$-butanol $(s-\mathrm{BuOH})$ for $\mathbf{1 0}$ with added phenylbis(2,4,6-trimethylbenzoyl)phosphine oxide (BAPO) photoinitiator $\left(\lambda_{\max }=317 \mathrm{~nm}\right)^{85}$ at $0.3 \mathrm{w} / \mathrm{w} \%$. The formulation $(0.32 \mathrm{~mL})$ was spuncoated onto PS substrates at $1250 \mathrm{rpm}$ and UV cured to the surface for $3 \mathrm{~min}$ at $0.158 \mathrm{~W} / \mathrm{cm}^{2}$ of UVA light. Drop-casting and dip-coating techniques achieved coatings that were thick, nonuniform, and led to significantly discoloured and burned coatings after UV curing. The substrate 
was washed twice thoroughly in a mixture of $\mathrm{MeOH} / \mathrm{dH}_{2} \mathrm{O}$, and the rinse solution was qualitatively tested for polymer that failed to graft to the surface by bromophenol blue (BPB) staining. The dianionic purple indicator dye undergoes anionic exchange with the chlorine on the quaternary species and undergoes a color change to blue. ${ }^{53}$ After two washes, the rinse solution no longer stained blue in the presence of BPB, an indication that all ungrafted polymers were removed. The resultant surface was observed to be clear with a tinge of yellow and was glossy in comparison to an uncoated surface. To visualize these coatings, the plastic substrates were submerged in a $4 \mathrm{ppm}$ BPB solution overnight and excess BPB dye was rinsed off with $\mathrm{dH}_{2} \mathrm{O}$. The plastic substrates coated with polymers 6-11 before and after BPB staining are displayed in Figure 2.6.

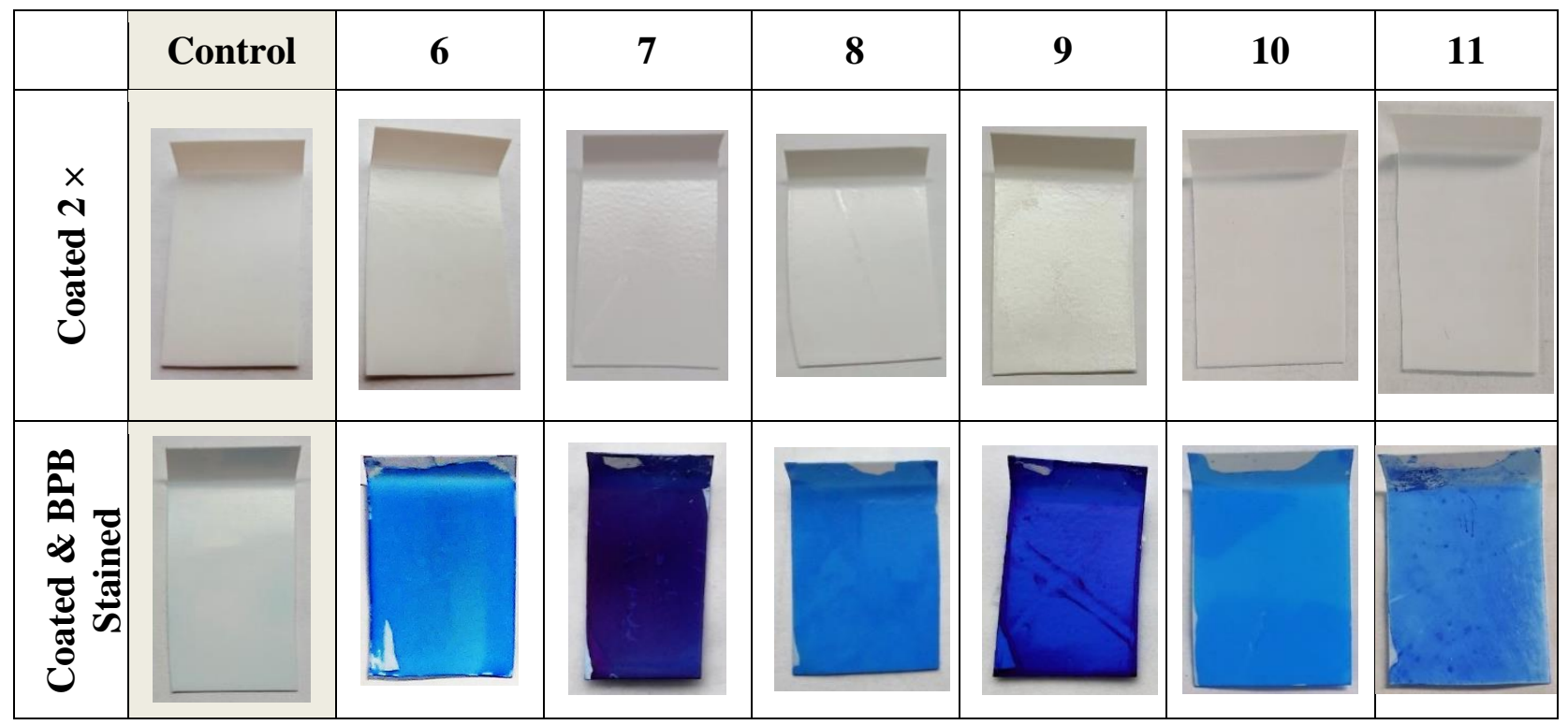

Figure 2.6 PS substrates coated with polymers 6-11 before and after BPB staining compared to the uncoated control 


\subsection{Coating Robustness}

\subsubsection{Solvent Resistance}

Solvent resistance of small molecule and polymeric quaternary ammonium treated surfaces were evaluated against $\mathrm{dH}_{2} \mathrm{O}$, IPA, MeOH, and Windex ${ }^{\circledR}$ following ASTM D5402-15. ${ }^{86}$ For comparison, the small molecule coating of C18 BP-QAC were subjected to solvent resistance testing against $\mathrm{dH}_{2} \mathrm{O}, \mathrm{IPA}, \mathrm{MeOH}$, and Windex®, double rubbed with solvent soaked cotton and stained with BPB. The small molecule C18 BP-QAC coatings underwent one double wet rub and substantial coating loss was seen when compared to the unrubbed control coating displayed in Figure 2.7. The durability of polymeric surfaces were assessed by using coating $\mathbf{6}$ as a representative sample, seen in Figure 2.8. The polymeric coatings were found to be considerably robust against $\mathrm{dH}_{2} \mathrm{O}$, experiencing some loss after 350 wet double rubs. These coatings were successfully found to be more robust than the small molecule coatings previously reported.

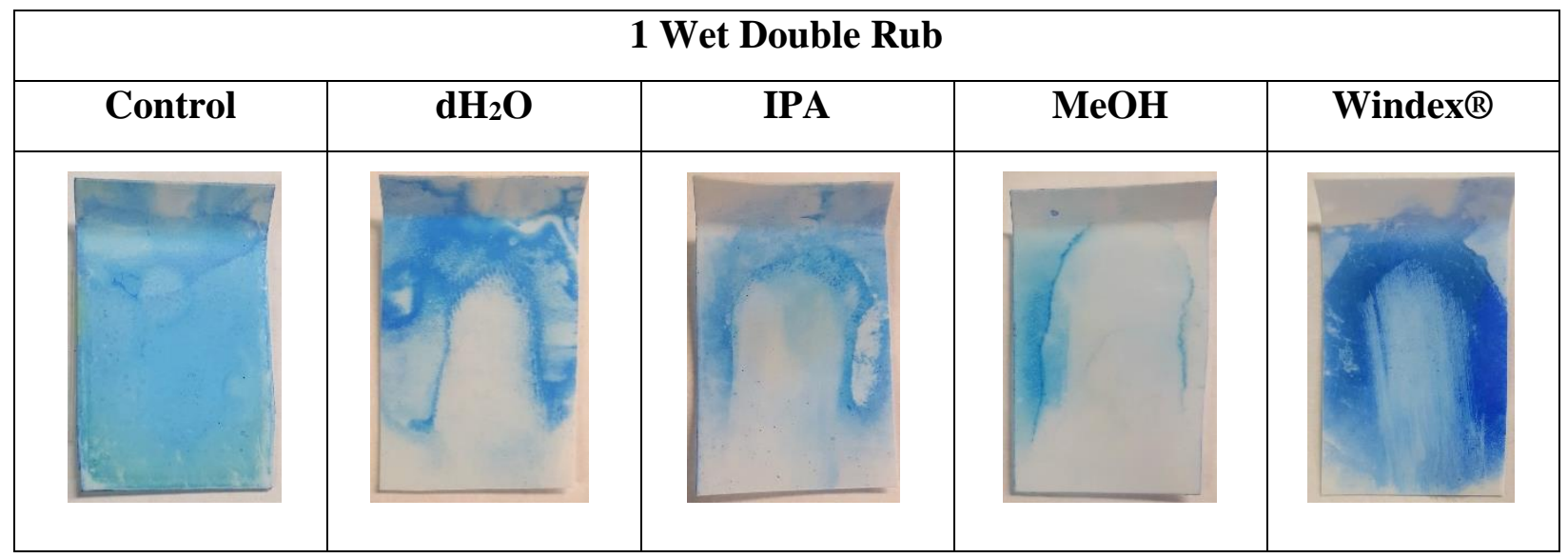

Figure 2.7 C18 BP-QAC small molecule coating on PS after subject to solvent resistance testing followed by BPB staining. Treated surfaces were double rubbed once with a cotton fabric soaked with $\mathrm{dH}_{2} \mathrm{O}, \mathrm{IPA}, \mathrm{MeOH}$, and Windex ${ }^{\circledR}$ with a pressure of $\sim 10 \mathrm{~N}$. 


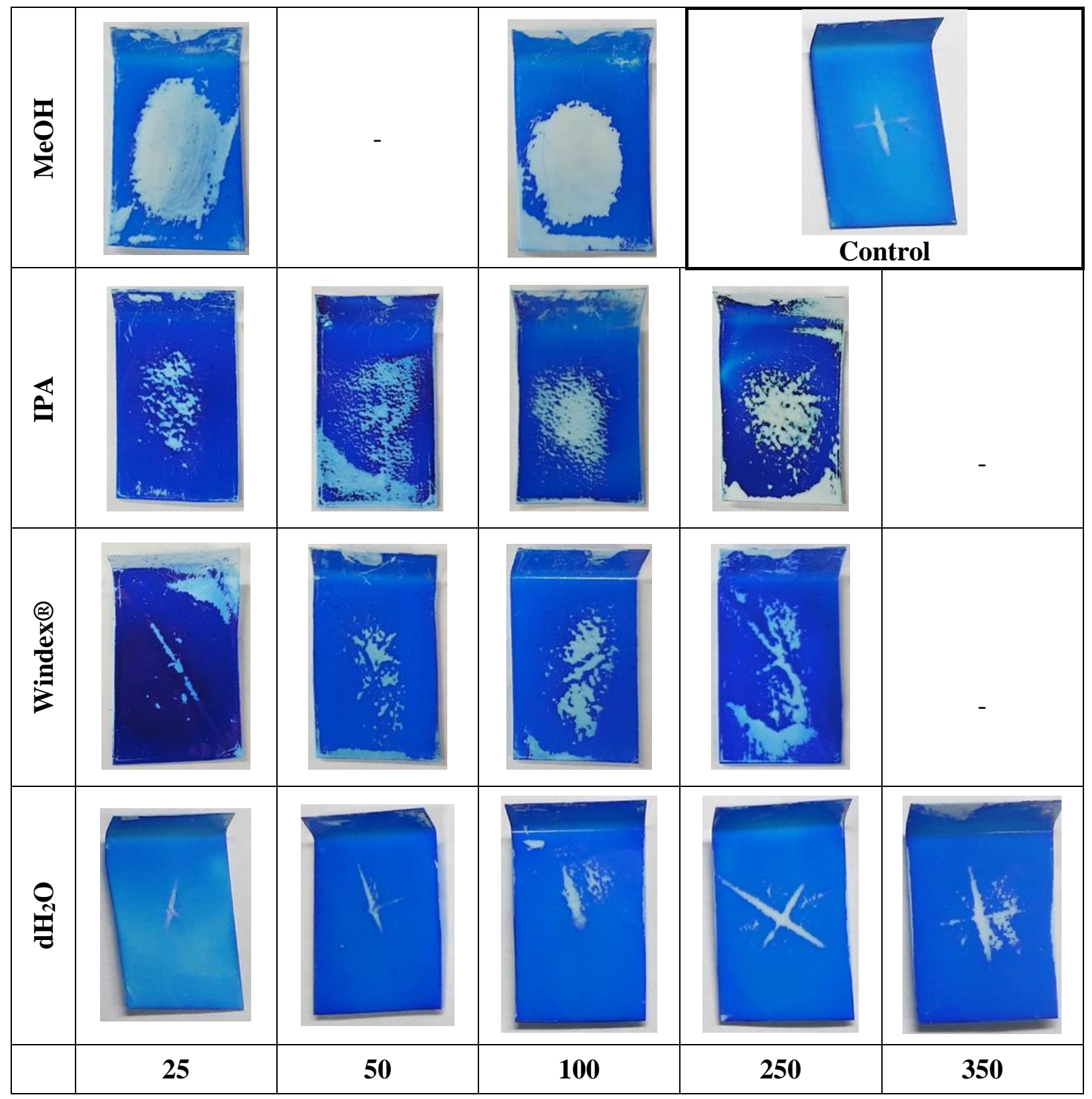

Figure 2.8 Polymeric coatings of 6 on PS after undergoing solvent resistance testing with $\mathrm{dH}_{2} \mathrm{O}$, IPA, $\mathrm{MeOH}$, and Windex ${ }^{\circledR}$ followed by BPB staining. 


\subsubsection{Adhesive Testing}

Coating adhesive testing was carried out following ASTM D3359-17. ${ }^{87}$ Using coating 6 on PS as a representative sample, the coating underwent crosshatching described in the standard operating procedure. The 3M Scotch Tape was evenly applied over the crosshatch and removed at a $180^{\circ}$ angle, parallel to the surface followed by BPB staining for coating visualization. When BAPO initiator was not added to the coating formulation, the coating was easily removed, as shown in Figure 2.9(a). Incorporation of BAPO in the coating formulation improved the adhesion of the coating significantly, as seen by the enduring coating after rapid tape removal (Figure 2.9(b)).

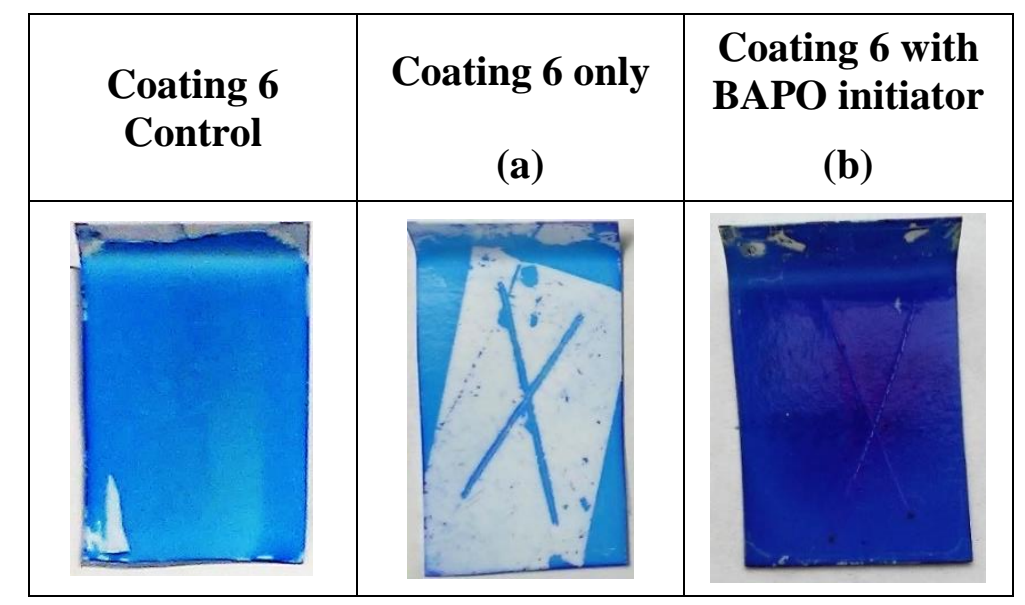

Figure 2.9 Results of the tape test for adhesion evaluation of double coating 6 as a representative sample

\subsection{Characterization of Polymeric Coatings}

\subsubsection{Cationic Charge Density Determination}

Cationic charge density of coatings 6-11 on PS were quantified indirectly by the fluorescein staining/destaining method described in previous work. ${ }^{3}$ The cationic charge density test indicated that coatings with bulkier substituents, $\mathbf{8}$ and $\mathbf{9}$, had similar quaternary densities to coatings of less 
bulky substituents, $\mathbf{6}$ and $\mathbf{7}$. Surfaces $\mathbf{1 0}$ and $\mathbf{1 1}$ possessed the least charge dense coatings, with values comparable to the previously reported small molecule coatings, C18 BP-QAC and BPtosylsulfonamide (Table 2.9)..$^{53,79}$

\subsubsection{Contact Angle Determination}

Cationic charge availability at the surface can be represented by surface wettability, which was obtained by water contact angle determination. A higher degree of wetting specified by a low contact angle indicates the higher population of charges at the surface. The water contact angle of coatings 6-11 are tabulated in Table 2.9. The water contact angle obtained of coatings of the bulkier species, $\mathbf{8}$ and $\mathbf{9}$, were found to be slightly higher than their small molecule counterparts as well as the Arthrobacter sp. active coatings, $\mathbf{6}$ and $\mathbf{7}$. The lower wettability of surfaces $\mathbf{8}$ and $\mathbf{9}$ suggests a lower amount of charges available at the coating interface. Surfaces $\mathbf{1 0}$ and $\mathbf{1 1}$ had the most hydrophobic properties, signifying the lack of cationic charges at the interface and presence of hydrophobic chains. High water contact angles of coating $\mathbf{1 1}$ are due to low surface energy as an effect of the fluorinated pendants.

Table 2.9 Charge density and advancing contact angle of PS substrate coated with 6-11

\begin{tabular}{|c|c|c|}
\hline Coating & Charge Density $\left(\left[\mathbf{Q}^{+} \mathbf{c m}^{-2}\right)\right.$ & $\begin{array}{c}\text { Advancing Contact Angle } \\
(\mathbf{d e g})\end{array}$ \\
\hline $\mathbf{6}$ & $(2.50 \pm 0.25) \times 10^{17}$ & $26.6 \pm 8.8$ \\
\hline $\mathbf{7}$ & $(3.23 \pm 0.22) \times 10^{17}$ & $39.0 \pm 1.1$ \\
\hline $\mathbf{8}$ & $(4.90 \pm 0.33) \times 10^{16}$ & $66.2 \pm 1.3$ \\
\hline $\mathbf{9}$ & $(5.26 \pm 0.40) \times 10^{16}$ & $66.1 \pm 1.5$ \\
\hline $\mathbf{1 0}$ & $(8.18 \pm 0.49) \times 10^{15}$ & $86.3 \pm 3.1$ \\
\hline $\mathbf{1 1}$ & $(1.98 \pm 0.30) \times 10^{16}$ & $100.3 \pm 7.6$ \\
\hline C18 BP-QAC & $(1.15 \pm 0.18) \times 10^{16}$ & $56.7 \pm 1.0$ \\
\hline BP-Tosylsulfonamide $^{\mathbf{7 9}}$ & $(2.16 \pm 0.66) \times 10^{15}$ & $51.1 \pm 6.5$ \\
\hline BP-CF3Benzylsulfonamide $^{\mathbf{7 9}}$ & $(1.92 \pm 0.37) \times 10^{15}$ & $73.9 \pm 2.1$ \\
\hline
\end{tabular}




\subsubsection{Surface Topography Analysis}

Atomic force microscopy (AFM) was used to analyze surface roughness and coating thickness. Single coated surfaces of $\mathbf{6 - 9}$ were studied as representative samples and the root mean square (RMS) roughness and coating thickness values are tabulated in Table 2.10. AFM images of uncoated control polystyrene and treated surface $\mathbf{6}$ and $\mathbf{9}$ are shown in Figure 2.10. Peculiar honeycomb-like patterning can be seen for surface $\mathbf{9}$, which may be the result of phase separation of the sulfonamide pendent from the polymer backbone, suggested by the presence of two $T_{g}$ events discussed earlier.

Table 2.10 RMS Roughness and coating thicknesses of uncoated control, single coated 6-9, and small molecule references on PS

\begin{tabular}{|l|c|c|}
\hline Coating & RMS Roughness of Surface (nm) & Coating Thickness (nm) \\
\hline Uncoated Control & 83.6 & - \\
\hline Poly(VBBP-NEt 3 ) (6) & 23.5 & $385 \pm 39$ \\
\hline Poly(VBBP-PMe3) (7) & 62.0 & $348 \pm 55$ \\
\hline Poly(VBBP-NC12) (8) & 40.2 & $330 \pm 32$ \\
\hline Poly(VBBP-TsSA) (9) & 39.1 & $213 \pm 26$ \\
\hline C18 BP-QAC $^{53, a}$ & $130.6^{b}$ & $366 \pm 148$ \\
\hline
\end{tabular}

${ }^{a}$ Double coated $\quad{ }^{b}$ Control RMS roughness $=5.1 \mathrm{~nm}$

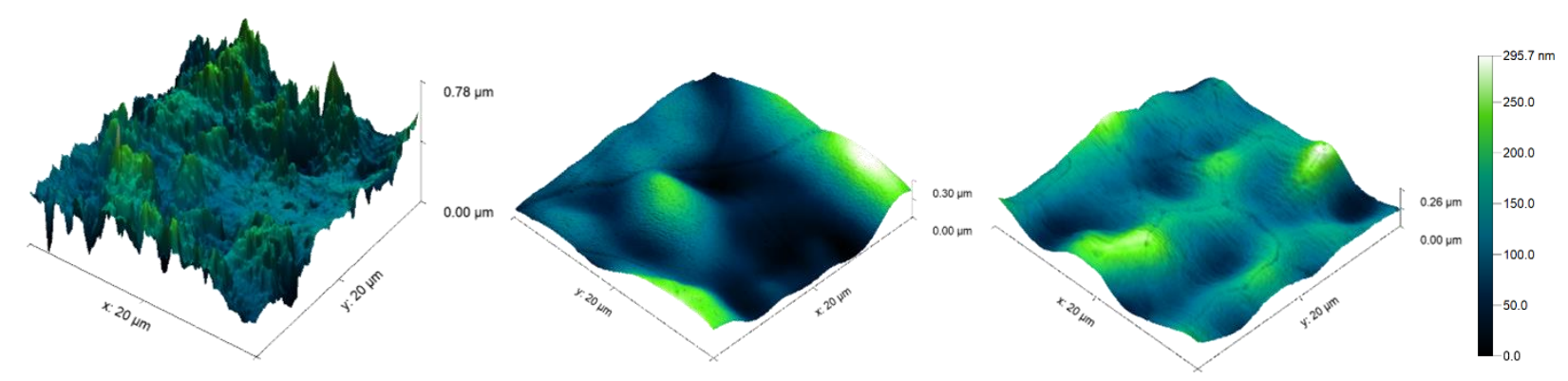

Figure 2.10 AFM images of uncoated PS control (left), PS coated with poly(VBBP-NEt 3 ) (6) (middle) and poly(VBBP-TsSA) (9) (right). The treated surfaces are seen to be smoother than the uncoated PS surface. 


\subsection{Biological Testing of Coated Surfaces}

2.18.1 Large Drop Inoculum Bioactivity Analysis at the Solid/air Interface

Surfaces coated with polymers 6-11 underwent large drop inoculum (LDI) testing to evaluate the antibacterial efficacy at the solid/air interface over a $3 \mathrm{~h}$ period. Antibacterial properties of surfaces were tested against Arthrobacter sp. (IAI-3) as the gram-positive model, portrayed by average cell survival in Figure 2.11(a). The coatings of short alkyl chain quaternary species, triethylammonium (6) and trimethylphosphonium (7), demonstrated complete kill of bacterial cells on the coated surfaces in comparison to the uncoated, control surfaces. The tosylsulfonamide (9) species demonstrated significant reduction of Arthrobacter sp. bacterial cells at a $95 \%$ confidence level. Over a prolonged exposure time of 24 h, coating 9 demonstrated significant kill at a $95 \%$ confidence level but did not completely reduce the bacterial load, depicted in Figure 2.12. While this bacterial reduction is statistically significant, a similar testing protocol, JIS Z 2801, specifies that a colony-forming unit (CFU) reduction of at least $10^{2}$ is determinant of antibacterial efficacy. ${ }^{88} \mathrm{~A}$ reduction of less than $10^{2} \mathrm{CFU}$ is observed for the surface treated with 9, inconclusive of antibacterial activity without further investigations.

The remaining bulky quaternary species, C12 ammonium (8), C18 ammonium (10), and $\mathrm{CF}_{3}$-benzylsulfonamide (11) possessed no antibacterial properties against Arthrobacter sp., contrary to previous work. Additionally, coatings 6-11 were inactive against the gram-negative model, E. coli wild type 36 (wt36), indicated by the lack of cell reduction of the treated in comparison to the control surface, depicted in Figure 2.11(b). 
(a) Coating 6-11 against Arthrobacter sp.

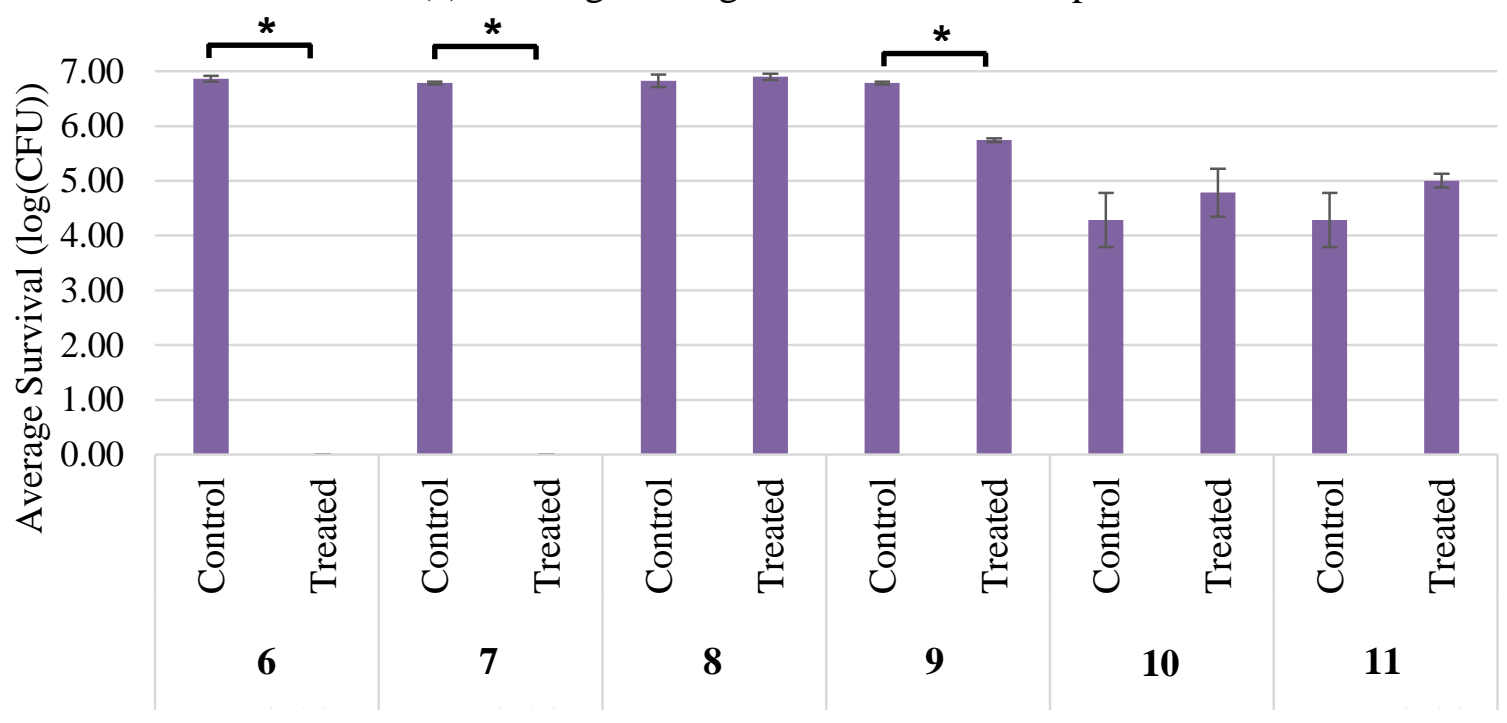

(b) Coating 6-11 against $E$. coli (wt36)

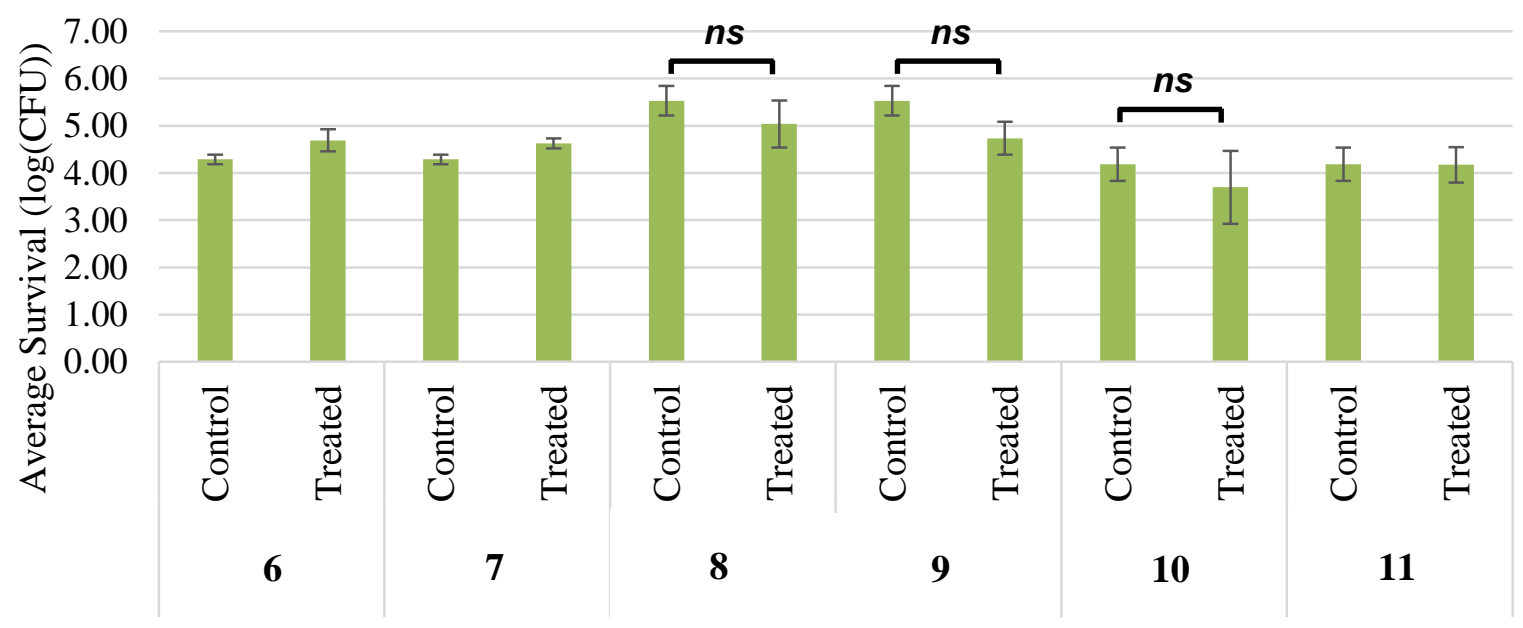

Figure 2.11 Graphs depicting average bacterial cell survival on coatings 6-11 against; (a) Arthrobacter sp. and (b) E. coli wt36. The surfaces were inoculated with a $100 \mu \mathrm{L}$ droplet of $10^{7}$ CFU bacterial suspension for $3 \mathrm{~h}$ until desiccation. Surfaces of $\mathbf{6}$ and $\mathbf{7}$ demonstrated antibacterial activity only against the gram-positive model as seen by the lack of cell survival in comparison to the control surface. The E. coli on coatings 6-11 experienced survived over the testing period, indicating an inactivity against gram-negative bacteria. (t-test, ${ }^{*} p<0.05$, ns $p>0.95$ ) 


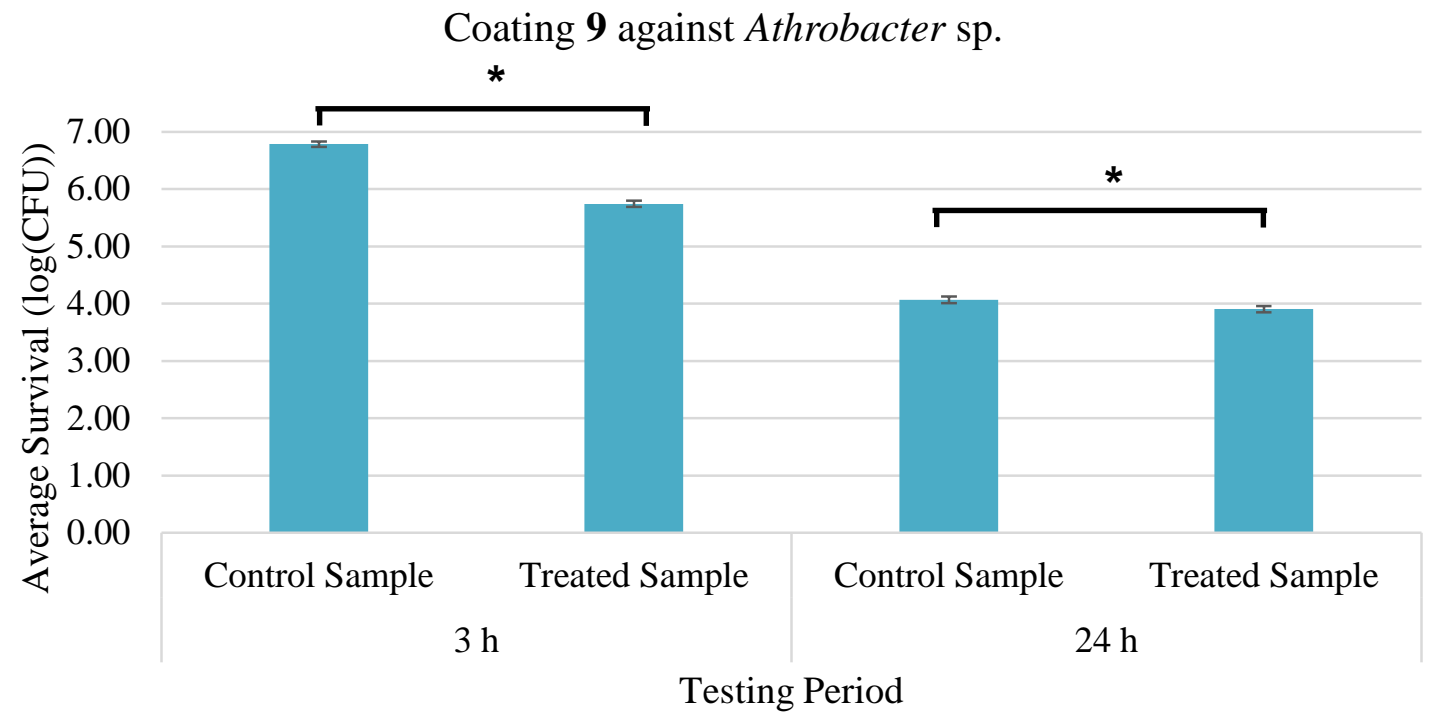

Figure 2.12 Graph depicting coating 9 tested against Arthrobacter sp. at the $3 \mathrm{~h}$ and $24 \mathrm{~h}$ time point. Coatings were found to significantly reduce the bacterial load. (t-test, $* p<0.05$ )

The antibacterial efficacy at the solid/air interface of these polymeric coatings were found to be considerably more inferior to the previous small molecule coatings formulated by our group. Of all the polymeric coatings, the short chain species, $\mathbf{6}$ and 7, were found to be the only bioactive surface, successfully reducing all $10^{7} \mathrm{CFU}$ gram-positive Arthrobacter sp. deposited on the surface. Coatings 6 and 7 had a higher degree of quaternization unimpeded by sterics of the short chain amine/phosphine, resulting in cationic charge dense coatings a magnitude higher than our previous C18 BP-QAC small molecule coating formulation (Figure 1.14 (c)). Additionally, the degree of wettability of coatings $6\left(26.6^{\circ}\right)$ and $7\left(39.0^{\circ}\right)$ were higher than the previously successful C18 BP-QAC $\left(56.7^{\circ}\right)$, indicating more charge availability at the surface. ${ }^{53}$ The high degree of cationic charge and lack of long alkyl chains available for cell wall penetration collectively suggest that the phospholipid sponge effect is the primary mechanism of action for these short chain species against gram-positive bacteria. In this mechanism, the negatively charged peptidoglycan found abundantly in gram-positive bacteria are adsorbed by the cationic surface. ${ }^{27}$ 
When tested against the gram-negative model, E. coli wt36, coatings 6 and 7 demonstrated no bacterial cell reduction at the solid/air interface. It is well known that gram-negative bacteria are less susceptible to cationic compounds due to an additional outer membrane which is absent in gram-positive species. ${ }^{18}$ Cationic species with long alkyl groups such as C12 (8) and tosylsulfonamide (9) were introduced to encourage antibacterial properties against the gramnegative model by including a polymeric spacer group. Surfaces treated with $\mathbf{8}$ and $\mathbf{9}$ were found to have ineffective antibacterial properties against both gram-positive and -negative bacteria. Our previous work with C18 BP-QAC small molecule coatings formulated by the Foucher group with $n$-alkyl chain lengths of 12 or 18 demonstrated antibacterial activity against all gram-negative and -positive models, P. aeruginosa, L. monocytogenes, and Arthrobacter sp. ${ }^{53}$ Likewise, our most recent small molecule coatings based on sulfonamides were found to be antibacterial, completely reducing $10^{5}-10^{7} \mathrm{CFU}$ of Arthrobacter sp., S. aureus, and E. coli (D5H $\left.\alpha\right){ }^{79}$ These conflicting results indicate a significant structure-activity relationship between polymeric and small molecule coatings for antibacterial activity.

Similar to 6 and 7, the coatings of 8 and $\mathbf{9}$ possessed high cationic charge, with densities five times higher than the C18 BP-QAC small molecule coating and one magnitude higher than the BP-tosylsulfonamide small molecule coating. ${ }^{53,79}$ Conversely, the wettability of coatings 8 and 9 were found to be lower than surfaces 6,7 , and the previous small molecule coatings signaled by the higher water contact angles of $66.2^{\circ}(8)$ and $66.1^{\circ}(\mathbf{9})$, an indication of low charge availability at the interface. The cationic charge density and water contact angle values are usually found to be in agreement, but for surfaces $\mathbf{8}$ and $\mathbf{9}$, these properties were found to be inconsistent. This peculiarity can be rationalized by the cationic charge density method. In this protocol, fluorescein undergoes anionic exchange with the chlorine counter ion at a one-to-one ratio, indirectly 
quantifying cationic charges accessible to the fluorescein molecule. Fluorescein has a size of $\sim 8 \AA$ and is able to interact with cationic groups well below the surface. The water contact angle only probes the charge availability at the interface, providing a perhaps more accurate representation of surface properties. For coatings $\mathbf{8}$ and $\mathbf{9}$, the combination of high charge density but low wettability and lack of antibacterial properties is suggestive of charge burial below the surface, possibly due to the backbone of the polymer, illustrated in Figure 2.13(a). While the coating possesses sufficient cationic charge density, the charges are unavailable to interact with bacteria which are considerably larger, at around 1-2 $\mu \mathrm{m}$ in size.

(a)

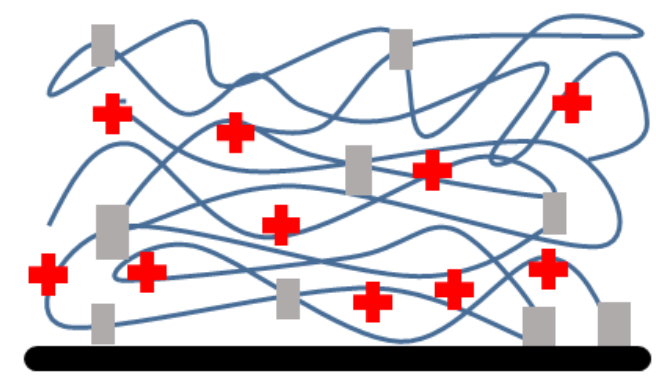

(b)

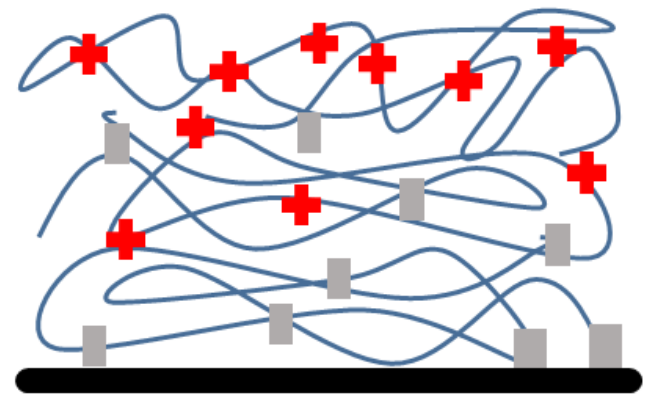

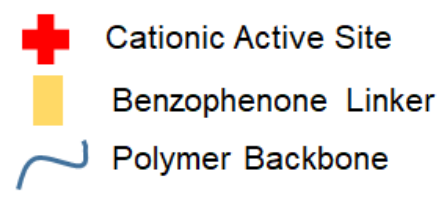

Figure 2.13 Illustration depicting (a) cationic charge burial, hindering cell wall interaction with cationic active sites and (b) cationic active sites self-enriched at the surface.

In hopes of encouraging the cationic functionalities to organize at the surface by phase separation, $\mathrm{C} 18$ ammonium (10) and $\mathrm{CF}_{3}$-benzylsulfonamide (11) quaternary coatings were formulated, illustrated in Figure 2.13(b). The long hydrophobic C18 chains are expected to selfenrich the surface as air is hydrophobic. The fluorinated functionality is expected to spontaneously migrate to the surface since interfacial tension between fluorinated compounds and air are low. ${ }^{89}$ The coating formulations possessed low wettability as indicated by the high water contact angle 
of coatings $\mathbf{1 0}\left(86.3^{\circ}\right)$ and $\mathbf{1 1}\left(100.3^{\circ}\right)$, suggesting organization of the hydrophobic and fluorinated functionalities at the surface. While these coatings had a comparable charge density to the C18 BP-QAC and BP-tosylsulfonamide small molecule coatings, surfaces $\mathbf{1 0}$ and $\mathbf{1 1}$ were found to possess no antibacterial activity against the gram-positive model (Arthrobacter sp.) and the gramnegative model (E. coli wt36).

While robust and charge dense, quaternary ammonium/phosphonium random block copolymer coatings of 6-11 were found to be consistently ineffective as antibacterial surfaces. The antibacterial efficacy of these surfaces were found to be heavily dependent on the bulkiness of the hydrophobic groups on the cationic site. For small molecule coatings, the structure-activity relationship of surface bound small molecule coatings seemly mimic quaternary compounds in solution. Quaternary compounds with $n$-alkyl chain lengths of less than 4 are ineffective as antibacterials when in solution ${ }^{28}$, as seen in the surface attached benzophenone C12 and C18 ammonium small molecule coatings. In contrast, these polymeric analogs in this work demonstrated some antibacterial activity, when the hydrophobic substituent were short alkyl chains. Bulkier substituents such as the $\mathrm{C} 12$ and tosylsulfonamide analogs failed to kill bacterial cells, and even with substituents that encouraged surface self-enrichment, bulkier groups such as $\mathrm{C} 18$ and $\mathrm{CF}_{3}$-benzylsulfonamide species were ineffective as antibacterial surfaces. This behaviour is a departure from to the behaviour of our previous small molecule coatings. ${ }^{53,79}$

The unanticipated trend of the poor antibacterial efficacy of these cationic coatings with bulky functionalities is rather reflective to work previously done by Tiller and colleagues on surface immobilized $n$-alkyl poly(vinylpyridinium) (PVP+) brushes formulated by a "grafting from" approach. Using spray inoculation methods, antibacterial testing of PVP+ possessing alkyl chain lengths of over 6 were found to be inactive against $S$. aureus, while alkyl chain lengths of 3 , 
4, and 6 were active. ${ }^{64} \mathrm{~A}$ statistical decrease of surviving cells was found when hexyl-PVP+ brushes were further tested against gram-positive models (S. aureus, Staphylococcus epidermidis) and gram-negative models (E. coli, P. aeruginosa) ${ }^{64}$ Tiller et al. attributed the lack of activity of their quaternary PVP+ brushes with alkyl chains lengths of over 6 to polymer aggregations of the hydrophobic chains, preventing interaction of the cationic groups with bacterial cells. ${ }^{64}$ The bulky substituents of the polymeric formulations in this work may also be rendered biologically ineffective due to the formation of interdigitating networks obscuring cationic charges at the interface (Figure 2.14). As indicated by the high cationic charge density, the polymeric coatings possessed more active sites and resultantly more hydrophobic substituents than the small molecule coatings. The lower amount of cationic active sites of the small molecule coatings may result in a more sparse assembly of cationic sites. Additionally, there are more hydrophobic regions in the polymeric analogs from the polymeric backbone, naturally absent in the small molecule coatings. The hydrophobic groups interlock to make the cationic charges inaccessible for activity against bacteria. 


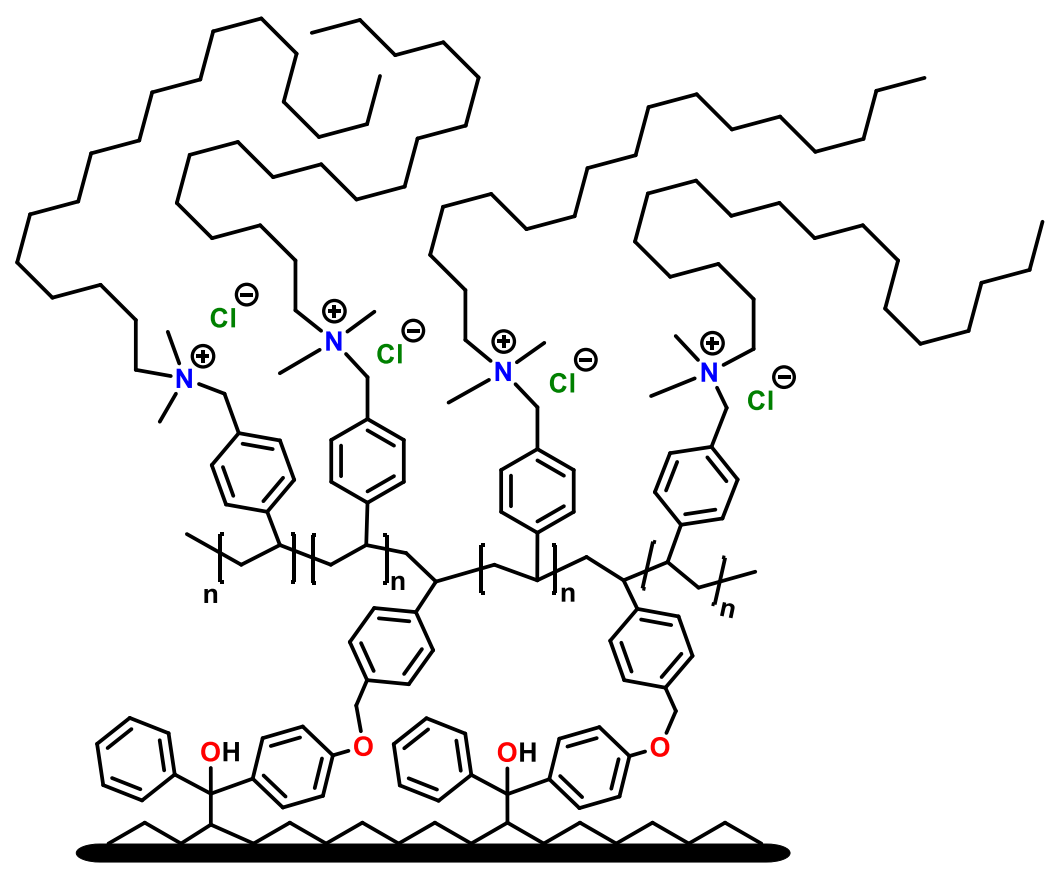

Figure 2.14 Illustration of a coating of high cationic loading depicting hydrophobic chain aggregation preventing charge availability at the surface

Recently, multiple literature sources indicate the relationship between surface topography and antibacterial activity. ${ }^{65,90-92}$ AFM images of surfaces 6-9 were obtained to evaluate coating quality, surface roughness, and relation to respective antibacterial efficacy. The bacterially ineffective polymeric coatings possessed an RMS roughness of 23.5-60.0 nm whereas the C18 BPQAC small molecule coating was found to have a RMS roughness over double of that at 130.6 $\mathrm{nm}$. The RMS roughness and respective bacterial behaviour at the surface are reflective of work by Ivanova and colleagues on the relationship between roughness and antibacterial properties of titanium surfaces..$^{90,91}$ Titanium treated by mechano-chemical polishing was found to have lower roughness parameters than the untreated surface. These surfaces were inoculated and incubated with $S$. aureus and unattached cells were washed off after $18 \mathrm{~h}$. The polished surfaces were found to have retained double the amount of cells in comparison to the untreated, rougher titanium surface. ${ }^{90}$ Other work by Ivanova et al. investigated bacterial adhesion and surface roughness of titanium coatings on a glass substrate. ${ }^{91}$ Titanium was coated on the surface by plasma vapour 
deposition at varying thicknesses corresponding to different roughness values and inoculated with S. aureus. Through cell staining, Ivanova and colleagues reported coatings of higher roughness parameters had higher bacterial cell adhesion of $S$. aureus after washing. Through residue staining techniques, higher amounts of EPS secretions were found on the surface of less rough coatings, indicating higher levels of biofilm growth on the surface. ${ }^{91}$ The smooth polymeric coatings 6-9 possessing low surface roughness may encourage biofilm formation whereas the rougher small molecule coatings deterred biofilm growth. The facilitated biofilm formation on polymeric coatings may contribute to the low antibacterial efficacy of the surface.

The difference in bioactivity between the polymeric and small molecule coatings may also be magnified due to the nature of surface roughness and antibacterial activity. Chatterjee et al. reported etching of aluminum alloy surfaces to modify surface topography for micro- and nanoscale roughness. ${ }^{65}$ The treated alloy surface was found to lyse E. coli and S. aureus after a $4 \mathrm{~h}$ period, decreasing cells by $94 \%$ and $90 \%$ respectively when compared to the untreated surface. ${ }^{65}$ Scanning electron microscopy imaging of inoculated treated surfaces showed the flattening and distortion of cells, unlike the morphology of healthy cells, indicating cell lysis from surface roughness. ${ }^{65}$

\subsubsection{Dynamic Shake Flask Method}

While previous antibacterial SIPN formulations by Cuthbert et al. ${ }^{82}$ are similar to the surfaces described in this work, the polymers in this work unexpectedly possess a lack of bioactivity. The differing bioactivity may be the result of the choice of antibacterial testing method. The SIPN surfaces underwent antimicrobial testing via DSF method ASTM 2149-13a, where the films were ground to a fine powder and inoculated and incubated in solution. Though the 
liquid/solid interface of the powder was being studied in this method, the ground powder provides more surface area than a true film. The LDI protocol determines bioactivity of a surface with better real world representation. Imitating droplet transmission, the inoculum only interacts with the available surface area as a droplet. To demonstrate the complications of the DSF method, the coating of poly(VBBP-NC12) (8) was subjected to DSF bioactivity testing against Arthrobacter sp. While coating $\mathbf{8}$ failed to reduce both gram-positive and negative cells when tested under the LDI protocol, the coating successfully reduced all bacterial cells via the DSF method, depicted in Figure 2.15. The successful reduction of cells using this technique raises concerns over the choice of antibacterial testing methods for coatings and surfaces and their true efficacy in real world situations.

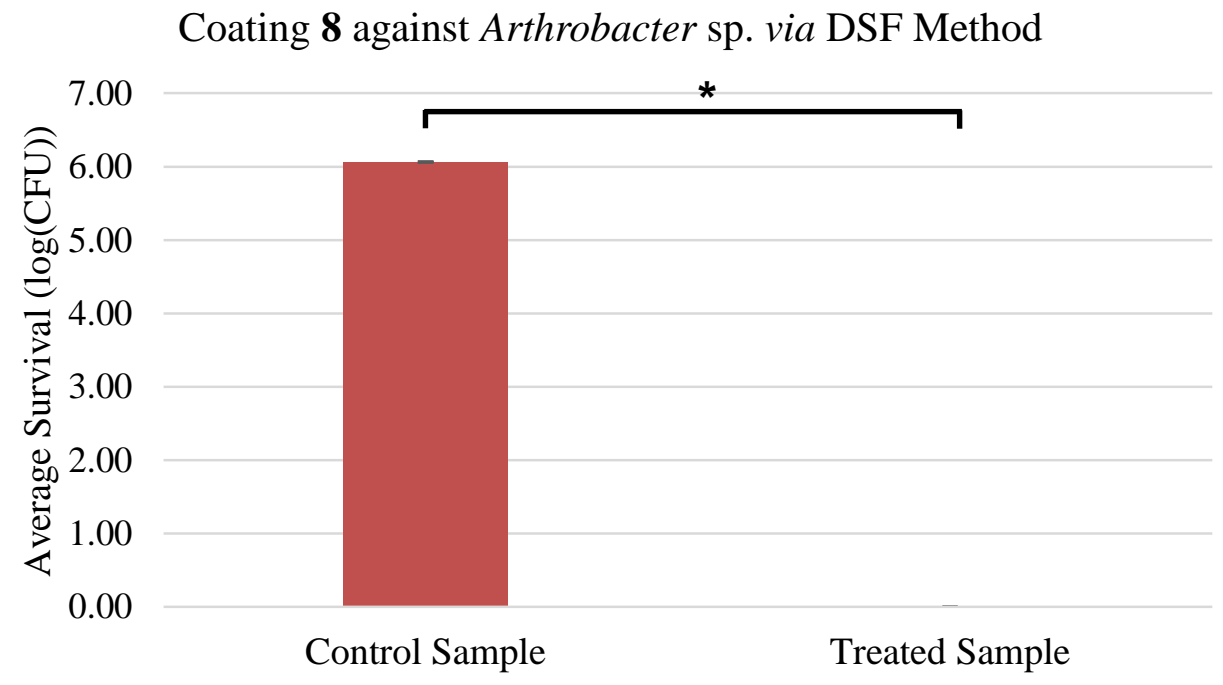

Figure 2.15 Graph depicting average bacterial cell survival of coating 8 against Arthrobacter sp. Film coatings were grounded to a powder and inoculated with $100 \mu \mathrm{L}$ of $10^{5} \mathrm{CFU}$ bacterial suspension, shaken for $1 \mathrm{~h}$. Coating $\mathbf{8}$ demonstrated antibacterial activity only against the grampositive model as seen by the lack of cell survival of the treated sample in comparison to the control. (t-test, $* p<0.05$ ) 


\subsection{Low Quaternary Ammonium Loading Random Block Copolymer}

Tiller et al. attributed their biologically inactive brushes with substituent alkyl chains of over 6 to hydrophobic interactions and chain aggregation. In their hypothesis, the alkyl chains interlock to prevent charge interaction with bacterial cell walls at the surface. In effort to avoid chain interlocking, we attempted to formulate a coating with a lower quaternary loading, therefore leading to a lower chain available for interlocking.

\subsubsection{Post-polymerization Modification of Poly $(V B C-B P)$ (2)}

Following the method previously described in section 2.1.4.1, a $10 \mathrm{~mol} \%$ loading of the poly(VBBP-NC18) (12) was synthesized (Scheme 2.8). The solvent was removed and the crude mixture was purified by precipitation into excess cold Et2O from DCM. The product was confirmed by NMR analysis.

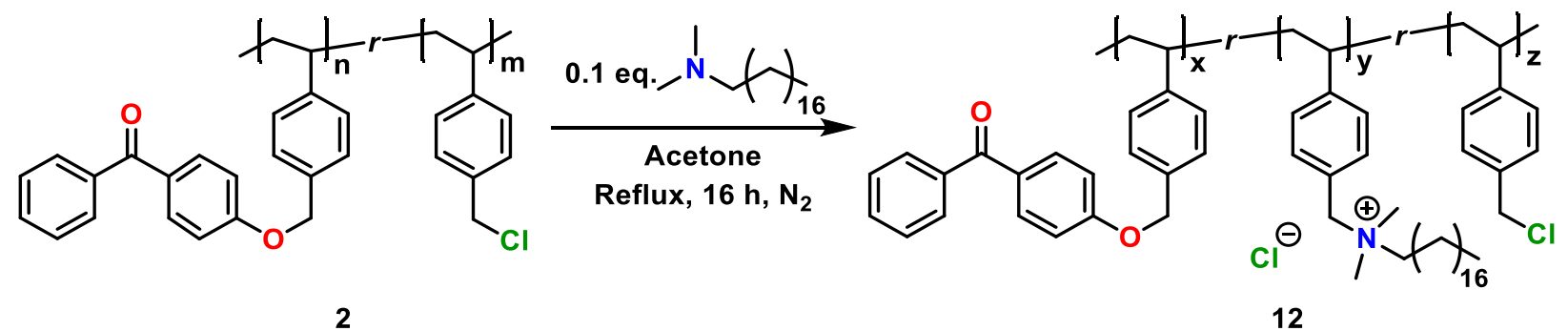

Scheme 2.8 Post-polymerization modification for synthesis of poly(VBBP-10\%-NC18) (12)

\subsubsection{Characterization of Poly(VBBP-10\%-NC18) (12)}

NMR analysis confirmed the final quaternary ammonium loading of the random block copolymer and structure, seen in Figure A 17. Proton integrations from the ${ }^{1} \mathrm{H}$ NMR was used to estimate the percent loading of benzophenone and quaternary ammonium. The protons on the terminal carbon corresponds to the most downfield triplet signal at $0.87 \mathrm{ppm}$. The protons on the 
carbon chain and the protons on the polymer backbone appear at the 1.03-2.15 ppm range. The $\mathrm{CH}_{2}$ and methyl protons adjacent to quaternary ammonium group appear upfield in the 2.80-3.76 ppm range due to the proximity to the more electronegative ammonium group. The next downfield signals at 4.49 and $5.02 \mathrm{ppm}$ correspond to the $\mathrm{CH}_{2}$ protons adjacent to the chlorine and benzophenone substituent, respectively. The remaining signals in the 6.03-7.98 ppm correspond to the aryl protons of the styrene and benzophenone groups. Integrations of the signals corresponding to the methylene groups and protons on the terminal carbon indicated a VBBP and quaternary ammonium loading of $13 \%$ and $14 \%$, respectively, confirmation of a low quaternary ammonium composition polymer.

\subsubsection{Coating Formulation of Poly(VBBP-10\%-NC18) (12)}

The low quaternary ammonium loading of polymer resulted in a fairly non-polar compound, limiting the casting solvent choices as alcoholic solutions were too polar. Toluene was chosen due to its higher boiling point in comparison to other non-polar solvents. The polymer was spin-casted onto PP instead of PS as toluene readily dissolves PS plastic. The polymer before quaternization (poly(VBC-BP)) was also casted on PP as a comparison piece. The polymer was UV cured with a dosage of $30 \mathrm{~J}$ and unbound compound was washed off with toluene. The PP substrate was double coated and BPB stained overnight, displayed in Figure 2.16. The PP treated with the quaternary ammonium compound was stained blue, indicating presence of cationic charges in the coating. As expected, poly(VBC-BP) coating did not demonstrate any charges at the surface. 


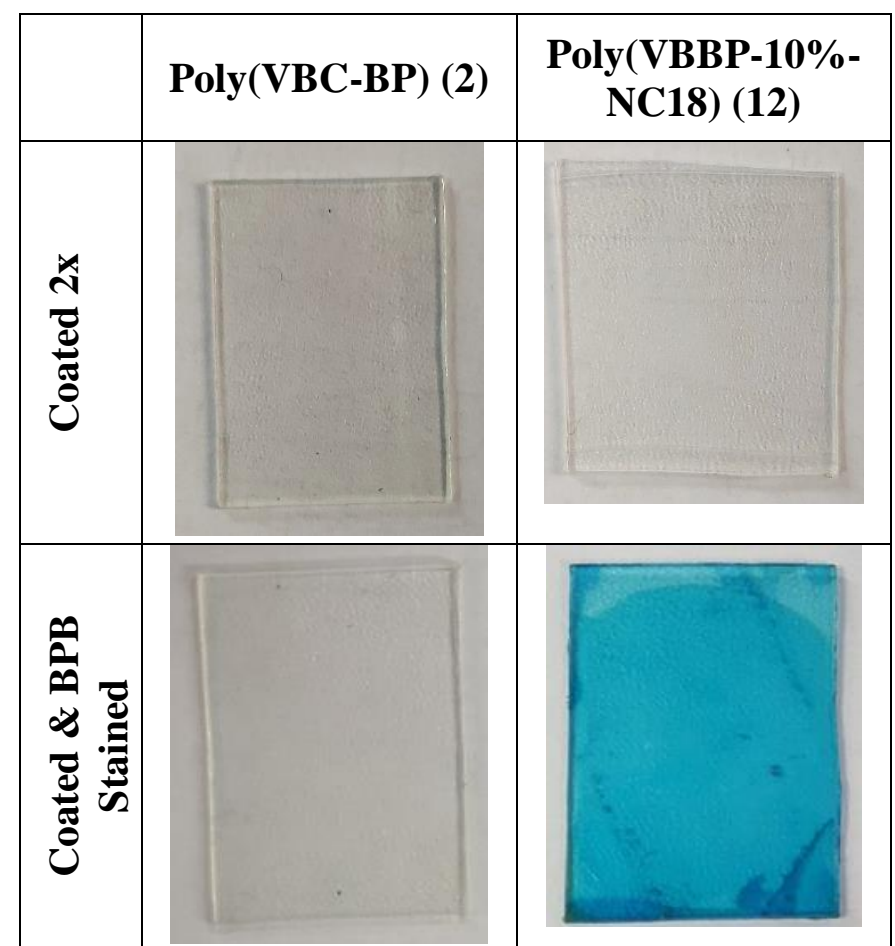

Figure 2.16 PP substrates coated with 2 and 12 before and after BPB staining

\subsubsection{Physical and Biological Properties Poly(VBBP-10\%-NC18) (12)}

The cationic charge density for surfaces treated with 12 was found to be $(5.38 \pm 0.64) \times$ $10^{14}\left[\mathrm{Q}^{+}\right] / \mathrm{cm}^{2}$, one magnitude lower than the suggested cationic charge density for effective bacterial kill. Charge availability was evaluated by advancing water contact angle determination, tabulated in Table 2.11. Poly(VBC-BP) (2) and 12 coatings had similar high angles $\left(82.4^{\circ}\right.$ and $81.9^{\circ}$, respectively), indicative of low wettability properties. While low degree of wettability suggests the lack of cationic charge at the solid/air interface, the cationic group of $\mathbf{1 2}$ possess long hydrophobic C18 chains that may offset the degree of wettability.

Table 2.11 Advancing contact angle of $\mathbf{2}$ and $\mathbf{1 2}$ double coated on PP

\begin{tabular}{|c|c|c|}
\hline & Poly(VBC-BP) (2) & Poly(VBBP-10\%-NC18) (12) \\
\hline Advancing Water Contact Angle & $82.8 \pm 2.4^{\circ}$ & $81.9 \pm 1.3^{\circ}$ \\
\hline
\end{tabular}


Coatings 2 and 12 were then tested against gram-positive Arthrobacter sp. following LDI protocol over a $3 \mathrm{~h}$ period. Surfaces treated with $\mathbf{2}$ served as a negative control as $\mathbf{2}$ does not possess any active quaternary ammonium/phosphonium groups. For coating 12, average bacterial cell survival of the uncoated control and treated sample are represented in Figure 2.17. Over the desiccation period, there was no significant reduction of bacterial cells at a confidence level of 95 $\%$, as indicated by the unchanged average survival log value between the control and treated sample. The low percentage loading of quaternary ammonium proved to be an ineffective method to induce antibacterial activity, suggesting alternative factors for inactivity. The random block structure of the polymer allows for cationic active sites to be buried by the polymeric backbone, prohibiting sufficient cell wall-charge interactions. Modifying the overall polymeric structure to encourage charge accessibility may prompt antibacterial activity.

Coatings 2 and $\mathbf{1 2}$ against Athrobacter sp.

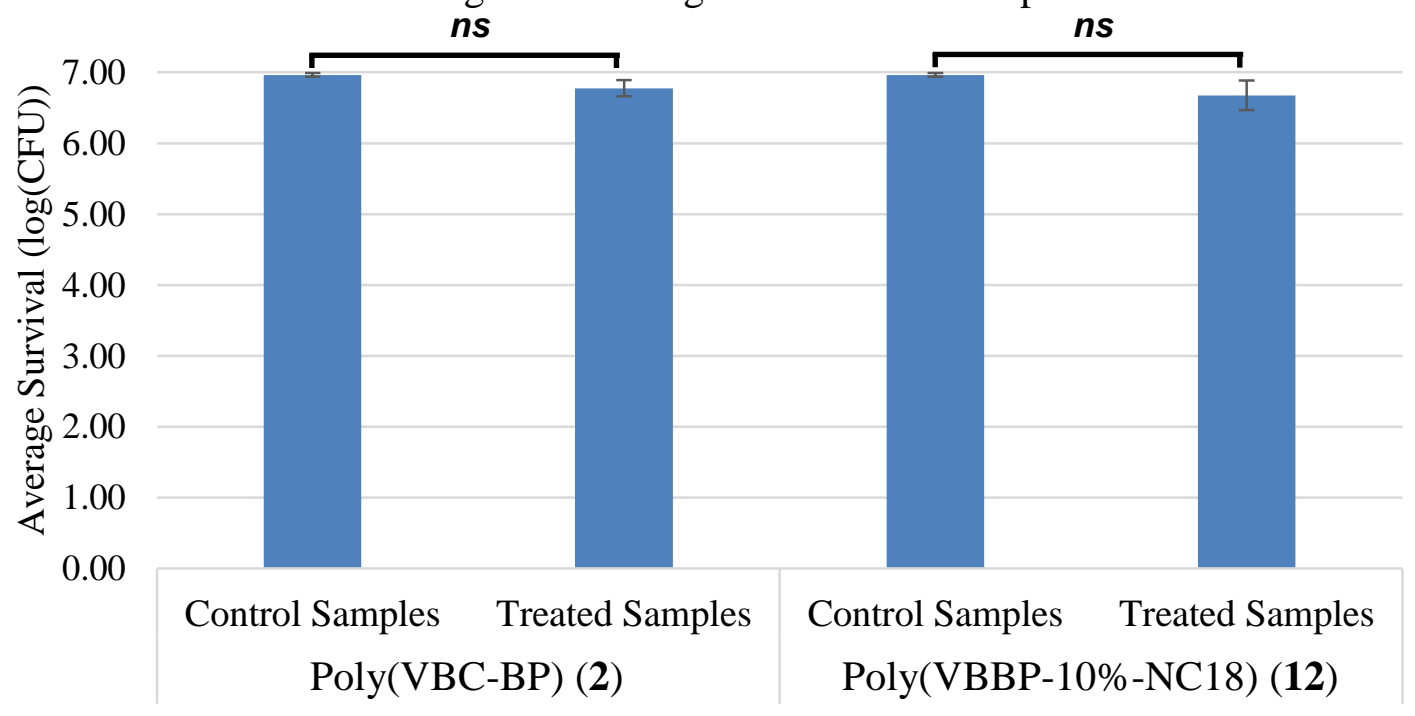

Figure 2.17 Graph depicting average bacterial cell survival over $3 \mathrm{~h}$ period of control and treated surfaces of coatings 2 and $\mathbf{1 2}$ on PP against $10^{7} \mathrm{CFU}$ loading of Arthrobacter sp. There was no significant cell reduction observed for both coatings. (t-test, ${ }^{*} p<0.05$, ns $p>0.95$ ) 


\subsection{Quaternary Phosphonium Diblock Copolymer Brush Coatings}

The work described earlier are random block copolymers, with cationic active sites and benzophenone functionalities evenly distributed along the polymer backbone. Behaving as anchors, benzophenone crosslinking to the surface and to the polymer backbone may have led to the burial of active sites. In hopes of increasing charge availability and avoid charge burial under the surface, surface attached cationic brushes with benzophenone moieties were explored. These brushes were formulated by synthesis of A-B diblock copolymers, where A consisted of low molecular weight homopolymer poly(VBBP) and section B comprised of cationic active sites, depicted in Figure 2.18.

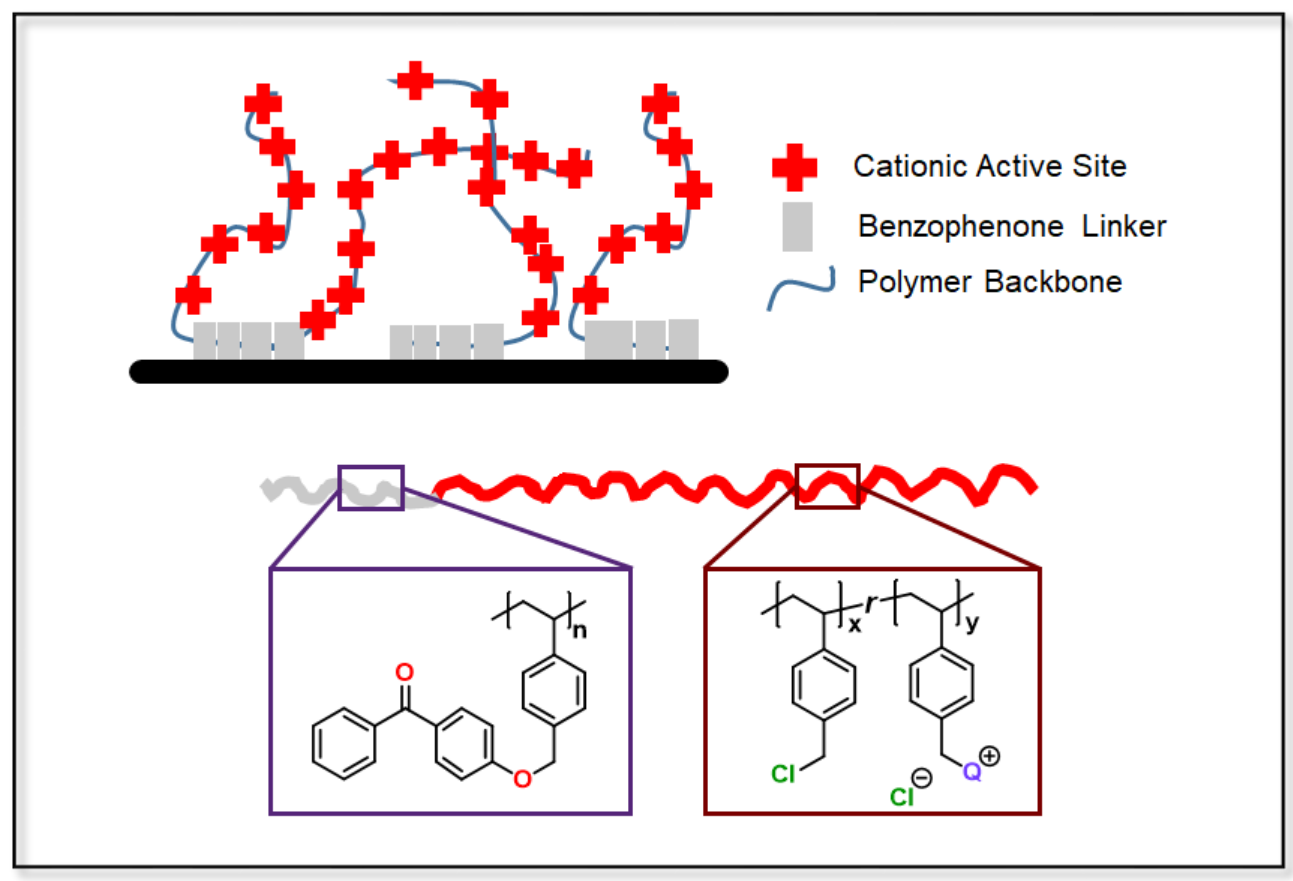

Figure 2.18 Illustration of cationic copolymer brush coatings 


\subsubsection{RAFT Polymerization for Synthesis of Poly $(V B B P-\mathrm{b}-V B C)$ (14)}

Poly(VBBP) (13) was first synthesized by RAFT polymerization initiated by AIBN using RAFT agent CTA1 at $2.1 \mathrm{~mol} \%$ for $24 \mathrm{~h}$ in THF at $63{ }^{\circ} \mathrm{C}$ (Scheme 2.9). The reaction had a high percent conversion of $78 \%$ determined by ${ }^{1} \mathrm{H}$ NMR analysis. The proton NMR spectra of $\mathbf{1 3}$ (Figure A 19) indicate polymerization of the monomer due to broadened signals, disappearance of vinyl protons (Figure 2.19(a)), and appearance of backbone protons in the 1-2 ppm range (Figure 2.19(b)). GPC analysis determined successful polymerization successfully achieved a low molecular weight polymer of $3.2 \mathrm{kDa}$ at a modest dispersity of $D=1.20$. Thermoanalysis of homopolymer $\mathbf{1 3}$ indicated a $T_{g}$ of $71.9^{\circ} \mathrm{C}$, considerably lower than $\mathbf{2}$ due to the low molecular weight nature of $\mathbf{1 3 .}$

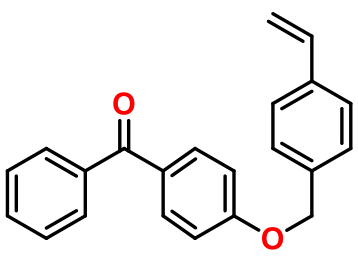

1

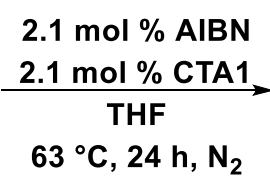

$63^{\circ} \mathrm{C}, 24 \mathrm{~h}, \mathrm{~N}_{2}$

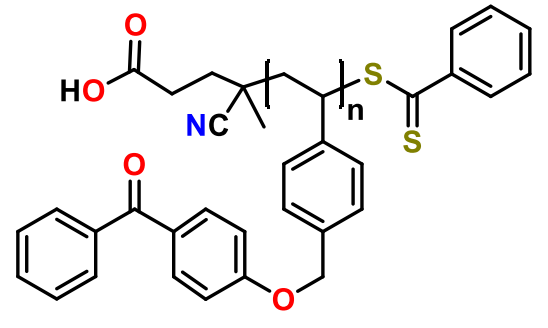

13

Scheme 2.9 RAFT polymerization for the synthesis of low molecular weight poly(VBBP) homopolymer (13)

Bulk copolymerization of poly(VBBP) (13) was continued with VBC for $24 \mathrm{~h}$ to synthesize poly(VBBP-b-VBC) (14, Scheme 2.10) with resultant polymer properties summarized in Table 2.12. Proton NMR analysis indicated the poly(VBC) in the polymer sample at a $97 \%$ loading when comparing the integrations of the $\mathrm{CH}_{2}$ signal of the VBC (4.25-4.69 ppm) to VBBP (4.85$5.29 \mathrm{ppm}$ ), accentuated in Figure 2.19(c). The addition of VBC repeat units on the VBBP homopolymer was confirmed by a unimodal GPC trace. An overlaid GPC trace of the starting polymer 13 and resultant copolymer 14 can be seen in Figure B 5. The resultant copolymer had a 
$\mathrm{M}_{\mathrm{w}}$ of $60.7 \mathrm{kDa}$, increasing the molecular weight by $57.5 \mathrm{kDa}$ or by $376 \mathrm{VBC}$ units. Proton NMR analysis of the $\mathrm{CH}_{2}$ protons indicated an addition of $374 \mathrm{VBC}$ units, closely aligned with GPC $\mathrm{M}_{\mathrm{w}}$ data. The secondary polymerization decreased polymer $D$ from 1.20 to a narrow $\oslash$ of 1.05 . The yield of this reaction was found to be $27 \%$, calculated from the moles of $\mathbf{1 3}$ polymer chains. The low reaction yield may be the result of terminated poly(VBBP) chains that failed to reinitiate in the copolymerization with VBC. The high molecular weight difference between homopolymer 13 and diblock copolymer $\mathbf{1 4}$ allowed for low molecular weight $\mathbf{1 3}$ to be removed in the purification step, leaving high molecular weight $\mathbf{1 4}$ at low dispersity. Thermoanalysis by DSC indicated a $T_{g}$ at $99.8{ }^{\circ} \mathrm{C}$, increased from the poly(VBBP) homopolymer (Figure C 6). The presence of a $T_{g}$ indicated an amorphous polymer.

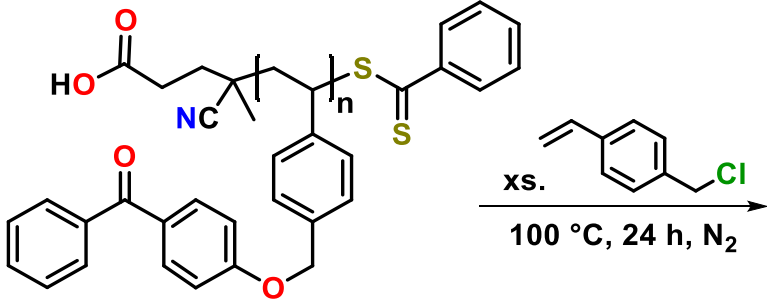

13

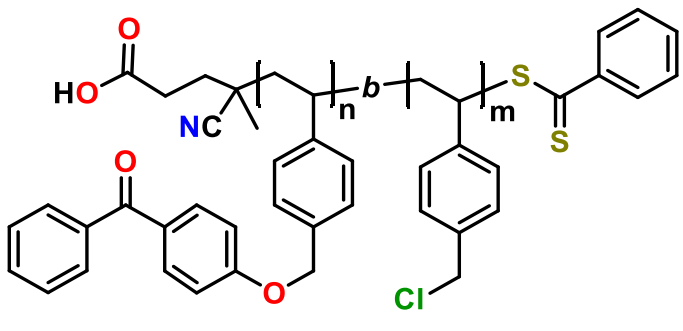

14

Scheme 2.10 Continued bulk RAFT copolymerization of poly(VBBP) (13) with VBC in the synthesis of poly(VBBP- $b$-VBC) (14) 
(a) (b)

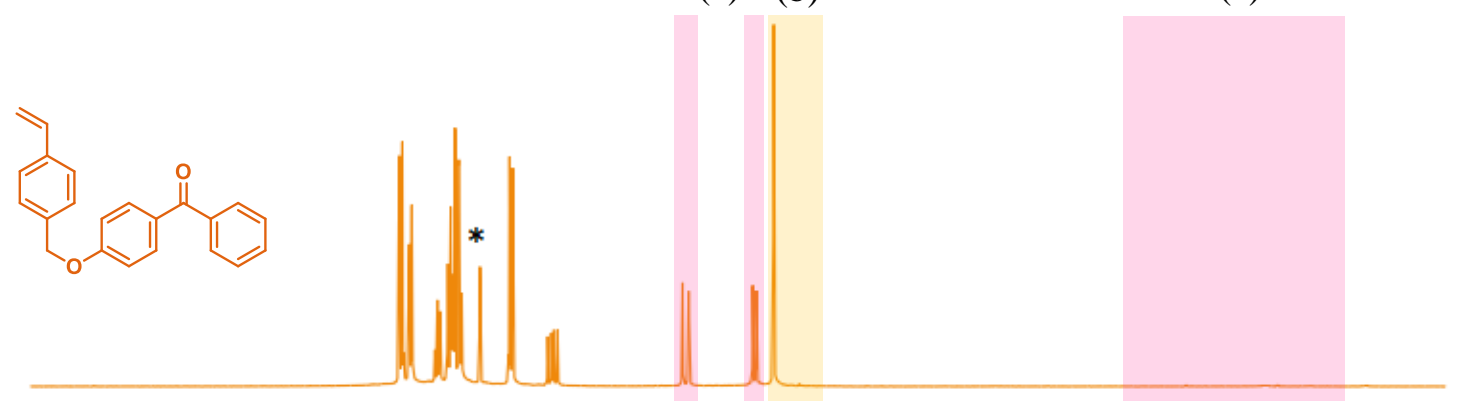

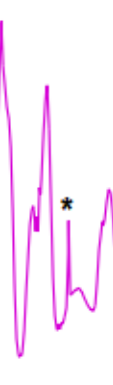

(c)

(c)

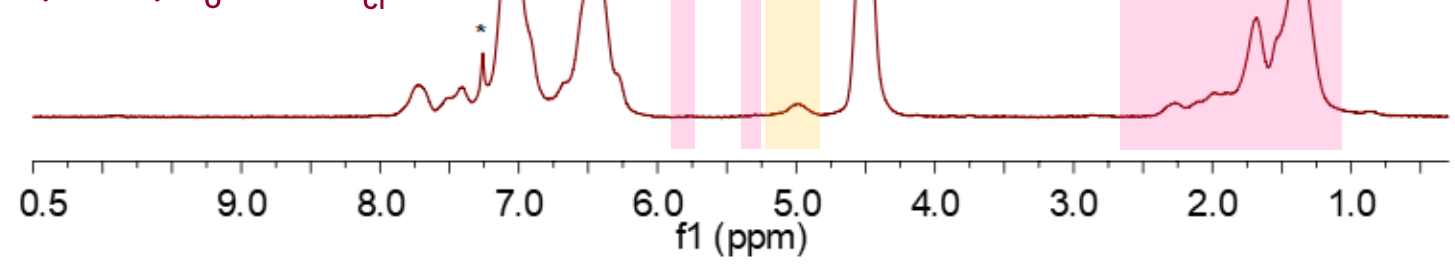

Figure 2.19 Stacked ${ }^{1} \mathrm{H}$ NMR spectra of VBBP monomer 1 , homopolymer 13 , and diblock copolymer $14\left(\mathrm{CDCl}_{3}\right)$

Table 2.12 Polymer properties before and after continued bulk copolymerization

\begin{tabular}{|l|c|c|c|c|c|}
\hline Polymer & \% Conversion & $\mathbf{M}_{\mathbf{w}}(\mathbf{k D a})$ & Repeat Units & $\boldsymbol{\theta}$ & $\boldsymbol{T}_{\boldsymbol{g}}\left({ }^{\circ} \mathbf{C}\right)$ \\
\hline Poly(VBBP) (13) & $78 \%$ & 3.2 & 10 & 1.20 & 71.9 \\
\hline Poly(VBBP-b-VBC) (14) & $27 \%$ & 60.7 & 376 & 1.05 & 99.8 \\
\hline
\end{tabular}




\subsubsection{Post-polymerization Quaternization of Poly(VBBP-b-VBC) (14) and Poly $(V B C-B P)$ (2)}

The copolymer was then subjected to post-polymerization modification with a phosphine species. As discussed earlier, Tiller and colleagues found bacterial inactivity when the n-alkyl substituents on the cationic sites consisted of $n>6 .{ }^{64}$ The short chain species, trimethylphosphonium, in the random block copolymer coating performed well against grampositive species, but the short alkyl chain led to a polar substituent. When substituted on the A-B diblock, poly(VBBP- $b$-VBC) (14), the large difference in polarity between the two end of the copolymer chain resulted in solubility issues where neither polar nor non-polar solvents are able to solubilize the compounds leading to characterization and coating formulation issues. Alternatively, tributylphosphine possesses hydrophobic n-alkyl chains where $n<6$, an ideal candidate for cationic substitution. Diblock copolymer 14 was quaternized with tributylphosphine overnight in dry acetone under nitrogen to synthesize poly(VBBP- $\left.b-\mathrm{P}^{n} \mathrm{Bu}_{3}\right)(\mathbf{1 5}$, Scheme 2.11(a)). Due to the product was purified by precipitation from DCM into cold excess $\mathrm{Et}_{2} \mathrm{O}$ thrice. The quaternization reaction yielded $71 \%$ diblock copolymer quaternary phosphonium.

For a fair comparison to the activity and properties of diblock copolymer brushes $\mathbf{1 5}$, the random block copolymer equivalent was formulated. Poly(VBC-BP) (2) was phosphinated with tributylphosphine to yield poly $\left(\mathrm{VBBP}-r-\mathrm{P}^{n} \mathrm{Bu}_{3}\right)(\mathbf{1 6})$ under the same reaction conditions and workup as above, yielding $89 \%$ (Scheme 2.11(b)). 
(a)

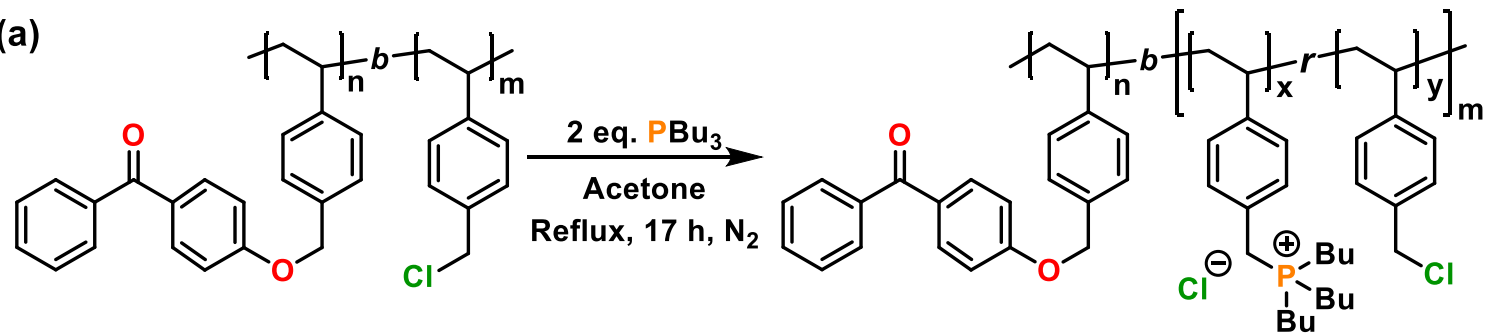

14

15

(b)

(b)

2

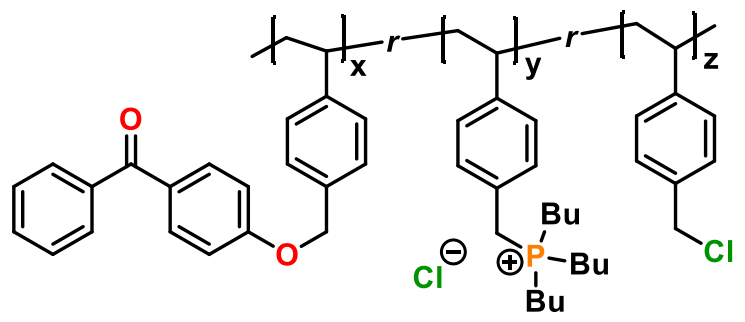

16

Scheme 2.11 Partial quaternization of (a) 14 to synthesize poly(VBBP- $b-\mathrm{P}^{n} \mathrm{Bu}_{3}$ ) (15) and (b) 2 to synthesize poly(VBBP- $\left.b-\mathrm{P}^{n} \mathrm{Bu}_{3}\right)(\mathbf{1 6})$ with tributylphosphine

\subsubsection{Characterization of Poly $\left(V B B P-\mathrm{b}-P^{\mathrm{n}} B u_{3}\right)(\mathbf{1 5})$ and Poly $\left(V B B P-\mathrm{r}-P^{\mathrm{n}} B u_{3}\right)(\mathbf{1 6})$}

Quaternization of $\mathbf{2}$ and $\mathbf{1 4}$ was confirmed by proton and phosphorous NMR analysis. Similar to the random block copolymers described in section 2.14 , the signal corresponding to the methylene groups adjacent to the benzophenone moiety is found most downfield in comparison to the other functionalities at $4.93 \mathrm{ppm}$, in accordance to its electron withdrawing properties. The $\mathrm{CH}_{2}$ adjacent to the least electronegative substituent, phosphonium, corresponds to the upfield signal at 2.49 , overlapped with the signal corresponding to H19 on the butyl chain. Due to the overlap and inconsistent integration values as a result of inadequate solubility, proton integrations could not be relied upon for the determination of functionality composition. Phosphorus NMR showed a strong broad signal at $31.29 \mathrm{ppm}$, corresponding to the phosphonium on the polymer. Weak signals at 38.05 and 48.66 ppm indicated presence of residual tributylphosphine oxide trapped within the polymer. Elemental analysis was used in the determination of VBBP and 
quaternary phosphonium percent loading, summarized in Table D 3. VBBP and quaternary phosphonium percent loadings were estimated to be $9.7 \%$ and $49 \%$, respectively. The low cationic loading of phosphonium is a result to the bulky alkyl groups around the phosphine, hindering full substitution of polymer 14.

Alike diblock copolymer 15, quaternization was established by proton and phosphorous NMR analysis. The proton spectrum was reflective of $\mathbf{1 5}$, but due to disperse quaternary phosphonium groups throughout the polymer, solubility did not hinder polymer composition estimation. Proton integrations provided an estimated percent loading of VBBP and quaternary phosphonium of $7.4 \%$ and $52 \%$, respectively (Table D 4). The ${ }^{31} \mathrm{P}$ NMR spectrum displayed a broad signal at $31.44 \mathrm{ppm}$, indicating the phosphonium polymer peak. The low signal at 38.01 ppm corresponded to trace tributylphosphine oxide trapped within the polymer. Elemental analysis (Table D 3) estimated the VBBP and quaternary phosphonium percent loading to be $8.5 \%$ and 67 $\%$, respectively.

\subsubsection{Coating Preparation of Poly $\left(V B B P-\mathrm{b}-P^{\mathrm{n}} B u_{3}\right)(15)$ and Poly $\left(V B B P-\mathrm{r}-P^{\mathrm{n}} B u_{3}\right)(16)$}

The brushes were spin coated twice onto PS substrates as described earlier as a $10 \mathrm{w} / \mathrm{v} \%$ with $0.3 \mathrm{w} / \mathrm{w} \%$ BAPO in EtOH followed by UV curing of $30 \mathrm{~J}$. Unbound compound was removed by $\mathrm{EtOH} / \mathrm{H}_{2} \mathrm{O}$ washes until the wash solution no longer contained polymer indicated by BPB staining. PS substrates coated with $\mathbf{1 5}$ and $\mathbf{1 6}$ stained and unstained are pictured in Figure 2.20. Both $\mathrm{P}^{n} \mathrm{Bu}_{3}$ species demonstrated even coating and good coverage on the PS substrate. After UV curing, the coatings were colourless and experienced no discolouration. 


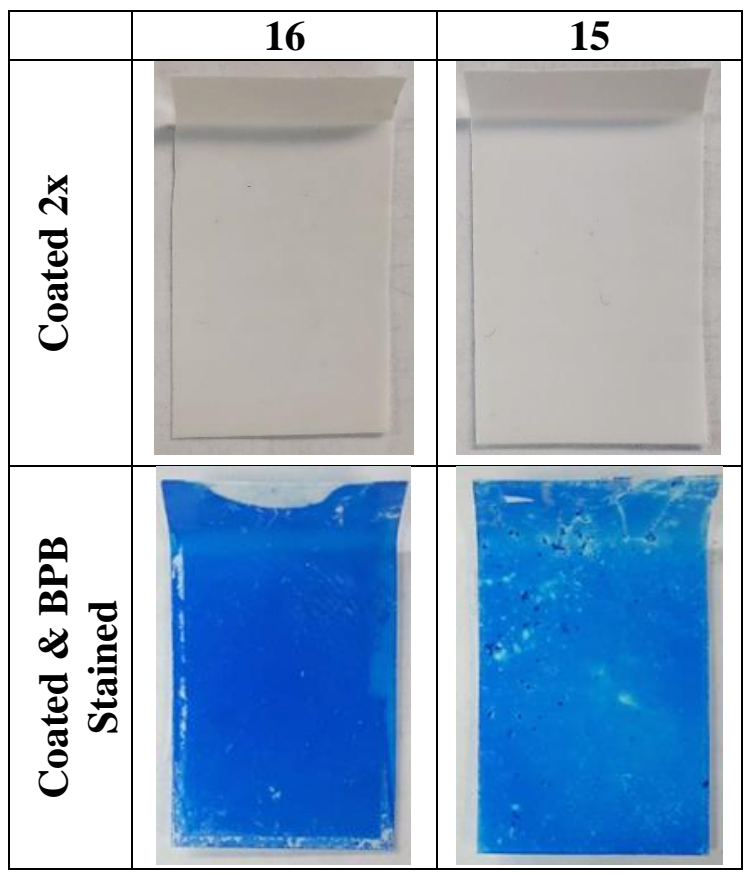

Figure 2.20 $\mathrm{P}^{n} \mathrm{Bu}_{3}$ species 15 and 16 coated and BPB stained PS substrates

\subsubsection{Properties of Surfaces Treated with $\mathbf{1 5}$ and $\mathbf{1 6}$}

Cationic charge density and advancing water contact angle of coatings $\mathbf{1 5}$ and $\mathbf{1 6}$ are tabulated in Table 2.13. The cationic charge densities of $\mathrm{P}^{n} \mathrm{Bu}_{3}$ coatings were found to be similar, with the same magnitude as the bioactive coatings 6 and 7. The advancing water contact angle of the treated surfaces were obtained to determine charge availability at the surface. As expected, the $\mathrm{P}^{n} \mathrm{Bu}_{3}$ brush (15) coating was found to have a higher degree of wettability as indicated by the low contact angle of $36.8^{\circ}$. The surface treated with the $\mathrm{P}^{n} \mathrm{Bu}_{3}$ random block copolymer (16) possessed a lower degree of wettability, demonstrated by the higher contact angle $\left(64.3^{\circ}\right)$. The high wettability of the brush surface suggest the availability of charges at the solid/air interface. 
Table 2.13 Advancing water contact angle and cationic charge density of PS substrates coated with $\mathrm{P}^{n} \mathrm{Bu}_{3}$ species 15 and $\mathbf{1 6}$

\begin{tabular}{|l|c|c|}
\hline & Charge Density $\left(\left[\mathbf{Q}^{+}\right] \mathbf{c m}^{-2}\right)$ & Advancing Water Contact Angle \\
\hline Poly(VBBP-b-P $\mathbf{P}^{n}$ Bu3) (15) & $(2.64 \pm 0.15) \times 10^{17}$ & $36.8 \pm 2.2^{\circ}$ \\
\hline Poly(VBBP-r-P $\mathbf{P}^{n}$ Bu3) (16) & $(1.96 \pm 0.06) \times 10^{17}$ & $64.3 \pm 2.2^{\circ}$ \\
\hline
\end{tabular}

\subsubsection{Biological Testing of Surfaces 15 and 16}

Poly $\left(\mathrm{VBBP}-b-\mathrm{P}^{n} \mathrm{Bu}_{3}\right)(\mathbf{1 5})$ and poly $\left(\mathrm{VBBP}-r-\mathrm{P}^{n} \mathrm{Bu}_{3}\right)(\mathbf{1 6})$ were subjected to antibacterial testing by the LDI protocol to determine bioactivity at the solid/air interface, as described above. Using Arthrobacter sp. as the gram-positive model and E. coli wt36 as the gram-negative model, the coatings were subjected to testing over a $3 \mathrm{~h}$ period and represented in Figure 2.21. The brushes of 15 completely reduced Arthrobacter sp. at the surface compared to the uncoated control PS substrate. Coating 16 demonstrated significant partial reduction against Arthrobacter sp. at the 95 $\%$ confidence level but failed to kill all bacterial cells on the surface. Surface $\mathbf{1 5}$ outperformed the polymeric coating of $\mathbf{1 6}$, signifying a relationship between charge availability and antibacterial efficacy. When tested against $E$. coli wt36, both $\mathrm{P}^{n} \mathrm{Bu}_{3}$ species failed to significantly reduce bacterial cells, as seen in the random block copolymers discussed in section 2.1. While both phosphonium species performed well against the gram-positive model, the gram-negative model experienced no cell death, characteristic of the E.coli wt36 resistance against quaternary compounds. 
(a) Coatings of $\mathbf{1 5}$ and $\mathbf{1 6}$ against Arthrobacter sp.

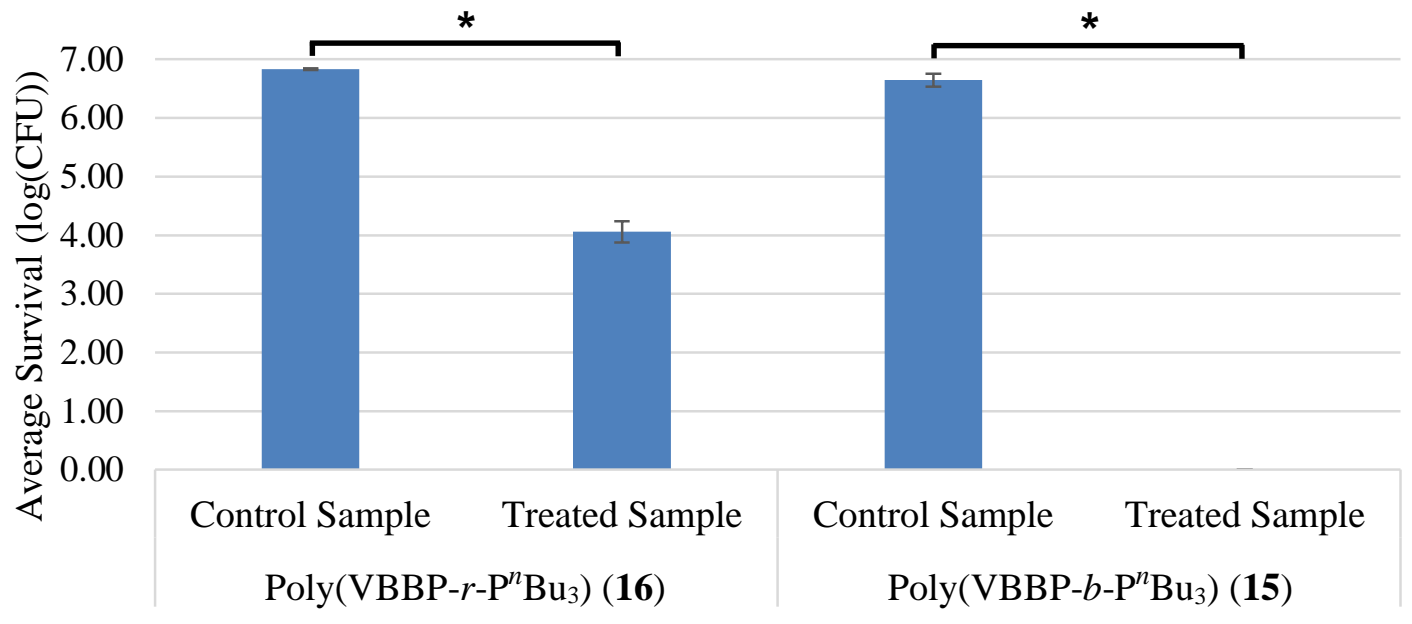

(b) Coatings of $\mathbf{1 5}$ and $\mathbf{1 6}$ against E. coli wt36

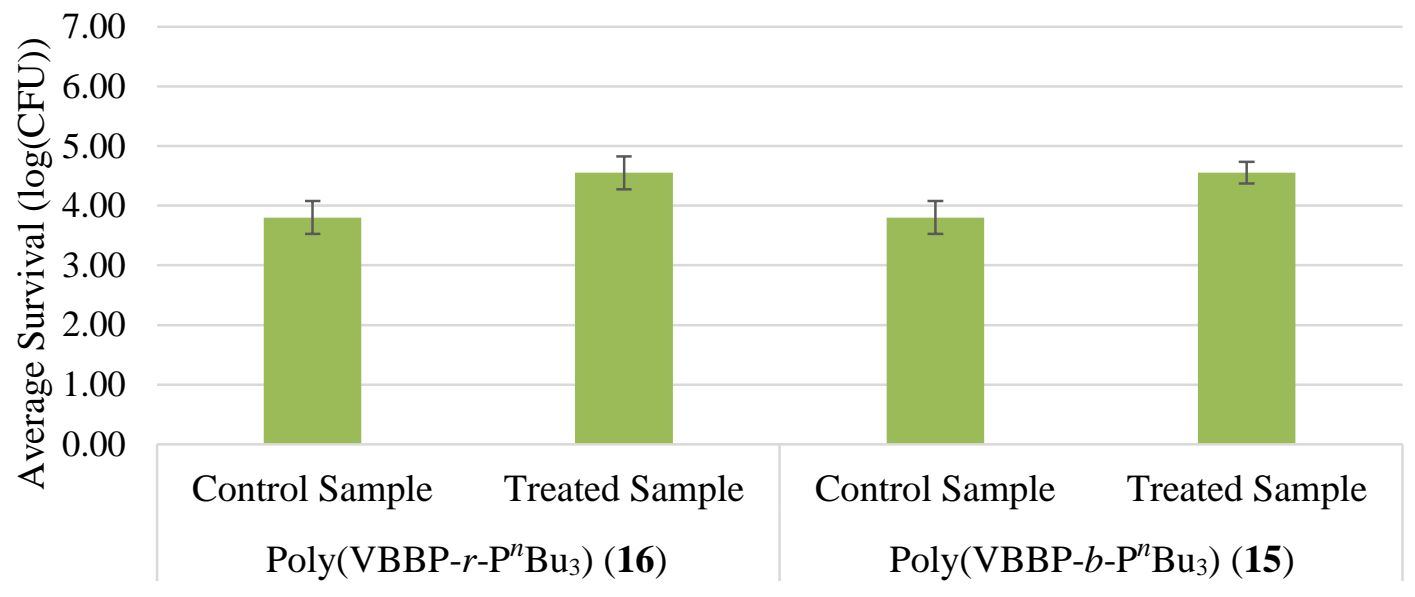

Figure 2.21 Graphs depicting bacterial cell survival when coatings of $\mathrm{P}^{n} \mathrm{Bu}_{3}$ species were tested against (a) Arthrobacter sp. and (b) E. coli wt36. Surfaces were inoculated with $100 \mu \mathrm{L}$ droplet of $10^{7} \mathrm{CFU}$ bacterial suspension for $3 \mathrm{~h}$ until desiccation. Brushes of $\mathbf{1 5}$ reduced all gram-positive bacteria on the surface while $\mathbf{1 6}$ significantly reduced bacteria. Both $\mathrm{P}^{n} \mathrm{Bu}_{3}$ species failed to reduce E. coli cells on the surface. (t-test, ${ }^{*} p<0.05$ ) 


\subsection{Quaternary Ammonium/Phosphonium Monomers and Polymerizations}

The persistent problem experienced in this work has been the insolubility of the polymers due to their polar cationic substituents and non-polar polymer backbone and hydrophobic alkyl chains, leading to difficult characterization of all formulations. Preparation of polymer brushes with cationic active sites with short alkyl chains were especially challenging as both polar and non-polar solvent were unsuitable for solubilization. While the brushes of $\mathbf{1 5}$ discussed in section 2.3 was soluble in $\mathrm{EtOH}$ at $10 \mathrm{w} / \mathrm{v} \%$, the formation of micelles (illustrated in Figure 2.22) was indicated by the presence of a light blue tinge (Figure 2.23). Micelle formation may have led to adverse effects in the coating step, averting benzophenone attachment to the substrate surface, possibly decreasing durability of the coating.

Quaternization as a post-polymerization step is less ideal as the resultant product would only be partially substituted, the degree of which would be dependent on substituent bulkiness. Full substitution of quaternary ammonium/phosphonium may be achieved by polymerization of the cationic monomer analog. The fully cationic polymer will be easily soluble in polar solvents, improving characterization and coating formulation. Herein, a range of cationic monomers were synthesized and subsequent polymerization are described. 

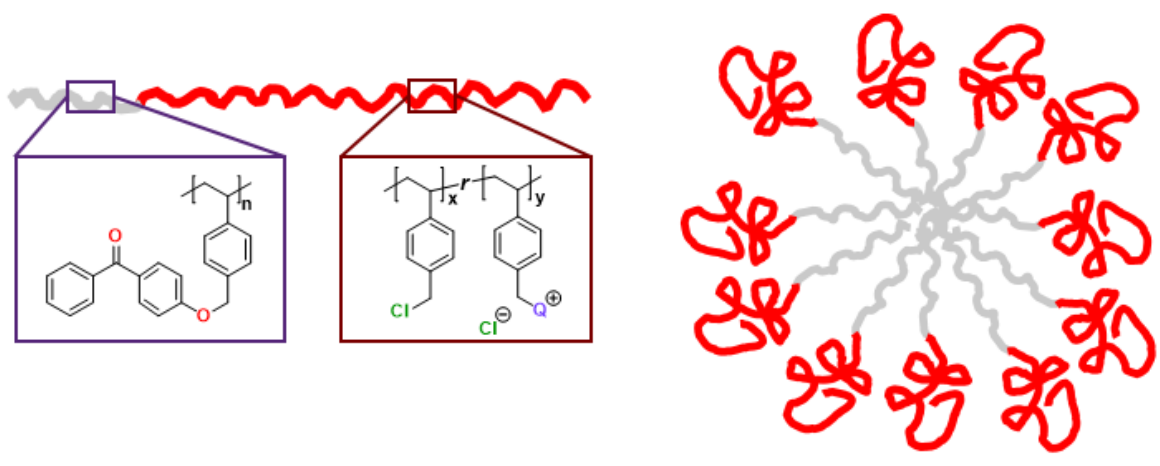

Figure 2.22 Micelle formation from A-B diblock copolymers in a polar solution where the Ablock is the hydrophobic benzophenone repeat units (grey) and the B-block is the hydrophilic cationic species (red).

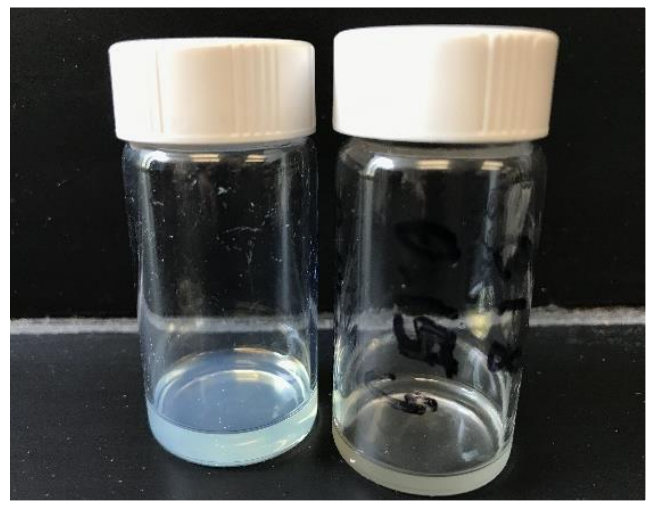

Figure 2.23 Left: $\mathrm{P}^{n} \mathrm{Bu}_{3}$ diblock copolymer 15 in $10 \mathrm{w} / \mathrm{v} \% \mathrm{EtOH}$ solution presented as a light blue tinge, indicting micelle formation. Right: $\mathrm{P}^{n} \mathrm{Bu}_{3}$ random block copolymer 16 in 10 w/v \% EtOH appearing as a clear yellow solution.

\subsubsection{Synthesis of Quaternary Ammonium and Phosphonium Monomers}

\subsubsection{4-Vinylbenzyl Triethylammonium Chloride (17)}

4-Vinylbenzyl triethylammonium chloride (17) was synthesized by an adapted procedure reported by Kaur et al. (Scheme 2.12). ${ }^{93}$ A range of reaction conditions and work-up procedures were then attempted, summarized in Table 2.14. When the reaction was carried out in the most polar solvent, $\mathrm{MeOH}$ (Entry 4, Table 2.14). the reaction achieved the highest yield at $89 \%$ in agreement with literature. ${ }^{26}$ High solvent polarity resulting in high reaction rates of the Menshutkin 
reaction can be attributed to stabilization of the reaction path of ion separation by the solvent. ${ }^{94}$ Purification was carried out by precipitation into cold $\mathrm{Et}_{2} \mathrm{O}$ afforded by the high polarity of quaternary ammonium compound. From ${ }^{1} \mathrm{H}$ NMR analysis, the new signals indicated the addition of triethylamine. The signal at $1.42 \mathrm{ppm}$ integrating for 9 protons represent the protons on the terminal carbons and $3.40 \mathrm{ppm}$ integrating for 6 protons for the protons on the secondary carbon of the three ethyl groups. The structure was further confirmed by single crystal X-ray diffraction, represented by the ORTEP diagram in Figure 2.24. The single crystal was grown by interface mixing of DCM and hexanes. The C1-N1 bond length was observed to be 1.555(11) A, similar to the reported $\mathrm{C}_{4}-\mathrm{N}^{+}$bond lengths of ammonium salts, $1.510 \AA .{ }^{95}$ The compound was found to decompose at $200{ }^{\circ} \mathrm{C}$, making it and the polymers derived from it, a less ideal candidate as an additive for injection molding. The product was stored under a nitrogen environment due to its hygroscopic properties from the quaternary ammonium group. When improperly stored, the product absorbed moisture from the atmosphere and converted to a gel.

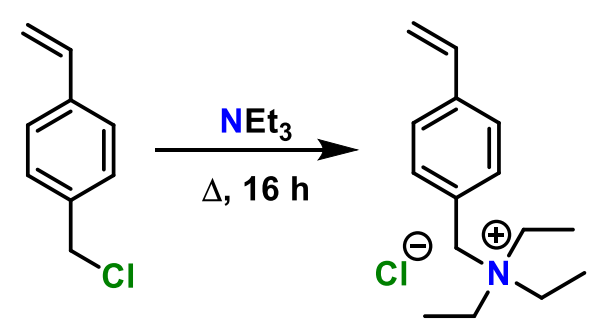

17

Scheme 2.12 Quaternization reaction of triethylamine with VBC for synthesis of $\mathbf{1 7}$ 
Table 2.14 Reaction and work-up conditions and subsequent percent yield to synthesize (17)

\begin{tabular}{|c|c|c|c|c|}
\hline Entry & Rxn Temp. & Solvent & Work-up & \% Yield \\
\hline $\mathbf{1}$ & $30{ }^{\circ} \mathrm{C}$ & $\mathrm{MeOH}$ & Precipitated into cold $\mathrm{Et}_{2} \mathrm{O}$ & $73 \%$ \\
\hline $\mathbf{2}$ & Refluxed & Acetone & Redissolve in DCM, precipitate in $\mathrm{Et}_{2} \mathrm{O}$ & $67 \%$ \\
\hline $\mathbf{3}$ & Refluxed & $\mathrm{MeCN}$ & As above & $74 \%$ \\
\hline $\mathbf{4}$ & $30^{\circ} \mathrm{C}$ & $\mathrm{MeOH}$ & As above & $89 \%$ \\
\hline
\end{tabular}

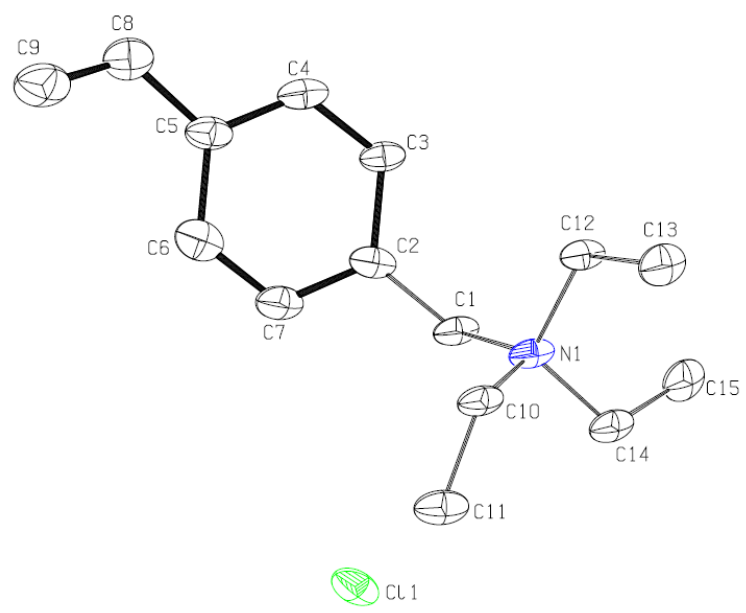

Figure 2.24 ORTEP representation of monomer 17

\subsubsection{4-Vinylbenzyl Tributylphosphonium Chloride (18)}

The synthesis of 4-vinylbenzyl tributylphosphonium (18) was performed following the procedure previously reported by Cuthbert et al., illustrated in Scheme $2.13 .{ }^{82}$ The reaction was refluxed overnight in $\mathrm{MeCN}$ under a nitrogen atmosphere. The crude product was cleaned by precipitation into cold $\mathrm{Et}_{2} \mathrm{O}$. Proton $\mathrm{NMR}$ analysis of product was similar to the monomer 17, but with additional signals corresponding to the butyl groups on the phosphonium. The triplet signal corresponding to 9 protons most upfield at $0.95 \mathrm{ppm}$ represent the protons at the terminal carbons of the butyl chains. At $1.50 \mathrm{ppm}$, the multiplet integrates for $12 \mathrm{H}$ are assigned to protons at the second and third carbons on the alkyl chains. The multiplet integrating for $6 \mathrm{H}$ at $2.15 \mathrm{ppm}$ 
correspond to the protons on the carbon of the butyl chains closest to the phosphonium group which experience more deshielding than the rest of the alkyl chain. Protons of the $\mathrm{CH}_{2}$ adjacent to the phosphonium are assigned to the signal at $3.69 \mathrm{ppm}$. The proximity of the protons to the phosphonium and benzyl group result in more deshielding, leading to a more downfield signal compared to protons at position 8 . Due to its vicinity to the phosphorous atom, the protons at the $\mathrm{CH}_{2}$ position also experience ${ }^{2} J_{\mathrm{PH}}$ coupling, presenting a doublet at $3.69 \mathrm{ppm}$. The signals found further downfield correctly integrate for the vinylbenzyl group as described earlier. Phosphorous NMR analysis confirmed the presence of only one phosphorus signal at $32.58 \mathrm{ppm}$, typical of quaternary phosphonium shifts. Single crystal analysis also confirmed the structure, shown in Figure 2.25. Using the same interface mixing technique as described above, the single crystal of 18 was grown from DCM and hexanes. The C1A-P1A bond length was observed to be 1.8025(17) $\AA$, similar to the reported carbon-quaternary phosphonium $\mathrm{C}_{4}-\mathrm{P}^{+}$bond length of $1.800 \AA .{ }^{95}$ Unlike monomer $\mathbf{1 7}, \mathbf{1 8}$ possessed a melting point at $121.2-123.1^{\circ} \mathrm{C}$, likely due to the presence of longer alkyl chains leading to improved van der Waals interactions. ${ }^{96}$ Similar to monomer $\mathbf{1 7}$, the compound was found to be hygroscopic and readily absorbed moisture from the air and converted to a gel when improperly stored under moisture free conditions.

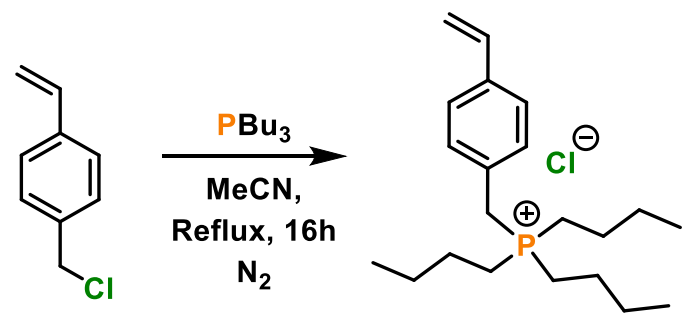

Scheme 2.13 Synthesis of monomer 18 


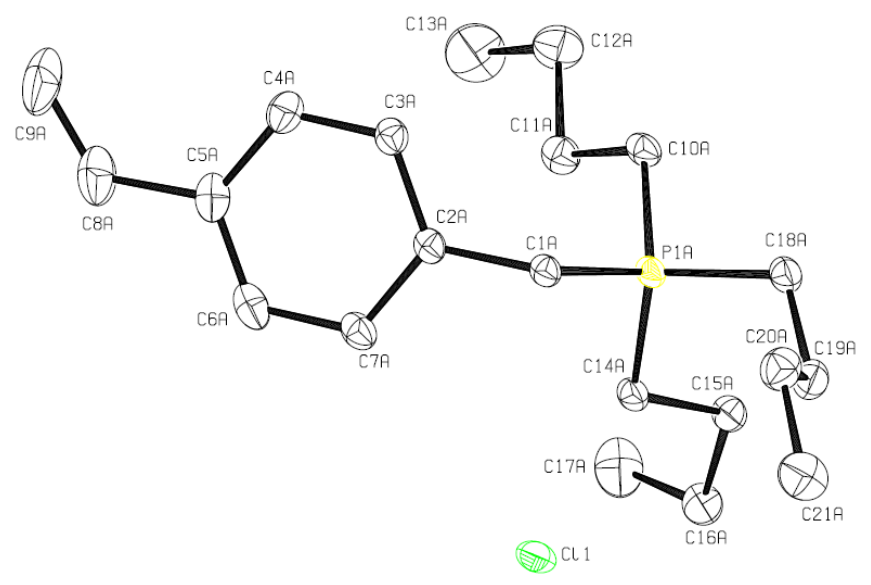

Figure 2.25 ORTEP representation of tributylphosphonium monomer (18)

\subsubsection{4-Vinylbenzyl Trimethylphosphonium Chloride (19)}

The phosphination of VBC was carried out with trimethylphopshine as a $1 \mathrm{M}$ solution in toluene (19, Scheme 2.14). Attempted reaction conditions for this synthesis can be found in Table 2.15. In the first attempt (Entry 1, Table 2.15), the reaction was carried out at rt over 3 days with no additional solvent other than the toluene solution. While the reaction was successful, it was low yielding at $53 \%$. The $S_{N} 2$ reaction requires a more polar solution, so for the second attempt (Entry 2, Table 2.15), additional $\mathrm{MeCN}$ was added, creating a tol/MeCN mixture. The reaction was also heated to $40{ }^{\circ} \mathrm{C}$, dramatically increasing the yield to $95 \%$. Residual trimethylphosphine, toluene, and $\mathrm{MeCN}$ were removed by reduced pressure and oxidized trimethylphosphine was removed by a series of THF washes. The product was confirmed by proton NMR analysis. The protons on the methyl groups correspond to the doublet most upfield at $2.13 \mathrm{ppm}$. The signal integrates for $9 \mathrm{H}$ and due to its vicinity to the phosphorous atom, ${ }^{2} J_{\mathrm{PH}}$ coupling is presented as a doublet. The remainder of signals are similar to the vinylbenzyl portion as described above. ${ }^{31} \mathrm{P}$ NMR analysis of 19 indicated one phosphorous environment at $26.75 \mathrm{ppm}$, in range of phosphonium signals. ${ }^{13} \mathrm{C}$ 
NMR analysis of $\mathbf{1 9}$ was carried out. The most upfield signal at $7.96 \mathrm{ppm}$ corresponds to the terminal carbons of the trimethylphosphine substituent. The proximity of the terminal carbons to the phosphorus atom resulted in ${ }^{1} J_{\mathrm{PC}}$ coupling, presenting as a doublet. The $\mathrm{CH}_{2}$ adjacent to the phosphonium group correspond to a doublet at $29.86 \mathrm{ppm}$, which also experiences ${ }^{1} J_{\mathrm{PC}}$ coupling. The remainder of signals on the ${ }^{13} \mathrm{C}$ NMR spectrum are assigned to the vinylbenzyl group as described earlier. The monomer salt was observed to decompose at $167{ }^{\circ} \mathrm{C}$, possibly as a result of the lack of alkyl chains, unlike the tributylphosphonium monomer. The hygroscopic monomer was stored under nitrogen and absorbed moisture from the air to convert to a gel when improperly stored.

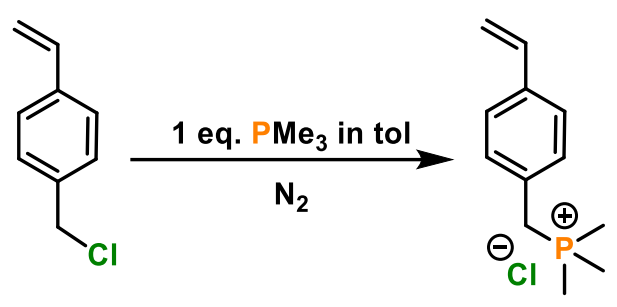

19

Scheme 2.14 Reaction scheme for synthesis of monomer 19

Table 2.15 Reaction conditions for the synthesis of monomer 19.

\begin{tabular}{|c|c|c|c|c|}
\hline Entry & Rxn Temp. & Solvent & Rxn Time & \% Yield \\
\hline $\mathbf{1}$ & $\mathrm{rt}$ & Tol only & 3 days & $53 \%$ \\
\hline $\mathbf{2}$ & $40^{\circ} \mathrm{C}$ & Tol \& MeCN & $4.5 \mathrm{~h}$ & $95 \%$ \\
\hline
\end{tabular}

\subsubsection{4-Vinylbenzyl Diethylsulfonium Chloride (20)}

Monomer 20 was synthesized following a procedure first reported by Hatch et al. ${ }^{97}$ The sulfonium species was synthesized from VBC and diethylsulfide in a mixture of water and $\mathrm{MeOH}$ at $50{ }^{\circ} \mathrm{C}$ for $24 \mathrm{~h}$ (Scheme 2.15). The product was purified by precipitation into excess cold $\mathrm{Et}_{2} \mathrm{O}$ 
from minimal MeOH. Proton NMR analysis confirmed synthesis of the product (Figure A 45). The protons on the terminal carbon on the ethyl groups correspond to the most upfield signal at $1.39 \mathrm{ppm}$. The methylene protons correspond to the downfield peak at $3.45 \mathrm{ppm}$, indicating lower electronegativity than the chlorine of the VBC starting material. The neighbouring multiplet signal at $3.63 \mathrm{ppm}$ is assigned to $\mathrm{H} 8$, splitting with H9. The remaining signals correspond to the remaining vinylbenzyl group, as described for similar monomers described above. Over the course of a month, the product was found to revert back to starting material, even when in cold storage under a nitrogen atmosphere. The instability of the product is characteristic of sulfonium salts. ${ }^{98,99}$ Anionic exchange of chlorine with a less nucleophilic anion such as tetrafluoroborate can improve the stability of the sulfonium compound.

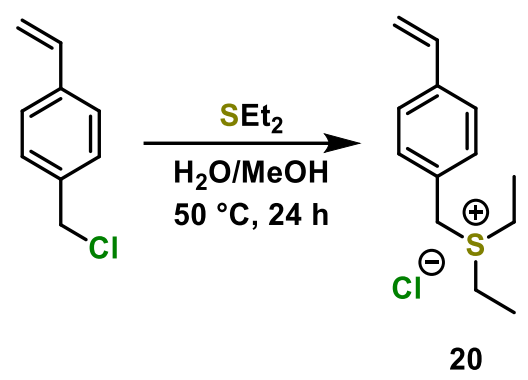

Scheme 2.15 Synthesis of monomer 20

\subsubsection{4-Vinylbenzyl Dimethyloctadecylammonium Chloride (21)}

Quaternary ammonium monomer with a long $n$-alkyl chain substituent of $n=18,21$, was synthesized following a similar procedure described above. VBC was aminated with $N, N-$ dimethyloctadecylamine by heating in $\mathrm{MeOH}$ overnight at $50^{\circ} \mathrm{C}$ (Scheme 2.16). The product was purified by precipitation into cold $\mathrm{Et}_{2} \mathrm{O}$. Proton NMR analysis confirmed synthesis of 21. The protons $\mathrm{H} 12$ on the terminal carbon are assigned to the most upfield signal at $0.83 \mathrm{ppm}$. Protons 
of the long alkyl chain are found in the 1-2 ppm range, indicated by the integration of 32 protons. The downfield singlet at $3.25 \mathrm{ppm}$ integrate for 6 protons, corresponding to the methyl groups on the quaternary ammonium group. Protons at position H9 is assigned to the multiplet signal at 3.46 ppm due to splitting with neighbouring protons on the alkyl chain integrating for 2 protons. The remaining signals appropriately correspond to the vinyl benzyl group, similar to the monomers discussed above.
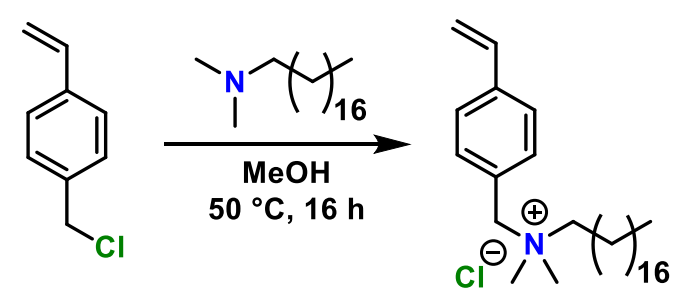

21

Scheme 2.16 Synthesis of monomer 21

\subsubsection{4-Vinylbenzyl Diethylamine (22)}

The synthesis of $\mathbf{2 2}$ followed a procedure reported by Su et al. ${ }^{100}$ VBC was aminated with diethyamine in the presence of $\mathrm{K}_{2} \mathrm{CO}_{3}$, illustrated in Scheme 2.17. Unreacted VBC was removed by flash column chromatography. ${ }^{1} \mathrm{H} \mathrm{NMR}\left(\mathrm{CDCl}_{3}\right)$ analysis confirmed synthesis of the product. The protons on the terminal carbon of the ethyl groups correspond to the upfield triplet signal at $1.08 \mathrm{ppm}$ which was integrated for 6 protons. The neighbouring proton signal at $2.56 \mathrm{ppm}$ integrating for $4 \mathrm{H}$ was assigned to the adjacent proton from the terminal carbon on the ethyl substituents. This quartet signal splitting corresponds to the terminal proton signal. The remaining signals correspond to vinylbenzyl group as described above. The carbon NMR of the monomer was also obtained for structure confirmation. The terminal carbons on the ethyl groups are assigned 
to the shift at $11.65 \mathrm{ppm}$. The neighbouring carbon correspond to the signal at $46.62 \mathrm{ppm}$. The rest of the signals correctly correspond to the vinylbenzyl group, as discussed earlier. NMR analysis are in agreement with literature. ${ }^{100}$

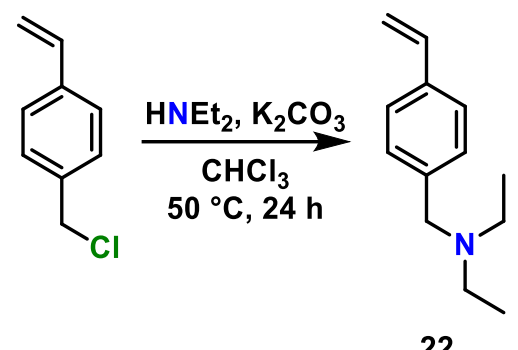

Scheme 2.17 Synthesis of monomer precursor 22

\subsubsection{NMP of Quaternary Ammonium/Phosphonium Monomers}

2.4.2.1 Aqueous NMP for Synthesis of Poly(vinylbenzyl-triethylammonium chloride) (23)

The NMP of monomer 17 was carried out following a procedure previously outlined by Kaur et al. synthesize poly(vinylbenzyl-triethylammonium chloride) (poly(VBNEt $), 23) .{ }^{93}$ The solution polymerization was redox initiated with potassium persulfate and mediated with TEMPO in a mixture of water and ethylene glycol. The reaction was heated to $125^{\circ} \mathrm{C}$ for $24 \mathrm{~h}$ (Scheme 2.18). Proton NMR analysis confirmed the formation of polymer due to the disappearance of vinyl signals in the 5-6 ppm range and appearance of broadened signals. Integrations of the proton NMR also indicate the polymerization of the quaternary monomer. The most upfield signal at $1.31 \mathrm{ppm}$ integrate for $12 \mathrm{H}$, corresponding to protons at the $\mathrm{C} 1, \mathrm{C} 2$, and terminal $\mathrm{C} 9$ positions. The protons at $\mathrm{C} 8$ are assigned to the signal at $3.18 \mathrm{ppm}$, integrating for $6 \mathrm{H}$. The $\mathrm{CH}_{2}$ group adjacent to the nitrogen atom is represented by the downfield signal at $4.37 \mathrm{ppm}$. Protons on the aryl group appear at 6.77 and $7.33 \mathrm{ppm}$ integrating for 4 protons together. While NMR analysis is indicative of 
polymer, characterization by GPC was not performed due to the lack of solubility in the THF eluent, and lack of access to a water-based GPC instrument. Thermoanalysis of the homopolymer determined a $T_{g}$ at $112.5^{\circ} \mathrm{C}$ and $T_{m}$ at $216.3{ }^{\circ} \mathrm{C}$ (Figure C 7), again unlike the monomer which was found to decompose at $200{ }^{\circ} \mathrm{C}$. The presence of a high $T_{m}$ make homopolymer a promising candidate as an additive in injection molding.

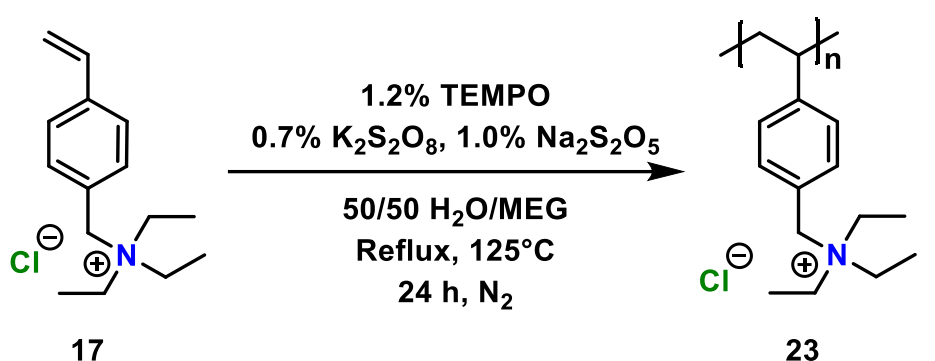

Scheme 2.18 Synthesis of Poly(VBNEt 3 ) (23)

2.4.2.2 Aqueous NMP for Synthesis of Poly(vinylbenzyl-tributylphosphonium chloride) (24)

Monomer $\mathbf{1 8}$ was polymerized by NMP, redox initiated by potassium persulfate and mediated by TEMPO at $125{ }^{\circ} \mathrm{C}$ similar to the synthesis of poly(vinylbenzyl-tributylphosphonium chloride) (poly(VBPBu$), 24$, Scheme 2.19). Proton and phosphorous NMR indicated the polymerization of the quaternary phosphonium monomer. The NMR signals are seen to broaden after polymerization. Figure 2.26 show the ${ }^{31} \mathrm{P}$ NMR of the monomer in comparison to the polymer. The signal around $32 \mathrm{ppm}$ significantly broadens post-polymerization, indicative of polymer. Full NMR spectra can be seen in Figure A 51. On the ${ }^{1} \mathrm{H}$ NMR, the signals in the upfield range $0.5-1.7 \mathrm{ppm}$ corresponded to the butyl substituents on the phosphonium and the polymer backbone at position $\mathrm{H} 1$ and $\mathrm{H} 2$. The remaining $\mathrm{VBC}$ signals are similar to the reported triethylammonium polymer described above. The ${ }^{13} \mathrm{C}$ NMR spectrum was obtained and can be 
seen in Figure A 52. The terminal carbon correspond to the signal most upfield at $12.81 \mathrm{ppm}$. The $\mathrm{C} 8$ atom was assigned to the doublet at $17.83 \mathrm{ppm}$, experiencing carbon-phosphorous ${ }^{1} \mathrm{~J}$ coupling to the phosphonium atom. The multiplet signal at $23.30 \mathrm{ppm}$ represents $\mathrm{C} 9$ and $\mathrm{C} 10$, which experience ${ }^{2} J$ and ${ }^{3} J$ carbon-phosphorous coupling, respectively. Due to low resolution, C9 and C10 could not be exactly assigned as it did not appear in the 2D HSQC NMR spectrum. The broad signal at $26.32 \mathrm{ppm}$ correspond to C7, as indicated by the 2D HSQC NMR spectrum. The next downfield signal at $40.14 \mathrm{ppm}$ is seen to be very broad, corresponding to $\mathrm{C} 1$ and $\mathrm{C} 2$ of the polymer backbone.
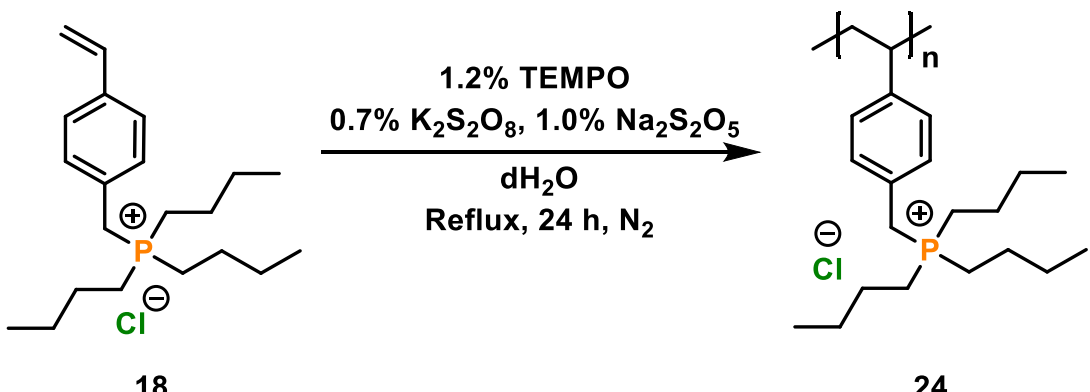

24

Scheme 2.19 Synthesis of poly $\left(\mathrm{VBPBu}_{3}\right)(\mathbf{2 4})$

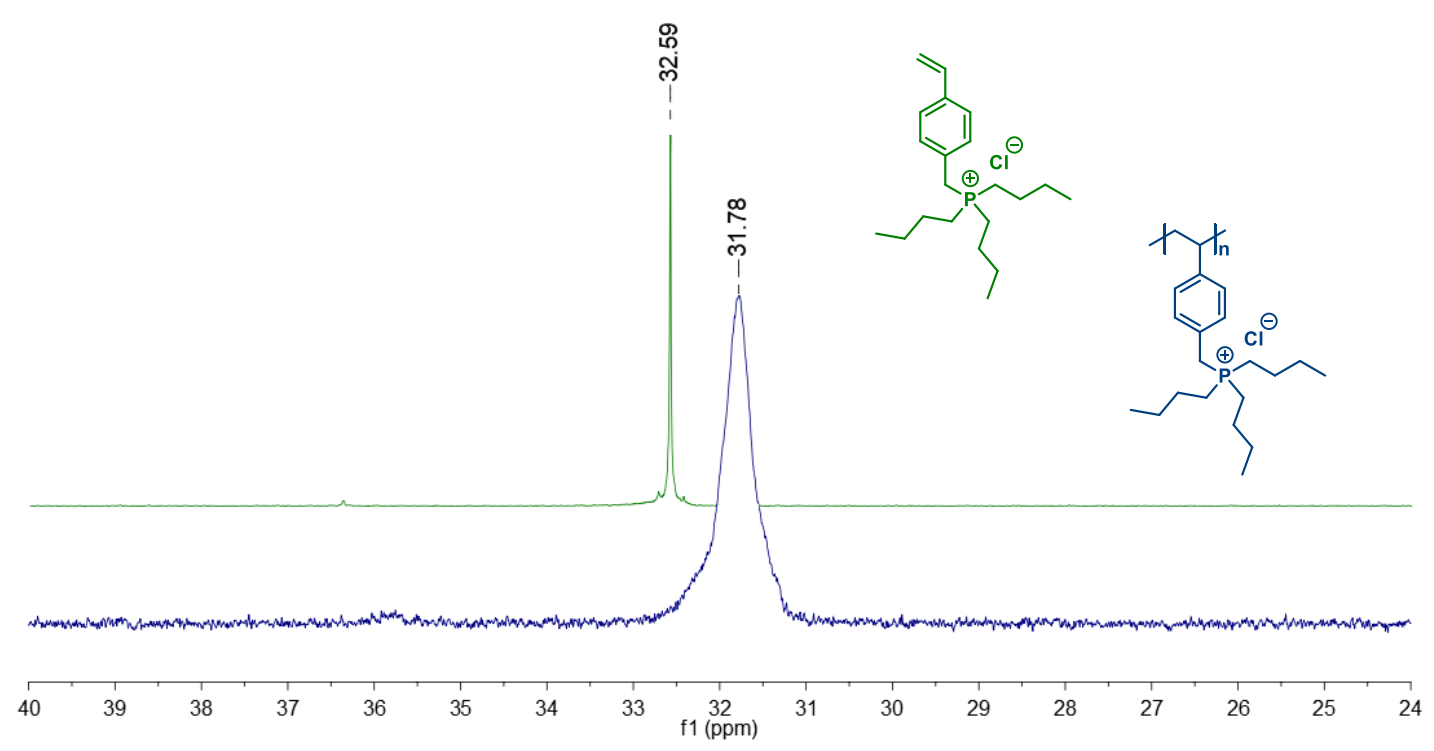

Figure 2.26 Stacked ${ }^{31} \mathrm{P}$ NMR spectra of monomer 18 and polymer $24\left(\mathrm{D}_{2} \mathrm{O}\right)$ 
2.4.2.3 Aqueous NMP for Synthesis of Poly(vinylbenzyl-trimethylphosphonium chloride) (25)

Following similar reaction conditions outlined in section 2.42.1, monomer 19 was polymerized with $\mathrm{K}_{2} \mathrm{~S}_{2} \mathrm{O}_{8} / \mathrm{Na}_{2} \mathrm{~S}_{2} \mathrm{O}_{5}$ redox initiators and mediated by TEMPO in $\mathrm{dH}_{2} \mathrm{O}$ at $125^{\circ} \mathrm{C}$ for $24 \mathrm{~h}$ to synthesize poly(vinylbenzyl-trimethylphosphonium chloride (poly(VBPMe 3 ), 25 , Scheme 2.20). NMR analysis confirmed polymerization by broadened proton signals and the disappearance of the vinyl signals. Phosphorous NMR spectrum showed one broad signal at 25.75 ppm, corresponding to the quaternary phosphonium group on the polymer (Figure A 56).

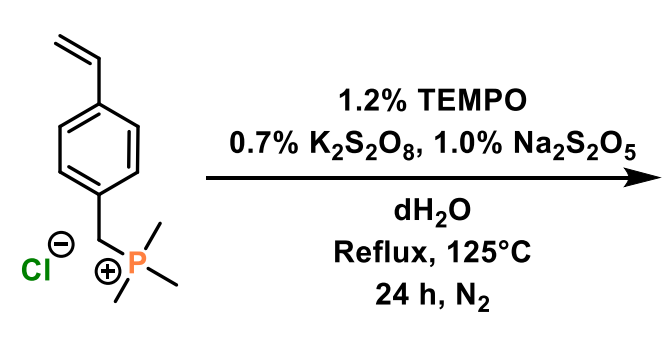

19

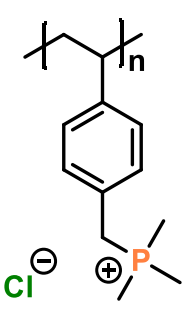

25

Scheme 2.20 Synthesis of poly(VBPMe 3$)(25)$

\subsubsection{Attempted aqueous NMP of monomer $\mathbf{2 0}$}

The polymerization of the sulfonium monomer $\mathbf{2 0}$ was attempted following the same reaction conditions outlined for polymer 17 (Scheme 2.21). The reaction formed a gel-like precipitate which was found to be insoluble in a range of solvents (ie. DMF, MeCN, THF, DMSO, $\mathrm{MeOH}$ ). Insolubility prevented purification and characterization of the product. The lack of solubility suggests the possibility of polymer crosslinking. To probe the polymerization of sulfonium monomers, a 50/50 ratio of sulfonium monomer and ammonium monomer or VBC as 
a miniemulsion polymerization may be explored under similar reaction conditions. Unfortunately due to time restraints, this was not attempted.

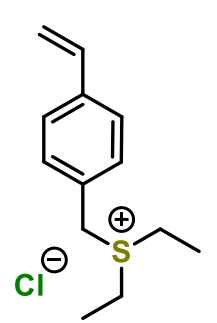

20

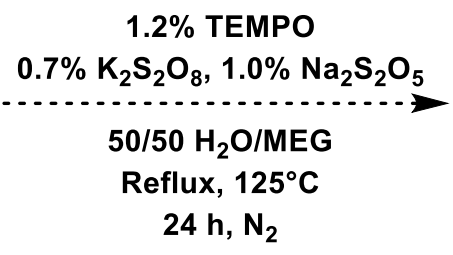

$24 \mathrm{~h}, \mathrm{~N}_{2}$

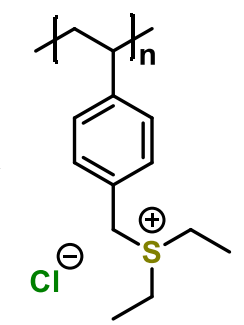

Scheme 2.21 Attempted polymerization of sulfonium monomer 20 


\section{CONCLUSION}

In this work, a range of quaternary ammonium and phosphonium random block copolymer were synthesized for thin film polymer coatings. These coatings were found to be robust against water and Windex ${ }^{\circledR}$ but possessed selective antibacterial properties. Polymer analogs of short $n$ alkyl chains were the only antibacterial coatings, active only against the gram-positive model. Species with bulkier substituents were inactive against both bacterial models, Arthrobacter $\mathrm{sp}$. and E. coli wt36. We contribute the inactivity to charge burial from bulky, hydrophobic substituents and the low degree of coating roughness.

Random block copolymer thin films with a low loading of quaternary ammonium with C18 alkyl chain substituent were synthesized to investigate charge burial attributed by the bulky substituent. The antibacterial efficacy remained inactive, unchanged from the high quaternary ammonium loading coatings. The sustained inactivity suggests that substituent bulkiness is not the major contributor to charge burial. The polymeric backbone may be the major hydrophobic attributor to charge inaccessibility at the surface.

Coatings of polymer brushes and random block copolymers with quaternary phosphonium pendants were formulated to explore charge availability at the interface. These surfaces were both highly charge dense at $10^{17}\left[\mathrm{Q}^{+}\right] \mathrm{cm}^{-2}$ but the brush coatings had a drastically higher contact angle, indicating that charges were made more accessible due to the brush structures. Antibacterial activity of the brushes was improved compared to the random block copolymer equivalent when tested against the gram-positive model, establishing the problem to be charge burial and inaccessibility. However, the brush coatings continued to fail when antibacterial efficacy was evaluated against the gram-negative species, E. coli wt36. 
Lastly, quaternary ammonium and phosphonium monomers were prepared for CRP polymerization. These fully quaternized polymers aim to resolve a persistent solubility issue encountered throughout this work. Full solubility of the polymers for formulation of antibacterial coatings can vastly improve compound characterization as well as coating quality. Higher compound polarity can allow for the use of more passive and environmentally friendly solvents such as water for coating formulation and disposal.

\subsection{Future Work}

Although this work produced robust, solvent resistant coatings, these surfaces were found to be biologically inactive. This work demonstrated the complexity of antimicrobial polymer coating design and the many facets required for consideration. An aspect worth further investigation is the effect of surface roughness. Electrospray deposition of the formulation may provide rougher coatings and result in higher antibacterial efficacy. Preliminary work with electrospray coating polymer brushes $\mathbf{1 5}$ at $1 \mathrm{w} / \mathrm{v} \% \mathrm{EtOH}$ solution can be seen in Figure 3.1. The contact angle was found to be slightly lower than the spin coat deposition of the same formulation, tabulated in Table 3.1. The difference in wettability suggests varying surface topography, a promising indication of increased surface roughness. These coatings should be evaluated for antibacterial efficacy and roughness parameters. Rougher coatings can also be induced by casting thinner films on substrates with higher roughness parameters, and the results from this roughness study can provide insight into better coating design. 


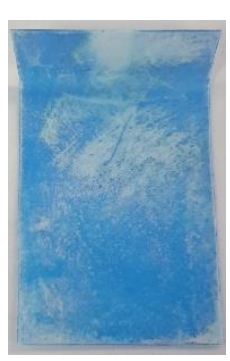

Figure 3.1 PS substrate coated by electrospray deposition of diblock copolymer $\mathbf{1 5}$ and stained with BPB

Table 3.1 Advancing water contact angle of PS substrates coated with $\mathbf{1 5}$ by spin-coating and electrospray deposition

\begin{tabular}{|c|c|c|}
\hline & Spin-Coating Deposition & Electrospray Deposition \\
\hline Advancing Water Contact Angle & $61.3 \pm 2.0^{\circ}$ & $69.7 \pm 5.0^{\circ}$ \\
\hline
\end{tabular}

Due to solubility issues experienced throughout this work, polymer brushes synthesized from cationic monomers can provide fully soluble polymers and avoid the formation of micelles. The quaternary ammonium analog of benzophenone monomer 27 can be synthesized by amination of (3-bromopropoxy)benzophenone under similar condition reactions described for quaternization (Scheme 3.1).

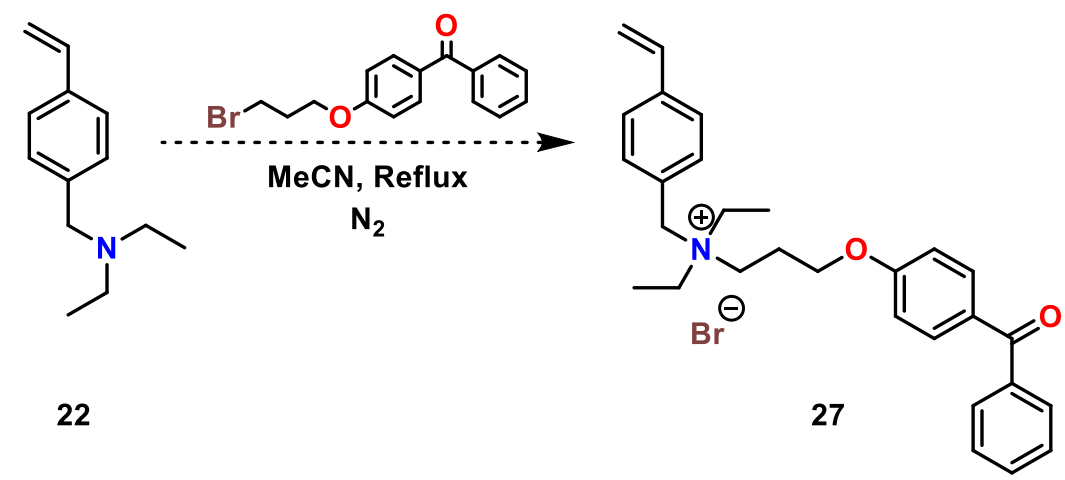

Scheme 3.1 Quaternization reaction for synthesis of benzophenone quaternary ammonium monomer 27

By RAFT polymerization, cationic monomer such as $\mathbf{2 1}$ can undergo homopolymerization initiated by AIBN and mediated by RAFT agent 2-methyl-2[(dodecylsulfanylthiocarbonyl)sulfanyl]propanoic acid (CTA3) for synthesis for 26 (Scheme 3.2). 
RAFT agent CTA3 has been shown to be successful in mediating homopolymerization of styrenic quaternary phosphonium compounds with long alkyl chain substituents. ${ }^{82}$ Use of RAFT polymerization techniques will result in a living polymer that can undergo further polymerization.

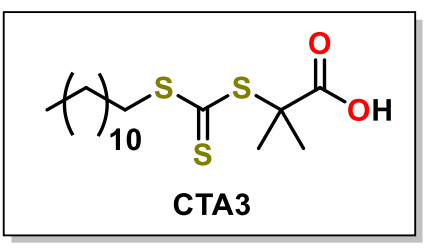

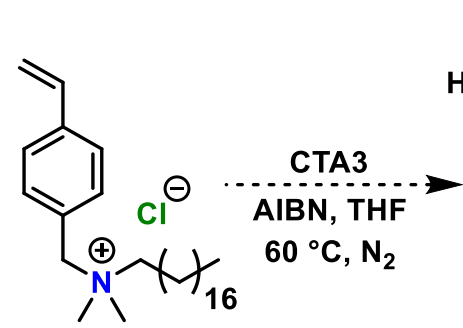

21

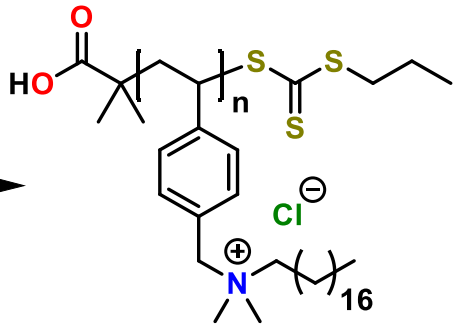

26

Scheme 3.2 RAFT polymerization of $\mathbf{2 1}$ for synthesis of homopolymer $\mathbf{2 6}$

The quaternary ammonium benzophenone monomer $\mathbf{2 7}$ can then be copolymerized with homopolymer 21, previously synthesized by RAFT polymerization (28, Scheme 3.3). The continued polymerization will synthesize A-B diblock copolymers for formulation of polymeric brush coatings. Polymer films can be casted by spin coating or electrospray deposition and evaluated for biological efficacy. 
<smiles>CC(SC(=S)SC(=O)C(C)(C)C)c1ccc(CN(C)C)cc1</smiles>

26<smiles>C=Cc1ccc(C[N+](CC)(CC)CCCOc2ccc(C(=O)c3ccccc3)cc2)cc1</smiles>

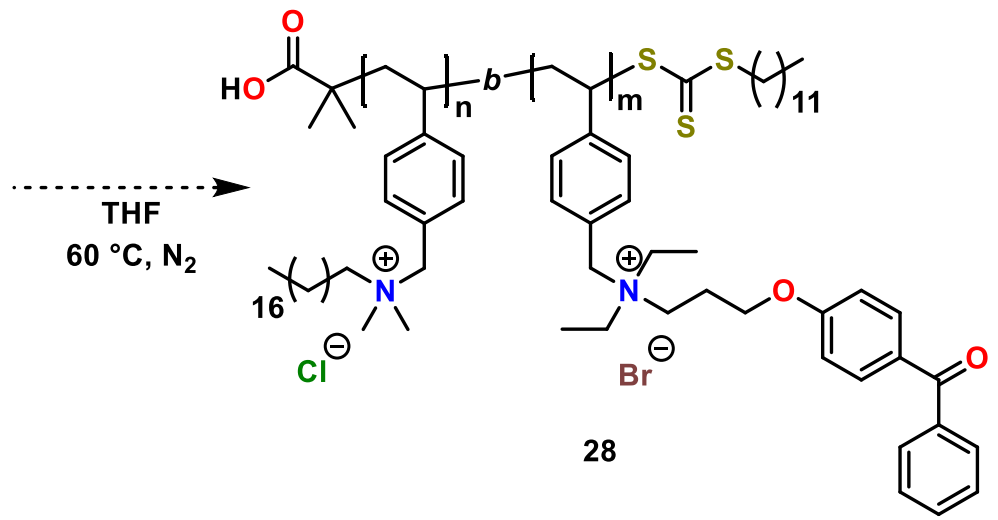

Scheme 3.3 Continued RAFT copolymerization to incorporate benzophenone monomer into polymer chain $(\mathbf{2 8})$

Thermoanalysis of many polymers described in this work indicate good thermal stability and may be suitable for injection molding. Small molecule QACs are hindered from wide use incorporation into thermoplastics due to their low thermal stability. These quaternary ammonium and phosphonium polymers with benzophenone linkages are promising as non-leaching additives and warrant investigation. 


\section{EXPERIMENTAL METHODS}

\subsection{General Methodology}

Acetone was dried over anhydrous calcium sulfate, decanted, and distilled. Anhydrous $\mathrm{MeOH}$ was used directly from the Aldrich Sure/Seal container. Hexanes, tetrahydrofuran (THF), and $\mathrm{Et}_{2} \mathrm{O}$ were purchased from Sigma Aldrich and dried using an MBraun MBM-SPS solvent system. All other solvents and reagents were reagent grade and used as supplied. Stock plastic polystyrene (cat. 89106-754) was obtained from VWR International.

Flash column chromatography was carried out on silica gel (Silica Gel 60, 40-63 $\mu \mathrm{m}$, EMD). Reactions and chromatographic purifications were monitored by thin layer chromatography (TLC). Silica-coated aluminum plates (Alugram Sil G/UV254, Macherey-Nagel) were used for TLC tests. Plates visualized by UV light or ninhydrin staining and heated with a heat gun.

Characterization by ${ }^{1} \mathrm{H},{ }^{13} \mathrm{C},{ }^{31} \mathrm{P}$, and $2 \mathrm{D}$ NMR (Nuclear Magnetic Resonance) spectroscopy was performed in deuterated chloroform $\left(\mathrm{CDCl}_{3}\right)$, methanol $\left(\mathrm{MeOD}-\mathrm{d}_{4}\right)$, or dimethyl sulfoxide (DMSO- $\mathrm{d}_{6}$ ) on the Bruker AVII-400 MHz spectrometer at room temperature unless otherwise stated. ${ }^{1} \mathrm{H}$ and ${ }^{13} \mathrm{C}$ spectra were referenced to residual $\mathrm{CHCl}_{3}(7.26 \mathrm{ppm}$ and $77.00 \mathrm{ppm}$, respectively), $\mathrm{MeOH}$ (3.31 ppm and $49.00 \mathrm{ppm}$, respectively), or DMSO (2.50 ppm and 39.52 ppm, respectively) solvent signals. ${ }^{31} \mathrm{P}$ NMR was referenced against the internal standard, phosphoric acid $\left(\mathrm{H}_{3} \mathrm{PO}_{4}\right)$. NMR data are reported as: chemical shift $\delta(\mathrm{ppm})$, multiplicity, coupling constant $J(\mathrm{~Hz})$, and number of protons. Mass spectrometry data was obtained on an Agilent 6538 UHD Q-TOF mass spectrometer at the Advanced Instrumentation for Molecular Structure Mass 
Spectrometry Laboratory. Gel permeation chromatography (GPC) analyses were performed in THF (HPLC-grade, $1.0 \mathrm{~mL} / \mathrm{min}$ ) using a Viscotek Triple Model 302 Detector system equipped with a Refractive Index Detector (RI), a four-capillary differential viscometer (VISC), and a right angle $\left(90^{\circ}\right)$ laser light scattering detector. GPC columns were calibrated against broad and narrow polystyrene standards (American Polymer Standards). Structure elucidation by $\mathrm{x}$-ray crystallography was performed by Dr. Alan Lough at the University of Toronto on a Bruker Kappa CCD diffractometer. Differential scanning calorimetry (DSC) was completed on a DSC Q20 TA Instruments at a heating rate of $20^{\circ} \mathrm{C} / \mathrm{min}$ under a nitrogen atmosphere in an aluminum Tzero pan with approximately $8 \mathrm{mg}$ of sample. Elemental analysis was performed by Atlantic Microlab Inc. in Norcross, GA, USA. 


\subsection{Synthetic Work}

\subsubsection{Synthesis of VBBP Monomer (1)}

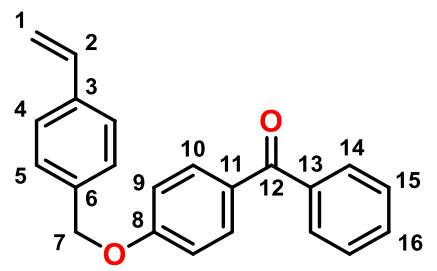

VBBP was prepared by a modified version of a method first described by Lin et al.$^{84} \mathrm{~A}$ solution of 4-hydroxybenzophenone (4.03 g, $0.02 \mathrm{~mol}), \mathrm{K}_{2} \mathrm{CO}_{3}(5.58 \mathrm{~g}, 0.04 \mathrm{~mol})$, and $\mathrm{KI}$ (4.36 g, $0.03 \mathrm{~mol}$ ) in $60 \mathrm{~mL}$ of $\mathrm{MeCN}$ was refluxed for $2.5 \mathrm{~h}$. A solution of $3.80 \mathrm{~mL}$ 4-vinylbenzyl chloride $(0.03 \mathrm{~mol})$ in $40 \mathrm{~mL} \mathrm{MeCN}$ was added dropwise to the mixture and refluxed for $24 \mathrm{~h}$ at $80{ }^{\circ} \mathrm{C}$. The reaction mixture was slowly brought to room temperature and gravity filtered. The solvent was removed from the filtrate by rotary evaporation and redissolved in $150 \mathrm{~mL} \mathrm{DCM}$ and $\sim 180 \mathrm{~mL}$ of $\mathrm{dH}_{2} \mathrm{O}$ was added. The mixture was extracted and washed thrice with DCM $(50 \mathrm{~mL})$ and dried over magnesium sulfate $\left(\mathrm{MgSO}_{4}\right)$. After removal of $\mathrm{MgSO}_{4}$ by gravity filtration, the solvent was removed by rotatory evaporation followed by drying in vacuo leaving a mustard yellow powder. The crude product was purified by recrystallization from high boiling $\left(60-90^{\circ} \mathrm{C}\right)$ petroleum ether after refluxing for $4 \mathrm{~h}$ and hot filtered. The mixture was cooled and the solid was collected by vacuum filtration, followed by cold petroleum ether wash. The recrystallization step was repeated once more and dried in vacuo at $80{ }^{\circ} \mathrm{C}$, yielding creamy yellow lustrous flakes. Yield $82 \%$ (5.33 g); melting point 104.0-105.0 ${ }^{\circ} \mathrm{C} .{ }^{1} \mathrm{H}$ NMR (400 MHz, $\left.\mathrm{CDCl}_{3}, \delta\right): 5.13(\mathrm{~s}, 2 \mathrm{H}, \mathrm{H} 7), 5.28\left(\mathrm{~d},{ }^{2} J_{\mathrm{HH}}\right.$ $\left.=12.0 \mathrm{~Hz}, 1 \mathrm{H}, \mathrm{H} 1_{\mathrm{cis}}\right), 5.79\left(\mathrm{~d},{ }^{2} J_{\mathrm{HH}}=20.0 \mathrm{~Hz}, 1 \mathrm{H}, \mathrm{H} 1_{\text {trans }}\right), 6.74\left(\mathrm{dd},{ }^{3} J_{\mathrm{HH}}=17.4 \mathrm{~Hz},{ }^{3} J_{\mathrm{HH}}=10.8\right.$ $\mathrm{Hz}, 1 \mathrm{H}, \mathrm{H} 2), 7.04\left(\mathrm{~d},{ }^{3} J_{\mathrm{HH}}=8.8 \mathrm{~Hz}, 2 \mathrm{H}, \mathrm{H} 10\right), 7.41\left(\mathrm{~d},{ }^{3} J_{\mathrm{HH}}=8.0 \mathrm{~Hz}, 2 \mathrm{H}, \mathrm{H} 4\right), 7.45\left(\mathrm{~d},{ }^{3} J_{\mathrm{HH}}=\right.$ 
$8.0 \mathrm{~Hz}, 2 \mathrm{H}, \mathrm{H} 5), 7.48\left(\mathrm{t},{ }^{3} J_{\mathrm{HH}}=8.0 \mathrm{~Hz}, 2 \mathrm{H}, \mathrm{H} 15\right), 7.57\left(\mathrm{t},{ }^{3} J_{\mathrm{HH}}=8.0 \mathrm{~Hz}, 1 \mathrm{H}, \mathrm{H} 16\right), 7.77\left(\mathrm{~d},{ }^{3} J_{\mathrm{HH}}\right.$ $=8.0 \mathrm{~Hz}, 2 \mathrm{H}, \mathrm{H} 14), 7.84\left(\mathrm{~d},{ }^{3} J_{\mathrm{HH}}=8.0 \mathrm{~Hz}, 2 \mathrm{H}, \mathrm{H} 9\right) \mathrm{ppm} .{ }^{13} \mathrm{C}\left\{{ }^{1} \mathrm{H}\right\} \mathrm{NMR}\left(101 \mathrm{MHz}, \mathrm{CDCl}_{3}, \delta\right)$ : 69.89 (C7), 114.33 (C1), 114.39 (C10), 126.49 (C5), 127.68 (C4), 128.16 (C15), 129.70 (C14), 130.35 (C11), 131.88 (C16), 132.53 (C9), 135.66 (C6), 136.29 (C2), 137.58 (C3), 138.20 (C13), 162.28 (C8), 195.49 (C12) ppm. HRMS (ESI-Q-TOF) $m / z\left[\mathrm{M}^{+} \mathrm{H}\right]^{+}$calcd for $\mathrm{C}_{22} \mathrm{H}_{18} \mathrm{O}_{2}$ : 315.1380, found: 315.1371 . (Agrees with literature ${ }^{84}$ ) 


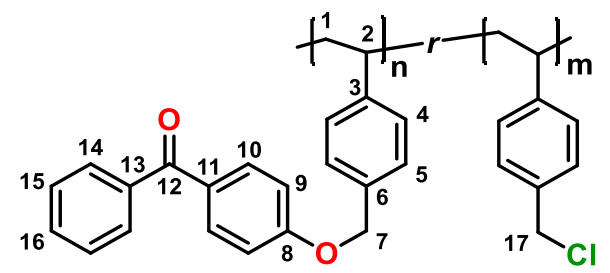

In a flame-dried, $150 \mathrm{~mL}$ round bottom with a magnetic stir bar, $0.52 \mathrm{~g}$ of monomer 1 (1.6 mmol) was dissolved in $4.6 \mathrm{~mL}$ VBC (0.033 mol) with $30 \mathrm{mg}$ (2,2,6,6-tetramethylpiperidin-1yl)oxyl (TEMPO, $0.19 \mathrm{mmol}$ ) and $59 \mathrm{mg}$ benzoyl peroxide (BPO, $0.24 \mathrm{mmol})$. The flask was evacuated and back filled with nitrogen followed by degassing of the reaction mixture with nitrogen over ice for $20 \mathrm{~min}$. The reaction was stirred in a preheated oil bath at $125{ }^{\circ} \mathrm{C}$ for $5 \mathrm{~h}$, forming a viscous, orange resin. The reaction mixture was diluted in $\sim 25 \mathrm{~mL}$ THF and purified by precipitation into $\sim 1 \mathrm{~L}$ cold $\mathrm{MeOH}$. The mixture was decanted and the resultant solid was collected by vacuum filtration followed by drying in vacuo at $60^{\circ} \mathrm{C}$, yielding a fine off-white powder. Yield $77 \%(5.21 \mathrm{~g}) . \mathrm{PDI}=1.06 . \mathrm{M}_{\mathrm{w}}=31,000 \mathrm{Da} . T_{\mathrm{g}}=110.8{ }^{\circ} \mathrm{C} .{ }^{1} \mathrm{H}$ NMR $\left(400 \mathrm{MHz}, \mathrm{CDCl}_{3}, \delta\right): 1.05-$ 1.55 (br m, 36 H, H2), 1.62-2.06 (br m, 18 H, H1), 4.17-4.76 (br s, 34 H, H17), 4.91-5.22 (br s, 2 H, H7), 6.19-6.77 (br m, 36 H, H4), 6.86-7.23 (br m, 36 H, H5), 7.33-7.66 (br m, 5 H, H14-16), 7.67-7.97 (br m, 4 H, H9-10). E.A.: Anal. Calcd. C, 72.34; H, 5.92. Found: C, 72.34, H: 5.85. 


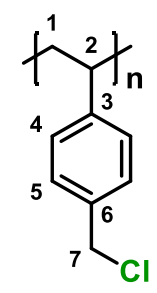

VBC (1.0 mL, $7.3 \mathrm{mmol})$ was added to a flame-dried Schlenk flask under nitrogen. In the glovebox, AIBN (5 mg, $0.03 \mathrm{mmol})$ or BPO $(7-11 \mathrm{mg}, 0.03-0.044 \mathrm{mmol})$ and CTA1 (8-12 mg, 0.03-0.043 mmol) or CTA2 (12-17 $\mathrm{mg}, 0.029-0.043 \mathrm{mmol})$ was added to the flask. The flask was brought out of the glovebox and the reaction mixture was degassing with nitrogen over ice for 20 min. The reaction was stirred in a preheated oil bath at $80-100{ }^{\circ} \mathrm{C}$ for $5-24 \mathrm{~h}$, forming a viscous, pink (CTA1) or yellow (CTA2) resin. The reaction mixture was diluted in $\sim 7 \mathrm{~mL}$ THF and purified by precipitation into $\sim 250 \mathrm{~mL}$ cold $\mathrm{MeOH}$. The mixture was decanted and the precipitate was collected by vacuum filtration followed by drying in vacuo at $50{ }^{\circ} \mathrm{C}$, yielding a fine pale pink (CTA1) or pale yellow (CTA2) powder. Yield 28-82 \% (0.31-0.91 g). $Đ=1.02-1.07 . \mathrm{M}_{\mathrm{w}}=4150-$ 35000 Da. ${ }^{1} \mathrm{H}$ NMR (400 MHz, $\mathrm{CDCl}_{3}, \delta$ ): 1.07-2.50 (br m, 3 H, H1, H2), 4.23-4.72 (br s, $2 \mathrm{H}$, H7), 6.13-6.81 (br m, 2 H, H4), 6.82-7.24 (br m, 2 H, H5) ppm. (Agrees with literature ${ }^{101}$ ) 


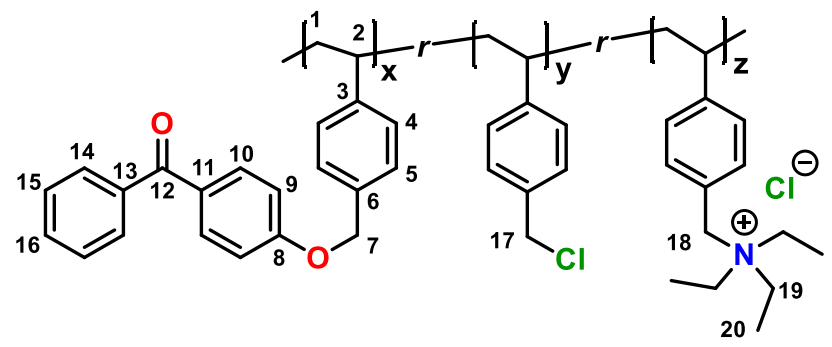

Poly(VBC-BP) (2) (4.81 g, $0.0297 \mathrm{~mol})$ was added to a flame-dried, $150 \mathrm{~mL}$ three-neck round bottom equipped with a condenser. The flask was quickly flame-dried again and back filled with nitrogen. Acetone $(30 \mathrm{~mL})$ and triethylamine $(7.8 \mathrm{~mL}, 0.056 \mathrm{~mol})$ were added to the flask under nitrogen via syringe. The reaction mixture was refluxed under nitrogen for $2 \mathrm{~h}$ forming a yellow precipitate. Anhydrous $\mathrm{MeOH}(20 \mathrm{~mL})$ was added via syringe to dissolve the precipitate and the reaction continued for $22 \mathrm{~h}$. The reaction solvent was removed in vacuo and redissolved in $\sim 30 \mathrm{~mL} \mathrm{MeOH}$. The mixture was purified by precipitation into excess cold THF. The precipitate was collected by vacuum filtration and dried in vacuo at $60{ }^{\circ} \mathrm{C}$. The resultant powder was purified once more by precipitation from isopropanol into cold hexanes and the precipitate was collected by vacuum filtration and dried in vacuo at $60{ }^{\circ} \mathrm{C}$, yielding a fine white powder. Yield: >99\% (7.34 g). $T_{\mathrm{g}}=92.5{ }^{\circ} \mathrm{C} \cdot T_{m}=218.5{ }^{\circ} \mathrm{C} .{ }^{1} \mathrm{H}$ NMR $\left(400 \mathrm{MHz}, \mathrm{DMSO}-\mathrm{d}_{6}, \delta\right.$ ): 0.56-1.96 (br m, $155 \mathrm{H}, \mathrm{H} 1$, H2, H20), 2.84-3.59 (br s, 67 H, H19), 4.17-5.02 (br s, 27 H, H17, H18), 5.03-5.43 (br s, 2 H, H7), 6.00-8.17 (br m, 67 H, H4, H5, H9, H10, H14-H16). E.A.: Anal. Calcd. C: 71.63, H: 9.17, N: 4.26. Found: C: $65.16, \mathrm{H}: 9.26, \mathrm{~N}: 4.31$. 


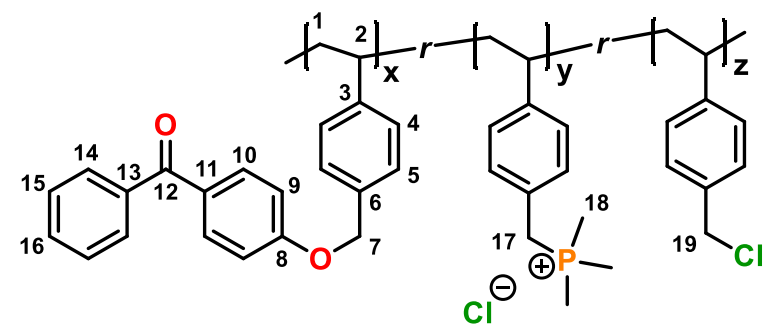

Copolymer 2 (2.16 g, $0.0133 \mathrm{~mol})$ was added to a flame-dried, $100 \mathrm{~mL}$ round bottom flask. The flask was quickly flame-dried again and back filled with nitrogen. After purging three times, $20 \mathrm{~mL}$ of $1 \mathrm{M}$ trimethylphosphine in toluene $(0.020 \mathrm{~mol})$ and $\mathrm{MeCN}(20 \mathrm{~mL})$ were added to the flask under nitrogen via syringe. The reaction mixture was heated to $40{ }^{\circ} \mathrm{C}$ for $4 \mathrm{~h}$ forming an offwhite precipitate. The solvent was removed in vacuo and redissolved in $\sim 15 \mathrm{~mL}$ EtOH. The mixture was purified by precipitation into excess cold hexanes. The resultant mixture was decanted and the precipitate collected was dried in vacuo at $60{ }^{\circ} \mathrm{C}$, yielding a fine off-white powder. Yield $68 \%$ (2.01 g). ${ }^{1} \mathrm{H}$ NMR (400 MHz, MeOD, $\delta$ ): 1.32-2.56 (br m, 245 H, H1, H2, H19), 3.67-4.31 (br s, 37 H, H18), 4.45-4.75 (br s, 4 H, H17), 4.97-5.55 (br s, 2 H, H7), 6.15-7.99 (br m, 104 H, H4, H5, H9, H10, H14-H16). ${ }^{31} \mathrm{P}\left\{{ }^{1} \mathrm{H}\right\}$ NMR (162 MHz, MeOD, $)$ ) 26.91 ppm. E.A.: Anal. Calcd. C: 64.60, H: 7.86. Found: C: 61.34, H: 7.87. 


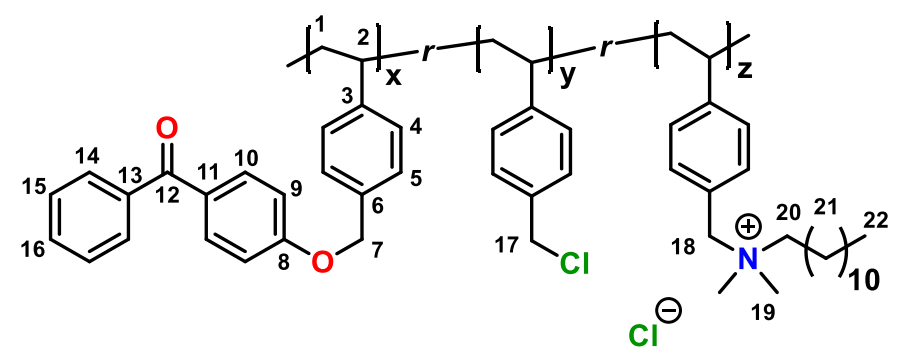

Copolymer $2(0.51 \mathrm{~g}, 3.1 \mathrm{mmol})$ was added to a flame-dried, $50 \mathrm{~mL}$ three-neck round bottom flask equipped with a reflux condenser. The flask was quickly flame-dried again and back filled with nitrogen. The copolymer was dissolved in $5 \mathrm{~mL}$ acetone and $N, N$ dimethyldodecylamine $(1.7 \mathrm{~mL}, 6.2 \mathrm{mmol})$ was added to the flask under nitrogen via syringe. The reaction mixture was refluxed for $4 \mathrm{~h}$ forming an off-white precipitate. $\mathrm{MeOH}(5 \mathrm{~mL})$ was added to dissolve the precipitate and the reaction continued for $20 \mathrm{~h}$. The solvent was removed in vacuo and redissolved in $\sim 10 \mathrm{~mL} \mathrm{MeOH}$ and purified by precipitation into excess cold $\mathrm{Et}_{2} \mathrm{O}$. The precipitate was decanted and purified once more by dissolution in $\sim 8 \mathrm{~mL} \mathrm{MeOH}$ and minimal THF $(\sim 2 \mathrm{~mL})$ followed by precipitation into excess cold hexanes. The resultant mixture was decanted, and the precipitate collected was dried in vacuo at $60{ }^{\circ} \mathrm{C}$, yielding a fine white powder. Yield 57 $\%(0.48 \mathrm{~g}) \cdot T_{m}=210.9{ }^{\circ} \mathrm{C} .{ }^{1} \mathrm{H}$ NMR $(400 \mathrm{MHz}, \mathrm{MeOD}, \delta): 0.74-1.00$ (br m, $\left.27 \mathrm{H}, \mathrm{H} 22\right), 1.01-$ 2.19 (br m, 184 H, H1, H2, H20, H21), 2.71-3.19 (br m, 48 H, H19), 3.33-3.66 (br s, 15 H, H18), 4.28-4.78 (br s, 15 H, H17), 5.00-5.46 (br s, 2 H, H7), 6.13-8.30 (br m, 3 H, H4, H5, H9, H10, H14-16) ppm. E.A.: Anal. Calcd. C: 75.37, H: 10.19, N: 3.23. Found: C: 74.62, H: 12.11, N: 4.00. 


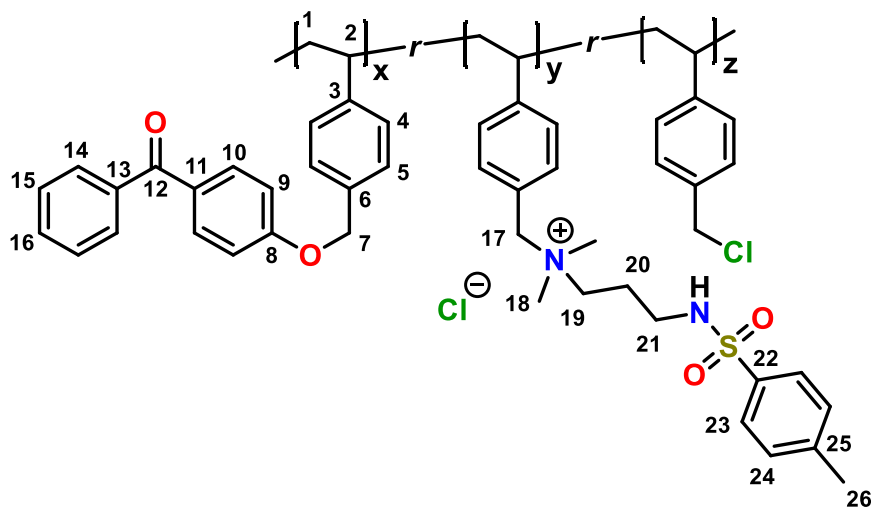

$N$-3-(dimethylamino)propyl)-4-methylbenzenesulfonamide was synthesized following a procedure previously described by Saettone et al. ${ }^{102}$ Copolymer $2(3.01 \mathrm{~g}, 0.0187 \mathrm{~mol})$ was added to a flame-dried, $250 \mathrm{~mL}$ three-neck round bottom flask equipped with a reflux condenser. The flask was quickly flame-dried again and back filled with nitrogen. The copolymer was dissolved in $20 \mathrm{~mL}$ dry acetone and $\mathrm{N}$-3-(dimethylamino)propyl)-4-methylbenzenesulfonamide $(7.20 \mathrm{~g}$, $0.0281 \mathrm{~mol}$ ) in $25 \mathrm{~mL}$ acetone was added to the flask under nitrogen via syringe. The reaction mixture was refluxed for $1 \mathrm{~h}$ forming a yellow precipitate. The solvent was removed by vacuum, and the precipitate was redissolved in $\sim 30 \mathrm{~mL} \mathrm{MeOH}$ and $\sim 5 \mathrm{~mL}$ THF and purified by precipitation into excess cold hexanes. The mixture was decanted, and the precipitate collected was dried in vacuo at $65{ }^{\circ} \mathrm{C}$, yielding a fine white powder. Yield: $55 \%(3.87 \mathrm{~g}) . T_{g l}=119.9^{\circ} \mathrm{C}, T_{g 2}=158.5$ ${ }^{\circ}$ C. E.A.: Anal. Calcd. C: 62.52, H: 7.31, N: 5.99. Found: C: 60.08, H: 7.17, N: 6.02. 


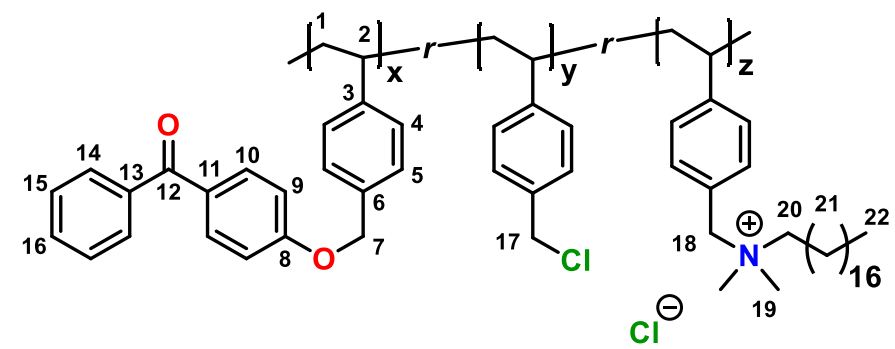

Polymer $2(3.00 \mathrm{~g}, 0.0187 \mathrm{~mol})$ was added to a flame-dried three-neck round bottom flask equipped with a reflux condenser. The flask was purged thrice and back filled with nitrogen. Dry acetone $(\sim 20 \mathrm{~mL})$ and $N, N$-dimethyloctadecylamine $(11.13 \mathrm{~g}, 0.0374 \mathrm{~mol})$ was added via syringe and refluxed for $16 \mathrm{~h}$. Once cool, the solvent was removed under vacuum leaving a gel crude mixture. The reaction mixture was purified by precipitation into excess cold $\mathrm{Et}_{2} \mathrm{O}(\sim 1 \mathrm{~L})$ from dry $\mathrm{CHCl}_{3}$. The solution was removed by canula transfer, and the precipitate was dried in vacuo at 50 ${ }^{\circ} \mathrm{C}$, leaving a fine white powder. Yield: $72 \%(4.72 \mathrm{~g}) .{ }^{1} \mathrm{H}$ NMR (400 MHz, $\left.\mathrm{CDCl}_{3}, \delta\right): 0.72-0.91$ (br m, 3 H, H22), 0.95-2.28 (br m, 31 H, H1, H2, H20, H21), 2.76-4.22 (br m, 6 H, H18, H19), 4.47-5.53 (br m, 1 H, H7, H17), 6.01-8.24 (br m, 3 H, H4, H5, H9, H10, H14-16). E.A.: Anal. Calcd. C: 77.38, H: 10.94, N: 2.73. Found: C: 76.21, H: 13.41, N: 3.01. 


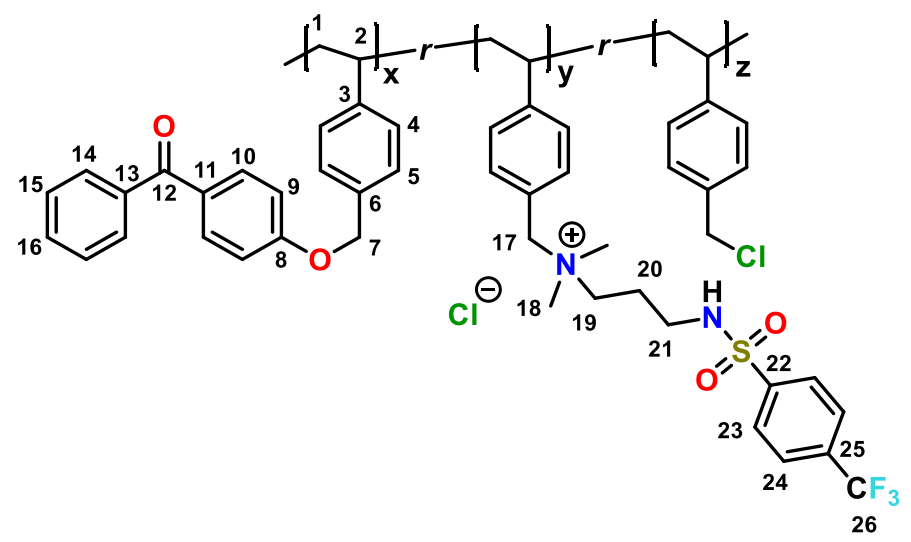

In a flame-dried three-neck round bottom flask equipped with a reflux condenser, 2 (2.00 g, $0.0125 \mathrm{~mol})$ was added and the flask was purged thrice. $N$-(3-(dimethylamino)propyl)-4(trifluoromethyl)benzenesulfonamide $(5.80 \mathrm{~g}, 0.0188 \mathrm{~mol})$ in $15 \mathrm{~mL}$ dry acetone was added to the flask via syringe under nitrogen. The flask was refluxed for $1 \mathrm{~h}$ and once cooled, the solvent was removed under reduced pressure. The crude mixture was purified by precipitation from a mixture of $\sim 15 \mathrm{~mL} \mathrm{MeOH}$ and $\sim 8 \mathrm{~mL}$ THF into cold excess $\mathrm{Et}_{2} \mathrm{O}$. The solution was removed by cannula transfer and the precipitate was dried in vacuo at $50{ }^{\circ} \mathrm{C}$. The purification step was repeated four times, yielding an off-white powder. Yield: $61 \%(3.23 \mathrm{~g}) .{ }^{19} \mathrm{~F}$ NMR (376 MHz, MeOD, $\delta$ ): 64.32 ppm. E.A.: Anal. Calcd. C: 56.66, H: 8.12, N: 4.94. Found: C: 58.00, H: 6.79, N: 6.31. 


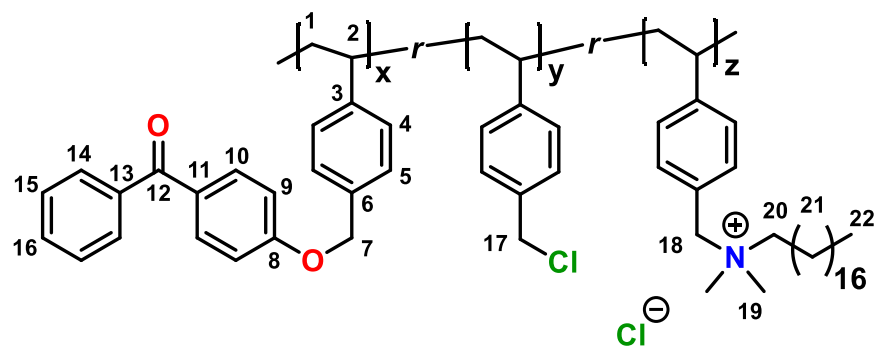

In a flame-dried three-neck round bottom equipped with a reflux condenser, $1.01 \mathrm{~g}$ of $\mathbf{2}$ $(6.55 \mathrm{mmol})$ was added, and the reaction flask was purged with nitrogen thrice. Dry acetone $(9$ $\mathrm{mL})$ and $0.17 \mathrm{~g} N, N$-dimethyloctadecylamine $(0.62 \mathrm{mmol})$ was via syringe under nitrogen. The reaction was refluxed for $16 \mathrm{~h}$, and once cooled, the solvent was removed under reduced pressure, leaving a creamy yellow thick gel. The crude product was purified by precipitation into cold excess $\mathrm{Et}_{2} \mathrm{O}$ from DCM. The solution was decanted and precipitate was dried in vacuo at $50{ }^{\circ} \mathrm{C}$. The purification step was repeated twice, yielding a fine off-white powder. Yield: $69 \%(0.83 \mathrm{~g}) .{ }^{1} \mathrm{H}$ NMR (400 MHz, $\left.\mathrm{CDCl}_{3}, \delta\right): 0.87$ (t, $\left.{ }^{2} J_{\mathrm{CH}}=8.0 \mathrm{~Hz}, 3 \mathrm{H}, \mathrm{H} 22\right), 1.03-2.15$ (br m, $50 \mathrm{H}, \mathrm{H} 1, \mathrm{H} 2$, H20, H21), 2.80-3.76 (m, 8 H, H18, H19), 4.33-4.66 (s, 12 H, H17), 4.87-5.16 (s, 1.8 H H7), 6.037.98 (m, 36.5 H, H4, H5, H9, H10, H14-16) ppm. 


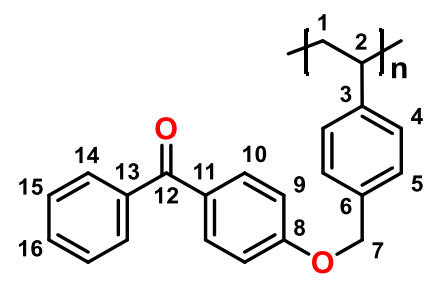

In a flame-dried $50 \mathrm{~mL}$ Schlenk flask, $3.00 \mathrm{~g}$ of $\operatorname{VBBP}(\mathbf{1}, 9.54 \mathrm{mmol})$ was dissolved in 8 $\mathrm{mL}$ THF under nitrogen. In a nitrogen atmosphere, AIBN (33 mg, $0.20 \mathrm{mmol})$ and CTA1 (55 mg, $0.20 \mathrm{mmol}$ ) were added to the reaction flask and degassed with bubbling $\mathrm{N}_{2}$ for $20 \mathrm{~min}$. The reaction mixture was stirred under $\mathrm{N}_{2}$ in a preheated oil bath at $63{ }^{\circ} \mathrm{C}$ for $24 \mathrm{~h}$. Once cooled, an additional 1-2 $\mathrm{mL}$ of THF was added and precipitated into excess cold methanol $(\sim 1 \mathrm{~L})$. The precipitate was collected by vacuum filtration and dried in vacuo at $50{ }^{\circ} \mathrm{C}$, yielding a fine light pink powder. Yield $70 \%(2.11 \mathrm{~g}) . \mathrm{PDI}=1.20 . \mathrm{M}_{\mathrm{w}}=3,200 \mathrm{Da} \cdot T_{\mathrm{g}}=71.9{ }^{\circ} \mathrm{C} .{ }^{1} \mathrm{H}$ NMR $(400 \mathrm{MHz}$, $\mathrm{CDCl}_{3}, \delta$ ): 0.72-2.52 (br m, $3 \mathrm{H}, \mathrm{H} 1, \mathrm{H} 2$ ), 4.72-5.31 (br s, $2 \mathrm{H}, \mathrm{H} 7$ ), 6.28-8.15 (br m, $13 \mathrm{H}, \mathrm{H} 4$, H5, H9, H10, H14-H16) 


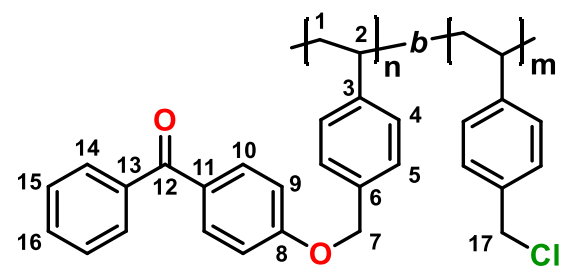

Poly(VBBP) (13) (3.2 kDa, $\left.0.10 \mathrm{~g}, 3.310^{-2} \mathrm{mmol}\right)$ was added to a flame-dried $25 \mathrm{~mL}$ round bottom. The flask was purged thrice with nitrogen and $5.0 \mathrm{~mL}$ VBC $(0.036 \mathrm{~mol})$ was added to dissolve 13 under nitrogen. The reaction mixture was stirred for $24 \mathrm{~h}$ in a preheated oil bath at 100 ${ }^{\circ} \mathrm{C}$. Once cooled, $\sim 15 \mathrm{~mL}$ THF was added to the mixture and precipitated into cold excess methanol $(\sim 600 \mathrm{~mL})$. The precipitate was collected by vacuum filtration and dried in vacuo at $50^{\circ} \mathrm{C}$, yielding a fine white powder. Yield $27 \%(0.59 \mathrm{~g}) . \mathrm{PDI}=1.05 . \mathrm{M}_{\mathrm{w}}=60,700 \mathrm{Da} \cdot T_{\mathrm{g}}=99.8{ }^{\circ} \mathrm{C} .{ }^{1} \mathrm{H} \mathrm{NMR}$ (400 MHz, $\mathrm{CDCl}_{3}, \delta$ ): 0.75-2.45 (br m, 99 H, H1, H2), 4.25-4.69 (br s, $62 \mathrm{H}, \mathrm{H} 17$ ), 4.85-5.29 (br s, 4 H, H7), 5.94-8.24 (br m, 141 H, H4, H5, H9, H10, H14-16). 


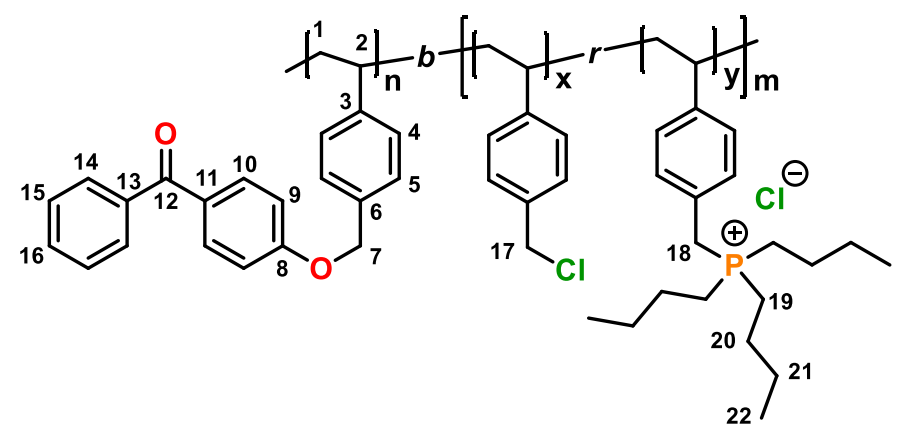

Poly(VBBP- $b$-VBC) $(\mathbf{1 4}, 0.75 \mathrm{~g}, 4.7 \mathrm{mmol})$ was added to a three neck round bottom equipped with a reflux condenser. The reaction flask was purged thrice with nitrogen and $4 \mathrm{~mL}$ dry acetone was added via septum. Tributylphosphine $(2.2 \mathrm{~mL}, 9.1 \mathrm{mmol})$ was added to the solution via septum, and the reaction was refluxed for $17 \mathrm{~h}$, forming a pale yellow gel. Once cooled, acetone was removed under reduced pressure, and the crude mixture was redissolved in DCM $(\sim 8 \mathrm{~mL})$. The solution was purified by precipitation into excess cold ether $(\sim 500 \mathrm{~mL})$. The mother liquor was removed by cannula transfer and the precipitate was dried in vacuo at $50{ }^{\circ} \mathrm{C}$, yielding a fine white powder. Yield $71 \%(0.89 \mathrm{~g}) .{ }^{1} \mathrm{H}$ NMR $\left(400 \mathrm{MHz}, \mathrm{CDCl}_{3}, \delta\right): 0.57-1.08$ (br m, H2, H22), 1.09-2.00 (br m, H1, H20, H21), 2.09-2.77 (br m, H18, H19), 3.95-4.78 (br s, H17) 4.79-5.20 (br s, H7), 5.86-8.06 (br m, H4, H5, H9, H10, H14-16) ppm. ${ }^{31} \mathrm{P}\left\{{ }^{1} \mathrm{H}\right\} \mathrm{NMR}(162 \mathrm{MHz}$, $\mathrm{CDCl}_{3}, \delta$ ): 31.29 ppm. E.A.: Anal. Calcd. C: 72.49, H: 8.69. Found: C: 72.08, H: 9.12. 


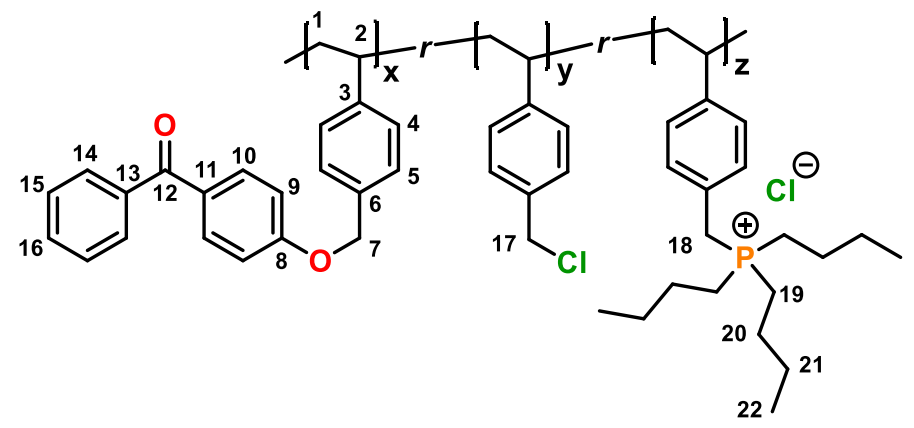

Copolymer $2(1.51 \mathrm{~g}, 9.83 \mathrm{mmol})$ was charged in a flame-dried three neck round bottom and purged thrice with $\mathrm{N}_{2}$. Under nitrogen, $2.80 \mathrm{~mL}$ tributylphosphine $(13.9 \mathrm{mmol})$ and $10 \mathrm{~mL}$ dry acetone was added to the flask via syringe. The reaction mixture was refluxed for $16 \mathrm{~h}$, forming a light yellow gel precipitate. Once cooled, the solvent was removed under reduced pressure, and the crude product was purified by precipitation into cold $\mathrm{Et}_{2} \mathrm{O}$ from DCM. The precipitate was isolated by decanting and dried in vacuo at $50{ }^{\circ} \mathrm{C}$, yielding a fine white powder. Yield $=89 \%$ (2.52 g) ${ }^{1} \mathrm{H}$ NMR (400 MHz, $\mathrm{CDCl}_{3}, \delta$ ): 0.12-1.04 (br s, $\left.102 \mathrm{H}, \mathrm{H} 2, \mathrm{H} 22\right), 1.05-1.89$ (br s, $134 \mathrm{H}$, H1, H20, H21), 1.90-3.40 (br m, 55 H, H18, H19), 3.56-4.76 (br s, 11 H, H7), 5.67-8.01 (br m, 37 H, H4, H5, H9, H10, H14-H16) ppm. ${ }^{31} \mathrm{P}\left\{{ }^{1} \mathrm{H}\right\}$ NMR (162 MHz, $\left.\mathrm{CDCl}_{3}, \delta\right): 31.44$ ppm. E.A.: Anal. Calcd. C: 72.17, H: 9.31. Found: C: 71.46, H: 10.53. 


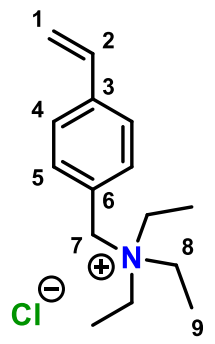

Triethylamine $(0.43 \mathrm{~mL}, 3.0 \mathrm{mmol})$ was added to a solution of VBC $(0.46 \mathrm{~mL}, 3.0 \mathrm{mmol})$ in $1.5 \mathrm{~mL}$ methanol and heated to $30^{\circ} \mathrm{C}$ overnight. The solvent was removed in vacuo and redissolved in minimal DCM $(1 \mathrm{~mL})$. The resultant mixture was purified by precipitation into cold $\mathrm{Et}_{2} \mathrm{O}(10 \mathrm{~mL})$, filtered, and dried in vacuo, yielding a fine white powder. fine white powder. Yield $89 \%(0.68 \mathrm{~g}){ }^{1} \mathrm{H}$ NMR (400 MHz, $\left.\mathrm{CDCl}_{3}, \delta\right): 1.42\left(\mathrm{t},{ }^{3} \mathrm{~J}_{\mathrm{HH}}=8.0 \mathrm{~Hz}, 9 \mathrm{H}\right), 3.40\left(\mathrm{q},{ }^{3} J_{\mathrm{HH}}=\right.$ $8.0 \mathrm{~Hz}, 6 \mathrm{H}, \mathrm{H} 8$ ), 4.77 (s, $2 \mathrm{H}, \mathrm{H} 7$ ), 5.31 (d, $\left.{ }^{3} J_{\text {Hlcis-H2 }}=12.0 \mathrm{~Hz}, 1 \mathrm{H}, \mathrm{H} 1 \mathrm{cis}\right), 5.76$ (d, ${ }^{3} J_{\text {Hltrans-H2 }}$ $\left.=16.0 \mathrm{~Hz}, 1 \mathrm{H}, \mathrm{H}_{1 \text { trans }}\right), 6.65\left(\mathrm{dd},{ }^{3} J_{H 2-H 1 t r a n s}=16.0 \mathrm{~Hz},{ }^{3} J_{H 2-\mathrm{Hlcis}}=12.0 \mathrm{~Hz}, 1 \mathrm{H}, \mathrm{H} 2\right), 7.38(\mathrm{~d}$, $\left.{ }^{3} J_{H H}=8.0 \mathrm{~Hz}, 2 \mathrm{H}, \mathrm{H} 4\right), 7.48\left(\mathrm{~d},{ }^{3} J_{H H}=8.0 \mathrm{~Hz}, 2 \mathrm{H}, \mathrm{H} 5\right) \mathrm{ppm} .{ }^{13} \mathrm{C}\left\{{ }^{1} \mathrm{H}\right\} \mathrm{NMR}(101 \mathrm{MHz}$, $\left.\mathrm{CDCl}_{3}, \delta\right): 8.43$ (s, C9), 52.81 (s, C8), 61.03 (s, C7), 116.22 (s, C1), 126.34 (s, C3), 126.86 (s,

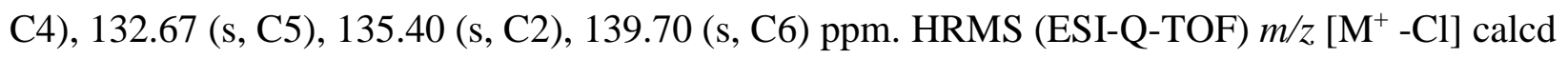
for $\mathrm{C}_{15} \mathrm{H}_{24} \mathrm{~N}$ : 218.1903, found: 218.1907. (Agrees with literature ${ }^{93}$ ) 


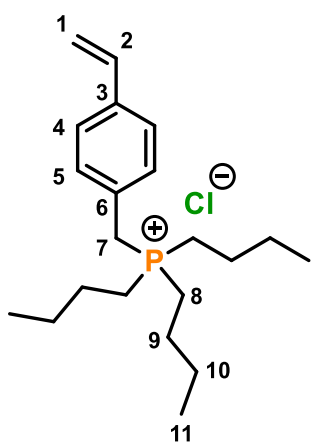

Tributylphosphine $(0.83 \mathrm{~mL}, 3 \mathrm{mmol})$ was added to a solution of 4-vinylbenzyl chloride (0.46 mL, $3 \mathrm{mmol})$ in $2.5 \mathrm{~mL}$ acetonitrile under nitrogen. The solution was refluxed for 16 hours. Solvent was removed in vacuo and redissolved in minimal DCM $(\sim 1 \mathrm{~mL})$. The resultant solution was purified by precipitation into cold $\mathrm{Et}_{2} \mathrm{O}$ followed by filtering, washing, and drying in vacuo, yielding a fine white powder. Yield $91 \%(0.97 \mathrm{~g})$. Melting point: $121.2-123.1{ }^{\circ} \mathrm{C} .{ }^{1} \mathrm{H}$ NMR $\left(\mathrm{D}_{2} \mathrm{O}, 400 \mathrm{MHz}, \delta\right): 0.95\left(\mathrm{t},{ }^{3} J_{\mathrm{HH}}=8.0 \mathrm{~Hz}, 9 \mathrm{H}, \mathrm{H} 11\right), 1.50(\mathrm{~m}, 12 \mathrm{H}, \mathrm{H} 9, \mathrm{H} 10), 2.15(\mathrm{~m}, 6 \mathrm{H}$, H8), $3.69\left(\mathrm{~d},{ }^{2} J_{\mathrm{HP}}=16 \mathrm{~Hz}, 2 \mathrm{H}, \mathrm{H} 7\right), 5.40\left(\mathrm{~d},{ }^{3} J_{\text {HIcis }-H 2}=12.0 \mathrm{~Hz}, 1 \mathrm{H}, \mathrm{H} 1 \mathrm{cis}\right), 5.92\left(\mathrm{~d},{ }^{3} J_{\text {Hltrans-H2 }}\right.$ $\left.=16.0 \mathrm{~Hz}, 1 \mathrm{H}, \mathrm{H}_{1 \text { trans }}\right), 6.83\left(\mathrm{dd},{ }^{3} J_{H 2-H \text { trans }}=16.0 \mathrm{~Hz},{ }^{3} J_{H 2-\mathrm{H} 1 \mathrm{cis}}=12.0 \mathrm{~Hz}, 1 \mathrm{H}, \mathrm{H} 2\right), 7.34(\mathrm{~d}$, $\left.{ }^{3} J_{H H}=8.0 \mathrm{~Hz}, 2 \mathrm{H}, \mathrm{H} 4\right), 7.58\left(\mathrm{~d},{ }^{3} J_{H H}=8.0 \mathrm{~Hz}, 2 \mathrm{H}, \mathrm{H} 5\right) \mathrm{ppm} .{ }^{13} \mathrm{C}\left\{{ }^{1} \mathrm{H}\right\} \mathrm{NMR}\left(101 \mathrm{MHz}, \mathrm{D}_{2} \mathrm{O}\right.$,

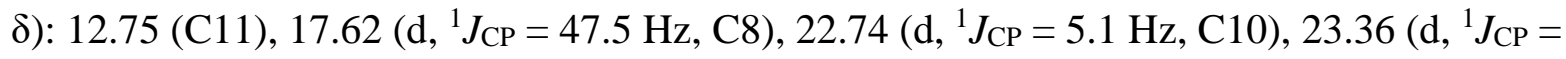
$16.2 \mathrm{~Hz}, \mathrm{C} 9), 25.93\left(\mathrm{~d},{ }^{1} J_{\mathrm{CP}}=45.5 \mathrm{~Hz}, \mathrm{C} 7\right), 115.00(\mathrm{C} 1), 127.06\left(\mathrm{~d},{ }^{4} J_{\mathrm{CP}}=3.0 \mathrm{~Hz}, \mathrm{C} 4\right), 128.36$ $\left(\mathrm{d},{ }^{5} J_{\mathrm{CP}}=9.1 \mathrm{~Hz}, \mathrm{C} 3\right), 130.37\left(\mathrm{~d},{ }^{3} J_{\mathrm{CP}}=5.1 \mathrm{~Hz}, \mathrm{C} 5\right), 135.97(\mathrm{C} 2), 137.26\left(\mathrm{~d},{ }^{2} J_{\mathrm{CP}}=4.0 \mathrm{~Hz}, \mathrm{C} 6\right)$ ppm. ${ }^{31} \mathrm{P}\left\{{ }^{1} \mathrm{H}\right\}$ NMR $\left(162 \mathrm{MHz}, \mathrm{D}_{2} \mathrm{O}, \delta\right): 32.58 \mathrm{ppm}$. HRMS (ESI-Q-TOF) $m / z\left[\mathrm{M}^{+}-\mathrm{Cl}\right]$ calcd for $\mathrm{C}_{21} \mathrm{H}_{36} \mathrm{P}: 319.2549$, found: 319.2555 . (Agrees with literature ${ }^{103}$ ) 


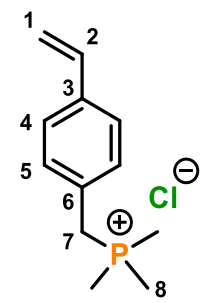

In a flame-dried microwave pressure vial, $3.29 \mathrm{~mL}$ of trimethylphosphine in toluene (1 $\mathrm{M}, 3.29 \mathrm{mmol})$ was added to a solution of 4-vinylbenzyl chloride $(0.52 \mathrm{~mL}, 3.3 \mathrm{mmol})$ in $3 \mathrm{~mL}$ anhydrous acetonitrile under nitrogen. The solution was stirred at $40{ }^{\circ} \mathrm{C}$ for 4.5 hours forming a white precipitate. Solvent and residual trimethylphosphine was removed under vacuum and the crude product was washed and filtered with anhydrous THF thrice followed by drying in vacuo, yielding a white powder. Yield $95 \%(0.71 \mathrm{~g}){ }^{1} \mathrm{H} \mathrm{NMR}\left(400 \mathrm{MHz}, \mathrm{CDCl}_{3}, \delta\right): 2.12\left(\mathrm{~d},{ }^{2} J_{\mathrm{HP}}=\right.$ 16.0 Hz, $9 \mathrm{H}, \mathrm{H} 8), 4.25\left(\mathrm{~d},{ }^{2} J_{\mathrm{HP}}=16.0,2 \mathrm{H}, \mathrm{H} 7\right), 5.29\left(\mathrm{~d},{ }^{3} J_{\text {Hlcis-H2 }}=12.0 \mathrm{~Hz}, 1 \mathrm{H}, \mathrm{H} 1 \mathrm{cis}\right), 5.75$ $\left(\mathrm{d},{ }^{3} J_{H 1 \text { trans }-H 2}=16.0 \mathrm{~Hz}, 1 \mathrm{H}, \mathrm{H}_{1 \text { trans }}\right), 6.68\left(\mathrm{dd},{ }^{3} J_{H 2-H 1 \text { trans }}=16.0 \mathrm{~Hz},{ }^{3} J_{H 2-H 1 \text { cis }}=12.0 \mathrm{~Hz}, 1 \mathrm{H}\right.$, H2), 7.36 (m, 4 H, H4, H5) ppm. ${ }^{13} \mathrm{C}\left\{{ }^{1} \mathrm{H}\right\}$ NMR $\left(101 \mathrm{MHz}, \mathrm{CDCl}_{3}, \delta\right): 7.96\left(\mathrm{~d},{ }^{1} J_{\mathrm{CP}}=87.5 \mathrm{~Hz}\right.$, C8), $29.86\left(\mathrm{~d},{ }^{1} J_{\mathrm{CP}}=49.5 \mathrm{~Hz}, \mathrm{C} 7\right), 114.71$ (s, C1), 126.88 (s, C4), 127.99 (s, C3), 130.36 (s, C5), 135.75 (s, C2), 137.35 (s, C6) ppm. ${ }^{31} \mathrm{P}\left\{{ }^{1} \mathrm{H}\right\} \mathrm{NMR}\left(162 \mathrm{MHz}, \mathrm{CDCl}_{3}, \delta\right): 26.57$ ppm. HRMS (ESI-Q-TOF) $m / z$ [M $\left.\mathrm{M}^{+} \mathrm{Cl}\right]$ calcd for $\mathrm{C}_{12} \mathrm{H}_{18} \mathrm{P}: 193.1141$, found: 193.1144 . 


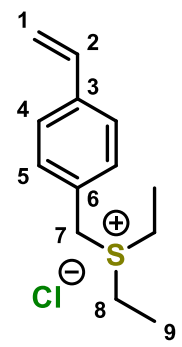

In a $25 \mathrm{~mL}$ round bottom, $2.0 \mathrm{~mL} \mathrm{VBC} \mathrm{(14} \mathrm{mmol),} 3.1 \mathrm{~mL}$ diethylsulfide ( $28 \mathrm{mmol}), 2.4$

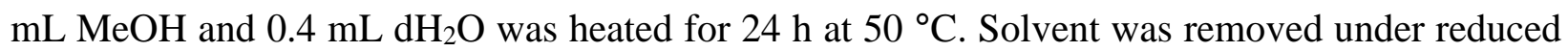
pressure leaving a creamy yellow gel. The crude product was purified by precipitation into cold excess $\mathrm{Et}_{2} \mathrm{O}$ from $\mathrm{MeOH}$. The solution was decanted and the precipitate was dried in vacuo, yielding a tacky off-white powder. Yield: $72 \%(2.5 \mathrm{~g}){ }^{1} \mathrm{H}$ NMR $\left(400 \mathrm{MHz}, \mathrm{CDCl}_{3}, \delta\right): 1.39(\mathrm{t}$, $\left.{ }^{2} J_{\mathrm{HH}}=8.0 \mathrm{~Hz}, 6 \mathrm{H}, \mathrm{H} 9\right), 3.45(\mathrm{~s}, 2 \mathrm{H}, \mathrm{H} 7), 3.63(\mathrm{~m}, 2 \mathrm{H}, \mathrm{H} 8), 5.29\left(\mathrm{~d},{ }^{3} J_{\text {HIcis-H2 }}=12.0 \mathrm{~Hz}, 1 \mathrm{H}\right.$, H1 $\left.1_{\text {cis }}\right), 5.74\left(\mathrm{~d},{ }^{3} J_{H 1 \text { trans }-H 2}=16.0 \mathrm{~Hz}, 1 \mathrm{H}, \mathrm{H}_{1 \text { trans }}\right), 6.65$ (dd, ${ }^{3} J_{H 2-H 1 t r a n s}=16.0 \mathrm{~Hz},{ }^{3} J_{H 2-H 1 \text { cis }}=12.0$ $\mathrm{Hz}, 1 \mathrm{H}, \mathrm{H} 2), 7.37$ (d, 2 H, H4), 7.56 (d, 2 H, H5) ppm. 


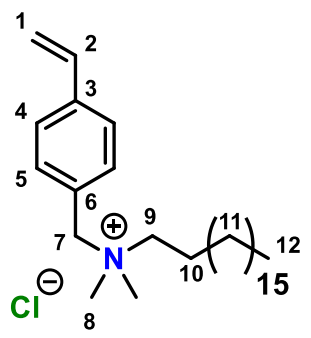

In a $25 \mathrm{~mL}$ flame-dried round bottom, VBC $(3.3 \mathrm{~mL}, 23.5 \mathrm{mmol})$ was added to a solution of $\mathrm{N}, \mathrm{N}$-dimethyloctadecylamine $(7 \mathrm{~g}, 8.75 \mathrm{~mL}, 23.5 \mathrm{mmol})$ in $16 \mathrm{~mL}$ methanol and stirred at 50 ${ }^{\circ} \mathrm{C}$ overnight. The solvent was removed in vacuo, and the crude product was purified by precipitation in excess cold diethyl ether $(\sim 15 \mathrm{~mL})$ from dichloromethane $(\sim 1 \mathrm{~mL})$. The solution was decanted and the precipitate was dried in vacuo. Yield 87\% (8.46 g); white powder; melting point: $174-176{ }^{\circ} \mathrm{C} .{ }^{1} \mathrm{H}$ NMR $\left(400 \mathrm{MHz}, \mathrm{CDCl}_{3}, \delta\right): 0.83\left(\mathrm{t},{ }^{2} J_{\mathrm{HH}}=8.0 \mathrm{~Hz}, 3 \mathrm{H}, \mathrm{H} 12\right), 1.11-1.34$ (m, 30 H, H11), 1.73 (s, 2 H, H10), 3.25 (s, 6 H, H8), 3.38-3.55 (s, 2 H, H9), 5.03 (s, 2 H, H7), $5.30\left(\mathrm{~d},{ }^{3} J_{H 1 c i s-H 2}=8.0 \mathrm{~Hz}, 1 \mathrm{H}, \mathrm{H} 1 \mathrm{cis}\right), 5.76\left(\mathrm{~d},{ }^{3} J_{H 1 t r a n s-H 2}=20.0 \mathrm{~Hz}, 1 \mathrm{H}, \mathrm{H}_{1 \text { trans }}\right), 6.66\left(\mathrm{dd},{ }^{3} J_{H 2-}\right.$ HItrans $\left.=20.0 \mathrm{~Hz},{ }^{3} J_{H 2-\mathrm{H} 1 \mathrm{cis}}=8.0 \mathrm{~Hz}, 1 \mathrm{H}, \mathrm{H} 2\right), 7.38\left(\mathrm{~d},{ }^{2} J_{\mathrm{HH}}=8.0 \mathrm{~Hz}, 2 \mathrm{H}, \mathrm{H} 4\right), 7.58\left(\mathrm{~d},{ }^{2} J_{\mathrm{HH}}=8.0\right.$ Hz, 2 H, H5) ppm. (Agrees with literature ${ }^{104}$ ) 


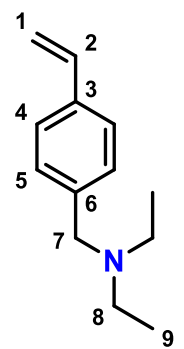

VBC (1.08 g, $7.10 \mathrm{mmol}), \mathrm{K}_{2} \mathrm{CO}_{3}(1.98 \mathrm{~g}, 14.2 \mathrm{mmol})$, diethylamine (1.50 mL, 14.2 mmol), and $7.5 \mathrm{~mL} \mathrm{CHCl}_{3}$ were added to a $25 \mathrm{~mL}$ round bottom. The reaction was stirred at $50{ }^{\circ} \mathrm{C}$

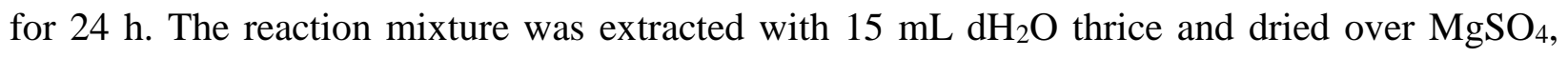
filtered, and solvent removed by rotary evaporation leaving a light orange oil. Excess diethylamine was removed by reduced pressure. The crude product was then purified by flash column chromatography with methanol, ethyl acetate and, hexanes (MeOH/EtOAC/Hexanes, 1:49.5:49.5, $\left.\mathrm{R}_{\mathrm{f}}=0.26\right)$ as eluents. The product was dried in vacuo, yielding a light orange oil. Yield: $88 \%$ (1.18 g) ${ }^{1} \mathrm{H} \mathrm{NMR}\left(400 \mathrm{MHz}, \mathrm{CDCl}_{3}, \delta\right): 1.08\left(\mathrm{t},{ }^{3} J_{\mathrm{HH}}=8.0 \mathrm{~Hz}, 6 \mathrm{H}, \mathrm{H} 9\right), 2.57\left(\mathrm{q},{ }^{3} J_{\mathrm{HH}}=8.0 \mathrm{~Hz}, 4 \mathrm{H}\right.$, H8), 3.59 (s, $2 \mathrm{H}, \mathrm{H} 7), 5.24$ (d, $\left.{ }^{3} J_{\text {HIcis-H2 }}=12.0 \mathrm{~Hz}, 1 \mathrm{H}, \mathrm{H} 1 \mathrm{cis}\right), 5.76\left(\mathrm{~d},{ }^{3} J_{H 1 t r a n s-H 2}=16.0 \mathrm{~Hz}, 1\right.$ $\left.\mathrm{H}, \mathrm{H}_{1 \text { trans }}\right), 6.75\left(\mathrm{dd},{ }^{3} J_{\mathrm{H} 2-\mathrm{Hltrans}}=16.0 \mathrm{~Hz},{ }^{3} J_{\mathrm{H} 2 \text {-H1cis }}=12.0 \mathrm{~Hz}, 1 \mathrm{H}, \mathrm{H} 2\right), 7.33\left(\mathrm{~d},{ }^{3} J_{\mathrm{HH}}=8.0 \mathrm{~Hz}, 2\right.$ $\mathrm{H}, \mathrm{H} 4), 7.39\left(\mathrm{~d},{ }^{3} \mathrm{JHH}_{\mathrm{HH}}=8.0 \mathrm{~Hz}, 2 \mathrm{H}, \mathrm{H} 5\right) \mathrm{ppm} .{ }^{13} \mathrm{C}\left\{{ }^{1} \mathrm{H}\right\} \mathrm{NMR}\left(101 \mathrm{MHz}, \mathrm{CDCl}_{3}, \delta\right): 11.85$ (s, C9), 46.62 (s, C8), 57.20 (s, C7), 112.94 (s, C1), 125.66 (s, C4), 128.88 (s, C5), 135.99 (s, C3), 136.61 (s, C2), 139.61 (s, C6) ppm. (Agrees with literature value ${ }^{100}$ ) 


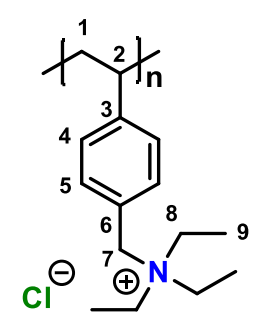

The homopolymerization of monomer $\mathbf{1 7}$ was performed following a procedure previously reported by Kaur et al..$^{93}$ Monomer (0.50 g, $\left.2.0 \mathrm{mmol}\right)$ and TEMPO (3.7 $\left.\mathrm{mg}, 0.024 \mathrm{mmol}\right)$ was dissolved in $1.2 \mathrm{~mL}$ of a 1:1 $\mathrm{dH}_{2} \mathrm{O}$ :ethylene glycol solution in a $25 \mathrm{~mL}$ three neck round bottom. The flask was warmed at $60{ }^{\circ} \mathrm{C}$ solubilize the monomer, and $4.0 \mathrm{mg}$ of $\mathrm{K}_{2} \mathrm{~S}_{2} \mathrm{O}_{5}(0.015 \mathrm{mmol})$ and $3.6 \mathrm{mg}$ of $\mathrm{Na}_{2} \mathrm{~S}_{2} \mathrm{O}_{5}(0.019 \mathrm{mmol})$ was added to the flask and purged with nitrogen three times. The reaction mixture was then degassed with $\mathrm{N}_{2}$ for $20 \mathrm{~min}$, and heated in a preheated oil bath at 125 ${ }^{\circ} \mathrm{C}$ for $24 \mathrm{~h}$. The crude mixture was then purified by precipitation into cold THF. The solution was decanted and the purification step was repeated thrice. The precipitate was dried in vacuo at $50{ }^{\circ} \mathrm{C}$ yielding a fine white powder. Yield: $82 \%(0.42 \mathrm{~g}) T_{g}=115.6{ }^{\circ} \mathrm{C} . T_{m}=216.3{ }^{\circ} \mathrm{C} .{ }^{1} \mathrm{H}$ NMR $(400$ MHz, $\mathrm{D}_{2} \mathrm{O}, \delta$ ): 0.88-1.84 (br m, 12 H, H1, H2, H9), 2.82-3.45 (br s, $6 \mathrm{H}, \mathrm{H} 8$ ), 4.18-4.59 (br s, 2 H, H7), 6.41-7.68 (br m, 4 H, H4, H5) ppm. (Agrees with literature ${ }^{105}$ ) 


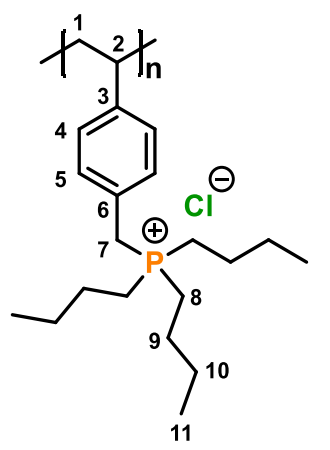

In a three-neck flask equipped with a condenser, $0.30 \mathrm{~g}$ tributylphosphonium monomer (0.85 mmol), $1.6 \mathrm{mg}$ TEMPO (0.010 mmol), $1.7 \mathrm{mg} \mathrm{K} \mathrm{S}_{2} \mathrm{O}_{5}(6.3 \mu \mathrm{mol})$ and $1.6 \mathrm{mg} \mathrm{of} \mathrm{Na}_{2} \mathrm{~S}_{2} \mathrm{O}_{5}$ $(8.2 \mu \mathrm{mol})$ was dissolved in $2 \mathrm{~mL} \mathrm{dH} \mathrm{H}_{2} \mathrm{O}$. The solution was gently warmed to aid with solubilization of the monomer and degassed with $\mathrm{N}_{2}$ for $20 \mathrm{~min}$. The reaction proceeded for $24 \mathrm{~h}$ in a preheated oil bath set at $125^{\circ} \mathrm{C}$. Once cooled, the solvent was removed under reduced pressure leaving a light orange gel. The crude product was purified by precipitation into cold excess $\mathrm{Et}_{2} \mathrm{O}$ from $\mathrm{MeOH}$. The solution was decanted and the precipitate was dried in vacuo at $50{ }^{\circ} \mathrm{C}$. The product was purified twice more, yielding a fine white powder. Yield: $67 \%$ (0.18 g). ${ }^{1} \mathrm{H}$ NMR (400 MHz, $\mathrm{D}_{2} \mathrm{O}, \delta$ ): 0.66-1.11 (br s, $10 \mathrm{H}, \mathrm{H} 2, \mathrm{H} 11$ ), 1.22-1.77 (br s, 15 H, H1, H9, H10), 1.94-2.43 (br s, 7 H, H8), 3.50-4.12 (br s, 2 H, H7), 6.26-6.97 (br m, 2 H, H4), 6.98-7.61 (br m, 4 H, H5) ppm. ${ }^{13} \mathrm{C}\left\{{ }^{1} \mathrm{H}\right\}$ NMR (101 MHz, $\left.\mathrm{D}_{2} \mathrm{O}, \delta\right): 12.81$ (s, C11), $17.83\left(\mathrm{~d},{ }^{1} J_{\mathrm{CP}}=46.5 \mathrm{~Hz}, \mathrm{C} 8\right) 23.13$ (m, C9, C10), 26.32 (br s, C7), 40.14 (br s, C1, C2), 126.11-132.01 (br m, C3, C4, C5), 144.97 (br s, C6) ppm. ${ }^{31} \mathrm{P}\left\{{ }^{1} \mathrm{H}\right\}$ NMR (162 MHz, $\left.\mathrm{D}_{2} \mathrm{O}, \delta\right): 31.77 \mathrm{ppm}$. (Agrees with literature ${ }^{106}$ ) 


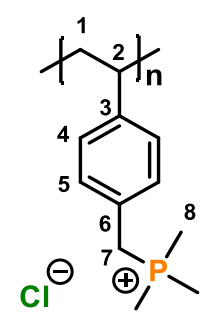

In a three-neck round bottom, $0.501 \mathrm{~g}$ of monomer 19 (2.19 mmol), $4.1 \mathrm{mg}$ TEMPO (26 $\mu \mathrm{mol})$, $4.4 \mathrm{mg} \mathrm{K} \mathrm{S}_{2} \mathrm{O}_{5}(16 \mu \mathrm{mol})$, and $4.0 \mathrm{mg}$ of $\mathrm{Na}_{2} \mathrm{~S}_{2} \mathrm{O}_{5}(21 \mu \mathrm{mol})$ were dissolved in $3.3 \mathrm{~mL}$ $\mathrm{dH}_{2} \mathrm{O}$. The reaction mixture was gently heated to aid in solubilization of monomer and degassed with $\mathrm{N}_{2}$ over ice for $20 \mathrm{~min}$. The reaction was refluxed in a preheated oil bath set at $125^{\circ} \mathrm{C}$ for 24 h under $\mathrm{N}_{2}$. Once cooled, the solvent was removed under reduced pressure leaving a creamy yellow gel. The crude product was redissolved and precipitated into cold excess DCM and precipitate collected. The product was purified twice and dried in vacuo at $40{ }^{\circ} \mathrm{C}$ yielding a fine white powder. Yield: $72 \%$ (0.36 g). ${ }^{1} \mathrm{H}$ NMR (400 MHz, $\mathrm{D}_{2} \mathrm{O}, \delta$ ): 0.84-2.20 (br m, $\left.12 \mathrm{H}, \mathrm{H} 1, \mathrm{H} 2, \mathrm{H} 8\right), 3.31-3.95$ (br s, $2 \mathrm{H}, \mathrm{H} 7)$, 6.13-7.52 (br m, 4 H, H4, H5) ppm. ${ }^{13} \mathrm{C}\left\{{ }^{1} \mathrm{H}\right\}$ NMR (101 MHz, $\left.\mathrm{D}_{2} \mathrm{O}, \delta\right): 6.93$ (d, $\left.{ }^{1} J_{\mathrm{CP}}=55.6 \mathrm{~Hz}, \mathrm{C} 8\right), 29.62\left(\mathrm{~d},{ }^{1} J_{\mathrm{PC}}=55.6 \mathrm{~Hz}, \mathrm{C} 7\right), 40.22(\mathrm{br} \mathrm{s}, \mathrm{C} 1, \mathrm{C} 2), 125.55(\mathrm{br} \mathrm{s}, \mathrm{C} 3), 127.68-$ 131.29 (br m, C4, C5), 145.42 (br s, C6) ppm. ${ }^{31} \mathrm{P}\left\{{ }^{1} \mathrm{H}\right\}$ NMR (162 MHz, $\mathrm{D}_{2} \mathrm{O}, \delta$ ): $25.75 \mathrm{ppm}$. (Agrees with literature ${ }^{105}$ ) 


\subsection{Preparation of Coatings}

The quaternary polymers were dissolved at $10 \%(\mathrm{w} / \mathrm{v})$ in $\mathrm{EtOH}(\mathbf{6 , 7}, \mathbf{1 5}, \mathbf{1 6})$, a mixture of $\mathrm{MeOH} / \mathrm{EtOH}(\mathbf{8}, \mathbf{9}, \mathbf{1 1}), s-\mathrm{BuOH}(\mathbf{1 0})$, or tol $(\mathbf{2}, \mathbf{1 2})$ with 0.3 mas \% of the UV photoinitiator, BAPO. A uniform film was created by spin-coating $0.32 \mathrm{~mL}$ of the solution deposited on a piece of plastic substrate of $3 \mathrm{~cm} \times 2 \mathrm{~cm}$ at $1250 \mathrm{rpm}$ for $10 \mathrm{~s}$. The substrates were UV-cured using a Novacure spot curing system with a mercury-arc discharge lamp for approximately 3 min, $10 \mathrm{~s}$ with the energy and power density of UVA $\left(9.994 \mathrm{~J} / \mathrm{cm}^{2}, 0.158 \mathrm{~W} / \mathrm{cm}^{2}\right), \mathrm{UVB}\left(1.071 \mathrm{~J} / \mathrm{cm}^{2}, 0\right.$ $\left.\mathrm{W} / \mathrm{cm}^{2}\right)$, and UVV $\left(8.728 \mathrm{~J} / \mathrm{cm}^{2}, 0.140 \mathrm{~W} / \mathrm{cm}^{2}\right)$, determined by EIT UV Power Puck II. The surfaces were washed twice with a mixture of $\mathrm{MeOH}$ and distilled water $\left(\mathrm{dH}_{2} \mathrm{O}\right)$. Once dry, a second coating $(0.36 \mathrm{~mL}$ of solution) was deposited and the UV-cure procedure repeated. 


\subsection{Antibacterial Testing of Coated Surfaces}

\subsection{Large Drop Inoculum Antimicrobial Testing of Coated Surfaces}

Bacterial test species were grown overnight in $10 \mathrm{~mL}$ of $0.3 \%(\mathrm{w} / \mathrm{v})$ tryptic soy broth (EMD Millipore) at $30{ }^{\circ} \mathrm{C}$ (Arthrobacter sp.) or $37^{\circ} \mathrm{C}$ (P. aeruginosa and E. coli) in a shaking incubator. Cultures were washed twice with sterile tap water by centrifugation at $9000 \mathrm{G}$. The cultures were diluted and used to inoculate the coated plastic substrates. Antimicrobial efficacy of the surfaces at the solid/air interface were determined via the LDI protocol, a modified ISO 22196/JIS Z 2801 standard procedure, illustrated in Figure 4.1. ${ }^{56,88}$ Triplicate treated and control samples were inoculated with $100 \mu \mathrm{L}$ of bacterial dilution $\left(10^{7} \mathrm{CFU}\right)$, and after desiccation of the droplet on the sample (typically $3 \mathrm{~h}$ ), surviving cells were quantified by spot plating, described in previous work. ${ }^{79}$
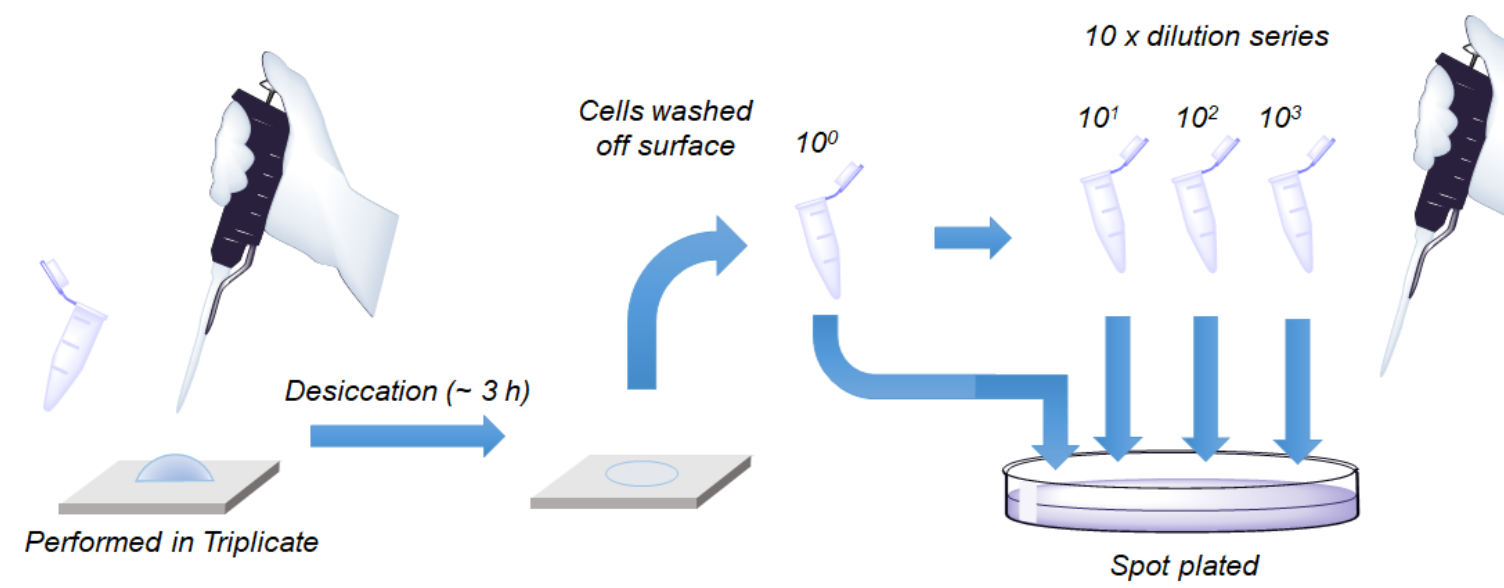

Figure 4.1 Large drop inoculum protocol. ${ }^{59}$ 


\subsection{Dynamic shake flask testing method of coated surfaces}

The dynamic shake flask method was based off of procedures described in ASTM 214913a and Cuthbert et al., illustrated in Figure 4.2. ${ }^{82}$ Films were formed by depositing $0.2 \mathrm{~mL}$ of 25 w/v \% polymer in $\mathrm{EtOH}$ on a glass slide. The polymer was sandwiched between two glass slides with one layer of electrical tape as a spacer. The slides and film were cured with $30 \mathrm{~J}$ of UV on both sides and dried in the oven for $1 \mathrm{~h}$. The film was removed from the glass, washed with water for $5 \mathrm{~h}$, and dried at $50{ }^{\circ} \mathrm{C}$ overnight. The film was ground to a fine powder in a mortar pestle. Bacterial test species (Arthrobacter sp.) were grown and prepared as described above in $0.3 \mathrm{mM}$ phosphate buffered saline (PBS) and diluted to $10^{5} \mathrm{CFU}$. Triplicate sample $(2 \mathrm{mg})$ and control were inoculated with $100 \mu \mathrm{L}$ of the bacterial dilution and vortexed for $1 \mathrm{~h}$. Samples were diluted to $1 \mathrm{~mL}$ and serial diluted. Surviving cells were quantified by spot plating, as described above.
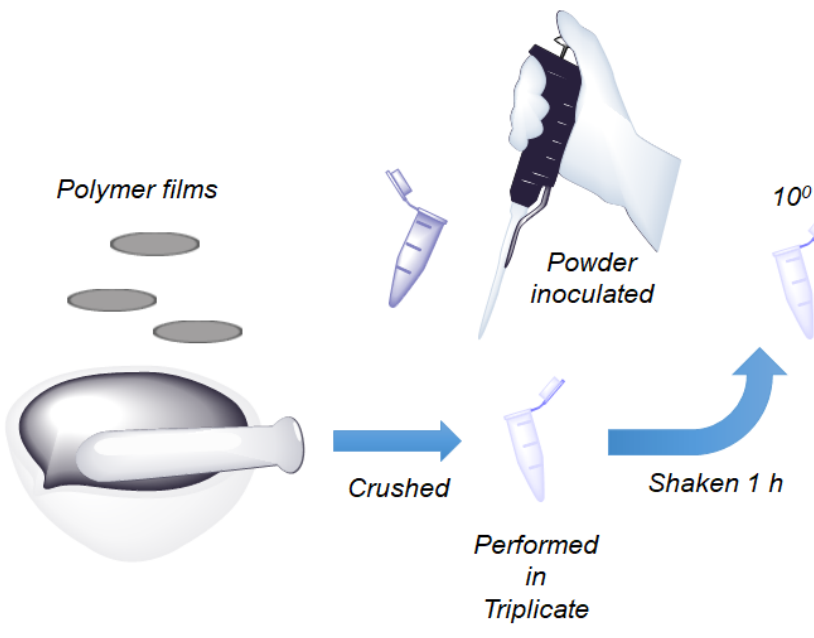

Figure 4.2 Dynamic shake flask method 


\subsection{Determination of Coating Properties}

\subsection{Cationic Charge Density Determination}

The density of cationic charges $\left(\left[\mathrm{Q}^{+}\right] \mathrm{cm}^{-2}\right)$ of PS substrates coated and UV cured with polymers 6-12, 15, and 16 were quantified following previously described fluorescein staining technique. ${ }^{53,107}$ Triplicate control and treated $4 \mathrm{~cm}^{2}$ samples were stained in $1 \% \mathrm{w} / \mathrm{v}$ aqueous fluorescein sodium salt solution on an orbital shaker at $150 \mathrm{rpm}$ for $24 \mathrm{~h}$. Excess unbound fluorescein was exhaustively rinsed off with $\mathrm{dH}_{2} \mathrm{O}$ before destaining. The stain was removed and collected by sonication in $9 \mathrm{~mL} 1 \% \mathrm{w} / \mathrm{v}$ cetyl trimethylammonium bromide (CTAB) in $10 \% \mathrm{w} / \mathrm{v}$ phosphate buffered saline (PBS) (BioShop cat. PBS404.100) solution for 1-2 h. The CTAB/fluorescein solution was replaced with $9 \mathrm{~mL}$ of fresh CTAB/PBS solution and further sonicated for $1 \mathrm{~h}$. The aliquots of CTAB/fluorescein was collected in a $25 \mathrm{~mL}$ volumetric flask and diluted to mark with fresh CTAB/PBS solution to form the stock solution. The solution was further diluted 10 fold and analyzed by UV-vis spectroscopy using an Agilent Cary $60 \mathrm{UV}$-Vis Spectrophotometer and quartz $1 \mathrm{~cm}$ path length (L) cuvette. The absorbance recorded at $\lambda_{\max }=$ $501 \mathrm{~nm}\left(\mathrm{~A}_{501}\right)$ was used to calculate the fluorescein concentration $\left(\mathrm{C}_{\text {fluorescein }}\right.$, $\left.\mathrm{mol} \mathrm{L}^{-1}\right)$ by the BeerLambert law (Eq. 1) with an extinction coefficient of $\varepsilon=77,000 \mathrm{M}^{-1} \mathrm{~cm}^{-1}\left(\varepsilon_{501}\right)$.

$$
\mathrm{C}_{\text {fluorescein }}=\frac{\mathrm{A}_{501}}{\varepsilon_{501}} \times \mathrm{L}
$$

Charge density $\left(\left[\mathrm{Q}^{+}\right],\left[\mathrm{Q}^{+}\right] \mathrm{cm}^{-2}\right)$ was then calculated from the fluorescein concentration following Eq. 2 below:

$$
\left[\mathrm{Q}^{+}\right]=\frac{\mathrm{C}_{\text {fluorescein }} \times D \times V \times \mathrm{N}}{A}
$$


Where $D$ represents the dilution (10), $V$ represents volume of the stock solution $(0.025 \mathrm{~L})$, $\mathrm{N}$ represents Avogado's number $\left(6.022 \times 10^{23}\right)$, and $A$ represents surface area of the coated substrate $\left(4 \mathrm{~cm}^{2}\right)$.

\subsection{Advancing Water Contact Angle Measurements}

The water contact angle determination were performed on PS substrates treated with compounds 6-12, 15, and 16 following ASTM D7334 at a humidity approximately $50 \%$. Droplets of $5 \mu \mathrm{L}$ were visualized on the surface using a Teli CCD camera with a Sony CMA-D camera adapter. Triplicate measurements were taken using software tool SCA20.

\subsection{Coating Preparation for AFM imaging}

Coatings were prepared in a similar fashion described above by spin coating 6-9 in a 10 w/v \% EtOH solution on PS substrates. The coating was UV cured with a dosage of $30 \mathrm{~J}$ with half the surface photomasked for coating thickness determination. Unbound and uncured polymer was thoroughly washed away until the wash solution did not stain blue with BPB.

\subsection{Solvent Resistance Testing}

PS substrates double coated with 6 were subjected to solvent resistance testing against $\mathrm{dH}_{2} \mathrm{O}, \mathrm{MeOH}$, IPA, and Windex ${ }^{\circledR}$ following ASTM D5402-15. ${ }^{86}$ Using a cotton fabric soaked with the respective solvent, the PS substrate underwent a series of double rubs on a top loading balance with a pressure of $\sim 10 \mathrm{~N}$. Force applied was determined by the balance, applying a pressure equivalent to $1-1.6 \mathrm{~kg}$. Coating integrity was visualized with BPB staining overnight and visually evaluated. 


\subsection{Adhesion Testing}

PS samples double coated with $\mathbf{6}$ were subjected to adhesion analysis by tape test. Following ASTM D3359-17 $7^{87}$, the coating was crosshatched with a scalpel to form a cross with an acute angle running vertical on the surface. 3M Scotch Tape was applied evenly along the acute

angle and the tape was rapidly removed at angle parallel to the surface. Coating integrity was visually determined after BPB staining for coating visualization. 


\section{APPENDIX}

Appendix A: NMR Spectra and Mass Spectra

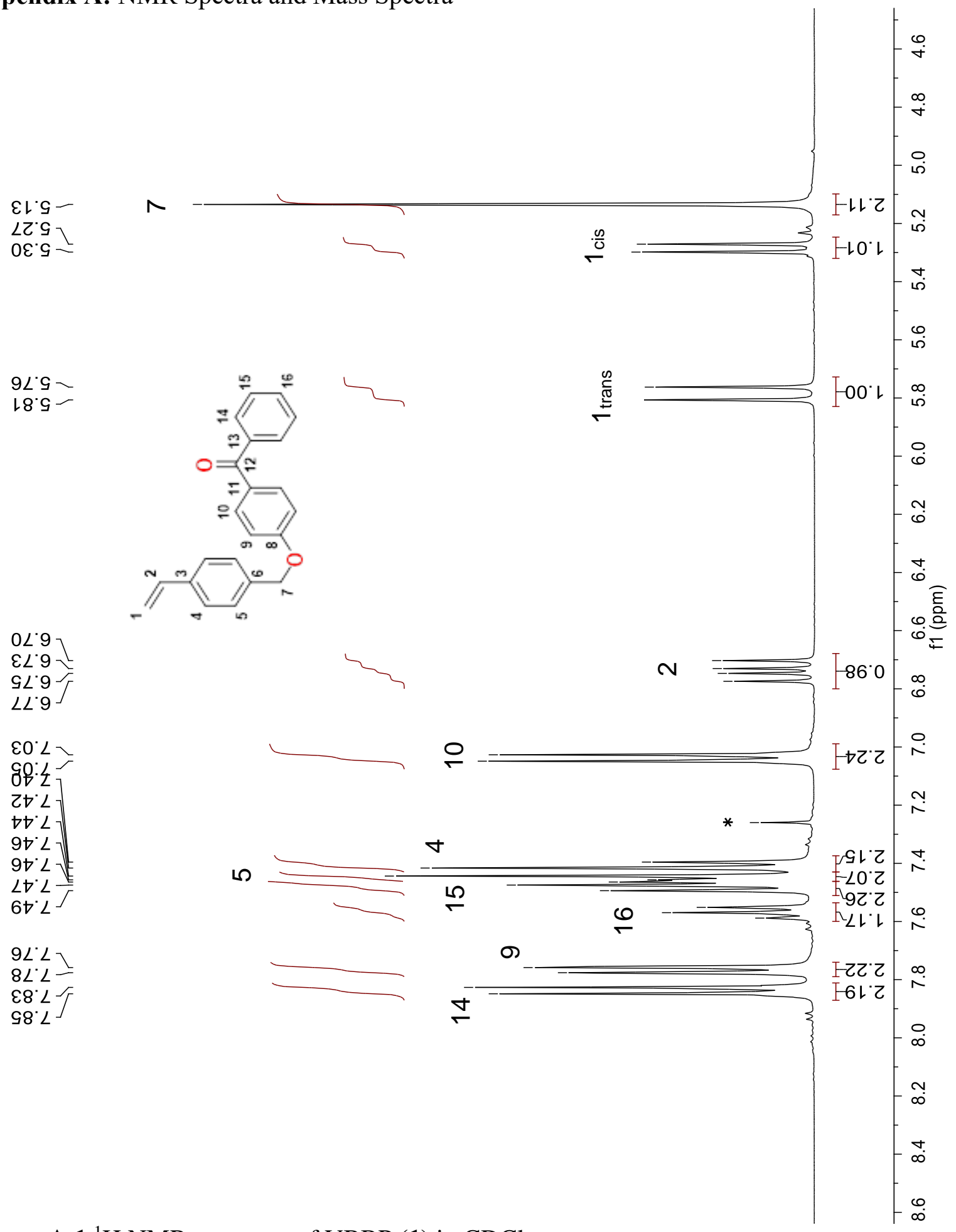

Figure A $1{ }^{1} \mathrm{H}$ NMR spectrum of $\operatorname{VBBP}(\mathbf{1})$ in $\mathrm{CDCl}_{3}$ 


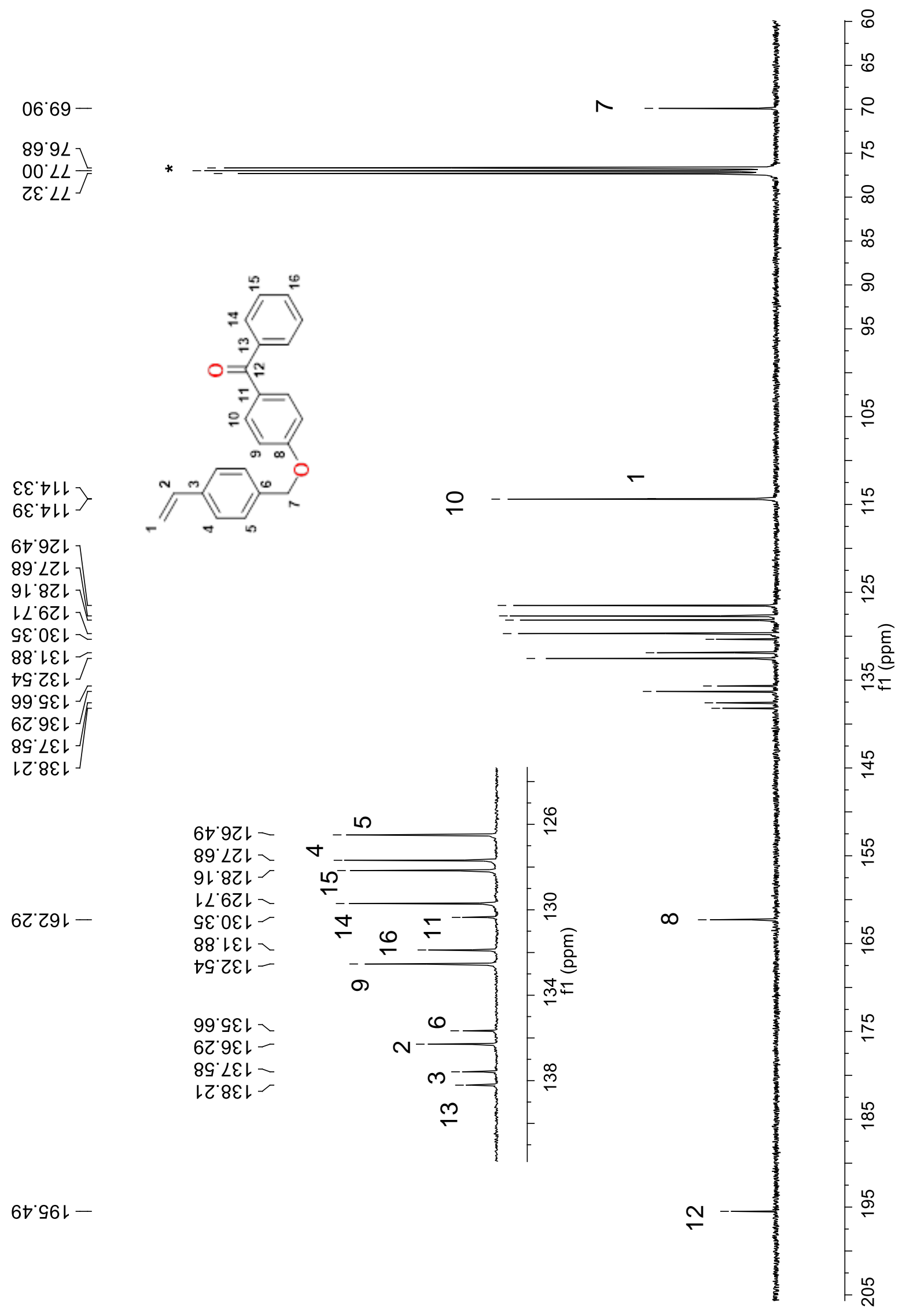

Figure A $2{ }^{13} \mathrm{C}$ NMR spectrum of $\operatorname{VBBP}(\mathbf{1})$ in $\mathrm{CDCl}_{3}$ 


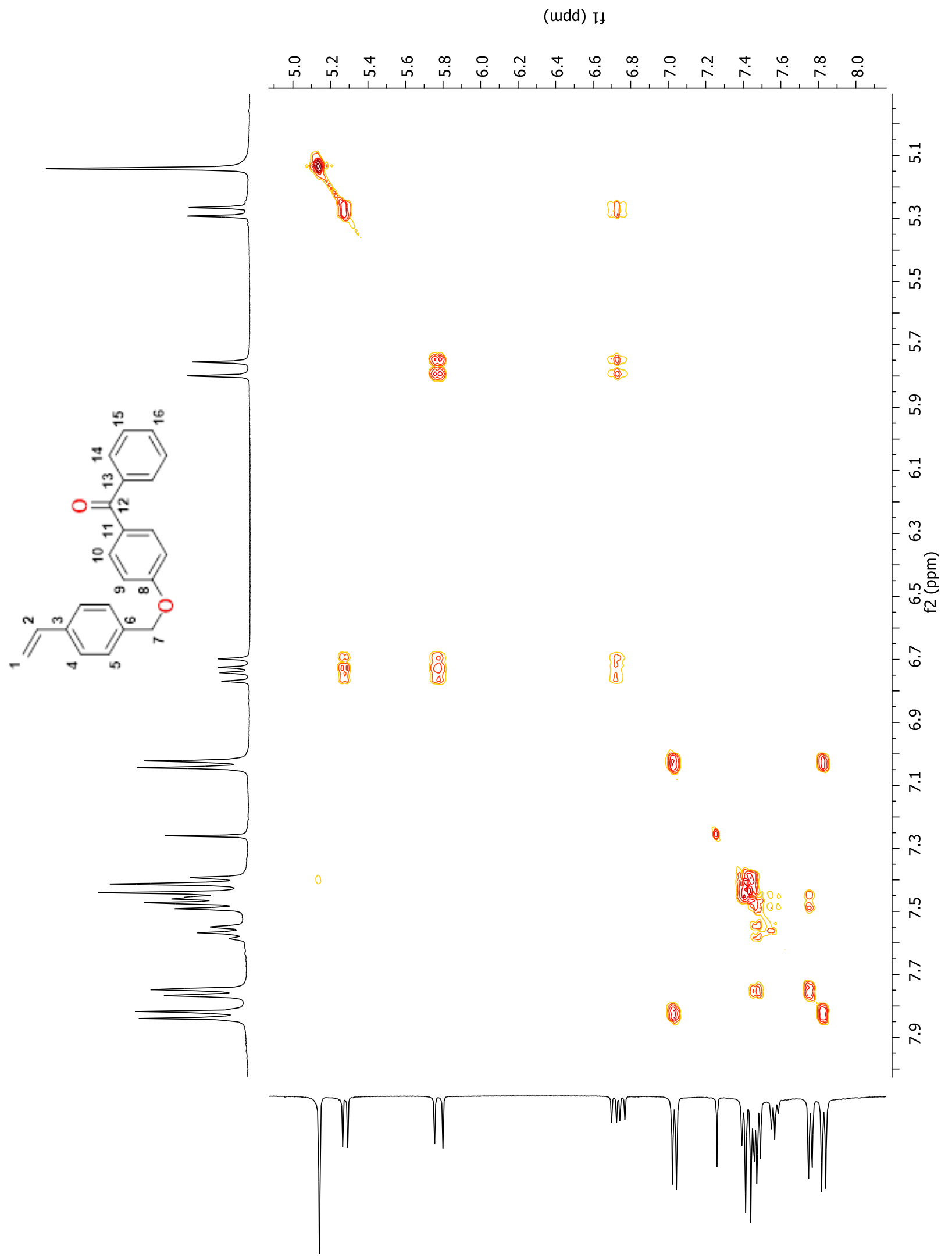

Figure A 3 2D COSY NMR spectrum of $\operatorname{VBBP}(\mathbf{1})$ in $\mathrm{CDCl}_{3}$ 


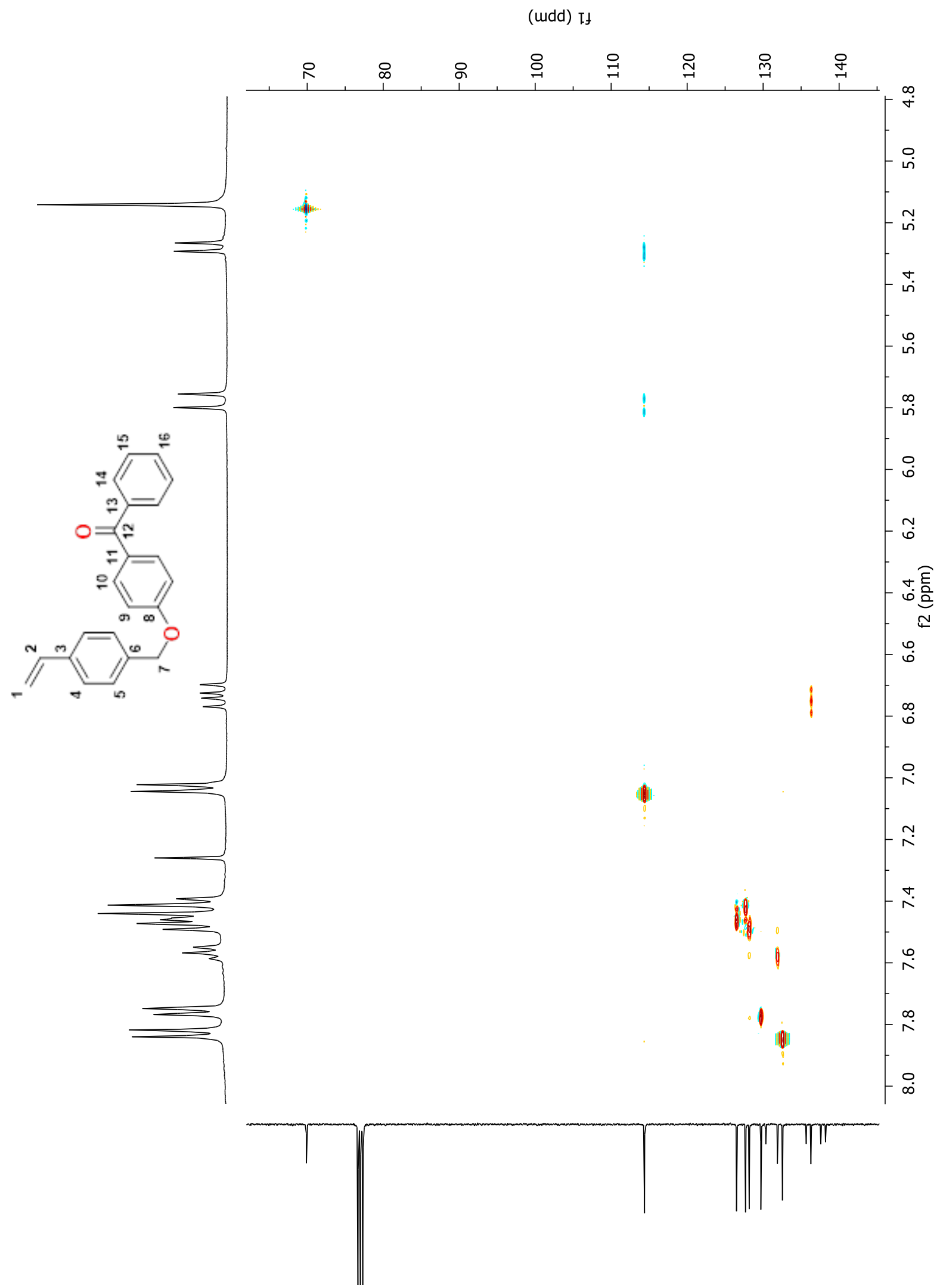

Figure A 4 2D HSQC NMR spectrum of $\mathrm{VBBP}(\mathbf{1})$ in $\mathrm{CDCl}_{3}$ 


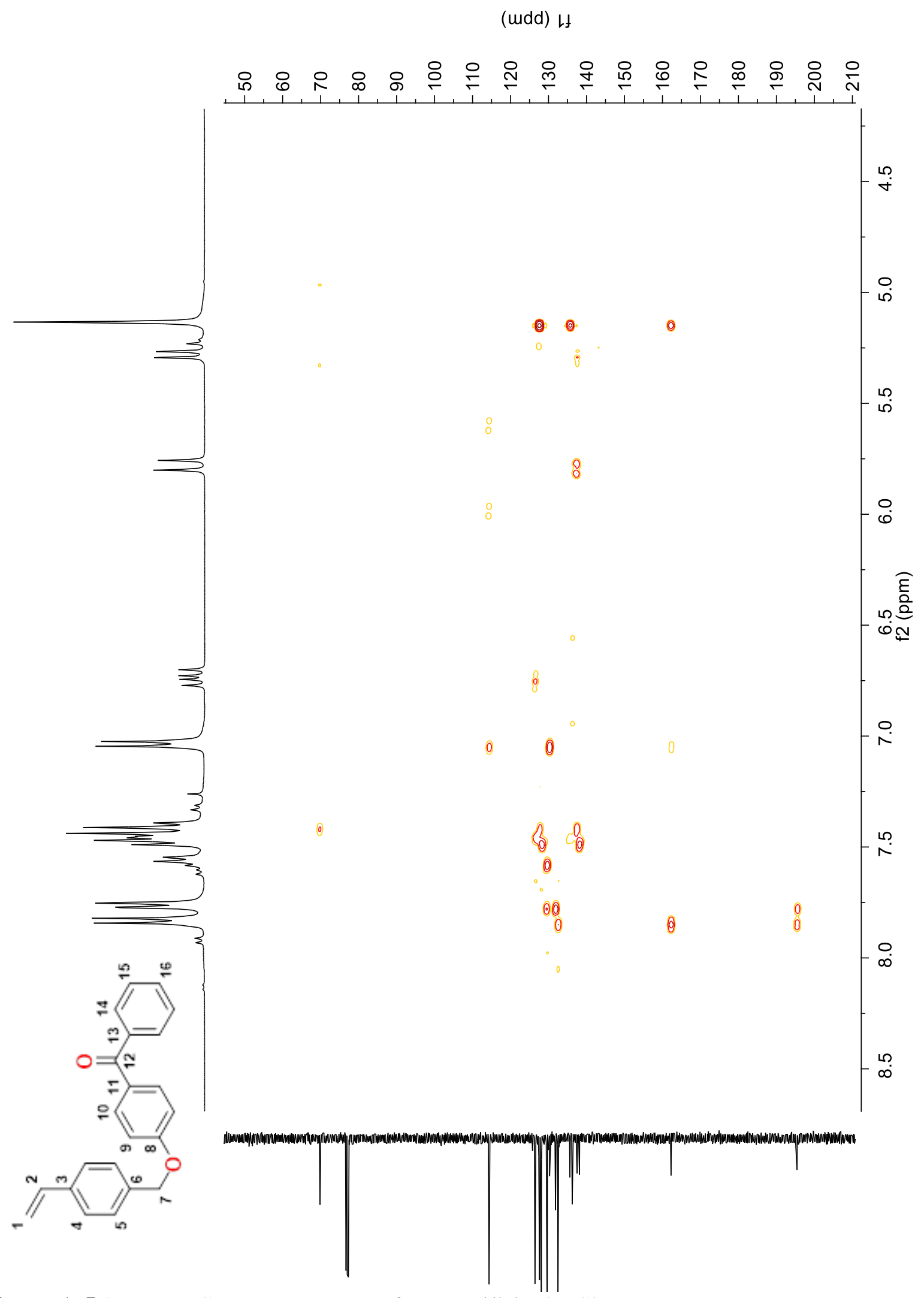

Figure A 5 2D HMBC NMR spectrum of $\mathrm{VBBP}(\mathbf{1})$ in $\mathrm{CDCl}_{3}$ 


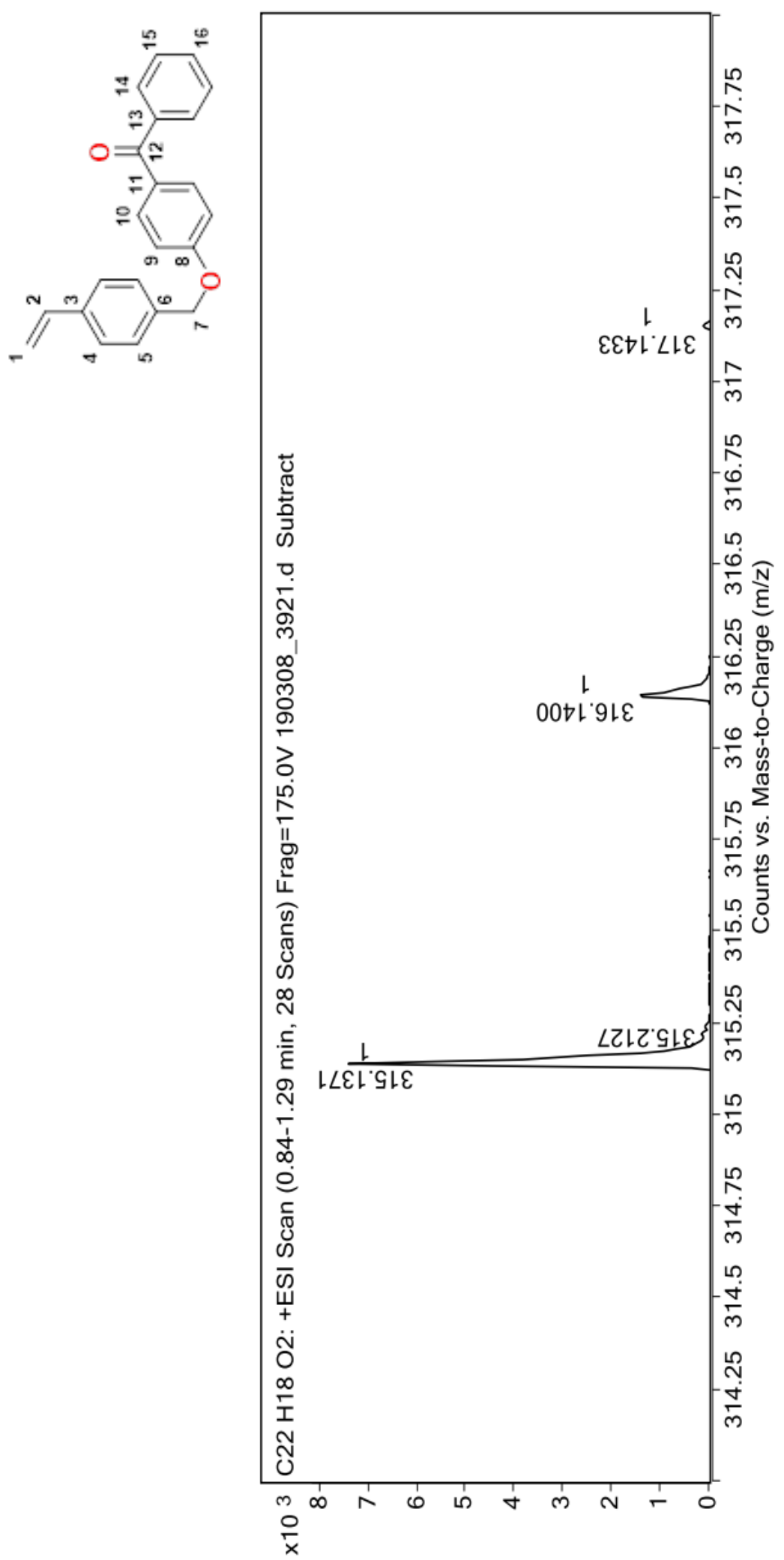

Figure A 6 ESI-Q-TOF MS of VBBP (1) 


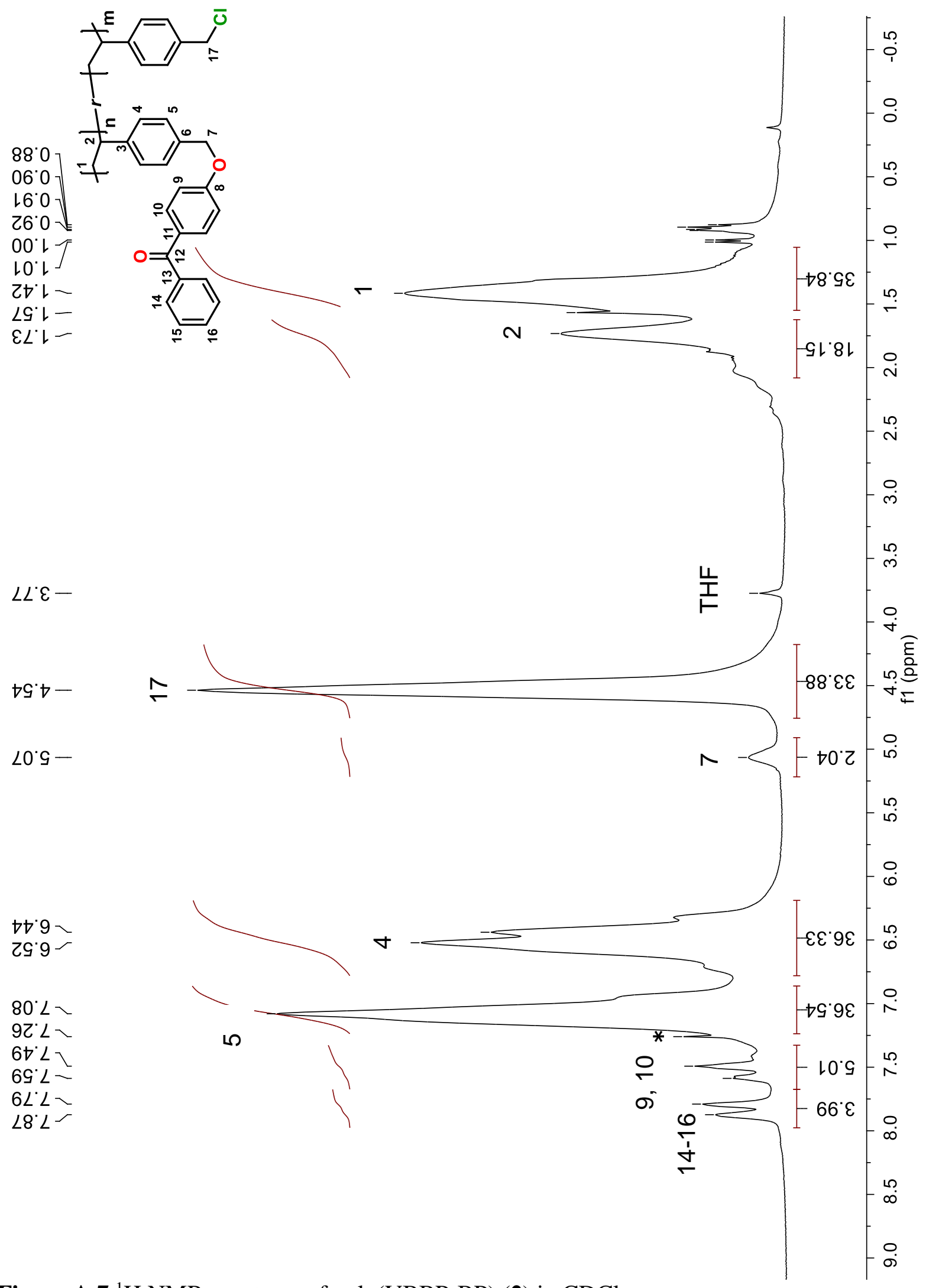

Figure A $7{ }^{1} \mathrm{H}$ NMR spectrum of poly(VBBP-BP) (2) in $\mathrm{CDCl}_{3}$ 


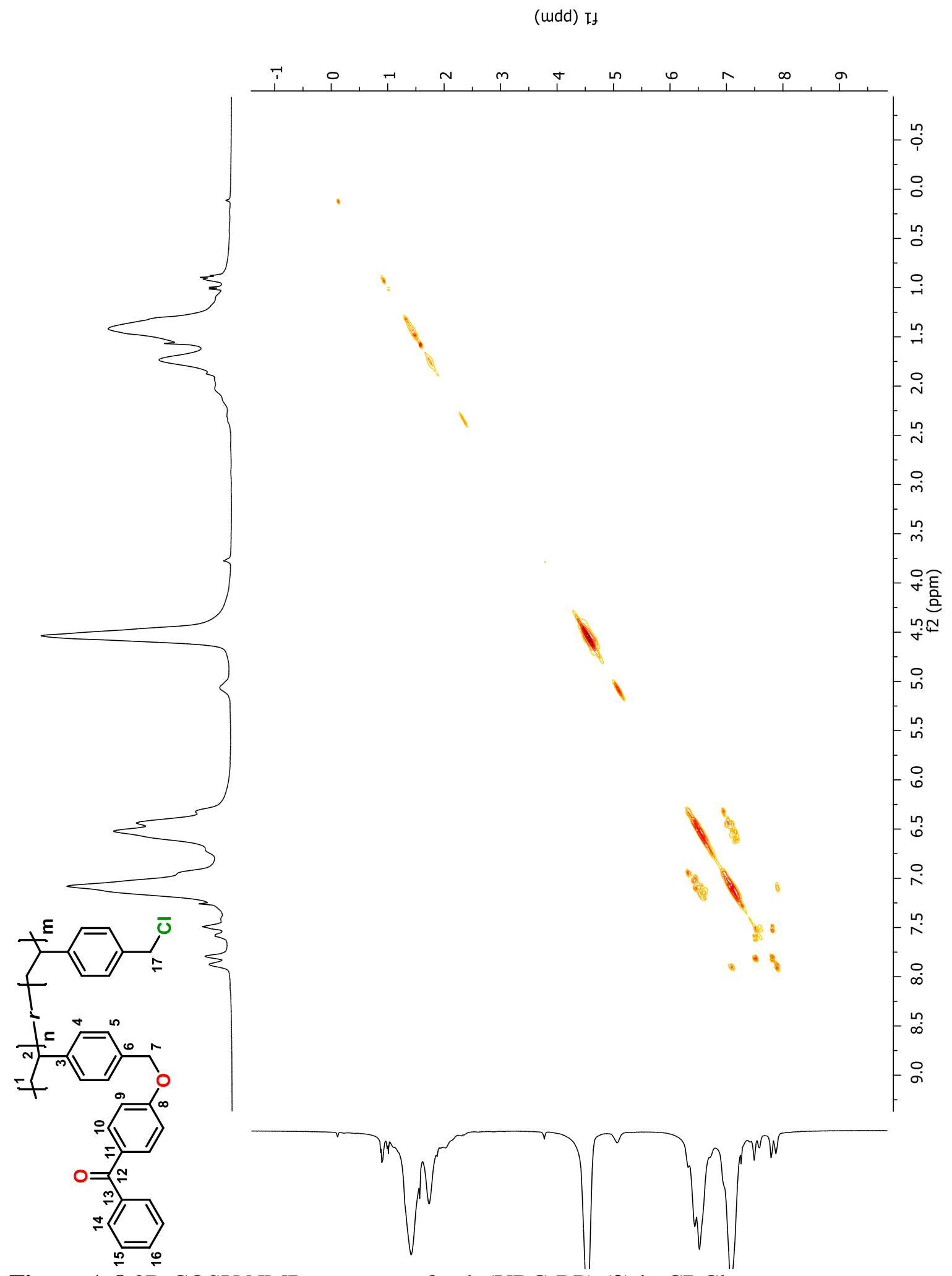

Figure A 8 2D COSY NMR spectrum of poly(VBC-BP) (2) in $\mathrm{CDCl}_{3}$ 
$80^{\circ}-$

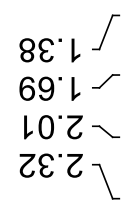

乙ง'॰ー

$82^{\circ} 9$ $0 t^{\circ} \cdot 9-2$

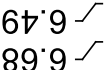
26.9 $90 \mathrm{~L}$ $92 \mathrm{~L} S$ $0 D^{\circ} L$

$\nabla G: L$ $98^{\circ} \mathrm{L}$

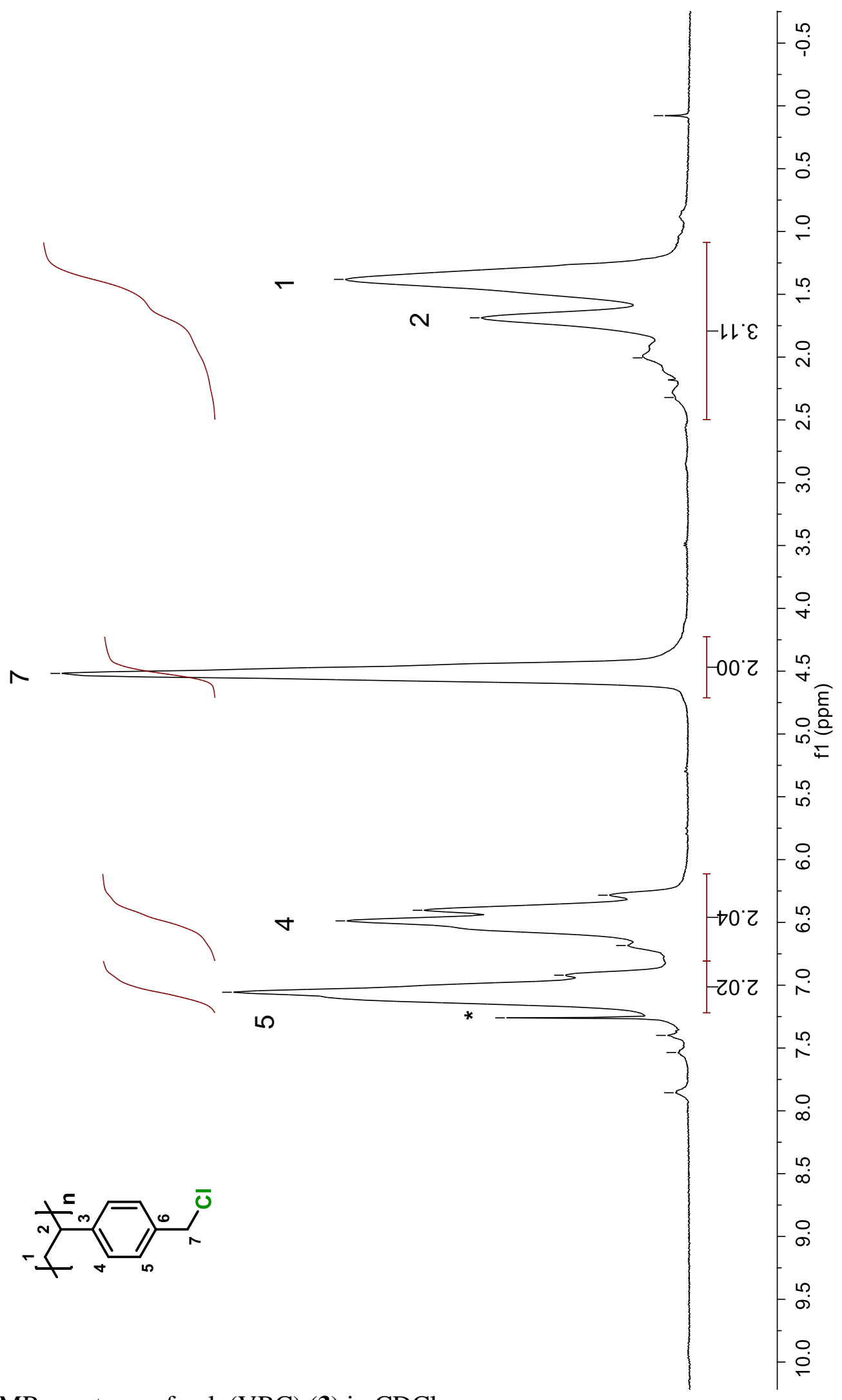

Figure A $9{ }^{1} \mathrm{H}$ NMR spectrum of poly(VBC) (3) in $\mathrm{CDCl}_{3}$ 


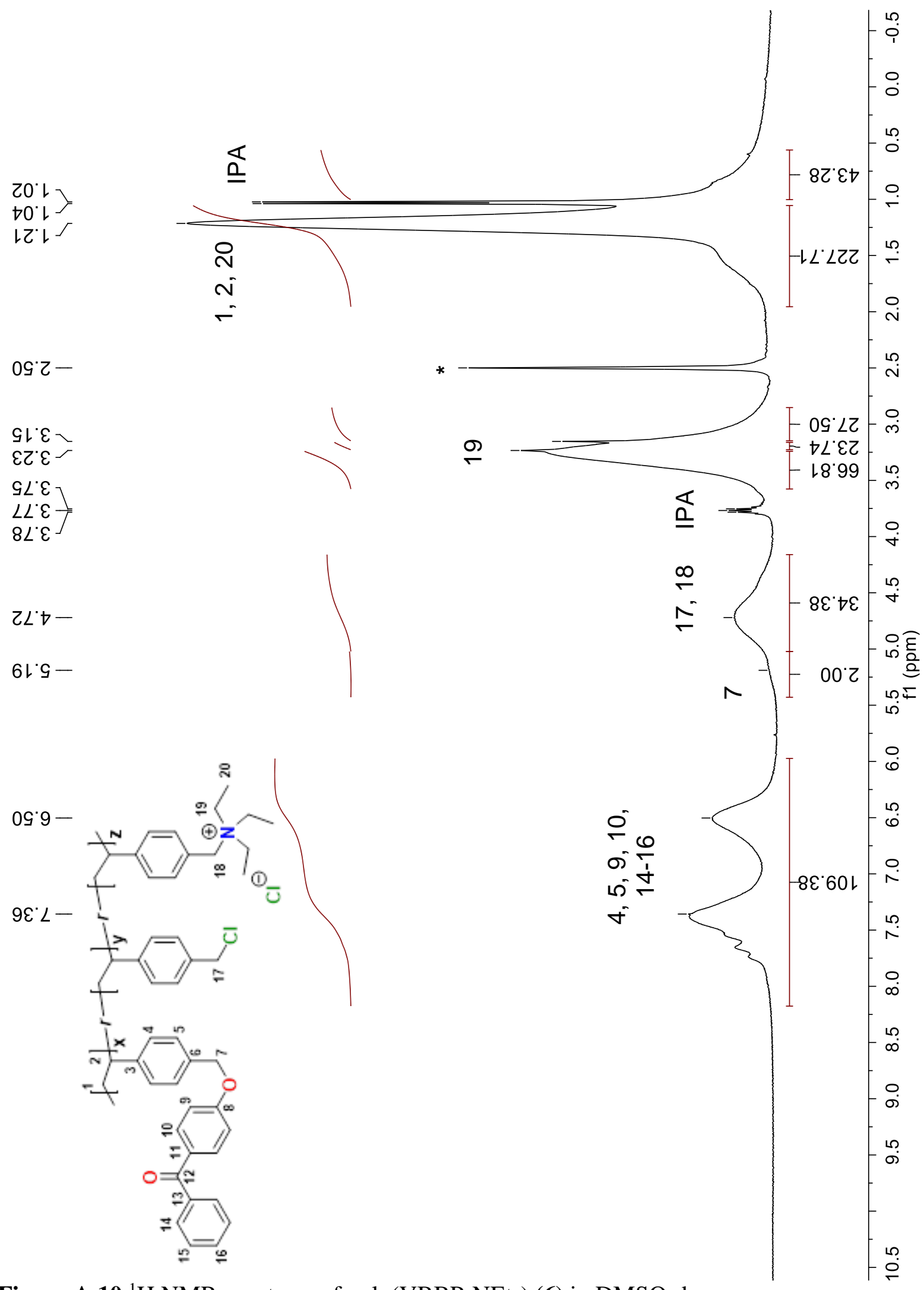

Figure A $10{ }^{1} \mathrm{H}$ NMR spectrum of poly(VBBP-NEt 3$)(6)$ in DMSO-d 6 


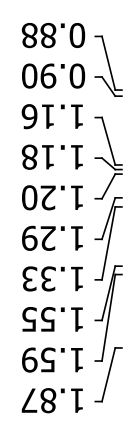

โ $\varepsilon^{\prime} \varepsilon$

ธะ' $\varepsilon$ ᄂ $6 S^{\prime} \varepsilon$

$09^{\circ} \varepsilon-$

29. $\varepsilon-$

$\left.\succ 9^{\circ} \varepsilon\right]$

$98^{\circ} \varepsilon$

$08^{\circ} \bullet-$

$2 Z^{\circ} \mathrm{S}-$

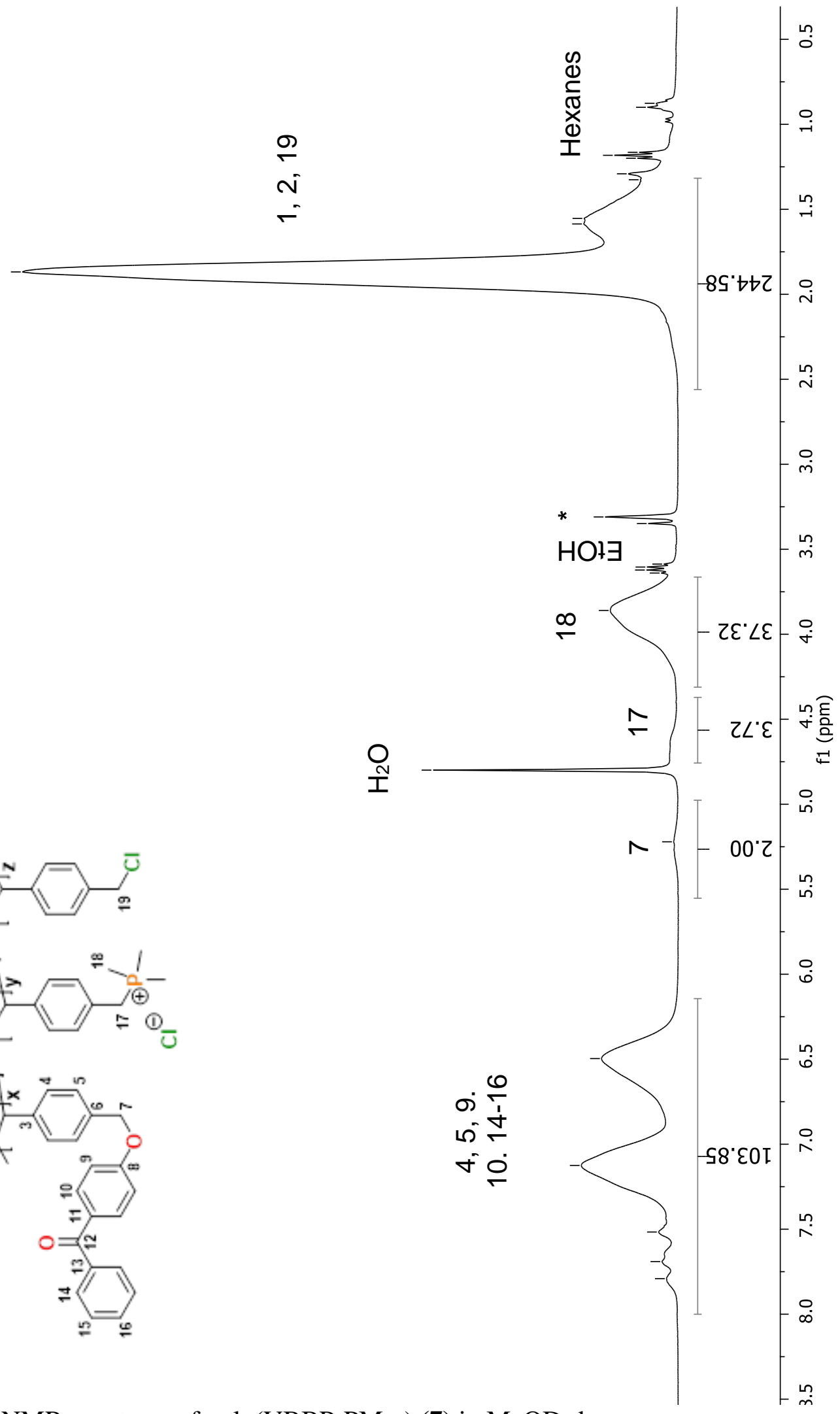

Figure A 11. ${ }^{1} \mathrm{H}$ NMR spectrum of poly(VBBP-PMe 3$)(7)$ in $\mathrm{MeOD}-\mathrm{d}_{4}$ 


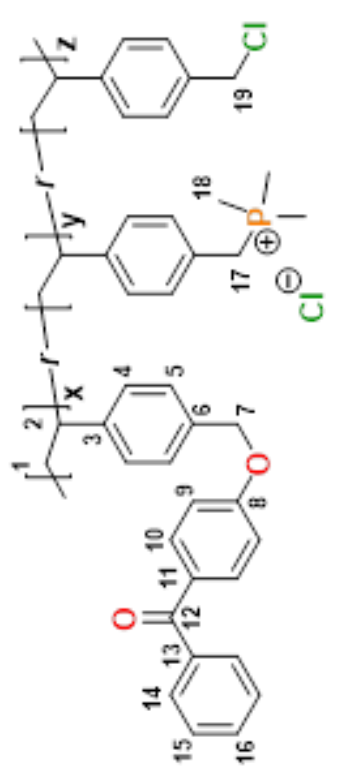

$16^{\circ} 92-$

Figure A 12. ${ }^{31} \mathrm{P}$ NMR spectrum of poly(VBBP-PMe 3 ) (7) in MeOD- $\mathrm{d}_{4}$ 


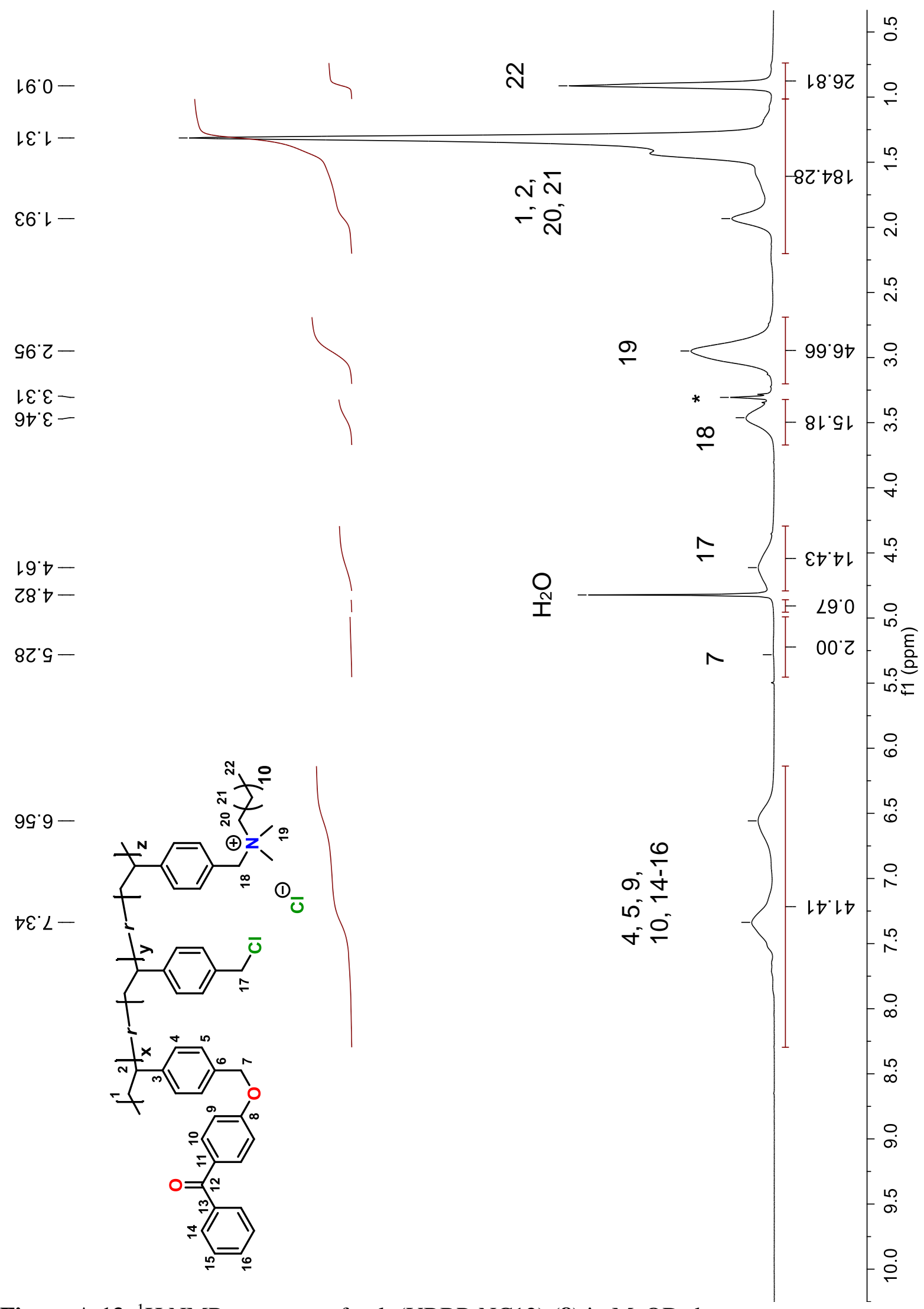

Figure A 13. ${ }^{1} \mathrm{H}$ NMR spectrum of poly(VBBP-NC12) (8) in MeOD-d 4 


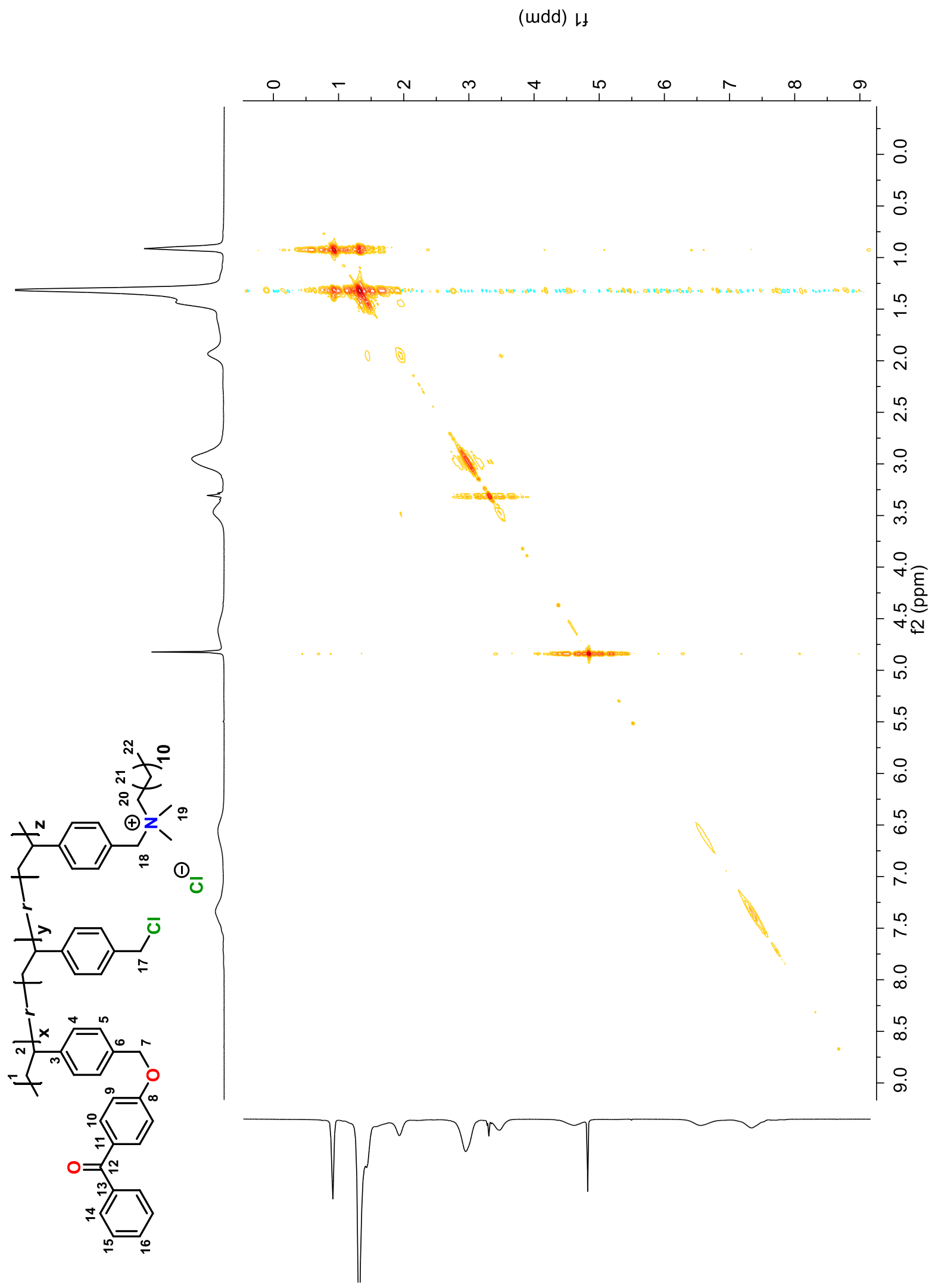

Figure A 14. 2D COSY NMR spectrum of poly(VBBP-NC12) (8) in MeOD-d 


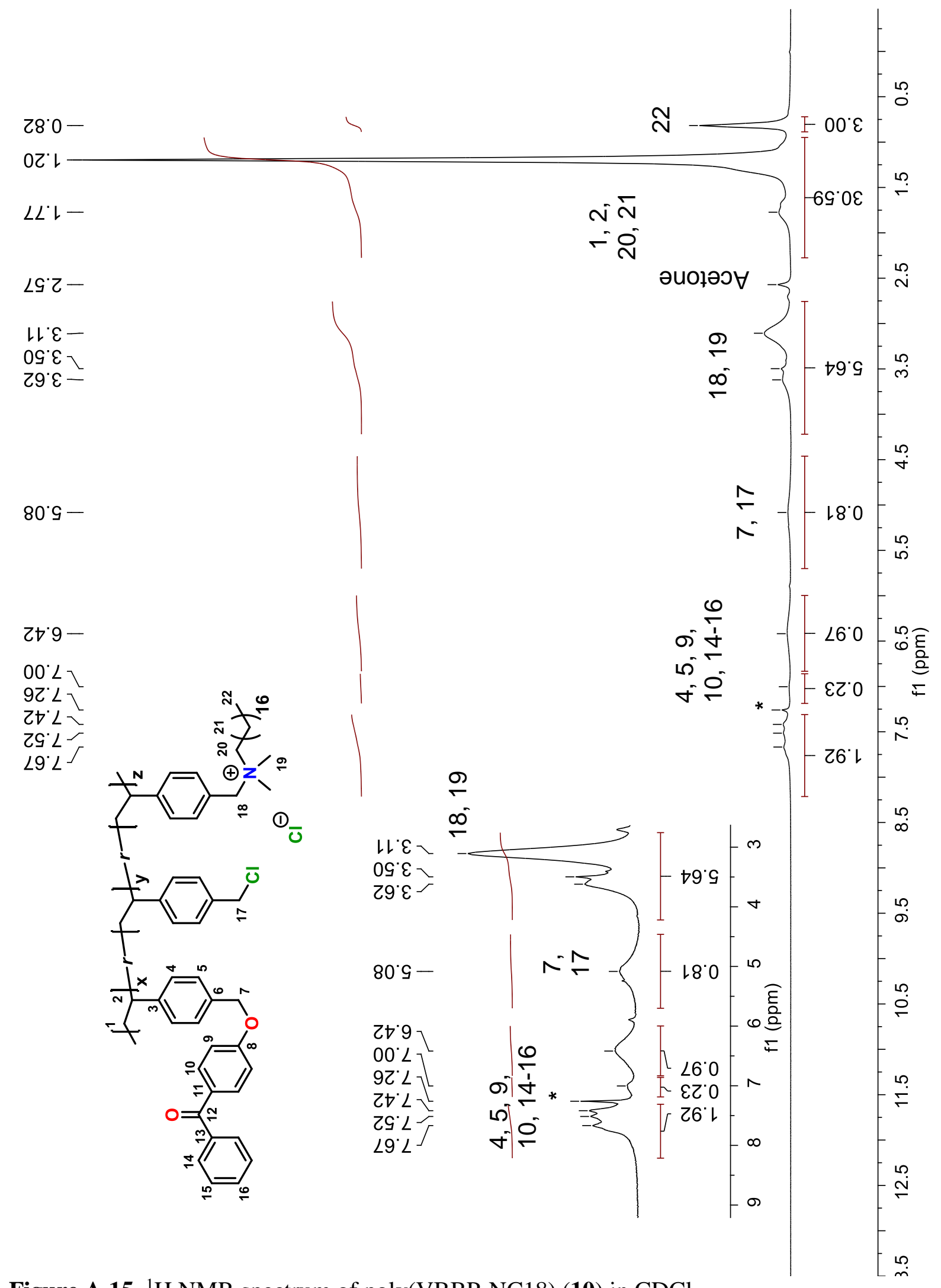

Figure A 15. ${ }^{1} \mathrm{H}$ NMR spectrum of poly(VBBP-NC18) (10) in $\mathrm{CDCl}_{3}$ 


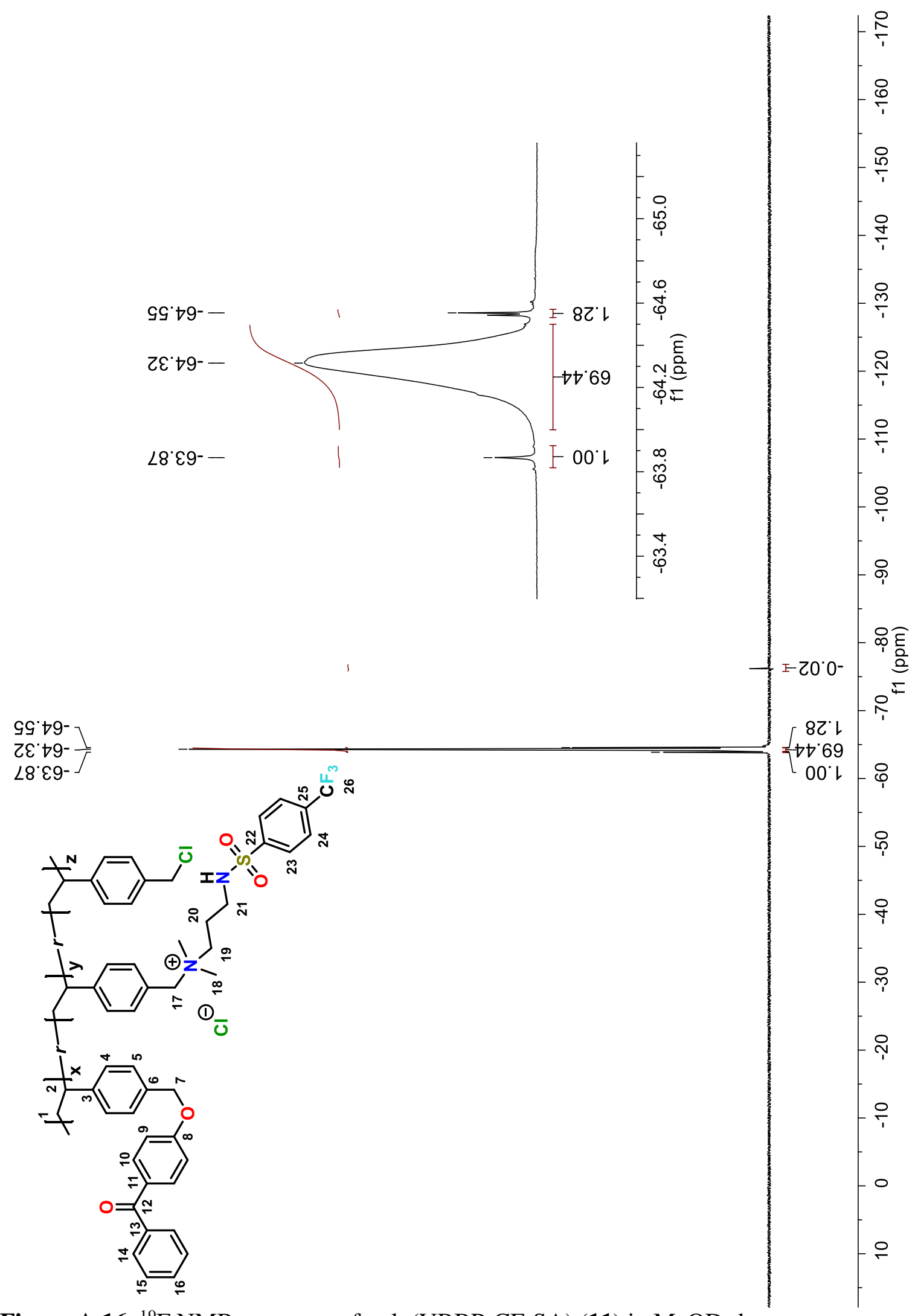

Figure A 16. ${ }^{19} \mathrm{~F}$ NMR spectrum of poly $\left(\mathrm{VBBP}-\mathrm{CF}_{3} \mathrm{SA}\right)(\mathbf{1 1})$ in $\mathrm{MeOD}-\mathrm{d}_{4}$ 


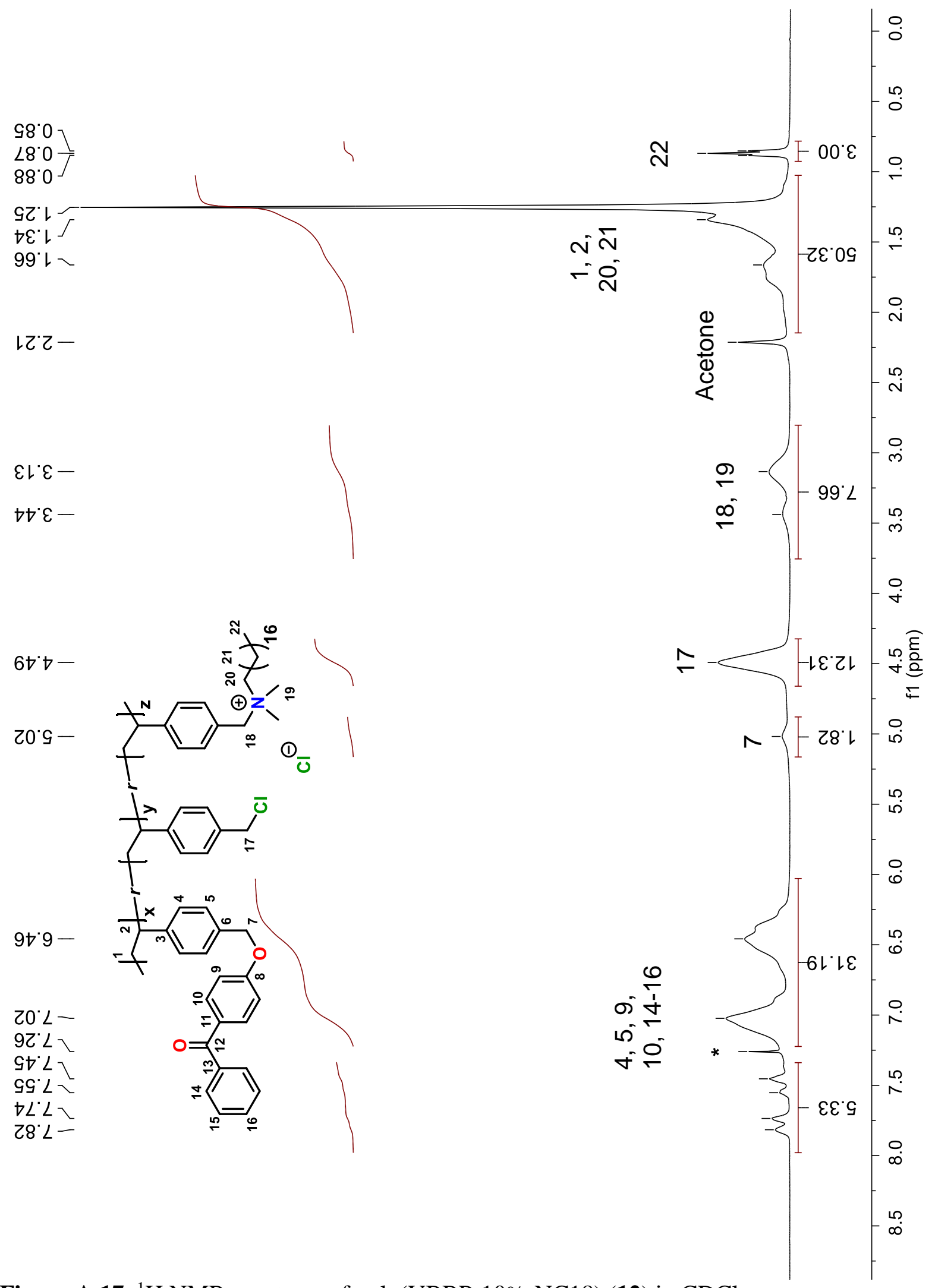

Figure A 17. ${ }^{1} \mathrm{H}$ NMR spectrum of poly(VBBP-10\%-NC18) (12) in $\mathrm{CDCl}_{3}$ 


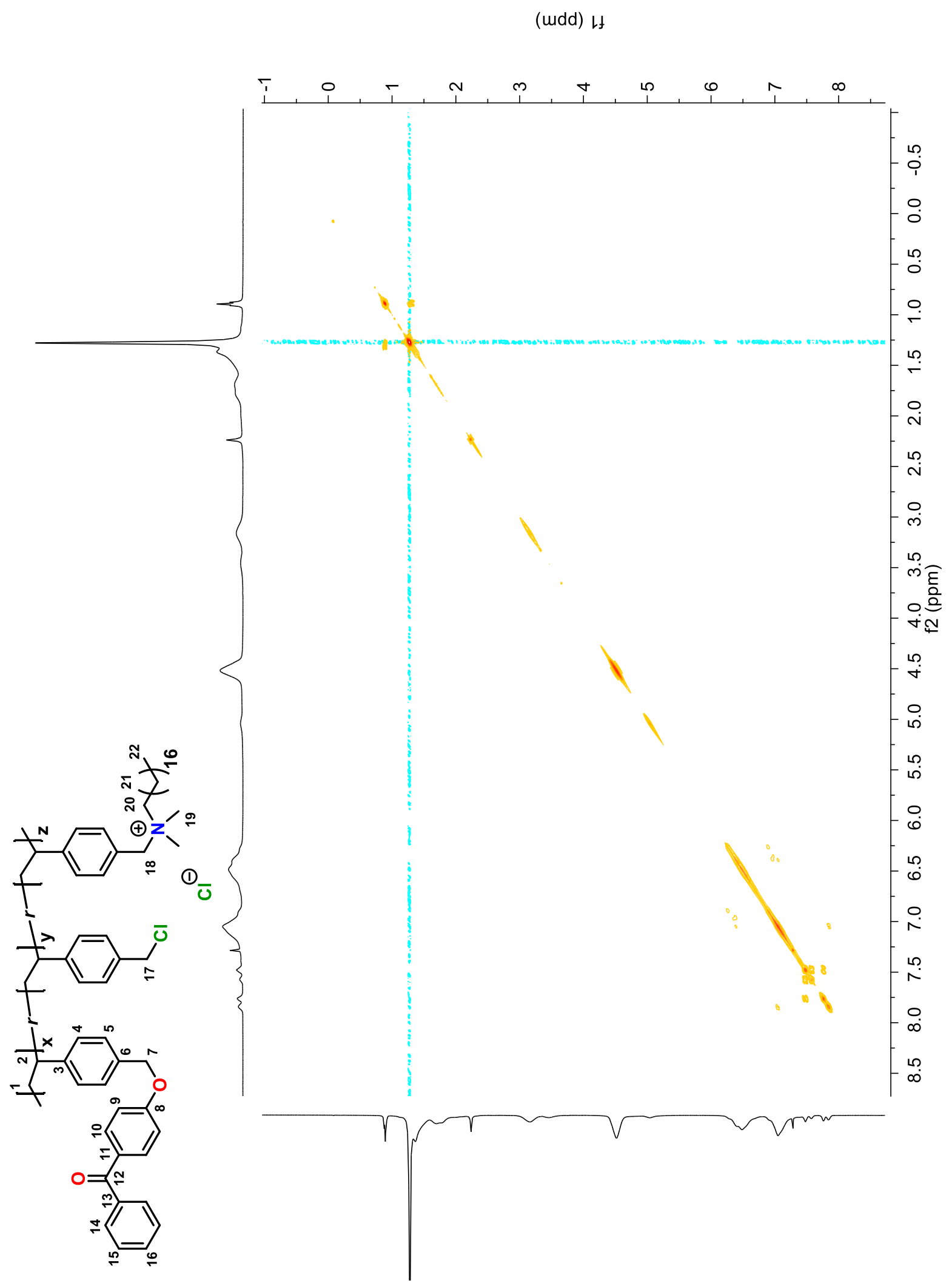

Figure A 18. 2D COSY NMR spectrum of poly(VBBP-10\%-NC18) (12) in $\mathrm{CDCl}_{3}$ 


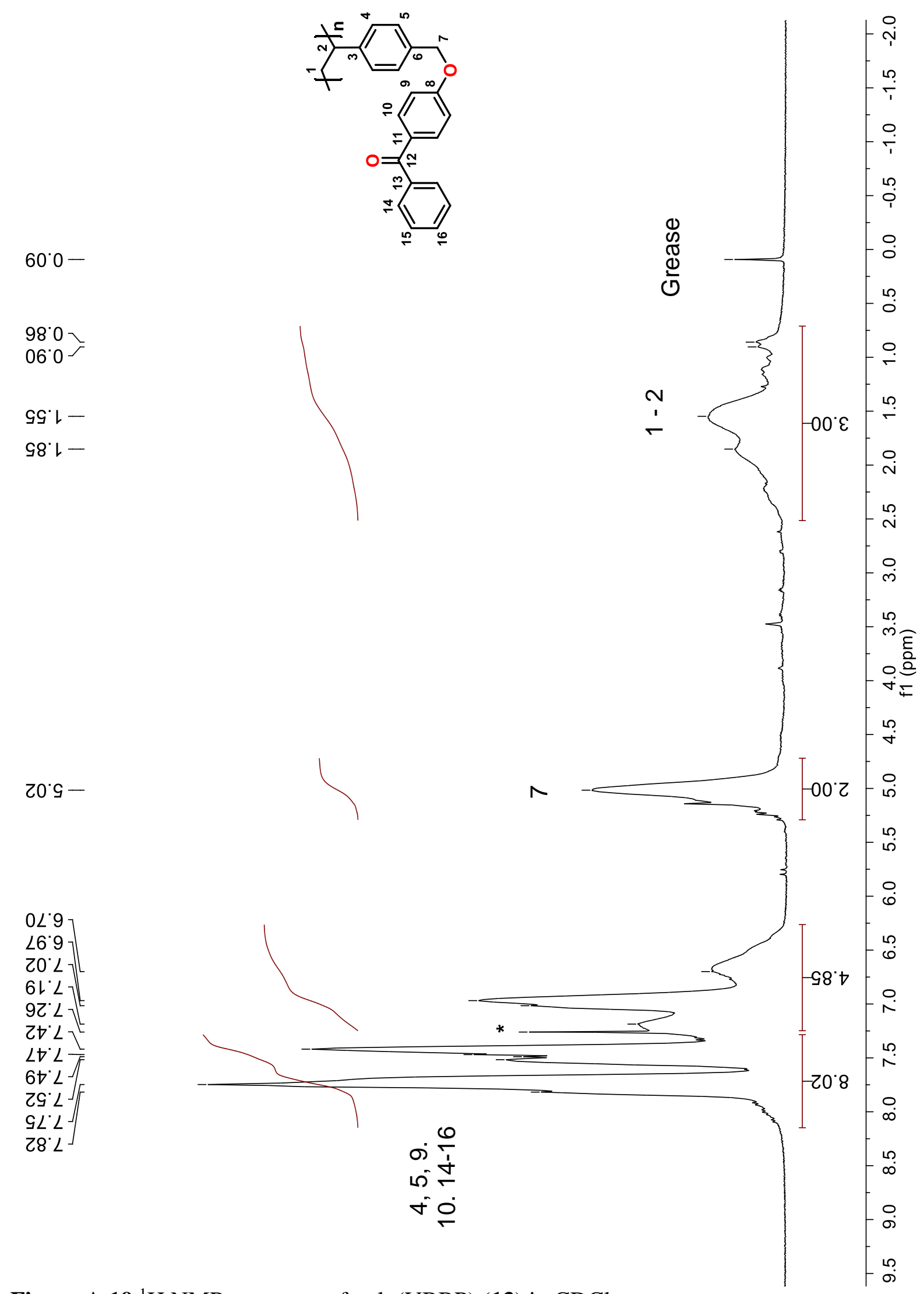

Figure A $19{ }^{1} \mathrm{H}$ NMR spectrum of poly(VBBP) (13) in $\mathrm{CDCl}_{3}$ 


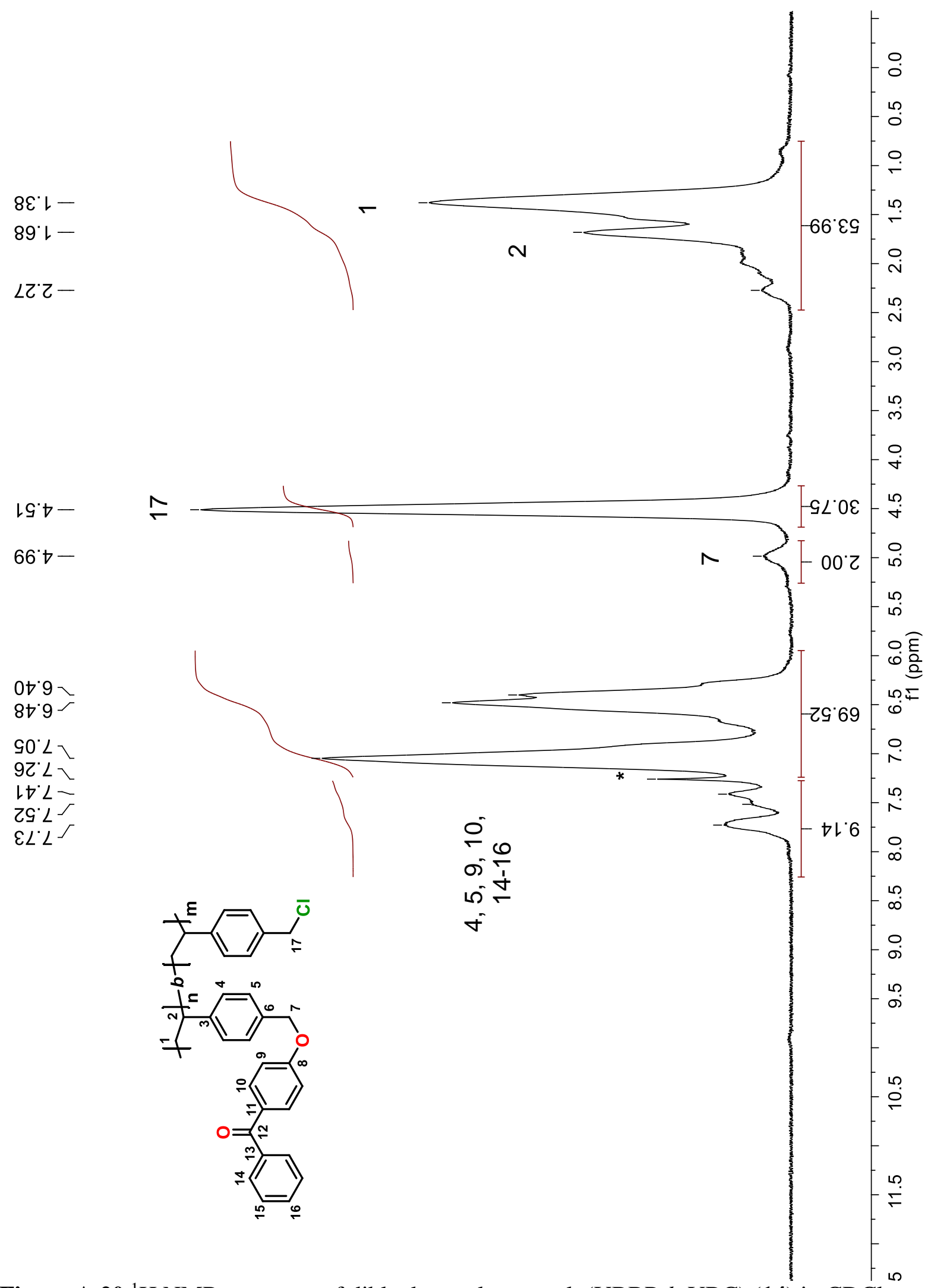

Figure A $20{ }^{1} \mathrm{H}$ NMR spectrum of diblock copolymer poly(VBBP- $b$-VBC) (14) in $\mathrm{CDCl}_{3}$ 


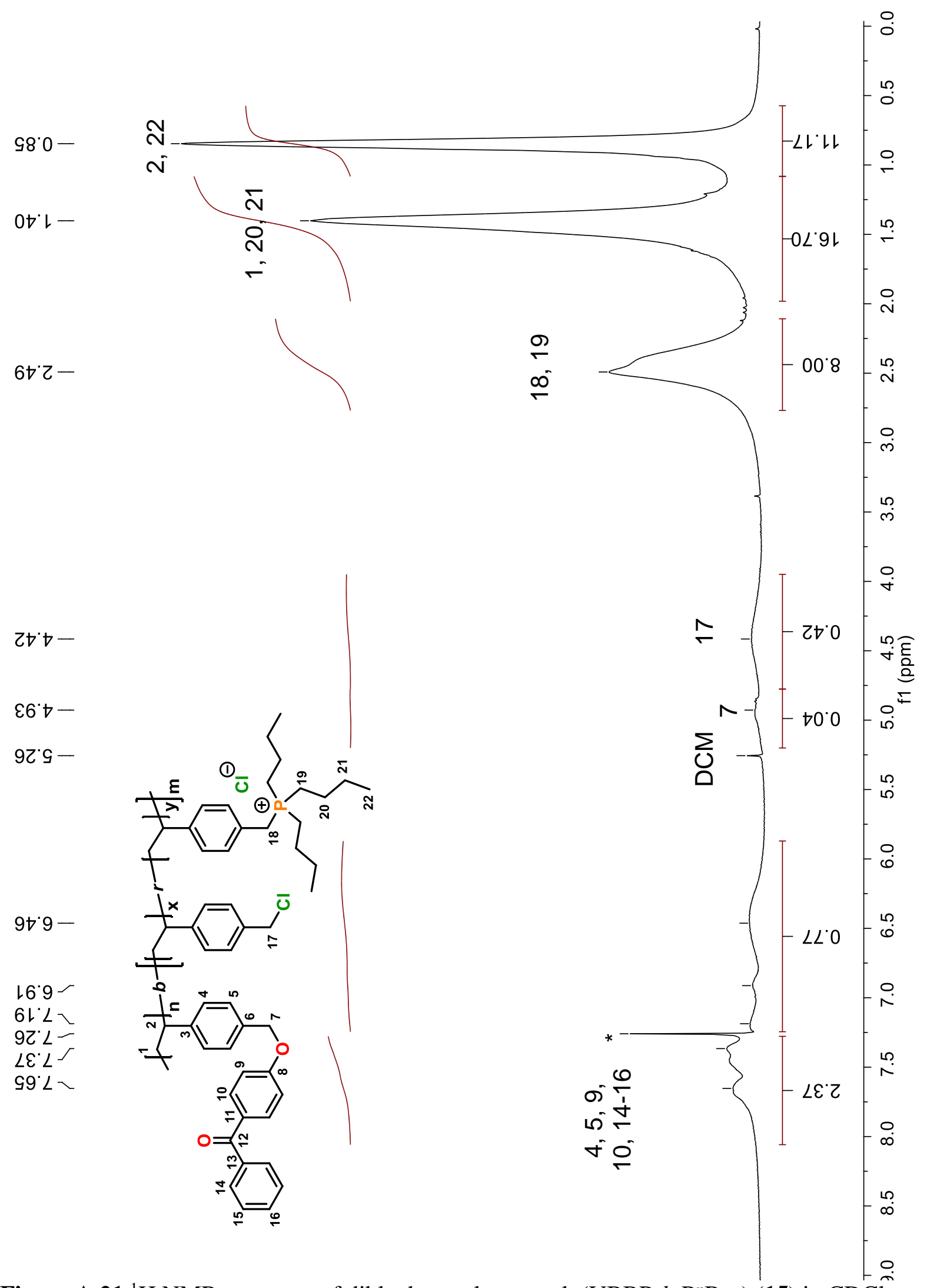

Figure A $21{ }^{1} \mathrm{H}$ NMR spectrum of diblock copolymer poly(VBBP- $\left.b-\mathrm{P}^{n} \mathrm{Bu}_{3}\right)(\mathbf{1 5})$ in $\mathrm{CDCl}_{3}$ 


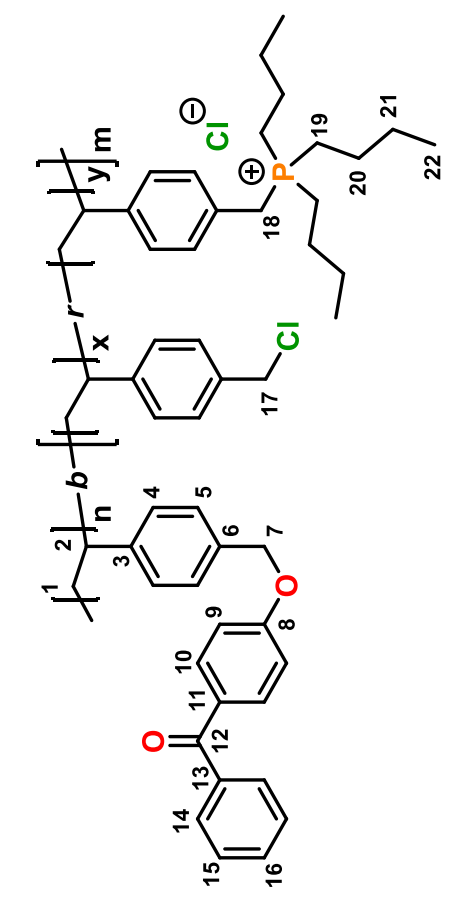

$6 乙^{\circ}\left\llcorner\varepsilon^{\top}\right.$

$90.8 \varepsilon-$

$99^{\circ} 8 \mathrm{~V}$ 久

Figure A $22{ }^{31} \mathrm{P}$ NMR spectrum of poly(VBBP- $\left.b-\mathrm{P}^{n} \mathrm{Bu}_{3}\right)(\mathbf{1 5})$ in $\mathrm{CDCl}_{3}$

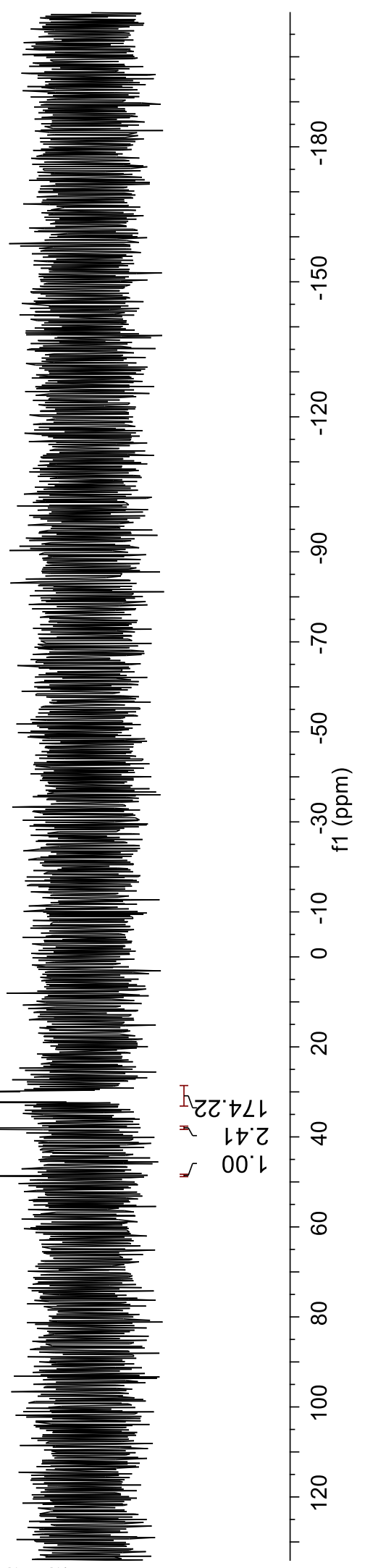




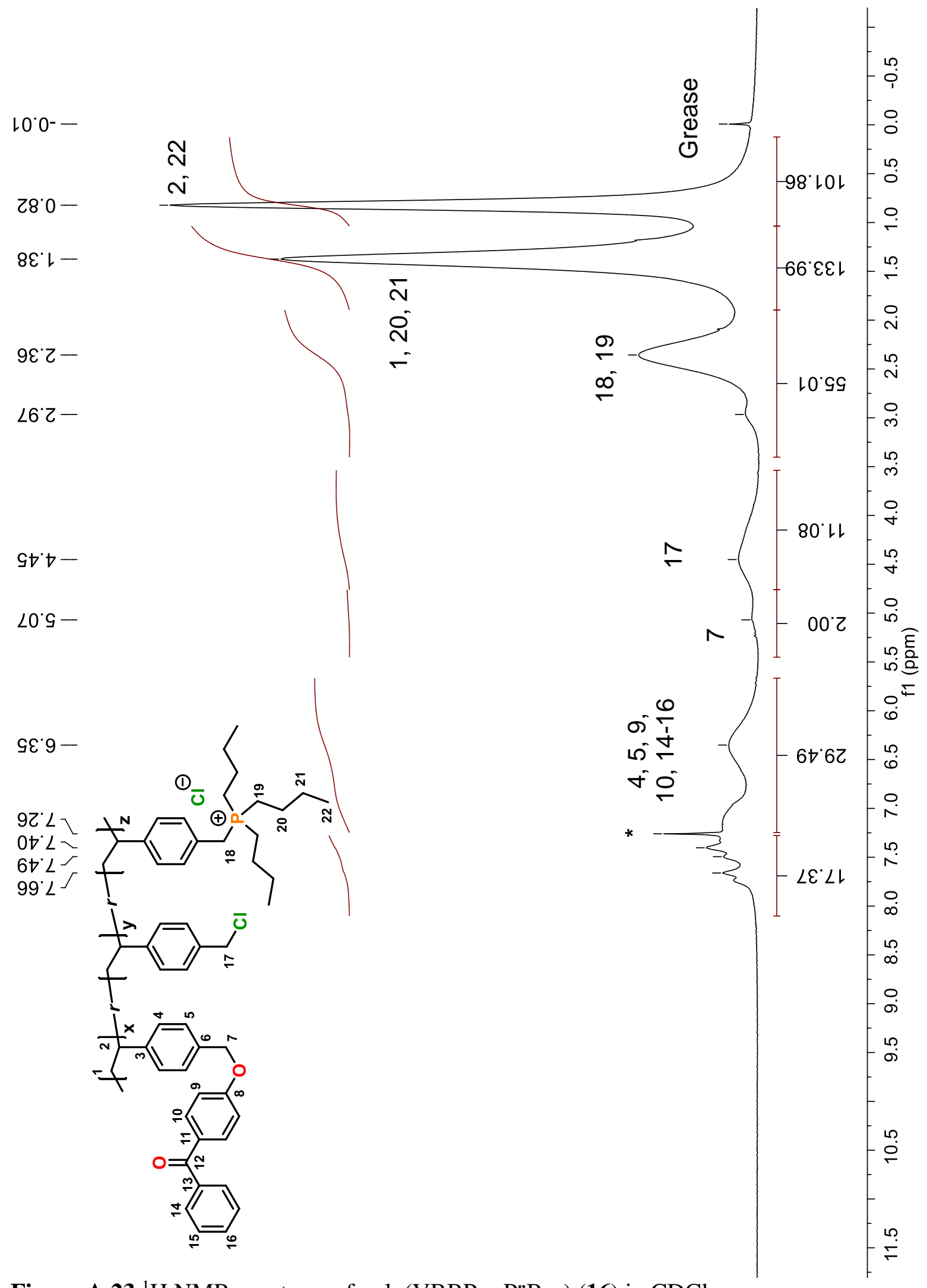

Figure A $23{ }^{1} \mathrm{H}$ NMR spectrum of poly(VBBP- $r$ - $\left.\mathrm{P}^{n} \mathrm{Bu}_{3}\right)(\mathbf{1 6})$ in $\mathrm{CDCl}_{3}$ 

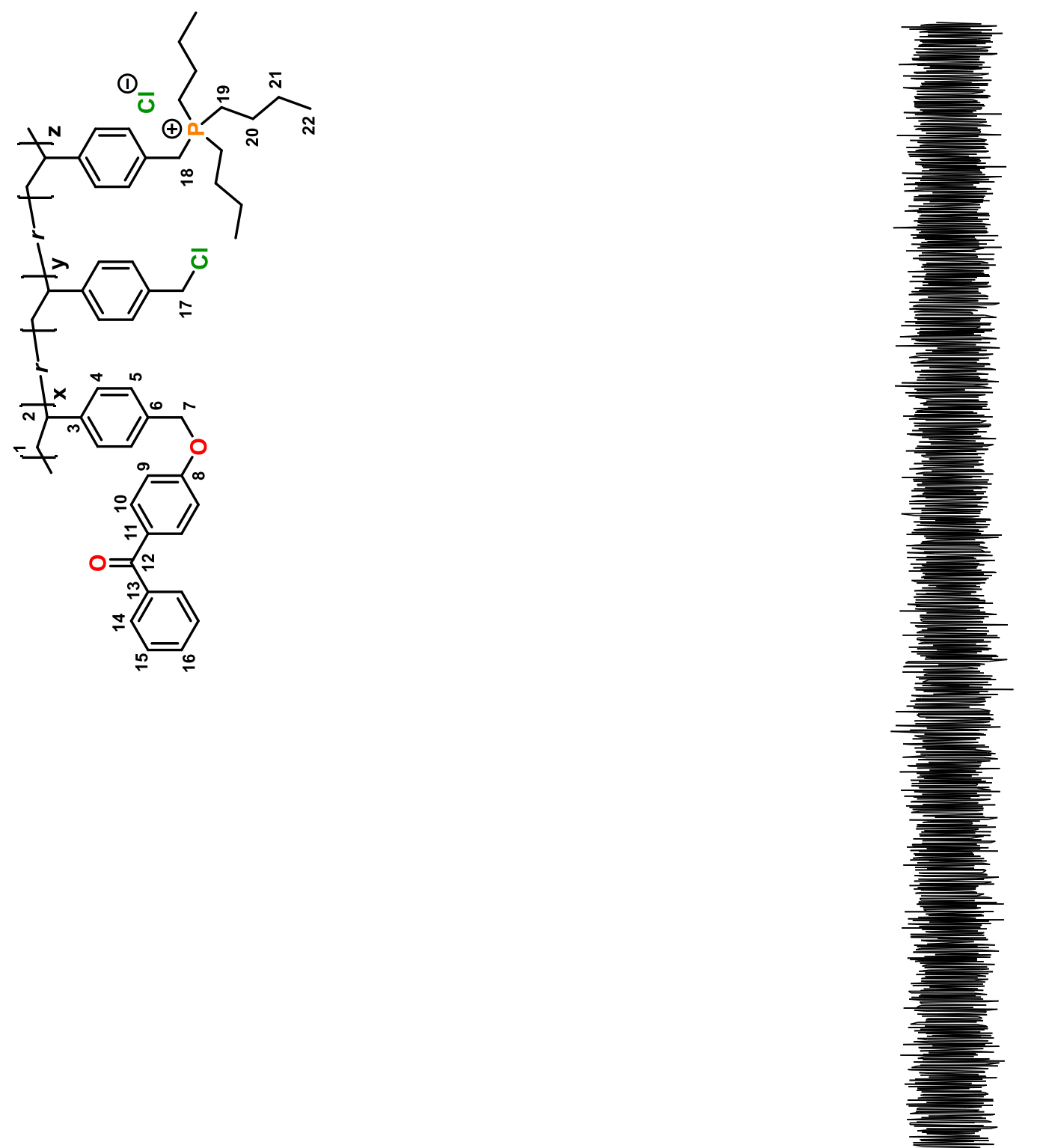

ฤฤเย-

$10.8 \varepsilon-$

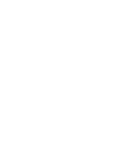




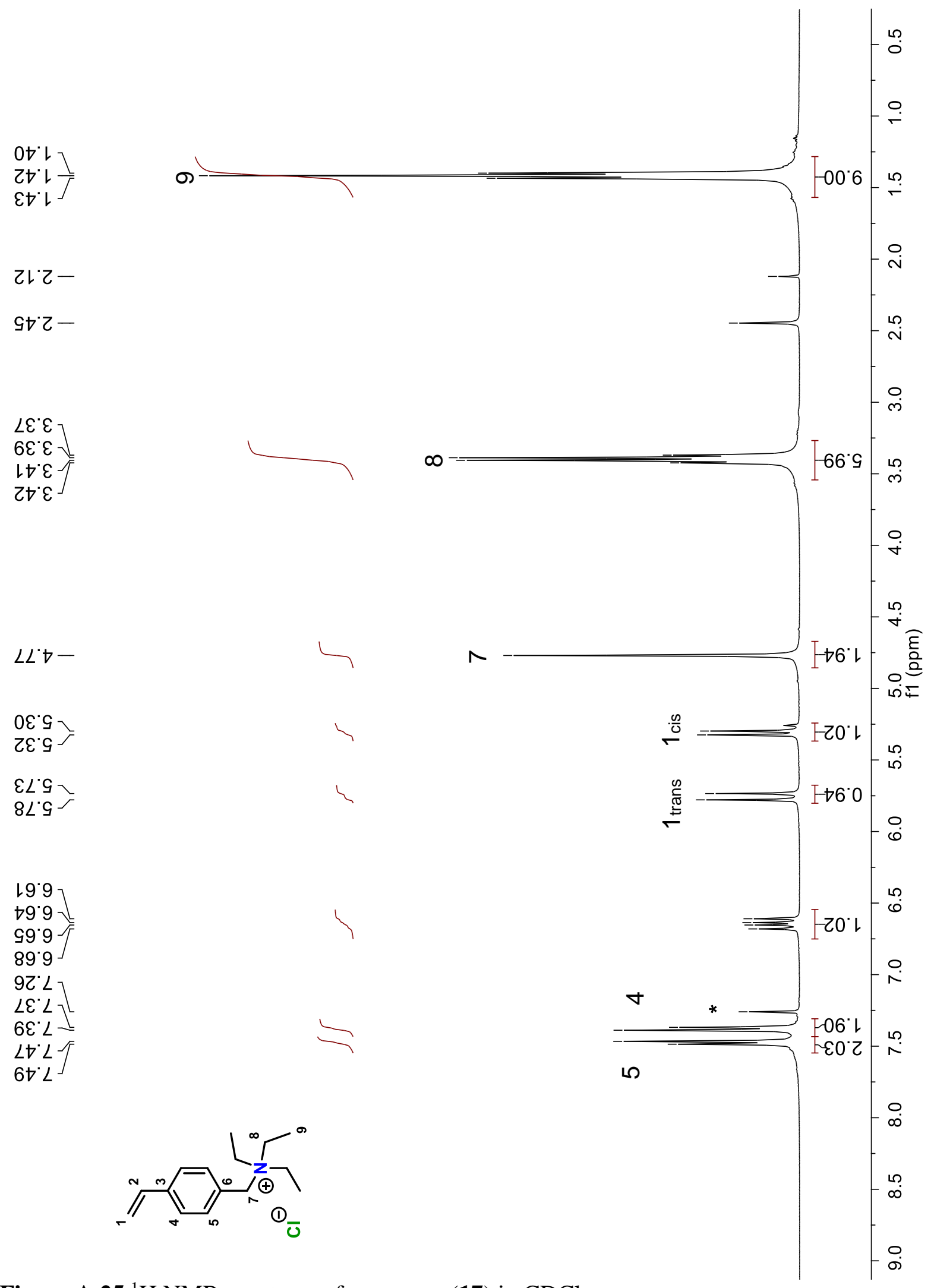

Figure A $25{ }^{1} \mathrm{H}$ NMR spectrum of monomer (17) in $\mathrm{CDCl}_{3}$ 


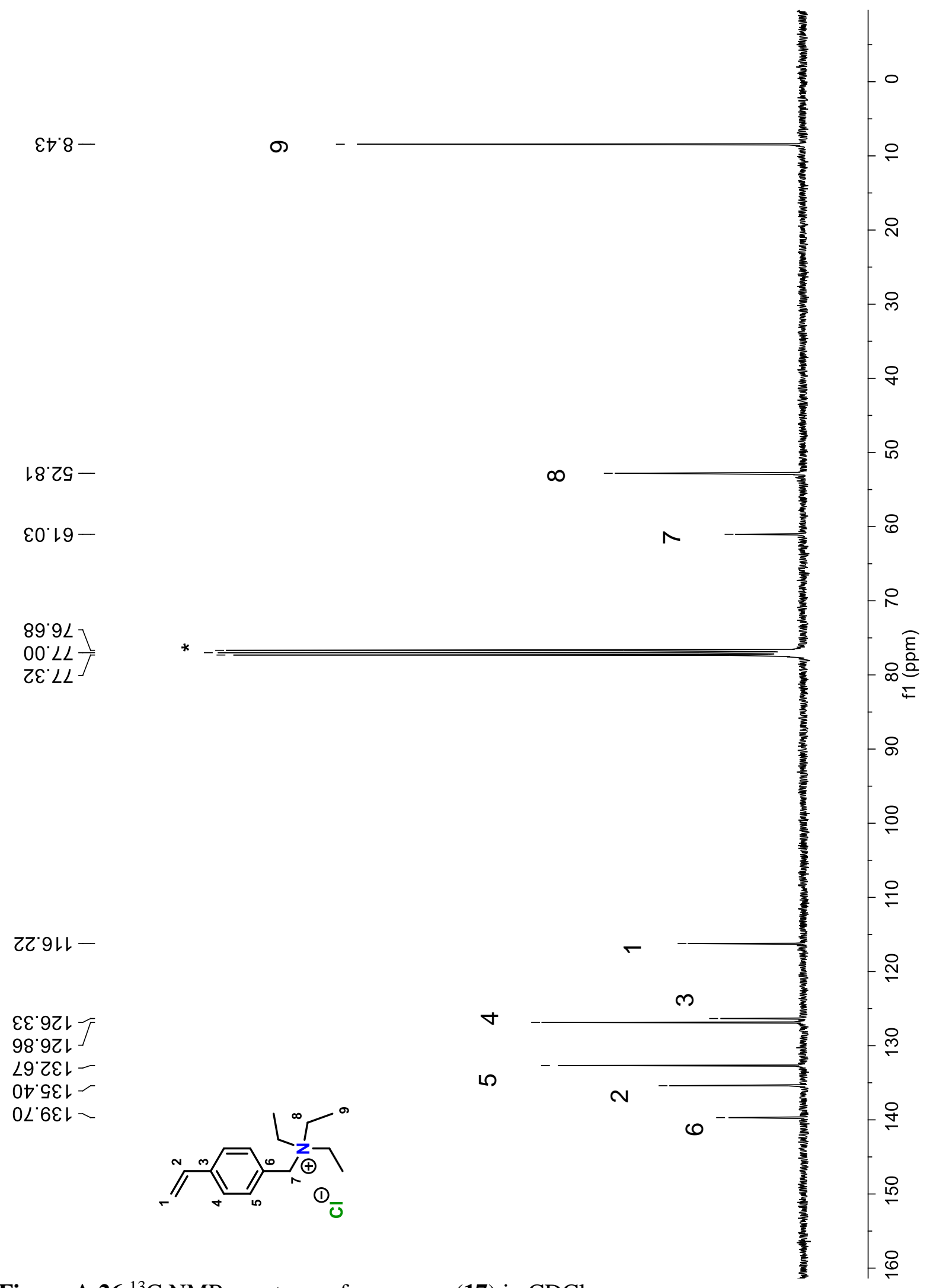

Figure A $26{ }^{13} \mathrm{C}$ NMR spectrum of monomer (17) in $\mathrm{CDCl}_{3}$ 


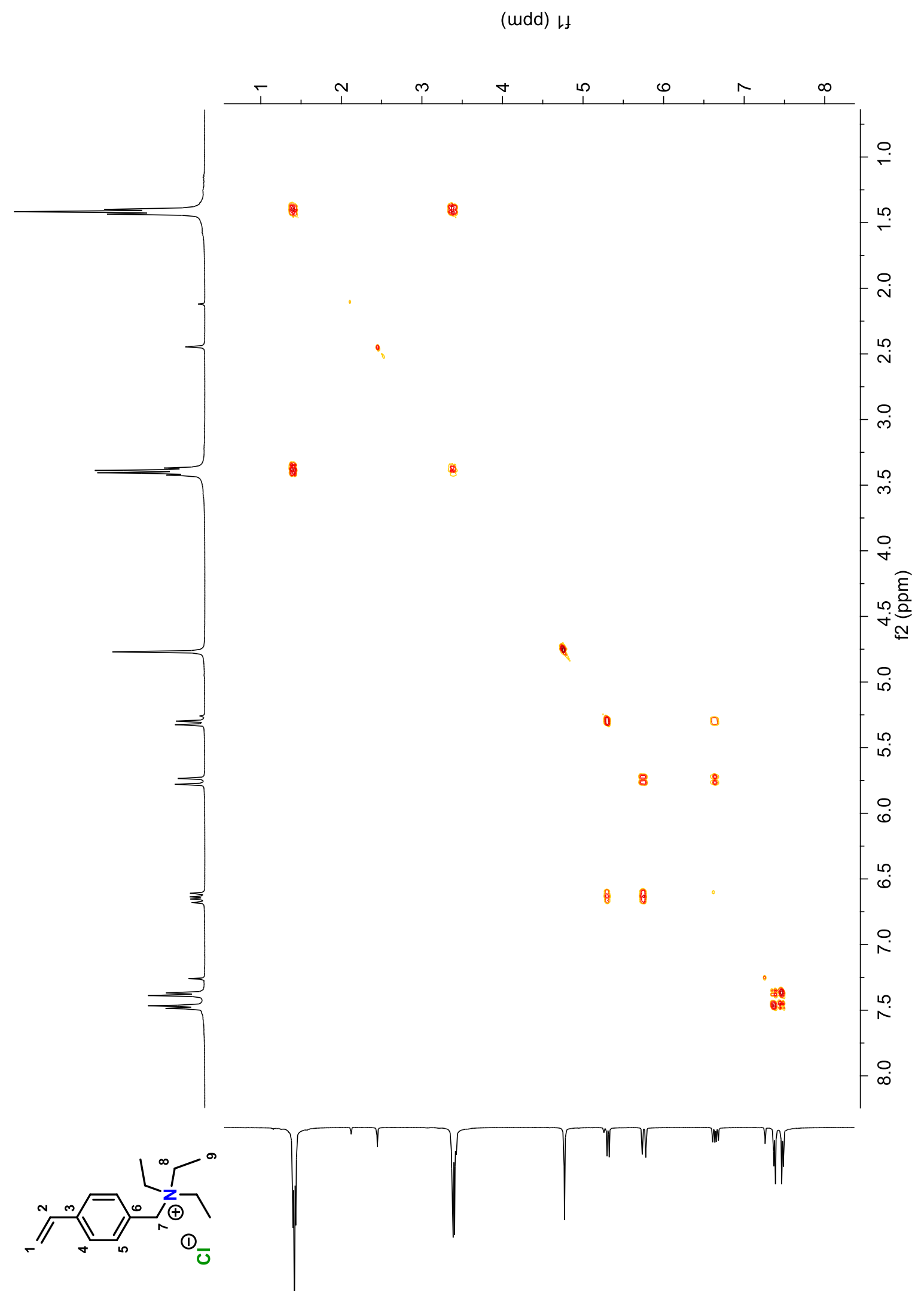

Figure A 27 2D COSY NMR spectrum of monomer 17 in $\mathrm{CDCl}_{3}$ 


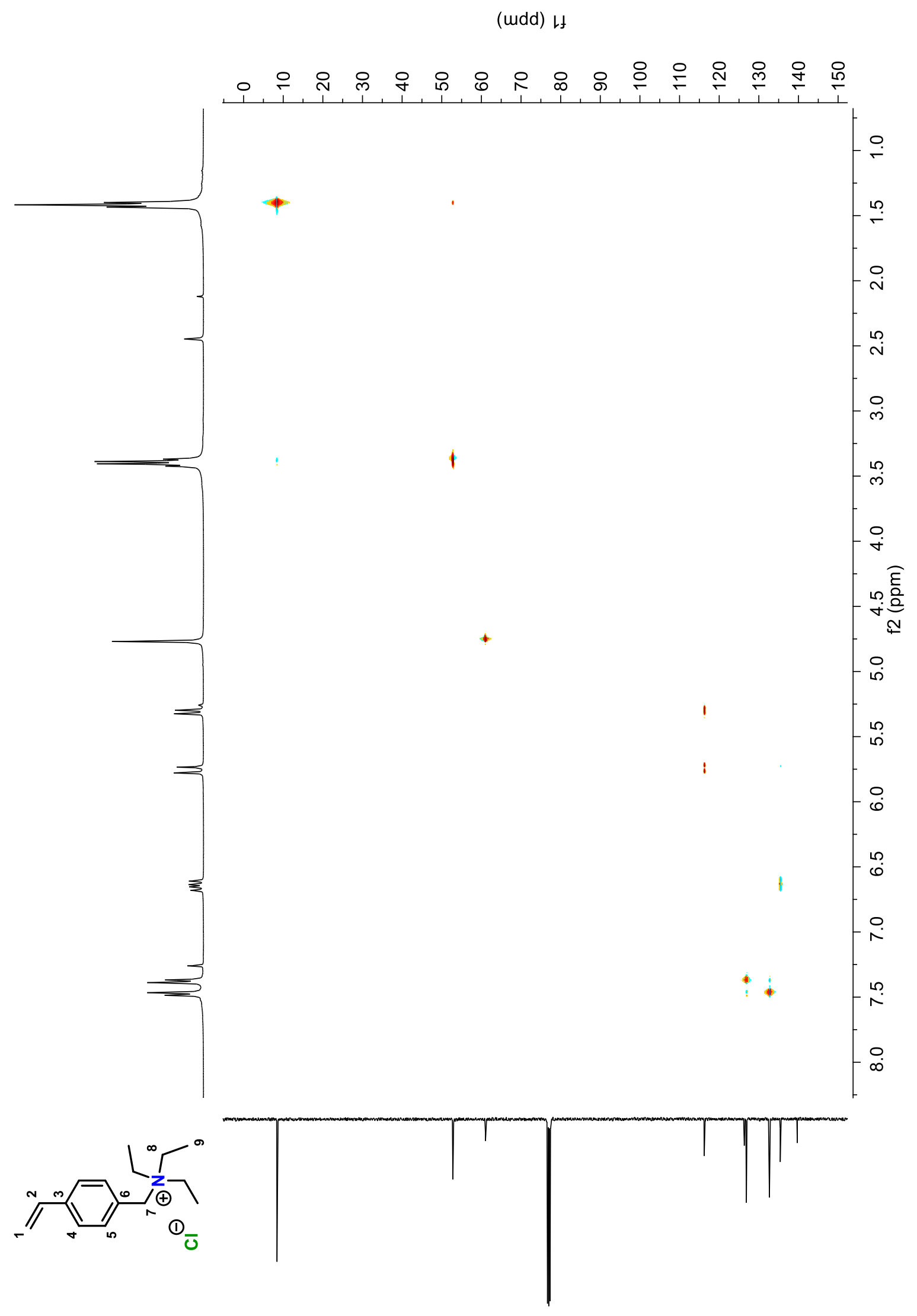

Figure A 28 2D HSQC NMR spectrum of monomer 17 in $\mathrm{CDCl}_{3}$ 


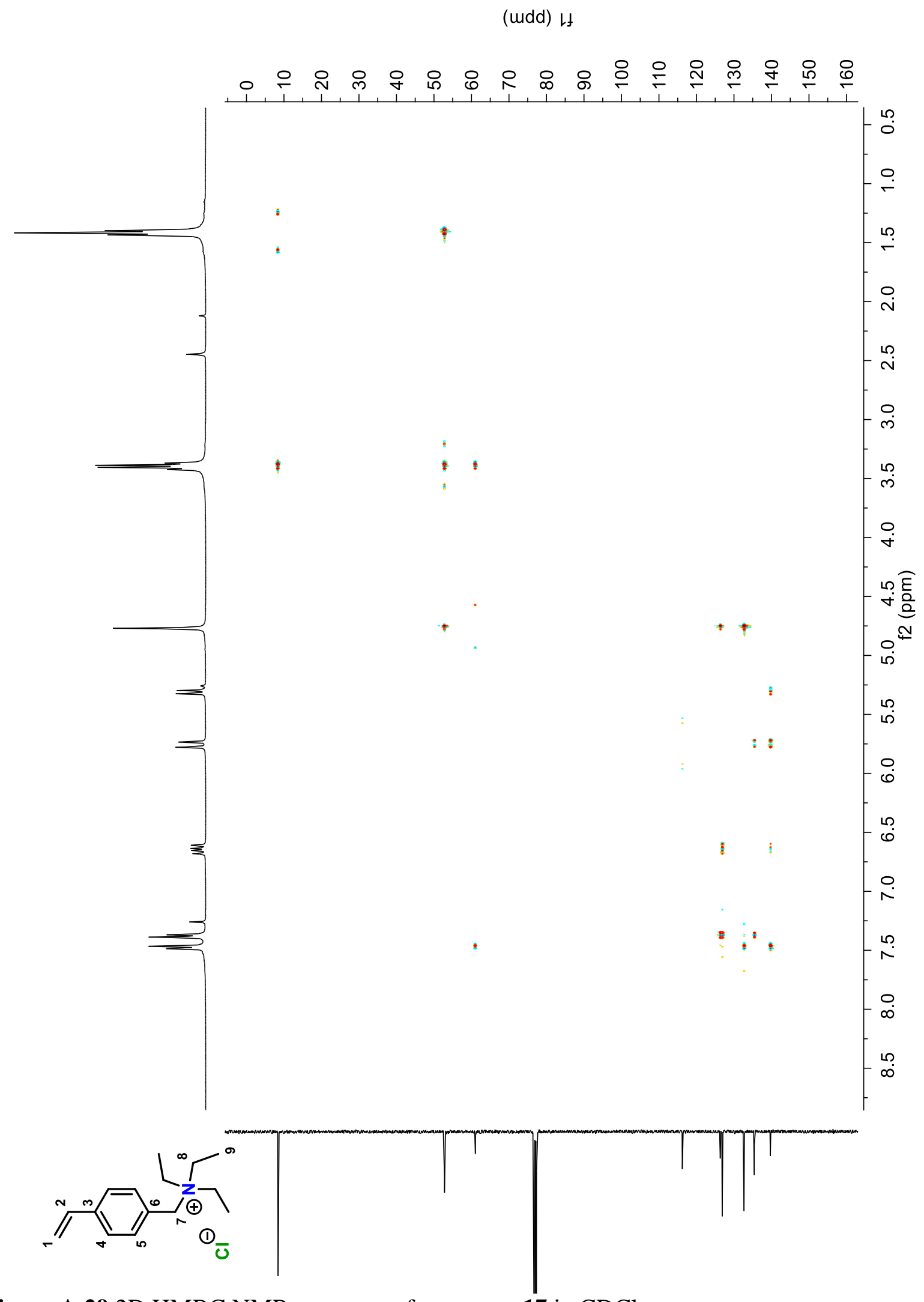

Figure A 29 2D HMBC NMR spectrum of monomer 17 in $\mathrm{CDCl}_{3}$ 


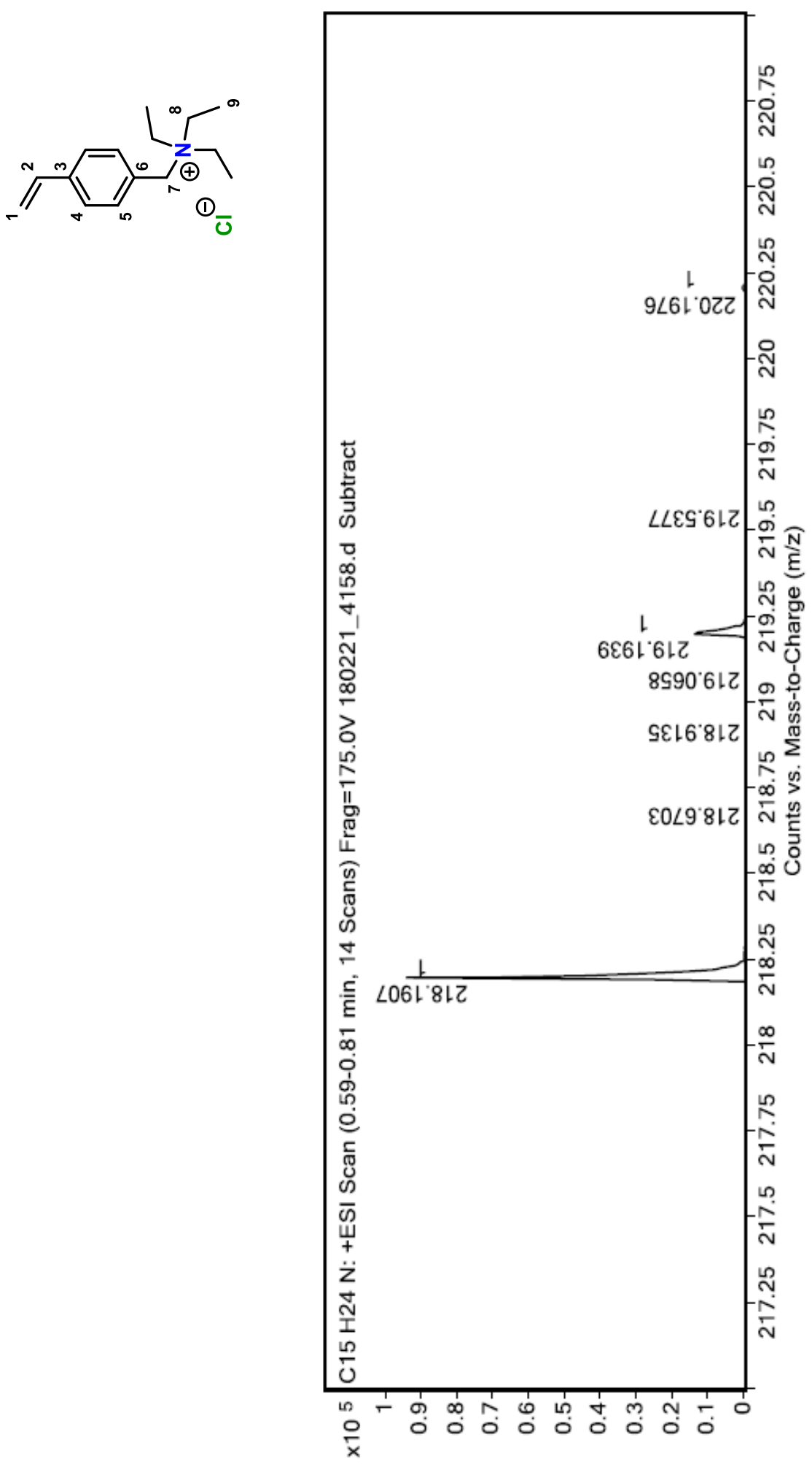

Figure A 30 HRMS ESI-Q-TOF spectrum of monomer 17 


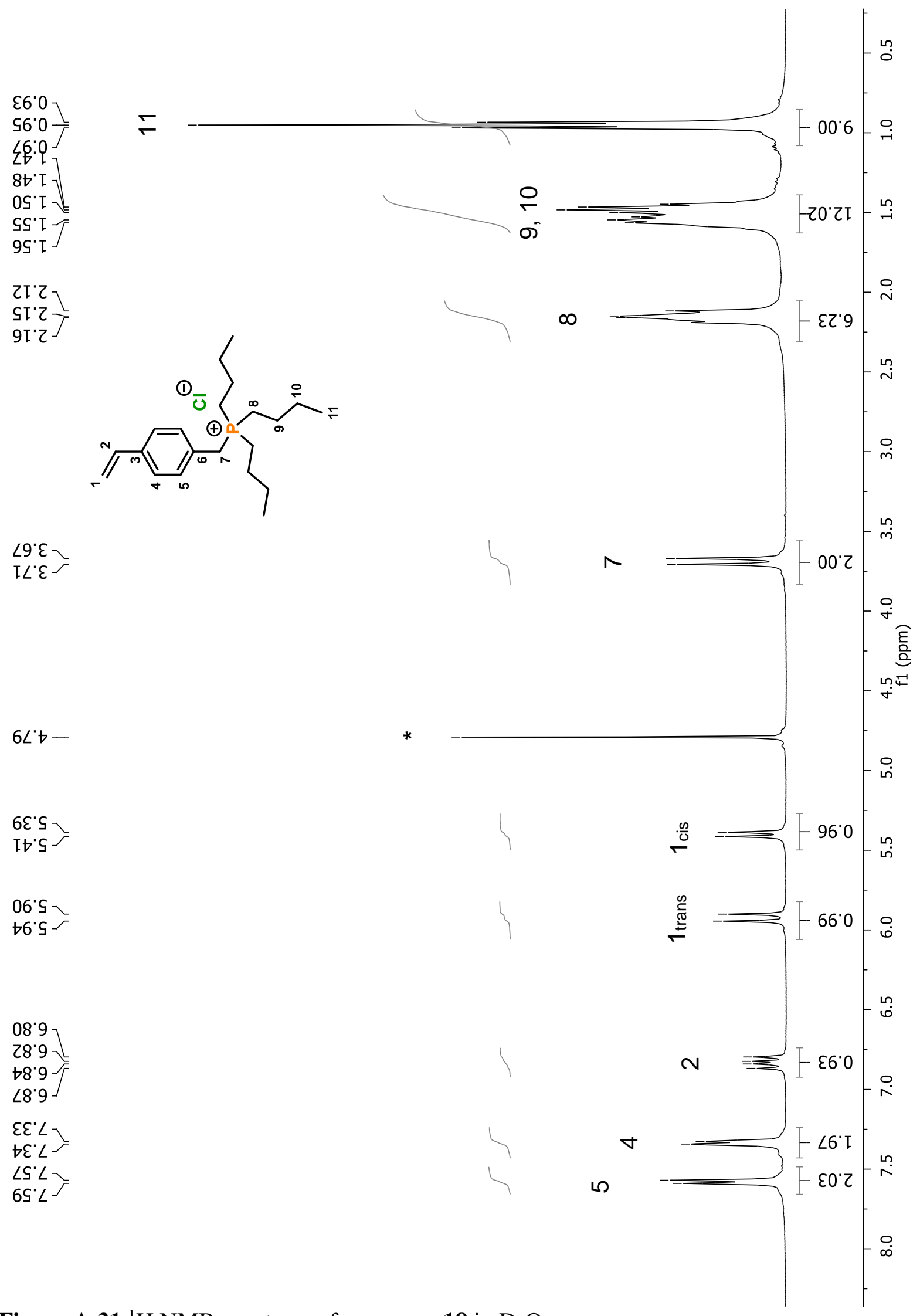

Figure A $31{ }^{1} \mathrm{H}$ NMR spectrum of monomer 18 in $\mathrm{D}_{2} \mathrm{O}$ 


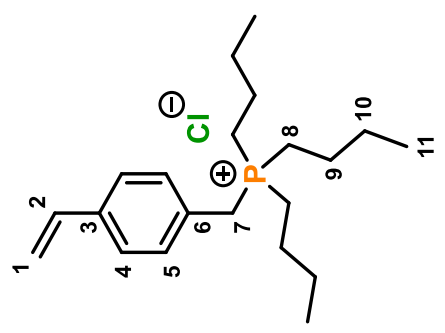

$8 \varsigma^{\prime} Z \varepsilon-$

Figure A $32{ }^{31} \mathrm{P}$ NMR spectrum of monomer 18 in $\mathrm{D}_{2} \mathrm{O}$ 


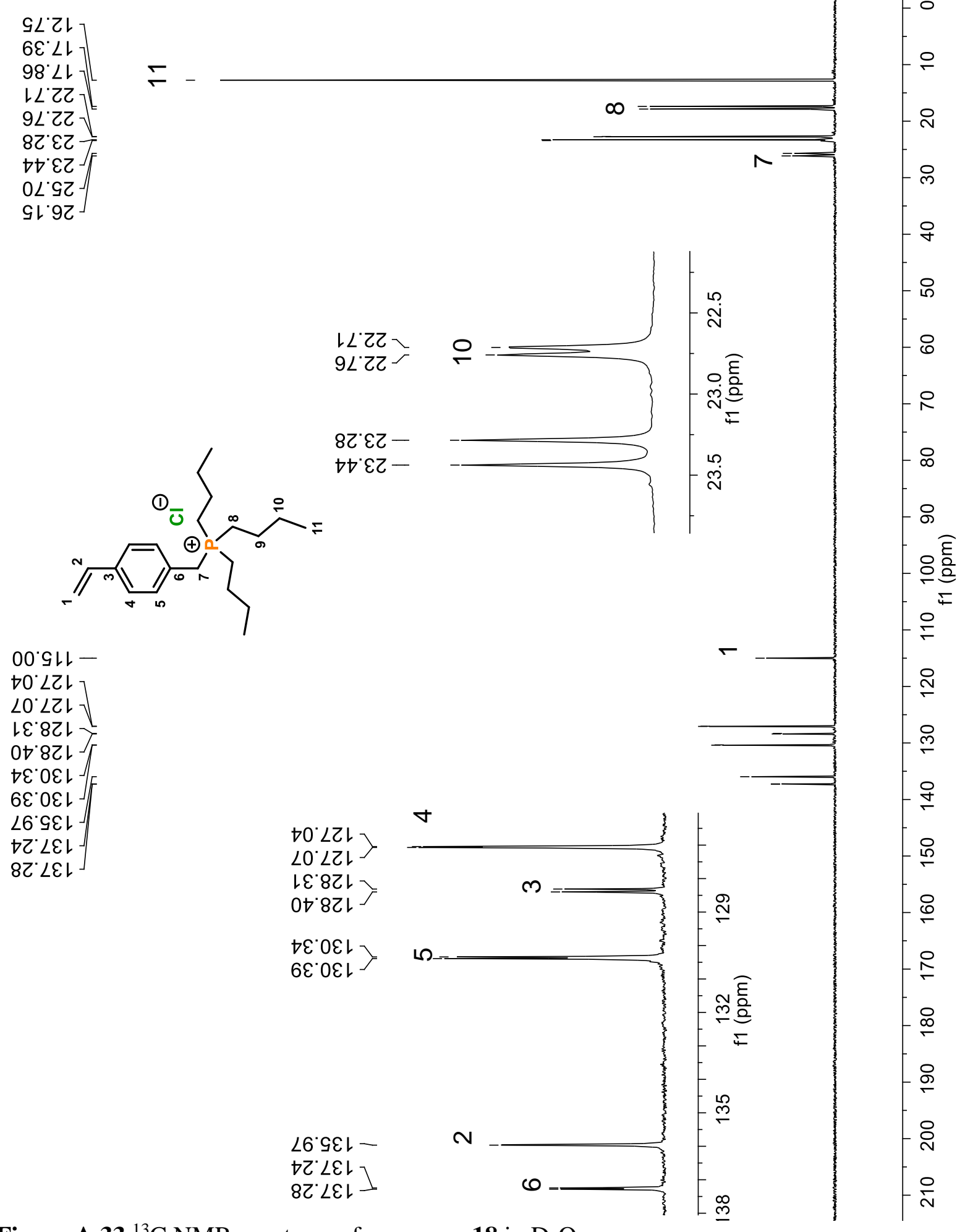

Figure A $33{ }^{13} \mathrm{C}$ NMR spectrum of monomer 18 in $\mathrm{D}_{2} \mathrm{O}$ 


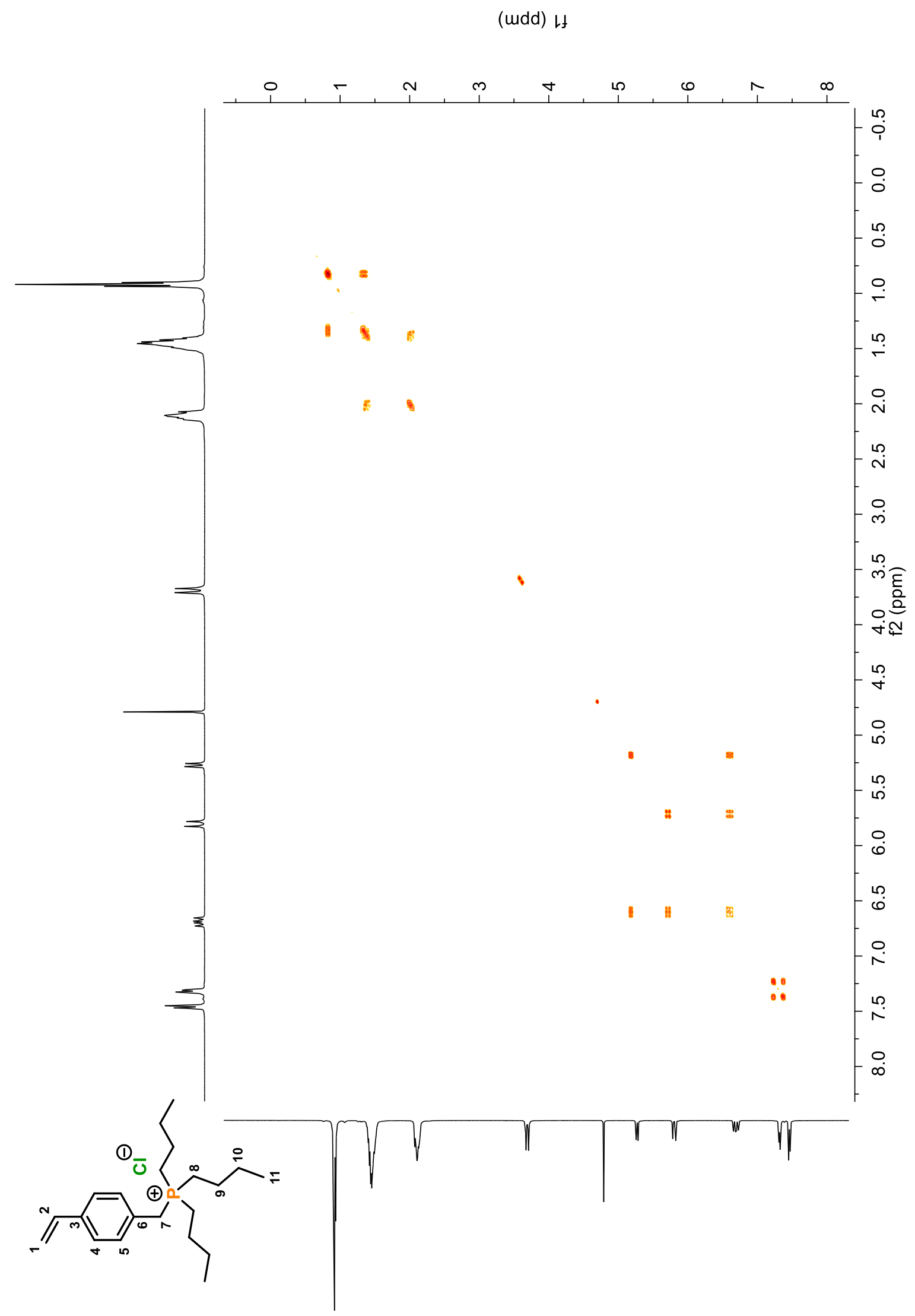

Figure A 34 2D COSY NMR spectrum of monomer 18 in $\mathrm{D}_{2} \mathrm{O}$ 


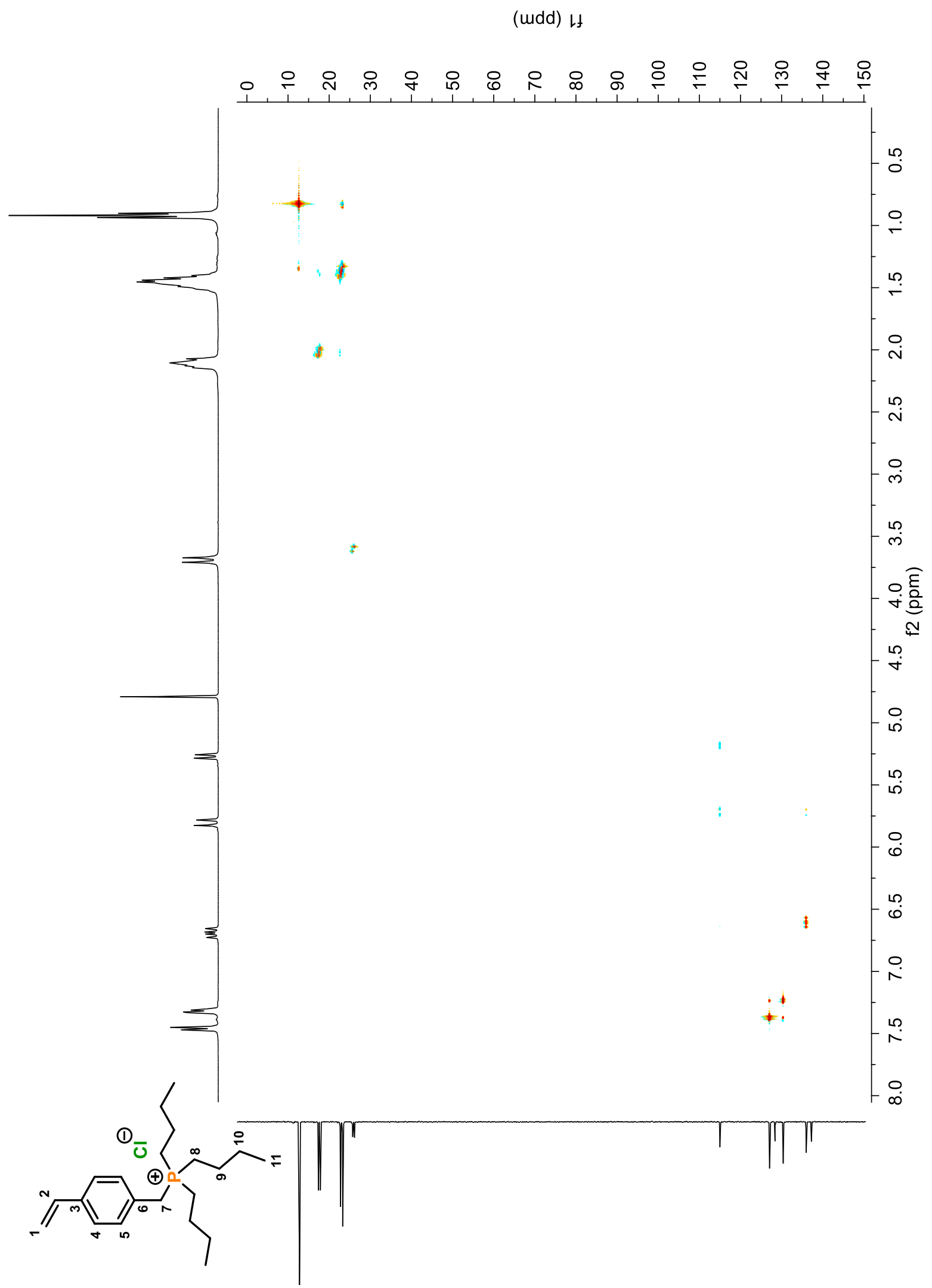

Figure A 35 2D HSQC NMR spectrum of monomer 18 in $\mathrm{D}_{2} \mathrm{O}$ 


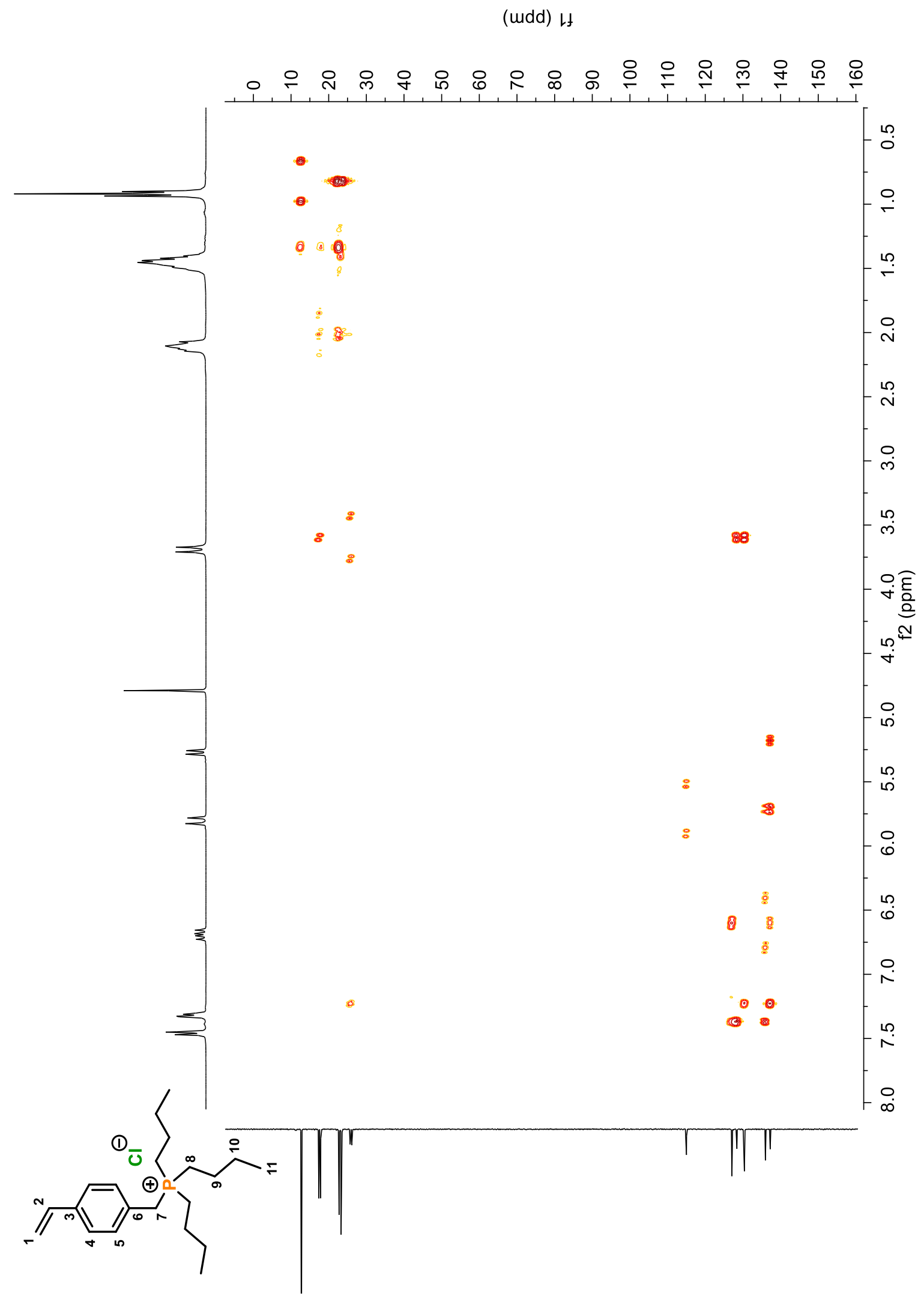

Figure A 36 2D HMBC NMR spectrum of monomer 18 in $\mathrm{D}_{2} \mathrm{O}$ 


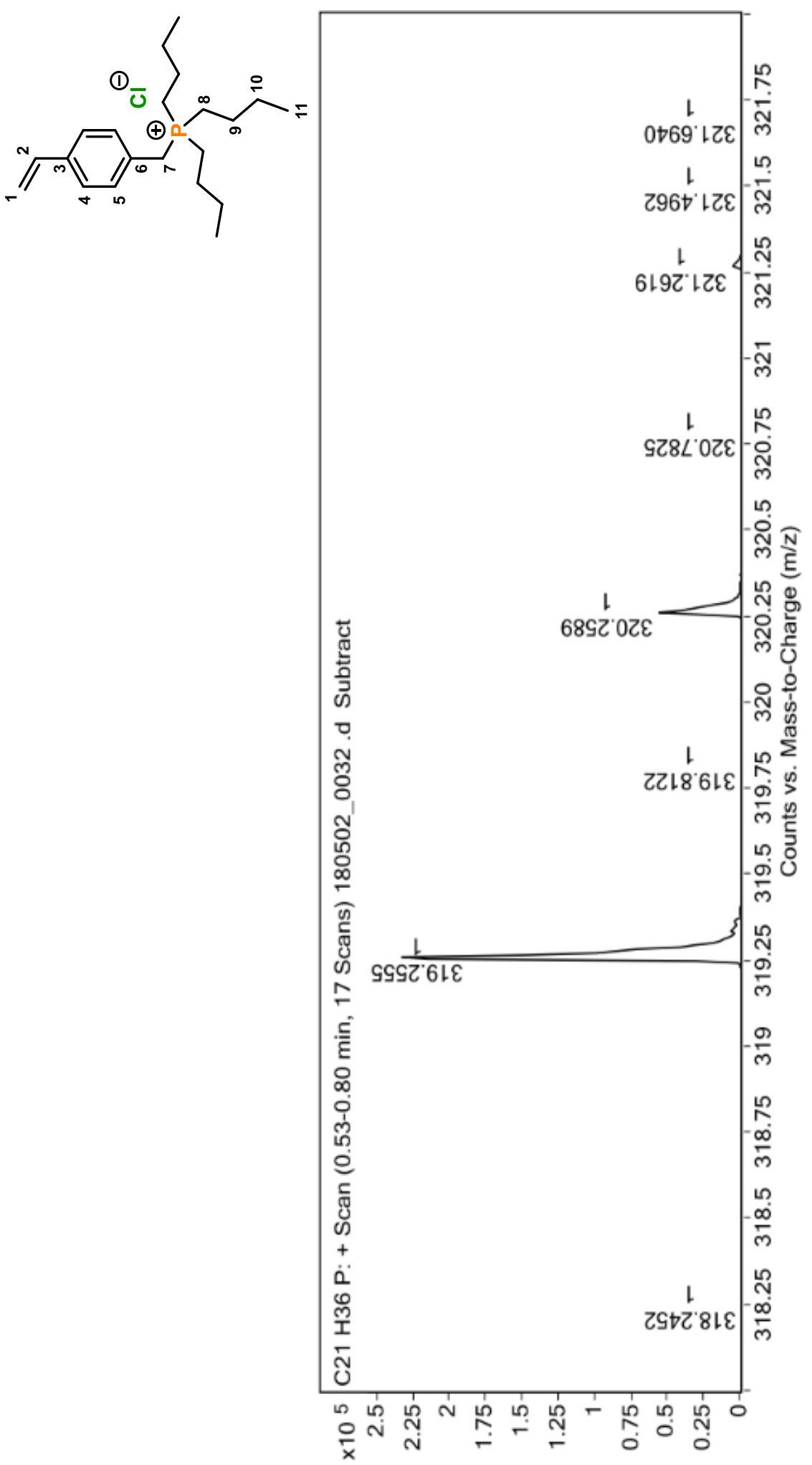

Figure A 37 HRMS ESI-Q-TOF spectrum of monomer 18 
$20^{\circ} 2$

112

si'z

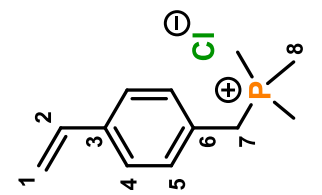

$\varepsilon Z^{\prime} \downarrow$

しでャノ

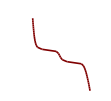

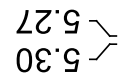

$\varepsilon L^{\circ} \mathrm{G}$

$\angle L S^{\prime}$

$79^{\circ} 9$

$\angle 9 \cdot 9$

$89.9-1$

$\left.9 Z^{\circ} L\right]$

$\left.\subseteq \varepsilon^{\circ} L\right]$

$9 \varepsilon<\frac{}{T}$

$\angle E^{\circ} \angle$

$8 \varepsilon^{\circ} L$

$0 D^{\circ} L$

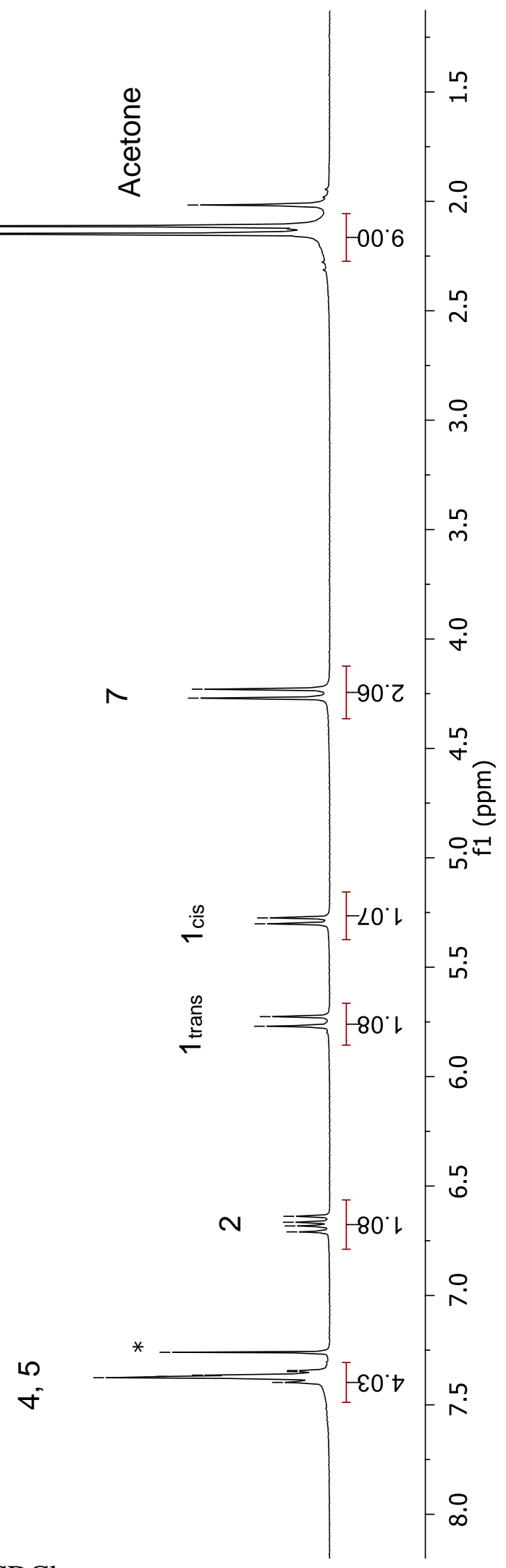

Figure A $38{ }^{1} \mathrm{H}$ NMR spectrum of monomer 19 in $\mathrm{CDCl}_{3}$ 

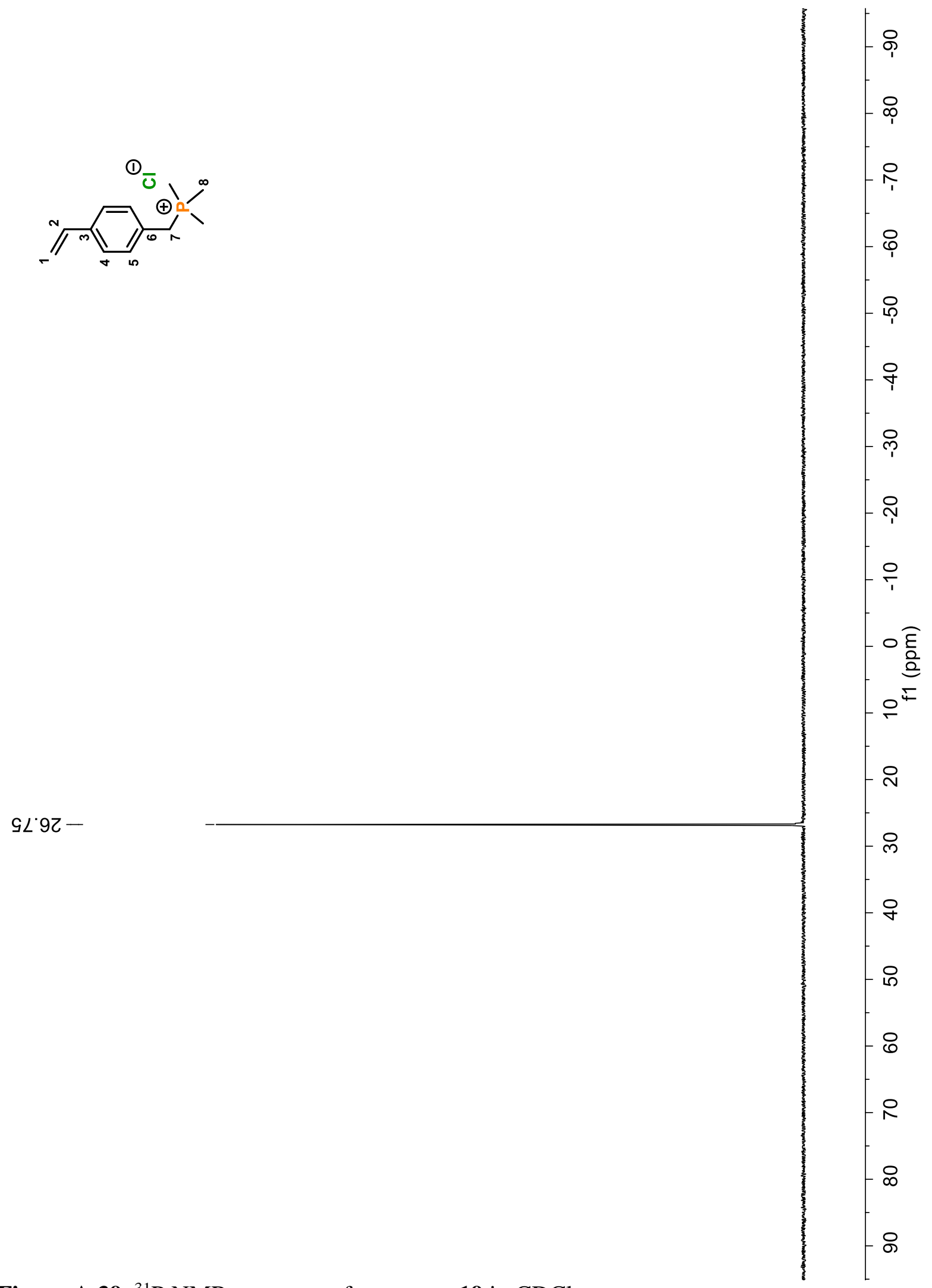

Figure A $39{ }^{31} \mathrm{P}$ NMR spectrum of monomer 19 in $\mathrm{CDCl}_{3}$ 


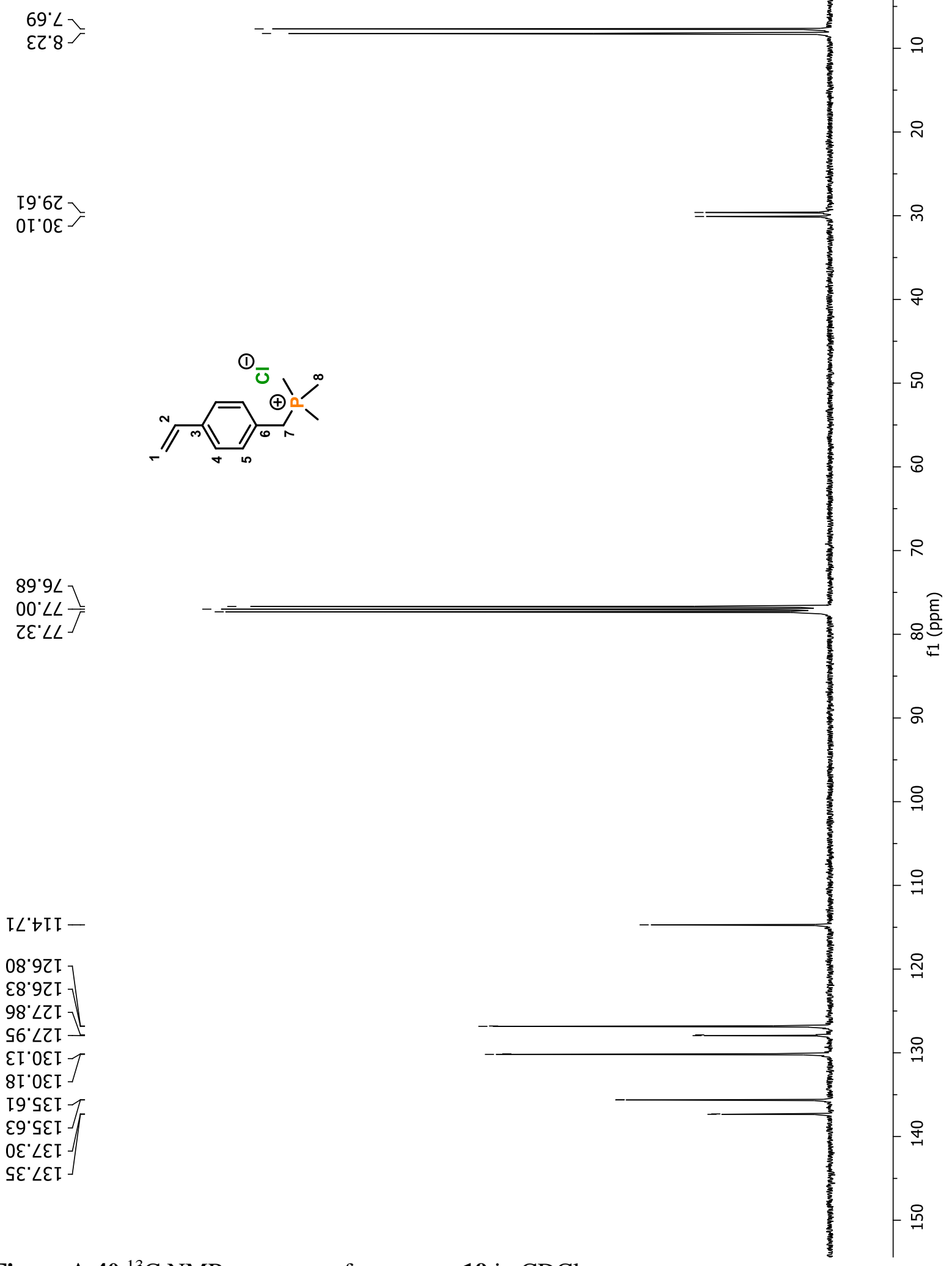

Figure A $40{ }^{13} \mathrm{C}$ NMR spectrum of monomer 19 in $\mathrm{CDCl}_{3}$ 


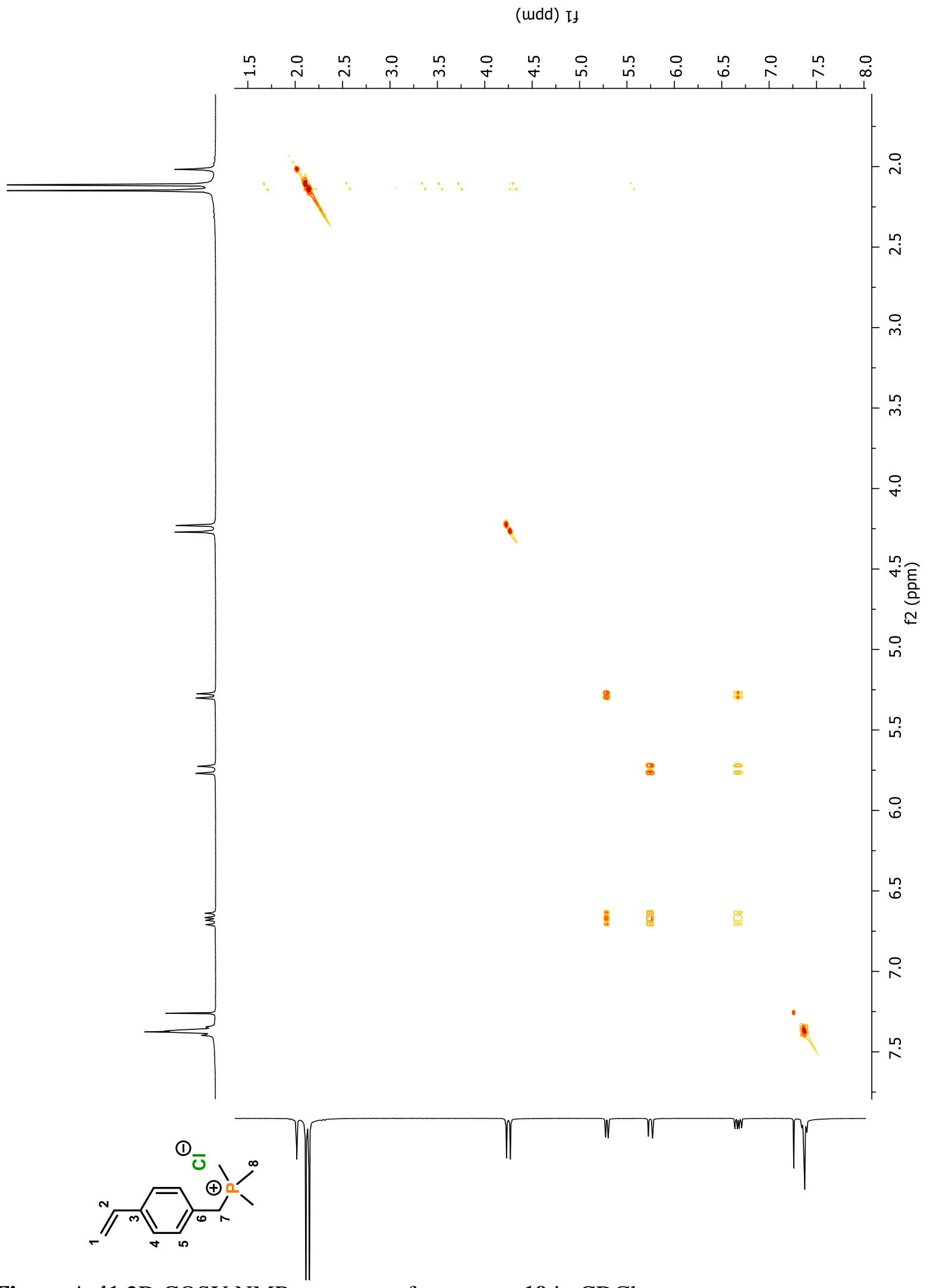

Figure A 41 2D COSY NMR spectrum of monomer 19 in $\mathrm{CDCl}_{3}$ 


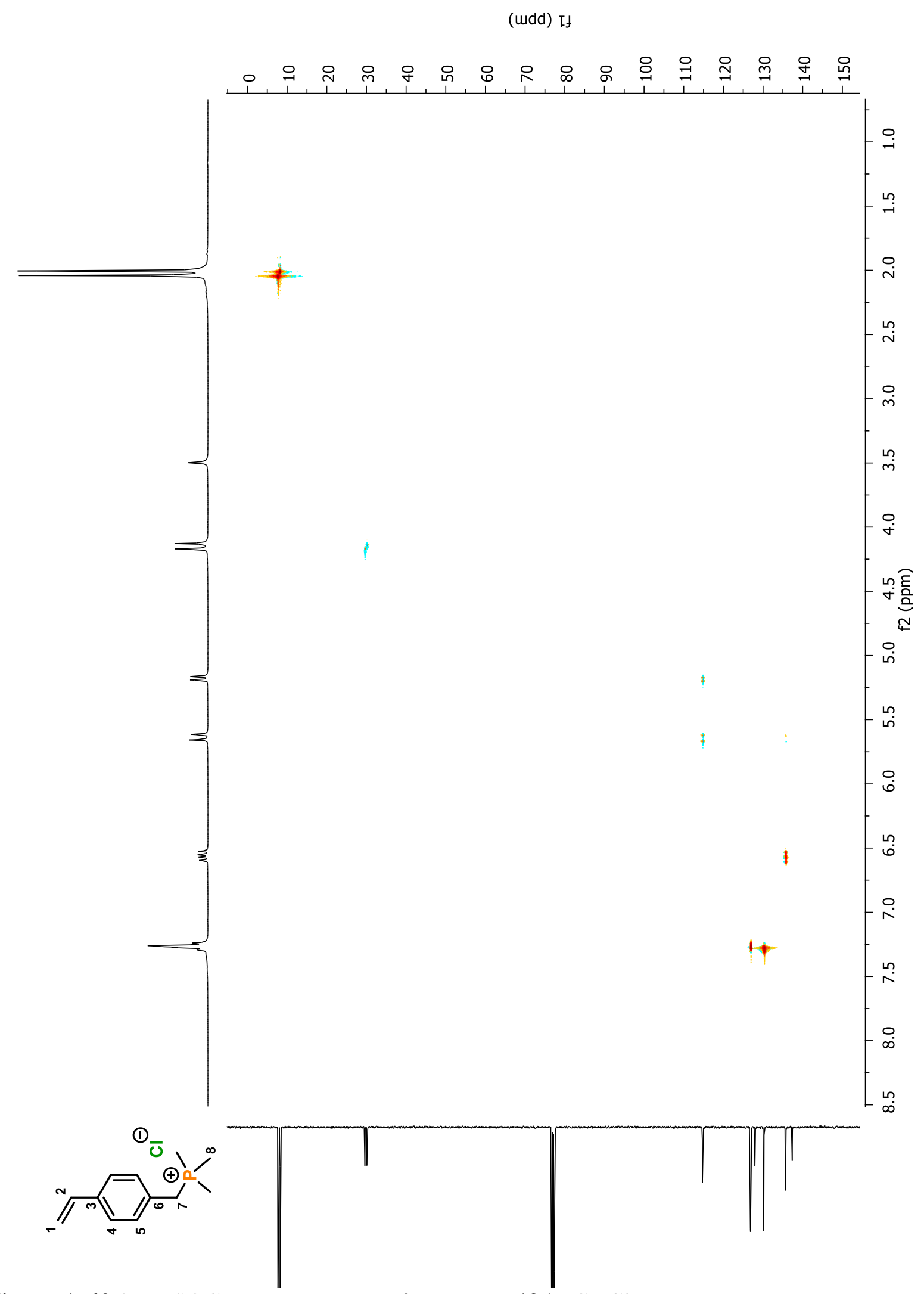

Figure A 42 2D HSQC NMR spectrum of monomer 19 in $\mathrm{CDCl}_{3}$ 
(mdd) H

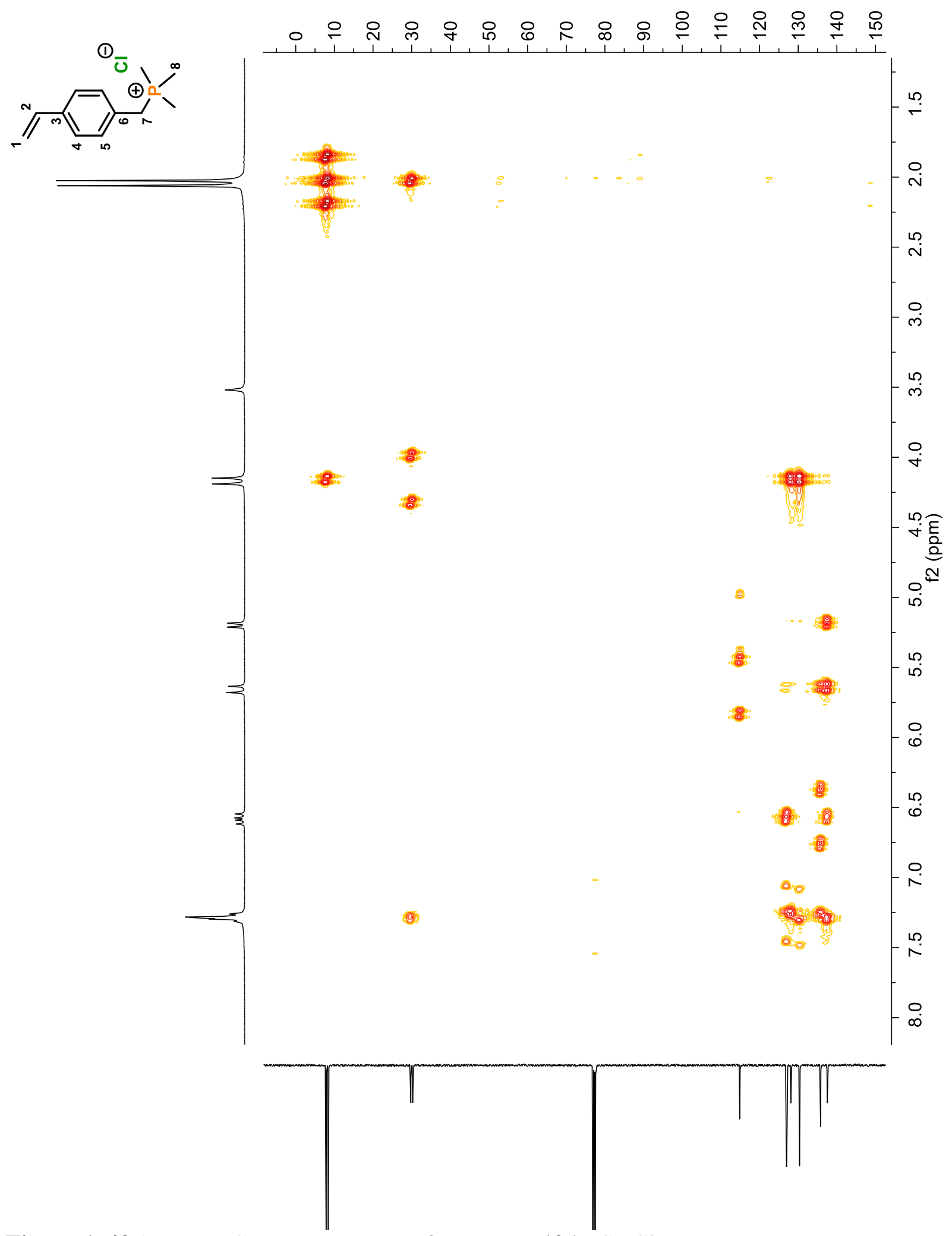

Figure A 43 2D HMBC NMR spectrum of monomer 19 in $\mathrm{CDCl}_{3}$ 

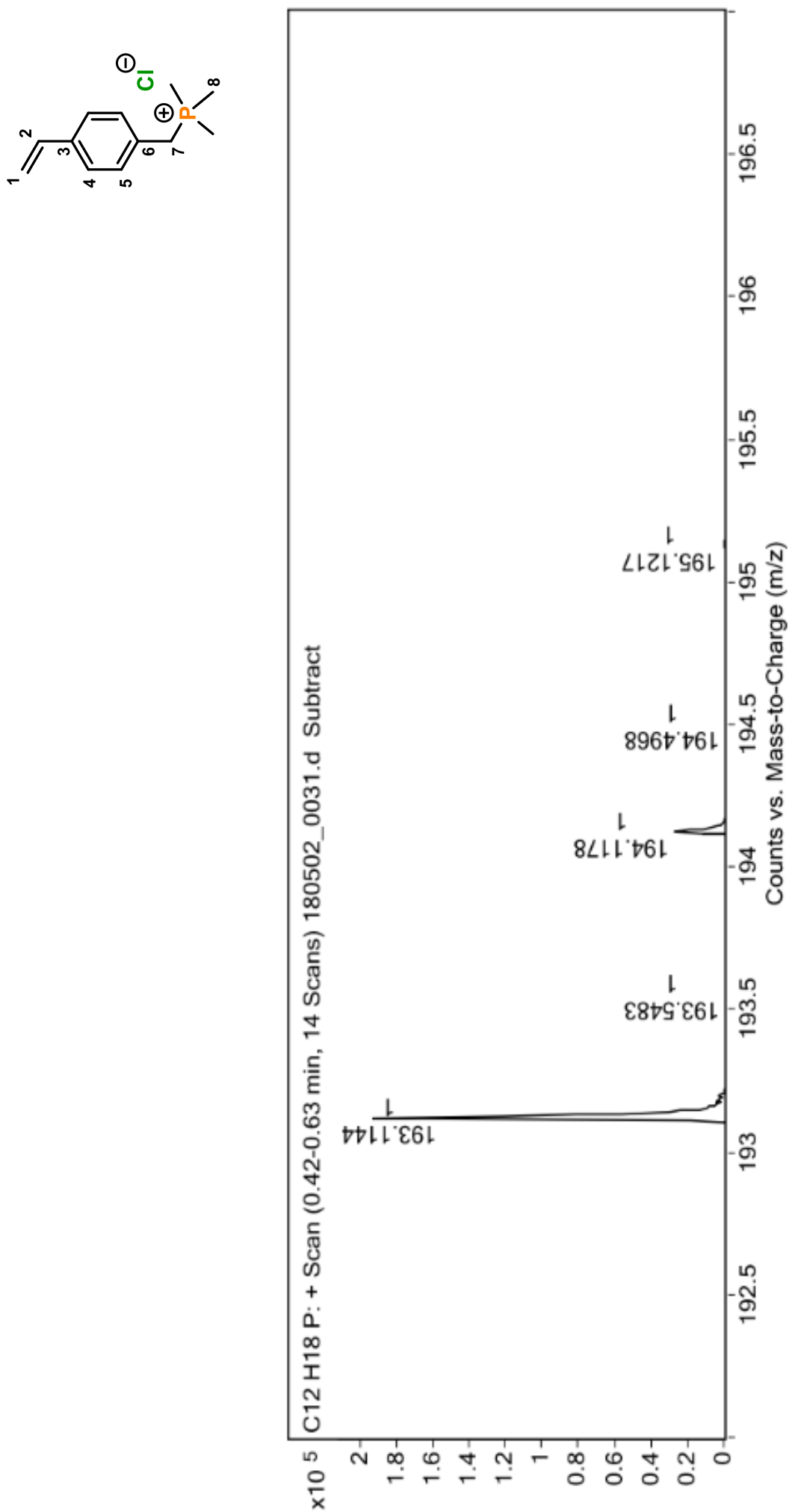

Figure A 44 HRMS ESI-Q-TOF spectrum of monomer 19 


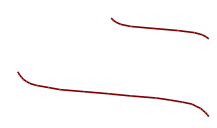

ZL'G $9 L^{\circ} \mathrm{S}^{\top}$
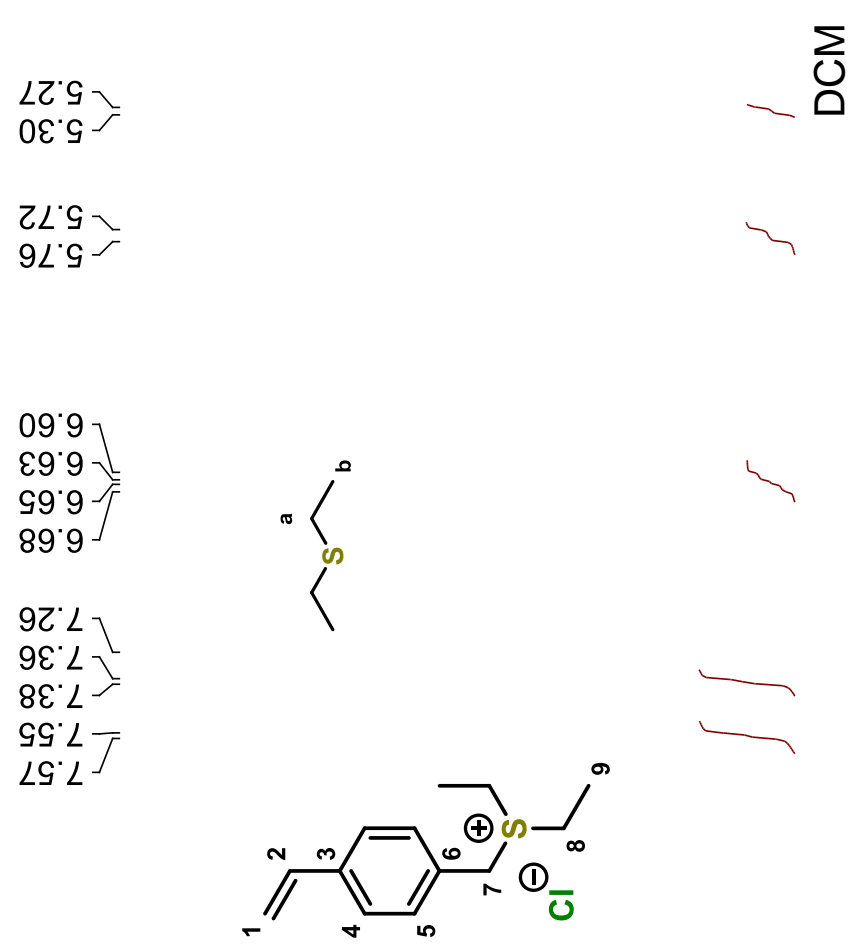

ช

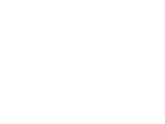




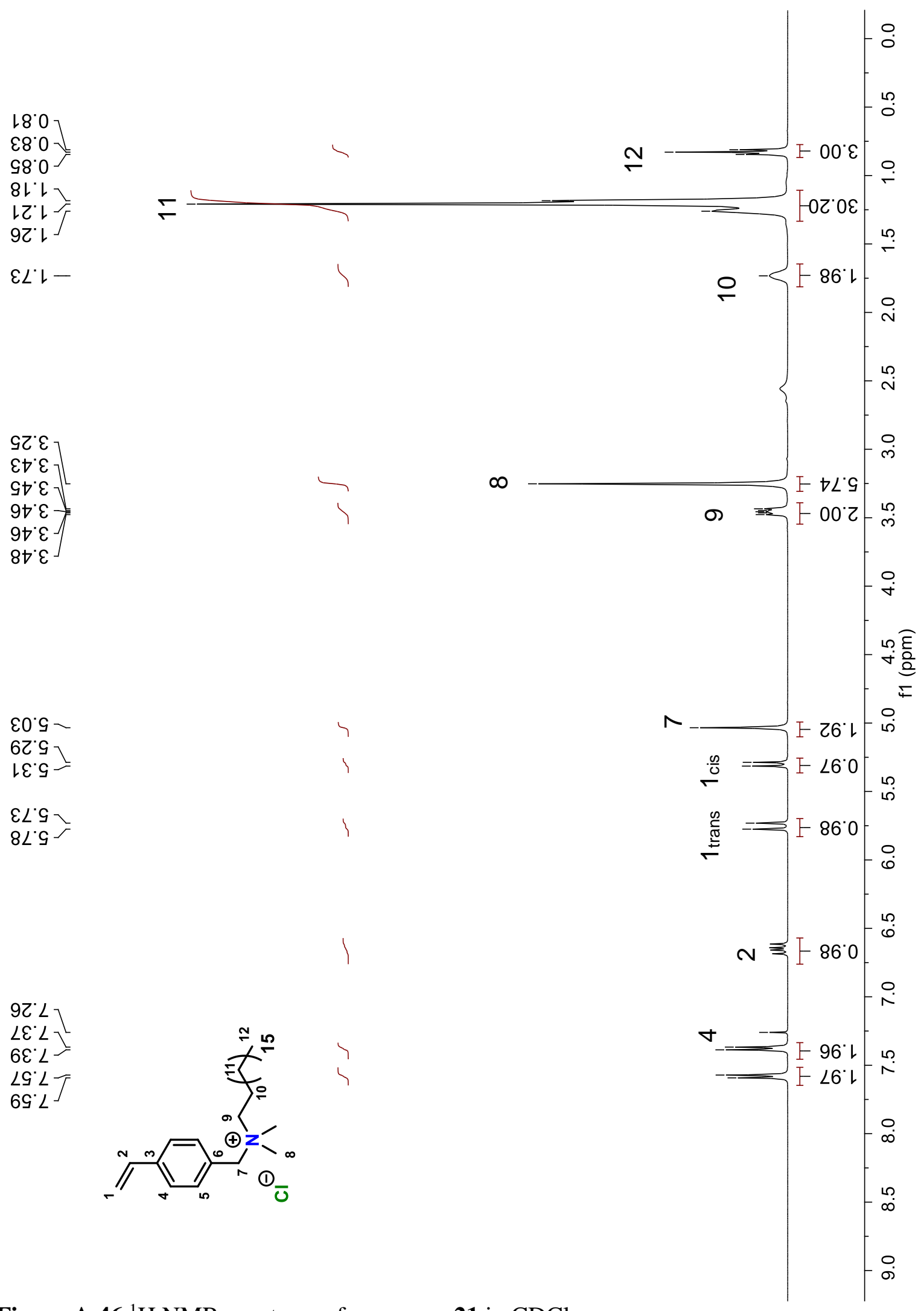

Figure A $46{ }^{1} \mathrm{H}$ NMR spectrum of monomer 21 in $\mathrm{CDCl}_{3}$ 


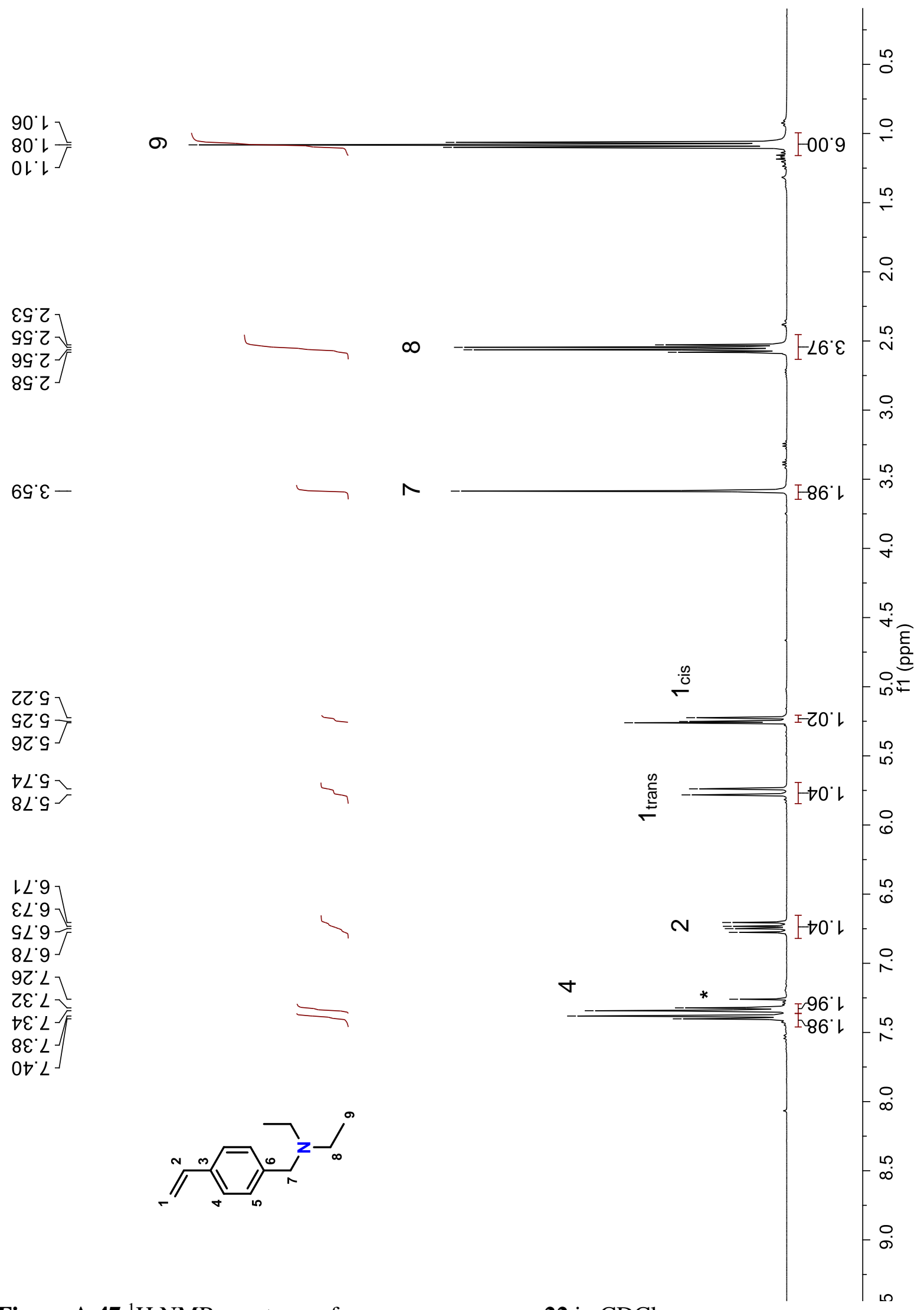

Figure A $47{ }^{1} \mathrm{H}$ NMR spectrum of monomer precursor 22 in $\mathrm{CDCl}_{3}$ 


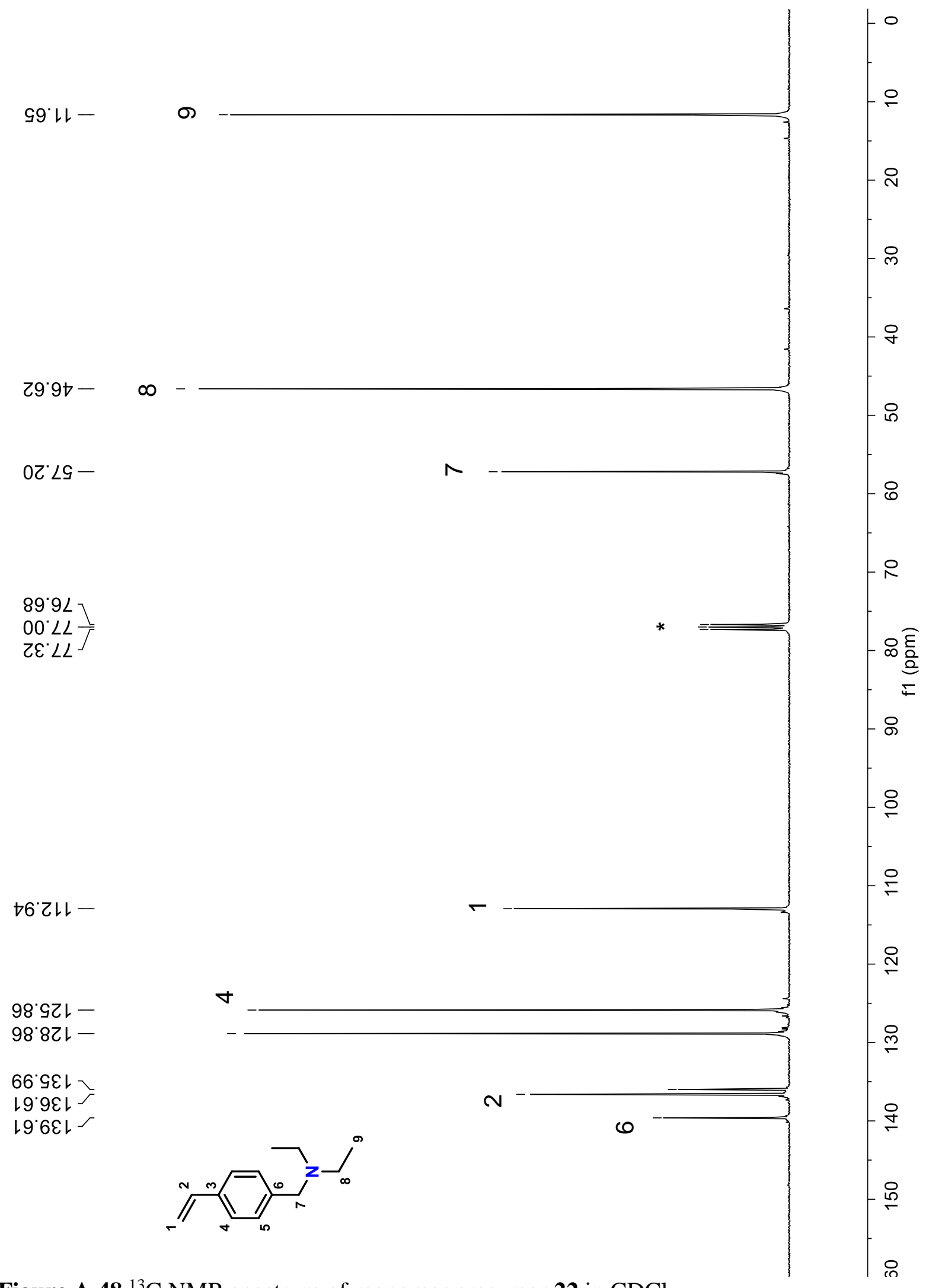

Figure A $48{ }^{13} \mathrm{C}$ NMR spectrum of monomer precursor 22 in $\mathrm{CDCl}_{3}$ 


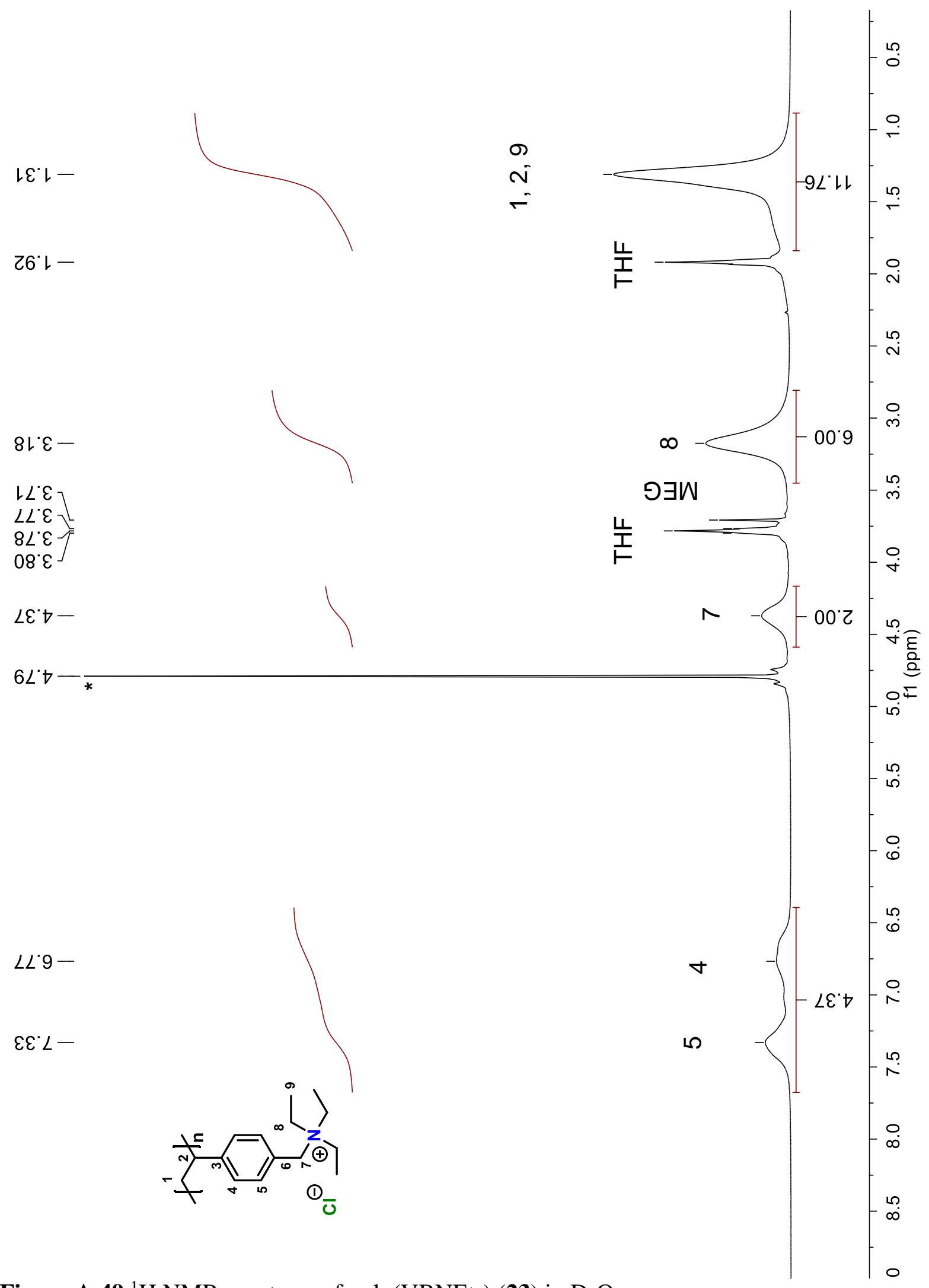

Figure A $49{ }^{1} \mathrm{H}$ NMR spectrum of poly(VBNEt 3$)(23)$ in $\mathrm{D}_{2} \mathrm{O}$ 


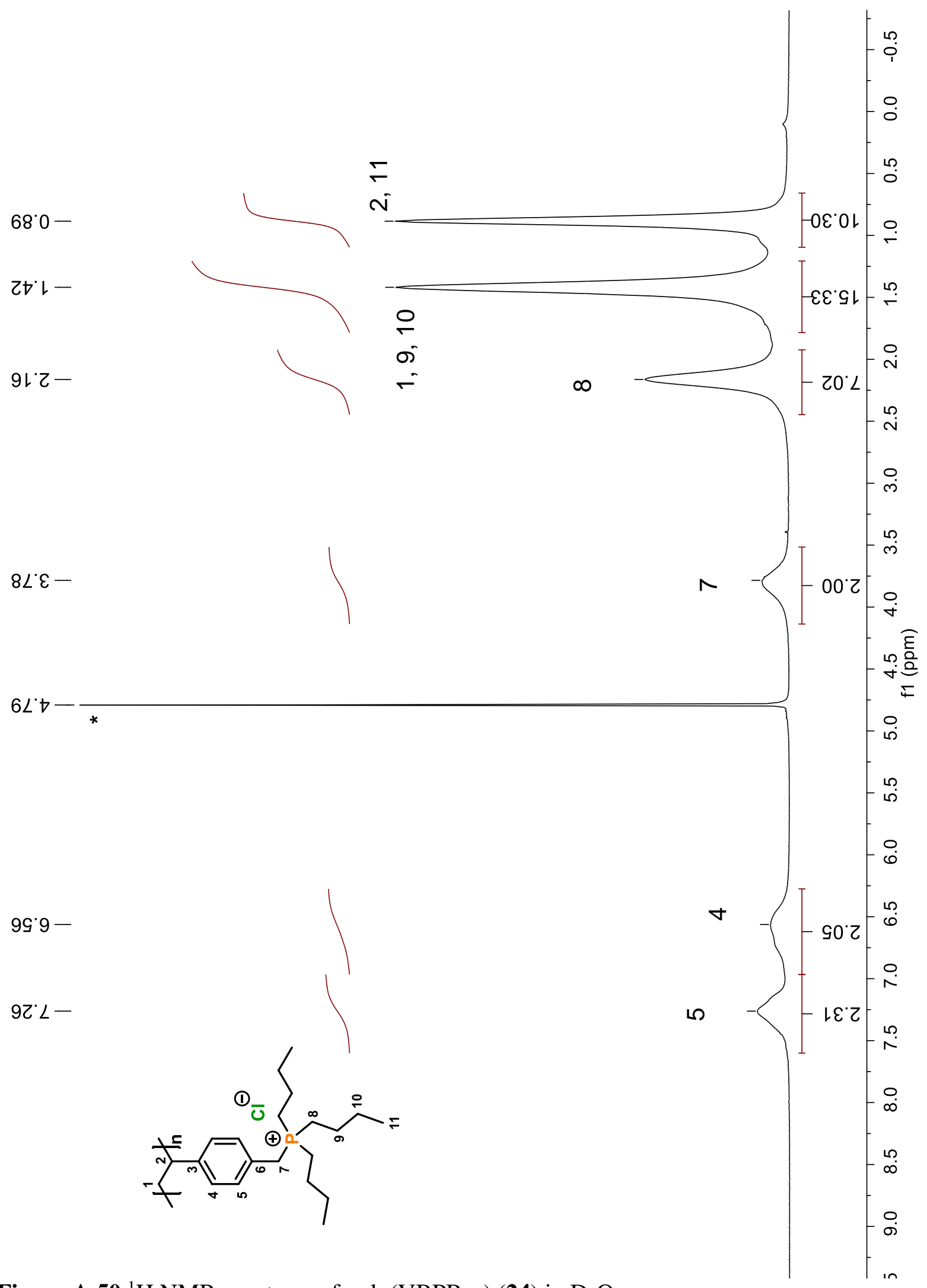

Figure A $50{ }^{1} \mathrm{H}$ NMR spectrum of poly $\left(V_{B P B u}\right)(24)$ in $\mathrm{D}_{2} \mathrm{O}$ 

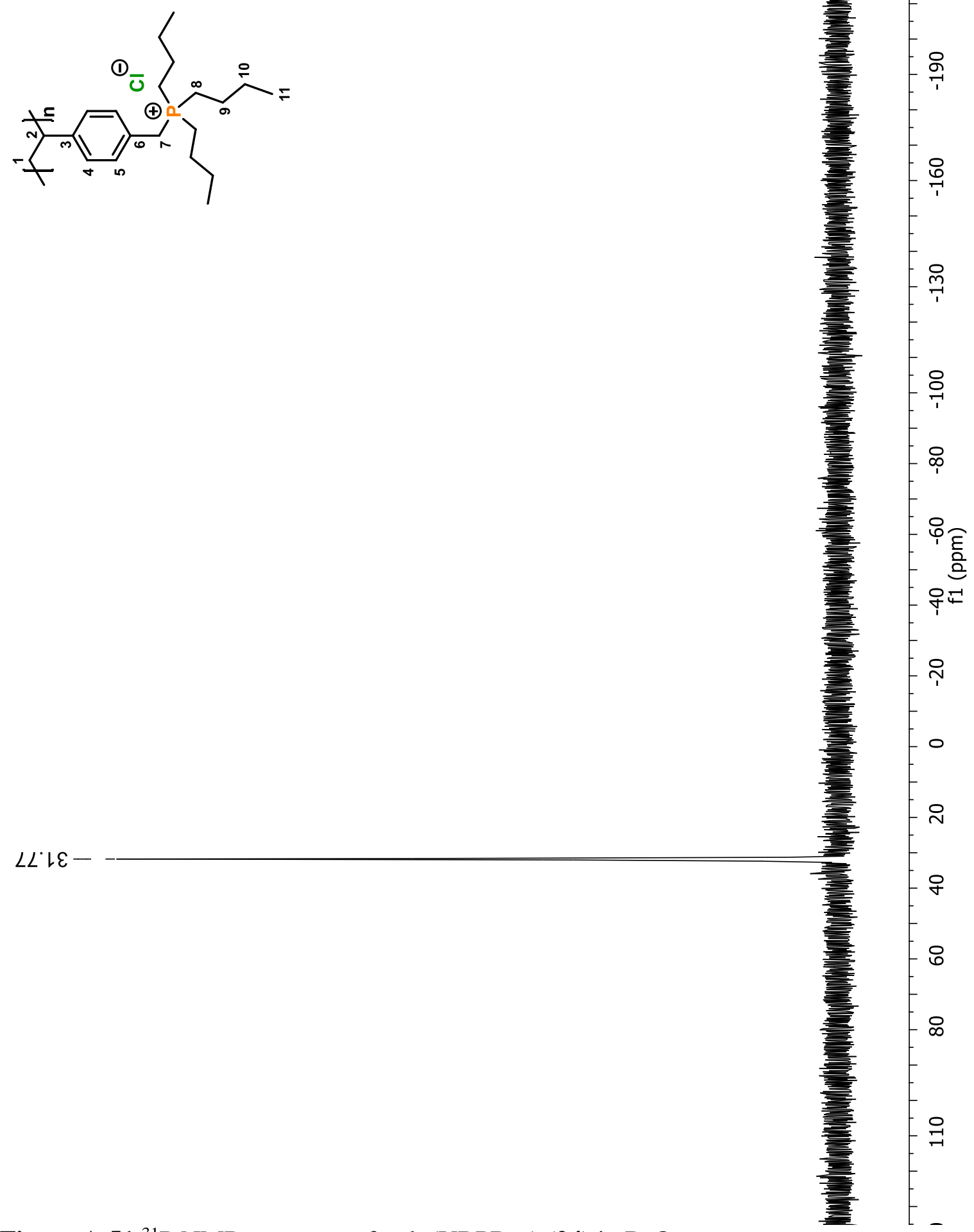

Figure A $51{ }^{31} \mathrm{P}$ NMR spectrum of poly $\left(\mathrm{VBPBu}_{3}\right)(\mathbf{2 4})$ in $\mathrm{D}_{2} \mathrm{O}$ 


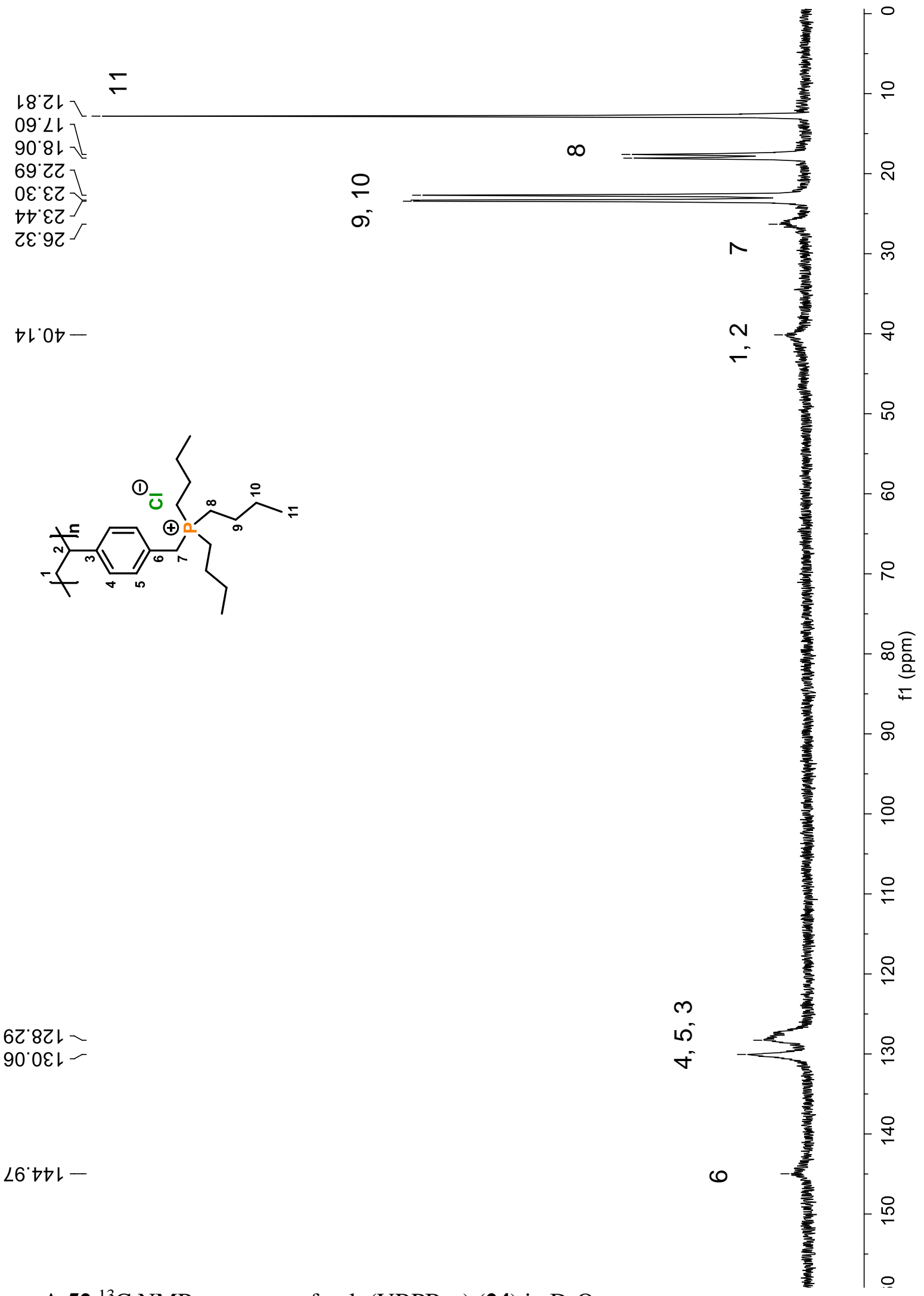

Figure A $52{ }^{13} \mathrm{C}$ NMR spectrum of poly $\left(\mathrm{VBPBu}_{3}\right)(\mathbf{2 4})$ in $\mathrm{D}_{2} \mathrm{O}$ 


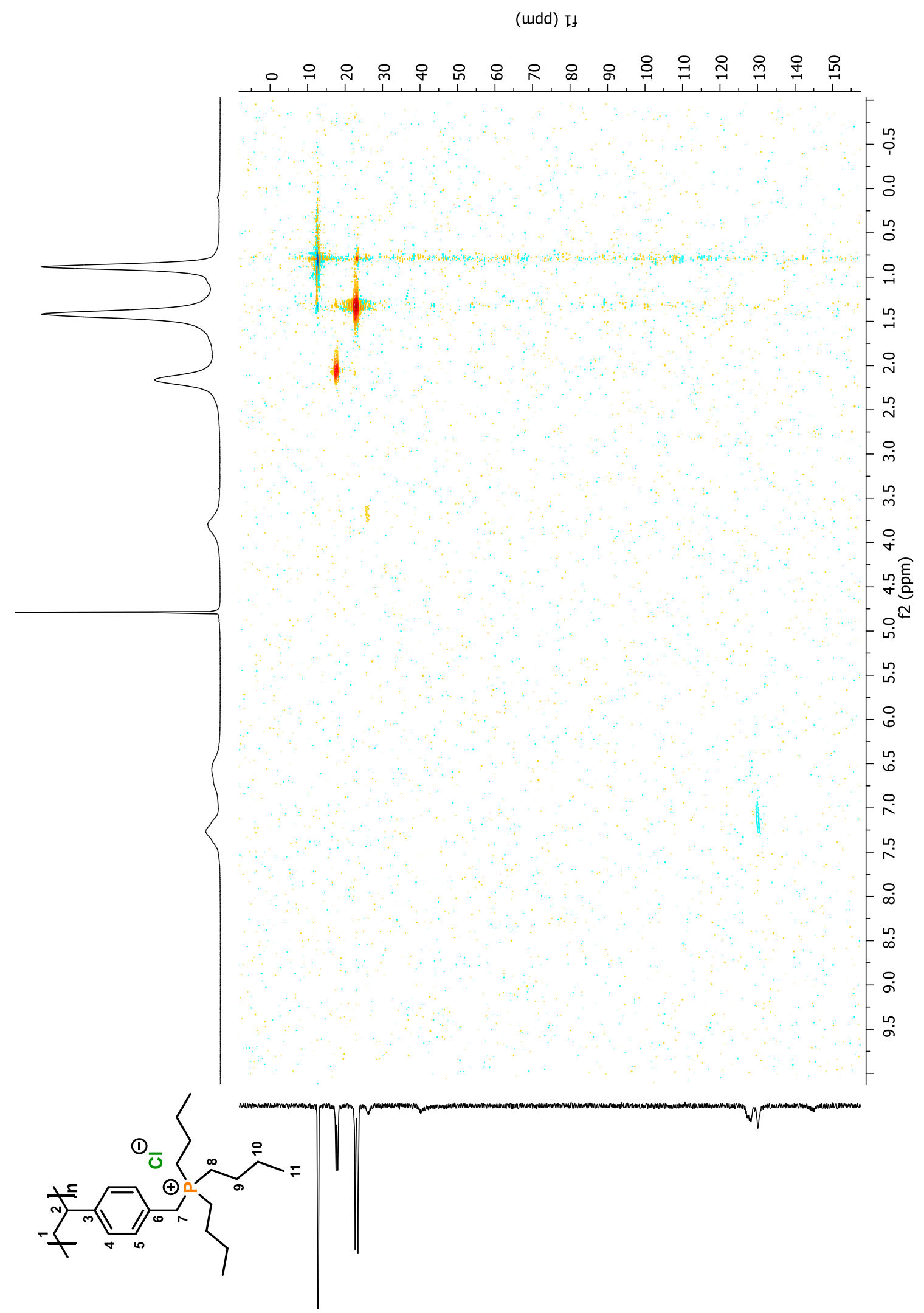

Figure A 53 2D HSQC NMR spectrum of poly $\left(\mathrm{VBPBu}_{3}\right)(\mathbf{2 4})$ in $\mathrm{D}_{2} \mathrm{O}$ 


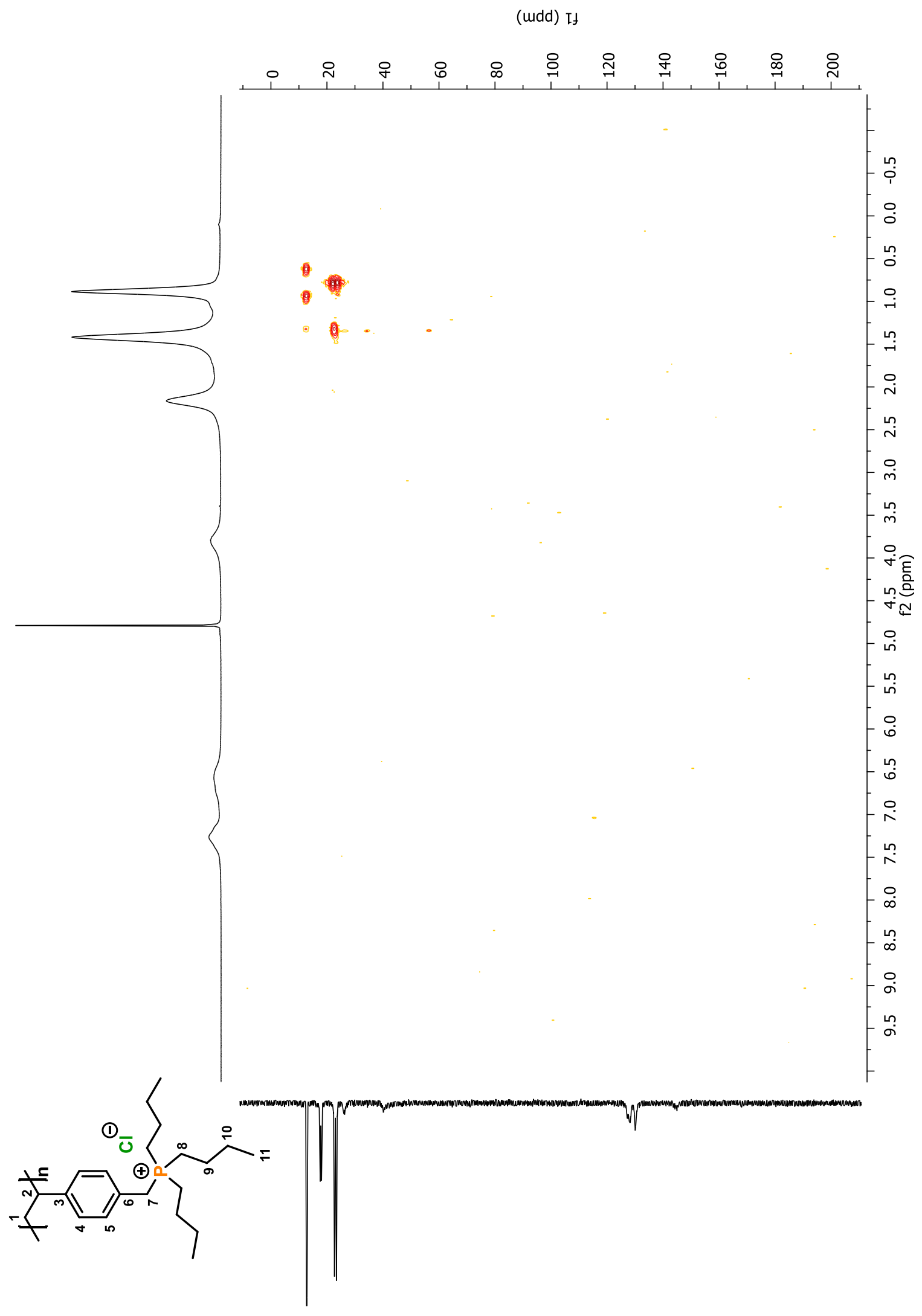

Figure A 54 2D HMBC NMR spectrum of poly(VBPBu 3$)(24)$ in $\mathrm{D}_{2} \mathrm{O}$ 


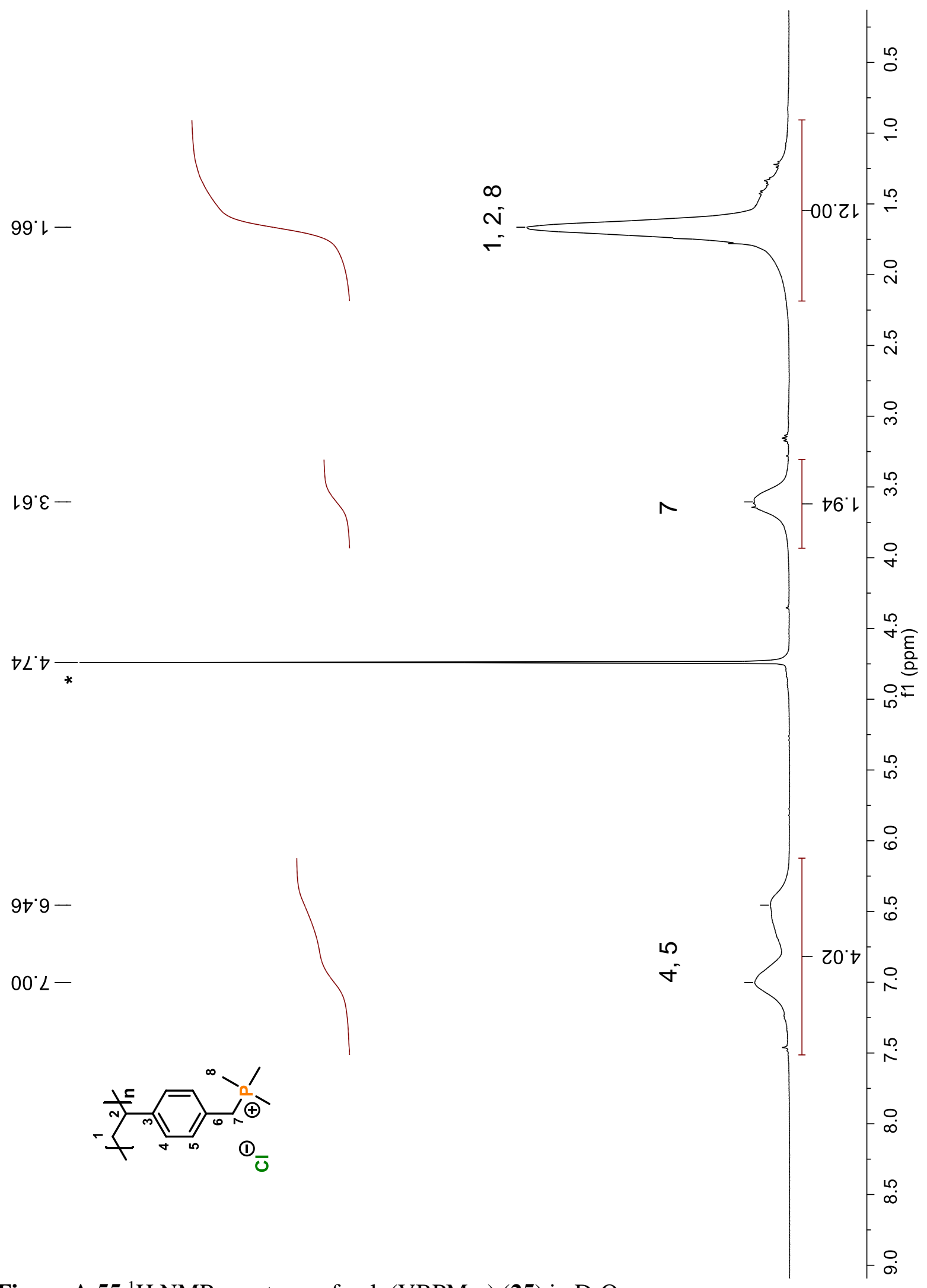

Figure A $55{ }^{1} \mathrm{H}$ NMR spectrum of poly(VBPMe $)(25)$ in $\mathrm{D}_{2} \mathrm{O}$ 

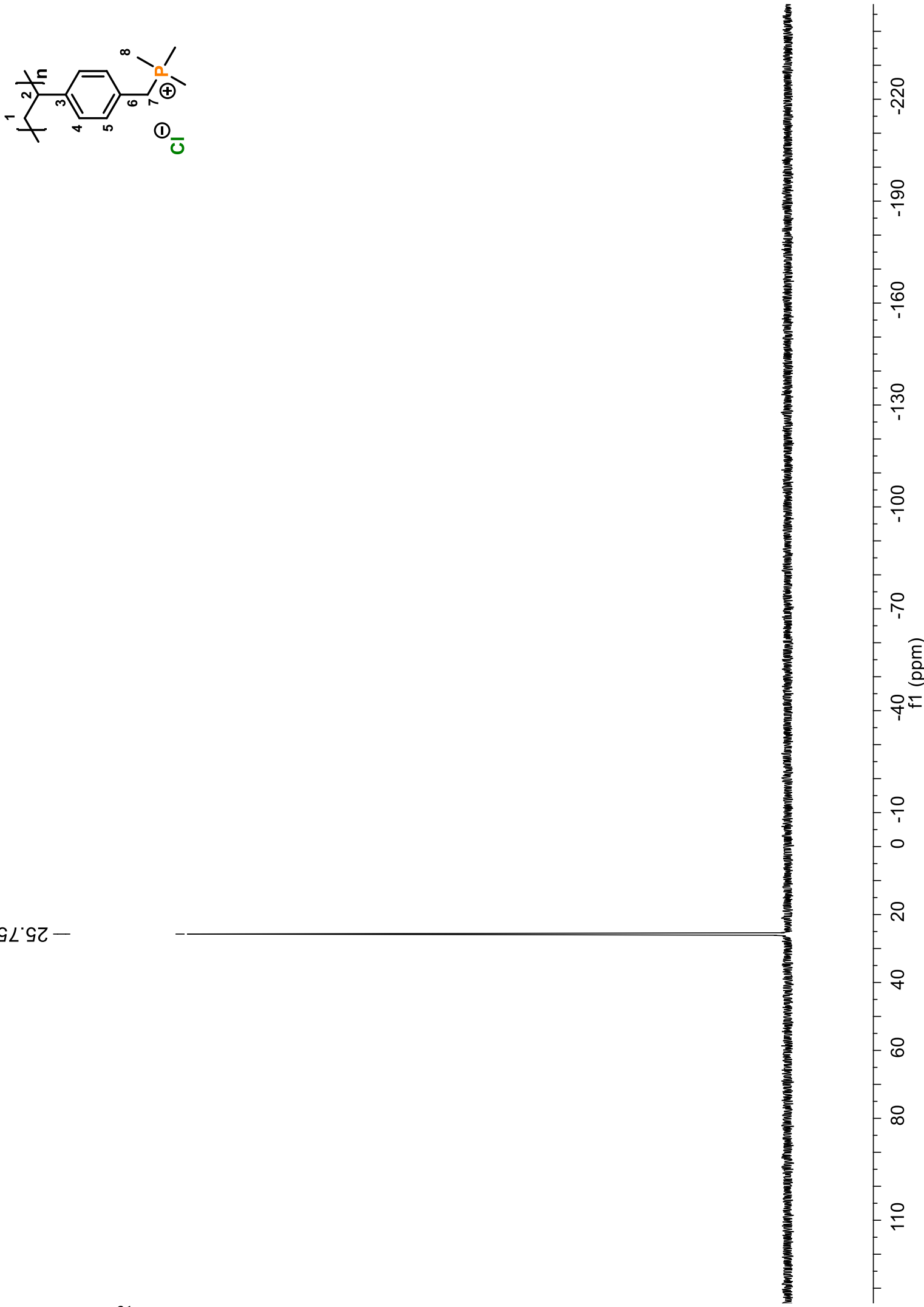

Figure A $56{ }^{31} \mathrm{P}$ NMR spectrum of poly $\left(\mathrm{VBPMe}_{3}\right)(\mathbf{2 5})$ in $\mathrm{D}_{2} \mathrm{O}$ 


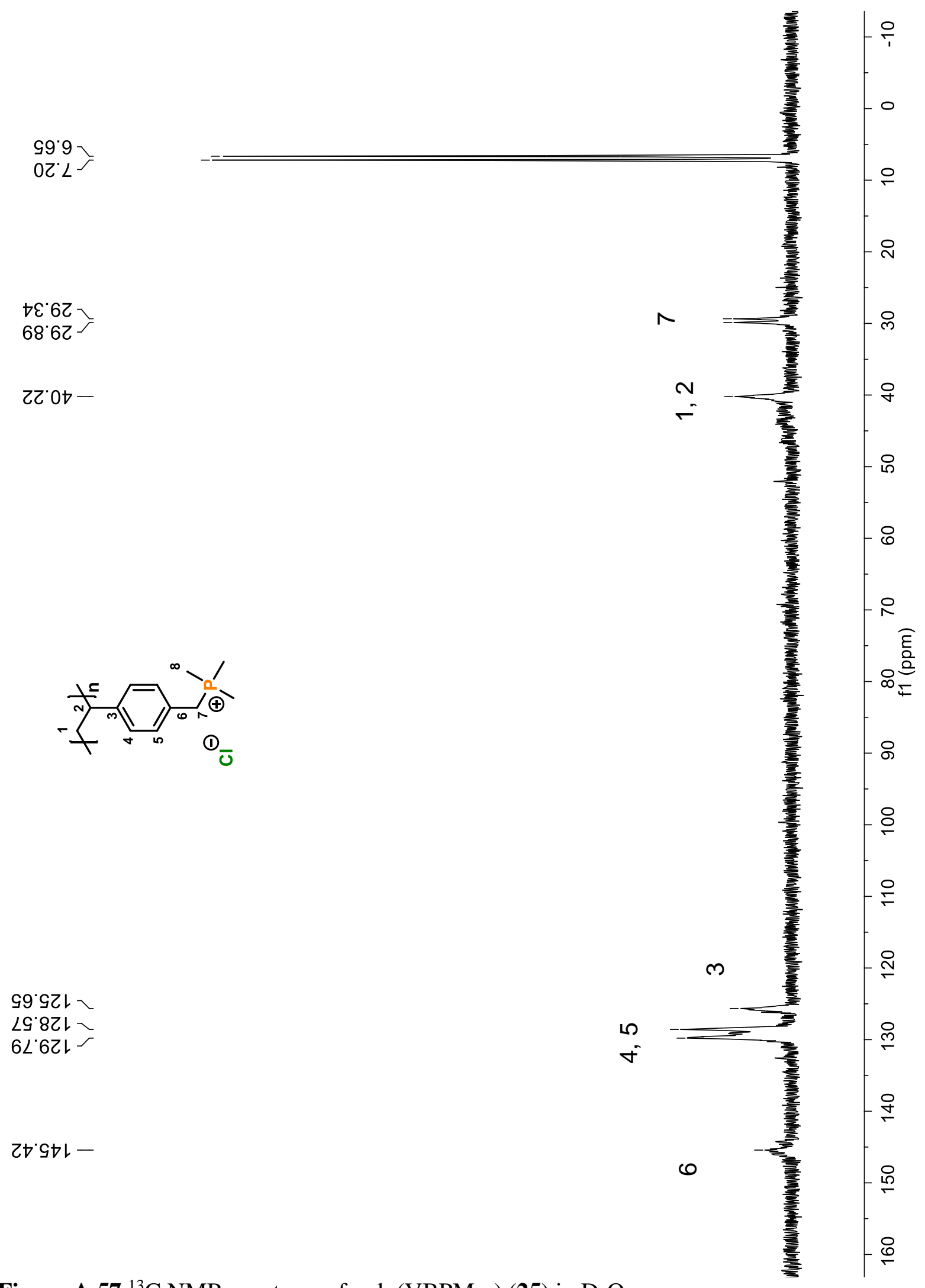

Figure A $57{ }^{13} \mathrm{C}$ NMR spectrum of poly(VBPMe 3$)(\mathbf{2 5})$ in $\mathrm{D}_{2} \mathrm{O}$ 


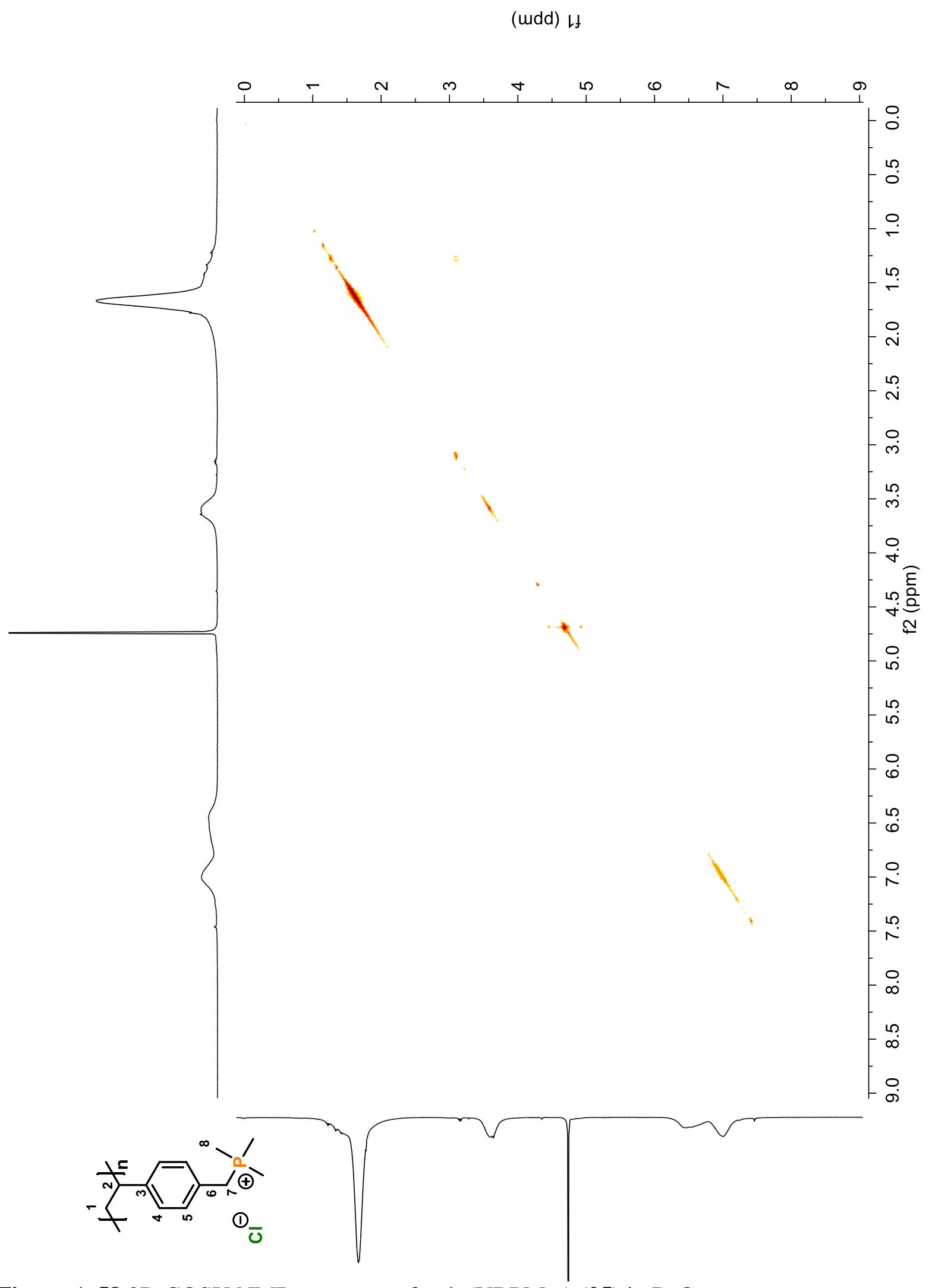

Figure A 58 2D COSY NMR spectrum of poly(VBPMe 3$)(\mathbf{2 5})$ in $\mathrm{D}_{2} \mathrm{O}$ 


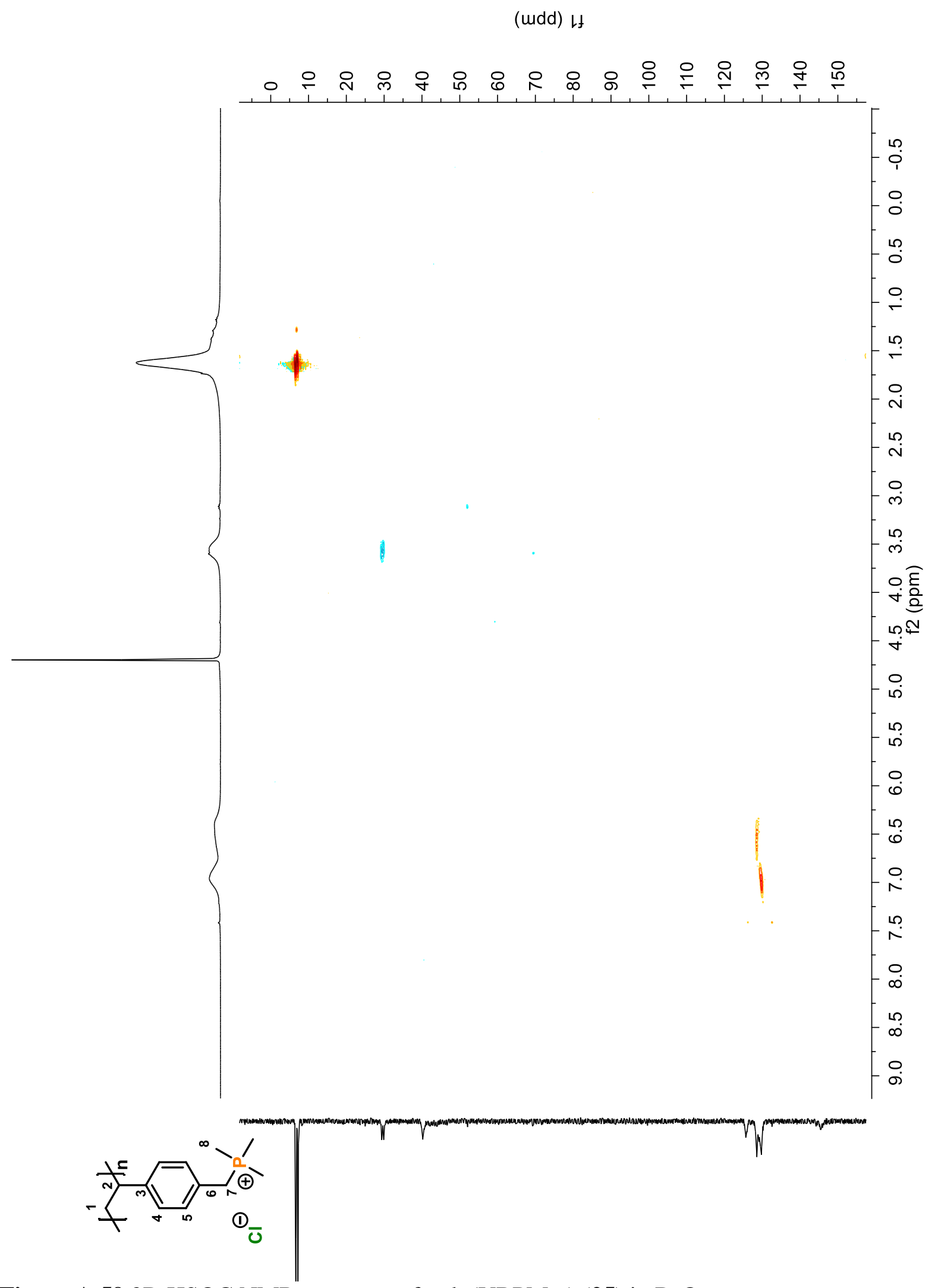

Figure A 59 2D HSQC NMR spectrum of poly(VBPMe 3$)(25)$ in $\mathrm{D}_{2} \mathrm{O}$ 


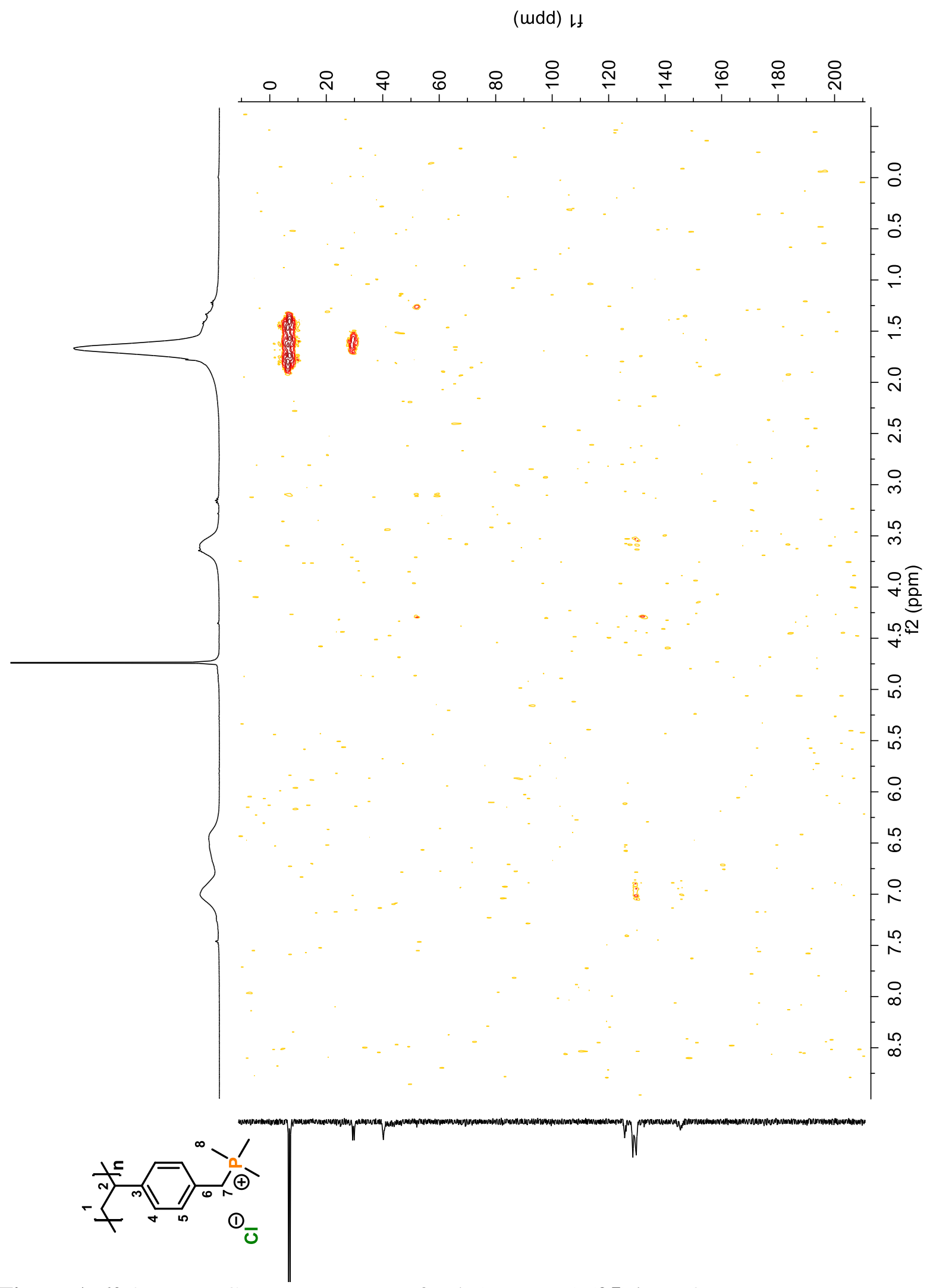

Figure A 60 2D HMBC NMR spectrum of poly(VBPMe $\left.{ }_{3}\right)(\mathbf{2 5})$ in $\mathrm{D}_{2} \mathrm{O}$ 
GPC Trace of Poly(VBC-BP) (2)

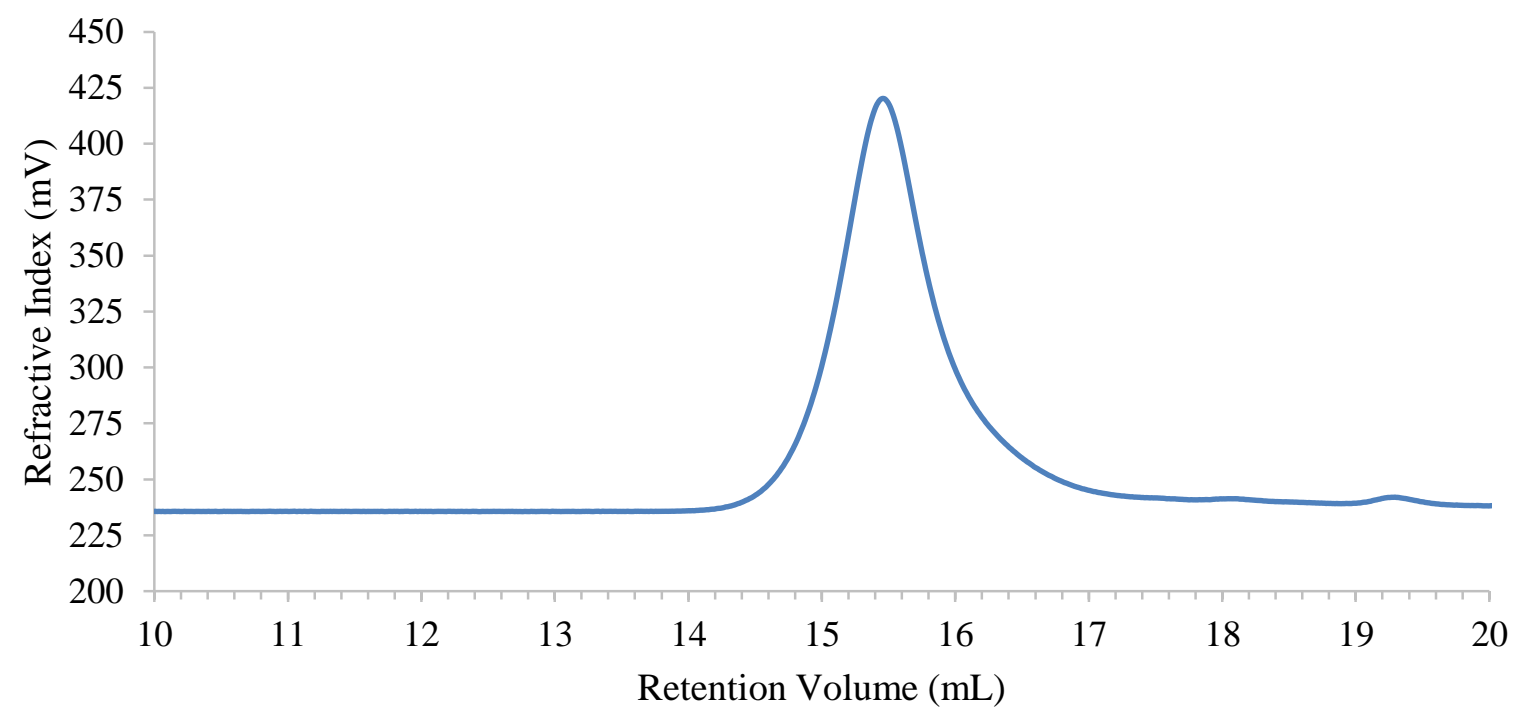

Figure B 1. GPC trace of poly(VBC-BP) (2)

GPC Trace of RAFT polymerization conditions with RAFT Agent CTA1

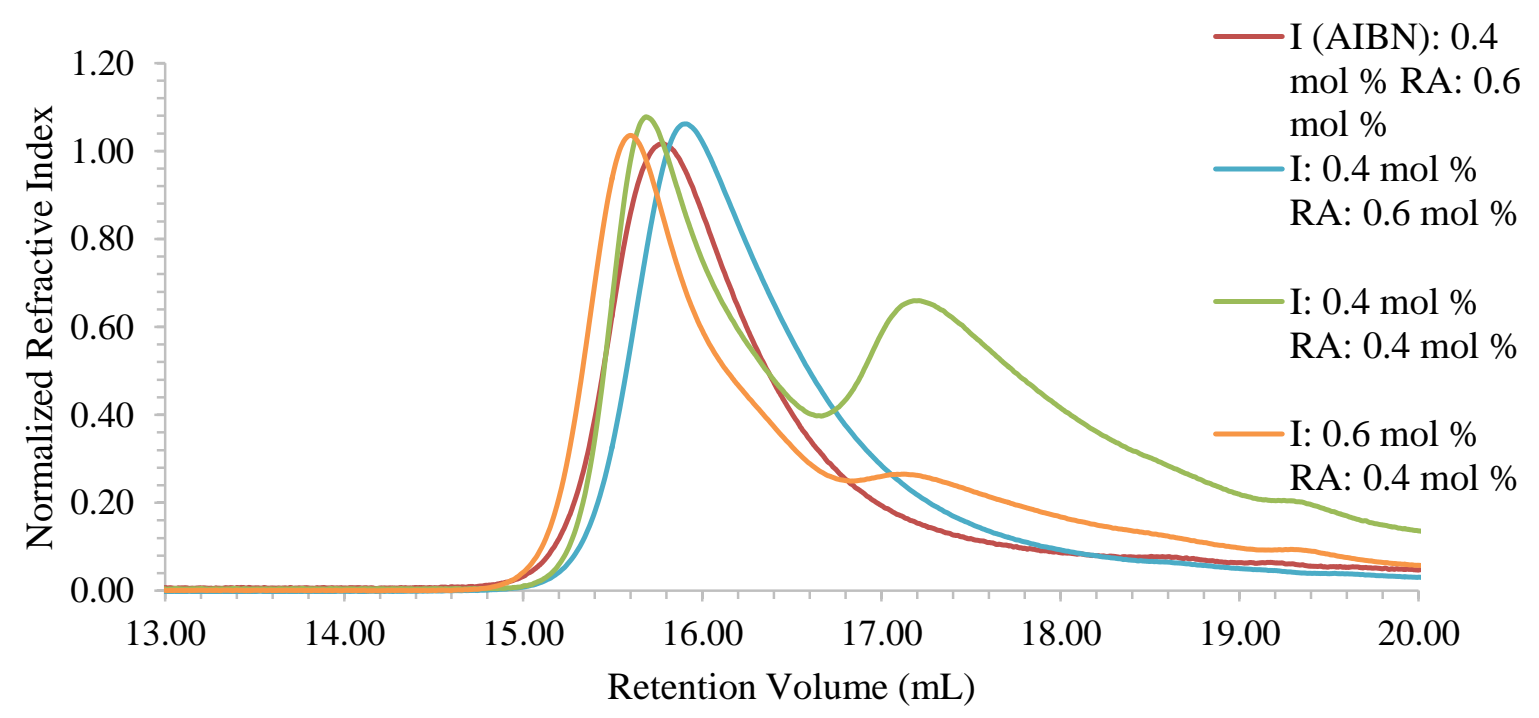

Figure B 2. GPC trace of polymers 3 synthesized by RAFT polymerization with varying reaction conditions using RAFT agent CTA1. 


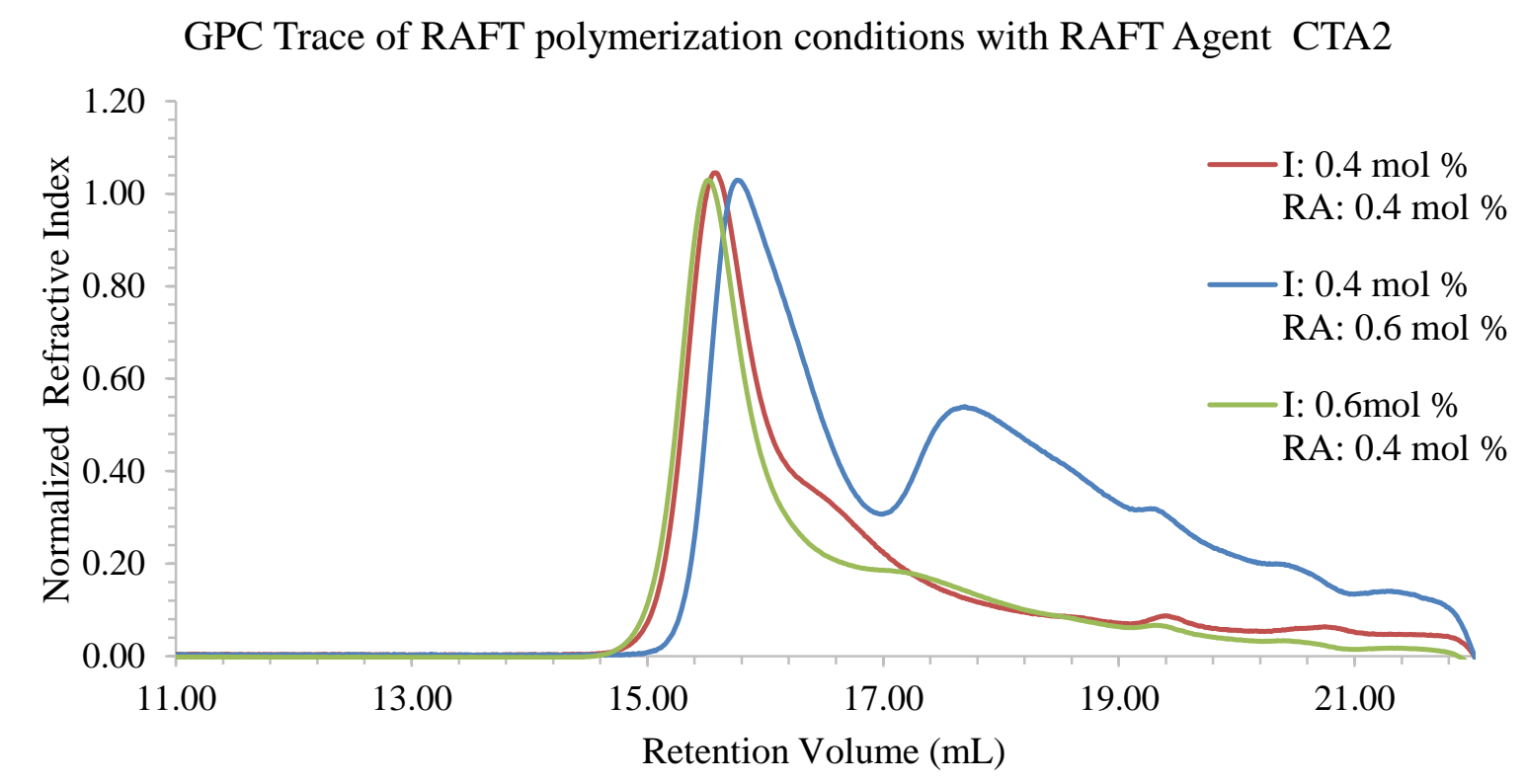

Figure B 3 GPC trace of polymers 4 synthesized by RAFT polymerization with varying reaction conditions using RAFT agent CTA2

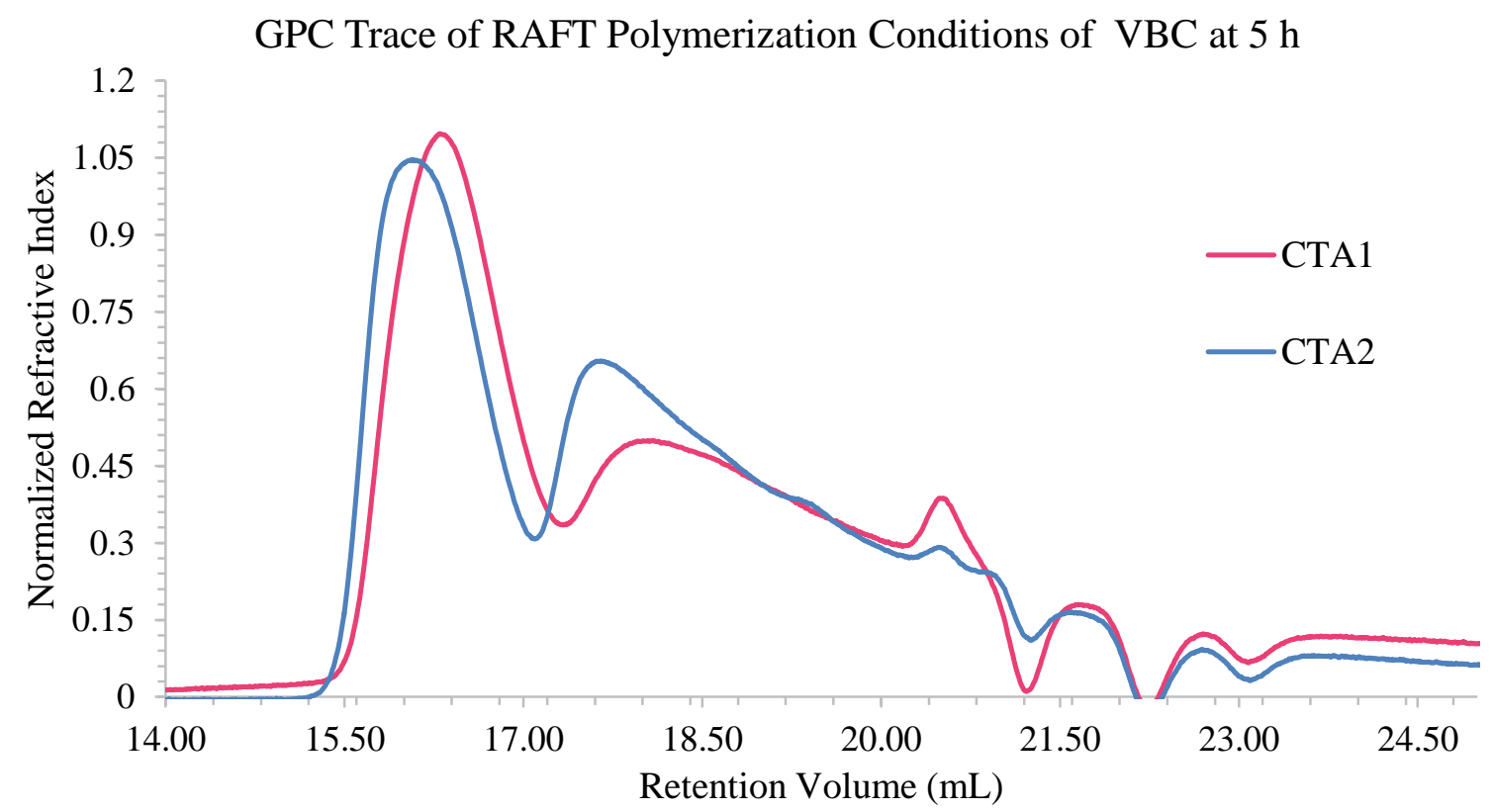

Figure B 4 GPC trace of polymers 5 synthesized by RAFT polymerization mediated by CTA1 and CTA 2 for $5 \mathrm{~h}$ 
GPC Trace of VBBP Homopolymer Copolymerization in the Synthesis of the Diblock Copolymer

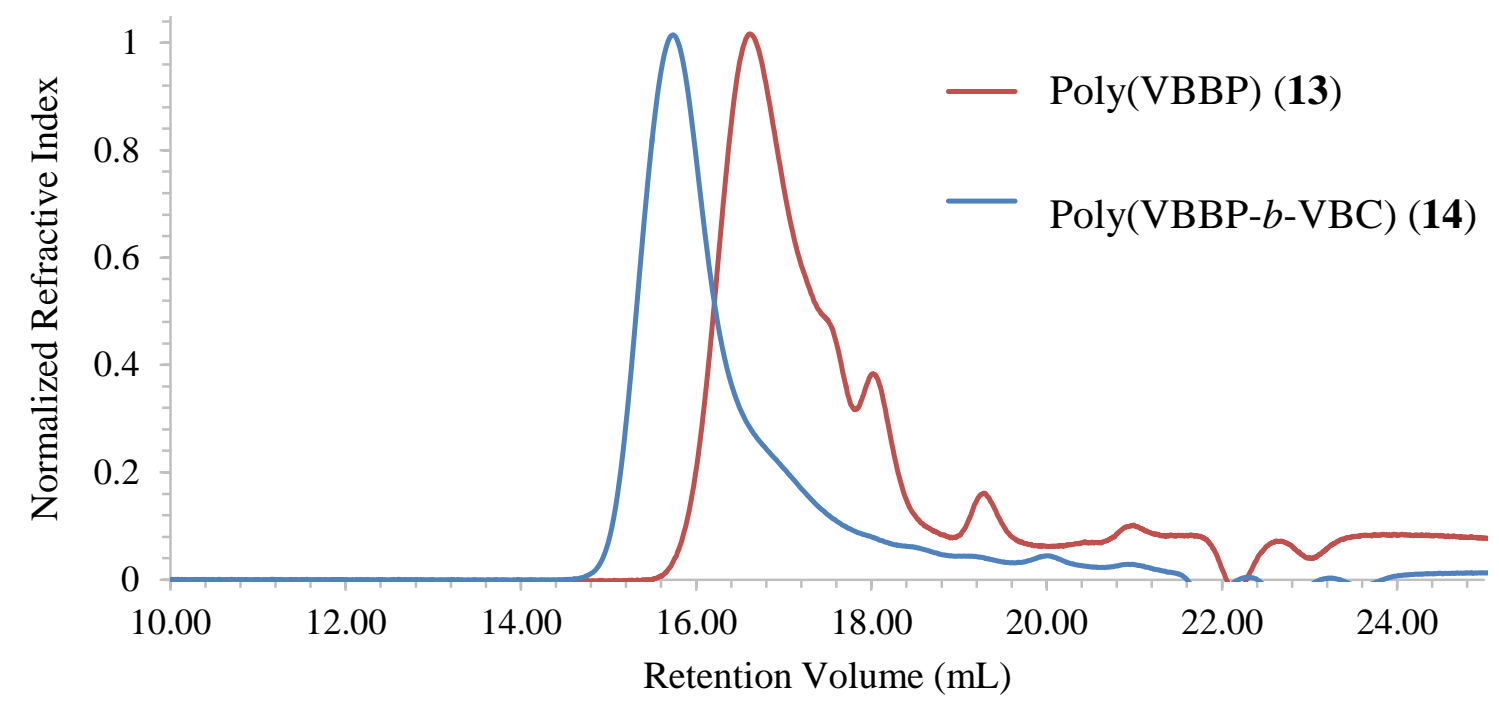

Figure B 5 GPC trace of starting homopolymer 13 and diblock copolymer after copolymerization (14) 
Appendix C: Thermoanalysis by DSC

DSC Trace of Poly(VBC-BP) (2)

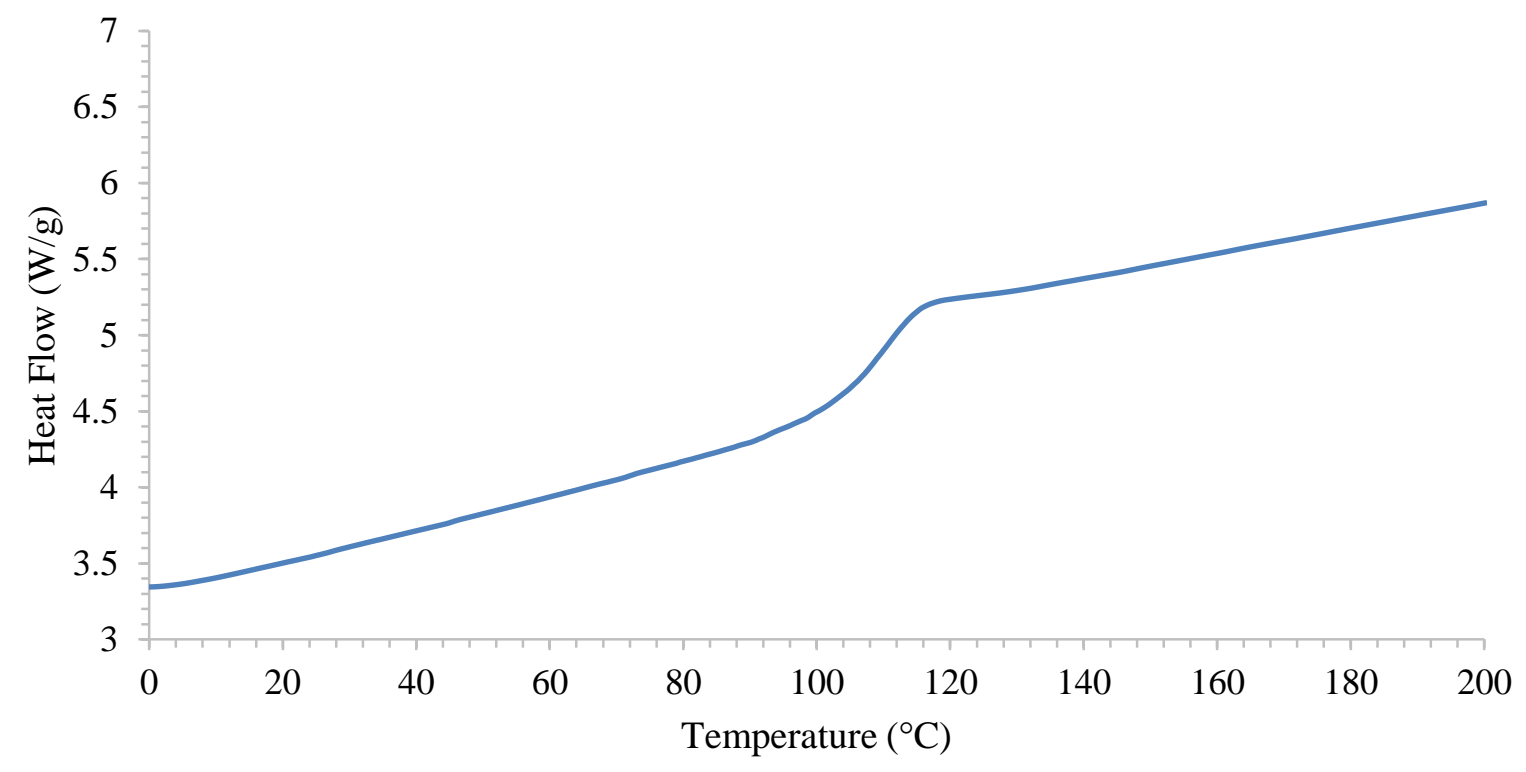

Figure C 1. DSC trace of poly(VBC-BP) (2)

DSC Trace of Poly(VBBP-NEt 3 ) (6)

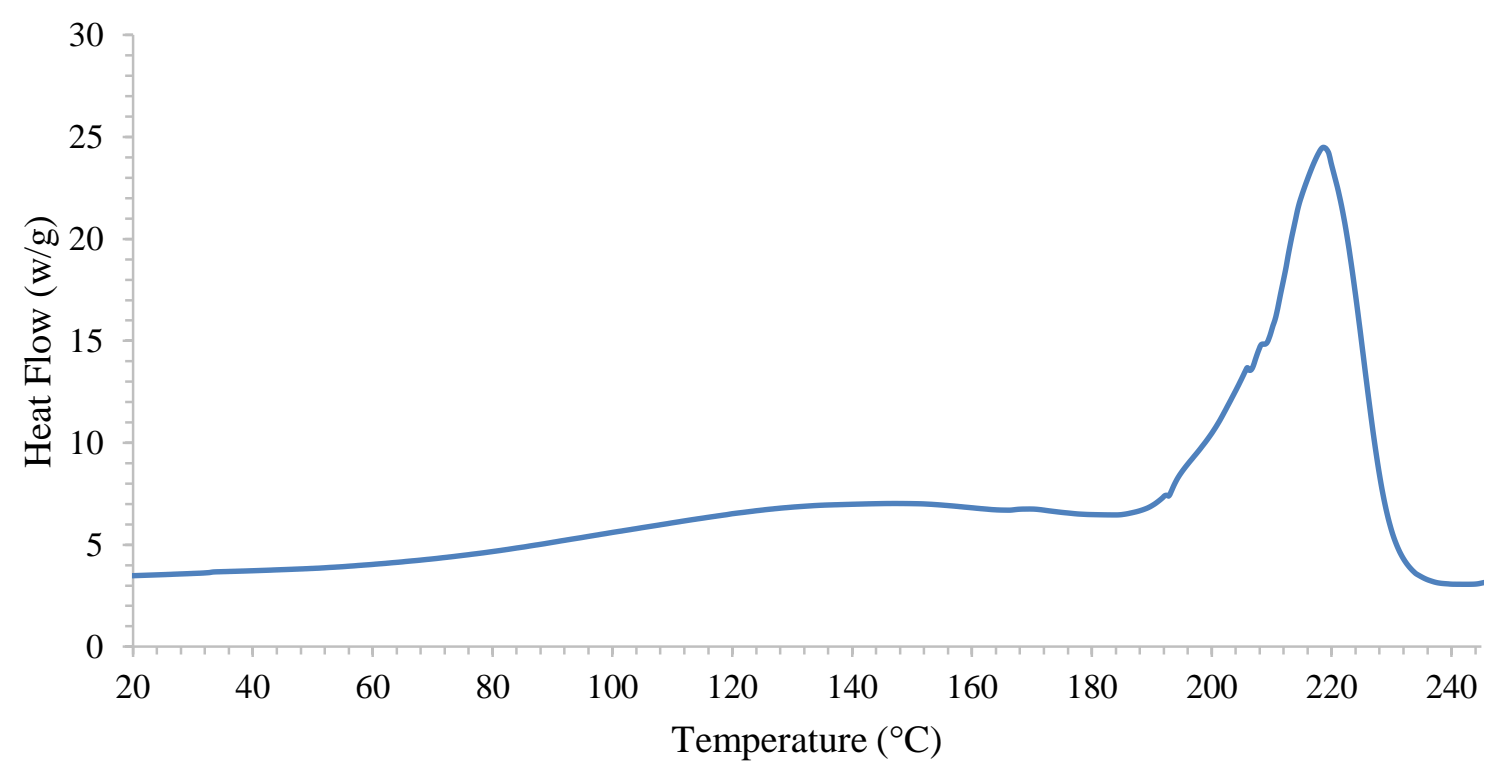

Figure C 2. DSC trace of poly $\left(\mathrm{VBBP}^{\left.-\mathrm{NEt}_{3}\right)(6)}\right.$ 
DSC Trace of Poly(VBBP-NC12) (8)

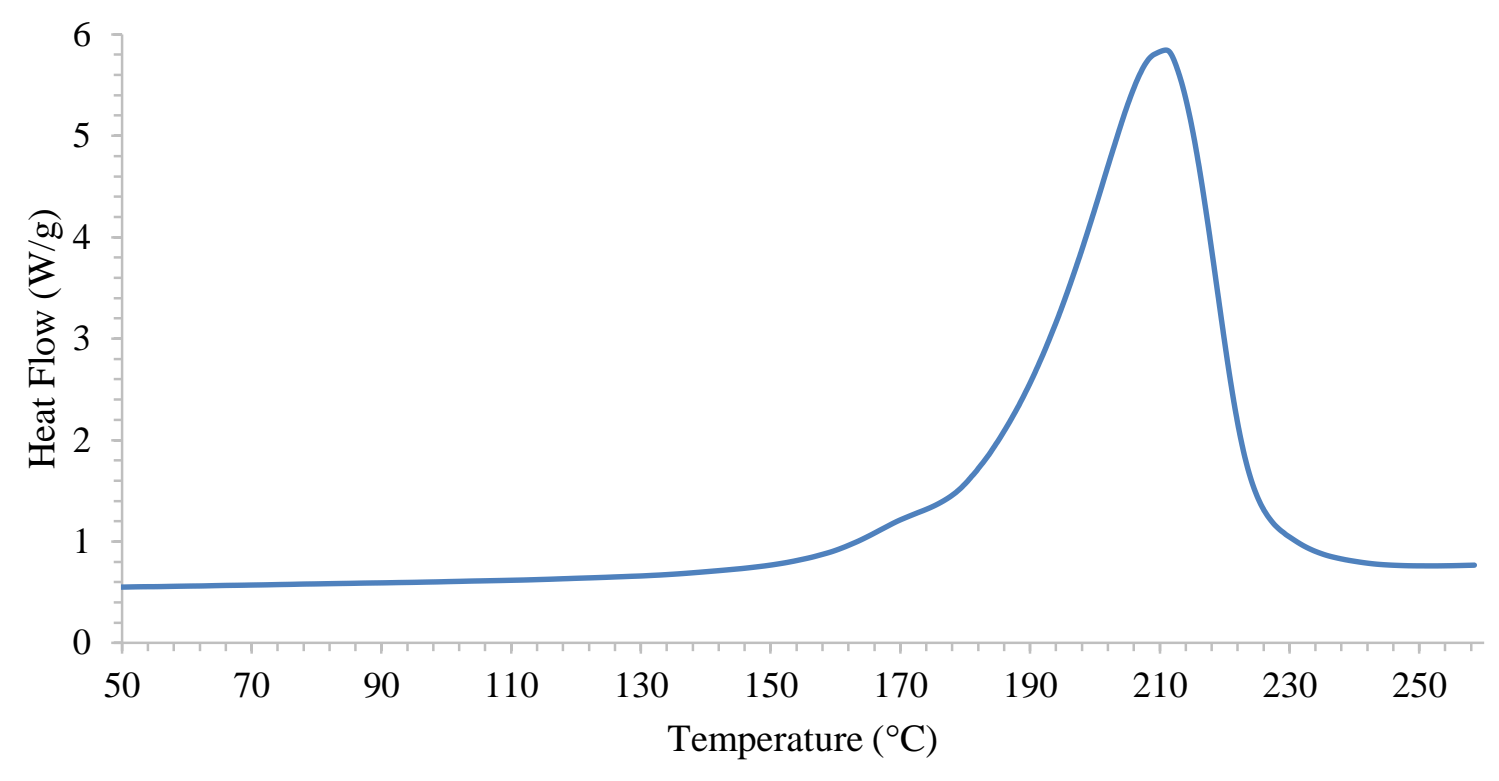

Figure C 3. DSC trace of poly(VBBP-NC12) (8)

DSC Trace of Poly(VBBP-TsSA) (9)

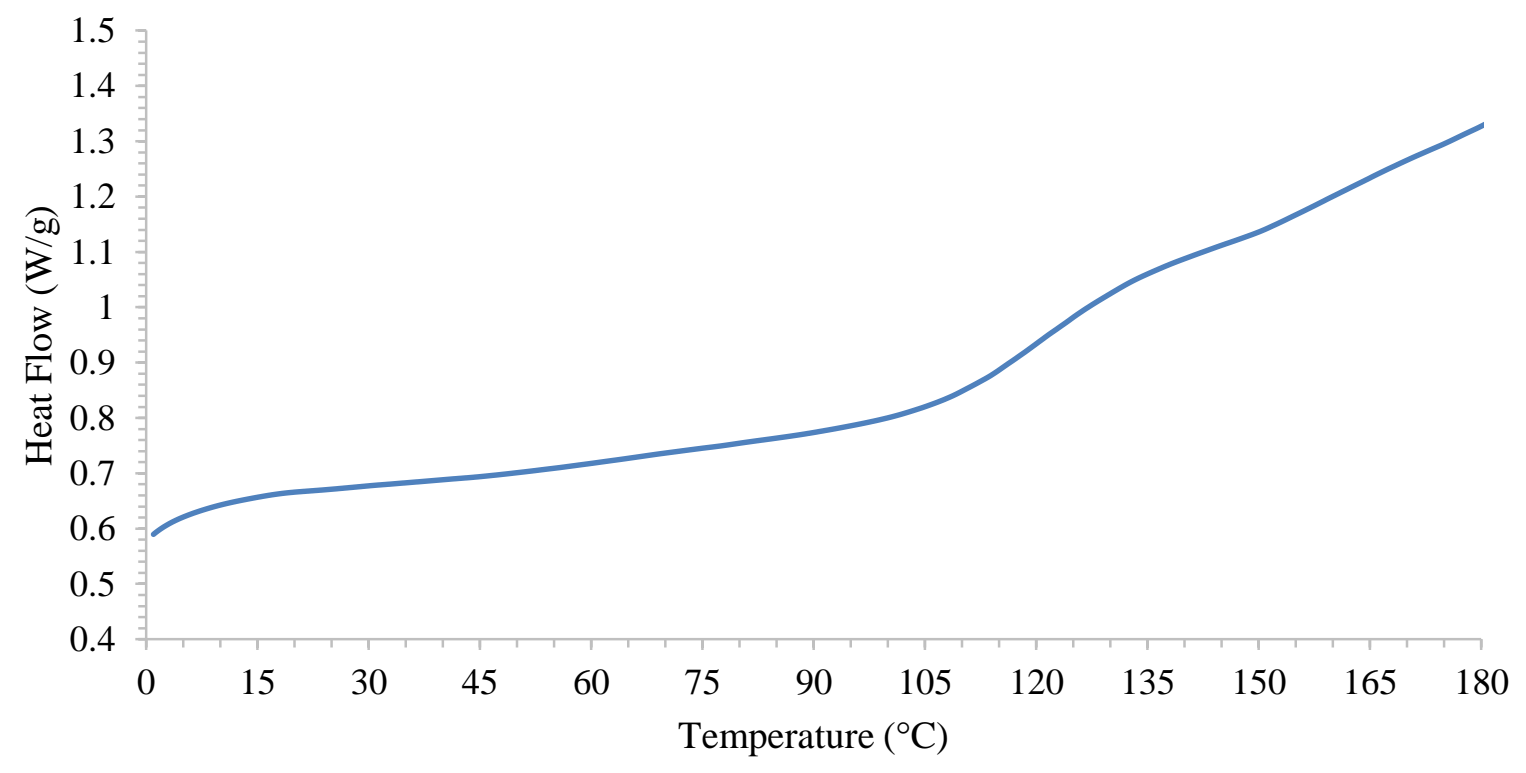

Figure C 4. DSC Trace of poly(VBBP-TsSA) (9) 


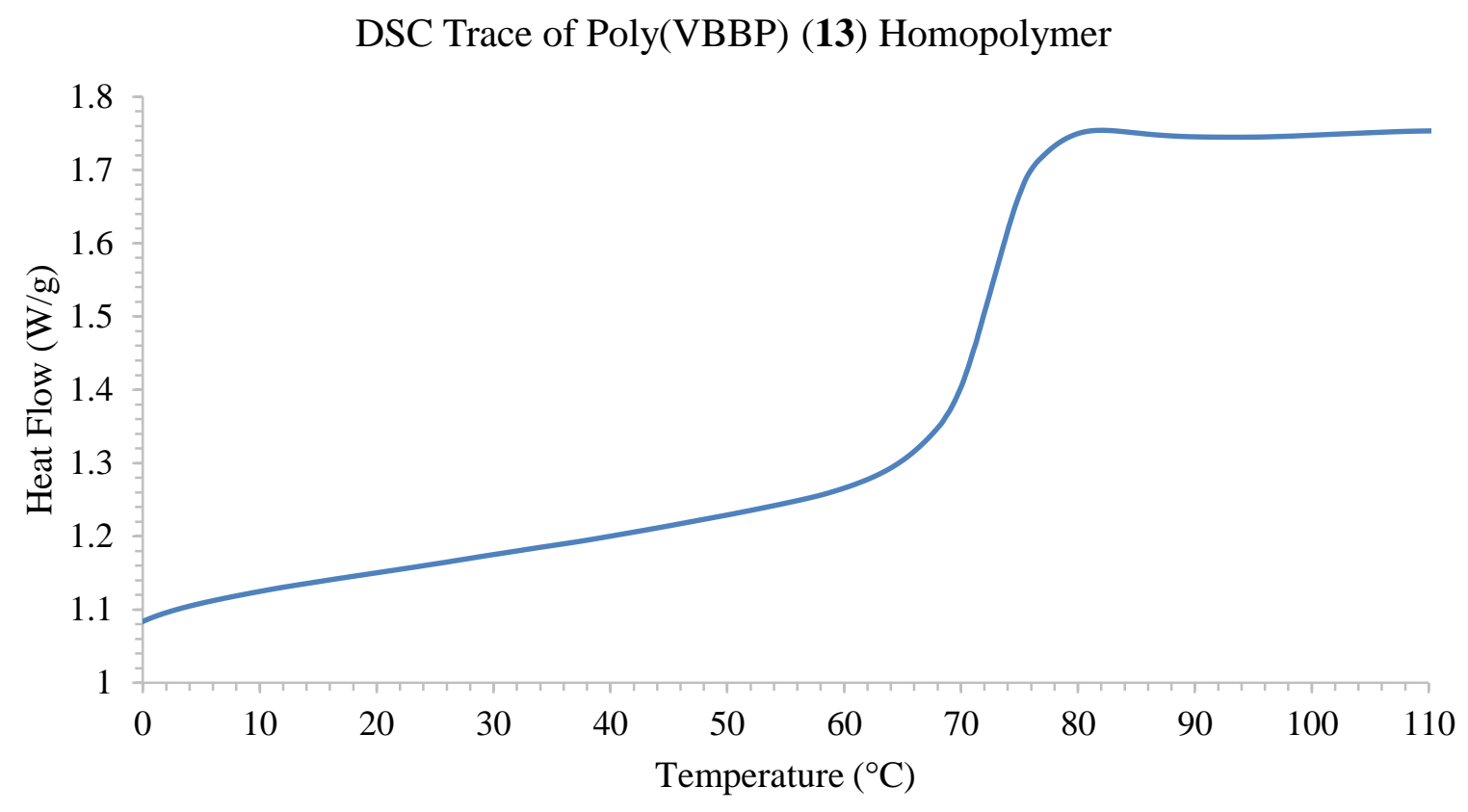

Figure C 5 DSC trace of poly(VBBP) (13) homopolymer

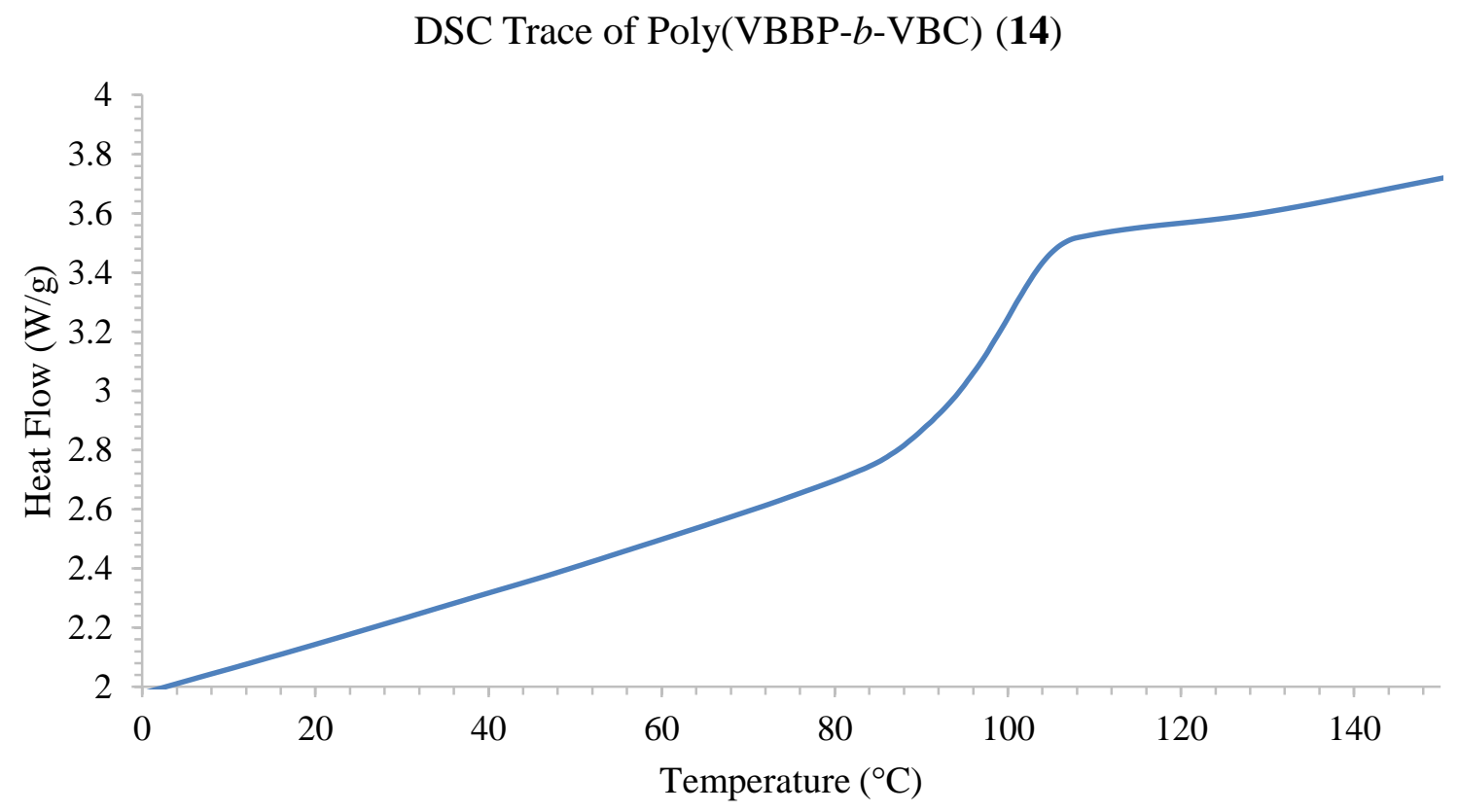

Figure C 6 DSC trace of diblock copolymer poly(VBBC- $b$-VBC) (14) 
DSC Trace of Poly(VBNEt $\left.{ }_{3}\right)$ (23)

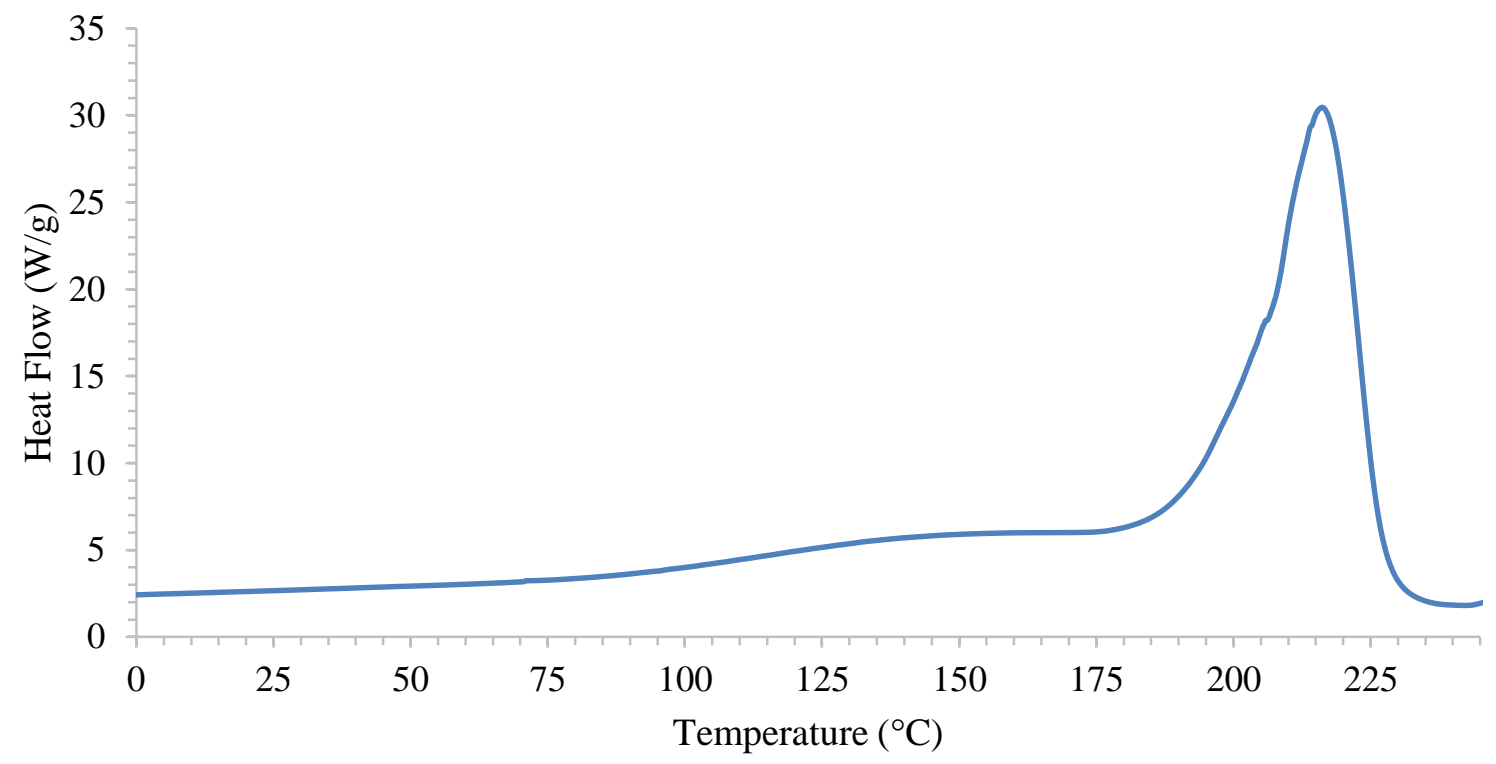

Figure C 7 DSC trace of poly(VBNEt 3 ) (23) 
Appendix D: Composition Estimation by $1 \mathrm{H} \mathrm{NMR}$ and Elemental Analysis

Table D 1 Data obtained from proton integration analysis for composition estimates of polymers 6-11

\begin{tabular}{|c|c|c|c|c|c|c|c|}
\hline \multirow{2}{*}{ Polymer } & \multirow{2}{*}{ Solvent } & \multicolumn{2}{|c|}{${ }^{\mathbf{H}}\left(\mathbf{C H}_{2}\right) \boldsymbol{\delta}(\mathbf{p p m})$} & \multicolumn{2}{c|}{ Estimated \% Loading } & \multirow{2}{*}{ VBBP:VBC:Q } \\
\cline { 3 - 7 } & & $\boldsymbol{V B B P}$ & $\boldsymbol{Q}$ & $\boldsymbol{V B C}$ & $\boldsymbol{V B B P}$ & $\boldsymbol{Q}$ & \\
\hline \multirow{2}{*}{$\mathbf{6}$} & $\mathbf{D M S O - d}_{\mathbf{6}}$ & 5.20 & 4.72 & 4.72 & $12 \%$ & $60 \%$ & $2: 5: 10$ \\
\cline { 2 - 7 } & $\mathbf{M e O D}$ & 5.25 & 4.50 & 4.50 & $7.7 \%$ & $69 \%$ & $1: 3: 9$ \\
\hline $\mathbf{7}$ & MeOD & 5.22 & 3.86 & 4.64 & $4.7 \%$ & $87 \%$ & $1: 2: 19$ \\
\hline $\mathbf{8}$ & $\mathbf{M e O D}$ & 5.28 & 3.48 & 4.61 & $5.7 \%$ & $49 \%$ & $1: 8: 8.6$ \\
\hline $\mathbf{9}^{\boldsymbol{a}}$ & - & - & - & - & - & - & - \\
\hline $\mathbf{1 0}$ & $\mathbf{C D C l}_{3}$ & 5.08 & 3.62 & 5.08 & $-{ }^{a}$ & $55 \%$ & $4: 5$ \\
\hline $\mathbf{1 1}^{\boldsymbol{a}}$ & - & - & - & - & - & - & - \\
\hline
\end{tabular}

${ }^{a}$ Undetermined due to solubility issues

Table D 2 Elemental analysis data of C, H, and N for estimation of polymer composition (6-11)

\begin{tabular}{|c|c|c|c|c|c|c|c|}
\hline \multicolumn{2}{|c|}{ Polymer } & \multirow{3}{*}{$\begin{array}{c}\text { C } \\
65.16 \\
\end{array}$} & \multirow{3}{*}{$\begin{array}{c}\mathbf{H} \\
9.26 \\
\end{array}$} & \multirow{4}{*}{$\begin{array}{c}\mathbf{N} \\
4.31 \\
4.26\end{array}$} & \multicolumn{2}{|c|}{$\begin{array}{c}\text { Estimated \% } \\
\text { Loading }\end{array}$} & \multirow{4}{*}{$\begin{array}{c}\text { VBBP:VBC:Q } \\
2: 2: 17\end{array}$} \\
\hline \multirow{3}{*}{6} & & & & & & & \\
\hline & Found & & & & \multirow{2}{*}{$9.5 \%$} & \multirow{2}{*}{$81 \%$} & \\
\hline & Theory & 71.63 & 9.17 & & & & \\
\hline \multirow{2}{*}{7} & Found & 61.34 & 7.87 & - & \multirow{2}{*}{$4.7 \%$} & \multirow{2}{*}{$88 \%$} & \multirow{2}{*}{$2: 3: 38$} \\
\hline & Theory & 64.60 & 7.86 & - & & & \\
\hline \multirow{2}{*}{8} & Found & 74.62 & 12.11 & 4.00 & \multirow{2}{*}{$8 \%$} & \multirow{2}{*}{$75 \%$} & \multirow{2}{*}{$1: 2: 9.5$} \\
\hline & Theory & 75.37 & 10.19 & 3.23 & & & \\
\hline \multirow{2}{*}{9} & Found & 60.08 & 7.17 & 6.02 & \multirow{2}{*}{$5 \%$} & \multirow{2}{*}{$85 \%$} & \multirow{2}{*}{$1: 2: 17$} \\
\hline & Theory & 62.52 & 7.31 & 5.99 & & & \\
\hline \multirow{2}{*}{10} & Found & 76.21 & 13.41 & 3.01 & \multirow{2}{*}{$7.7 \%$} & \multirow{2}{*}{$62 \%$} & \multirow{2}{*}{$1: 4: 8$} \\
\hline & Theory & 77.39 & 10.94 & 2.73 & & & \\
\hline \multirow{2}{*}{11} & Found & 58.00 & 6.79 & 6.31 & \multirow{2}{*}{$5.1 \%$} & \multirow{2}{*}{$85 \%$} & \multirow{2}{*}{$1: 2: 16.5$} \\
\hline & Theory & 56.66 & 8.12 & 4.94 & & & \\
\hline
\end{tabular}


Table D 3 Elemental analysis data of $\mathrm{C}$ and $\mathrm{H}$ for estimation for composition estimations of polymers $\mathbf{1 5}$ and $\mathbf{1 6}$

\begin{tabular}{|c|c|c|c|c|c|c|}
\hline \multirow{2}{*}{\multicolumn{2}{|c|}{ Polymer }} & \multirow[t]{2}{*}{$\mathbf{C}$} & \multirow[t]{2}{*}{$\mathbf{H}$} & \multicolumn{2}{|c|}{$\begin{array}{c}\text { Estimated \% } \\
\text { Loading }\end{array}$} & \multirow[t]{2}{*}{ VBBP:VBC:Q } \\
\hline & & & & VBBP & $Q$ & \\
\hline \multirow{2}{*}{15} & Found & 72.08 & 9.18 & \multirow{2}{*}{$9.7 \%$} & \multirow{2}{*}{$49 \%$} & \multirow{2}{*}{$1.4: 6: 7$} \\
\hline & Theory & 72.49 & 8.69 & & & \\
\hline \multirow{2}{*}{16} & Found & 71.46 & 10.53 & \multirow{2}{*}{$8.5 \%$} & \multirow{2}{*}{$67 \%$} & \multirow{2}{*}{$1.4: 4: 11$} \\
\hline & Theory & 72.17 & 9.31 & & & \\
\hline
\end{tabular}

Table D 4 Proton integration data for composition estimates of polymer 16

\begin{tabular}{|c|c|c|c|c|c|c|c|}
\hline \multirow{2}{*}{ Polymer } & \multirow{2}{*}{ Solvent } & \multicolumn{2}{|c|}{$\mathbf{1}_{\mathbf{H}}\left(\mathrm{CH}_{2}\right) \boldsymbol{\delta}(\mathbf{p p m})$} & \multicolumn{2}{c|}{ Estimated \% Loading } & \multirow{2}{*}{ VBBP:VBC:Q } \\
\cline { 3 - 7 } & & $\boldsymbol{V B B P}$ & $\boldsymbol{Q}$ & $V B C$ & $\boldsymbol{V B B P}$ & $\boldsymbol{Q}$ & \\
\hline $\mathbf{1 6}$ & $\mathbf{C D C l}_{3}$ & 5.07 & 2.97 & 4.45 & $7.4 \%$ & $52 \%$ & $1: 5.5: 7$ \\
\hline
\end{tabular}


Appendix E: AFM Images

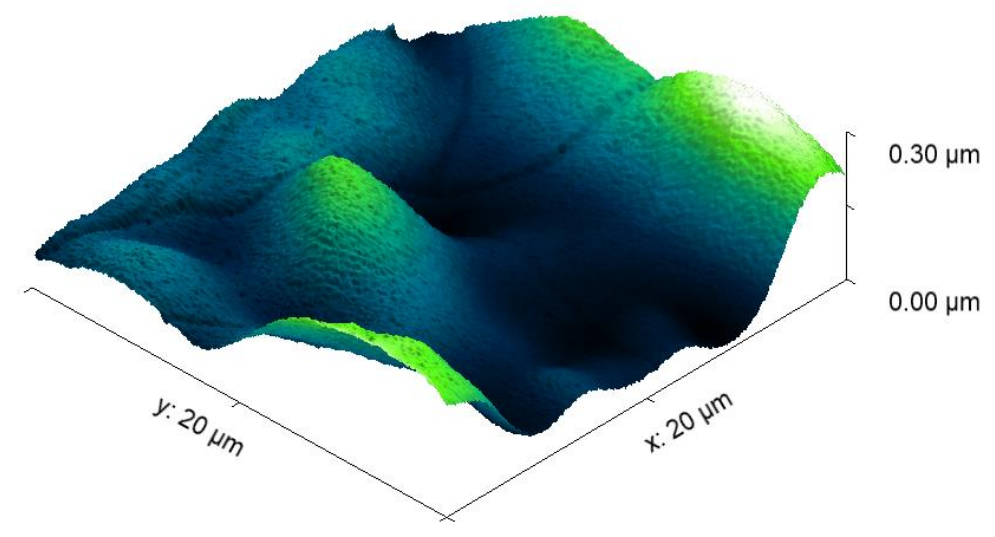

Figure E 1 AFM image of PS coated with poly(VBBP-PMe 3 ) (7)

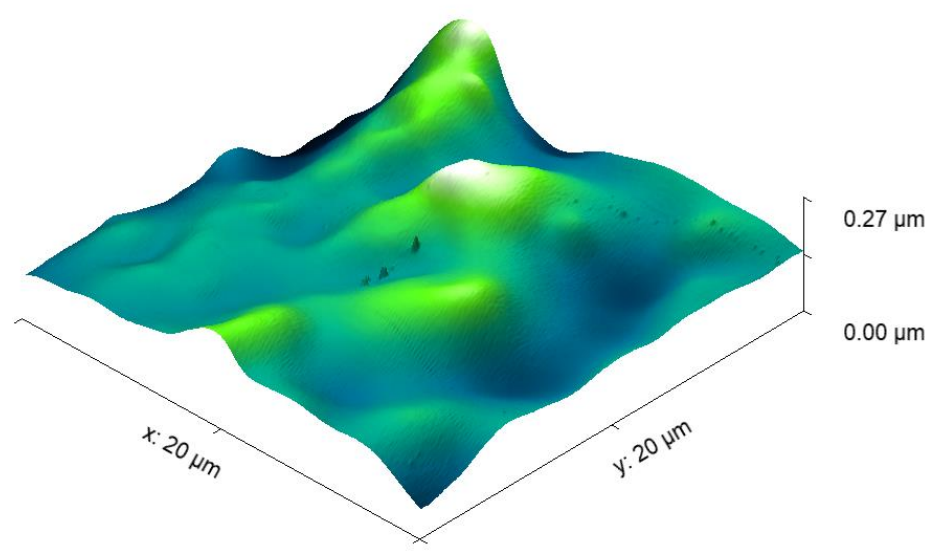

Figure E 2 AFM image of PS coated with poly(VBBP-NC12) (8) 
Appendix F: Biological Data

Table F 1 Colony-forming unit (CFU) from antibacterial testing by LDI method against Arthrobacter sp. Control and treated surfaces (coatings 2, 6-12, 15, 16) was inoculated with $10 \mu \mathrm{L}$ droplet of $10^{7} \mathrm{CFU}$ in sterile tap water for $3 \mathrm{~h}$

\begin{tabular}{|c|c|c|c|c|c|}
\hline \multirow[b]{2}{*}{ Coating } & & \multicolumn{2}{|c|}{ Control } & \multicolumn{2}{|c|}{ Treated } \\
\hline & & Total CFU & $\begin{array}{c}\text { Log Total } \\
(\log (\mathrm{CFU}))\end{array}$ & Total CFU & $\begin{array}{c}\text { Log Total } \\
(\log (C F U))\end{array}$ \\
\hline \multirow{7}{*}{2} & Trial 1 & $8.30 \mathrm{E}+06$ & 6.92 & $4.20 \mathrm{E}+06$ & 6.62 \\
\hline & Trial 2 & $9.25 \mathrm{E}+06$ & 6.97 & $5.05 \mathrm{E}+06$ & 6.70 \\
\hline & Trial 3 & $1.02 \mathrm{E}+07$ & 7.01 & $1.00 \mathrm{E}+07$ & 7.00 \\
\hline & Average & $9.25 \mathrm{E}+06$ & 6.96 & $6.42 \mathrm{E}+06$ & 6.78 \\
\hline & Std Error & $5.48 \mathrm{E}+05$ & 0.03 & $1.81 \mathrm{E}+06$ & 0.11 \\
\hline & t-statistic & \multicolumn{4}{|c|}{2.151382} \\
\hline & t-critical & \multicolumn{4}{|c|}{2.91998558} \\
\hline \multirow{7}{*}{6} & Trial 1 & $5.70 \mathrm{E}+06$ & 6.76 & 0 & 0 \\
\hline & Trial 2 & $8.15 \mathrm{E}+06$ & 6.91 & 0 & 0 \\
\hline & Trial 3 & $8.25 \mathrm{E}+06$ & 6.92 & 0 & 0 \\
\hline & Average & $7.37 \mathrm{E}+06$ & 6.86 & 0 & 0 \\
\hline & Std Error & $8.34 \mathrm{E}+05$ & 0.05 & 0 & 0 \\
\hline & t-statistic & \multicolumn{4}{|c|}{8.834700769} \\
\hline & t-critical & \multicolumn{4}{|c|}{2.91998558} \\
\hline \multirow{7}{*}{7} & Trial 1 & $5.50 \mathrm{E}+06$ & 6.74 & 0 & 0 \\
\hline & Trial 2 & $6.00 \mathrm{E}+06$ & 6.78 & 0 & 0 \\
\hline & Trial 3 & $6.80 \mathrm{E}+06$ & 6.83 & 0 & 0 \\
\hline & Average & $6.10 \mathrm{E}+06$ & 6.78 & 0 & 0 \\
\hline & Std Error & $3.79 \mathrm{E}+05$ & 0.03 & 0 & 0 \\
\hline & t-statistic & \multicolumn{4}{|c|}{16.1122515857589} \\
\hline & t-critical & \multicolumn{4}{|c|}{2.91998558035373} \\
\hline \multirow{7}{*}{8} & Trial 1 & $9.65 \mathrm{E}+06$ & 6.98 & $1.01 \mathrm{E}+07$ & 7.00 \\
\hline & Trial 2 & $7.75 \mathrm{E}+06$ & 6.89 & $7.90 \mathrm{E}+06$ & 6.90 \\
\hline & Trial 3 & $4.05 \mathrm{E}+06$ & 6.61 & $6.30 \mathrm{E}+06$ & 6.80 \\
\hline & Average & $7.15 \mathrm{E}+06$ & 6.83 & $8.08 \mathrm{E}+06$ & 6.90 \\
\hline & Std Error & $1.64 \mathrm{E}+06$ & 0.11 & $1.09 \mathrm{E}+06$ & 0.06 \\
\hline & t-statistic & \multicolumn{4}{|c|}{-1.40927894} \\
\hline & t-critical & \multicolumn{4}{|c|}{2.91998558} \\
\hline \multirow{5}{*}{9} & Trial 1 & $5.50 \mathrm{E}+06$ & 6.74 & $5.30 \mathrm{E}+05$ & 5.72 \\
\hline & Trial 2 & $6.00 \mathrm{E}+06$ & 6.78 & $5.00 \mathrm{E}+05$ & 5.70 \\
\hline & Trial 3 & $6.80 \mathrm{E}+06$ & 6.83 & $6.40 \mathrm{E}+05$ & 5.81 \\
\hline & Average & $6.10 \mathrm{E}+06$ & 6.78 & $5.57 \mathrm{E}+05$ & 5.74 \\
\hline & Std Error & $3.79 \mathrm{E}+05$ & 0.03 & $4.26 \mathrm{E}+04$ & 0.03 \\
\hline
\end{tabular}




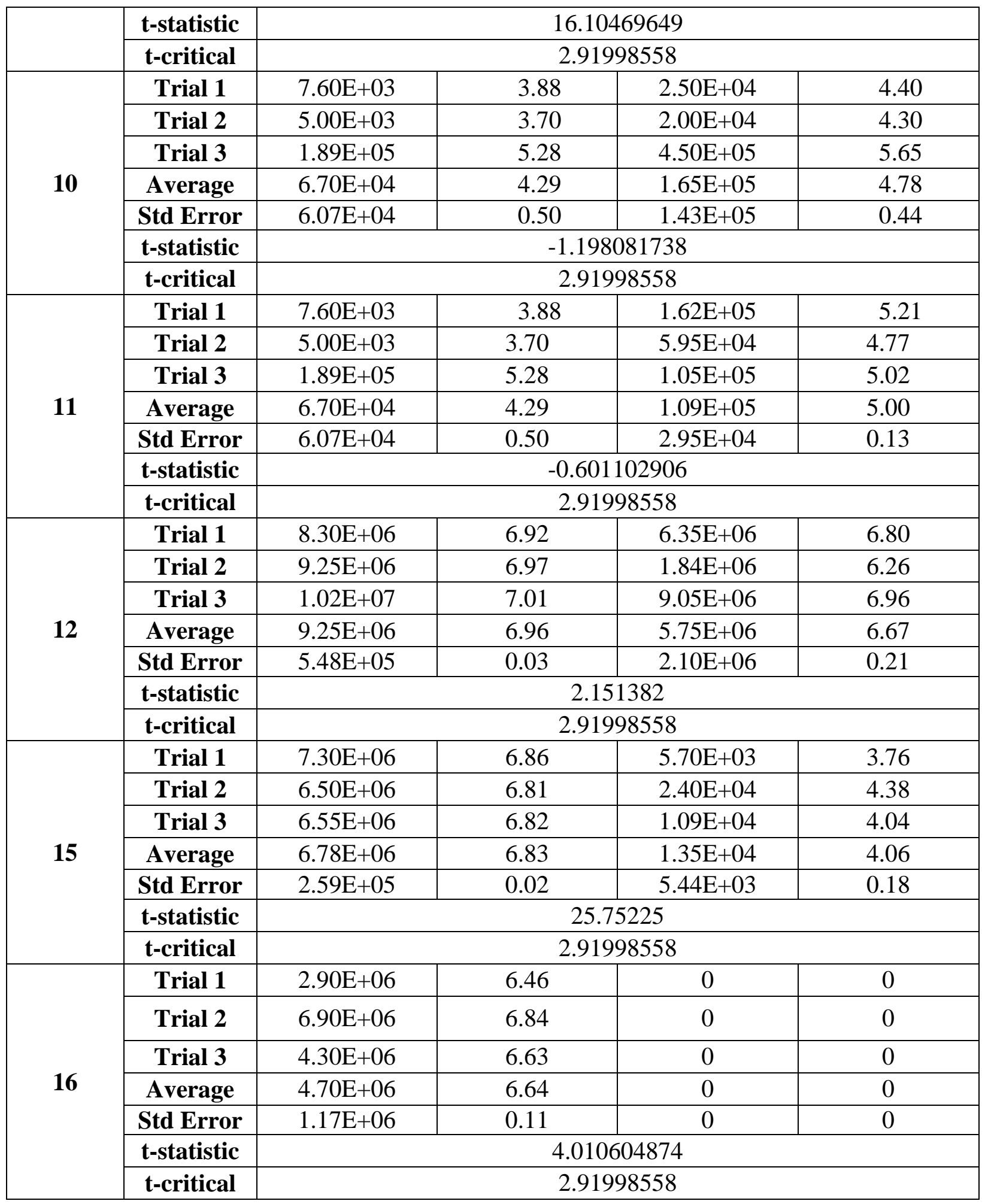


Table F 2 Colony-forming unit (CFU) from antibacterial testing by LDI method against E. coli wt36. Control and treated surfaces (coatings $\mathbf{2 , \mathbf { 6 - 1 2 }}, \mathbf{1 5}, \mathbf{1 6}$ ) was inoculated with $10 \mu \mathrm{L}$ droplet of $10^{7} \mathrm{CFU}$ in sterile tap water for $3 \mathrm{~h}$

\begin{tabular}{|c|c|c|c|c|c|}
\hline \multirow[b]{2}{*}{ Coating } & & \multicolumn{2}{|c|}{ Control } & \multicolumn{2}{|c|}{ Treated } \\
\hline & & Total CFU & $\begin{array}{c}\log \text { Total } \\
(\log (C F U))\end{array}$ & Total CFU & $\begin{array}{c}\log \text { Total } \\
(\log (C F U))\end{array}$ \\
\hline \multirow{7}{*}{6} & Trial 1 & $1.22 \mathrm{E}+04$ & 4.09 & $9.30 \mathrm{E}+04$ & 4.97 \\
\hline & Trial 2 & $2.65 \mathrm{E}+04$ & 4.42 & $7.65 \mathrm{E}+04$ & 4.88 \\
\hline & Trial 3 & $2.27 \mathrm{E}+04$ & 4.36 & $1.68 \mathrm{E}+04$ & 4.22 \\
\hline & Average & $2.04 \mathrm{E}+04$ & 4.29 & $6.21 \mathrm{E}+04$ & 4.69 \\
\hline & Std Error & $4.26 \mathrm{E}+03$ & 0.10 & $2.32 \mathrm{E}+04$ & 0.24 \\
\hline & t-statistic & \multicolumn{4}{|c|}{-1.641179397} \\
\hline & t-critical & \multicolumn{4}{|c|}{2.91998558} \\
\hline \multirow{7}{*}{7} & Trial 1 & $1.22 \mathrm{E}+04$ & 4.09 & $5.20 \mathrm{E}+04$ & 4.72 \\
\hline & Trial 2 & $2.65 \mathrm{E}+04$ & 4.42 & $5.60 \mathrm{E}+04$ & 4.75 \\
\hline & Trial 3 & $2.27 \mathrm{E}+04$ & 4.36 & $2.64 \mathrm{E}+04$ & 4.42 \\
\hline & Average & $2.04 \mathrm{E}+04$ & 4.29 & $4.48 \mathrm{E}+04$ & 4.63 \\
\hline & Std Error & $4.26 \mathrm{E}+03$ & 0.10 & $9.29 \mathrm{E}+03$ & 0.10 \\
\hline & t-statistic & \multicolumn{4}{|c|}{-2.267095283} \\
\hline & t-critical & \multicolumn{4}{|c|}{2.91998558} \\
\hline \multirow{7}{*}{8} & Trial 1 & $1.37 \mathrm{E}+05$ & 5.14 & $1.37 \mathrm{E}+05$ & 5.14 \\
\hline & Trial 2 & $2.03 \mathrm{E}+05$ & 5.31 & $1.37 \mathrm{E}+04$ & 4.14 \\
\hline & Trial 3 & $1.41 \mathrm{E}+06$ & 6.15 & $7.00 \mathrm{E}+05$ & 5.85 \\
\hline & Average & $5.83 \mathrm{E}+05$ & 5.53 & $2.83 \mathrm{E}+05$ & 5.04 \\
\hline & Std Error & $4.14 \mathrm{E}+05$ & 0.31 & $2.11 \mathrm{E}+05$ & 0.50 \\
\hline & t-statistic & \multicolumn{4}{|c|}{1.413756022} \\
\hline & t-critical & \multicolumn{4}{|c|}{2.91998558} \\
\hline \multirow{7}{*}{9} & Trial 1 & $1.37 \mathrm{E}+05$ & 5.14 & $1.14 \mathrm{E}+04$ & 4.05 \\
\hline & Trial 2 & $2.03 \mathrm{E}+05$ & 5.31 & $9.45 \mathrm{E}+04$ & 4.98 \\
\hline & Trial 3 & $1.41 \mathrm{E}+06$ & 6.15 & $1.52 \mathrm{E}+05$ & 5.18 \\
\hline & Average & $5.83 \mathrm{E}+05$ & 5.53 & $8.58 \mathrm{E}+04$ & 4.74 \\
\hline & Std Error & $4.14 \mathrm{E}+05$ & 0.31 & $4.07 \mathrm{E}+04$ & 0.35 \\
\hline & t-statistic & \multicolumn{4}{|c|}{1.307596778} \\
\hline & t-critical & \multicolumn{4}{|c|}{2.91998558} \\
\hline \multirow{7}{*}{10} & Trial 1 & $3.60 \mathrm{E}+03$ & 3.56 & $5.10 \mathrm{E}+04$ & 4.71 \\
\hline & Trial 2 & $6.05 E+04$ & 4.78 & $1.63 \mathrm{E}+04$ & 4.21 \\
\hline & Trial 3 & $1.66 \mathrm{E}+04$ & 4.22 & $1.50 \mathrm{E}+02$ & 2.18 \\
\hline & Average & $2.69 \mathrm{E}+04$ & 4.19 & $2.25 \mathrm{E}+04$ & 3.70 \\
\hline & Std Error & $1.72 \mathrm{E}+04$ & 0.35 & $1.50 \mathrm{E}+04$ & 0.77 \\
\hline & t-statistic & \multicolumn{4}{|c|}{0.163406293} \\
\hline & t-critical & \multicolumn{4}{|c|}{2.91998558} \\
\hline
\end{tabular}




\begin{tabular}{|c|c|c|c|c|c|}
\hline \multirow{7}{*}{11} & Trial 1 & $3.60 \mathrm{E}+03$ & 3.56 & $8.45 \mathrm{E}+04$ & 4.93 \\
\hline & Trial 2 & $6.05 \mathrm{E}+04$ & 4.78 & $6.95 \mathrm{E}+03$ & 3.84 \\
\hline & Trial 3 & $1.66 \mathrm{E}+04$ & 4.22 & $5.70 \mathrm{E}+03$ & 3.76 \\
\hline & Average & $2.69 \mathrm{E}+04$ & 4.19 & $3.24 \mathrm{E}+04$ & 4.17 \\
\hline & Std Error & $1.72 \mathrm{E}+04$ & 0.35 & $2.61 \mathrm{E}+04$ & 0.38 \\
\hline & t-statistic & \multicolumn{4}{|c|}{-0.138232695} \\
\hline & t-critical & \multicolumn{4}{|c|}{2.91998558} \\
\hline \multirow{7}{*}{15} & Trial 1 & $3.70 \mathrm{E}+03$ & 3.57 & $4.90 \mathrm{E}+04$ & 4.69 \\
\hline & Trial 2 & $2.25 \mathrm{E}+04$ & 4.35 & $5.30 \mathrm{E}+04$ & 4.72 \\
\hline & Trial 3 & $3.05 \mathrm{E}+03$ & 3.48 & $1.74 \mathrm{E}+04$ & 4.24 \\
\hline & Average & $9.73 \mathrm{E}+03$ & 3.80 & $3.98 \mathrm{E}+04$ & 4.55 \\
\hline & Std Error & $6.36 \mathrm{E}+03$ & 0.28 & $1.13 \mathrm{E}+04$ & 0.16 \\
\hline & t-statistic & \multicolumn{4}{|c|}{-3.35663} \\
\hline & t-critical & \multicolumn{4}{|c|}{2.91998558} \\
\hline \multirow{7}{*}{16} & Trial 1 & $3.70 \mathrm{E}+03$ & 3.57 & $7.95 \mathrm{E}+04$ & 4.90 \\
\hline & Trial 2 & $2.25 \mathrm{E}+04$ & 4.35 & $1.97 \mathrm{E}+04$ & 4.29 \\
\hline & Trial 3 & $3.05 \mathrm{E}+03$ & 3.48 & $5.55 \mathrm{E}+04$ & 4.74 \\
\hline & Average & $9.73 \mathrm{E}+03$ & 3.80 & $5.16 \mathrm{E}+04$ & 4.65 \\
\hline & Std Error & $6.36 \mathrm{E}+03$ & 0.28 & $1.74 \mathrm{E}+04$ & 0.18 \\
\hline & t-statistic & \multicolumn{4}{|c|}{-1.79436} \\
\hline & t-critical & \multicolumn{4}{|c|}{2.91998558} \\
\hline
\end{tabular}

Table F 3 Colony-forming unit (CFU) from antibacterial testing by DSF method against Arthrobacter sp. Control and treated film (coating 8) was inoculated with $10 \mu \mathrm{L}$ solution of $10^{5}$ $\mathrm{CFU}$ in $0.3 \mathrm{mM} \mathrm{KH}_{2} \mathrm{PO}_{4}$ for $1 \mathrm{~h}$

\begin{tabular}{|c|c|c|c|c|c|}
\hline \multirow{3}{*}{ Coating } & \multicolumn{2}{|c|}{ Control } & \multicolumn{2}{c|}{ Treated } \\
\cline { 3 - 6 } & & Total CFU & $\begin{array}{c}\text { Log Total } \\
(\log (\boldsymbol{C F U}))\end{array}$ & Total CFU & $\begin{array}{c}\text { Log Total } \\
(\log (\boldsymbol{C F U}))\end{array}$ \\
\hline \multirow{4}{*}{8} & Trial 1 & $1.13 \mathrm{E}+06$ & 6.05 & $<10^{1}$ & - \\
\cline { 2 - 6 } & Trial 2 & $1.15 \mathrm{E}+06$ & 6.06 & $<10^{1}$ & - \\
\cline { 2 - 6 } & Trial 3 & $1.19 \mathrm{E}+06$ & 6.07 & $<10^{1}$ & - \\
\cline { 2 - 6 } & Average & $1.16 \mathrm{E}+06$ & 6.06 & $<10^{1}$ & - \\
\cline { 2 - 6 } & Std Error & $1.61 \mathrm{E}+04$ & 0.01 & - & - \\
\cline { 2 - 6 } & t-statistic & \multicolumn{4}{|c|}{71.86075} \\
\cline { 2 - 6 } & t-critical & \multicolumn{2}{|c|}{2.91998558} \\
\hline
\end{tabular}


Appendix G: X-Ray Crystallography Data

Table G 1 Crystal data and structure refinement for $\mathbf{1 7}$

Identification code

Empirical formula

Formula weight

Temperature

Wavelength

Crystal system

Space group

Unit cell dimensions

Volume

Z

Density (calculated)

Absorption coefficient

$\mathrm{F}(000)$

Crystal size

Theta range for data collection

Index ranges

Reflections collected

Independent reflections

Completeness to theta $=67.679^{\circ}$

Absorption correction

Max. and min. transmission

Refinement method

Data / restraints / parameters

Goodness-of-fit on $\mathrm{F}^{2}$

Final $\mathrm{R}$ indices [I>2sigma(I)]

$\mathrm{R}$ indices (all data)

Absolute structure parameter

Extinction coefficient

Largest diff. peak and hole d1877_a

$\mathrm{C} 15 \mathrm{H} 24 \mathrm{ClN}$

253.80

150(2) K

$1.54178 \AA$

Orthorhombic

$\mathrm{P} 212121$

$\mathrm{a}=7.9778(2) \AA \quad \mathrm{a}=90^{\circ}$.

$\mathrm{b}=12.7633(4) \AA \quad \mathrm{b}=90^{\circ}$.

$\mathrm{c}=14.0317(5) \AA \quad \mathrm{g}=90^{\circ}$.

1428.75(8) $\AA^{3}$

4

$1.180 \mathrm{Mg} / \mathrm{m}^{3}$

$2.177 \mathrm{~mm}^{-1}$

552

$0.120 \times 0.100 \times 0.060 \mathrm{~mm}^{3}$

4.683 to $68.148^{\circ}$.

$-9<=\mathrm{h}<=9,-15<=\mathrm{k}<=15,-16<=1<=16$

21967

$2544[\mathrm{R}(\mathrm{int})=0.0774]$

$98.7 \%$

Semi-empirical from equivalents

0.7529 and 0.6064

Full-matrix least-squares on $\mathrm{F}^{2}$

2544 / 286 / 218

1.152

$\mathrm{R} 1=0.0927, \mathrm{wR} 2=0.2017$

$\mathrm{R} 1=0.1015, \mathrm{wR} 2=0.2068$

$0.103(10)$

$\mathrm{n} / \mathrm{a}$

0.356 and -0.644 e. $\AA^{-3}$ 
Table G 2 Atomic coordinated and equivalent isotropic displacement parameters for $\mathbf{1 7}$

\begin{tabular}{lclll}
\hline & $\mathrm{x}$ & $\mathrm{y}$ & $\mathrm{z}$ & $\mathrm{U}(\mathrm{eq})$ \\
\hline $\mathrm{Cl}(1)$ & & & & \\
$\mathrm{C}(2)$ & $8584(2)$ & $9467(2)$ & $4853(2)$ & $61(1)$ \\
$\mathrm{C}(3)$ & $6111(9)$ & $7052(7)$ & $3015(8)$ & $46(2)$ \\
$\mathrm{C}(4)$ & $4949(10)$ & $6325(7)$ & $2680(6)$ & $39(2)$ \\
$\mathrm{C}(5)$ & $4120(10)$ & $6482(8)$ & $1821(7)$ & $48(2)$ \\
$\mathrm{C}(6)$ & $4367(11)$ & $7380(7)$ & $1267(7)$ & $48(2)$ \\
$\mathrm{C}(7)$ & $5509(11)$ & $8123(8)$ & $1630(8)$ & $55(3)$ \\
$\mathrm{C}(8)$ & $6354(12)$ & $7959(7)$ & $2485(8)$ & $56(3)$ \\
$\mathrm{C}(9)$ & $3437(13)$ & $7464(9)$ & $336(8)$ & $59(3)$ \\
$\mathrm{C}(1)$ & $3690(13)$ & $8194(9)$ & $-296(10)$ & $77(4)$ \\
$\mathrm{N}(1)$ & $6994(9)$ & $6911(6)$ & $3937(6)$ & $42(2)$ \\
$\mathrm{C}(10)$ & $8602(11)$ & $6234(6)$ & $3821(7)$ & $43(2)$ \\
$\mathrm{C}(11)$ & $9810(13)$ & $6696(10)$ & $3119(9)$ & $46(2)$ \\
$\mathrm{C}(12)$ & $10717(17)$ & $7682(11)$ & $3370(12)$ & $55(4)$ \\
$\mathrm{C}(13)$ & $8112(14)$ & $5142(7)$ & $3495(11)$ & $44(2)$ \\
$\mathrm{C}(14)$ & $9532(18)$ & $4371(11)$ & $3395(15)$ & $59(3)$ \\
$\mathrm{C}(15)$ & $9413(13)$ & $6169(10)$ & $4804(9)$ & $46(2)$ \\
$\mathrm{C}(1 \mathrm{~A})$ & $8492(19)$ & $5516(12)$ & $5524(10)$ & $51(3)$ \\
$\mathrm{N}(1 \mathrm{~A})$ & $6994(9)$ & $6911(6)$ & $3937(6)$ & $42(2)$ \\
$\mathrm{C}(10 \mathrm{~A})$ & $8610(14)$ & $6259(8)$ & $4129(9)$ & $45(2)$ \\
$\mathrm{C}(11 \mathrm{~A})$ & $10012(14)$ & $6664(15)$ & $3495(11)$ & $47(2)$ \\
$\mathrm{C}(12 \mathrm{~A})$ & $9890(30)$ & $6405(19)$ & $2457(12)$ & $53(4)$ \\
$\mathrm{C}(13 \mathrm{~A})$ & $8309(17)$ & $5115(9)$ & $3916(15)$ & $46(2)$ \\
$\mathrm{C}(14 \mathrm{~A})$ & $9780(20)$ & $4392(12)$ & $4040(20)$ & $47(4)$ \\
$\mathrm{C}(15 \mathrm{~A})$ & $9141(17)$ & $6382(13)$ & $5157(10)$ & $43(2)$ \\
& $8150(30)$ & $5828(17)$ & $5906(12)$ & $48(4)$ \\
\hline & & & &
\end{tabular}


Table G 3 Bond lengths $[\AA]$ and angles $\left[{ }^{\circ}\right]$ for $\mathbf{1 7}$

\begin{tabular}{ll}
\hline $\mathrm{C}(2)-\mathrm{C}(7)$ & $1.390(14)$ \\
$\mathrm{C}(2)-\mathrm{C}(3)$ & $1.393(11)$ \\
$\mathrm{C}(2)-\mathrm{C}(1 \mathrm{~A})$ & $1.483(12)$ \\
$\mathrm{C}(2)-\mathrm{C}(1)$ & $1.483(12)$ \\
$\mathrm{C}(3)-\mathrm{C}(4)$ & $1.389(13)$ \\
$\mathrm{C}(3)-\mathrm{H}(3 \mathrm{~A})$ & 0.9500 \\
$\mathrm{C}(4)-\mathrm{C}(5)$ & $1.399(14)$ \\
$\mathrm{C}(4)-\mathrm{H}(4 \mathrm{~A})$ & 0.9500 \\
$\mathrm{C}(5)-\mathrm{C}(6)$ & $1.409(13)$ \\
$\mathrm{C}(5)-\mathrm{C}(8)$ & $1.507(14)$ \\
$\mathrm{C}(6)-\mathrm{C}(7)$ & $1.392(15)$ \\
$\mathrm{C}(6)-\mathrm{H}(6 \mathrm{~A})$ & 0.9500 \\
$\mathrm{C}(7)-\mathrm{H}(7 \mathrm{~A})$ & 0.9500 \\
$\mathrm{C}(8)-\mathrm{C}(9)$ & $1.302(15)$ \\
$\mathrm{C}(8)-\mathrm{H}(8 \mathrm{~A})$ & 0.9500 \\
$\mathrm{C}(9)-\mathrm{H}(9 \mathrm{~A})$ & 0.9500 \\
$\mathrm{C}(9)-\mathrm{H}(9 \mathrm{~B})$ & 0.9500 \\
$\mathrm{C}(1)-\mathrm{N}(1)$ & $1.555(11)$ \\
$\mathrm{C}(1)-\mathrm{H}(1 \mathrm{~A})$ & 0.9900 \\
$\mathrm{C}(1)-\mathrm{H}(1 \mathrm{~B})$ & 0.9900 \\
$\mathrm{~N}(1)-\mathrm{C}(10)$ & $1.499(10)$ \\
$\mathrm{N}(1)-\mathrm{C}(12)$ & $1.518(9)$ \\
$\mathrm{N}(1)-\mathrm{C}(14)$ & $1.525(10)$ \\
$\mathrm{C}(10)-\mathrm{C}(11)$ & $1.494(12)$ \\
$\mathrm{C}(10)-\mathrm{H}(10 \mathrm{~A})$ & 0.9900 \\
$\mathrm{C}(10)-\mathrm{H}(10 \mathrm{D})$ & 0.9900 \\
$\mathrm{C}(11)-\mathrm{H}(11 \mathrm{~A})$ & 0.9800 \\
$\mathrm{C}(11)-\mathrm{H}(11 \mathrm{~B})$ & 0.9800 \\
$\mathrm{C}(11)-\mathrm{H}(11 \mathrm{C})$ & 0.9800 \\
$\mathrm{C}(12)-\mathrm{C}(13)$ & $1.507(11)$ \\
$\mathrm{C}(12)-\mathrm{H}(12 \mathrm{~A})$ & 0.9900 \\
$\mathrm{C}(12)-\mathrm{H}(12 \mathrm{~B})$ & 0.9900 \\
$\mathrm{C}(13)-\mathrm{H}(13 \mathrm{~A})$ & 0.9800 \\
&
\end{tabular}




\begin{tabular}{|c|c|}
\hline $\mathrm{C}(13)-\mathrm{H}(13 \mathrm{~B})$ & 0.9800 \\
\hline C(13)-H(13C) & 0.9800 \\
\hline $\mathrm{C}(14)-\mathrm{C}(15)$ & $1.501(12)$ \\
\hline $\mathrm{C}(14)-\mathrm{H}(14 \mathrm{~A})$ & 0.9900 \\
\hline C(14)-H(14B) & 0.9900 \\
\hline $\mathrm{C}(15)-\mathrm{H}(15 \mathrm{~A})$ & 0.9800 \\
\hline $\mathrm{C}(15)-\mathrm{H}(15 \mathrm{~B})$ & 0.9800 \\
\hline $\mathrm{C}(15)-\mathrm{H}(15 \mathrm{C})$ & 0.9800 \\
\hline $\mathrm{C}(1 \mathrm{~A})-\mathrm{N}(1 \mathrm{~A})$ & $1.558(12)$ \\
\hline $\mathrm{C}(1 \mathrm{~A})-\mathrm{H}(1 \mathrm{AA})$ & 0.9900 \\
\hline $\mathrm{C}(1 \mathrm{~A})-\mathrm{H}(1 \mathrm{AB})$ & 0.9900 \\
\hline $\mathrm{N}(1 \mathrm{~A})-\mathrm{C}(12 \mathrm{~A})$ & $1.510(10)$ \\
\hline $\mathrm{N}(1 \mathrm{~A})-\mathrm{C}(14 \mathrm{~A})$ & $1.512(10)$ \\
\hline $\mathrm{N}(1 \mathrm{~A})-\mathrm{C}(10 \mathrm{~A})$ & $1.520(10)$ \\
\hline$C(10 A)-C(11 A)$ & $1.497(12)$ \\
\hline $\mathrm{C}(10 \mathrm{~A})-\mathrm{H}(10 \mathrm{~B})$ & 0.9900 \\
\hline $\mathrm{C}(10 \mathrm{~A})-\mathrm{H}(10 \mathrm{C})$ & 0.9900 \\
\hline $\mathrm{C}(11 \mathrm{~A})-\mathrm{H}(11 \mathrm{D})$ & 0.9800 \\
\hline $\mathrm{C}(11 \mathrm{~A})-\mathrm{H}(11 \mathrm{E})$ & 0.9800 \\
\hline $\mathrm{C}(11 \mathrm{~A})-\mathrm{H}(11 \mathrm{~F})$ & 0.9800 \\
\hline$C(12 A)-C(13 A)$ & $1.506(12)$ \\
\hline $\mathrm{C}(12 \mathrm{~A})-\mathrm{H}(12 \mathrm{C})$ & 0.9900 \\
\hline $\mathrm{C}(12 \mathrm{~A})-\mathrm{H}(12 \mathrm{D})$ & 0.9900 \\
\hline $\mathrm{C}(13 \mathrm{~A})-\mathrm{H}(13 \mathrm{D})$ & 0.9800 \\
\hline $\mathrm{C}(13 \mathrm{~A})-\mathrm{H}(13 \mathrm{E})$ & 0.9800 \\
\hline $\mathrm{C}(13 \mathrm{~A})-\mathrm{H}(13 \mathrm{~F})$ & 0.9800 \\
\hline$C(14 A)-C(15 A)$ & $1.493(12)$ \\
\hline $\mathrm{C}(14 \mathrm{~A})-\mathrm{H}(14 \mathrm{C})$ & 0.9900 \\
\hline $\mathrm{C}(14 \mathrm{~A})-\mathrm{H}(14 \mathrm{D})$ & 0.9900 \\
\hline $\mathrm{C}(15 \mathrm{~A})-\mathrm{H}(15 \mathrm{D})$ & 0.9800 \\
\hline $\mathrm{C}(15 \mathrm{~A})-\mathrm{H}(15 \mathrm{G})$ & 0.9800 \\
\hline $\mathrm{C}(15 \mathrm{~A})-\mathrm{H}(15 \mathrm{E})$ & 0.9800 \\
\hline $\mathrm{C}(7)-\mathrm{C}(2)-\mathrm{C}(3)$ & 117.8(9) \\
\hline$C(7)-C(2)-C(1 A)$ & $120.1(8)$ \\
\hline $\mathrm{C}(3)-\mathrm{C}(2)-\mathrm{C}(1 \mathrm{~A})$ & $122.0(9)$ \\
\hline
\end{tabular}




\begin{tabular}{|c|c|}
\hline$C(7)-C(2)-C(1)$ & $120.1(8)$ \\
\hline $\mathrm{C}(3)-\mathrm{C}(2)-\mathrm{C}(1)$ & $122.0(9)$ \\
\hline $\mathrm{C}(4)-\mathrm{C}(3)-\mathrm{C}(2)$ & $120.9(9)$ \\
\hline $\mathrm{C}(4)-\mathrm{C}(3)-\mathrm{H}(3 \mathrm{~A})$ & 119.5 \\
\hline $\mathrm{C}(2)-\mathrm{C}(3)-\mathrm{H}(3 \mathrm{~A})$ & 119.5 \\
\hline$C(3)-C(4)-C(5)$ & $122.2(8)$ \\
\hline $\mathrm{C}(3)-\mathrm{C}(4)-\mathrm{H}(4 \mathrm{~A})$ & 118.9 \\
\hline $\mathrm{C}(5)-\mathrm{C}(4)-\mathrm{H}(4 \mathrm{~A})$ & 118.9 \\
\hline $\mathrm{C}(4)-\mathrm{C}(5)-\mathrm{C}(6)$ & 116.2(9) \\
\hline$C(4)-C(5)-C(8)$ & $118.0(8)$ \\
\hline$C(6)-C(5)-C(8)$ & $125.7(10)$ \\
\hline$C(7)-C(6)-C(5)$ & $121.6(10)$ \\
\hline$C(7)-C(6)-H(6 A)$ & 119.2 \\
\hline $\mathrm{C}(5)-\mathrm{C}(6)-\mathrm{H}(6 \mathrm{~A})$ & 119.2 \\
\hline$C(2)-C(7)-C(6)$ & 121.2(9) \\
\hline $\mathrm{C}(2)-\mathrm{C}(7)-\mathrm{H}(7 \mathrm{~A})$ & 119.4 \\
\hline $\mathrm{C}(6)-\mathrm{C}(7)-\mathrm{H}(7 \mathrm{~A})$ & 119.4 \\
\hline $\mathrm{C}(9)-\mathrm{C}(8)-\mathrm{C}(5)$ & $124.4(10)$ \\
\hline $\mathrm{C}(9)-\mathrm{C}(8)-\mathrm{H}(8 \mathrm{~A})$ & 117.8 \\
\hline $\mathrm{C}(5)-\mathrm{C}(8)-\mathrm{H}(8 \mathrm{~A})$ & 117.8 \\
\hline $\mathrm{C}(8)-\mathrm{C}(9)-\mathrm{H}(9 \mathrm{~A})$ & 120.0 \\
\hline $\mathrm{C}(8)-\mathrm{C}(9)-\mathrm{H}(9 \mathrm{~B})$ & 120.0 \\
\hline $\mathrm{H}(9 \mathrm{~A})-\mathrm{C}(9)-\mathrm{H}(9 \mathrm{~B})$ & 120.0 \\
\hline $\mathrm{C}(2)-\mathrm{C}(1)-\mathrm{N}(1)$ & $111.6(6)$ \\
\hline $\mathrm{C}(2)-\mathrm{C}(1)-\mathrm{H}(1 \mathrm{~A})$ & 109.3 \\
\hline $\mathrm{N}(1)-\mathrm{C}(1)-\mathrm{H}(1 \mathrm{~A})$ & 109.3 \\
\hline $\mathrm{C}(2)-\mathrm{C}(1)-\mathrm{H}(1 \mathrm{~B})$ & 109.3 \\
\hline $\mathrm{N}(1)-\mathrm{C}(1)-\mathrm{H}(1 \mathrm{~B})$ & 109.3 \\
\hline $\mathrm{H}(1 \mathrm{~A})-\mathrm{C}(1)-\mathrm{H}(1 \mathrm{~B})$ & 108.0 \\
\hline $\mathrm{C}(10)-\mathrm{N}(1)-\mathrm{C}(12)$ & $109.2(6)$ \\
\hline $\mathrm{C}(10)-\mathrm{N}(1)-\mathrm{C}(14)$ & $110.1(7)$ \\
\hline $\mathrm{C}(12)-\mathrm{N}(1)-\mathrm{C}(14)$ & $109.4(6)$ \\
\hline $\mathrm{C}(10)-\mathrm{N}(1)-\mathrm{C}(1)$ & $112.3(6)$ \\
\hline $\mathrm{C}(12)-\mathrm{N}(1)-\mathrm{C}(1)$ & 109.2(6) \\
\hline $\mathrm{C}(14)-\mathrm{N}(1)-\mathrm{C}(1)$ & $106.6(6)$ \\
\hline $\mathrm{C}(11)-\mathrm{C}(10)-\mathrm{N}(1)$ & 119.2(9) \\
\hline
\end{tabular}




$\begin{array}{ll}\mathrm{C}(11)-\mathrm{C}(10)-\mathrm{H}(10 \mathrm{~A}) & 107.5 \\ \mathrm{~N}(1)-\mathrm{C}(10)-\mathrm{H}(10 \mathrm{~A}) & 107.5 \\ \mathrm{C}(11)-\mathrm{C}(10)-\mathrm{H}(10 \mathrm{D}) & 107.5 \\ \mathrm{~N}(1)-\mathrm{C}(10)-\mathrm{H}(10 \mathrm{D}) & 107.5 \\ \mathrm{H}(10 \mathrm{~A})-\mathrm{C}(10)-\mathrm{H}(10 \mathrm{D}) & 107.0 \\ \mathrm{C}(10)-\mathrm{C}(11)-\mathrm{H}(11 \mathrm{~A}) & 109.5 \\ \mathrm{C}(10)-\mathrm{C}(11)-\mathrm{H}(11 \mathrm{~B}) & 109.5 \\ \mathrm{H}(11 \mathrm{~A})-\mathrm{C}(11)-\mathrm{H}(11 \mathrm{~B}) & 109.5 \\ \mathrm{C}(10)-\mathrm{C}(11)-\mathrm{H}(11 \mathrm{C}) & 109.5 \\ \mathrm{H}(11 \mathrm{~A})-\mathrm{C}(11)-\mathrm{H}(11 \mathrm{C}) & 109.5 \\ \mathrm{H}(11 \mathrm{~B})-\mathrm{C}(11)-\mathrm{H}(11 \mathrm{C}) & 109.5 \\ \mathrm{C}(13)-\mathrm{C}(12)-\mathrm{N}(1) & 115.7(8) \\ \mathrm{C}(13)-\mathrm{C}(12)-\mathrm{H}(12 \mathrm{~A}) & 108.4 \\ \mathrm{~N}(1)-\mathrm{C}(12)-\mathrm{H}(12 \mathrm{~A}) & 108.4 \\ \mathrm{C}(13)-\mathrm{C}(12)-\mathrm{H}(12 \mathrm{~B}) & 108.4 \\ \mathrm{~N}(1)-\mathrm{C}(12)-\mathrm{H}(12 \mathrm{~B}) & 108.4 \\ \mathrm{H}(12 \mathrm{~A})-\mathrm{C}(12)-\mathrm{H}(12 \mathrm{~B}) & 107.4 \\ \mathrm{C}(12)-\mathrm{C}(13)-\mathrm{H}(13 \mathrm{~A}) & 109.5 \\ \mathrm{C}(12)-\mathrm{C}(13)-\mathrm{H}(13 \mathrm{~B}) & 109.5 \\ \mathrm{H}(13 \mathrm{~A})-\mathrm{C}(13)-\mathrm{H}(13 \mathrm{~B}) & 109.5 \\ \mathrm{C}(12)-\mathrm{C}(13)-\mathrm{H}(13 \mathrm{C}) & 109.5 \\ \mathrm{H}(13 \mathrm{~A})-\mathrm{C}(13)-\mathrm{H}(13 \mathrm{C}) & 109.5 \\ \mathrm{H}(13 \mathrm{~B})-\mathrm{C}(13)-\mathrm{H}(13 \mathrm{C}) & 109.5 \\ \mathrm{C}(15)-\mathrm{C}(14)-\mathrm{N}(1) & 115.5(8) \\ \mathrm{C}(15)-\mathrm{C}(14)-\mathrm{H}(14 \mathrm{~A}) & 108.4 \\ \mathrm{~N}(1)-\mathrm{C}(14)-\mathrm{H}(14 \mathrm{~A}) & 108.4 \\ \mathrm{C}(15)-\mathrm{C}(14)-\mathrm{H}(14 \mathrm{~B}) & 108.4 \\ \mathrm{~N}(1)-\mathrm{C}(14)-\mathrm{H}(14 \mathrm{~B}) & 108.4 \\ \mathrm{H}(14 \mathrm{~A})-\mathrm{C}(14)-\mathrm{H}(14 \mathrm{~B}) & 107.5 \\ \mathrm{C}(14)-\mathrm{C}(15)-\mathrm{H}(15 \mathrm{~A}) & 109.5 \\ \mathrm{C}(14)-\mathrm{C}(15)-\mathrm{H}(15 \mathrm{~B}) & 109.5 \\ \mathrm{H}(15 \mathrm{~A})-\mathrm{C}(15)-\mathrm{H}(15 \mathrm{~B}) & 109.5 \\ \mathrm{C}(14)-\mathrm{H}(15 \mathrm{C}) & 109.5 \\ & 109.5 \\ \mathrm{H}) & 127.5(7)\end{array}$




\begin{tabular}{|c|c|}
\hline $\mathrm{C}(2)-\mathrm{C}(1 \mathrm{~A})-\mathrm{H}(1 \mathrm{AA})$ & 105.4 \\
\hline $\mathrm{N}(1 \mathrm{~A})-\mathrm{C}(1 \mathrm{~A})-\mathrm{H}(1 \mathrm{AA})$ & 105.4 \\
\hline $\mathrm{C}(2)-\mathrm{C}(1 \mathrm{~A})-\mathrm{H}(1 \mathrm{AB})$ & 105.4 \\
\hline $\mathrm{N}(1 \mathrm{~A})-\mathrm{C}(1 \mathrm{~A})-\mathrm{H}(1 \mathrm{AB})$ & 105.4 \\
\hline $\mathrm{H}(1 \mathrm{AA})-\mathrm{C}(1 \mathrm{~A})-\mathrm{H}(1 \mathrm{AB})$ & 106.0 \\
\hline$C(12 A)-N(1 A)-C(14 A)$ & $109.5(8)$ \\
\hline $\mathrm{C}(12 \mathrm{~A})-\mathrm{N}(1 \mathrm{~A})-\mathrm{C}(10 \mathrm{~A})$ & $109.3(7)$ \\
\hline$C(14 A)-N(1 A)-C(10 A)$ & $108.5(8)$ \\
\hline $\mathrm{C}(12 \mathrm{~A})-\mathrm{N}(1 \mathrm{~A})-\mathrm{C}(1 \mathrm{~A})$ & $110.5(7)$ \\
\hline $\mathrm{C}(14 \mathrm{~A})-\mathrm{N}(1 \mathrm{~A})-\mathrm{C}(1 \mathrm{~A})$ & $110.0(7)$ \\
\hline $\mathrm{C}(10 \mathrm{~A})-\mathrm{N}(1 \mathrm{~A})-\mathrm{C}(1 \mathrm{~A})$ & $109.0(7)$ \\
\hline$C(11 A)-C(10 A)-N(1 A)$ & $116.5(10)$ \\
\hline$C(11 A)-C(10 A)-H(10 B)$ & 108.2 \\
\hline $\mathrm{N}(1 \mathrm{~A})-\mathrm{C}(10 \mathrm{~A})-\mathrm{H}(10 \mathrm{~B})$ & 108.2 \\
\hline$C(11 A)-C(10 A)-H(10 C)$ & 108.2 \\
\hline $\mathrm{N}(1 \mathrm{~A})-\mathrm{C}(10 \mathrm{~A})-\mathrm{H}(10 \mathrm{C})$ & 108.2 \\
\hline$H(10 B)-C(10 A)-H(10 C)$ & 107.3 \\
\hline$C(10 A)-C(11 A)-H(11 D)$ & 109.5 \\
\hline $\mathrm{C}(10 \mathrm{~A})-\mathrm{C}(11 \mathrm{~A})-\mathrm{H}(11 \mathrm{E})$ & 109.5 \\
\hline$H(11 D)-C(11 A)-H(11 E)$ & 109.5 \\
\hline$C(10 A)-C(11 A)-H(11 F)$ & 109.5 \\
\hline $\mathrm{H}(11 \mathrm{D})-\mathrm{C}(11 \mathrm{~A})-\mathrm{H}(11 \mathrm{~F})$ & 109.5 \\
\hline $\mathrm{H}(11 \mathrm{E})-\mathrm{C}(11 \mathrm{~A})-\mathrm{H}(11 \mathrm{~F})$ & 109.5 \\
\hline $\mathrm{C}(13 \mathrm{~A})-\mathrm{C}(12 \mathrm{~A})-\mathrm{N}(1 \mathrm{~A})$ & $116.5(9)$ \\
\hline $\mathrm{C}(13 \mathrm{~A})-\mathrm{C}(12 \mathrm{~A})-\mathrm{H}(12 \mathrm{C})$ & 108.2 \\
\hline $\mathrm{N}(1 \mathrm{~A})-\mathrm{C}(12 \mathrm{~A})-\mathrm{H}(12 \mathrm{C})$ & 108.2 \\
\hline $\mathrm{C}(13 \mathrm{~A})-\mathrm{C}(12 \mathrm{~A})-\mathrm{H}(12 \mathrm{D})$ & 108.2 \\
\hline $\mathrm{N}(1 \mathrm{~A})-\mathrm{C}(12 \mathrm{~A})-\mathrm{H}(12 \mathrm{D})$ & 108.2 \\
\hline $\mathrm{H}(12 \mathrm{C})-\mathrm{C}(12 \mathrm{~A})-\mathrm{H}(12 \mathrm{D})$ & 107.3 \\
\hline $\mathrm{C}(12 \mathrm{~A})-\mathrm{C}(13 \mathrm{~A})-\mathrm{H}(13 \mathrm{D})$ & 109.5 \\
\hline $\mathrm{C}(12 \mathrm{~A})-\mathrm{C}(13 \mathrm{~A})-\mathrm{H}(13 \mathrm{E})$ & 109.5 \\
\hline $\mathrm{H}(13 \mathrm{D})-\mathrm{C}(13 \mathrm{~A})-\mathrm{H}(13 \mathrm{E})$ & 109.5 \\
\hline $\mathrm{C}(12 \mathrm{~A})-\mathrm{C}(13 \mathrm{~A})-\mathrm{H}(13 \mathrm{~F})$ & 109.5 \\
\hline $\mathrm{H}(13 \mathrm{D})-\mathrm{C}(13 \mathrm{~A})-\mathrm{H}(13 \mathrm{~F})$ & 109.5 \\
\hline $\mathrm{H}(13 \mathrm{E})-\mathrm{C}(13 \mathrm{~A})-\mathrm{H}(13 \mathrm{~F})$ & 109.5 \\
\hline $\mathrm{C}(15 \mathrm{~A})-\mathrm{C}(14 \mathrm{~A})-\mathrm{N}(1 \mathrm{~A})$ & $118.3(10)$ \\
\hline
\end{tabular}


C(15A)-C(14A)-H(14C) 107.7

$\mathrm{N}(1 \mathrm{~A})-\mathrm{C}(14 \mathrm{~A})-\mathrm{H}(14 \mathrm{C}) \quad 107.7$

C(15A)-C(14A)-H(14D) 107.7

$\mathrm{N}(1 \mathrm{~A})-\mathrm{C}(14 \mathrm{~A})-\mathrm{H}(14 \mathrm{D}) \quad 107.7$

$\mathrm{H}(14 \mathrm{C})-\mathrm{C}(14 \mathrm{~A})-\mathrm{H}(14 \mathrm{D}) \quad 107.1$

$\mathrm{C}(14 \mathrm{~A})-\mathrm{C}(15 \mathrm{~A})-\mathrm{H}(15 \mathrm{D}) \quad 109.5$

$\mathrm{C}(14 \mathrm{~A})-\mathrm{C}(15 \mathrm{~A})-\mathrm{H}(15 \mathrm{G}) \quad 109.5$

$\mathrm{H}(15 \mathrm{D})-\mathrm{C}(15 \mathrm{~A})-\mathrm{H}(15 \mathrm{G}) \quad 109.5$

$\mathrm{C}(14 \mathrm{~A})-\mathrm{C}(15 \mathrm{~A})-\mathrm{H}(15 \mathrm{E}) \quad 109.5$

$\mathrm{H}(15 \mathrm{D})-\mathrm{C}(15 \mathrm{~A})-\mathrm{H}(15 \mathrm{E}) \quad 109.5$

$\mathrm{H}(15 \mathrm{G})-\mathrm{C}(15 \mathrm{~A})-\mathrm{H}(15 \mathrm{E}) \quad 109.5$

Table G 4 Anisotropic displacement parameters for $\mathbf{1 7}$

\begin{tabular}{lcccccc}
\hline & $\mathrm{U}^{11}$ & $\mathrm{U}^{22}$ & $\mathrm{U}^{33}$ & $\mathrm{U}^{23}$ & $\mathrm{U}^{13}$ & $\mathrm{U}^{12}$ \\
\hline $\mathrm{Cl}(1)$ & $22(1)$ & $37(1)$ & $123(2)$ & $-4(1)$ & $3(1)$ & $-2(1)$ \\
$\mathrm{C}(2)$ & $19(4)$ & $34(4)$ & $84(7)$ & $-25(5)$ & $2(4)$ & $7(3)$ \\
$\mathrm{C}(3)$ & $28(4)$ & $49(5)$ & $41(5)$ & $-19(4)$ & $3(4)$ & $-4(4)$ \\
$\mathrm{C}(4)$ & $30(4)$ & $60(6)$ & $55(6)$ & $-24(5)$ & $0(4)$ & $-7(4)$ \\
$\mathrm{C}(5)$ & $30(4)$ & $48(5)$ & $64(6)$ & $-17(5)$ & $4(4)$ & $-4(4)$ \\
$\mathrm{C}(6)$ & $36(5)$ & $46(5)$ & $84(8)$ & $-16(5)$ & $13(5)$ & $6(4)$ \\
$\mathrm{C}(7)$ & $29(4)$ & $41(5)$ & $97(8)$ & $-23(5)$ & $-10(6)$ & $7(4)$ \\
$\mathrm{C}(8)$ & $40(5)$ & $71(7)$ & $66(7)$ & $-7(5)$ & $8(5)$ & $-4(5)$ \\
$\mathrm{C}(9)$ & $40(5)$ & $81(8)$ & $110(10)$ & $-13(7)$ & $-1(7)$ & $-4(6)$ \\
$\mathrm{C}(1)$ & $20(3)$ & $41(3)$ & $67(4)$ & $-30(3)$ & $-9(3)$ & $11(2)$ \\
$\mathrm{N}(1)$ & $21(2)$ & $42(3)$ & $66(4)$ & $-29(3)$ & $-9(3)$ & $11(3)$ \\
$\mathrm{C}(10)$ & $22(3)$ & $47(4)$ & $70(5)$ & $-25(4)$ & $-10(4)$ & $8(4)$ \\
$\mathrm{C}(11)$ & $29(6)$ & $65(7)$ & $72(8)$ & $-30(7)$ & $5(6)$ & $-2(6)$ \\
$\mathrm{C}(12)$ & $25(4)$ & $39(4)$ & $67(5)$ & $-29(4)$ & $-9(4)$ & $11(3)$ \\
$\mathrm{C}(13)$ & $43(6)$ & $55(6)$ & $80(8)$ & $-22(7)$ & $-9(7)$ & $19(6)$ \\
$\mathrm{C}(14)$ & $24(4)$ & $47(4)$ & $67(5)$ & $-24(4)$ & $-11(4)$ & $13(4)$ \\
$\mathrm{C}(15)$ & $32(6)$ & $55(7)$ & $66(8)$ & $-10(6)$ & $-6(6)$ & $19(6)$ \\
& & & & & &
\end{tabular}




$\begin{array}{lllllll}\mathrm{C}(1 \mathrm{~A}) & 20(3) & 41(3) & 67(4) & -30(3) & -9(3) & 11(2) \\ \mathrm{N}(1 \mathrm{~A}) & 22(3) & 43(3) & 69(4) & -26(3) & -9(3) & 11(3) \\ \mathrm{C}(10 \mathrm{~A}) & 23(4) & 46(4) & 71(5) & -24(5) & -9(4) & 9(4) \\ \mathrm{C}(11 \mathrm{~A}) & 32(7) & 55(8) & 73(9) & -18(8) & -6(8) & 9(8) \\ \mathrm{C}(12 \mathrm{~A}) & 25(4) & 41(4) & 70(5) & -29(5) & -8(4) & 10(4) \\ \mathrm{C}(13 \mathrm{~A}) & 31(7) & 39(7) & 71(8) & -27(8) & -15(7) & 18(6) \\ \mathrm{C}(14 \mathrm{~A}) & 20(4) & 41(4) & 67(5) & -26(4) & -9(4) & 16(4) \\ \mathrm{C}(15 \mathrm{~A}) & 30(7) & 42(8) & 73(9) & -13(7) & -9(7) & 17(7)\end{array}$

Table G 5 Hydrogen coordinates and isotropic displacement parameters for $\mathbf{1 7}$

\begin{tabular}{|c|c|c|c|c|}
\hline & $\mathrm{x}$ & $\mathrm{y}$ & $\mathrm{z}$ & $\mathrm{U}(\mathrm{eq})$ \\
\hline $\mathrm{H}(3 \mathrm{~A})$ & 4721 & 5713 & 3044 & 47 \\
\hline $\mathrm{H}(4 \mathrm{~A})$ & 3361 & 5961 & 1602 & 58 \\
\hline $\mathrm{H}(6 \mathrm{~A})$ & 5706 & 8750 & 1283 & 66 \\
\hline $\mathrm{H}(7 \mathrm{~A})$ & 7110 & 8478 & 2711 & 67 \\
\hline $\mathrm{H}(8 \mathrm{~A})$ & 2610 & 6949 & 200 & 71 \\
\hline $\mathrm{H}(9 \mathrm{~A})$ & 4507 & 8721 & -183 & 92 \\
\hline $\mathrm{H}(9 \mathrm{~B})$ & 3058 & 8199 & -869 & 92 \\
\hline $\mathrm{H}(1 \mathrm{~A})$ & 6231 & 6569 & 4397 & 51 \\
\hline $\mathrm{H}(1 \mathrm{~B})$ & 7304 & 7606 & 4196 & 51 \\
\hline $\mathrm{H}(10 \mathrm{~A})$ & 10664 & 6155 & 2976 & 55 \\
\hline $\mathrm{H}(10 \mathrm{D})$ & 9186 & 6827 & 2521 & 55 \\
\hline $\mathrm{H}(11 \mathrm{~A})$ & 11454 & 7883 & 2843 & 83 \\
\hline $\mathrm{H}(11 \mathrm{~B})$ & 9903 & 8242 & 3488 & 83 \\
\hline $\mathrm{H}(11 \mathrm{C})$ & 11389 & 7567 & 3946 & 83 \\
\hline $\mathrm{H}(12 \mathrm{~A})$ & 7292 & 4854 & 3955 & 52 \\
\hline $\mathrm{H}(12 \mathrm{~B})$ & 7539 & 5202 & 2871 & 52 \\
\hline $\mathrm{H}(13 \mathrm{~A})$ & 9090 & 3694 & 3182 & 89 \\
\hline $\mathrm{H}(13 \mathrm{~B})$ & 10339 & 4634 & 2926 & 89 \\
\hline $\mathrm{H}(13 \mathrm{C})$ & 10091 & 4285 & 4012 & 89 \\
\hline $\mathrm{H}(14 \mathrm{~A})$ & 10557 & 5880 & 4730 & 55 \\
\hline
\end{tabular}




\begin{tabular}{lrrll}
$\mathrm{H}(14 \mathrm{~B})$ & 9525 & 6889 & 5060 & 55 \\
$\mathrm{H}(15 \mathrm{~A})$ & 9106 & 5522 & 6129 & 77 \\
$\mathrm{H}(15 \mathrm{~B})$ & 7367 & 5805 & 5621 & 77 \\
$\mathrm{H}(15 \mathrm{C})$ & 8401 & 4795 & 5291 & 77 \\
$\mathrm{H}(1 \mathrm{AA})$ & 6141 & 6630 & 4381 & 51 \\
$\mathrm{H}(1 \mathrm{AB})$ & 7252 & 7628 & 4163 & 51 \\
$\mathrm{H}(10 \mathrm{~B})$ & 10057 & 7436 & 3559 & 56 \\
$\mathrm{H}(10 \mathrm{C})$ & 11086 & 6383 & 3738 & 56 \\
$\mathrm{H}(11 \mathrm{D})$ & 10853 & 6703 & 2119 & 80 \\
$\mathrm{H}(11 \mathrm{E})$ & 9884 & 5642 & 2375 & 80 \\
$\mathrm{H}(11 \mathrm{~F})$ & 8851 & 6699 & 2196 & 80 \\
$\mathrm{H}(12 \mathrm{C})$ & 7394 & 4865 & 4335 & 55 \\
$\mathrm{H}(12 \mathrm{D})$ & 7910 & 5055 & 3251 & 55 \\
$\mathrm{H}(13 \mathrm{D})$ & 9448 & 3674 & 3882 & 71 \\
$\mathrm{H}(13 \mathrm{E})$ & 10692 & 4611 & 3612 & 71 \\
$\mathrm{H}(13 \mathrm{~F})$ & 10175 & 4420 & 4700 & 71 \\
$\mathrm{H}(14 \mathrm{C})$ & 10319 & 6145 & 5209 & 51 \\
$\mathrm{H}(14 \mathrm{D})$ & 9127 & 7140 & 5310 & 51 \\
$\mathrm{H}(15 \mathrm{D})$ & 8633 & 5977 & 6534 & 73 \\
$\mathrm{H}(15 \mathrm{G})$ & 6987 & 6071 & 5889 & 73 \\
$\mathrm{H}(15 \mathrm{E})$ & 8184 & 5071 & 5788 & 73 \\
\hline
\end{tabular}

Table G 6 Torsion angles $\left[{ }^{\circ}\right]$ for $\mathbf{1 7}$

\begin{tabular}{lc}
\hline $\mathrm{C}(7)-\mathrm{C}(2)-\mathrm{C}(3)-\mathrm{C}(4)$ & $2.9(12)$ \\
$\mathrm{C}(1 \mathrm{~A})-\mathrm{C}(2)-\mathrm{C}(3)-\mathrm{C}(4)$ & $179.1(8)$ \\
$\mathrm{C}(1)-\mathrm{C}(2)-\mathrm{C}(3)-\mathrm{C}(4)$ & $179.1(8)$ \\
$\mathrm{C}(2)-\mathrm{C}(3)-\mathrm{C}(4)-\mathrm{C}(5)$ & $-1.9(13)$ \\
$\mathrm{C}(3)-\mathrm{C}(4)-\mathrm{C}(5)-\mathrm{C}(6)$ & $0.0(13)$ \\
$\mathrm{C}(3)-\mathrm{C}(4)-\mathrm{C}(5)-\mathrm{C}(8)$ & $178.6(8)$ \\
$\mathrm{C}(4)-\mathrm{C}(5)-\mathrm{C}(6)-\mathrm{C}(7)$ & $0.8(13)$ \\
$\mathrm{C}(8)-\mathrm{C}(5)-\mathrm{C}(6)-\mathrm{C}(7)$ & $-177.7(9)$ \\
$\mathrm{C}(3)-\mathrm{C}(2)-\mathrm{C}(7)-\mathrm{C}(6)$ & $-2.2(13)$
\end{tabular}




\begin{tabular}{|c|c|}
\hline$C(1 A)-C(2)-C(7)-C(6)$ & $-178.4(8)$ \\
\hline $\mathrm{C}(1)-\mathrm{C}(2)-\mathrm{C}(7)-\mathrm{C}(6)$ & $-178.4(8)$ \\
\hline$C(5)-C(6)-C(7)-C(2)$ & $0.3(14)$ \\
\hline$C(4)-C(5)-C(8)-C(9)$ & $-171.9(10)$ \\
\hline $\mathrm{C}(6)-\mathrm{C}(5)-\mathrm{C}(8)-\mathrm{C}(9)$ & $6.6(16)$ \\
\hline $\mathrm{C}(7)-\mathrm{C}(2)-\mathrm{C}(1)-\mathrm{N}(1)$ & $-97.7(9)$ \\
\hline $\mathrm{C}(3)-\mathrm{C}(2)-\mathrm{C}(1)-\mathrm{N}(1)$ & $86.2(9)$ \\
\hline $\mathrm{C}(2)-\mathrm{C}(1)-\mathrm{N}(1)-\mathrm{C}(10)$ & $58.0(9)$ \\
\hline $\mathrm{C}(2)-\mathrm{C}(1)-\mathrm{N}(1)-\mathrm{C}(12)$ & $-63.2(9)$ \\
\hline $\mathrm{C}(2)-\mathrm{C}(1)-\mathrm{N}(1)-\mathrm{C}(14)$ & $178.7(7)$ \\
\hline $\mathrm{C}(12)-\mathrm{N}(1)-\mathrm{C}(10)-\mathrm{C}(11)$ & $-168.9(11)$ \\
\hline $\mathrm{C}(14)-\mathrm{N}(1)-\mathrm{C}(10)-\mathrm{C}(11)$ & $-48.7(14)$ \\
\hline $\mathrm{C}(1)-\mathrm{N}(1)-\mathrm{C}(10)-\mathrm{C}(11)$ & $69.9(13)$ \\
\hline $\mathrm{C}(10)-\mathrm{N}(1)-\mathrm{C}(12)-\mathrm{C}(13)$ & $59.7(15)$ \\
\hline $\mathrm{C}(14)-\mathrm{N}(1)-\mathrm{C}(12)-\mathrm{C}(13)$ & $-60.9(14)$ \\
\hline $\mathrm{C}(1)-\mathrm{N}(1)-\mathrm{C}(12)-\mathrm{C}(13)$ & $-177.2(12)$ \\
\hline C(10)-N(1)-C(14)-C(15) & $-167.4(10)$ \\
\hline C(12)-N(1)-C(14)-C(15) & $-47.4(13)$ \\
\hline $\mathrm{C}(1)-\mathrm{N}(1)-\mathrm{C}(14)-\mathrm{C}(15)$ & $70.5(11)$ \\
\hline $\mathrm{C}(7)-\mathrm{C}(2)-\mathrm{C}(1 \mathrm{~A})-\mathrm{N}(1 \mathrm{~A})$ & $-99.9(10)$ \\
\hline $\mathrm{C}(3)-\mathrm{C}(2)-\mathrm{C}(1 \mathrm{~A})-\mathrm{N}(1 \mathrm{~A})$ & $84.1(11)$ \\
\hline $\mathrm{C}(2)-\mathrm{C}(1 \mathrm{~A})-\mathrm{N}(1 \mathrm{~A})-\mathrm{C}(12 \mathrm{~A})$ & $-62.4(12)$ \\
\hline $\mathrm{C}(2)-\mathrm{C}(1 \mathrm{~A})-\mathrm{N}(1 \mathrm{~A})-\mathrm{C}(14 \mathrm{~A})$ & $176.5(9)$ \\
\hline $\mathrm{C}(2)-\mathrm{C}(1 \mathrm{~A})-\mathrm{N}(1 \mathrm{~A})-\mathrm{C}(10 \mathrm{~A})$ & $57.7(11)$ \\
\hline $\mathrm{C}(12 \mathrm{~A})-\mathrm{N}(1 \mathrm{~A})-\mathrm{C}(10 \mathrm{~A})-\mathrm{C}(11 \mathrm{~A})$ & $48.1(19)$ \\
\hline $\mathrm{C}(14 \mathrm{~A})-\mathrm{N}(1 \mathrm{~A})-\mathrm{C}(10 \mathrm{~A})-\mathrm{C}(11 \mathrm{~A})$ & $167.4(15)$ \\
\hline $\mathrm{C}(1 \mathrm{~A})-\mathrm{N}(1 \mathrm{~A})-\mathrm{C}(10 \mathrm{~A})-\mathrm{C}(11 \mathrm{~A})$ & $-72.8(17)$ \\
\hline $\mathrm{C}(14 \mathrm{~A})-\mathrm{N}(1 \mathrm{~A})-\mathrm{C}(12 \mathrm{~A})-\mathrm{C}(13 \mathrm{~A})$ & $-60.7(19)$ \\
\hline $\mathrm{C}(10 \mathrm{~A})-\mathrm{N}(1 \mathrm{~A})-\mathrm{C}(12 \mathrm{~A})-\mathrm{C}(13 \mathrm{~A})$ & $58(2)$ \\
\hline $\mathrm{C}(1 \mathrm{~A})-\mathrm{N}(1 \mathrm{~A})-\mathrm{C}(12 \mathrm{~A})-\mathrm{C}(13 \mathrm{~A})$ & $177.9(17)$ \\
\hline $\mathrm{C}(12 \mathrm{~A})-\mathrm{N}(1 \mathrm{~A})-\mathrm{C}(14 \mathrm{~A})-\mathrm{C}(15 \mathrm{~A})$ & $-47.4(17)$ \\
\hline $\mathrm{C}(10 \mathrm{~A})-\mathrm{N}(1 \mathrm{~A})-\mathrm{C}(14 \mathrm{~A})-\mathrm{C}(15 \mathrm{~A})$ & $-166.6(14)$ \\
\hline$C(1 \mathrm{~A})-\mathrm{N}(1 \mathrm{~A})-\mathrm{C}(14 \mathrm{~A})-\mathrm{C}(15 \mathrm{~A})$ & $74.3(16)$ \\
\hline
\end{tabular}


Table G 7 Crystal data and structure refinement for $\mathbf{1 9}$

Identification code

Empirical formula

Formula weight

Temperature

Wavelength

Crystal system

Space group

Unit cell dimensions

Volume

$\mathrm{Z}$

Density (calculated)

Absorption coefficient

$\mathrm{F}(000)$

Crystal size

Theta range for data collection

Index ranges

Reflections collected

Independent reflections

Completeness to theta $=67.243^{\circ}$

Absorption correction

Max. and min. transmission

Refinement method

Data / restraints / parameters

Goodness-of-fit on $\mathrm{F}^{2}$

Final $R$ indices [I $>2 \operatorname{sigma}(\mathrm{I})]$

$\mathrm{R}$ indices (all data)

Extinction coefficient

Largest diff. peak and hole d1876_a

C21 H36 Cl P

354.92

150(2) K

$1.54178 \AA$

Triclinic

$\mathrm{P}-1$

$\mathrm{a}=12.6560(5) \AA \quad \mathrm{a}=89.733(2)^{\circ}$.

$\mathrm{b}=13.1527(5) \AA \quad \mathrm{b}=77.265(2)^{\circ}$.

$\mathrm{c}=13.6289(5) \AA \quad \mathrm{g}=89.302(2)^{\circ}$.

2212.70(15) $\AA^{3}$

4

$1.065 \mathrm{Mg} / \mathrm{m}^{3}$

$2.175 \mathrm{~mm}^{-1}$

776

$0.530 \times 0.170 \times 0.010 \mathrm{~mm}^{3}$

3.324 to $67.243^{\circ}$.

$-14<=\mathrm{h}<=15,-15<=\mathrm{k}<=15,-16<=\mathrm{l}<=16$

50255

$7798[\mathrm{R}(\mathrm{int})=0.0679]$

$98.3 \%$

Semi-empirical from equivalents

0.7529 and 0.5374

Full-matrix least-squares on $\mathrm{F}^{2}$

7798 / 134 / 507

1.029

$\mathrm{R} 1=0.0391, \mathrm{wR} 2=0.0938$

$\mathrm{R} 1=0.0550, \mathrm{wR} 2=0.1037$

$\mathrm{n} / \mathrm{a}$

0.285 and -0.313 e. $\AA^{-}-3$ 
Table G 8 Atomic coordinates and equivalent isotropic displacement parameters for $\mathbf{1 9}$

\begin{tabular}{|c|c|c|c|c|}
\hline & $\mathrm{x}$ & $\mathrm{y}$ & $\mathrm{z}$ & $\mathrm{U}(\mathrm{eq})$ \\
\hline $\mathrm{Cl}(1)$ & $4698(1)$ & $4251(1)$ & $3106(1)$ & $40(1)$ \\
\hline $\mathrm{Cl}(2)$ & $5437(1)$ & $736(1)$ & $7937(1)$ & $34(1)$ \\
\hline $\mathrm{P}(1 \mathrm{~A})$ & $5452(1)$ & $1930(1)$ & $985(1)$ & $25(1)$ \\
\hline $\mathrm{C}(1 \mathrm{~A})$ & $5446(2)$ & $1770(1)$ & $2300(1)$ & $26(1)$ \\
\hline $\mathrm{C}(2 \mathrm{~A})$ & $6553(2)$ & $1643(1)$ & $2526(1)$ & $26(1)$ \\
\hline$C(3 A)$ & $7013(2)$ & $678(2)$ & $2546(1)$ & $31(1)$ \\
\hline $\mathrm{C}(4 \mathrm{~A})$ & $8020(2)$ & $546(2)$ & $2768(2)$ & $39(1)$ \\
\hline $\mathrm{C}(5 \mathrm{~A})$ & $8596(2)$ & $1378(2)$ & 2992(2) & $42(1)$ \\
\hline$C(6 A)$ & $8139(2)$ & $2338(2)$ & $2962(2)$ & $43(1)$ \\
\hline$C(7 A)$ & $7130(2)$ & $2477(2)$ & $2735(1)$ & $34(1)$ \\
\hline $\mathrm{C}(8 \mathrm{~A})$ & $9649(2)$ & $1266(2)$ & $3278(2)$ & $64(1)$ \\
\hline $\mathrm{C}(9 \mathrm{~A})$ & $10114(2)$ & 421(3) & $3489(3)$ & $82(1)$ \\
\hline$C(10 A)$ & $6268(2)$ & $975(2)$ & $228(1)$ & $31(1)$ \\
\hline $\mathrm{C}(11 \mathrm{~A})$ & $7480(2)$ & $1198(2)$ & $-83(2)$ & $36(1)$ \\
\hline $\mathrm{C}(12 \mathrm{~A})$ & $8115(2)$ & $341(2)$ & $-698(2)$ & $54(1)$ \\
\hline$C(13 A)$ & $9313(2)$ & $564(3)$ & $-997(3)$ & $83(1)$ \\
\hline $\mathrm{C}(14 \mathrm{~A})$ & $5958(2)$ & 3171(2) & $588(1)$ & $30(1)$ \\
\hline$C(15 A)$ & $5832(2)$ & $3455(2)$ & $-477(1)$ & $34(1)$ \\
\hline$C(16 A)$ & $6454(2)$ & $4412(2)$ & $-873(2)$ & $46(1)$ \\
\hline $\mathrm{C}(17 \mathrm{~A})$ & $7670(2)$ & $4245(2)$ & $-1161(2)$ & $67(1)$ \\
\hline$C(18 A)$ & $4090(2)$ & $1818(2)$ & $811(2)$ & $33(1)$ \\
\hline C(19A) & $3283(2)$ & $2638(2)$ & 1341(2) & $39(1)$ \\
\hline$C(20 A)$ & $2676(2)$ & $2354(2)$ & $2386(2)$ & $45(1)$ \\
\hline$C(21 A)$ & $1926(2)$ & $3206(2)$ & 2889(2) & $57(1)$ \\
\hline $\mathrm{P}(1 \mathrm{~B})$ & $4552(1)$ & $3079(1)$ & $6179(1)$ & $29(1)$ \\
\hline$C(1 C)$ & $4555(2)$ & $3208(2)$ & 7496(1) & $30(1)$ \\
\hline$C(2 C)$ & $3463(7)$ & $3196(6)$ & $8239(10)$ & $30(2)$ \\
\hline$C(3 C)$ & $2904(5)$ & $4078(5)$ & $8631(5)$ & $36(2)$ \\
\hline$C(4 C)$ & 1887(4) & $4054(5)$ & $9284(5)$ & $41(1)$ \\
\hline$C(5 C)$ & $1355(4)$ & $3148(5)$ & $9564(5)$ & $41(1)$ \\
\hline$C(6 C)$ & 1906(7) & $2268(6)$ & $9167(9)$ & $40(2)$ \\
\hline
\end{tabular}




\begin{tabular}{|c|c|c|c|c|}
\hline$C(7 C)$ & $2928(10)$ & 2291(7) & $8532(18)$ & $34(2)$ \\
\hline$C(8 C)$ & $303(5)$ & $3105(6)$ & $10300(6)$ & $60(2)$ \\
\hline $\mathrm{C}(9 \mathrm{C})$ & $-157(5)$ & $3835(5)$ & $10910(4)$ & $71(2)$ \\
\hline $\mathrm{C}(1 \mathrm{~B})$ & $4555(2)$ & $3208(2)$ & $7496(1)$ & $30(1)$ \\
\hline $\mathrm{C}(2 \mathrm{~B})$ & $3425(6)$ & $3283(6)$ & $8107(9)$ & $31(2)$ \\
\hline $\mathrm{C}(3 \mathrm{~B})$ & $2945(4)$ & $4240(5)$ & $8290(5)$ & $37(1)$ \\
\hline$C(4 B)$ & $1890(4)$ & $4304(4)$ & $8838(5)$ & $43(1)$ \\
\hline$C(5 B)$ & $1342(4)$ & $3441(5)$ & $9241(5)$ & $42(1)$ \\
\hline$C(6 B)$ & $1834(7)$ & $2493(5)$ & $9061(8)$ & $42(2)$ \\
\hline$C(7 B)$ & $2883(10)$ & $2413(6)$ & $8493(16)$ & $37(2)$ \\
\hline $\mathrm{C}(8 \mathrm{~B})$ & $225(4)$ & $3528(6)$ & $9839(5)$ & $68(2)$ \\
\hline $\mathrm{C}(9 \mathrm{~B})$ & $-240(6)$ & $2888(6)$ & $10544(5)$ & $79(2)$ \\
\hline $\mathrm{C}(10 \mathrm{~B})$ & $3674(2)$ & $4005(2)$ & $5790(2)$ & $38(1)$ \\
\hline$C(11 B)$ & $2466(2)$ & $3756(2)$ & $6033(2)$ & $48(1)$ \\
\hline $\mathrm{C}(12 \mathrm{~B})$ & $1771(2)$ & $4664(2)$ & $5865(3)$ & $74(1)$ \\
\hline$C(13 B)$ & $565(3)$ & $4448(3)$ & $6159(4)$ & $114(2)$ \\
\hline$C(14 B)$ & $4116(2)$ & $1820(2)$ & $5964(1)$ & $31(1)$ \\
\hline$C(15 B)$ & $4264(2)$ & $1558(2)$ & $4844(1)$ & $37(1)$ \\
\hline$C(16 B)$ & $3687(2)$ & $575(2)$ & $4700(2)$ & $48(1)$ \\
\hline $\mathrm{C}(17 \mathrm{~B})$ & $2476(2)$ & $712(2)$ & $4860(2)$ & $64(1)$ \\
\hline $\mathrm{C}(18 \mathrm{~B})$ & $5907(2)$ & $3266(2)$ & $5474(2)$ & $37(1)$ \\
\hline$C(19 B)$ & $6740(2)$ & $2480(2)$ & $5688(2)$ & $42(1)$ \\
\hline$C(20 B)$ & $7379(5)$ & $2857(4)$ & $6397(4)$ & $49(2)$ \\
\hline$C(21 B)$ & $8216(7)$ & $2072(7)$ & $6573(6)$ & $62(2)$ \\
\hline$C(19 C)$ & $6740(2)$ & $2480(2)$ & $5688(2)$ & $42(1)$ \\
\hline$C(20 C)$ & 7797(9) & $2874(6)$ & $5848(14)$ & $74(4)$ \\
\hline $\mathrm{C}(21 \mathrm{C})$ & $8553(15)$ & $2066(11)$ & $6139(15)$ & $93(5)$ \\
\hline
\end{tabular}


Table G 9 Bond lengths $[\AA]$ and angles $\left[{ }^{\circ}\right]$ for $\mathbf{1 9}$

\begin{tabular}{|c|c|}
\hline$P(1 \mathrm{~A})-\mathrm{C}(10 \mathrm{~A})$ & $1.7948(19)$ \\
\hline $\mathrm{P}(1 \mathrm{~A})-\mathrm{C}(14 \mathrm{~A})$ & $1.797(2)$ \\
\hline $\mathrm{P}(1 \mathrm{~A})-\mathrm{C}(18 \mathrm{~A})$ & $1.800(2)$ \\
\hline $\mathrm{P}(1 \mathrm{~A})-\mathrm{C}(1 \mathrm{~A})$ & $1.8025(17)$ \\
\hline $\mathrm{C}(1 \mathrm{~A})-\mathrm{C}(2 \mathrm{~A})$ & $1.507(2)$ \\
\hline $\mathrm{C}(1 \mathrm{~A})-\mathrm{H}(1 \mathrm{AA})$ & 0.9900 \\
\hline $\mathrm{C}(1 \mathrm{~A})-\mathrm{H}(1 \mathrm{AB})$ & 0.9900 \\
\hline $\mathrm{C}(2 \mathrm{~A})-\mathrm{C}(7 \mathrm{~A})$ & $1.389(3)$ \\
\hline $\mathrm{C}(2 \mathrm{~A})-\mathrm{C}(3 \mathrm{~A})$ & $1.393(3)$ \\
\hline$C(3 \mathrm{~A})-\mathrm{C}(4 \mathrm{~A})$ & $1.382(3)$ \\
\hline $\mathrm{C}(3 \mathrm{~A})-\mathrm{H}(3 \mathrm{AA})$ & 0.9500 \\
\hline$C(4 A)-C(5 A)$ & $1.394(3)$ \\
\hline $\mathrm{C}(4 \mathrm{~A})-\mathrm{H}(4 \mathrm{AA})$ & 0.9500 \\
\hline$C(5 A)-C(6 A)$ & $1.387(3)$ \\
\hline$C(5 A)-C(8 A)$ & $1.474(3)$ \\
\hline $\mathrm{C}(6 \mathrm{~A})-\mathrm{C}(7 \mathrm{~A})$ & $1.389(3)$ \\
\hline C(6A)-H(6AA) & 0.9500 \\
\hline $\mathrm{C}(7 \mathrm{~A})-\mathrm{H}(7 \mathrm{AA})$ & 0.9500 \\
\hline $\mathrm{C}(8 \mathrm{~A})-\mathrm{C}(9 \mathrm{~A})$ & $1.311(4)$ \\
\hline $\mathrm{C}(8 \mathrm{~A})-\mathrm{H}(8 \mathrm{AA})$ & 0.9500 \\
\hline $\mathrm{C}(9 \mathrm{~A})-\mathrm{H}(9 \mathrm{~A} 1)$ & 0.9500 \\
\hline $\mathrm{C}(9 \mathrm{~A})-\mathrm{H}(9 \mathrm{~A} 2)$ & 0.9500 \\
\hline$C(10 A)-C(11 A)$ & $1.530(3)$ \\
\hline $\mathrm{C}(10 \mathrm{~A})-\mathrm{H}(10 \mathrm{~A})$ & 0.9900 \\
\hline $\mathrm{C}(10 \mathrm{~A})-\mathrm{H}(10 \mathrm{~B})$ & 0.9900 \\
\hline$C(11 A)-C(12 A)$ & $1.519(3)$ \\
\hline $\mathrm{C}(11 \mathrm{~A})-\mathrm{H}(11 \mathrm{~A})$ & 0.9900 \\
\hline $\mathrm{C}(11 \mathrm{~A})-\mathrm{H}(11 \mathrm{~B})$ & 0.9900 \\
\hline$C(12 A)-C(13 A)$ & $1.512(4)$ \\
\hline $\mathrm{C}(12 \mathrm{~A})-\mathrm{H}(12 \mathrm{~A})$ & 0.9900 \\
\hline $\mathrm{C}(12 \mathrm{~A})-\mathrm{H}(12 \mathrm{~B})$ & 0.9900 \\
\hline $\mathrm{C}(13 \mathrm{~A})-\mathrm{H}(13 \mathrm{~A})$ & 0.9800 \\
\hline $\mathrm{C}(13 \mathrm{~A})-\mathrm{H}(13 \mathrm{~B})$ & 0.9800 \\
\hline
\end{tabular}




\begin{tabular}{|c|c|}
\hline $\mathrm{C}(13 \mathrm{~A})-\mathrm{H}(13 \mathrm{C})$ & 0.9800 \\
\hline $\mathrm{C}(14 \mathrm{~A})-\mathrm{C}(15 \mathrm{~A})$ & $1.540(3)$ \\
\hline $\mathrm{C}(14 \mathrm{~A})-\mathrm{H}(14 \mathrm{~A})$ & 0.9900 \\
\hline $\mathrm{C}(14 \mathrm{~A})-\mathrm{H}(14 \mathrm{~B})$ & 0.9900 \\
\hline$C(15 A)-C(16 A)$ & $1.524(3)$ \\
\hline $\mathrm{C}(15 \mathrm{~A})-\mathrm{H}(15 \mathrm{~A})$ & 0.9900 \\
\hline $\mathrm{C}(15 \mathrm{~A})-\mathrm{H}(15 \mathrm{~B})$ & 0.9900 \\
\hline$C(16 A)-C(17 A)$ & $1.516(4)$ \\
\hline $\mathrm{C}(16 \mathrm{~A})-\mathrm{H}(16 \mathrm{~A})$ & 0.9900 \\
\hline $\mathrm{C}(16 \mathrm{~A})-\mathrm{H}(16 \mathrm{~B})$ & 0.9900 \\
\hline $\mathrm{C}(17 \mathrm{~A})-\mathrm{H}(17 \mathrm{~A})$ & 0.9800 \\
\hline $\mathrm{C}(17 \mathrm{~A})-\mathrm{H}(17 \mathrm{~B})$ & 0.9800 \\
\hline $\mathrm{C}(17 \mathrm{~A})-\mathrm{H}(17 \mathrm{C})$ & 0.9800 \\
\hline $\mathrm{C}(18 \mathrm{~A})-\mathrm{C}(19 \mathrm{~A})$ & $1.543(3)$ \\
\hline $\mathrm{C}(18 \mathrm{~A})-\mathrm{H}(18 \mathrm{~A})$ & 0.9900 \\
\hline $\mathrm{C}(18 \mathrm{~A})-\mathrm{H}(18 \mathrm{~B})$ & 0.9900 \\
\hline$C(19 A)-C(20 A)$ & $1.510(3)$ \\
\hline C(19A)-H(19A) & 0.9900 \\
\hline $\mathrm{C}(19 \mathrm{~A})-\mathrm{H}(19 \mathrm{~B})$ & 0.9900 \\
\hline$C(20 A)-C(21 A)$ & $1.523(3)$ \\
\hline $\mathrm{C}(20 \mathrm{~A})-\mathrm{H}(20 \mathrm{~A})$ & 0.9900 \\
\hline $\mathrm{C}(20 \mathrm{~A})-\mathrm{H}(20 \mathrm{~B})$ & 0.9900 \\
\hline $\mathrm{C}(21 \mathrm{~A})-\mathrm{H}(21 \mathrm{~A})$ & 0.9800 \\
\hline $\mathrm{C}(21 \mathrm{~A})-\mathrm{H}(21 \mathrm{~B})$ & 0.9800 \\
\hline $\mathrm{C}(21 \mathrm{~A})-\mathrm{H}(21 \mathrm{C})$ & 0.9800 \\
\hline $\mathrm{P}(1 \mathrm{~B})-\mathrm{C}(18 \mathrm{~B})$ & $1.790(2)$ \\
\hline$P(1 B)-C(10 B)$ & $1.795(2)$ \\
\hline $\mathrm{P}(1 \mathrm{~B})-\mathrm{C}(14 \mathrm{~B})$ & $1.7966(19)$ \\
\hline $\mathrm{P}(1 \mathrm{~B})-\mathrm{C}(1 \mathrm{C})$ & $1.8054(18)$ \\
\hline $\mathrm{P}(1 \mathrm{~B})-\mathrm{C}(1 \mathrm{~B})$ & $1.8054(18)$ \\
\hline $\mathrm{C}(1 \mathrm{C})-\mathrm{C}(2 \mathrm{C})$ & $1.523(5)$ \\
\hline $\mathrm{C}(1 \mathrm{C})-\mathrm{H}(1 \mathrm{CA})$ & 0.9900 \\
\hline $\mathrm{C}(1 \mathrm{C})-\mathrm{H}(1 \mathrm{CB})$ & 0.9900 \\
\hline$C(2 C)-C(7 C)$ & $1.390(6)$ \\
\hline $\mathrm{C}(2 \mathrm{C})-\mathrm{C}(3 \mathrm{C})$ & $1.397(6)$ \\
\hline$C(3 C)-C(4 C)$ & $1.394(6)$ \\
\hline
\end{tabular}




\begin{tabular}{|c|c|}
\hline $\mathrm{C}(3 \mathrm{C})-\mathrm{H}(3 \mathrm{CA})$ & 0.9500 \\
\hline$C(4 C)-C(5 C)$ & $1.386(6)$ \\
\hline $\mathrm{C}(4 \mathrm{C})-\mathrm{H}(4 \mathrm{CA})$ & 0.9500 \\
\hline$C(5 C)-C(6 C)$ & $1.393(6)$ \\
\hline$C(5 C)-C(8 C)$ & $1.482(6)$ \\
\hline $\mathrm{C}(6 \mathrm{C})-\mathrm{C}(7 \mathrm{C})$ & $1.389(6)$ \\
\hline $\mathrm{C}(6 \mathrm{C})-\mathrm{H}(6 \mathrm{CA})$ & 0.9500 \\
\hline $\mathrm{C}(7 \mathrm{C})-\mathrm{H}(7 \mathrm{CA})$ & 0.9500 \\
\hline $\mathrm{C}(8 \mathrm{C})-\mathrm{C}(9 \mathrm{C})$ & $1.316(7)$ \\
\hline $\mathrm{C}(8 \mathrm{C})-\mathrm{H}(8 \mathrm{CA})$ & 0.9500 \\
\hline C(9C)-H(9C1) & 0.9500 \\
\hline $\mathrm{C}(9 \mathrm{C})-\mathrm{H}(9 \mathrm{C} 2)$ & 0.9500 \\
\hline $\mathrm{C}(1 \mathrm{~B})-\mathrm{C}(2 \mathrm{~B})$ & $1.490(5)$ \\
\hline $\mathrm{C}(1 \mathrm{~B})-\mathrm{H}(1 \mathrm{BA})$ & 0.9900 \\
\hline $\mathrm{C}(1 \mathrm{~B})-\mathrm{H}(1 \mathrm{BB})$ & 0.9900 \\
\hline $\mathrm{C}(2 \mathrm{~B})-\mathrm{C}(7 \mathrm{~B})$ & $1.383(6)$ \\
\hline $\mathrm{C}(2 \mathrm{~B})-\mathrm{C}(3 \mathrm{~B})$ & $1.390(5)$ \\
\hline$C(3 B)-C(4 B)$ & $1.380(5)$ \\
\hline $\mathrm{C}(3 \mathrm{~B})-\mathrm{H}(3 \mathrm{BA})$ & 0.9500 \\
\hline$C(4 B)-C(5 B)$ & $1.385(6)$ \\
\hline $\mathrm{C}(4 \mathrm{~B})-\mathrm{H}(4 \mathrm{BA})$ & 0.9500 \\
\hline$C(5 B)-C(6 B)$ & $1.385(6)$ \\
\hline $\mathrm{C}(5 \mathrm{~B})-\mathrm{C}(8 \mathrm{~B})$ & $1.471(6)$ \\
\hline$C(6 B)-C(7 B)$ & $1.385(6)$ \\
\hline $\mathrm{C}(6 \mathrm{~B})-\mathrm{H}(6 \mathrm{BA})$ & 0.9500 \\
\hline $\mathrm{C}(7 \mathrm{~B})-\mathrm{H}(7 \mathrm{BA})$ & 0.9500 \\
\hline$C(8 B)-C(9 B)$ & $1.316(7)$ \\
\hline $\mathrm{C}(8 \mathrm{~B})-\mathrm{H}(8 \mathrm{BA})$ & 0.9500 \\
\hline C(9B)-H(9B1) & 0.9500 \\
\hline C(9B)-H(9B2) & 0.9500 \\
\hline$C(10 B)-C(11 B)$ & $1.530(3)$ \\
\hline $\mathrm{C}(10 \mathrm{~B})-\mathrm{H}(10 \mathrm{C})$ & 0.9900 \\
\hline$C(10 B)-H(10 D)$ & 0.9900 \\
\hline$C(11 B)-C(12 B)$ & $1.521(4)$ \\
\hline $\mathrm{C}(11 \mathrm{~B})-\mathrm{H}(11 \mathrm{C})$ & 0.9900 \\
\hline $\mathrm{C}(11 \mathrm{~B})-\mathrm{H}(11 \mathrm{D})$ & 0.9900 \\
\hline
\end{tabular}




\begin{tabular}{|c|c|}
\hline $\mathrm{C}(12 \mathrm{~B})-\mathrm{C}(13 \mathrm{~B})$ & $1.520(5)$ \\
\hline $\mathrm{C}(12 \mathrm{~B})-\mathrm{H}(12 \mathrm{C})$ & 0.9900 \\
\hline $\mathrm{C}(12 \mathrm{~B})-\mathrm{H}(12 \mathrm{D})$ & 0.9900 \\
\hline $\mathrm{C}(13 \mathrm{~B})-\mathrm{H}(13 \mathrm{D})$ & 0.9800 \\
\hline $\mathrm{C}(13 \mathrm{~B})-\mathrm{H}(13 \mathrm{E})$ & 0.9800 \\
\hline $\mathrm{C}(13 \mathrm{~B})-\mathrm{H}(13 \mathrm{~F})$ & 0.9800 \\
\hline$C(14 B)-C(15 B)$ & $1.537(2)$ \\
\hline $\mathrm{C}(14 \mathrm{~B})-\mathrm{H}(14 \mathrm{C})$ & 0.9900 \\
\hline $\mathrm{C}(14 \mathrm{~B})-\mathrm{H}(14 \mathrm{D})$ & 0.9900 \\
\hline$C(15 B)-C(16 B)$ & $1.527(3)$ \\
\hline $\mathrm{C}(15 \mathrm{~B})-\mathrm{H}(15 \mathrm{C})$ & 0.9900 \\
\hline $\mathrm{C}(15 \mathrm{~B})-\mathrm{H}(15 \mathrm{D})$ & 0.9900 \\
\hline$C(16 B)-C(17 B)$ & $1.508(4)$ \\
\hline $\mathrm{C}(16 \mathrm{~B})-\mathrm{H}(16 \mathrm{C})$ & 0.9900 \\
\hline $\mathrm{C}(16 \mathrm{~B})-\mathrm{H}(16 \mathrm{D})$ & 0.9900 \\
\hline $\mathrm{C}(17 \mathrm{~B})-\mathrm{H}(17 \mathrm{D})$ & 0.9800 \\
\hline $\mathrm{C}(17 \mathrm{~B})-\mathrm{H}(17 \mathrm{E})$ & 0.9800 \\
\hline $\mathrm{C}(17 \mathrm{~B})-\mathrm{H}(17 \mathrm{~F})$ & 0.9800 \\
\hline $\mathrm{C}(18 \mathrm{~B})-\mathrm{C}(19 \mathrm{C})$ & $1.540(3)$ \\
\hline $\mathrm{C}(18 \mathrm{~B})-\mathrm{C}(19 \mathrm{~B})$ & $1.540(3)$ \\
\hline $\mathrm{C}(18 \mathrm{~B})-\mathrm{H}(18 \mathrm{C})$ & 0.9900 \\
\hline $\mathrm{C}(18 \mathrm{~B})-\mathrm{H}(18 \mathrm{D})$ & 0.9900 \\
\hline$C(19 B)-C(20 B)$ & $1.480(5)$ \\
\hline C(19B)-H(19C) & 0.9900 \\
\hline $\mathrm{C}(19 B)-H(19 D)$ & 0.9900 \\
\hline$C(20 B)-C(21 B)$ & $1.525(6)$ \\
\hline $\mathrm{C}(20 \mathrm{~B})-\mathrm{H}(20 \mathrm{C})$ & 0.9900 \\
\hline $\mathrm{C}(20 \mathrm{~B})-\mathrm{H}(20 \mathrm{D})$ & 0.9900 \\
\hline $\mathrm{C}(21 \mathrm{~B})-\mathrm{H}(21 \mathrm{D})$ & 0.9800 \\
\hline $\mathrm{C}(21 \mathrm{~B})-\mathrm{H}(21 \mathrm{E})$ & 0.9800 \\
\hline $\mathrm{C}(21 \mathrm{~B})-\mathrm{H}(21 \mathrm{~F})$ & 0.9800 \\
\hline$C(19 C)-C(20 C)$ & $1.501(7)$ \\
\hline $\mathrm{C}(19 \mathrm{C})-\mathrm{H}(19 \mathrm{E})$ & 0.9900 \\
\hline C(19C)-H(19F) & 0.9900 \\
\hline$C(20 C)-C(21 C)$ & $1.529(8)$ \\
\hline $\mathrm{C}(20 \mathrm{C})-\mathrm{H}(20 \mathrm{E})$ & 0.9900 \\
\hline
\end{tabular}




\begin{tabular}{|c|c|}
\hline $\mathrm{C}(20 \mathrm{C})-\mathrm{H}(20 \mathrm{~F})$ & 0.9900 \\
\hline $\mathrm{C}(21 \mathrm{C})-\mathrm{H}(21 \mathrm{G})$ & 0.9800 \\
\hline $\mathrm{C}(21 \mathrm{C})-\mathrm{H}(21 \mathrm{H})$ & 0.9800 \\
\hline $\mathrm{C}(21 \mathrm{C})-\mathrm{H}(21 \mathrm{I})$ & 0.9800 \\
\hline $\mathrm{C}(10 \mathrm{~A})-\mathrm{P}(1 \mathrm{~A})-\mathrm{C}(14 \mathrm{~A})$ & $109.87(9)$ \\
\hline$C(10 A)-P(1 A)-C(18 A)$ & $107.80(9)$ \\
\hline $\mathrm{C}(14 \mathrm{~A})-\mathrm{P}(1 \mathrm{~A})-\mathrm{C}(18 \mathrm{~A})$ & $109.55(10)$ \\
\hline$C(10 A)-P(1 A)-C(1 A)$ & $111.63(9)$ \\
\hline $\mathrm{C}(14 \mathrm{~A})-\mathrm{P}(1 \mathrm{~A})-\mathrm{C}(1 \mathrm{~A})$ & $108.94(8)$ \\
\hline $\mathrm{C}(18 \mathrm{~A})-\mathrm{P}(1 \mathrm{~A})-\mathrm{C}(1 \mathrm{~A})$ & $109.02(9)$ \\
\hline $\mathrm{C}(2 \mathrm{~A})-\mathrm{C}(1 \mathrm{~A})-\mathrm{P}(1 \mathrm{~A})$ & $114.48(12)$ \\
\hline $\mathrm{C}(2 \mathrm{~A})-\mathrm{C}(1 \mathrm{~A})-\mathrm{H}(1 \mathrm{AA})$ & 108.6 \\
\hline $\mathrm{P}(1 \mathrm{~A})-\mathrm{C}(1 \mathrm{~A})-\mathrm{H}(1 \mathrm{AA})$ & 108.6 \\
\hline$C(2 A)-C(1 A)-H(1 A B)$ & 108.6 \\
\hline $\mathrm{P}(1 \mathrm{~A})-\mathrm{C}(1 \mathrm{~A})-\mathrm{H}(1 \mathrm{AB})$ & 108.6 \\
\hline $\mathrm{H}(1 \mathrm{AA})-\mathrm{C}(1 \mathrm{~A})-\mathrm{H}(1 \mathrm{AB})$ & 107.6 \\
\hline$C(7 A)-C(2 A)-C(3 A)$ & $118.52(17)$ \\
\hline$C(7 A)-C(2 A)-C(1 A)$ & $121.13(17)$ \\
\hline$C(3 A)-C(2 A)-C(1 A)$ & $120.33(16)$ \\
\hline$C(4 A)-C(3 A)-C(2 A)$ & $121.10(18)$ \\
\hline $\mathrm{C}(4 \mathrm{~A})-\mathrm{C}(3 \mathrm{~A})-\mathrm{H}(3 \mathrm{AA})$ & 119.5 \\
\hline $\mathrm{C}(2 \mathrm{~A})-\mathrm{C}(3 \mathrm{~A})-\mathrm{H}(3 \mathrm{AA})$ & 119.5 \\
\hline$C(3 A)-C(4 A)-C(5 A)$ & $120.7(2)$ \\
\hline $\mathrm{C}(3 \mathrm{~A})-\mathrm{C}(4 \mathrm{~A})-\mathrm{H}(4 \mathrm{AA})$ & 119.7 \\
\hline $\mathrm{C}(5 \mathrm{~A})-\mathrm{C}(4 \mathrm{~A})-\mathrm{H}(4 \mathrm{AA})$ & 119.7 \\
\hline$C(6 A)-C(5 A)-C(4 A)$ & $117.98(19)$ \\
\hline$C(6 A)-C(5 A)-C(8 A)$ & $119.6(2)$ \\
\hline$C(4 A)-C(5 A)-C(8 A)$ & $122.4(2)$ \\
\hline$C(5 A)-C(6 A)-C(7 A)$ & $121.7(2)$ \\
\hline$C(5 A)-C(6 A)-H(6 A A)$ & 119.2 \\
\hline$C(7 A)-C(6 A)-H(6 A A)$ & 119.2 \\
\hline$C(6 A)-C(7 A)-C(2 A)$ & $120.0(2)$ \\
\hline $\mathrm{C}(6 \mathrm{~A})-\mathrm{C}(7 \mathrm{~A})-\mathrm{H}(7 \mathrm{AA})$ & 120.0 \\
\hline $\mathrm{C}(2 \mathrm{~A})-\mathrm{C}(7 \mathrm{~A})-\mathrm{H}(7 \mathrm{AA})$ & 120.0 \\
\hline $\mathrm{C}(9 \mathrm{~A})-\mathrm{C}(8 \mathrm{~A})-\mathrm{C}(5 \mathrm{~A})$ & $127.4(3)$ \\
\hline
\end{tabular}




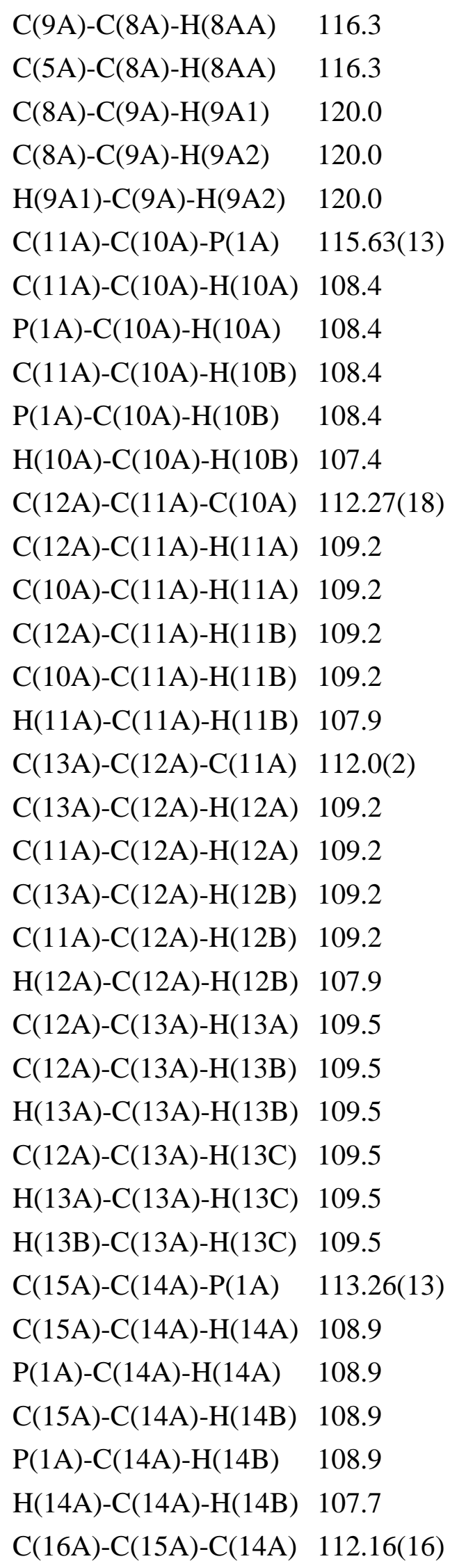




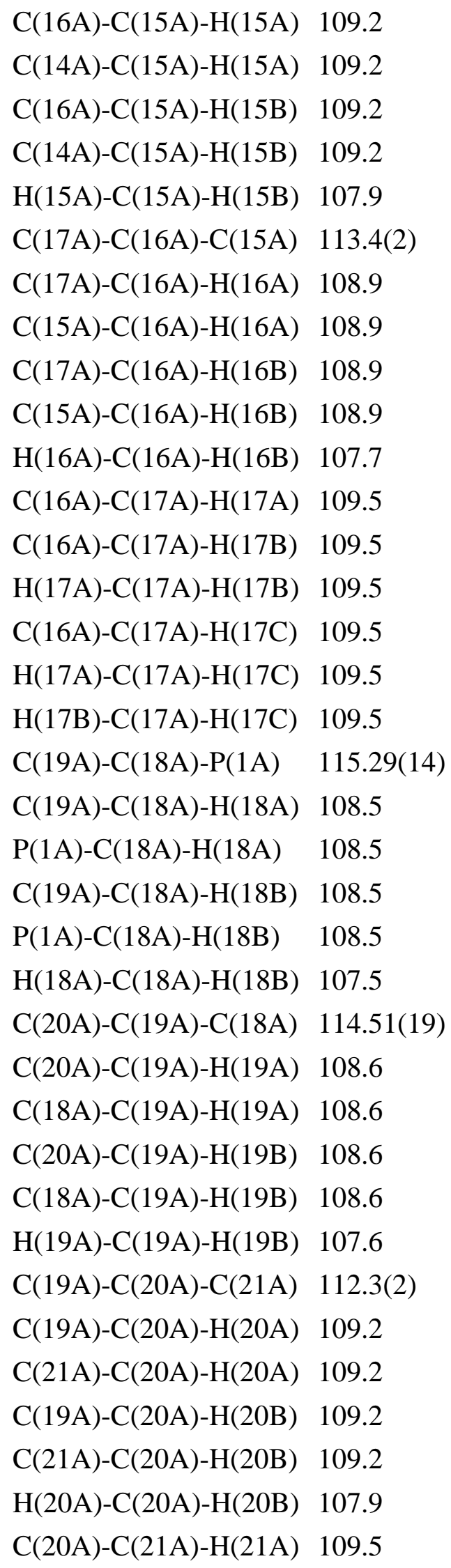




\begin{tabular}{|c|c|}
\hline $\mathrm{C}(20 \mathrm{~A})-\mathrm{C}(21 \mathrm{~A})-\mathrm{H}(21 \mathrm{~B})$ & 109.5 \\
\hline $\mathrm{H}(21 \mathrm{~A})-\mathrm{C}(21 \mathrm{~A})-\mathrm{H}(21 \mathrm{~B})$ & 109.5 \\
\hline$C(20 A)-C(21 A)-H(21 C)$ & 109.5 \\
\hline $\mathrm{H}(21 \mathrm{~A})-\mathrm{C}(21 \mathrm{~A})-\mathrm{H}(21 \mathrm{C})$ & 109.5 \\
\hline$H(21 B)-C(21 A)-H(21 C)$ & 109.5 \\
\hline $\mathrm{C}(18 \mathrm{~B})-\mathrm{P}(1 \mathrm{~B})-\mathrm{C}(10 \mathrm{~B})$ & $109.02(10)$ \\
\hline$C(18 B)-P(1 B)-C(14 B)$ & $109.93(10)$ \\
\hline$C(10 B)-P(1 B)-C(14 B)$ & $109.84(10)$ \\
\hline $\mathrm{C}(18 \mathrm{~B})-\mathrm{P}(1 \mathrm{~B})-\mathrm{C}(1 \mathrm{C})$ & $107.90(9)$ \\
\hline $\mathrm{C}(10 \mathrm{~B})-\mathrm{P}(1 \mathrm{~B})-\mathrm{C}(1 \mathrm{C})$ & $111.41(9)$ \\
\hline $\mathrm{C}(14 \mathrm{~B})-\mathrm{P}(1 \mathrm{~B})-\mathrm{C}(1 \mathrm{C})$ & $108.72(9)$ \\
\hline $\mathrm{C}(18 \mathrm{~B})-\mathrm{P}(1 \mathrm{~B})-\mathrm{C}(1 \mathrm{~B})$ & $107.90(9)$ \\
\hline$C(10 B)-P(1 B)-C(1 B)$ & $111.41(9)$ \\
\hline $\mathrm{C}(14 \mathrm{~B})-\mathrm{P}(1 \mathrm{~B})-\mathrm{C}(1 \mathrm{~B})$ & $108.72(9)$ \\
\hline $\mathrm{C}(2 \mathrm{C})-\mathrm{C}(1 \mathrm{C})-\mathrm{P}(1 \mathrm{~B})$ & $117.3(6)$ \\
\hline $\mathrm{C}(2 \mathrm{C})-\mathrm{C}(1 \mathrm{C})-\mathrm{H}(1 \mathrm{CA})$ & 108.0 \\
\hline $\mathrm{P}(1 \mathrm{~B})-\mathrm{C}(1 \mathrm{C})-\mathrm{H}(1 \mathrm{CA})$ & 108.0 \\
\hline $\mathrm{C}(2 \mathrm{C})-\mathrm{C}(1 \mathrm{C})-\mathrm{H}(1 \mathrm{CB})$ & 108.0 \\
\hline $\mathrm{P}(1 \mathrm{~B})-\mathrm{C}(1 \mathrm{C})-\mathrm{H}(1 \mathrm{CB})$ & 108.0 \\
\hline $\mathrm{H}(1 \mathrm{CA})-\mathrm{C}(1 \mathrm{C})-\mathrm{H}(1 \mathrm{CB})$ & 107.2 \\
\hline$C(7 C)-C(2 C)-C(3 C)$ & $115.4(5)$ \\
\hline$C(7 C)-C(2 C)-C(1 C)$ & $121.3(6)$ \\
\hline$C(3 C)-C(2 C)-C(1 C)$ & $123.3(6)$ \\
\hline$C(4 C)-C(3 C)-C(2 C)$ & $122.5(5)$ \\
\hline $\mathrm{C}(4 \mathrm{C})-\mathrm{C}(3 \mathrm{C})-\mathrm{H}(3 \mathrm{CA})$ & 118.8 \\
\hline $\mathrm{C}(2 \mathrm{C})-\mathrm{C}(3 \mathrm{C})-\mathrm{H}(3 \mathrm{CA})$ & 118.8 \\
\hline$C(5 C)-C(4 C)-C(3 C)$ & $121.8(5)$ \\
\hline $\mathrm{C}(5 \mathrm{C})-\mathrm{C}(4 \mathrm{C})-\mathrm{H}(4 \mathrm{CA})$ & 119.1 \\
\hline $\mathrm{C}(3 \mathrm{C})-\mathrm{C}(4 \mathrm{C})-\mathrm{H}(4 \mathrm{CA})$ & 119.1 \\
\hline$C(4 C)-C(5 C)-C(6 C)$ & $115.8(5)$ \\
\hline$C(4 C)-C(5 C)-C(8 C)$ & $122.4(5)$ \\
\hline$C(6 C)-C(5 C)-C(8 C)$ & $121.6(6)$ \\
\hline$C(7 C)-C(6 C)-C(5 C)$ & $122.4(6)$ \\
\hline $\mathrm{C}(7 \mathrm{C})-\mathrm{C}(6 \mathrm{C})-\mathrm{H}(6 \mathrm{CA})$ & 118.8 \\
\hline $\mathrm{C}(5 \mathrm{C})-\mathrm{C}(6 \mathrm{C})-\mathrm{H}(6 \mathrm{CA})$ & 118.8 \\
\hline$C(6 C)-C(7 C)-C(2 C)$ & $122.1(6)$ \\
\hline
\end{tabular}




\begin{tabular}{|c|c|}
\hline $\mathrm{C}(6 \mathrm{C})-\mathrm{C}(7 \mathrm{C})-\mathrm{H}(7 \mathrm{CA})$ & \\
\hline $\mathrm{C}(2 \mathrm{C})-\mathrm{C}(7 \mathrm{C})-\mathrm{H}(7 \mathrm{CA})$ & 118.9 \\
\hline$C(9 C)-C(8 C)-C(5 C)$ & $126.9(7)$ \\
\hline $\mathrm{C}(9 \mathrm{C})-\mathrm{C}(8 \mathrm{C})-\mathrm{H}(8 \mathrm{CA})$ & 16.5 \\
\hline $\mathrm{C}(5 \mathrm{C})-\mathrm{C}(8 \mathrm{C})-\mathrm{H}(8 \mathrm{CA})$ & 16.5 \\
\hline $\mathrm{C}(8 \mathrm{C})-\mathrm{C}(9 \mathrm{C})-\mathrm{H}(9 \mathrm{C} 1)$ & 20.0 \\
\hline $\mathrm{C}(8 \mathrm{C})-\mathrm{C}(9 \mathrm{C})-\mathrm{H}(9 \mathrm{C} 2)$ & 0.0 \\
\hline $\mathrm{H}(9 \mathrm{C} 1)-\mathrm{C}(9 \mathrm{C})-\mathrm{H}(9 \mathrm{C} 2)$ & 20.0 \\
\hline $\mathrm{C}(2 \mathrm{~B})-\mathrm{C}(1 \mathrm{~B})-\mathrm{P}(1 \mathrm{~B})$ & \\
\hline $\mathrm{C}(2 \mathrm{~B})-\mathrm{C}(1 \mathrm{~B})-\mathrm{H}(1 \mathrm{BA})$ & \\
\hline$P(1 B)-C(1 B)-H(1 B A)$ & \\
\hline $\mathrm{C}(2 \mathrm{~B})-\mathrm{C}(1 \mathrm{~B})-\mathrm{H}(1 \mathrm{BB})$ & 109.6 \\
\hline $\mathrm{P}(1 \mathrm{~B})-\mathrm{C}(1 \mathrm{~B})-\mathrm{H}(1 \mathrm{BB})$ & \\
\hline $\mathrm{H}(1 \mathrm{BA})-\mathrm{C}(1 \mathrm{~B})-\mathrm{H}(1 \mathrm{BB})$ & 108.1 \\
\hline$C(7 B)-C(2 B)-C(3 B)$ & \\
\hline$C(7 B)-C(2 B)-C(1 B)$ & $120.0(5)$ \\
\hline$C(3 B)-C(2 B)-C(1 B)$ & $118.7(5)$ \\
\hline$C(4 B)-C(3 B)-C(2 B)$ & $118.4(5)$ \\
\hline $\mathrm{C}(4 \mathrm{~B})-\mathrm{C}(3 \mathrm{~B})-\mathrm{H}(3 \mathrm{BA})$ & 120.8 \\
\hline $\mathrm{C}(2 \mathrm{~B})-\mathrm{C}(3 \mathrm{~B})-\mathrm{H}(3 \mathrm{BA})$ & 120.8 \\
\hline $\mathrm{C}(3 \mathrm{~B})-\mathrm{C}(4 \mathrm{~B})-\mathrm{C}(5 \mathrm{~B})$ & $120.8(4)$ \\
\hline $\mathrm{C}(3 \mathrm{~B})-\mathrm{C}(4 \mathrm{~B})-\mathrm{H}(4 \mathrm{BA})$ & \\
\hline $\mathrm{C}(5 \mathrm{~B})-\mathrm{C}(4 \mathrm{~B})-\mathrm{H}(4 \mathrm{BA})$ & 119.6 \\
\hline$C(4 B)-C(5 B)-C(6 B)$ & \\
\hline $\mathrm{C}(4 \mathrm{~B})-\mathrm{C}(5 \mathrm{~B})-\mathrm{C}(8 \mathrm{~B})$ & \\
\hline$C(6 B)-C(5 B)-C(8 B)$ & \\
\hline$C(7 B)-C(6 B)-C(5 B)$ & \\
\hline$C(7 B)-C(6 B)-H(6 B A)$ & 20.1 \\
\hline $\mathrm{C}(5 \mathrm{~B})-\mathrm{C}(6 \mathrm{~B})-\mathrm{H}(6 \mathrm{BA})$ & 120.1 \\
\hline$C(2 B)-C(7 B)-C(6 B)$ & $119.5(6$ \\
\hline $\mathrm{C}(2 \mathrm{~B})-\mathrm{C}(7 \mathrm{~B})-\mathrm{H}(7 \mathrm{BA})$ & 120.3 \\
\hline $\mathrm{C}(6 \mathrm{~B})-\mathrm{C}(7 \mathrm{~B})-\mathrm{H}(7 \mathrm{BA})$ & 120.3 \\
\hline $\mathrm{C}(9 \mathrm{~B})-\mathrm{C}(8 \mathrm{~B})-\mathrm{C}(5 \mathrm{~B})$ & \\
\hline $\mathrm{C}(9 \mathrm{~B})-\mathrm{C}(8 \mathrm{~B})-\mathrm{H}(8 \mathrm{BA})$ & 117.3 \\
\hline $\mathrm{C}(5 \mathrm{~B})-\mathrm{C}(8 \mathrm{~B})-\mathrm{H}(8 \mathrm{BA})$ & \\
\hline $\mathrm{C}(8 \mathrm{~B})-\mathrm{C}(9 \mathrm{~B})-\mathrm{H}(9 \mathrm{~B} 1)$ & 120.0 \\
\hline
\end{tabular}




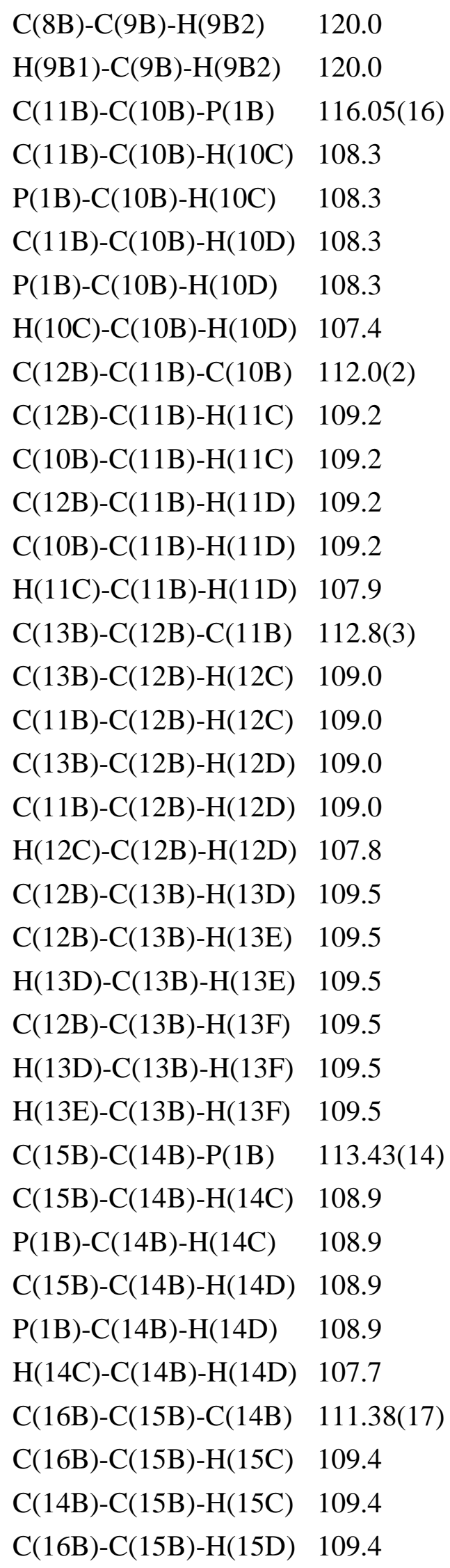




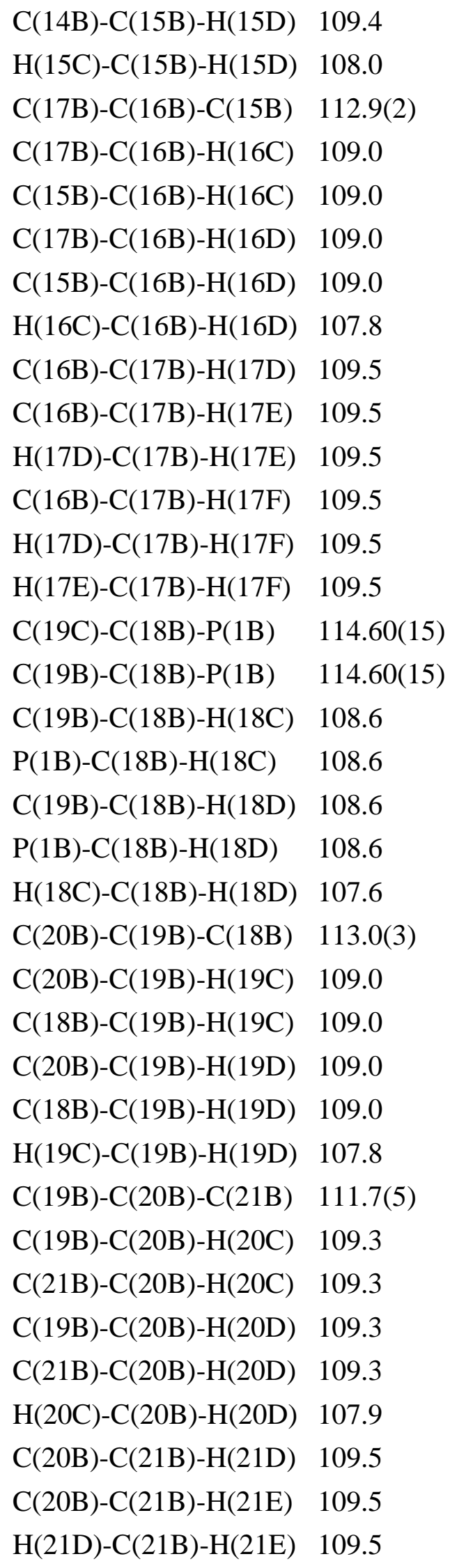




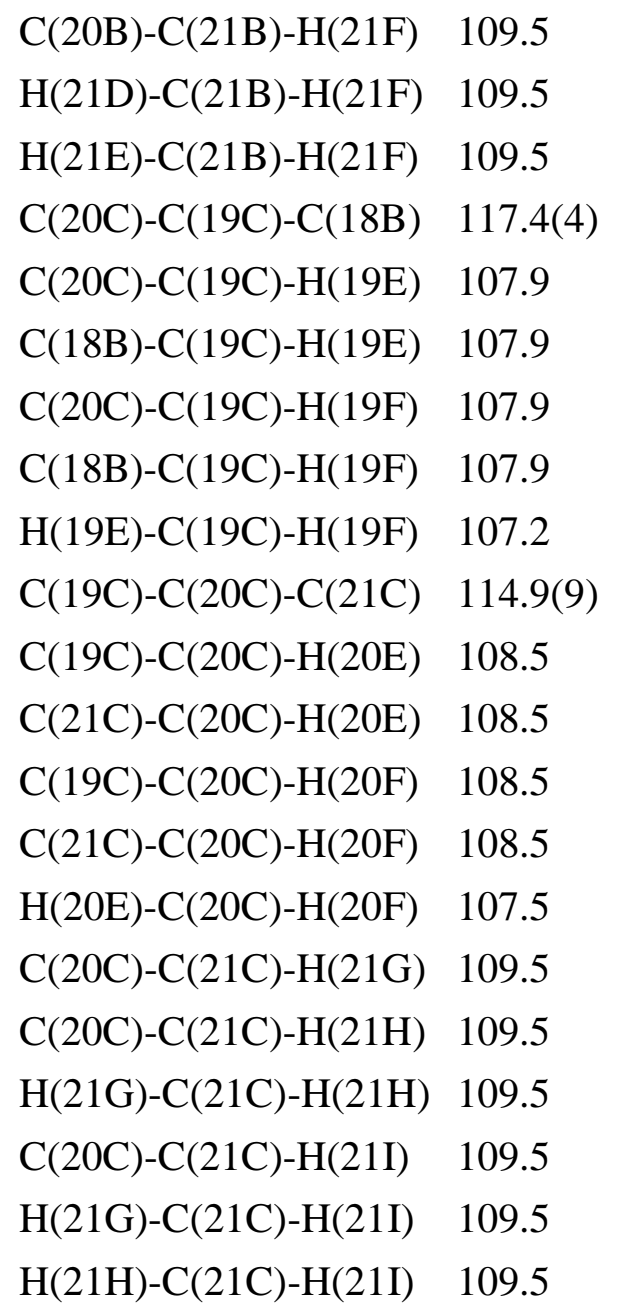


Table G 10 Anisotropic displacement parameters for $\mathbf{1 9}$

\begin{tabular}{|c|c|c|c|c|c|c|}
\hline & U11 & $\mathrm{U}^{22}$ & $\mathrm{U}^{33}$ & $\mathrm{U}^{23}$ & $\mathrm{U}^{13}$ & U12 \\
\hline $\mathrm{Cl}(1)$ & $58(1)$ & $25(1)$ & $40(1)$ & $-5(1)$ & $-18(1)$ & $-2(1)$ \\
\hline $\mathrm{Cl}(2)$ & $51(1)$ & $26(1)$ & $28(1)$ & $3(1)$ & $-16(1)$ & $-5(1)$ \\
\hline $\mathrm{P}(1 \mathrm{~A})$ & $35(1)$ & $23(1)$ & $20(1)$ & $0(1)$ & $-11(1)$ & $-4(1)$ \\
\hline$C(1 \mathrm{~A})$ & $33(1)$ & $25(1)$ & $21(1)$ & $1(1)$ & $-8(1)$ & $-4(1)$ \\
\hline $\mathrm{C}(2 \mathrm{~A})$ & $34(1)$ & $31(1)$ & $16(1)$ & $2(1)$ & $-6(1)$ & $-4(1)$ \\
\hline$C(3 A)$ & $34(1)$ & $33(1)$ & $27(1)$ & $3(1)$ & $-10(1)$ & $-4(1)$ \\
\hline $\mathrm{C}(4 \mathrm{~A})$ & $38(1)$ & $43(1)$ & $36(1)$ & $8(1)$ & $-10(1)$ & $1(1)$ \\
\hline$C(5 A)$ & $34(1)$ & $58(2)$ & $36(1)$ & $7(1)$ & $-11(1)$ & $-7(1)$ \\
\hline$C(6 A)$ & $44(1)$ & $51(2)$ & $36(1)$ & $1(1)$ & $-14(1)$ & $-18(1)$ \\
\hline$C(7 A)$ & $43(1)$ & $34(1)$ & $26(1)$ & $-1(1)$ & $-10(1)$ & $-7(1)$ \\
\hline $\mathrm{C}(8 \mathrm{~A})$ & $41(1)$ & $86(2)$ & $72(2)$ & $6(2)$ & $-25(1)$ & $-13(1)$ \\
\hline $\mathrm{C}(9 \mathrm{~A})$ & $48(2)$ & $110(3)$ & 101(2) & $21(2)$ & $-41(2)$ & $-4(2)$ \\
\hline$C(10 A)$ & $43(1)$ & $28(1)$ & $21(1)$ & $-2(1)$ & $-9(1)$ & $-4(1)$ \\
\hline $\mathrm{C}(11 \mathrm{~A})$ & $42(1)$ & $38(1)$ & $28(1)$ & $-2(1)$ & $-6(1)$ & $-2(1)$ \\
\hline$C(12 A)$ & $54(2)$ & $52(2)$ & $51(1)$ & $-10(1)$ & $-2(1)$ & $5(1)$ \\
\hline$C(13 A)$ & $55(2)$ & $97(3)$ & $90(2)$ & $-27(2)$ & $2(2)$ & $15(2)$ \\
\hline $\mathrm{C}(14 \mathrm{~A})$ & $39(1)$ & $27(1)$ & $26(1)$ & $-1(1)$ & $-14(1)$ & $-4(1)$ \\
\hline $\mathrm{C}(15 \mathrm{~A})$ & $48(1)$ & $30(1)$ & $28(1)$ & $7(1)$ & $-16(1)$ & $-6(1)$ \\
\hline$C(16 A)$ & $67(2)$ & $36(1)$ & $42(1)$ & $17(1)$ & $-25(1)$ & $-14(1)$ \\
\hline $\mathrm{C}(17 \mathrm{~A})$ & $66(2)$ & $76(2)$ & $63(2)$ & $31(2)$ & $-19(1)$ & $-31(2)$ \\
\hline $\mathrm{C}(18 \mathrm{~A})$ & $42(1)$ & $31(1)$ & $31(1)$ & $2(1)$ & $-16(1)$ & $-6(1)$ \\
\hline$C(19 A)$ & $38(1)$ & $38(1)$ & $43(1)$ & $6(1)$ & $-15(1)$ & $-2(1)$ \\
\hline$C(20 A)$ & $39(1)$ & $45(1)$ & $49(1)$ & $5(1)$ & $-10(1)$ & $-2(1)$ \\
\hline$C(21 \mathrm{~A})$ & $48(1)$ & $60(2)$ & $61(2)$ & $-6(1)$ & $-3(1)$ & $-5(1)$ \\
\hline $\mathrm{P}(1 \mathrm{~B})$ & $43(1)$ & $22(1)$ & $24(1)$ & $0(1)$ & $-12(1)$ & $-2(1)$ \\
\hline$C(1 C)$ & $38(1)$ & $27(1)$ & $28(1)$ & $-4(1)$ & $-14(1)$ & $0(1)$ \\
\hline$C(2 C)$ & $38(3)$ & $33(3)$ & $25(3)$ & $-4(2)$ & $-19(2)$ & $8(3)$ \\
\hline$C(3 C)$ & 49(3) & $33(3)$ & $31(3)$ & $-5(2)$ & $-16(2)$ & $2(2)$ \\
\hline$C(4 C)$ & $46(2)$ & $42(3)$ & $35(3)$ & $-2(2)$ & $-10(2)$ & $11(2)$ \\
\hline$C(5 C)$ & $40(2)$ & $46(3)$ & $40(3)$ & $7(2)$ & $-13(2)$ & $3(2)$ \\
\hline$C(6 C)$ & $43(3)$ & $41(3)$ & $40(3)$ & $4(3)$ & $-15(2)$ & $5(2)$ \\
\hline
\end{tabular}




$\begin{array}{lcccccc}\mathrm{C}(7 \mathrm{C}) & 40(3) & 36(3) & 30(3) & 3(3) & -14(3) & 7(3) \\ \mathrm{C}(8 \mathrm{C}) & 41(4) & 75(6) & 59(5) & 12(4) & -2(4) & 7(4) \\ \mathrm{C}(9 \mathrm{C}) & 53(4) & 97(6) & 55(4) & 14(3) & 3(3) & 11(3) \\ \mathrm{C}(1 \mathrm{~B}) & 38(1) & 27(1) & 28(1) & -4(1) & -14(1) & 0(1) \\ \mathrm{C}(2 \mathrm{~B}) & 36(3) & 41(3) & 19(3) & -10(2) & -14(2) & 1(2) \\ \mathrm{C}(3 \mathrm{~B}) & 43(2) & 38(3) & 31(3) & -11(2) & -8(2) & 2(2) \\ \mathrm{C}(4 \mathrm{~B}) & 42(2) & 43(3) & 42(3) & -12(2) & -3(2) & 6(2) \\ \mathrm{C}(5 \mathrm{~B}) & 35(2) & 49(3) & 41(3) & -5(2) & -4(2) & 1(2) \\ \mathrm{C}(6 \mathrm{~B}) & 37(3) & 52(3) & 36(3) & 3(3) & -11(2) & 0(3) \\ \mathrm{C}(7 \mathrm{~B}) & 40(3) & 48(4) & 24(3) & -4(3) & -7(3) & 5(3) \\ \mathrm{C}(8 \mathrm{~B}) & 43(3) & 74(5) & 77(5) & -18(4) & 8(3) & 0(3) \\ \mathrm{C}(9 \mathrm{~B}) & 56(4) & 98(6) & 67(4) & -19(4) & 25(3) & -15(4) \\ \mathrm{C}(10 \mathrm{~B}) & 54(1) & 26(1) & 39(1) & 1(1) & -22(1) & -1(1) \\ \mathrm{C}(11 \mathrm{~B}) & 55(1) & 41(1) & 57(1) & 5(1) & -31(1) & -2(1) \\ \mathrm{C}(12 \mathrm{~B}) & 65(2) & 56(2) & 111(3) & 8(2) & -42(2) & 11(2) \\ \mathrm{C}(13 \mathrm{~B}) & 63(2) & 121(4) & 169(4) & 17(3) & -47(2) & 20(2) \\ \mathrm{C}(14 \mathrm{~B}) & 48(1) & 26(1) & 22(1) & 0(1) & -11(1) & -4(1) \\ \mathrm{C}(15 \mathrm{~B}) & 57(1) & 31(1) & 22(1) & 0(1) & -10(1) & -7(1) \\ \mathrm{C}(16 \mathrm{~B}) & 83(2) & 35(1) & 27(1) & -2(1) & -16(1) & -19(1) \\ \mathrm{C}(17 \mathrm{~B}) & 92(2) & 60(2) & 52(2) & 18(1) & -42(1) & -35(2) \\ \mathrm{C}(18 \mathrm{~B}) & 51(1) & 29(1) & 31(1) & 4(1) & -9(1) & -4(1) \\ \mathrm{C}(19 \mathrm{~B}) & 44(1) & 38(1) & 40(1) & 3(1) & -3(1) & 1(1) \\ \mathrm{C}(20 \mathrm{~B}) & 48(3) & 52(3) & 49(3) & 1(2) & -16(2) & 3(2) \\ \mathrm{C}(21 \mathrm{~B}) & 51(3) & 73(4) & 65(4) & 8(3) & -19(3) & 12(3) \\ \mathrm{C}(19 \mathrm{C}) & 44(1) & 38(1) & 40(1) & 3(1) & -3(1) & 1(1) \\ \mathrm{C}(20 \mathrm{C}) & 86(8) & 40(5) & 116(11) & 2(6) & -63(7) & -2(5) \\ \mathrm{C}(21 \mathrm{C}) & 104(11) & 65(7) & 137(14) & 0(8) & -84(11) & 5(7)\end{array}$


Table G 11 Hydrogen coordinates and isotropic displacement parameters for 19

\begin{tabular}{|c|c|c|c|c|}
\hline & $\mathrm{x}$ & $\mathrm{y}$ & z & $\mathrm{U}(\mathrm{eq})$ \\
\hline $\mathrm{H}(1 \mathrm{AA})$ & 5084 & 2369 & 2672 & 31 \\
\hline $\mathrm{H}(1 \mathrm{AB})$ & 5010 & 1166 & 2555 & 31 \\
\hline $\mathrm{H}(3 \mathrm{AA})$ & 6629 & 100 & 2405 & 37 \\
\hline $\mathrm{H}(4 \mathrm{AA})$ & 8323 & -119 & 2768 & 46 \\
\hline $\mathrm{H}(6 \mathrm{AA})$ & 8526 & 2915 & 3100 & 51 \\
\hline $\mathrm{H}(7 \mathrm{AA})$ & 6834 & 3142 & 2722 & 41 \\
\hline $\mathrm{H}(8 \mathrm{AA})$ & 10034 & 1875 & 3314 & 77 \\
\hline $\mathrm{H}(9 \mathrm{~A} 1)$ & 9766 & -211 & 3465 & 99 \\
\hline $\mathrm{H}(9 \mathrm{~A} 2)$ & 10799 & 441 & 3666 & 99 \\
\hline $\mathrm{H}(10 \mathrm{~A})$ & 6176 & 325 & 605 & 37 \\
\hline $\mathrm{H}(10 \mathrm{~B})$ & 5990 & 878 & -389 & 37 \\
\hline $\mathrm{H}(11 \mathrm{~A})$ & 7585 & 1833 & -483 & 43 \\
\hline $\mathrm{H}(11 \mathrm{~B})$ & 7767 & 1305 & 528 & 43 \\
\hline $\mathrm{H}(12 \mathrm{~A})$ & 8008 & -295 & -300 & 65 \\
\hline $\mathrm{H}(12 \mathrm{~B})$ & 7833 & 237 & -1312 & 65 \\
\hline $\mathrm{H}(13 \mathrm{~A})$ & 9692 & -5 & -1391 & 125 \\
\hline $\mathrm{H}(13 \mathrm{~B})$ & 9424 & 1187 & -1402 & 125 \\
\hline $\mathrm{H}(13 \mathrm{C})$ & 9598 & 654 & -391 & 125 \\
\hline $\mathrm{H}(14 \mathrm{~A})$ & 5568 & 3682 & 1070 & 35 \\
\hline $\mathrm{H}(14 \mathrm{~B})$ & 6735 & 3197 & 608 & 35 \\
\hline $\mathrm{H}(15 \mathrm{~A})$ & 6097 & 2883 & -939 & 41 \\
\hline $\mathrm{H}(15 \mathrm{~B})$ & 5054 & 3564 & -467 & 41 \\
\hline $\mathrm{H}(16 \mathrm{~A})$ & 6201 & 4661 & -1469 & 56 \\
\hline $\mathrm{H}(16 \mathrm{~B})$ & 6287 & 4946 & -350 & 56 \\
\hline $\mathrm{H}(17 \mathrm{~A})$ & 8023 & 4885 & -1408 & 101 \\
\hline $\mathrm{H}(17 \mathrm{~B})$ & 7931 & 4014 & -571 & 101 \\
\hline $\mathrm{H}(17 \mathrm{C})$ & 7844 & 3729 & -1690 & 101 \\
\hline $\mathrm{H}(18 \mathrm{~A})$ & 4109 & 1845 & 81 & 40 \\
\hline $\mathrm{H}(18 \mathrm{~B})$ & 3813 & 1143 & 1060 & 40 \\
\hline $\mathrm{H}(19 \mathrm{~A})$ & 3685 & 3272 & 1382 & 47 \\
\hline
\end{tabular}




\begin{tabular}{|c|c|c|c|c|}
\hline $\mathrm{H}(19 \mathrm{~B})$ & 2751 & 2783 & 922 & 47 \\
\hline $\mathrm{H}(20 \mathrm{~A})$ & 3203 & 2186 & 2804 & 54 \\
\hline $\mathrm{H}(20 \mathrm{~B})$ & 2243 & 1739 & 2347 & 54 \\
\hline $\mathrm{H}(21 \mathrm{~A})$ & 1550 & 2988 & 3563 & 86 \\
\hline $\mathrm{H}(21 \mathrm{~B})$ & 2353 & 3812 & 2942 & 86 \\
\hline $\mathrm{H}(21 \mathrm{C})$ & 1393 & 3365 & 2486 & 86 \\
\hline $\mathrm{H}(1 \mathrm{CA})$ & 5007 & 2650 & 7681 & 36 \\
\hline $\mathrm{H}(1 \mathrm{CB})$ & 4915 & 3855 & 7585 & 36 \\
\hline $\mathrm{H}(3 \mathrm{CA})$ & 3228 & 4719 & 8446 & 44 \\
\hline $\mathrm{H}(4 \mathrm{CA})$ & 1550 & 4675 & 9543 & 49 \\
\hline $\mathrm{H}(6 \mathrm{CA})$ & 1572 & 1629 & 9337 & 48 \\
\hline $\mathrm{H}(7 \mathrm{CA})$ & 3273 & 1667 & 8290 & 41 \\
\hline $\mathrm{H}(8 \mathrm{CA})$ & -80 & 2484 & 10336 & 72 \\
\hline $\mathrm{H}(9 \mathrm{C} 1)$ & 191 & 4471 & 10905 & 85 \\
\hline $\mathrm{H}(9 \mathrm{C} 2)$ & -838 & 3725 & 11355 & 85 \\
\hline $\mathrm{H}(1 \mathrm{BA})$ & 4927 & 2612 & 7721 & 36 \\
\hline $\mathrm{H}(1 \mathrm{BB})$ & 4958 & 3825 & 7598 & 36 \\
\hline $\mathrm{H}(3 \mathrm{BA})$ & 3334 & 4836 & 8044 & 44 \\
\hline $\mathrm{H}(4 \mathrm{BA})$ & 1536 & 4948 & 8940 & 52 \\
\hline $\mathrm{H}(6 \mathrm{BA})$ & 1453 & 1900 & 9327 & 50 \\
\hline $\mathrm{H}(7 \mathrm{BA})$ & 3227 & 1766 & 8371 & 45 \\
\hline $\mathrm{H}(8 \mathrm{BA})$ & -196 & 4093 & 9706 & 82 \\
\hline $\mathrm{H}(9 \mathrm{~B} 1)$ & 152 & 2312 & 10702 & 95 \\
\hline $\mathrm{H}(9 \mathrm{~B} 2)$ & -967 & 3002 & 10895 & 95 \\
\hline $\mathrm{H}(10 \mathrm{C})$ & 3768 & 4662 & 6110 & 46 \\
\hline $\mathrm{H}(10 \mathrm{D})$ & 3911 & 4100 & 5053 & 46 \\
\hline $\mathrm{H}(11 \mathrm{C})$ & 2338 & 3185 & 5603 & 57 \\
\hline $\mathrm{H}(11 \mathrm{D})$ & 2247 & 3534 & 6743 & 57 \\
\hline $\mathrm{H}(12 \mathrm{C})$ & 1933 & 5246 & 6264 & 89 \\
\hline $\mathrm{H}(12 \mathrm{D})$ & 1964 & 4860 & 5146 & 89 \\
\hline $\mathrm{H}(13 \mathrm{D})$ & 156 & 5055 & 6036 & 172 \\
\hline $\mathrm{H}(13 \mathrm{E})$ & 396 & 3883 & 5756 & 172 \\
\hline $\mathrm{H}(13 \mathrm{~F})$ & 365 & 4268 & 6874 & 172 \\
\hline $\mathrm{H}(14 \mathrm{C})$ & 4530 & 1322 & 6282 & 38 \\
\hline $\mathrm{H}(14 \mathrm{D})$ & 3341 & 1756 & 6296 & 38 \\
\hline $\mathrm{H}(15 \mathrm{C})$ & 3972 & 2122 & 4495 & 44 \\
\hline
\end{tabular}




\begin{tabular}{|c|c|c|c|c|}
\hline $\mathrm{H}(15 \mathrm{D})$ & 5047 & 1482 & 4538 & 44 \\
\hline $\mathrm{H}(16 \mathrm{C})$ & 3976 & 318 & 4011 & 57 \\
\hline $\mathrm{H}(16 \mathrm{D})$ & 3846 & 57 & 5179 & 57 \\
\hline $\mathrm{H}(17 \mathrm{D})$ & 2148 & 61 & 4759 & 95 \\
\hline $\mathrm{H}(17 \mathrm{E})$ & 2181 & 952 & 5547 & 95 \\
\hline $\mathrm{H}(17 \mathrm{~F})$ & 2312 & 1213 & 4379 & 95 \\
\hline $\mathrm{H}(18 \mathrm{C})$ & 5906 & 3245 & 4748 & 44 \\
\hline $\mathrm{H}(18 \mathrm{D})$ & 6141 & 3953 & 5624 & 44 \\
\hline $\mathrm{H}(19 \mathrm{C})$ & 6356 & 1857 & 5970 & 50 \\
\hline $\mathrm{H}(19 \mathrm{D})$ & 7241 & 2294 & 5046 & 50 \\
\hline $\mathrm{H}(20 \mathrm{C})$ & 6883 & 3023 & 7047 & 58 \\
\hline$H(20 D)$ & 7752 & 3488 & 6125 & 58 \\
\hline $\mathrm{H}(21 \mathrm{D})$ & 8622 & 2347 & 7045 & 93 \\
\hline$H(21 E)$ & 7847 & 1451 & 6854 & 93 \\
\hline$H(21 F)$ & 8716 & 1915 & 5933 & 93 \\
\hline $\mathrm{H}(19 \mathrm{E})$ & 6395 & 2089 & 6294 & 50 \\
\hline $\mathrm{H}(19 \mathrm{~F})$ & 6901 & 1997 & 5118 & 50 \\
\hline $\mathrm{H}(20 \mathrm{E})$ & 7643 & 3395 & 6384 & 89 \\
\hline $\mathrm{H}(20 \mathrm{~F})$ & 8180 & 3212 & 5223 & 89 \\
\hline $\mathrm{H}(21 \mathrm{G})$ & 9220 & 2387 & 6228 & 139 \\
\hline $\mathrm{H}(21 \mathrm{H})$ & 8193 & 1738 & 6769 & 139 \\
\hline $\mathrm{H}(21 \mathrm{I})$ & 8731 & 1555 & 5605 & 139 \\
\hline
\end{tabular}


Table G 12 Torsion angles [ ${ }^{\circ}$ ] for 19

\begin{tabular}{|c|c|}
\hline $\mathrm{C}(10 \mathrm{~A})-\mathrm{P}(1 \mathrm{~A})-\mathrm{C}(1 \mathrm{~A})-\mathrm{C}(2 \mathrm{~A})$ & $49.70(16)$ \\
\hline $\mathrm{C}(14 \mathrm{~A})-\mathrm{P}(1 \mathrm{~A})-\mathrm{C}(1 \mathrm{~A})-\mathrm{C}(2 \mathrm{~A})$ & $-71.81(16)$ \\
\hline $\mathrm{C}(18 \mathrm{~A})-\mathrm{P}(1 \mathrm{~A})-\mathrm{C}(1 \mathrm{~A})-\mathrm{C}(2 \mathrm{~A})$ & $168.68(13)$ \\
\hline $\mathrm{P}(1 \mathrm{~A})-\mathrm{C}(1 \mathrm{~A})-\mathrm{C}(2 \mathrm{~A})-\mathrm{C}(7 \mathrm{~A})$ & $90.88(18)$ \\
\hline $\mathrm{P}(1 \mathrm{~A})-\mathrm{C}(1 \mathrm{~A})-\mathrm{C}(2 \mathrm{~A})-\mathrm{C}(3 \mathrm{~A})$ & $-90.14(19)$ \\
\hline$C(7 A)-C(2 A)-C(3 A)-C(4 A)$ & $0.0(3)$ \\
\hline$C(1 A)-C(2 A)-C(3 A)-C(4 A)$ & $-178.99(17)$ \\
\hline $\mathrm{C}(2 \mathrm{~A})-\mathrm{C}(3 \mathrm{~A})-\mathrm{C}(4 \mathrm{~A})-\mathrm{C}(5 \mathrm{~A})$ & $0.9(3)$ \\
\hline $\mathrm{C}(3 \mathrm{~A})-\mathrm{C}(4 \mathrm{~A})-\mathrm{C}(5 \mathrm{~A})-\mathrm{C}(6 \mathrm{~A})$ & $-1.5(3)$ \\
\hline$C(3 A)-C(4 A)-C(5 A)-C(8 A)$ & 177.1(2) \\
\hline$C(4 A)-C(5 A)-C(6 A)-C(7 A)$ & $1.2(3)$ \\
\hline$C(8 A)-C(5 A)-C(6 A)-C(7 A)$ & $-177.4(2)$ \\
\hline $\mathrm{C}(5 \mathrm{~A})-\mathrm{C}(6 \mathrm{~A})-\mathrm{C}(7 \mathrm{~A})-\mathrm{C}(2 \mathrm{~A})$ & $-0.3(3)$ \\
\hline$C(3 A)-C(2 A)-C(7 A)-C(6 A)$ & $-0.3(3)$ \\
\hline$C(1 A)-C(2 A)-C(7 A)-C(6 A)$ & $178.66(17)$ \\
\hline$C(6 A)-C(5 A)-C(8 A)-C(9 A)$ & $169.0(3)$ \\
\hline$C(4 A)-C(5 A)-C(8 A)-C(9 A)$ & $-9.6(4)$ \\
\hline $\mathrm{C}(14 \mathrm{~A})-\mathrm{P}(1 \mathrm{~A})-\mathrm{C}(10 \mathrm{~A})-\mathrm{C}(11 \mathrm{~A})$ & $36.21(17)$ \\
\hline $\mathrm{C}(18 \mathrm{~A})-\mathrm{P}(1 \mathrm{~A})-\mathrm{C}(10 \mathrm{~A})-\mathrm{C}(11 \mathrm{~A})$ & $155.53(15)$ \\
\hline $\mathrm{C}(1 \mathrm{~A})-\mathrm{P}(1 \mathrm{~A})-\mathrm{C}(10 \mathrm{~A})-\mathrm{C}(11 \mathrm{~A})$ & $-84.76(16)$ \\
\hline $\mathrm{P}(1 \mathrm{~A})-\mathrm{C}(10 \mathrm{~A})-\mathrm{C}(11 \mathrm{~A})-\mathrm{C}(12 \mathrm{~A})$ & $178.58(16)$ \\
\hline $\mathrm{C}(10 \mathrm{~A})-\mathrm{C}(11 \mathrm{~A})-\mathrm{C}(12 \mathrm{~A})-\mathrm{C}(13 \mathrm{~A})$ & $-179.6(2)$ \\
\hline $\mathrm{C}(10 \mathrm{~A})-\mathrm{P}(1 \mathrm{~A})-\mathrm{C}(14 \mathrm{~A})-\mathrm{C}(15 \mathrm{~A})$ & $66.49(17)$ \\
\hline $\mathrm{C}(18 \mathrm{~A})-\mathrm{P}(1 \mathrm{~A})-\mathrm{C}(14 \mathrm{~A})-\mathrm{C}(15 \mathrm{~A})$ & $-51.76(17)$ \\
\hline $\mathrm{C}(1 \mathrm{~A})-\mathrm{P}(1 \mathrm{~A})-\mathrm{C}(14 \mathrm{~A})-\mathrm{C}(15 \mathrm{~A})$ & $-170.94(14)$ \\
\hline $\mathrm{P}(1 \mathrm{~A})-\mathrm{C}(14 \mathrm{~A})-\mathrm{C}(15 \mathrm{~A})-\mathrm{C}(16 \mathrm{~A})$ & $-169.07(16)$ \\
\hline$C(14 A)-C(15 A)-C(16 A)-C(17 A)$ & $73.1(2)$ \\
\hline $\mathrm{C}(10 \mathrm{~A})-\mathrm{P}(1 \mathrm{~A})-\mathrm{C}(18 \mathrm{~A})-\mathrm{C}(19 \mathrm{~A})$ & $-175.36(15)$ \\
\hline $\mathrm{C}(14 \mathrm{~A})-\mathrm{P}(1 \mathrm{~A})-\mathrm{C}(18 \mathrm{~A})-\mathrm{C}(19 \mathrm{~A})$ & $-55.83(17)$ \\
\hline $\mathrm{C}(1 \mathrm{~A})-\mathrm{P}(1 \mathrm{~A})-\mathrm{C}(18 \mathrm{~A})-\mathrm{C}(19 \mathrm{~A})$ & $63.29(17)$ \\
\hline $\mathrm{P}(1 \mathrm{~A})-\mathrm{C}(18 \mathrm{~A})-\mathrm{C}(19 \mathrm{~A})-\mathrm{C}(20 \mathrm{~A})$ & $-91.8(2)$ \\
\hline$C(18 A)-C(19 A)-C(20 A)-C(21 A)$ & $177.70(19)$ \\
\hline $\mathrm{C}(18 \mathrm{~B})-\mathrm{P}(1 \mathrm{~B})-\mathrm{C}(1 \mathrm{C})-\mathrm{C}(2 \mathrm{C})$ & $173.3(4)$ \\
\hline
\end{tabular}




\begin{tabular}{|c|c|}
\hline $\mathrm{C}(10 \mathrm{~B})-\mathrm{P}(1 \mathrm{~B})-\mathrm{C}(1 \mathrm{C})-\mathrm{C}(2 \mathrm{C})$ & $53.7(4)$ \\
\hline $\mathrm{C}(14 \mathrm{~B})-\mathrm{P}(1 \mathrm{~B})-\mathrm{C}(1 \mathrm{C})-\mathrm{C}(2 \mathrm{C})$ & $-67.5(4)$ \\
\hline $\mathrm{P}(1 \mathrm{~B})-\mathrm{C}(1 \mathrm{C})-\mathrm{C}(2 \mathrm{C})-\mathrm{C}(7 \mathrm{C})$ & $79.0(14)$ \\
\hline $\mathrm{P}(1 \mathrm{~B})-\mathrm{C}(1 \mathrm{C})-\mathrm{C}(2 \mathrm{C})-\mathrm{C}(3 \mathrm{C})$ & $-97.6(11)$ \\
\hline $\mathrm{C}(7 \mathrm{C})-\mathrm{C}(2 \mathrm{C})-\mathrm{C}(3 \mathrm{C})-\mathrm{C}(4 \mathrm{C})$ & $1.0(16)$ \\
\hline $\mathrm{C}(1 \mathrm{C})-\mathrm{C}(2 \mathrm{C})-\mathrm{C}(3 \mathrm{C})-\mathrm{C}(4 \mathrm{C})$ & $177.7(9)$ \\
\hline $\mathrm{C}(2 \mathrm{C})-\mathrm{C}(3 \mathrm{C})-\mathrm{C}(4 \mathrm{C})-\mathrm{C}(5 \mathrm{C})$ & $-1.7(10)$ \\
\hline$C(3 C)-C(4 C)-C(5 C)-C(6 C)$ & $1.0(10)$ \\
\hline $\mathrm{C}(3 \mathrm{C})-\mathrm{C}(4 \mathrm{C})-\mathrm{C}(5 \mathrm{C})-\mathrm{C}(8 \mathrm{C})$ & $176.5(6)$ \\
\hline $\mathrm{C}(4 \mathrm{C})-\mathrm{C}(5 \mathrm{C})-\mathrm{C}(6 \mathrm{C})-\mathrm{C}(7 \mathrm{C})$ & $0.2(16)$ \\
\hline $\mathrm{C}(8 \mathrm{C})-\mathrm{C}(5 \mathrm{C})-\mathrm{C}(6 \mathrm{C})-\mathrm{C}(7 \mathrm{C})$ & $-175.3(14)$ \\
\hline$C(5 C)-C(6 C)-C(7 C)-C(2 C)$ & $-1(3)$ \\
\hline$C(3 C)-C(2 C)-C(7 C)-C(6 C)$ & $0(2)$ \\
\hline $\mathrm{C}(1 \mathrm{C})-\mathrm{C}(2 \mathrm{C})-\mathrm{C}(7 \mathrm{C})-\mathrm{C}(6 \mathrm{C})$ & $-176.6(16)$ \\
\hline$C(4 C)-C(5 C)-C(8 C)-C(9 C)$ & $-13.7(11)$ \\
\hline $\mathrm{C}(6 \mathrm{C})-\mathrm{C}(5 \mathrm{C})-\mathrm{C}(8 \mathrm{C})-\mathrm{C}(9 \mathrm{C})$ & 161.6(9) \\
\hline $\mathrm{C}(18 \mathrm{~B})-\mathrm{P}(1 \mathrm{~B})-\mathrm{C}(1 \mathrm{~B})-\mathrm{C}(2 \mathrm{~B})$ & $167.8(3)$ \\
\hline $\mathrm{C}(10 \mathrm{~B})-\mathrm{P}(1 \mathrm{~B})-\mathrm{C}(1 \mathrm{~B})-\mathrm{C}(2 \mathrm{~B})$ & $48.2(3)$ \\
\hline $\mathrm{C}(14 \mathrm{~B})-\mathrm{P}(1 \mathrm{~B})-\mathrm{C}(1 \mathrm{~B})-\mathrm{C}(2 \mathrm{~B})$ & $-73.0(3)$ \\
\hline $\mathrm{P}(1 \mathrm{~B})-\mathrm{C}(1 \mathrm{~B})-\mathrm{C}(2 \mathrm{~B})-\mathrm{C}(7 \mathrm{~B})$ & $90.9(13)$ \\
\hline $\mathrm{P}(1 \mathrm{~B})-\mathrm{C}(1 \mathrm{~B})-\mathrm{C}(2 \mathrm{~B})-\mathrm{C}(3 \mathrm{~B})$ & $-90.2(9)$ \\
\hline $\mathrm{C}(7 \mathrm{~B})-\mathrm{C}(2 \mathrm{~B})-\mathrm{C}(3 \mathrm{~B})-\mathrm{C}(4 \mathrm{~B})$ & $-2.5(15)$ \\
\hline $\mathrm{C}(1 \mathrm{~B})-\mathrm{C}(2 \mathrm{~B})-\mathrm{C}(3 \mathrm{~B})-\mathrm{C}(4 \mathrm{~B})$ & $178.7(8)$ \\
\hline $\mathrm{C}(2 \mathrm{~B})-\mathrm{C}(3 \mathrm{~B})-\mathrm{C}(4 \mathrm{~B})-\mathrm{C}(5 \mathrm{~B})$ & $3.3(9)$ \\
\hline $\mathrm{C}(3 \mathrm{~B})-\mathrm{C}(4 \mathrm{~B})-\mathrm{C}(5 \mathrm{~B})-\mathrm{C}(6 \mathrm{~B})$ & $-2.7(10)$ \\
\hline $\mathrm{C}(3 \mathrm{~B})-\mathrm{C}(4 \mathrm{~B})-\mathrm{C}(5 \mathrm{~B})-\mathrm{C}(8 \mathrm{~B})$ & $178.8(6)$ \\
\hline $\mathrm{C}(4 \mathrm{~B})-\mathrm{C}(5 \mathrm{~B})-\mathrm{C}(6 \mathrm{~B})-\mathrm{C}(7 \mathrm{~B})$ & $1.2(15)$ \\
\hline $\mathrm{C}(8 \mathrm{~B})-\mathrm{C}(5 \mathrm{~B})-\mathrm{C}(6 \mathrm{~B})-\mathrm{C}(7 \mathrm{~B})$ & $179.7(12)$ \\
\hline $\mathrm{C}(3 \mathrm{~B})-\mathrm{C}(2 \mathrm{~B})-\mathrm{C}(7 \mathrm{~B})-\mathrm{C}(6 \mathrm{~B})$ & $1(2)$ \\
\hline $\mathrm{C}(1 \mathrm{~B})-\mathrm{C}(2 \mathrm{~B})-\mathrm{C}(7 \mathrm{~B})-\mathrm{C}(6 \mathrm{~B})$ & $179.9(14)$ \\
\hline $\mathrm{C}(5 \mathrm{~B})-\mathrm{C}(6 \mathrm{~B})-\mathrm{C}(7 \mathrm{~B})-\mathrm{C}(2 \mathrm{~B})$ & $0(2)$ \\
\hline $\mathrm{C}(4 \mathrm{~B})-\mathrm{C}(5 \mathrm{~B})-\mathrm{C}(8 \mathrm{~B})-\mathrm{C}(9 \mathrm{~B})$ & $-153.7(7)$ \\
\hline $\mathrm{C}(6 \mathrm{~B})-\mathrm{C}(5 \mathrm{~B})-\mathrm{C}(8 \mathrm{~B})-\mathrm{C}(9 \mathrm{~B})$ & $27.9(11)$ \\
\hline $\mathrm{C}(18 \mathrm{~B})-\mathrm{P}(1 \mathrm{~B})-\mathrm{C}(10 \mathrm{~B})-\mathrm{C}(11 \mathrm{~B})$ & $160.15(16)$ \\
\hline $\mathrm{C}(14 \mathrm{~B})-\mathrm{P}(1 \mathrm{~B})-\mathrm{C}(10 \mathrm{~B})-\mathrm{C}(11 \mathrm{~B})$ & $39.64(19)$ \\
\hline $\mathrm{C}(1 \mathrm{C})-\mathrm{P}(1 \mathrm{~B})-\mathrm{C}(10 \mathrm{~B})-\mathrm{C}(11 \mathrm{~B})$ & $-80.89(17)$ \\
\hline
\end{tabular}




\begin{tabular}{lc}
$\mathrm{C}(1 \mathrm{~B})-\mathrm{P}(1 \mathrm{~B})-\mathrm{C}(10 \mathrm{~B})-\mathrm{C}(11 \mathrm{~B})$ & $-80.89(17)$ \\
$\mathrm{P}(1 \mathrm{~B})-\mathrm{C}(10 \mathrm{~B})-\mathrm{C}(11 \mathrm{~B})-\mathrm{C}(12 \mathrm{~B})$ & $168.74(19)$ \\
$\mathrm{C}(10 \mathrm{~B})-\mathrm{C}(11 \mathrm{~B})-\mathrm{C}(12 \mathrm{~B})-\mathrm{C}(13 \mathrm{~B})$ & $-176.8(3)$ \\
$\mathrm{C}(18 \mathrm{~B})-\mathrm{P}(1 \mathrm{~B})-\mathrm{C}(14 \mathrm{~B})-\mathrm{C}(15 \mathrm{~B})$ & $-52.26(18)$ \\
$\mathrm{C}(10 \mathrm{~B})-\mathrm{P}(1 \mathrm{~B})-\mathrm{C}(14 \mathrm{~B})-\mathrm{C}(15 \mathrm{~B})$ & $67.70(18)$ \\
$\mathrm{C}(1 \mathrm{C})-\mathrm{P}(1 \mathrm{~B})-\mathrm{C}(14 \mathrm{~B})-\mathrm{C}(15 \mathrm{~B})$ & $-170.16(15)$ \\
$\mathrm{C}(1 \mathrm{~B})-\mathrm{P}(1 \mathrm{~B})-\mathrm{C}(14 \mathrm{~B})-\mathrm{C}(15 \mathrm{~B})$ & $-170.16(15)$ \\
$\mathrm{P}(1 \mathrm{~B})-\mathrm{C}(14 \mathrm{~B})-\mathrm{C}(15 \mathrm{~B})-\mathrm{C}(16 \mathrm{~B})$ & $-168.91(16)$ \\
$\mathrm{C}(14 \mathrm{~B})-\mathrm{C}(15 \mathrm{~B})-\mathrm{C}(16 \mathrm{~B})-\mathrm{C}(17 \mathrm{~B})$ & $76.0(2)$ \\
$\mathrm{C}(10 \mathrm{~B})-\mathrm{P}(1 \mathrm{~B})-\mathrm{C}(18 \mathrm{~B})-\mathrm{C}(19 \mathrm{C})$ & $-176.59(15)$ \\
$\mathrm{C}(14 \mathrm{~B})-\mathrm{P}(1 \mathrm{~B})-\mathrm{C}(18 \mathrm{~B})-\mathrm{C}(19 \mathrm{C})$ & $-56.14(17)$ \\
$\mathrm{C}(1 \mathrm{~B})-\mathrm{P}(1 \mathrm{~B})-\mathrm{C}(18 \mathrm{~B})-\mathrm{C}(19 \mathrm{C})$ & $62.27(17)$ \\
$\mathrm{C}(10 \mathrm{~B})-\mathrm{P}(1 \mathrm{~B})-\mathrm{C}(18 \mathrm{~B})-\mathrm{C}(19 \mathrm{~B})$ & $-176.59(15)$ \\
$\mathrm{C}(14 \mathrm{~B})-\mathrm{P}(1 \mathrm{~B})-\mathrm{C}(18 \mathrm{~B})-\mathrm{C}(19 \mathrm{~B})$ & $-56.14(17)$ \\
$\mathrm{C}(1 \mathrm{C})-\mathrm{P}(1 \mathrm{~B})-\mathrm{C}(18 \mathrm{~B})-\mathrm{C}(19 \mathrm{~B})$ & $62.27(17)$ \\
$\mathrm{P}(1 \mathrm{~B})-\mathrm{C}(18 \mathrm{~B})-\mathrm{C}(19 \mathrm{~B})-\mathrm{C}(20 \mathrm{~B})$ & $-99.2(3)$ \\
$\mathrm{C}(18 \mathrm{~B})-\mathrm{C}(19 \mathrm{~B})-\mathrm{C}(20 \mathrm{~B})-\mathrm{C}(21 \mathrm{~B})$ & $-178.5(4)$ \\
$\mathrm{P}(1 \mathrm{~B})-\mathrm{C}(18 \mathrm{~B})-\mathrm{C}(19 \mathrm{C})-\mathrm{C}(20 \mathrm{C})$ & $-134.1(8)$ \\
$\mathrm{C}(18 \mathrm{~B})-\mathrm{C}(19 \mathrm{C})-\mathrm{C}(20 \mathrm{C})-\mathrm{C}(21 \mathrm{C})$ & $175.4(8)$ \\
& \\
\hline
\end{tabular}




\section{REFERENCES}

(1) Wessner, D. R.; Dupont, C.; Charles, T. C. Microbiology. In Microbiology; Wiley, 2013; p 56.

(2) Flemming, H. C.; Wingender, J. The biofilm matrix. Nat. Rev. Microbiol. 2010, 8, 623-633.

(3) Bryers, J. D. Medical biofilms. Biotechnol. Bioeng. 2008, 100, 1-18.

(4) Percival, S. L.; Suleman, L.; Donelli, G. Healthcare-Associated infections, medical devices and biofilms: Risk, tolerance and control. J. Med. Microbiol. 2015, 64, 323-334.

(5) Zoutman, D. E.; Ford, B. D.; Bryce, E.; Gourdeau, M.; Hébert, G.; Henderson, E.; Paton, S. The state of infection surveillance and control in Canadian acute care hospitals. Am. J. Infect. Control 2003, 31, 266-273.

(6) Mauldin, P. D.; Salgado, C. D.; Hansen, I. S.; Durup, D. T.; Bosso, J. A. Attributable hospital cost and length of stay associated with health care-associated infections caused by antibiotic-resistant gram-negative bacteria. Antimicrob. Agents Chemother. 2010, 54, 109115.

(7) Ventola, C. L. The Antibiotic Resistance Crisis. Pharm. Ther. 2015, 40, 278-283.

(8) Michael, C. A.; Dominey-Howes, D.; Labbate, M. The Antimicrobial Resistance Crisis: Causes, Consequences, and Management. Front. Public Heal. 2014, 2, 1-8.

(9) O’Neil, J. Antimicrobial Resistance: Tackling a Crisis for the Health and Wealth of Nations; 2014.

(10) Cornell, R. J.; Donaruma, L. G. 2-Methacryloxytropones. Intermediates for the Synthesis of Biologically Active Polymers. J. Med. Chem. 1965, 8, 388-390.

(11) Fu, E.; McCue, K.; Boesenberg, D. Chemical Disinfection of Hard Surfaces - Household, Industrial and Institutional Settings. In Handbook for Cleaning/Decontamination of 
Surfaces; Johansson, I., Somasundaran, P., Eds.; 2007; Vol. 1, pp 573-592.

(12) Ilker, M. F.; Nüsslein, K.; Tew, G. N.; Coughlin, E. B. Tuning the hemolytic and antibacterial activities of amphiphilic polynorbornene derivatives. J. Am. Chem. Soc. 2004, $126,15870-15875$.

(13) Kanazawa, A.; Ikeda, T.; Endo, T. Novel polycationic biocides: Synthesis and antibacterial activity of polymeric phosphonium salts. J. Polym. Sci. Part A Polym. Chem. 1993, 31, 335343.

(14) Xue, Y.; Xiao, H.; Zhang, Y. Antimicrobial polymeric materials with quaternary ammonium and phosphonium salts. Int. J. Mol. Sci. 2015, 16, 3626-3655.

(15) Carmona-Ribeiro, A. M.; de Melo Carrasco, L. D. Cationic antimicrobial polymers and their assemblies. Int. J. Mol. Sci. 2013, 14, 9906-9946.

(16) Jaeger, W.; Bohrisch, J.; Laschewsky, A. Synthetic polymers with quaternary nitrogen atoms-Synthesis and structure of the most used type of cationic polyelectrolytes. Prog. Polym. Sci. 2010, 35, 511-577.

(17) Zubris, D. L.; Minbiole, K. P. C.; Wuest, W. M. Polymeric Quaternary Ammonium Compounds: Versatile Antimicrobial Materials. Curr. Top. Med. Chem. 2017, 17, 305-318.

(18) Timofeeva, L.; Kleshcheva, N. Antimicrobial polymers: Mechanism of action, factors of activity, and applications. Appl. Microbiol. Biotechnol. 2011, 89, 475-492.

(19) Li, Y.; Kumar, K. N.; Dabkowski, J. M.; Corrigan, M.; Scott, R. W.; Nüsslein, K.; Tew, G. N. New bactericidal surgical suture coating. Langmuir 2012, 28, 12134-12139.

(20) Brogden, N. K.; Brogden, K. A. Will new generations of modified antimicrobial peptides improve their potential as pharmaceuticals? Int. J. Antimicrob. Agents 2011, 38, 217-225.

(21) Takahashi, H.; Caputo, G. A.; Vemparala, S.; Kuroda, K. Synthetic Random Copolymers 
as a Molecular Platform to Mimic Host-Defense Antimicrobial Peptides. Bioconjug. Chem. 2017, 28, 1340-1350.

(22) Guo, J.; Qin, J.; Ren, Y.; Wang, B.; Cui, H.; Ding, Y.; Mao, H.; Yan, F. Antibacterial activity of cationic polymers: Side-chain or main-chain type? Polym. Chem. 2018, 9, 46114616.

(23) Al-Badri, Z. M.; Som, A.; Lyon, S.; Nelson, C. F.; Nüsslein, K.; Tew, G. N. Investigating the effect of increasing charge density on the hemolytic activity of synthetic antimicrobial polymers. Biomacromolecules 2008, 9, 2805-2810.

(24) Lienkamp, K.; Madkour, A. E.; Musante, A.; Nelson, C. F.; Nüsslein, K.; Tew, G. N. Antimicrobial polymers prepared by ROMP with unprecedented selectivity: A molecular construction kit approach. J. Am. Chem. Soc. 2008, 130, 9836-9843.

(25) Colak, S.; Nelson, C. F.; Nusslein, K.; Tew, G. N. Hydrophilic modifications of an amphiphilic polynorbornene and the effects on its hemolytic and antibacterial activity. Biomacromolecules 2009, 10, 353-359.

(26) Solà, M.; Lledós, A.; Duran, M.; Bertrán, J.; Abboud, J. L. M. Analysis of Solvent Effects on the Menshutkin Reaction. J. Am. Chem. Soc. 1991, 113, 2873-2879.

(27) Siedenbiedel, F.; Tiller, J. C. Antimicrobial polymers in solution and on surfaces: Overview and functional principles. Polymers (Basel). 2012, 4, 46-71.

(28) Gilbert, P.; Moore, L. E. Cationic antiseptics: Diversity of action under a common epithet. J. Appl. Microbiol. 2005, 99, 703-715.

(29) Hadjesfandiari, N.; Yu, K.; Mei, Y.; Kizhakkedathu, J. N. Polymer brush-based approaches for the development of infection-resistant surfaces. J. Mater. Chem. B 2014, 2, 4968-4978.

(30) Tiller, J. C. Coatings for Prevention or Deactivation of Biological Contamination. In 
Developments in Surface Contamination and Cleaning; 2008; pp 1013-1065.

(31) Andresen, J. A.; Muir, D.; Ueno, D.; Darling, C.; Theobald, N.; Bester, K. Emerging pollutants in the north sea in comparison to Lake Ontario, Canada, data. Environ. Toxicol. Chem. 2007, 26, 1081-1089.

(32) Romao, C.; Miranda, C. A.; Silva, J.; Clementino, M. M.; de Filippis, I.; Asensi, M. Presence of qacE $\Delta 1$ Gene and Susceptibility to a Hospital Biocide in Clinical Isolates of Pseudomonas aeruginosa Resistant to Antibiotics. Curr. Microbiol. 2011, 63, 16-21.

Buffet-Bataillon, S.; Branger, B.; Cormier, M.; Bonnaure-Mallet, M.; Jolivet-Gougeon, A. Effect of higher minimum inhibitory concentrations of quaternary ammonium compounds in clinical E. coli isolates on antibiotic susceptibilities and clinical outcomes. J. Hosp. Infect. 2011, 79, 141-146.

(34) Taylor, N. G. H.; Verner-Jeffreys, D. W.; Baker-Austin, C. Aquatic systems: Maintaining, mixing and mobilising antimicrobial resistance? Trends Ecol. Evol. 2011, 26, 278-284.

(35) Isquith, A. J.; Abbott, E. A.; Walters, P. A. Surface-bonded antimicrobial activity of an organosilicon quaternary ammonium chloride. Appl. Microbiol. 1972, 24, 859-863.

(36) Porosa, L. M.; Mistry, K. B.; Mocella, A.; Deng, H.; Hamzehi, S.; Caschera, A.; Lough, A. J.; Wolfaardt, G.; Foucher, D. A. Synthesis, structures and properties of self-assembling quaternary ammonium dansyl fluorescent tags for porous and non-porous surfaces. $J$. Mater. Chem. B 2014, 2, 1509-1520.

(37) Appendini, P.; Hotchkiss, J. H. Review of antimicrobial food packaging. Innov. Food Sci. Emerg. Technol. 2002, 3, 113-126.

(38) Gottenbos, B.; Van Der Mei, H. C.; Klatter, F.; Nieuwenhuis, P.; Busscher, H. J. In vitro and in vivo antimicrobial activity of covalently coupled quaternary ammonium silane 
coatings on silicone rubber. Biomaterials 2002, 23, 1417-1423.

(39) Körner, M.; Prucker, O.; Rühe, J. Kinetics of the Generation of Surface-Attached Polymer Networks through C, H-Insertion Reactions. Macromolecules 2016, 49, 2438-2447.

(40) Ma, H.; Davis, R. H.; Bowman, C. N. A Novel Sequential Photoinduced Living Graft Polymerization. Macromolecules 2000, 33, 331-335.

(41) Edmondson, S.; Osborne, V. L.; Huck, W. T. S. Polymer brushes via surface-initiated polymerizations. Chem. Soc. Rev. 2004, 33, 14-22.

(42) Nesvadba, P. Radical Polymerization in Industry. In Encyclopedia of Radicals in Chemistry, Biology and Materials; Chatgilialoglu, C., Studer, A., Eds.; John Wiley \& Sons , Ltd., 2012; pp 1701-1736.

(43) Matyjaszewski, K.; Spanswick, J. Controlled/living radical polymerization. Mater. Today 2005, 8, 26-33.

(44) Otsu, T.; Yoshida, M. Role of initiator-transfer agent-terminator (iniferter) in radical polymerizations: Polymer design by organic disulfides as iniferters. Die Makromol. Chemie, Rapid Commun. 1982, 3, 127-132.

(45) Georges, M. K.; Veregin, R. P. N.; Kazmaier, P. M.; Hamer, G. K. Narrow Molecular Weight Resins by a Free-Radical Polymerization Process. Macromolecules 1993, 26, $2987-$ 2988.

(46) Hawker, C. J. Nitroxide Mediated Living Radical Polymerization. In Handbook of Radical Polymerization; Matyjaszewski, K., David, T. P., Eds.; John Wiley \& Sons, Inc., 2002; pp $463-522$.

(47) Kato, M.; Kamigaito, M.; Sawamoto, M.; Higashimura, T. Polymerization of Methyl Methacrylate with the Carbon Tetrachloride/Dichlorotris- 
(triphenylphosphine)ruthenium(II)/ Methylaluminum Bis(2,6-di-tert-butylphenoxide) Initiating System: Possibility of Living Radical Polymerization. Macromolecules 1995, 28, $1721-1723$.

(48) Wang, J. S.; Matyjaszewski, K. Controlled/“Living” Radical Polymerization. Halogen Atom Transfer Radical Polymerization Promoted by a $\mathrm{Cu}(\mathrm{I}) / \mathrm{Cu}(\mathrm{II})$ Redox Process. Macromolecules 1995, 28, 7901-7910.

(49) Chiefari, J.; Chong, Y. K.; Ercole, F.; Krstina, J.; Jeffery, J.; Le, T. P. T.; Mayadunne, R. T. A.; Meijs, G. F.; Moad, C. L.; Moad, G.; et al. Living free-radical polymerization by reversible addition - Fragmentation chain transfer: The RAFT process. Macromolecules 1998, 31, 5559-5562.

(50) Le, T. P.; Moad, G.; Rizzardo, E.; Thang, S. H. Polymerization with Living Characteristics. WO1998001478, Jan. 15, 1998.

(51) Corpart, P.; Charmot, D.; Zard, S. Z.; Biadatti, T.; Michelet, D. Method for Block Polymer Synthesis by Controlled Radical Polymerisation. U.S. Patent 6,153,705, Nov. 28, 2000., 1998.

(52) Willcock, H.; O'Reilly, R. K. End group removal and modification of RAFT polymers. Polym. Chem. 2010, 1, 149-157.

(53) Porosa, L.; Caschera, A.; Bedard, J.; Mocella, A.; Ronan, E.; Lough, A. J.; Wolfaardt, G.; Foucher, D. A. UV-Curable Contact Active Benzophenone Terminated Quaternary Ammonium Antimicrobials for Applications in Polymer Plastics and Related Devices. ACS Appl. Mater. Interfaces 2017, 9, 27491-27503.

(54) Thom, K. A.; Standiford, H. C.; Johnson, J. K.; Hanna, N.; Furuno, J. P. Effectiveness of an Antimicrobial Polymer to Decrease Contamination of Environmental Surfaces in the 
Clinical Setting. Infect. Control Hosp. Epidemiol. 2014, 35, 1060-1062.

(55) ASTM E2149-13a Standard Test Method for Determining the Antimicrobial Activity of Antimicrobial Agents Under Dynamic Contact Conditions, 2013.

(56) ISO 22196:2011 Measurement of Antibacterial Activity on the Plastics and Other NonPorous Surfaces, 2011.

(57) Eames, I.; Tang, J. W.; Li, Y.; Wilson, P. Airborne transmission of disease in hospitals. J. R. Soc. Interface 2009, 6, 697-702.

(58) Campos, M. D.; Zucchi, P. C.; Phung, A.; Leonard, S. N.; Hirsch, E. B. The activity of antimicrobial surfaces varies by testing protocol utilized. PLoS One 2016, 11, 1-11.

(59) Ronan, E.; Yeung, C. W.; Hausner, M.; Wolfaardt, G. M. Interspecies interaction extends bacterial survival at solid - air interfaces. Biofouling 2013, 29, 1087-1096.

(60) Lee, S. D.; Ryan, S. P.; Snyder, E. G. Development of an aerosol surface inoculation method for Bacillus spores. Appl. Environ. Microbiol. 2011, 77, 1638-1645.

(61) Milović, N. M.; Wang, J.; Lewis, K.; Klibanov, A. M. Immobilized N-alkylated polyethylenimine avidly kills bacteria by rupturing cell membranes with no resistance developed. Biotechnol. Bioeng. 2005, 90, 715-722.

(62) Zou, P.; Laird, D.; Riga, E. K.; Deng, Z.; Dorner, F.; Perez-Hernandez, H.-R.; GuevaraSolarte, D. L.; Steinberg, T.; Al-Ahmad, A.; Lienkamp, K. Antimicrobial and cellcompatible surface-attached polymer networks - how the correlation of chemical structure to physical and biological data leads to a modified mechanism of action. J. Mater. Chem. B $\mathbf{2 0 1 5}, 3,6224-6238$.

(63) Murata, H.; Koepsel, R. R.; Matyjaszewski, K.; Russell, A. J. Permanent, non-leaching antibacterial surfaces-2: How high density cationic surfaces kill bacterial cells. 
Biomaterials 2007, 28, 4870-4879.

(64) Tiller, J. C.; Liao, C. J.; Lewis, K.; Klibanov, A. M. Designing surfaces that kill bacteria on contact. Proc. Natl. Acad. Sci. U. S. A. 2001, 98, 5981-5985.

(65) Hasan, J.; Jain, S.; Padmarajan, R.; Purighalla, S.; Sambandamurthy, V. K.; Chatterjee, K. Multi-scale surface topography to minimize adherence and viability of nosocomial drugresistant bacteria. Mater. Des. 2018, 140, 332-344.

(66) Bouloussa, O.; Rondelez, F.; Semetey, V. A new, simple approach to confer permanent antimicrobial properties to hydroxylated surfaces by surface functionalization. Chem. Commun. 2008, 951-953.

(67) Bieser, A. M.; Tiller, J. C. Mechanistic Considerations on Contact-Active Antimicrobial Surfaces with Controlled Functional Group Densities. Macromol. Biosci. 2011, 11, 526534.

(68) Li, P.; Poon, Y. F.; Li, W.; Zhu, H.-Y.; Yeap, S. H.; Cao, Y.; Qi, X.; Zhou, C.; Lamrani, M.; Beuerman, R. W.; et al. A polycationic antimicrobial and biocompatible hydrogel with microbe membrane suctioning ability. Nat. Mater. 2011, 10, 149-156.

(69) Gao, J.; White, E. M.; Liu, Q.; Locklin, J. Evidence for the Phospholipid Sponge Effect as the Biocidal Mechanism in Surface-Bound Polyquaternary Ammonium Coatings with Variable Cross-Linking Density. ACS Appl. Mater. Interfaces 2017, 9, 7745-7751.

(70) Gottenbos, B.; Van Der Mei, H. C.; Klatter, F.; Nieuwenhuis, P.; Busscher, H. J. In vitro and in vivo antimicrobial activity of covalently coupled quaternary ammonium silane coatings on silicone rubber. Biomaterials 2002, 23, 1417-1423.

(71) Porosa, L.; Wolfaardt, G.; Foucher, D. Phosphorus Functional Antimicrobial Coatings for Metal Surfaces. U.S. Patent 15,235,240, Mar. 10, 2016. 
(72) Pasquier, N.; Keul, H.; Heine, E.; Moeller, M. From multifunctionalized poly(ethylene imine)s toward antimicrobial coatings. Biomacromolecules 2007, 8, 2874-2882.

(73) Yang, W. J.; Pranantyo, D.; Neoh, K. G.; Kang, E. T.; Teo, S. L. M.; Rittschof, D. Layerby-layer click deposition of functional polymer coatings for combating marine biofouling. Biomacromolecules 2012, 13, 2769-2780.

(74) Huang, J.; Murata, H.; Koepsel, R. R.; Russell, A. J.; Matyjaszewski, K. Antibacterial polypropylene via surface-initiated atom transfer radical polymerization. Biomacromolecules 2007, 8, 1396-1399.

(75) Kyomoto, M.; Moro, T.; Takatori, Y.; Kawaguchi, H.; Nakamura, K.; Ishihara, K. Selfinitiated surface grafting with poly(2-methacryloyloxyethyl phosphorylcholine) on poly(ether-ether-ketone). Biomaterials 2010, 31, 1017-1024.

(76) Gao, J.; Huddleston, N. E.; White, E. M.; Pant, J.; Handa, H.; Locklin, J. Surface Grafted Antimicrobial Polymer Networks with High Abrasion Resistance. ACS Biomater. Sci. Eng. 2016, 2, 1169-1179.

(77) Dhende, V. P.; Samanta, S.; Jones, D. M.; Hardin, I. R.; Locklin, J. One-step photochemical synthesis of permanent, nonleaching, ultrathin antimicrobial coatings for textiles and plastics. ACS Appl. Mater. Interfaces 2011, 3, 2830-2837.

(78) Locklin, J. J.; Dhende, V. Photochemical Cross-Linkable Polymers, Methods of Making Photochemical Cross-Linkable Polymers, Methods of Using Photochemical Cross-Linkable Polymers, and Methods of Making Articles Containing Photochemical Cross-Linkable Polymers. U.S. Patent 15,417,488, May 18, 2017.

(79) Caschera, A.; Mistry, K. B.; Bedard, J.; Ronan, E.; Syed, M. A.; Khan, A. U.; Lough, A. J.; Wolfaardt, G.; Foucher, D. A. Surface-attached sulfonamide containing quaternary 
ammonium antimicrobials for textiles and plastics. $R S C A d v \mathbf{2 0 1 9}, 9,3140-3150$.

(80) Kanazawa, A.; Ikeda, T.; Endo, T. Polymeric phosphonium salts as a novel class of cationic biocides. VII. Synthesis and antibacterial activity of polymeric phosphonium salts and their model compounds containing long alkyl chains. J. Appl. Polym. Sci. 1994, 53, 1237-1244.

(81) Kanazawa, A.; Ikeda, T.; Endo, T. Polymeric phosphonium salts as a novel class of cationic biocides. III. Immobilization of phosphonium salts by surface photografting and antibacterial activity of the surface-treated polymer films. J. Polym. Sci. Part A Polym. Chem. 1993, 31, 1467-1472.

(82) Cuthbert, T. J.; Harrison, T. D.; Ragogna, P. J.; Gillies, E. R. Synthesis, properties, and antibacterial activity of polyphosphonium semi-interpenetrating networks. J. Mater. Chem. B 2016, 4, 4872-4883.

(83) Kazmaier, P.; Daimon, K.; Georges, M.; Hamer, G.; Veregin, R. Nitroxide-Mediated "Living": Free Radical Polymerization:\&amp;nbsp; A Rapid Polymerization of (Chloromethyl)styrene for the Preparation of Random, Block, and Segmental Arborescent Polymers. Macromolecules 1997, 30, 2228-2231.

(84) Lin, H.; Wang, Y.; Gan, Y.; Hou, H.; Yin, J.; Jiang, X. Simultaneous Formation of a SelfWrinkled Surface and Silver Nanoparticles on a Functional Photocuring Coating. Langmuir 2015, 31, 11800-11808.

(85) Ikemura, K.; Ichizawa, K.; Yoshida, M.; Ito, S.; Endo, T. UV-VIS spectra and photoinitiation behaviors of acylphosphine oxide and bisacylphosphine oxide derivatives in unfilled, light-cured dental resins. Dent. Mater. J. 2009, 27, 765-774.

(86) ASTM D5402-15 Standard Practice for Assessing the Solvent Resistance of Organic Coatings Using Solvent Rubs, 2015. 
(87) ASTM D3359-17 Standard Test Methods for Rating Adhesion by Tape Test, 2017.

(88) JIS Z 2801 Test for Antimicrobial Activity of Plastics, 2010.

(89) Grainger, D. W.; Stewart, C. W. Fluorinated Coatings and Films: Motivation and Significance. In Fluorinated Surfaces, Coatings, and Films; Castner, D. G., Grainger, D. W., Eds.; American Chemical Society, 2001; pp 1-14.

(90) Truong, V. K.; Lapovok, R.; Estrin, Y. S.; Rundell, S.; Wang, J. Y.; Fluke, C. J.; Crawford, R. J.; Ivanova, E. P. Biomaterials The influence of nano-scale surface roughness on bacterial adhesion to ultrafine-grained titanium. Biomaterials 2010, 31, 3674-3683.

(91) Ivanova, E. P.; Truong, V. K.; Wang, J. Y.; Bemdt, C. C.; Jones, R. T.; Yusuf, I. I.; Peake, I.; Schmidt, H. W.; Fluke, C.; Barnes, D.; et al. Impact of nanoscale roughness of titanium thin film surfaces on bacterial Retention. Langmuir 2010, 26, 1973-1982.

(92) Crawford, R. J.; Webb, H. K.; Truong, V. K.; Hasan, J.; Ivanova, E. P. Surface topographical factors in fl uencing bacterial attachment. Adv. Colloid Interface Sci. 2012, $179-182,142-149$.

(93) Kaur, G.; Chang, S. L. Y.; Bell, T. D. M.; Hearn, M. T. W.; Saito, K. Bioinspired corecrosslinked micelles from thymine-functionalized amphiphilic block copolymers: Hydrogen bonding and photo-crosslinking study. J. Polym. Sci. Part A Polym. Chem. 2011, $49,4121-4128$.

(94) Reinheimer, J. D.; Harley, J. D.; Meers, W. W. Solvent Effects in the Menschutkin Reaction. J. Org. Chem. 1963, 28, 1575-1579.

(95) Allen, F. H.; Kennard, O.; Watson, D. G.; Brammer, L.; Orpen, A. G. Tables of Bond Lengths determined by X-Ray and Neutron Diffraction. Part 1. Bond Lengths in Organic Compounds. J. Chem. Soc. Perkin Trans. 2 1987, S1-S19. 
(96) Zhang, Y.; Maginn, E. J. Molecular dynamics study of the effect of alkyl chain length on melting points of [CnMIM][PF6] ionic liquids. Physcial Chem. Chem. Phys. 2014, 16, 13489-13499.

(97) Hatch, M. J.; Meyer, F. J.; Lloyd, W. D. Sulfonium polymers derived from Ar-vinylbenzyl chloride. I. Exploratory study of the preparation and properties of the monomers and polymers. J. Appl. Polym. Sci. 1969, 13, 721-744.

(98) Ray, F. E.; Levine, I. A mechanism for the reaction of organic halides with sulfides. Sulfonium compounds I. J. Org. Chem. 1937, 2, 267-275.

(99) Stirling, C. J. M. Sulfonium Salts. In Organic Chemistry of Sulfur; Oae, S., Ed.; Plenum Press: New York, 1977; pp 473-526.

(100) Su, Y.; Dan, M.; Xiao, X.; Wang, X.; Zhang, W. A New Thermo-responsive Block Copolymer with Tunable Upper Critical Solution Temperature and Lower Critical Solution Temperature in the Alcohol / Water Mixture. J. Polym. Sicience, Part A Polym. Chem. 2013, $51,4399-4412$.

(101) Balinge, K. R.; Datir, S. K.; Khajone, V. B.; Bhansali, K. J.; Khiratkar, A. G.; Bhagat, P. R. Iron(III)-salen complex on a polymer scaffold as heterogeneous catalyst for synthesis of benzimidazoles. Res. Chem. Intermed. 2019, 45, 155-168.

(102) Saettone, M. F.; Alderigi, C.; Giannaccini, B.; Anselmi, C.; Rossetti, M. G.; Scotton, M.; Cerini, R. Substantivity of sunscreens-preparation and evaluation of some quaternary ammonium benzophenone derivatives. Int. J. Cosmet. Sci. 1988, 10, 99-109.

(103) Zuo, Y.; Yu, J.; Liu, X.; Cao, P.; Song, P.; Wang, R.; Xiong, Y. Poly(ionic liquid)-based nanogels and their reversible photo-mediated association and dissociation. Polym. Chem. 2017, 8, 1146-1154. 
(104) Liu, Y.; Xiao, C.; Li, X.; Li, L.; Ren, X.; Liang, J.; Huang, T. S. Antibacterial efficacy of functionalized silk fabrics by radical copolymerization with quaternary ammonium salts. $J$. Appl. Polym. Sci. 2016, 133, 43450-43455.

(105) Hemp, S. T.; Zhang, M.; Allen, M. H.; Cheng, S.; Moore, R. B.; Long, T. E. Comparing ammonium and phosphonium polymerized ionic liquids: Thermal analysis, conductivity, and morphology. Macromol. Chem. Phys. 2013, 214, 2099-2107.

(106) Béland, V. A.; Ross, M. A. S.; Coady, M. J.; Guterman, R.; Ragogna, P. J. Patterned Phosphonium-Functionalized Photopolymer Networks as Ceramic Precursors. Chem. Mater. 2017, 29, 8884-8891.

(107) van de Lagemaat, M.; Grotenhuis, A.; van de Belt-Gritter, B.; Roest, S.; Loontjens, T. J. A.; Busscher, H. J.; van der Mei, H. C.; Ren, Y. Comparison of methods to evaluate bacterial contact-killing materials. Acta Biomater. 2017, 59, 139-147. 